\title{
GULF OF MEXICO SEAFLOOR STABILITY AND GAS HYDRATE MONITORING STATION PROJECT
}

FINAL TECHNICAL PROGRESS REPORT

FOR THE 12TH QUARTER

1 October 2003 through 31 March 2004

PREPARED BY THE MANAGEMENT TEAM

THE CENTER FOR MARINE RESOURCES \& ENVIRONMENTAL TECHNOLOGY

NOVEMBER 2004

COOPERATIVE AGREEMENT NUMBER:

DE-FC26-00NT40920

Prepared by

The Center for Marine Resources and Environmental Technology of

The University of Mississippi

This report was prepared with the support of the United States Department of Energy, under award No. DE-FC26-00NT40920. However, any opinions, findings, conclusions, or recommendations expressed herein are those of the authors and do not necessarily reflect the views of the DOE. DOE Award Number DE-FC26-00NT40920 is managed by the U.S. Department of Energy's National Energy Technology Laboratory. 
Subcontractors:

J. Robert Woolsey, Thomas M. McGee, Robin C. Buchannon, 220 Old

Chemistry, University of Mississippi, University, MS 38677

Task 1: Program Development

Harry Roberts, Coastal Studies Institute, Louisiana State University, 331 Old Geology Complex, Baton Rouge, LA, 70803

Task 2: Site Selection

Roger Sassen, Geochemical and Environmental Research Group, College of Geosciences, Texas A\&M University, 833 Graham Road, College Station, TX 77845

Task 3: Site Selection and the Characterization of Vent Gas, Gas Hydrate and Associated Sediments

James M. Brooks, TDI-Brooks International, 1902 Pinon Drive, College Station, TX 77845 and Ian MacDonald and Bernie Bernard, Geochemical and Environmental Research Group, College of Geosciences, Texas A\&M University, 833 Graham Road, College Station, TX 77845

Task 4: Heat Flow and Temperature Studies at "Bush Hill" Other and Other Hydrate Sites Using the Submersible Johnson Sea Link

Rudy Rogers, Department of Chemical Engineering, Mississippi State University, PO Drawer 9595, Mississippi State, MS 39762

Task 5: Gas Hydrate Formation and Decomposition in Ocean Sediments

Jean Whelan, Department of Marine Chemistry and Geochemistry, Fye Building MS \#4, Woods Hole Oceanographic Institution, 360 Woods Hole Road, Woods Hole, MA 02543

Task 6: Gas Hydrates - Organic Geochemistry

Jeffrey P. Chanton, Department of Oceanography, Florida State University, Tallahassee, FL 32306 and Christopher S. Martens, Department of Marine Sciences, University of North Carolina-Chapel Hill, Chapel Hill, NC 27599 Task 7: Monitoring Biogeochemical Processes Controlling Gas Hydrate

Formation, Deposition and Composition in the Gulf of Mexico

Marc Slattery, National Center for Natural Products Research, University of Mississippi, University, MS 38677

Task 8: Deep-Sea Microbes as sources of Biomedically Important Natural Products

Douglass Bartlett and Miriam Kastner, Scripps Institution of Oceanography, University of California-San Diego, La Jolla, CA 92093

Task 9: Scripps Institution of Oceanography Microbiology and Geochemistry

Joel S. Kuszmaul, Department of Geology and Geological Engineering, 118-A Carrier Hall, University of Mississippi, University, MS

Task 10: Establishing the Parameters Required for Spatial Models of Gas Hydrate Collections 


\section{DISCLAIMER}

This report was prepared as an account of work sponsored by an agency of the United States Government. Neither the United States Government nor any agency thereof, nor any of their employees, makes any warranty, express or implied, or assumes any legal liability or responsibility for the accuracy, completeness, or usefulness of any information, apparatus, product, or process disclosed, or represents that its use would not infringe privately owned rights. Reference herein to any specific commercial product, process, or service by trade name, trademark, manufacturer, or otherwise does not necessarily constitute or imply its endorsement, recommendation, or favoring by the United States Government or any agency thereof. The views and opinions of authors expressed herein do not necessarily state or reflect those of the United States Government or any agency thereof. 


\section{ABSTRACT}

The gas hydrates research Consortium (HRC), established and administered at the University if Mississippi's Center for Marine Research and Environmental Technology (CMRET) has been active on many fronts in FY 03. Extension of the original contract through March 2004, has allowed completion of many projects that were incomplete at the end of the original project period due, primarily, to severe weather and difficulties in rescheduling test cruises. The primary objective of the Consortium, to design and emplace a remote sea floor station for the monitoring of gas hydrates in the Gulf of Mexico by the year 2005 remains intact. However, the possibility of levering HRC research off of the Joint Industries Program (JIP) became a possibility that has demanded reevaluation of some of the fundamental assumptions of the station format. These provisions are discussed in Appendix A.

Landmark achievements of FY03 include:

- Continuation of Consortium development with new researchers and additional areas of research contribution being incorporated into the project. During this period, NOAA's National Undersea Research Program's (NURP) National Institute for Undersea Science and Technology (NIUST) became a Consortium funding partner, joining DOE and Minerals Management Service (MMS),

- Very successful annual and semiannual meetings in Oxford Mississippi in February and September, 2003,

- Collection of piston cores from MC798 in support of the effort to evaluate the site for possible monitoring station installation,

- Completion of the site evaluation effort including reports of all localities in the northern Gulf of Mexico where hydrates have been documented or are strongly suspected to exist on the sea floor or in the shallow subsurface,

- Collection and preliminary evaluation of vent gases and core samples of hydrate from sites in Green Canyon and Mississippi Canyon, northern Gulf of Mexico,

- Monitoring of gas activity on the sea floor, acoustically and thermally,

- Design, construction, and successful deployment of an in situ pore-water sampling device,

- Improvements to the original Raman spectrometer (methane sensor),

- Laboratory demonstration of the impact of bacterially-produced surfactants' rates of hydrate formation,

- Construction and sea floor emplacement and testing - with both watergun and ship noise sources - of the prototypal vertical line array (VLA),

- Initiation of studies of spatial controls on hydrates,

- Compilation and analyses of seismic data, including mapping of surface anomalies,

- Additional field verification (bottom samples recovered), in support of the site selection effort,

- Collection and preliminary analyses of gas hydrates from new sites that exhibit variant structures,

- Initial shear wave tests carried out in shallow water,

- Isolation of microbes for potential medicinal products development,

- Preliminary modeling of occurrences of gas hydrates. 
TABLE OF CONTENTS

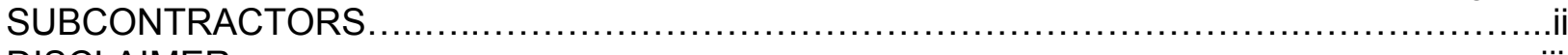

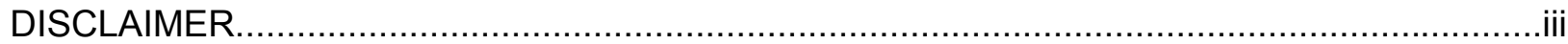

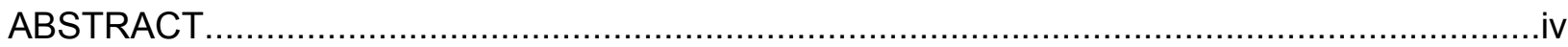

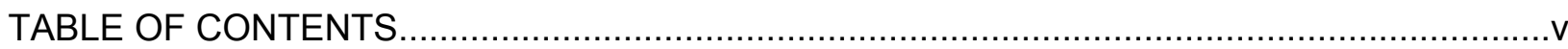

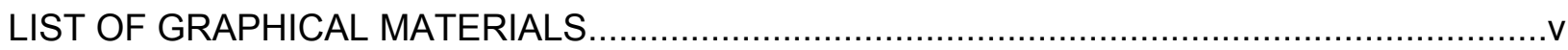

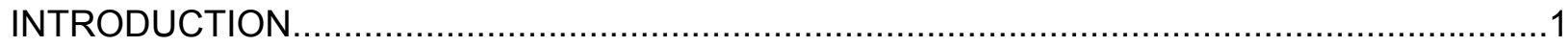

EXECUTIVE SUMMARY

EXPERIMENTAL

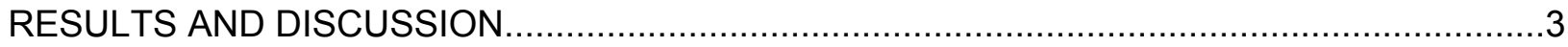

CONCLUSION

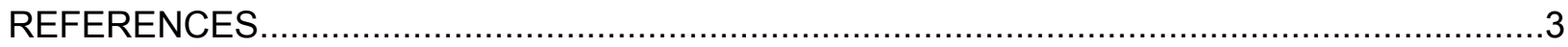

LIST OF ACRONYMS AND ABBREVIATIONS ..........................................................

APPENDIX: STATUS/PROGRESS REPORTS SUBMITTED BY THE SUBCONTRACTORS

Task 1: Program Development.............................................................

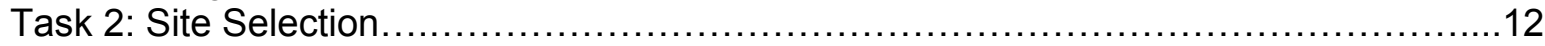

Task 3: Site Selection and Characterization of vent gas, gas hydrate and Associated Sediments ....................................................................... 12

Task 4: Heat Flow and Temperature Studies at Bush Hill and other Hydrate Sites using the Submersible, Johnson Sea Link ..............................................228

Task 5: Gas Hydrate Formation and Decomposition in Ocean Sediments...................265

Task 6: Gas Hydrates - Organic Geochemistry .........................................277

Task 7: Monitoring Biogeochemical Processes Controlling Gas Hydrate Formation, Deposition and Composition in the Gulf of Mexico.................................314

Task 8: Deep Sea Microbes as Sources of Biomedically-Important Natural Products....334

Task 9: Scripps Institute of Oceanography Microbiology and Geochemistry ................341

Task 10: Establishing the Parameters Required for Spatial Models of Gas Hydrate Collections

\section{LIST OF GRAPHICAL MATERIALS}

Figures appropriate to the individual subcontractor's research appear in those researchers' reports listed by task number. 


\section{INTRODUCTION}

Established in1999, the objective of the Gulf of Mexico gas hydrate monitoring station project is to emplace a remote, multi-sensor monitoring station at a selected location within the hydrate stability zone of the northern Gulf of Mexico. Plans for the station have produced subsets of research related to the establishment, emplacement and maintenance of the facility. These include physical, chemical, and biological components of the hydrocarbon system, location of the station, both geographically and within the water column, and means of accessing, assessing, and archiving the acquired data. Eventually this data base will be made available to groups investigating gas hydrate for energy resource potential and environmental impact.

Responsibility for oversight of each activity of the project is vested in a member of the project's Scientific Supervisory Board. The FY2002 board members were:

- Managing Director: Bob Woolsey, Center for Marine Resources and Environmental Technology, University of Mississippi, Oxford, MS.

- Geologic Setting: Harry Roberts, Coastal Studies Institute, Louisiana State University, Baton Rouge, LA.

- Vertical Line Arrays: Ross Chapman, School of Earth and Ocean Sciences, University of Victoria, British Columbia, Canada

- Water Currents: Vernon Asper, School of Marine Sciences, University of Southern Mississippi, Stennis Space Center, MS.

- Gas Bubble Studies: Ralph Goodman, School of Marine Sciences, University of Southern Mississippi, Stennis Space Center, MS.

- Geoelectric Systems: Rob Evans, Woods Hole Oceanographic Institution, Woods Hole, MA.

- Geochemistry: Roger Sassen, Geochemical and Environmental Research Group, Texas A\&M University, College Station, TX.

- Water Chemistry: Jean Whelan, Woods Hole Oceanographic Institute, Woods Hole, MA.

- Pore Water Chemistry: Jeff Chanton, Department of Oceanography, Florida State University, Tallahassee, FL.

- Laboratory Studies: Rudy Rogers, Chemical Engineering Department, Mississippi State University, Starkville, MS.

- Heat Flow Studies: Bernie Bernard, TDI-Brooks International Inc., College Station, TX.

- Pharmaceuticals: Marc Slattery, Pharmacognosy Department, University of Mississippi, Oxford, MS.

- Comparative Studies: Camelia Diaconescu, Department of Geological Sciences, University of South Carolina, Columbia, SC.

- Data Recovery: Paul Higley, Specialty Devices Inc. (SDI), Plano, TX.

- Site Surveys: Tom McGee, CMRET, University of Mississippi, Oxford, MS. 


\section{EXECUTIVE SUMMARY}

During FY 2003 all tasks described in the original contracts were either completed or funds were redirected to carry out research related to the eventual completion and installation of the monitoring station. Due to complications of funding and scheduling of research activities, the project work period was extended, mid-year, to March 31, 2003. Therefore, most component research activities had not been completed by the close fiscal year 2002. However, a number of significant achievements had been made and others were in progress at the close of the FY02 reporting period.

A large quantity of hydrocarbon fluids floats on the surface of the Gulf of Mexico. At certain locations, oil slicks are extensive enough to be seen from space. The origins of these slicks are natural seeps on the sea floor. Much of the oil rides up on gas bubbles, some of which may originate from the dissociation of natural gas hydrates. This is the basis of concern that drives a significant portion of the hydrate research being done in the Gulf. The Consortium continues to include studies of the properties of bubbles as priority investigation. While the study of the acoustical properties of bubbles, funded in FY01, is not a component of this report, the funding and the studies will resume in FY03. In addition, the Consortium is pursuing other possible bubble investigations, including the mechanical properties of bubbles, at great depth.

Hydrates have been observed and investigated directly by Consortium participants. Temperature probes were inserted directly into outcropping mounds on the sea floor of the Gulf of Mexico (GOM) as well as into nearby mud. Temperatures obtained from the hydrate mounds, the mud, and the water column directly above these sites will serve to advance current knowledge and thinking regarding thermal conductivity of hydrate deposits and their surroundings.

A device for sampling pore water in the upper meter of sea-floor sediment and returning the samples to the surface under in situ pressure was designed and built. This device was deployed, successfully, from the Johnson Sea Link. Initial results reveal the highest concentrations of dissolved hydrocarbons yet reported.

Design modifications to improve the resolution of the Raman spectrometer, built with DOE FY01 funding, were made. The device is intended to be mounted on a submersible vehicle so that it can be deployed to analyze hydrocarbons near sea-floor seeps. Outstanding obstacles include the need for this instrument to make measurements extremely rapidly so that variations can be detected over very small distances.

Laboratory studies of hydrate formation continue at Mississippi State University. This work has already demonstrated the significant impact of bacterially produced surfactants on the rate of hydrate formation. New studies address hydrate formation in relation to composition and configuration of host particles.

The prototype vertical line array (VLA) was completed, deployed and tested during FY02. Test tracks were run using both an $80 \mathrm{in}^{3}$ water gun and ship noise as acoustic sources. The VLA was deployed in 830 meters of water at a site where previous heat-flow measurements indicated that the base of the hydrate stability zone (BHSZ) is located about 400 meters below the sea floor. A deep-towed hydrophone was also deployed to provide surface-source/deepreceiver (SS/DR) profiles simultaneously with the VLA recordings. The data sets will be processed, analyzed for possible hydrate-related features, and compared.

The University of Mississippi and the University of Wales (Bangor) carried out shear wave studies using a hydrophone and a 3-component accelerometer mounted in a probe that was pushed into sediments of the shallow water in Mississippi Sound. Difficulties encountered in this test form the basis for work aimed at continuing to improve the sensitivity of this instrument.

A variety of samples was recovered from a number of environments known to contain gas hydrates. Testing of these samples is ongoing with plans to use the produced data to determine a sampling protocol for the summer 2003. 
Spatial analyses of hydrate-bearing deposits has begun and occurrences of hydrates in the Gulf of Mexico are being compared to those in other marine deposits in an effort to characterize these deposits.

Two problems that have not yet been addressed adequately by the consortium are:

1) supplying electrical power to sensors on the sea floor and

2) telemetering the resulting data to an onshore facility.

The possibility of using an existing oil production platform to solve both has been discussed but has not seemed very appealing because it would seriously limit the choice of monitoring station sites. Alternative solutions have been identified, tentatively, during the past few months:

1) Total power requirements would be rather modest if power generation could be done on the sea floor because the loss associated with transmission from surface to sea floor would be avoided. Sea-floor generation may be feasible using a type of microbial battery that has been under development for some years by Naval Research Laboratory (NRL).

2) The NOAA National Data Buoy Center at Stennis Space Center has expressed an interest in providing a buoy to bring the data to the surface. An interest has also been expressed in engineering the transmission of data over the NOAA satellite system to the Stennis facility.

\section{EXPERIMENTAL: CONTAINED IN PROGRESS-REPORTS FROM THE SUBCONTRACTORS THAT FOLLOW "RESULTS AND DISCUSSION"}

\section{RESULTS AND DISCUSSION: CONTAINED IN THE FOLLOWING PROGRESS-REPORTS FROM THE SUBCONTRACTORS}

\section{CONCLUSIONS}

The work of the HRC is now supported, approximately equally, by three federal agencies: MMS, DOE, and NIUST (NOAA-NURP). Sensor development has advanced to, in most cases, the sea-trials phase. Data acquisition and transfer needs are just beginning to be addressed and will have to be incorporated into the next round of proposals and funding requests. Affiliation with the JIP has necessitated changes in the plan for the Monitoring Station/Sea Floor Observatory (MS/SFO) deployment scheduling as well as many of the details of configuration.

\section{REFERENCES: LISTED IN THE INDIVIDUAL REPORTS OF THE SUBCONTRACTS}

\section{LIST OF ACRONYMS AND ABBREVIATIONS}

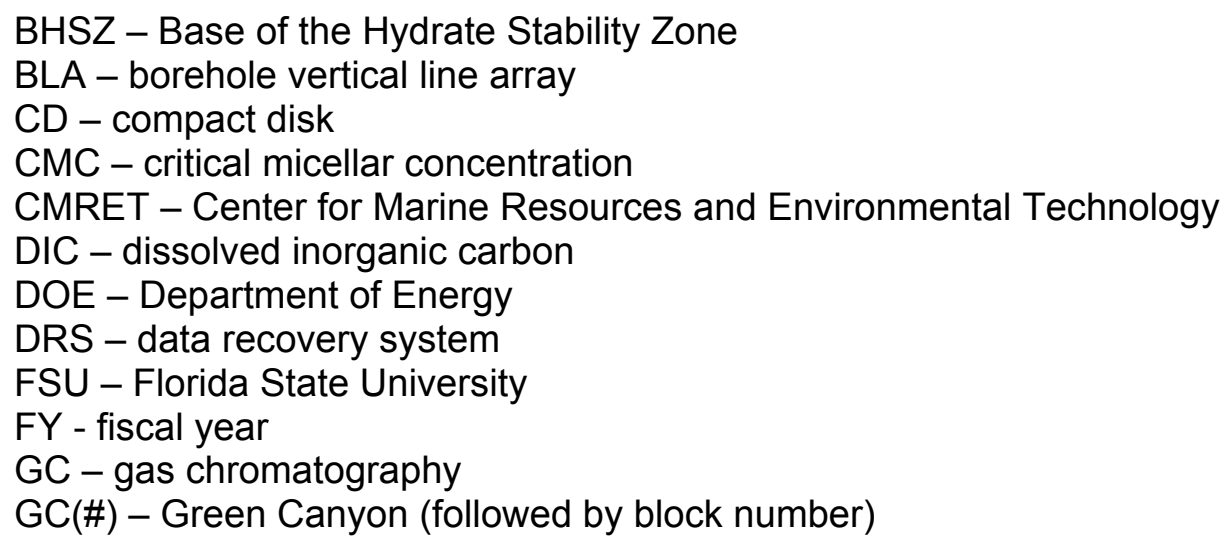


GHASTLI - Gas Hydrate and Sediment Test Laboratory Instrument GOM - Gulf of Mexico

HRC - Hydrates Research Consortium

HSZ - Hydrate Stability Zone

IDP - integrated data power unit

IRMS - isotope ratio mass spectrometer

JIP - Joint Industries Project

JSL - Johnson Sea Link

MC - Mississippi Canyon

METS - methane sensor available commercially

MMS - Minerals Management Service

MS/SFO - monitoring station or sea floor observatory

NIUST - National Institute for Undersea Science and Technology

NOAA - National Oceanographic and Atmospheric Administration

NRL - Naval Research Laboratory

NURP - National Undersea Research Program

$\mathrm{PCB}$ - pressure compensated battery

PI - Principal Investigator

PVC - polyvinyl chloride

ROV - Remotely Operated Vehicle

$\mathrm{RV}=\mathrm{R} / \mathrm{V}$ - Research Vessel

SDS - sodium dodecyl sulfate

SFO - Sea floor observatory

SRR - sulfate reduction rates

SS/DR - surface source/deep receiver

SSD - station service device

TOC - total sedimentary organic carbon

UM/UMiss - University of Mississippi

UNC - University of North Carolina

USGS - United States Geological Survey

UVic - University of Victoria (British Columbia)

VLA - Vertical Line Array 


\section{GULF OF MEXICO SEAFLOOR STABILITY AND GAS HYDRATE MONITORING STATION PROJECT}

Task 1: Program Development

FINAL TECHNICAL PROGRESS REPORT

DECEMBER 2003

\section{FOR THE 12TH QUARTER}

1 July 2003 through 30 September 2003

Principal Investigators: J.R. Woolsey, T.M. McGee, Robin Buchannon, Carol Blanton Lutken Mississippi Mineral Resources Institute and the Center for Marine Resources and

Environmental Technology, University of Mississippi

220 Old Chemistry Building

University, Mississippi 38677

DOE Award DE-FC26-00NT40920 


\begin{abstract}
Program development in FY03 included all proposal organization and reporting to the Department of Energy. The Annual Meeting of the Consortium, hosted by the Center for Marine Resources and Environmental Technology (CMRET), was held in Oxford, Mississippi, February 17-18, 2003 to define future needs. A semiannual meeting, also held in Oxford, was attended by some 30 participants, September 10-11, 2003, and served as a midyear evaluation point for the group. Since the FY02 contract had been extended to 30 September, 2003, then again to 31 March, 2004, progress reports were given by Consortium participants funded under both FY02 and FY03 DOE grants.
\end{abstract}

Program Development highlights include:

- A shallow (500-1000m water depth) and a deeper (>1000m water depth) water site are being further characterized,

- The funding granted by DOE to support investigation of the use of an established, industrial platform in the Gulf of Mexico was successfully redirected to fund a cruise to recover cores from the Mississippi Canyon, Northern Gulf of Mexico. Ten 2-8m cores were recovered from the Mississippi Canyon in January of 2004. The cores have been sampled and are currently being evaluated for various properties including age and their ability to "grow" hydrate in the laboratory.

- Establishment of the ChevronTexaco Joint Industries Project (JIP) has influenced many aspects of the Hydrates Monitoring Station research, including site selection. Scientists from the Consortium are playing active roles in the selection of the site that the JIP is scheduled to drill in the Gulf of Mexico in 2004.

\title{
TABLE OF CONTENTS
}

TITLE PAGE

\author{
ABSTRACT \\ TABLE OF CONTENTS \\ GRAPHICAL MATERIALS \\ INTRODUCTION \\ EXECUTIVE SUMMARY \\ EXPERIMENTAL \\ RESULTS AND DISCUSSION \\ CONCLUSION \\ REFERENCES \\ ACRONYMS/ABBREVIATIONS
}

\section{GRAPHICAL MATERIALS} observatory.

Figure 1-1. Diagram of the component parts of the monitoring station/sea floor 


\section{INTRODUCTION}

Established in1999, the objective of the Gulf of Mexico gas hydrate monitoring station project is to emplace a remote, multi-sensor monitoring station at a selected location within the hydrate stability zone of the northern Gulf of Mexico. Plans for the station have produced subsets of research related to the establishment, emplacement and maintenance of the facility. These include physical, chemical, and biological components of the hydrocarbon system, location of the station, both geographically and within the water column, and means of accessing, assessing, and archiving the acquired data. Eventually this data base will be made available to groups investigating gas hydrate for energy resource potential and environmental impact.

The CMRET, as the administrator of the Consortium, organizes an annual and a semiannual meeting each year, usually held in Oxford, Mississippi. The principal goals of these meetings are to maintain communications between investigators and to keep the project on task and on schedule. Attendees include researchers from academia as well as from industry and government and representatives from the Consortium funding agencies, MMS, DOE and, now, the National Oceanic and Atmospheric Administration's National Undersea Research Program's National Institute for Undersea Science and Technology (NOAA-NURP-NIUST). In addition, a compact disk including the agenda, attendees and contact information, presentations and related information from the meeting is produced and distributed to attendees and other interested parties.

The final project of this funding cycle involved the replacement of Conoco's platformbased work with the acquisition of cores from the sea floor in an area from which no core data were available. These cores, subsampled in June, 2004, will be used to establish dates of deposition for shallow units in Mississippi Canyon and to attempt to characterize the sediments in terms of potential for hosting hydrates.

\section{EXECUTIVE SUMMARY}

Program development in FY03 included all proposal organization and reporting to the Department of Energy. The Annual Meeting of the Consortium, hosted by the Center for Marine Resources and Environmental Technology (CMRET), was held in Oxford, Mississippi, February $17-18,2003$. This meeting was attended by over 40 active Consortium participants who collaborated on evaluation of the project to date and in defining future needs. Many of the needs were incorporated into summer research plans. A semiannual meeting, also held in Oxford, was attended by some 30 participants, September 10-11, 2003, and served as a midyear evaluation point for the group. Compact disks (CDs) including each meeting's agenda, presentations, attendees and contact information were produced and distributed to those who attended the meeting. The CDs have also been made available to any Consortium member who has requested them. Since the FY02 contract had been extended to 30 September, 2003, then again to 31 March, 2004, progress reports were given by Consortium participants funded under both FY02 and FY03 DOE grants. The following sections comprise highlights of the progress reported at these two meetings.

- Site selection has progressed to the point that a shallow (500-1000m water depth) and a deeper (>1000m water depth) water site are being further characterized. Hydrates are now known to occur either at the surface or within the shallow subsurface at both sites.

- The funding granted by DOE to support investigation of the use of an established, industrial platform in the Gulf of Mexico was successfully redirected to fund a cruise to recover cores from the Mississippi Canyon, Northern Gulf of Mexico. This is an area of known hydrate occurrence (Neurauter and Bryant, 1989) and one from which the 
CMRET has acquired much seismic data and had already contracted TDI Brooks, International to acquire heat flow data. No core data were available from this area so when the Conoco contract was negated with the August 30, 2002 merger transaction between Conoco Inc. and Phillips Petroleum, the CMRET applied to DOE to allow the redirection of funds. The result is that ten $2-8 \mathrm{~m}$ cores were recovered from the Mississippi Canyon in January of 2004. The cores have been sampled and are currently being evaluated for various properties including age (University of Southern Mississippi) and their ability to "grow" hydrate in the laboratory (Mississippi State University). Other researchers will perform additional investigations pending the results of these first studies.

- Establishment of the ChevronTexaco Joint Industries Project (JIP) has influenced many aspects of the Hydrates Monitoring Station research, including site selection. Scientists from the Consortium are playing active roles in the selection of the site that the JIP scheduled to drill in the Gulf of Mexico in 2004. Details of the major changes that cooperation with the JIP has necessitated follow:

\section{EXPERIMENTAL}

RECOVERY OF CORE MATERIAL FROM MISSISSIPPI CANYON 798

Carol Lutken, CMRET geologist and Charlotte Brunner, University of Southern Mississippi paleontologist and faculty member at Stennis Space Center campus, participated in the January cruise aboard TDI Brooks, International RV J.W. Powell (for vessel specifications, go to http://www.tdi-bi.com/vessels/powell.htm) in the northern Gulf of Mexico. The purposes of the cruise were several but the Consortium contracted with TDI for 3 days' coring time, waiving transit time as a favor, in support of the research efforts of the Consortium.

Core sites were selected following runs across features observed on 1998 Huntec boomer data and confirmed with onboard chirp sonar. Ten cores were recovered for the following reasons:

- The location was proximal to an area of reported hydrate outcrop,

- The location was distant from areas of reported hydrate outcrop and appeared, on the chirp records to represent continuous deposition and minimal deformation,

- The location was shown on chirp records to be an acoustic wipe-out zone,

- The location appeared, on chirp records, to have had younger units missing and so recovered samples might represent stratigraphically older units,

- The location was coincident with that of a vertical array deployment in 2002 or 2003.

All cores were recovered using 3 -inch diameter core-liners in the piston coring apparatus deployed from the Powell's starboard a-frame. Recovery ranged from $1.97 \mathrm{~m}$ to $7.13 \mathrm{~m}$. Following recovery, all cores were cut into $120 \mathrm{~cm}$ sections, capped, labeled and stored upright until they were off-loaded at Venice, La. Following their trip to Oxford, they were returned to Stennis where they were logged using the Naval Research Laboratory's logger. They were stored in an enclosed, temperature-controlled facility. In June, the core sections were split and one half archived. The other half was photographed, sampled, wrapped, and stored for possible future sampling. The samples were transported to Mississippi State University and to the University of Southern Mississippi, Stennis Space Center via automobile where they will be analyzed with regard to hydrate-producing/hosting potential and age analyses. 
CHANGES IN THE SFO DESIGN AND DEPLOYMENT SCHEDULE ARRAY DESIGN AND DEVELOPMENT

A most important development is the opportunity to deploy an array of sensors in a borehole that will be drilled by the Department of Energy/Joint Industry Project (DOE/JIP) Consortium. The borehole vertical line array (BLA) will consist of hydrophones, threecomponent accelerometers and temperature sensors that would remain in the hole after the drill stem is recovered, letting the hole collapse and making the installation permanent and thus providing a sub-seafloor component to the station.

The BLA has been funded, separately, by the DOE/JIP and it would not represent a cost increase to the station. The Consortium is, however, investigating the possibility of adding horizontal arrays to the station configuration. This would provide three-dimensional coverage of the water column, seafloor and sub-seafloor at the monitoring station site. It may be that some of the vertical arrays can be converted to horizontal arrays if this route is pursued.

\section{DATA RECOVERY}

External factors have also impacted the way monitoring station data will be recovered. For some time it has been hoped that a commercial service would be available in 2004 which would allow the station data-collecting unit to stream data onto an optic-fiber link for near-to-real time transmission to shore. It was learned in the autumn of 2003 , however, that the service would not become available until 2006 or later.

The use of a remotely operated vehicle (ROV) to download data directly from the station's data loggers was found to be prohibitively expensive due to the depth of water and the weight of the battery packs that would need to be exchanged. Therefore, until the optic fiber link becomes available, an integrated data power (IDP) module will stream data onto an optic-fiber data recovery system (DRS) which will be connected via optic fiber to an access connector. Whenever downloading is required, a system of buoys will bring the DRS access connector to the surface so that the data can be downloaded onto a computer in a boat. The system has been used successfully before and involves far less expense than repeated use of a deep-water ROV. The system has been dubbed the "Big M" and is illustrated in Fig.1-1.

\section{ELECTRICAL POWER FOR THE MS/SFO}

The Gulf of Mexico Hydrates Research Consortium funds the development of microbial batteries but it will be some time before this emerging technology can provide sufficient electrical power to the monitoring station. In the meantime, the IDP module will supply electricity to the station by exchanging the pressure compensated battery (PCB) component about once a year. This will involve unplugging the depleted PCB from the IDP and plugging in a fresh one. The emplacement and exchange of PCBs will be accomplished by a station service device (SSD). The SSD is a ROV-like system, especially designed for the task, that utilizes the power and weight of the payload PCB to supplement the maneuvering of the surface vessel to dock and enable the PCB exchange. The design somewhat restricts the radius of operation, but eliminates the costly power umbilical required by conventional deep ocean ROV's.

A docking station will be incorporated into the IDP module to facilitate changing the PCB's. The SSD will carry the recharged PCB unit to the sea floor and return with the depleted unit. In addition, the SSD will be capable of conducting other tasks such as taking pressure retained short cores and in situ pore-fluid samples. Perhaps most significantly, the SSD will be the means by which all station systems are connected to the IDP for data recovery and electrical power. 


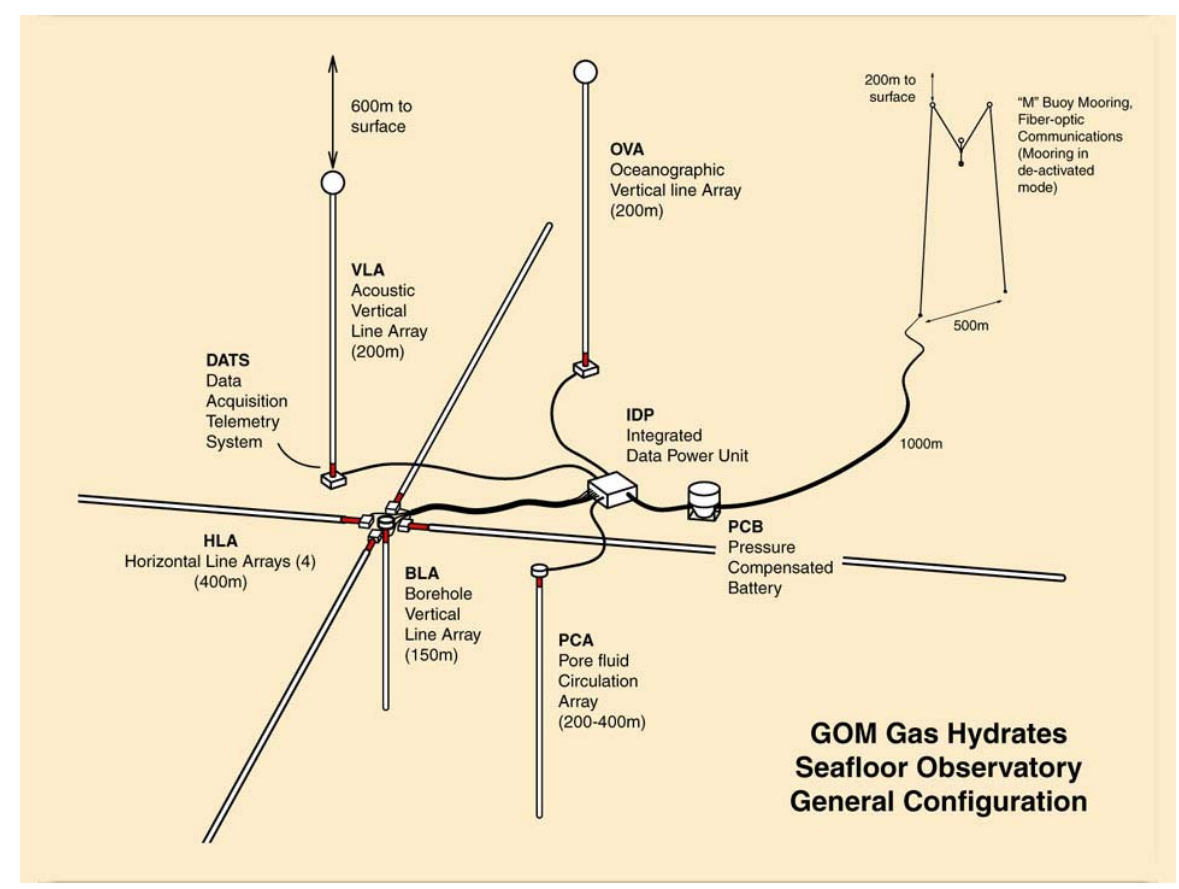

Figure 1-1. Diagram of the component parts of the monitoring station/sea floor observatory

\section{RESULTS AND DISCUSSION}

The Mississippi Canyon sample-analyses are underway. Preliminary indications are that the sediments recovered produce hydrates under laboratory conditions and that there are pre-Holocene sediments in our samples. More detail will appear in future reports. underway.

Results of the JIP decisions are, as yet, unknown. Drilling location discussions are still

\section{CONCLUSIONS}

Two meetings were held at which Consortium members presented results of their research and made significant progress toward surmounting some of the major obstacles remaining to MS/SFO installation on the sea floor. Small group sessions enabled new and continuing cooperations to be established or advance.

The first samples to which consortium members have had access have been recovered from the sea floor in Mississippi Canyon 798. Evaluation of these samples has commenced and will be included in FY03 reporting.

MS/SFO scheduling and physical modifications discussed herein are not intended to change the basic concepts, overall plans and mission for the monitoring station. Instead, they are expected to enhance the accomplishment of that mission. The MS/SFO will gain a significant degree of autonomy, provide time on the learning curve to deal with the large data sets generated by the station, provide an ROV-like SSD capable of conducting a wide range of support activities, and, probably most important, keep on task towards station deployment in 2005 and operation by 2006 . 


\section{REFERENCES}

Neurauter, T.W. and W.R.Bryant, 1989, "Gas hydrates and their association with mud diapir/mud volcanos on the Louisiana continental slope," Proc. $21^{\text {st }}$ Offshore Tech. Conf., Paper No. 5944, vol.1, 599-607.

\section{LIST OF ACRONYMS AND ABBREVIATIONS}

BLA - borehole vertical line array

CD - compact disk

CMRET - Center for Marine Resources and Environmental Technology

DOE - Department of Energy

DRS - data recovery system

FY - fiscal year

GOM - Gulf of Mexico

HRC - Hydrates Research Consortium

IDP - integrated data power unit

JIP - Joint Industries Project

MC - Mississippi Canyon

MMS - Minerals Management Service

MS/SFO - monitoring station or sea floor observatory

NIUST - National Institute for Undersea Science and Technology

NOAA - National Oceanographic and Atmospheric Administration

NURP - National Undersea Research Program

PCB - pressure compensated battery

ROV - Remotely Operated Vehicle

$\mathrm{RV}=\mathrm{R} / \mathrm{V}-$ Research Vessel

SFO - Sea floor observatory $=$ MS/SFO

SSD - station service device

UM/UMiss - University of Mississippi

VLA - Vertical Line Array 


\section{Site Selection and Characterization of Vent Gas, Gas Hydrate and Associated Sediments}

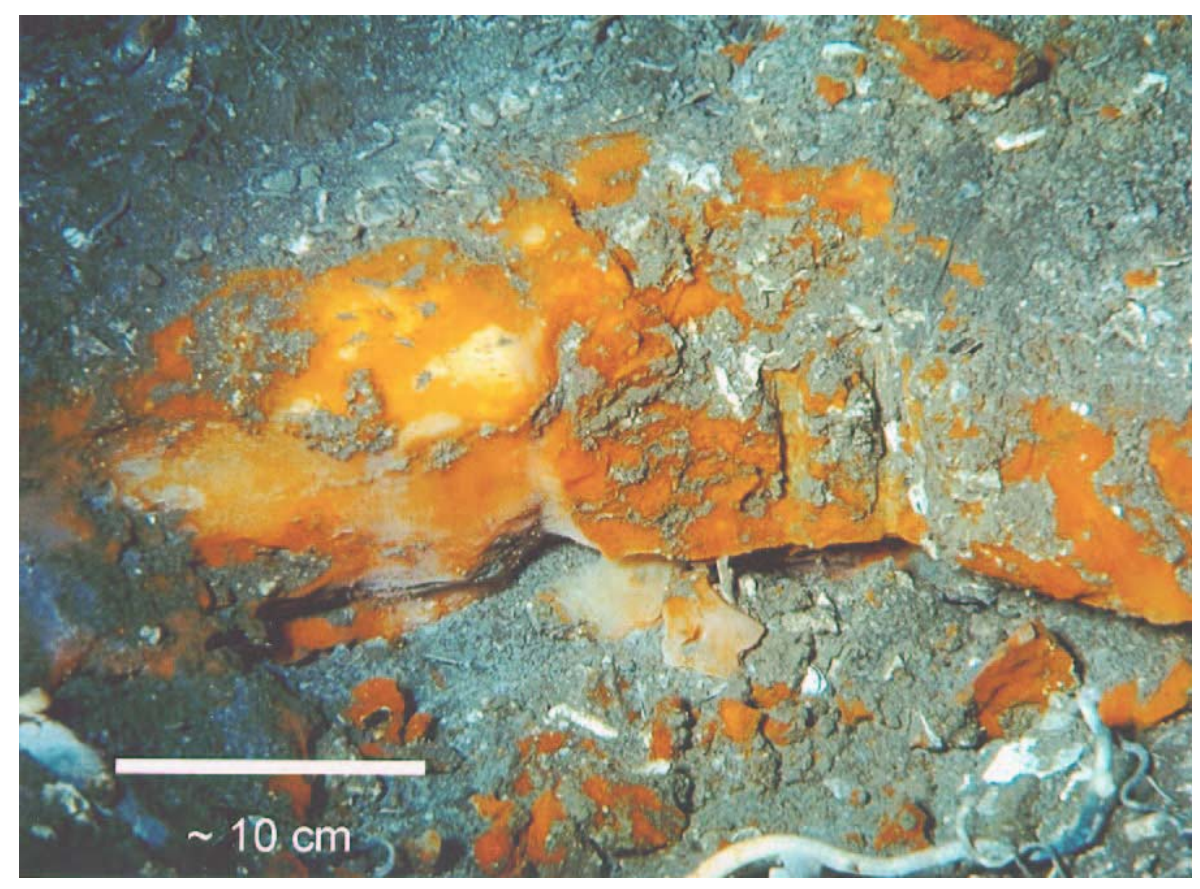

Annual Technical Report January 1, 2002 - June 30, 2004

Roger Sassen, Geochemical and Environmental Research Group, Texas A\&M University, College Station, Texas 77845 and

Harry H. Roberts, Department of Oceanography and Coastal Studies, Louisiana State University, Baton Rouge, Louisiana 70803

June 28, 2004

DOE Award \# DE-FC26-00NT40920 


\title{
SITE SELECTION AND CHARACTERIZATION OF VENT GAS, GAS HYDRATE AND ASSOCIATED SEDIMENTS
}

\author{
Principal Investigators: Roger Sassen, Geochemical and Environmental Research \\ Group, Texas A\&M University, College Station, Texas 77845 \\ and
}

Harry H. Roberts, Department of Oceanography and Coastal Studies, Louisiana State University, Baton Rouge, Louisiana 70803

\section{Table of Contents}

\author{
Abstract \\ Introduction \\ Executive Summary \\ Geologic Setting and Deep Fluid Sources \\ Review of Gas Hydrate in the Gulf of Mexico Slope \\ Experimental \\ Results and Discussion \\ Descriptions of JSL Sites with Gas Hydrate \\ Descriptions of JSL Sites without Gas Hydrate \\ Geochemistry of Vent Gas, Gas Hydrate, and Sediment Gas \\ Biodegradation of Crude Oil \\ Isotopic Properties of Authigenic Carbonate Rock \\ Sites Lacking Gas Hydrate \\ Conclusions \\ References \\ Bibliography \\ Acronyms and Abbreviations \\ List of Graphics
}

\begin{abstract}
$\underline{\text { ABSTRACT }}$
The total volume of the gas hydrate resource in the Gulf of Mexico slope is estimated to be equivalent to $\sim 1.0-1.4 \times 10 \mathrm{~m}_{3}$ of gas at STP including methane and other hydrate-forming hydrocarbons. The estimate of the Gulf slope hydrate resource is based solely on gas hydrate proven to occur in the mud matrix. There is no evidence as yet that gas hydrate fills pore-space in sands of the Gulf slope. Research on gas hydrate as an alternate energy source or drilling hazard in the Gulf slope should focus on mud as the dominant "reservoir" for gas hydrate. Bottom Simulating Reflectors (BSR) may be a secondary issue in the total energy resource estimate.

The discovery of gas hydrate at the sea floor greatly increases the probability of deep gas hydrate accumulations in the same area. As part of the present study, samples of intact massive gas hydrate were successfully collected from three (3) main sites along with the vent gas from which the gas hydrate crystallized and associated sediment to include
\end{abstract}


authigenic carbonate rock strongly depleted in ${ }^{13} \mathrm{C}$. The sites from which intact gas hydrate and vent gas were successfully recovered are Green Canyon (GC) Block 234, GC 232, and Mississippi Canyon (MC) Block 118. The combination of $38 \mathrm{kHz}$ imagery showing large gas plumes in the water column with conventional shallow seismic amplitudes may improve the probability of discovering new gas hydrate sites.

The molecular properties of vent gas provide strong evidence that gas hydrate is not decomposing at a significant rate in the Gulf of Mexico. Some minor decomposition of extremely shallow gas hydrate is likely to occur but deeply buried gas hydrate appears stable over long spans of time. Exposed or shallow gas hydrate may be affected by sea water temperature changes, but overall gas hydrate is apparently accumulating in the Gulf of Mexico slope at present. Thus monitoring of gas hydrate by seismic arrays should focus on the upper few meters of study sites.

Three "control" sites with strong geophysical anomalies were also sampled to determine why no gas hydrate occurs. The apparent lack of gas hydrate at the VK 826 and MC 885 sites may be partially attributed to low hydrocarbon gas flux but the carbonate-rich sites are also too shallow and warm for structure I or II gas hydrate to be stable. In addition, brines are likely to retard gas hydrate crystallization. The MC 709 is a major geophysical anomaly and may or may not contain any gas hydrate although there are areas with intermediate hydrocarbon flux within the Gas Hydrate Stability Zone. The main reason for the lack of abundant gas hydrate at MC 709 may be brine poisoning. More than 160 major gas seeps identified in the deep Gulf of Mexico slope appear to lack gas hydrate. Additional research on factors that prevent gas hydrate crystallization is justified.

\section{INTRODUCTION}

In multidisciplinary study of natural gas hydrate, one of the fundamental aspects is to document the basic molecular and isotopic properties of vent gas at the sea floor that gives rise to gas hydrate, the gas hydrate itself, and the sediments intimately associated with gas hydrate. All other research studies depend upon this type of basic information on gas hydrate for their context. This task was undertaken with collaboration from Dr. Harry Roberts of Louisiana State University, who apportioned dive time in the Johnson Sea-Link (JSL) research submersible during the mid-summer of 2002.

Samples of intact gas hydrate were successfully collected from three (3) main sites along with the vent gas from which the gas hydrate crystallized and associated sediment. The sites from which intact gas hydrate and vent gas were successfully recovered are Green Canyon (GC) Block 234, GC 232, and Mississippi Canyon (MC) Block 118 (Fig. 1). Specialized sampling equipment was used to include a high-pressure vessel designed to recover gas hydrate from the sea floor and to return it to the surface intact at temperature and pressure conditions approximately ambient to the deep sea floor.

The GC 234 site has been visited before and new data can be set in the context of previously published research to assess any detectable change in the gas hydrate system at this site. In contrast, gas hydrate was recovered for the first time from GC 232 and 
from MC 118. GC 232 is similar to the GC 234 site; the MC 118 site is the most eastward gas hydrate site ever discovered in the Gulf of Mexico. Preliminary molecular and isotopic analyses were performed to characterize the samples as examples of gas hydrate sites. The gas hydrate from these sites can be compared to gas hydrate from other sites in the Gulf of Mexico.

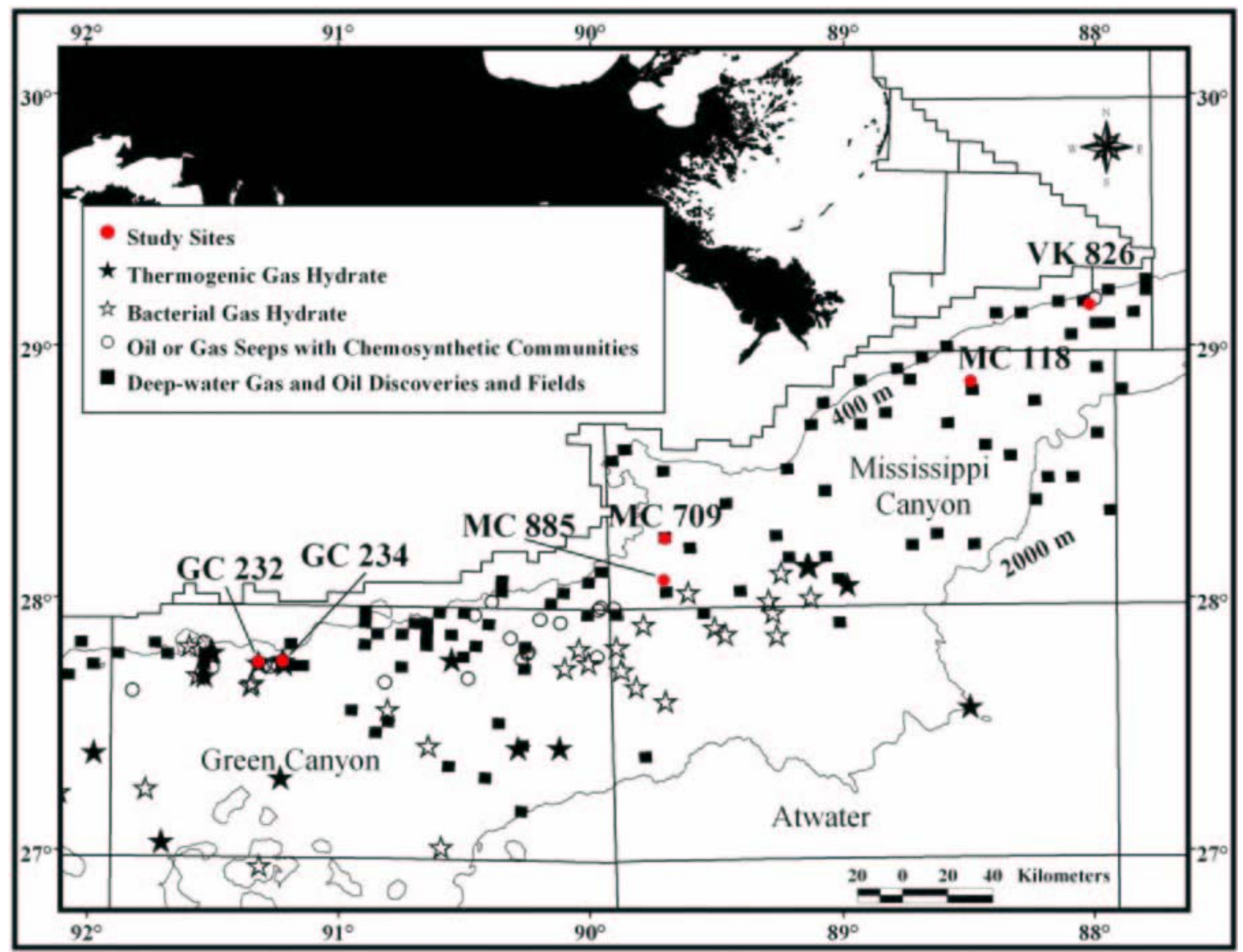

Figure 1. Map showing locations of main study sites of the Gulf slope with gas hydrate (GC 234, GC 232, and MC 118) within a region of has hydrate occurrences, seeps with chemosynthetic communities, and subsurface accumulations of oil and gas. Other study sites used as control sites without gas hydrate are also shown (MC 709, MC 885, VK 826). Hydrate map modified from Sassen et al. (2001a) and sites of chemosynthetic communities from MacDonald et al. (1996).

It is important to emphasized that piston cores taken at many gas-rich sites $(>160)$ within the Gas Hydrate Stability zone (GHSZ) of the Gulf of Mexico have not recovered gas hydrate (Sassen et al., 2002). Such sites are obvious geophysical anomalies and contain abundant free methane and other gases that form hydrate under proper conditions. The ratio of known gassy sites without gas hydrate to sites with gas hydrate is $\sim 2: 1$. Geophysics has limitations at present in direct sensing of gas hydrate that are not widely recognized. Why no hydrate occurs within the GHSZ where abundant free gas is available is an important question that demands a scientific explanation. 
A series of "control" sites lacking gas hydrate were characterized and sampled by the JSL dives in 2002 in order to assess the characteristics of sites that do not show any evidence of gas hydrate near the surface of the sea floor. The JSL sites without gas hydrate include MC 709, MC 885, and Viosca Knoll (VK) 826 (Fig. 1).

The ultimate rationale for the studies is to help determine characteristics of known sites for gas hydrate suitable for further study to include vertical seismic arrays intended to show changes related to instability. The question presupposes that gas hydrate can be directly detected by geophysics and that instability of gas hydrate is sufficient to be detectable. Another ultimate goal is to high-grade sites for deep rotary coring that will provide maximum information on gas hydrate as a drilling hazard and in terms of economic geology. The results of the present study are surprising.

\section{EXECUTIVE SUMMARY}

The total volume of the gas hydrate resource in the Gulf slope is estimated to be equivalent to $\sim 1.0-1.4 \times 10^{13} \mathrm{~m} 3$ of gas at STP including methane and other hydrate-forming hydrocarbons. The estimate of the Gulf slope hydrate resource is based solely on gas hydrate proven to occur in the mud matrix. It should be emphasized that nearly all known occurrences of gas hydrate in the Gulf of Mexico occur as vein-fillings and thick pavements of nearly pure gas hydrate in hemipelagic mud. Rarely, gas hydrate fills vuggy porosity in authigenic carbonate rock. There is no evidence, as yet, that gas hydrate fills pore-space in sands of the Gulf of Mexico slope. Thus, research on gas hydrate as an alternate energy source or drilling hazard in the Gulf slope should focus on mud and carbonate rock as the dominant "reservoir" for gas hydrate in the Gulf of Mexico. The study of Bottom Simulating Reflectors (BSR) may be a secondary issue when estimating the total hydrate energy resource of the Gulf of Mexico because they are so rare and are of limited size in the Gulf of Mexico slope.

As part of the present study, samples of intact massive gas hydrate were successfully collected from three (3) main sites along with the vent gas from which the gas hydrate crystallized and associated sediment to include authigenic carbonate rock strongly depleted in ${ }^{13} \mathrm{C}$. The sites from which intact gas hydrate and vent gas were successfully recovered are Green Canyon (GC) Block 234, GC 232, and Mississippi Canyon (MC) Block 118 (Fig. 1). The study sites appear representative of most other gas hydrate sites discovered elsewhere in the Gulf slope. It is now possible to synthesize available data to address important issues relating to gas hydrate as a potential energy source, and to suggest the best ways to study gas hydrate in the unique geologic setting of the Gulf of Mexico slope.

It has been suggested that decomposition of gas hydrate is an important process in nature at this point in geologic time, but there is no geochemical evidence to support the concept in the Gulf of Mexico. If gas is observed venting from over buried gas hydrate, it is sometimes assumed that the vent gas is from decomposition of gas hydrate. It should be emphasized that the composition of vent gas from all sites thus far studied in the Gulf of Mexico slope is consistent with gas hydrate stability over recent geologic time. If gas 
hydrate decomposition was geologically significant, then vent gas would show enrichment of hydrate-forming hydrocarbon gases such as ethane and propane. This molecular distribution is not observed, providing strong evidence that gas hydrate is not decomposing at a significant rate in the Gulf of Mexico. Instead, gas hydrate is apparently accumulating in the Gulf at present.

Some decomposition of extremely shallow gas hydrate is likely to occur but deeply buried gas hydrate appears stable in the Gulf of Mexico over long spans of time. Exposed or shallow gas may be affected by sea water temperature changes. The irregular surface of the sea floor at gas hydrate sites is partly the result of cyclical gas hydrate crystallization and decomposition in only a thin-layer nearest the sea floor. Thus, seismic evidence of gas hydrate instability is most likely to be observed in the top $\sim 2$ meters of sediment nearest the sea floor whereas deeper sediment should not show evidence of change on any short time scale.

Only a fraction of shallow amplitudes are likely to be related to gas hydrate because several factors appear to retard gas hydrate crystallization in complex natural settings. Brine poisoning may prevent gas from crystallizing as gas hydrate and may help explain $>160$ known gas seeps in the Gulf that lack any evidence of gas hydrate. Localized heating or insufficient gas flux may also contribute to the observed lack of gas hydrate.

The discovery of gas hydrate at the sea floor greatly increases the probability of deep gas hydrate accumulations in the same area. There are limits to the application of conventional seismic techniques because they do not directly indicate gas hydrate in shallow sediment of the Gulf of Mexico. It is more reasonable to suggest that seismic techniques can identify gassy seeps as sea floor amplitudes. The combination of $38 \mathrm{kHz}$ imagery showing large gas plumes in the water column with conventional shallow seismic amplitudes may improve the probability of discovering new gas hydrate sites.

Additional research to explain why gas hydrate fails to crystallize at many gas seeps in the Gulf of Mexico slope is justified. Although the VK 826 and MC 885 sites display obvious geophysical anomalies, no gas hydrate is present. The apparent lack of gas hydrate at the VK 826 and MC 885 sites may be partially attributed to insufficient hydrocarbon gas flux but the carbonate-rich sites are too shallow and warm for structure I or II gas hydrate to be stable. In addition, brines are present at both sites that are likely to retard gas hydrate crystallization. The MC 709 is a major geophysical anomaly and may or may not contain any gas hydrate although there are areas with intermediate hydrocarbon flux within the GHSZ. The main reason for the lack of abundant gas hydrate at MC 709 may be brine poisoning.

\section{GEOLOGIC SETTING AND DEEP FLUID SOURCES}

The main structural features of the northern Gulf of Mexico are salt basins including the large Gulf of Mexico Salt Basin that extends from the coastal salt-dome province to the lower continental slope, and a series of smaller interior salt basins that extend onshore from south Texas to Alabama. The basins formed during Late Triassic rifting and were 
floored by salt (Louanne/Werner formations) during Middle Jurassic marine incursions (Salvador, 1987). Structural style is profoundly influenced by the effects of salt movement driven by rapid deposition of siliciclastic sediment. The Gulf of Mexico continental shelf is now characterized by numerous salt domes, whereas the continental slope is affected by large sheet-like salt thrusts that extend from the shelf edge across the continental slope to the Sigsbee Escarpment, near the upper limit of the abyssal plain (Worrall and Snelson, 1989).

The geology of the Gulf of Mexico slope is conducive to seepage and venting from deeply buried petroleum systems to the sea floor because hydrocarbon generation is geologically recent within the deep sediment section beneath the salt thrust and on the upper abyssal plain (Sassen et al., 2001a,b,c,d). The framework of deep hydrocarbon migration involves vertical migration through salt withdrawal basins that pierce the large salt thrusts (Fig. 2). Rapid sedimentation in Pleistocene depocenters (Galloway et al., 2000) activates migration conduits from depth to the sea floor within the salt basins. Fracture zones associated with moving salt sheets, as well as active faults, provide efficient migration conduits for fluid flow to the sea floor including gas, oil, and brines. In some brine seeps, which may be associated with limited chemosynthetic communities, mineral assemblages include strontium-rich barite and radium (Fu and Aharon, 1998).

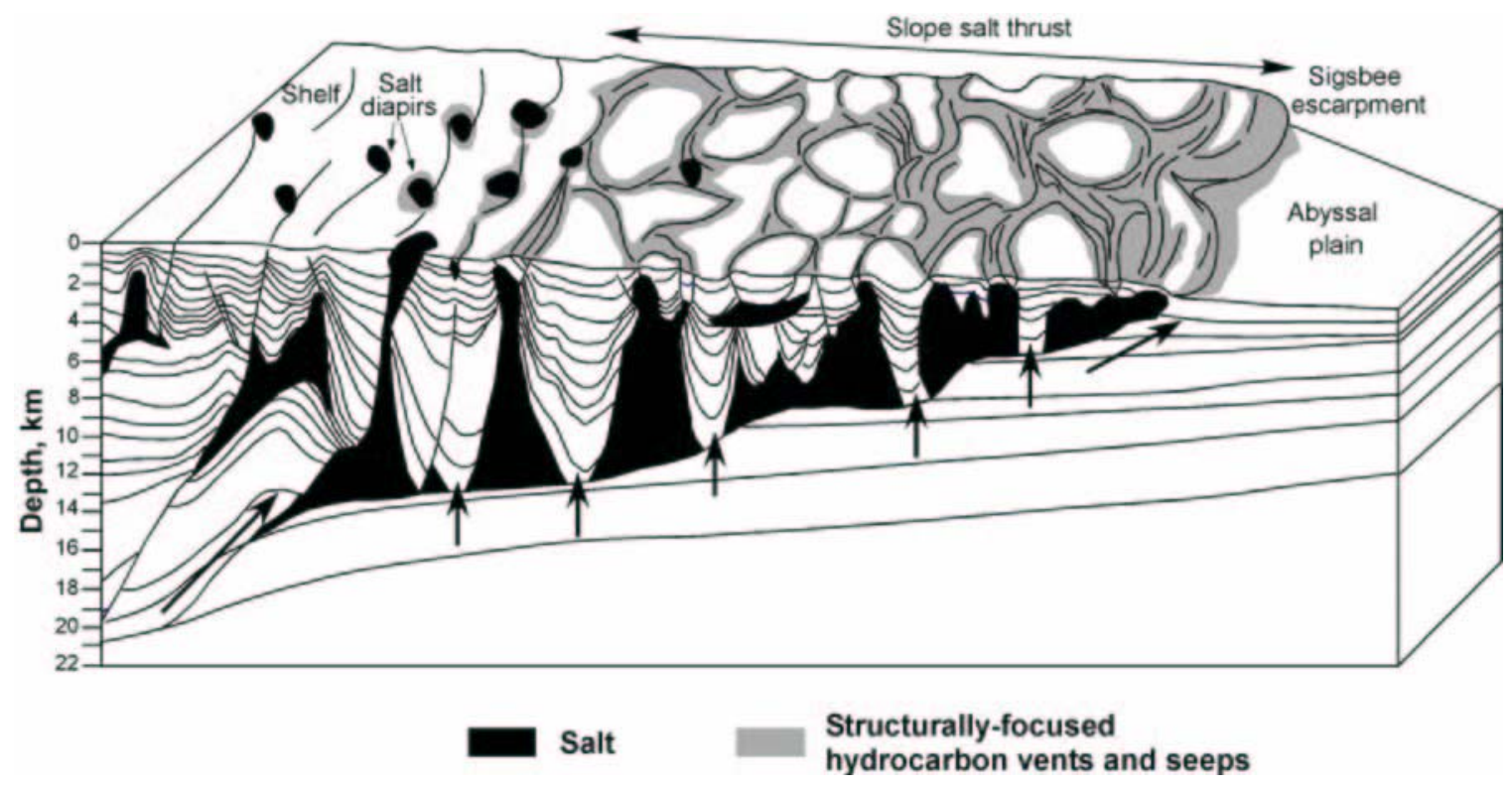

Figure 2. A conceptualized north-south cross-section of central Gulf slope from the shelf to the abyssal plain is shown. Numerous geologically young basins formed by salt withdrawal pierce the slope salt thrust. The drainage areas of recently mature Mesozoic source rocks are deeply buried beneath the allochthonous salt. Fluids migrate upward through holes in the salt thrust (arrows). Within basins, salt and related faults provide conduits for vertical migration of fluids to reservoirs and to the seafloor. Hydrocarbon vents and seeps are focused by the structure near the rims of salt withdrawal basins. Modified from Milkov and Sassen (2001). 
The sea floor shows markedly irregular bathymetry from structural deformation, faulting, fracturing, and slumping (Roberts and Carney, 1997). Massive hydrocarbon seepage manifests itself at the Gulf sea floor as gas hydrate, oil-stained sediments, authigenic carbonate rock with carbon depleted in ${ }^{13} \mathrm{C}$, and hydrocarbon-driven chemosynthetic communities (e.g. Aharon et al., 1997; Roberts and Aharon, 1994; MacDonald et al., 1989; Roberts and Carney, 1997; Sassen et al., 1999a).

Seeps and vents are not uniformly distributed across the Gulf slope. Because fluid flow from great depth is structurally focused, gas hydrate and chemosynthetic communities are concentrated along the rims of salt-withdrawal basins, over salt ridges, and near the faulted and folded margin of the Sigsbee Escarpment at the downdip limit of the Gulf of Mexico Salt Basin (Fig. 2). Seep-related features are infrequently observed within salt withdrawal basins themselves because such sediments are less deformed and usually lack major migration conduits to shallow sediments or the sea floor (Fig. 2).

\section{REVIEW OF GAS HYDRATE IN THE GULF OF MEXICO SLOPE}

The total volume of gas hydrate in the Gulf slope is estimated to be equivalent to $1.0-1.4$ $\mathrm{x} 10^{13} \mathrm{~m} 3$ of gas at STP including methane and other hydrate-forming hydrocarbons (Milkov and Sassen, 2001). The estimate of the Gulf slope hydrate resource is based solely on gas hydrate proven to occur in the mud matrix (e.g. Sassen et al., 1999a). All known occurrences of gas hydrate in the Gulf of Mexico occur as vein-fillings and thick pavements of nearly pure gas hydrate in hemipelagic mud. Occasionally, gas hydrate occur filling vuggy porosity in authigenic carbonate rock. There is no evidence that gas hydrate occurs as pore-space fillings in sands of the Gulf of Mexico slope. Thus, research in the Gulf slope should focus on mud and carbonate rock as "reservoirs" for gas hydrate in sediment.

This volume of gas in geologically shallow sediment dwarfs the volume of conventional reserves of oil and gas in deep subsurface reservoirs of the Gulf slope (Milkov and Sassen, 2002). It should be emphasized that the entire estimate of the Gulf of Mexico gas hydrate resource is based on vein-fillings and pavements of nearly pure gas hydrate in mud. None of this estimate includes gas hydrate in the pore throats of sand and none is related to Bottom Simulating Reflectors (BSRs). There is as yet no evidence of any gas hydrate in a BSR or in sand facies of the Gulf of Mexico.

Thermogenic (oil-related) gas and crude oil from the subsurface hydrocarbon system vents at the sea floor of the Gulf of Mexico continental slope, entering the water column as oil-coated bubbles that rise to the sea surface (Sassen et al., 2001a, b). Much of the thermogenic gas, strongly enriched in $13 \mathrm{C}$ relative to bacterial methane, bypasses sediment and vents to the water column and the atmosphere. Because research submersible platforms allow direct sampling of gas vents within $0.5 \mathrm{~m}$ of sea floor orifices (see Fig. 3), the molecular and isotopic properties of the hydrocarbon vent gas are well constrained. Some fraction of the vent gas crystallizes near the sea floor as structure II gas hydrate. Vent gas is the starting material from which other major carbon pools at chemosynthetic communities (Fig. 4) are derived, mainly by complex microbial 
processes that lead ultimately to geologically significant sequestration of carbon as authigenic carbonate rock depleted in 13C (Fig. 5).

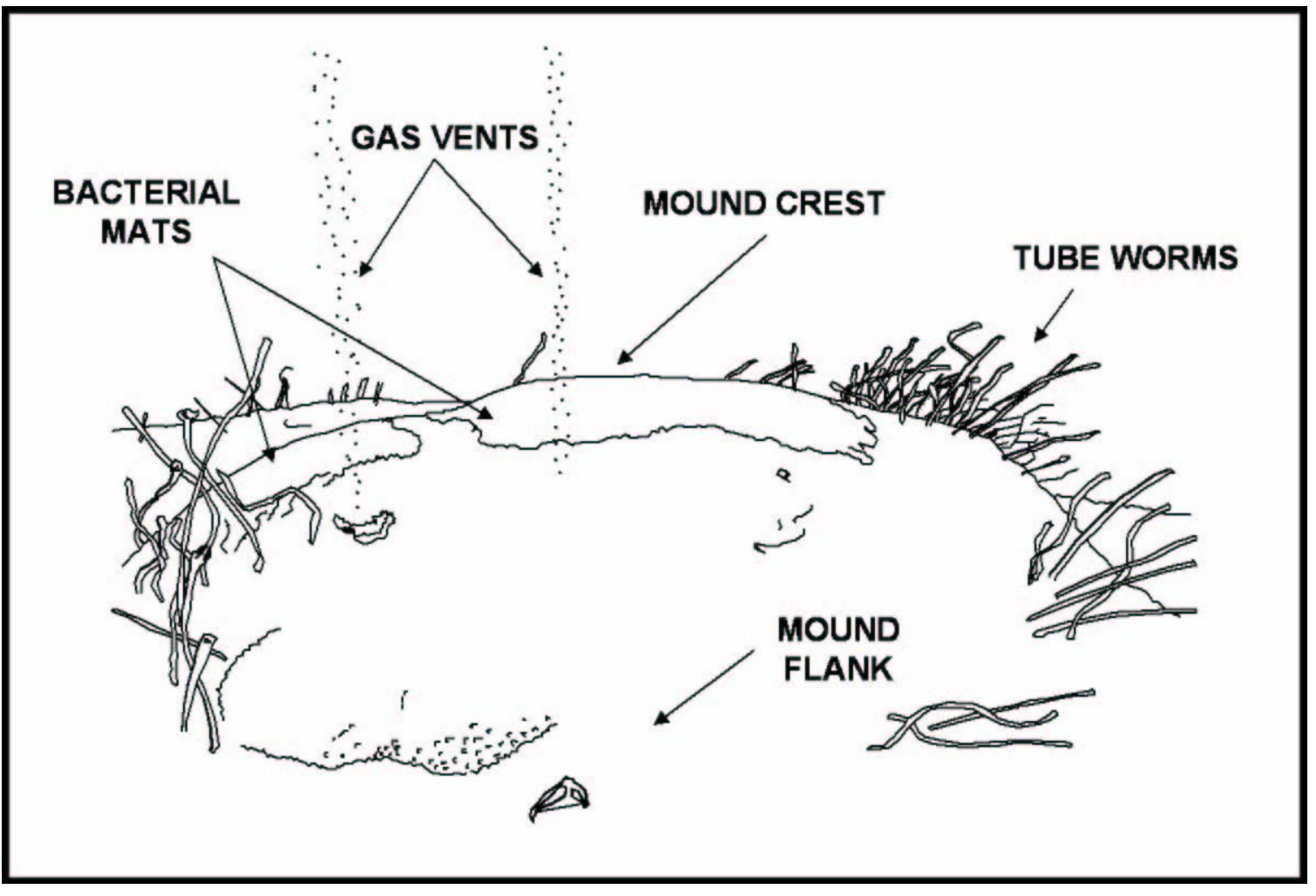

Figure 3. Features of a typical gas hydrate mound ( $\sim 2 \mathrm{~m}$ across) with gas vents and nearby chemosynthetic community of tubeworms (Sassen et al.,1999b).

Gas hydrate is an ice-like crystalline mineral in which hydrocarbon gases and nonhydrocarbon gases are held within rigid cages of water molecules (Fig. 6). Structure I gas hydrate has a body-centered cubic lattice, structure II gas hydrate has a diamond lattice, and structure H gas hydrate has a hexagonal lattice (Sloan, 1998). Structure I gas hydrate, which occurs in many basins, is usually predominately bacterial methane (Kvenvolden, 1993; 1995). The ${ }^{\mathrm{TM}{ }^{13}} \mathrm{C}$ and ${ }^{\mathrm{TM}} \mathrm{D}$ of bacterial methane from seafloor gas vents and structure I gas hydrate from the Gulf slope is thus far consistent with a source from methanogenesis via $\mathrm{CO}_{2}$ reduction in geologically shallow sediment (Sassen et al., 1999a, b). Both structure II and structure H gas hydrate are believed to co-exist in the Gulf at water depths as shallow as $540 \mathrm{~m}$ (Sassen and MacDonald, 1997). Structure II gas hydrate generally includes $\mathrm{C}_{1}-\mathrm{C}_{4}$ hydrocarbons (methane through isobutane) whereas structure $\mathrm{H}$ gas hydrate generally includes $\mathrm{C}_{1}-\mathrm{C}_{5}$ hydrocarbons (methane through isopentane) as significant components (Sloan, 1998).

The total number of documented gas hydrate sites on the deep Gulf of Mexico sea floor is $\sim 65$ but this is a minimum number. Known shallow or outcropping gas hydrate sites extend along the Gulf slope offshore Texas and Louisiana over a distance $>500 \mathrm{~km}$, and the maximum width of the belt is $>100 \mathrm{~km}$. Solid gas hydrate has been recovered from 

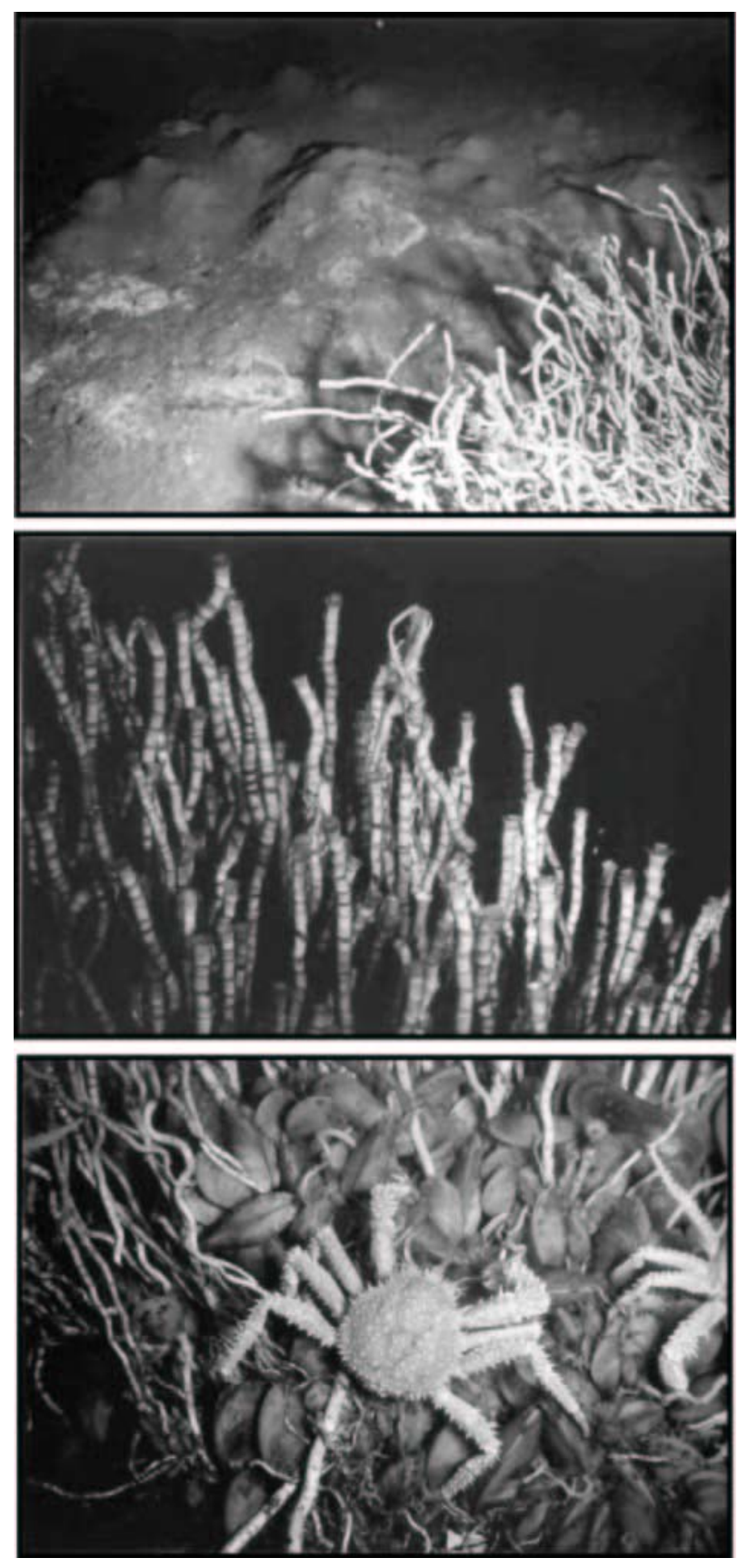

Figure 4. Photographs of tube worms and seep mussels from chemosynthetic communities are shown.

shallow sediments $(<6 \mathrm{~m})$ by piston coring and by research submersibles from $>50$ localities on the Gulf slope (Fig. 1). The distribution of mapped gas hydrate sites corresponds to a late Pleistocene depocenter (Galloway et al., 2000). The minimum observed water depth of occurrence of gas hydrate in the Gulf of Mexico is $\sim 440 \mathrm{~m}$ and the maximum depth is $>2,400 \mathrm{~m}$ (Sassen et al., 1999a). The thickness of the gas hydrate stability zone (GHSZ) increases with water depth. Calculations of stability, based on free gas with $90.4 \%$ methane, suggest that the thickness of the GHSZ in sediment may be as 
much as $\sim 450 \mathrm{~m}$ at $540 \mathrm{~m}$ water depth, and $>1 \mathrm{~km}$ at $1930 \mathrm{~m}$ water depth in the Gulf (Milkov and Sassen, 2000).

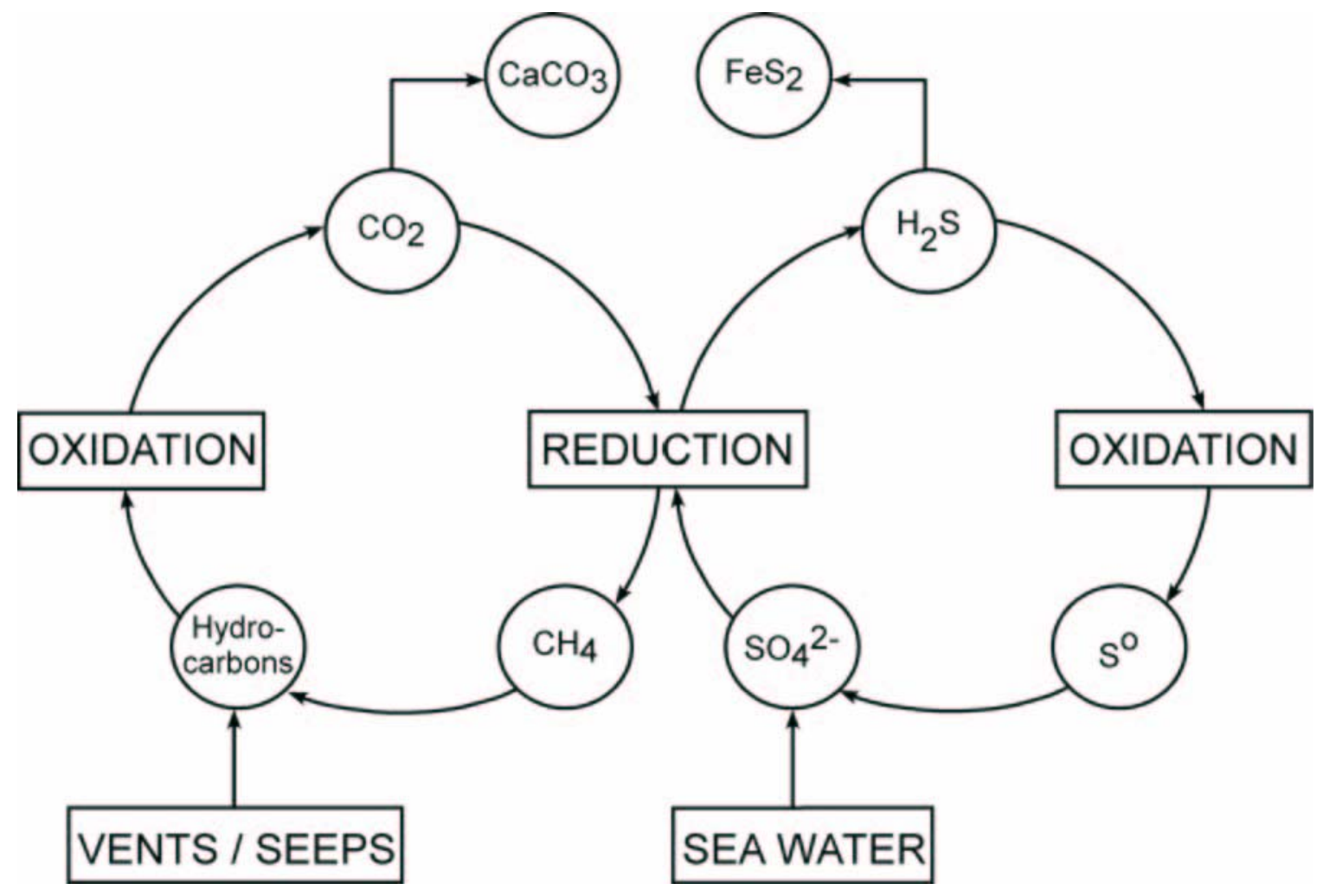

Figure 5. Summary diagram of the microbially-mediated carbon and sulfur cycles associated with hydrate-related chemosynthetic communities. These basic biogeochemical processes are generalized to occur at many other hydrocarbon vent, seep, gas hydrate, and chemosynthetic community sites globally.

In contrast to simple bacterial methane hydrate, thermogenic gas hydrate (methane through pentanes) preserves more complex information on source and stability because numerous hydrocarbon molecules of varying chemical properties are held within crystal lattices. The vent gas and gas hydrate are intimately associated with complex chemosynthetic communities whose initiation and stability depend upon hydrocarbon-driven microbial processes in sediment, which include microbial hydrocarbon oxidation, reduction of $\mathrm{CO}_{2}$ via methanogenesis, sulfate reduction, and sulfide oxidation (Sassen et al., 1993, 1994, 1999b). The molecular and isotopic properties of vent gas, intact gas hydrate, and free hydrocarbons in sediment trace the effects of microbial hydrocarbon oxidation and $\mathrm{CO}_{2}$ reduction in anaerobic sediment rich in $\mathrm{H}_{2} \mathrm{~S}$ (Fig. 5).

The gas hydrate environment encourages microbial activity. New perspectives on anaerobic methane oxidation result from studies in which methane represents the entire hydrocarbon pool (Valentine and Reeburgh, 2000). However, methane may be less abundant relative to total $\mathrm{C}_{2}-\mathrm{C}_{5}$ hydrocarbons in sediment of some Gulf slope chemosynthetic communities associated with structure II gas hydrate (Sassen et al., 
1999b). Fatty acid biomarkers strongly depleted in ${ }^{13} \mathrm{C}$ (Zhang et al., 2002) and RNA characterization (Lanoil et al., 2001) suggest the presence of methanogens (Archaea) in sediment associated with gas hydrate and chemosynthetic communities, where independent geochemical evidence of methane oxidation is compelling. However, there is also geochemical evidence that the $\mathrm{C}_{2}-\mathrm{C}_{5}$ hydrocarbons may be altered by microbial oxidation (Sassen et al., 1999b) in the same anaerobic setting.

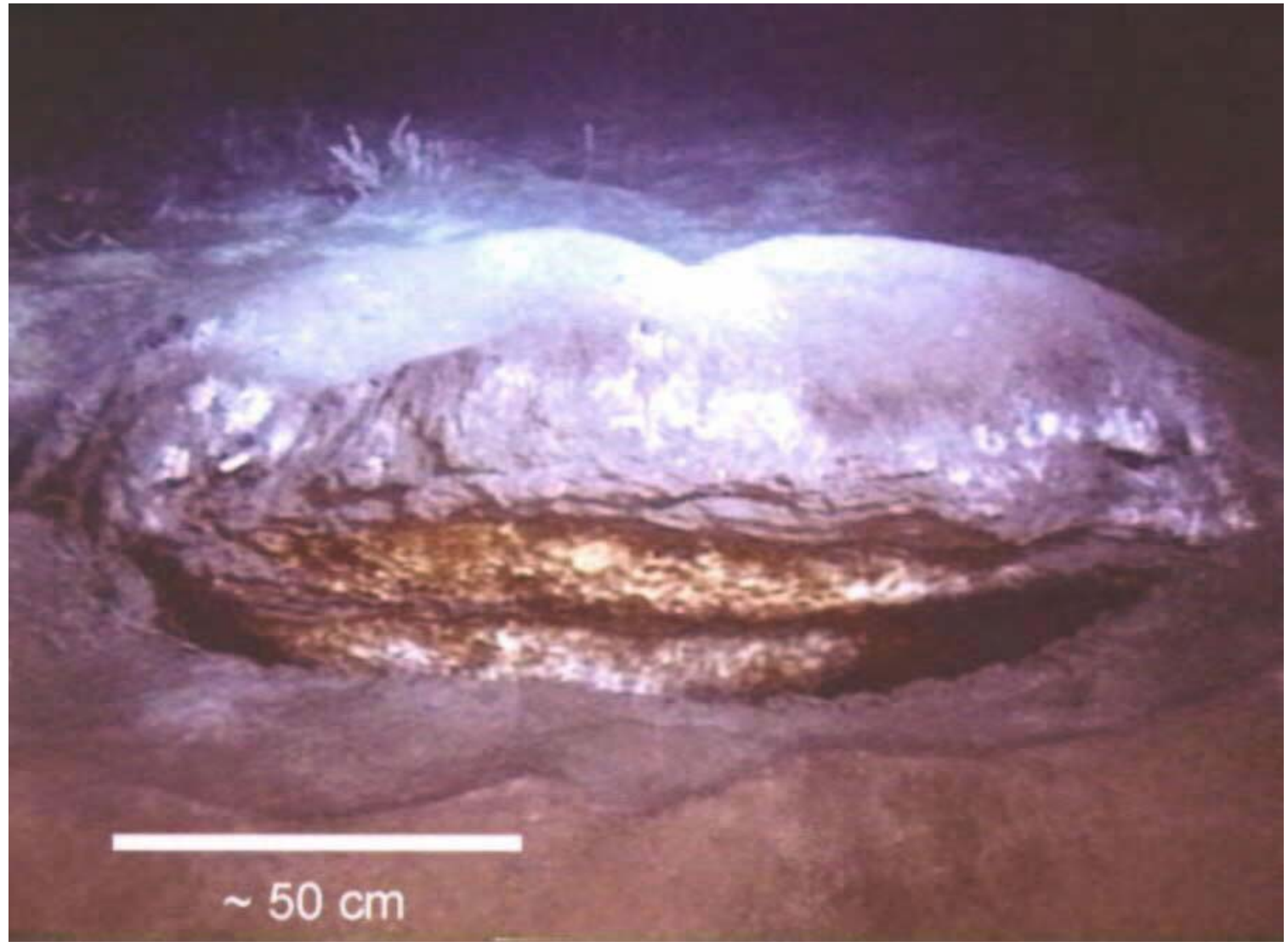

Figure 6. Photograph of structure II gas hydrate on a freshly exposed fault surface at GC 234. Massive vein-fillings of gas hydrate are separated by deformed hemipelagic mud, and the base of the exposure is under cut by flow of free gas. Note scale bar. Photograph by C.F. Fisher.

\section{EXPERIMENTAL}

Vent gas was collected using a research submersible by allowing the gas to displace ambient seawater in gas-tight sampling vessels within 0.5 to $1 \mathrm{~m}$ of the sea floor. Upon recovery to the sea surface, large aliquots of the gas sample $(\sim 240 \mathrm{ml})$ were transferred from the sampling vessel to pre-evacuated aluminum vacuum containers using a $60 \mathrm{ml}$ gas-tight syringe, and held at $-20^{\circ} \mathrm{C}$ until analysis. Sediment samples were collected using $30-\mathrm{cm}$ push cores on the sea floor. Sediment was immediately canned and frozen for later gas analysis. Analytical procedures for $\mathrm{C}_{1}-\mathrm{C}_{5}$ gas chromatography, and measurement of isotopic properties of hydrocarbon vent gases, and $\mathrm{C}_{15}+$ chromatography of oil from sediment are described by Sassen et al. (2004). An aliquot of sediment was 
frozen at collection, and later extracted in the laboratory with hexane (Soxhlet apparatus, 24 hours) to yield solvent-soluble components, mainly a mixture of crude oil with minor recent microbial lipids. The technique of Kennicutt et al. (1992) was used for $\mathrm{C}_{15}+$ chromatography of sediment extracts. Biomarker analyses were also performed by gas chromatography mass spectrometry (GCMS) using the techniques described by Sassen et al. (2001d). Authigenic carbonate, other authigenic minerals, and associated microbes were collected using the robot arm of the JSL research submersible. The d13C of carbonate was determined by Dr. Ethan Grossman of the Department of Geology and Geophysics, Texas A\&M University. Precision of $\mathrm{C}_{1}-\mathrm{C}_{5}$ gas chromatography is $\pm 5 \%$. The ${ }^{\mathrm{TM}{ }^{13} \mathrm{C}}$ values are reported as parts per thousand (\%o) relative to the PeeDee Belemnite standard (precision $= \pm 0.2 \%$ ), and the ${ }^{\mathrm{TM} D}$ values are reported as \%o relative to the standard mean ocean water $(\mathrm{SMOW})$ standard (precision $= \pm 5 \%$ ).

\section{RESULTS AND DISCUSSION}

\section{DESCRIPTIONS OF JSL SITES WITH GAS HYDRATE}

\section{Green Canyon 234}

1. GC 234 hydrate site is a useful case history with abundant gas hydrate: The GC 234 site has been well-documented by previous studies and serves as a useful example of a typical gas hydrate accumulation in the Gulf of Mexico slope. The location is $\left(27^{\circ} 44.8^{\prime} \mathrm{N}\right.$ and $\left.91^{\circ} 13.3^{\prime} \mathrm{W}\right)$. Research on the GC 234 site is extensive and shows great similarity to the better studied Bush Hill site (GC 185) which is nearby $\left(27^{\circ} 45.7^{\prime} \mathrm{N}\right.$ and $\left.91^{\circ} 30.5^{\prime} \mathrm{W}\right)$. Refer to Sassen et al. (2004) for an updated comparison of the GC 234 and Bush Hill gas hydrate sites. Water depth of both sites is $\sim 540$ meters and temperatures vary in the approximate $6-11^{\circ} \mathrm{C}$ range. Salinity of sediment pore water shows variation but overall is similar to that of sea water except in immediate proximity to gas hydrate surfaces.

2. Method of discovery: The first identification of the site was based on recovery of intact gas variant structure II gas hydrate by a piston core directed at a sea floor geophysical anomaly. No bottom simulating reflector (BSR) is detected although this is a major gas hydrate site. The finding by piston core led to further study by research submersibles. A gas hydrate outcrop literally covered with "ice worms" was described in 1997 by Fisher et al. (2000).

3. Gas Hydrate fabric: Gas hydrate outcrops on the sea floor where it forms as a consequence of active venting of oil-related gas from the deep subsurface. Veins and pavements of pure gas hydrate are up to tens of centimeters in thickness are most obvious (Fig. 6). Gas hydrate also occurs as nodules in sediment. Maximum depth in sediment known from piston cores of gas hydrate is $>4$ meters.

4. Gas Hydrate stability: In conformity with stability models (Milkov and Sassen, 2000; Milkov et al., 2000), water depth and temperatures suggest thermogenic gas hydrate (structure II and H hydrate) is stable while biogenic methane (structure I) is not. No structure I gas hydrate has been found at the GC 234 site.

5. Effects of water temperature fluctuations. The upper 0-2 meters of the gas hydrate is relatively unstable because of changes in sea water temperature (Fig 6; Milkov 
et al., 2000). Deeper gas hydrate is believed to be stable geologically. Research suggests that from the geologic perspective, GC 234 is not at equilibrium. Instead, venting drives gas hydrate accumulation. The volume of gas hydrate is increasing slowly at this point in geologic time as at the Bush Hill site (Sassen et al., 2001a). There is no geochemical evidence of significant gas hydrate decomposition below the shallow zone of instability ( $\sim 0$ to 2 meters).

6. Geologic occurrence: The gas hydrate occurs as veins and as laterally extensive pavements of nearly pure gas hydrate that apparently extend as a circular feature $\sim 100$ meters in diameter as determined from sea floor navigation and by $38 \mathrm{kHz}$ imagery of the sea floor. The host sediment is $\sim 100 \%$ hemipelagic clay with no detectably sand. Some hydrate occupies vuggy porosity in authigenic carbonate rock. No discrete sand is present. Salinity of sediment pore water varies but approximates that of normal sea water. The site is old, perhaps thousands of years in age.

7. Gas venting: The hydrate forms when thermogenic (oil-related $\mathrm{C}_{1}-\mathrm{C}_{5}$ hydrocarbons) gas vents from the deep surface petroleum system and the hydrocarbon gases react with water to crystallize as a mineral. The area shows a near-surface geophysical anomaly and is remotely sensed by $38 \mathrm{kHz}$ imagery (Fig. 7) which is consistent with an intermediate level of fluid flux including deep formation water, gas, and oil (see Roberts and Carney, 1997).

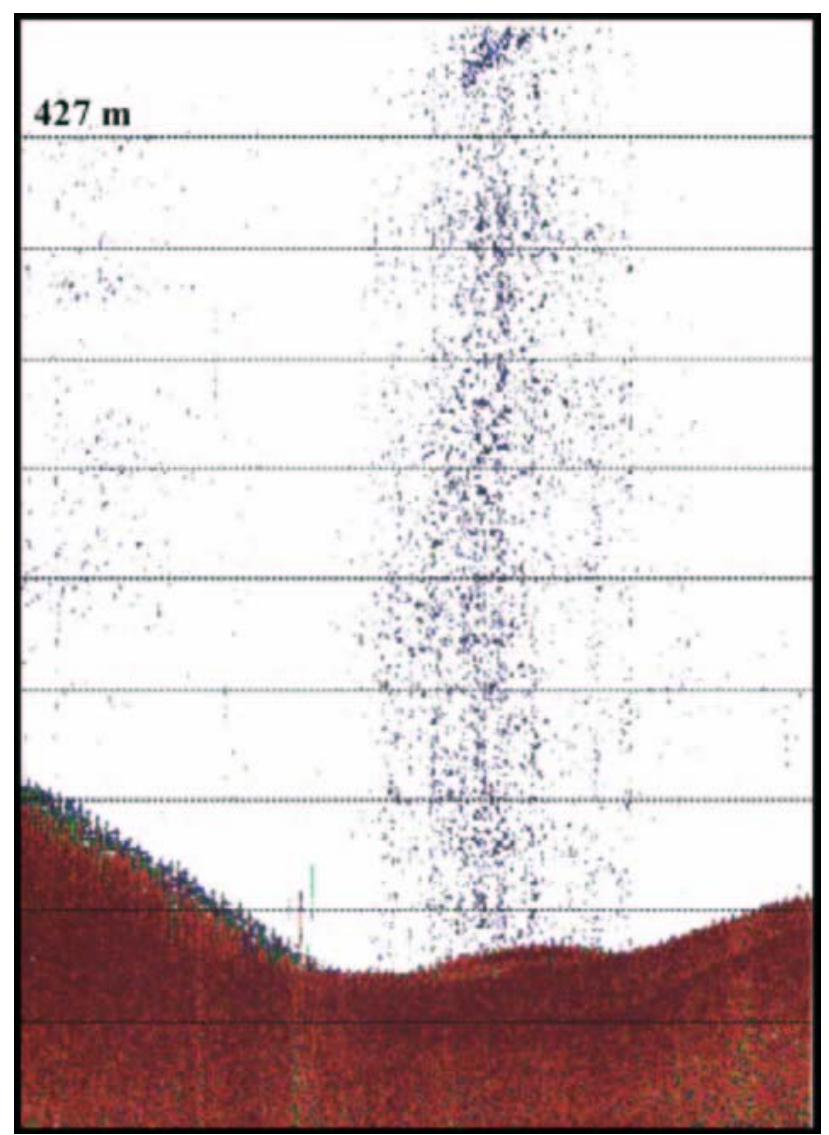

Figure 7. The $38 \mathrm{kHz}$ image shows rapid gas venting to the water column from the GC 234 site. 
8. Hydrocarbon Geochemistry: Thermogenic hydrocarbons vent from the deepsubsurface petroleum system to the sea floor. The $\mathrm{C}_{1}-\mathrm{C}_{5}$ hydrocarbons plus biodegraded crude oil vent from the deep subsurface through gas hydrate mounds $\sim 0.5$ to 1 meter across. Sediment is oil-stained. A review of data on gas hydrate, vent gas, and sediment samples is provided by Sassen et al. (2004).

9. Ecology: The GC 234 site has a lush chemosynthetic community dominated by white and orange bacterial mats (Beggiatoa), tube worms and mussels plus numerous other species similar to Bush Hill.

10. JSL samples: During JSL dives 4406 and 4407 in 2002, new samples of vent gas and intact gas hydrate were recovered intact from the sea floor. Push-core samples of gassy mud were collected at depths $(<30 \mathrm{~cm})$ in sediment. Authigenic carbonate rock samples were also collected.

\section{Green Canyon 232}

1. GC 232 hydrate site is a useful case history with abundant gas hydrate: The existence of GC 232 has been known for some time from piston cores (unpublished data) but no samples of vent gas or gas hydrate had been collected or subjected to molecular and isotopic analyses prior to the 2002 JSL dives. In many respects, GC 232 is a "twin" of the nearby GC 234 and Bush Hills sites, only being smaller in size. The location is $\left(27^{\circ} 44.5^{\prime} \mathrm{N}\right.$ and $\left.91^{\circ} 19.1^{\prime} \mathrm{W}\right)$. Water depth is $~$ 570 meters. A variant structure II gas hydrate with a component of structure $\mathrm{H}$ hydrate (a crystal intergrowth) occurs at GC 232.

2. Method of discovery: The first identification of the site was based on recovery of intact gas variant structure II gas hydrate by a piston core directed at a sea floor geophysical anomaly (unpublished data). No BSR is detected although this is a major gas hydrate site. The finding of gas hydrate by piston core led to further study by research submersibles.

3. Gas Hydrate fabric: Gas hydrate outcrops on the sea floor where it forms as a consequence of active venting of oil-related gas from the deep subsurface. Veins and pavements of pure gas hydrate are up to tens of centimeters in thickness are most obvious because they are exposed at the sea floor. Gas hydrate also occurs as isolated nodules in sediment and filling porosity in authigenic carbonate rock. Maximum depth of gas hydrate in sediment is unknown because no deeppenetration coring has been done at the site.

4. Gas Hydrate stability: Water depth and temperatures suggest thermogenic gas hydrate (structure II and $\mathrm{H}$ hydrate) is stable. Gas hydrate composed of pure biogenic methane (structure I) is not. Structure II/H gas hydrate has been recovered intact but no structure I gas hydrate has been found at the GC 232 site. The site is relatively old, perhaps thousands or tens of thousands of years in age.

5. Effects of water temperature changes: The upper 1-2 meters of the gas hydrate is relatively unstable because of changes in sea water temperature (see Milkov et al., 2000). This is readily apparent between dives because the morphology of gas hydrate outcrops changes in response to changes in water temperature. Deeper gas hydrate is believed to be stable geologically. The volume of gas hydrate is thought 
to be increasing slowly at this point in geologic time as at the Bush Hill site (Sassen et al., 2001a).

6. Geologic occurrence: The gas hydrate occurs as mounds, veins and as laterally extensive pavements of nearly pure gas hydrate that apparently extend as a circular feature $\sim 50$ to 75 meters in diameter as estimated from sea floor navigation and by $38 \mathrm{kHz}$ imagery of the sea floor. The host sediment is $\sim 100 \%$ hemipelagic clay with no detectable sand. Salinity is normal. Sediment contains abundant $\mathrm{H}_{2} \mathrm{~S}$ and is sometimes visibly oil-stained.

7. Gas venting: The hydrate forms when thermogenic (oil-related $\mathrm{C}_{1}-\mathrm{C}_{5}$ hydrocarbons) gas vents from the deep surface petroleum system and the hydrocarbon gases react with water to crystallize as a mineral. The area shows a near-surface geophysical anomaly and is remotely sensed by $38 \mathrm{kHz}$ imagery which shows a reproducible bubble plume in the water column (Fig. 8). Fluid including deep formation water, gas, and oil are introduced to shallow sediment and the water column. Flux of fluid is intermediate according to the classification of Roberts and Carney (1997).

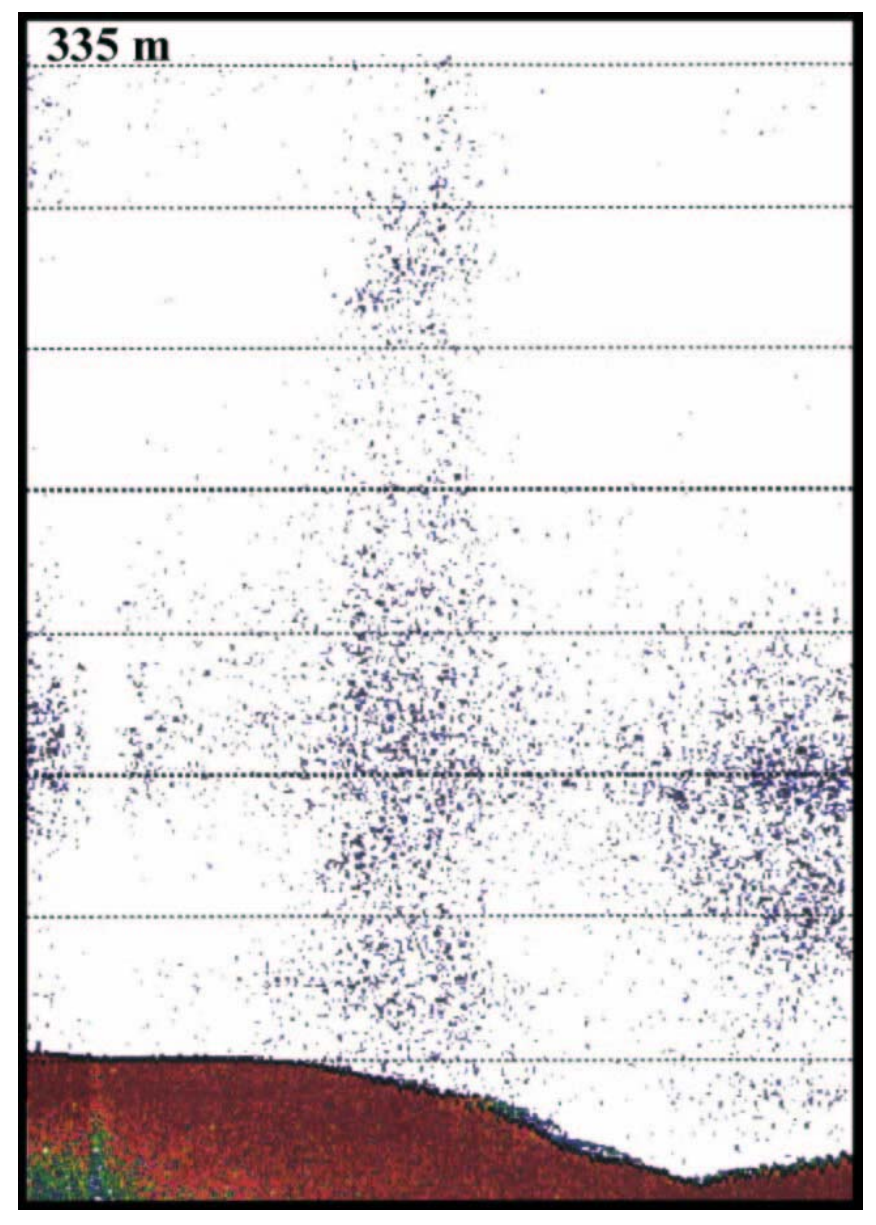

Figure 8 . The $38 \mathrm{kHz}$ imagery shows gas venting to the water column from the sea floor at GC 232. 
8. Ecology: The GC 232 site has a lush chemosynthetic community dominated by white and orange bacterial mats (Beggiatoa), tube worms and mussels plus numerous other species similar to both GC 234 and to Bush Hill. Ice worms were noted at the site in direct association with gas hydrate.

9. JSL samples: During JSL dives in 2002 (4401, 4402/4403, 4404, 4405 and later dives on other JSL cruises that year), new samples of vent gas and intact gas hydrate were recovered intact from the sea floor. Push-core samples of gassy mud were collected at depths $(<30 \mathrm{~cm})$ in sediment. Authigenic carbonate rock samples were also collected.

\section{Mississippi Canyon 118}

1. The MC 118 site contains significant gas hydrate: The MC 118 site has received little scientific study previously. Because the site is in an area distant from other known gas hydrate discoveries, the hydrocarbons from the MC 188 site may be from a different source rock and petroleum system that gave rise to gas hydrate in Green Canyon. MC 118 is the easternmost discovery of gas hydrate in the Gulf of Mexico slope. The location of the MC 118 site is $\sim 28^{\circ} 51.4^{\prime} \mathrm{N}$ and $88^{\circ} 29.5^{\prime} \mathrm{W}$. Maximum water depth at the site during JSL dives is $\sim 890$ meters. No samples of vent gas or gas hydrate had been collected or subjected to molecular and isotopic analyses prior to the 2002 JSL dives.

2. Method of discovery: The first identification of the site was on the basis of a sea floor geophysical anomaly in the form of bright amplitudes near the sea floor (unpublished data). There are no reported BSR's near the known hydrate site at MC 118. The geophysical finding led to further study by research submersible and in 2003 by the U.S. Navy nuclear research submarine NR-1 in a grant to Roger Sassen.

3. Gas Hydrate fabric: Gas hydrate outcrops on the sea floor where it forms as a consequence of slow venting of oil-related gas from the deep subsurface. Veins of pure gas hydrate outcrop in mud. Gas hydrate also occurs as small nodules in sediment. Maximum depth of gas hydrate in sediment is unknown.

4. Gas Hydrate stability: The upper 1-2 meters of the gas hydrate is relatively unstable because of changes in sea water temperature, but hypothesized deeper gas hydrate is believed to be stable over a relatively short span of geologic time, perhaps thousands of years.

5. Geologic occurrence: The gas hydrate occurs as mounds, veins of nearly pure gas hydrate that forms a circular sea floor feature only $\sim 25$ meters in diameter as estimated from sea floor navigation and by a later dive to the site by the U.S. Navy NR1 nuclear research submarine (unpublished data). The host sediment is $\sim 100 \%$ hemipelagic clay with no detectably sand. Authigenic carbonate rock is present. $\mathrm{H}_{2} \mathrm{~S}$ is present in sediment. Salinity is normal.

6. Gas venting: The hydrate forms when thermogenic gas rapidly vents from the deep surface petroleum system and the hydrocarbon gases react with water to crystallize structure II gas hydrate. Fluid flux, including deep formation water, gas, and oil is low to intermediate. Sediment is visibly oil-stained. 
7. Ecology: The MC 118 site has a complex chemosynthetic community dominated by bacterial mats (Beggiatoa), tube worms and mussels plus other species similar to both GC 234 and to Bush Hill.

8. JSL samples: During JSL dives in 2002, new samples of vent gas and intact gas hydrate were recovered from the sea floor. Push-core samples of gassy mud were collected in sediment $(<30 \mathrm{~cm})$.

\section{DESCRIPTIONS OF JSL SITES WITHOUT GAS HYDRATE}

\section{Mississippi Canyon 709}

1. The MC 709 site, although well studied, is not proven to contain gas hydrate: The site is characterized by intermediate flux gas and oil seepage and by complex chemosynthetic communities. The MC 709 area consists of a sea floor high over shallow salt that is draped with hemipelagic mud. The location is $\sim 28^{\circ} 13.9^{\prime} \mathrm{N}$ and $89^{\circ} 42.5^{\prime} \mathrm{W}$. Maximum water depth at the site during JSL dives is $\sim 658$ meters. Sea floor temperature is recorded at $6.8^{\circ} \mathrm{C}$ on JSL dive 4412 . Sea water salinity is normal but many brine seeps and brine pools are present in sediment at the site.

2. Method of discovery: The MC 709 site was first characterized as a gas and oil seep site by piston core and has been studied by research submersible for over a decade (see Roberts and Carney, 1997).

3. Gas hydrate stability: Free gas is present at the MC 709 site but no gas hydrate has been proven to occur there. Given water depth and temperature, all three types of gas hydrate are potentially stable in sediment at MC 709. Application of $38 \mathrm{kHz}$ imagery to detect significant gas bubble trains in the water column has been unsuccessful. The apparent lack of gas hydrate at the MC 709 site may relate to brine poisoning that retards the crystallization of gas hydrate. Gas may occur in high concentrations and oil-staining occurs in sediment.

4. Geologic occurrence: The host sediment is largely hemipelagic clay with no sand. Authigenic carbonate rock is present in abundance. Salinity appears to vary widely across the site as a consequence of active dissolution of the underlying salt. However, areas with high flux seepage are associated with extensive brine pools and flows of dense brine that erode gullies down slope.

5. Gas venting: Fluid flux from the deep subsurface is intermediate according to the classification of Roberts and Carney (1997). Small but active vents of gas are observed at several sites and the concentration of oil in sediments is sometimes high.

6. Ecology: The MC 709 site is characterized by white bacteria mats (Beggiatoa) and both chemosynthetic tubes worms and mussels. In brine seep areas, mussels may predominate and tube worms are absent. Gorgonians and coral are also present on carbonate outcrops along the periphery of the site. The site has a complex chemosynthetic community with some bacterial mats (Beggiatoa), tube worms and mussels, but the area is dissimilar to the classic chemosynthetic communities at GC 234, GC 232, and GC 185. 
7. JSL samples: During JSL dives late in $2002(4412,4413)$ samples of sediment were recovered intact from the sea floor using push-cores $(<30 \mathrm{~cm})$. Authigenic carbonate rock samples were also collected.

\section{Mississippi Canyon 885}

1. The MC 885 site contains no known gas hydrate: The MC 885 is a large diameter site composed of hemipelagic mud, $\mathrm{H}_{2} \mathrm{~S}$, and abundant authigenic carbonate rock. The location is $\sim 28^{\circ} 04.1^{\prime} \mathrm{N}$ and $89^{\circ} 43.3^{\prime} \mathrm{W}$ ). Maximum water depth at the site during JSL dives is $\sim 708$ meters. Sea floor temperature is recorded at $6.8^{\circ} \mathrm{C}$ on JSL dive 4409.

2. Method of discovery: The first identification of the site was on the basis of a sea floor geophysical anomaly (unpublished data). The geophysical finding led to further study by research submersible because of its unique biology.

3. Gas Hydrate stability: Free gas is present at the MC 885 site but no gas hydrate has been proven to occur there. Given water depth and temperature, all three types of gas hydrate are potentially stable. Application of $38 \mathrm{kHz}$ imagery to detect significant gas bubble trains in the water column has been unsuccessful. The apparent lack of gas hydrate at the MC 885 site may relate to insufficient hydrocarbon gas flux (Fig. 9) or to brine poisoning. Small volumes of gas and relatively low concentrations of oil occur in sediment.

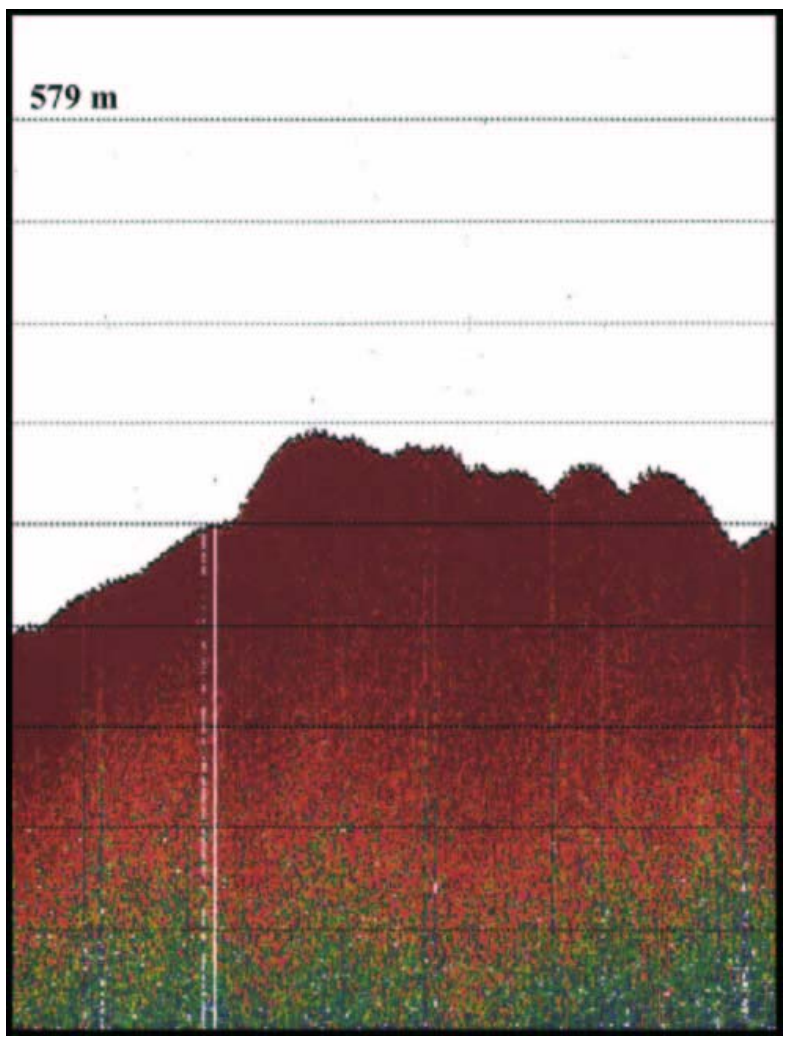

Figure 9. The $38 \mathrm{kHz}$ imagery of MC 885 with no gas vents resolved. 
4. Geologic occurrence: The host sediment is largely hemipelagic clay with no sand. Authigenic carbonate rock is present in unusually high abundance as is shell hash of chemosynthetic bivalves. Salinity may vary widely.

5. Gas venting: Fluid flux from the deep subsurface is slow according to the classification of Roberts and Carney (1997). Because the area is not mineralized by carbonate depleted in ${ }^{13} \mathrm{C}$, it may represent a relict seep site active in the near geologic past, perhaps during the Pleistocene.

6. Ecology: The MC 885 is highly mineralized and gorgonians and coral are quite common. The site has a complex chemosynthetic community with some bacterial mats (Beggiatoa), tube worms and mussels, but the area is dissimilar to the classic chemosynthetic communities associated with gas hydrate.

7. JSL samples: During JSL dives in $2002(4408,4409)$ samples of sediment were recovered intact from the sea floor using push-cores $(<30 \mathrm{~cm})$. Authigenic carbonate rock samples were also collected.

\section{Viosca Knoll 826}

1. The VK 826 site contains no gas hydrate: The VK 826 is a large diameter mineralized sea floor mound over shallow salt where hemipelagic mud partially covers abundant authigenic carbonate rock at $\sim 29^{\circ} 09.6^{\prime} \mathrm{N}$ and $88^{\circ} 01.3^{\prime} \mathrm{W}$. The authigenic carbonate rock covers a dome-shaped feature on the sea floor related to the crest of an underlying salt mass. Water depth of JSL dives is between $\sim 448$ meters at the crest of the dome and $\sim 560$ meters on the flank of the dome. A water temperature of $9.6^{\circ} \mathrm{C}$ was recorded during JSL dive 4410 .

2. Method of discovery: The first identification of the site was on the basis of a sea floor bathymetric high and as a geophysical anomaly near a sub-commercial oil discovery (unpublished data) followed by dredging of chemosynthetic fauna. The early findings led to further study by research submersible because of its unique biology.

3. Gas Hydrate stability: Free gas is present at the VK 826 site but no gas hydrate has been proven to occur there. Application of $38 \mathrm{kHz}$ imagery to detect significant gas bubble trains in the water column has been unsuccessful. The apparent lack of gas hydrate is found at the site may be partially attributed to insufficient hydrocarbon gas flux but much of the site is too shallow and warm for structure II gas hydrate to be stable. Small volumes of free gas and low concentrations of oil occur in sediment.

4. Geologic occurrence: Geologic structure is determined by an underlying salt body and long-term seepage of hydrocarbons that resulting in a "cap rock" of authigenic carbonate rock depleted in ${ }^{13} \mathrm{C}$. Authigenic carbonate rock is present in unusually high abundance. Shell hash from chemosynthetic bivalves is commonly noted. Salinity appears normal. The site is similar to the GC 140 area (see Roberts and Carney, 1997) where massive carbonate is found as a relic of much more active seepage in the geologic past.

5. Gas venting: Fluid flux from the deep subsurface is slow according to the classification of Roberts and Carney (1997). Because the area is highly 
mineralized by carbonate depleted in ${ }^{13} \mathrm{C}$, the area may represent a relict seep site active in the near geologic past. Dead bivalves and much shell hash are noted, perhaps indicating episodic and localized hydrocarbon venting in the recent past.

6. Ecology: Gorgonians and deep-water corals (including black coral) are common along with various bivalves and other organisms. The VK 826 site displays some bacterial mats (Beggiatoa), tube worms and mussels but the tube worms appear poorly developed. Overall, the site is dissimilar to the classic hydrate-related chemosynthetic communities at GC 234, GC 232, and GC 185.

7. JSL samples: During JSL dives in 2002 (4410, 4411), samples of mud sediment were recovered intact from the sea floor using push-cores $(<30 \mathrm{~cm})$. Authigenic carbonate rock samples were also collected.

\section{GEOCHEMISTRY OF VENT GAS, GAS HYDRATE, AND SEDIMENT GAS}

\section{Molecular Properties}

A vent is defined as a point orifice of fluid flow where water, gas, and oil enter the water column from the sea floor at a detectable rate which sometimes may be so rapid as to affect sea floor geology and create large mud volcanoes. It is necessary to analyze the molecular and isotopic compositions of vent gas because it is the starting material from which gas hydrate crystallizes. Much gas bypasses the sediment. Gas that is held in sediment is rapidly altered by microbial activity to allow development of the unique chemosynthetic communities of the Gulf of Mexico slope (e.g. Sassen et al., 2001b; Sassen et al., 2004)).

From the sphere of the JSL research submersible, vent gas appears as bubble trains of hydrocarbon gas that issue from the sea floor to the water column. Some vents have relatively low slow flux rates and are only detectable by careful visual observation of the sea floor for evidence of small bubble trains issuing from the sea floor to the water column. Other vents have flow rates that are orders of magnitude higher and are quite obvious during observation from the JSL. Some intermediate to high-flux vents may be documented by $38 \mathrm{kHz}$ imagery of the sea floor and water column.

The molecular properties of the vent gas associated with gas hydrate (see Data Appendix) are basically similar at GC 232, GC 234, and MC 118. In all cases, the vent gas is $>90 \%$ methane $\left(\mathrm{C}_{1}\right)$. Ethane $\left(\mathrm{C}_{2}\right)$ is next most abundant, followed by propane $\left(\mathrm{C}_{3}\right)$, the butanes $\left(i-\mathrm{C}_{4}\right.$ and $\left.n-\mathrm{C}_{4}\right)$, and the pentanes ( $i-\mathrm{C}_{5}$ and $n-\mathrm{C}_{5}$ and some neo-pentane). The relative abundance of the vent gas molecules simply decreases with increasing carbon number as is commonly noted in subsurface reservoirs of thermogenic gas of oil fields (e.g. Sassen et al., 2001b).

The molecular properties of samples collected during the 2002 JSL cruise reflects the narrow choice of sites on the upper Gulf slope within the shallow diving limits of the JSL research submersible ( $<1000$ meters) in an area where numerous oil fields have been discovered (Figure 1). The gas found venting to the water column in association with gas hydrate is oil-related and directly correlates with, for example, the subsurface reservoir 
gas of Jolliet Field in Green Canyon (Sassen et al., 2001b). If the vent gas has not leaked directly from reservoirs, it has migrated directly from subsurface conduits of the subsurface petroleum system. Data on one vent gas sample from MC 118 appear unreliable because of contamination with gas hydrate fragments during collection at the sea floor.

\section{Molecular Fractionation during Gas Hydrate Crystallization from Vent Gas}

The molecular properties of vent gas and the dominantly structure II gas hydrate that crystallizes from the vent gas differ greatly because of molecular fractionation (Sloan, 1998). For example, structure II gas hydrate preferentially encloses ethane, propane, and butanes from vent gas within its crystal structure (Fig. 10). On this basis, the relative abundance of methane in gas hydrate decreases in structure II gas hydrate relative to the vent gas from which it crystallized. In the gas hydrates collected from GC 234, GC 232, and MC 118 methane is the single most abundant molecule, followed by either propane or ethane, and then by isobutane which are the normal hydrocarbon constituents of structure II gas hydrate. However, none of the gas hydrate collected during the 2002 JSL cruise displays the ideal composition of pure "structure II" hydrate. The most obvious anomaly is that propane does not always show greater relative abundance than ethane. In addition, all the gas hydrate contains $n$-butane and some pentanes, molecules that are too large for inclusion in the cages of structure II gas hydrate. The anomalous compositions of Gulf of Mexico slope gas hydrate have been recently explained. X-ray diffraction shows that the typical Gulf of Mexico hydrate is a physical mixture of different crystal structures, explaining the observed molecular compositions of gas hydrate.

\section{Isotopes Fingerprint Vent Gas to Associated Gas Hydrate}

Methane gas from the Gulf of Mexico slope shows a wide range of isotopic properties from pure thermogenic methane to pure biogenic methane. The "heaviest" methane thus far measured in the Gulf slope has a $\delta^{13} \mathrm{C}$ of $-30.1 \%$ indicating a deep thermogenic origin, whereas the "lightest methane thus far analyzed has a $\delta^{13} \mathrm{C}$ of $-116.5 \%$ (Sassen et al, 2002). The mean $\delta^{13} \mathrm{C}$ of methane in the Gulf slope $(n=160)$ is $-74.0 \%$. One key observation is that the $\mathrm{C}_{2}-\mathrm{C}_{5}$ hydrocarbon gases and oil are commonly associated with methane, even when the methane is strongly depleted in ${ }^{13} \mathrm{C}$. The best explanation is that methane has different thermogenic and biogenic sources, mixing occurs during migration from depth, and microbial activity impacts isotopic properties either by methane oxidation or via methanogenesis (Sassen et al., 2003).

The $\delta^{13} \mathrm{C}$ of methane from vent gas and gas hydrate from individual sites are essentially the same, generally showing differences of $1 \%$ or less. This is because there is no isotopic fractionation during gas hydrate crystallization (see Sassen et al, 2004 and references therein). For example, the $\delta^{13} \mathrm{C}$ of methane of vent gas from GC 234 varies between $-48.5 \%$ to $-48.4 \%$ whereas that of closely associated gas hydrate is $-48.0 \%$ o (Fig. 10). At GC 232, the $\delta^{13} \mathrm{C}$ of methane of vent gas from varies between $-42.8 \%$ o to $43.0 \%$. Methane of closely associated gas hydrate has $\delta^{13} \mathrm{C}$ of $-42.7 \%$. Similarly, at $\mathrm{MC}$ 118 the $\delta^{13} \mathrm{C}$ of methane of vent gas is $-45.7 \%$ whereas that of closely associated gas 

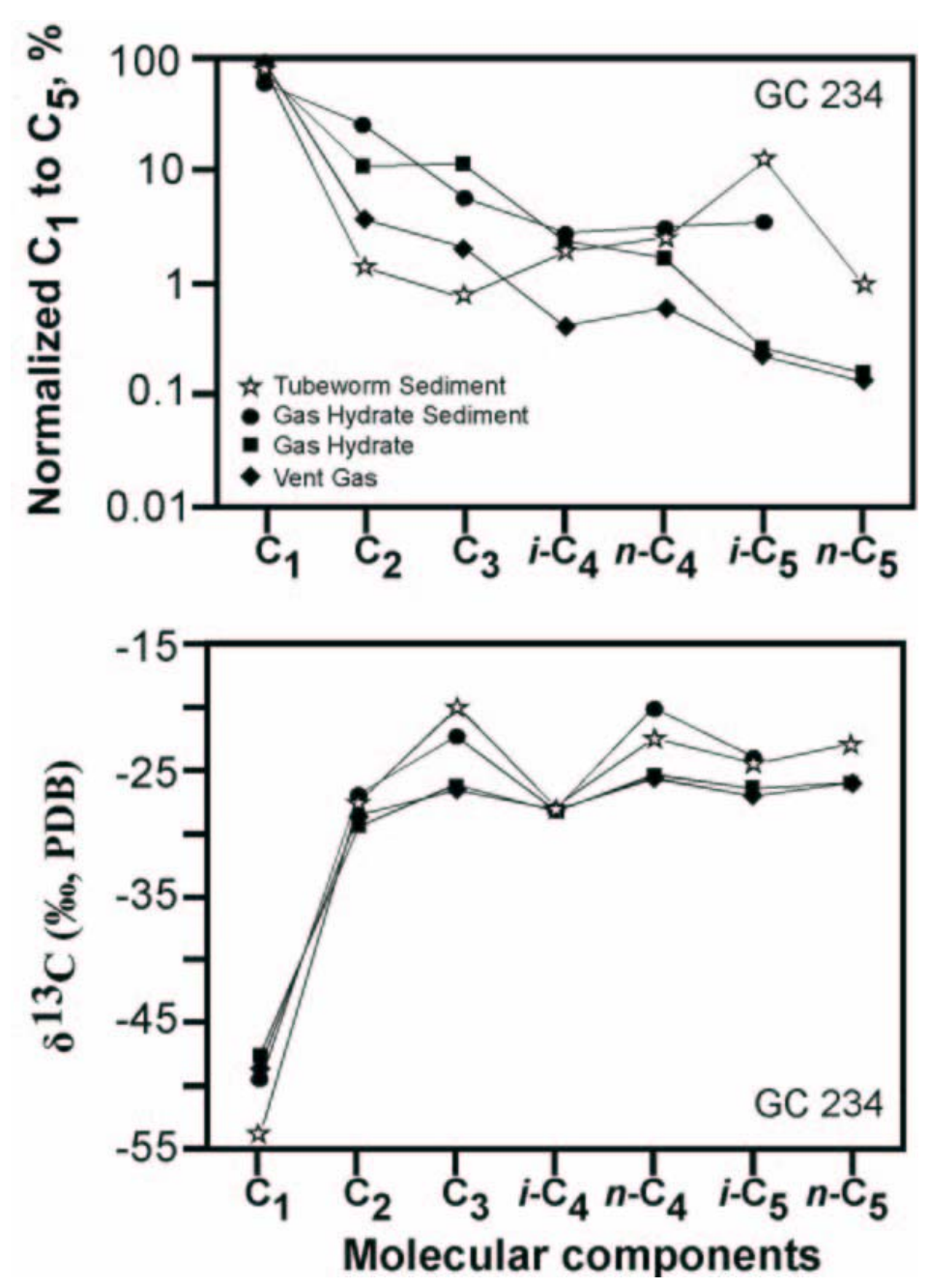

Figure 10. Summary diagrams of molecular and isotopic data from GC 234. Data from Sassen et al. 2004.

hydrate is $-45.8 \%$. The similarity of the hydrogen isotopic properties $(\delta \mathrm{D})$ of methane at each site establishes a correlation between vent gas and associated gas hydrate. Lastly, the $\delta^{13} \mathrm{C}$ of vent gas and hydrate of ethane and propane are so similar as to show a conclusive correlation. The isotopic data provides powerful evidence that at each study site, gas hydrate crystallizes from the adjacent vent gas. Moreover, once held in the crystal structure of gas hydrate, hydrocarbon gases appear protected from microbial oxidation, preserving important geochemical data (Sassen et al., 2001e).

All of the hydrocarbon gases from GC 234, GC 232, and MC 118 are of thermogenic sources, and were formed at great depth and high temperatures from thermally mature source rocks for oil. None of the vent gases encountered on the 2002 JSL cruise contain significant biogenic methane. This is not representative of the Gulf as a whole. There 
are many seeps and vents of fossil biogenic methane, as well as mixtures of biogenic and thermogenic gas in vents in the deep subsurface (see Sassen et al., 2003).

\section{Bacterial oxidation of free hydrocarbon gas in hydrate-associated sediments}

Free hydrocarbon gas in sediment is rapidly destroyed by bacterial oxidation that is coupled with reduction of sulfate to yield the $\mathrm{H}_{2} \mathrm{~S}$ common at many gas hydrate sites. To monitor the fate of hydrocarbons in sediment, canned sediment samples from GC 234, GC 232, and from MC 118 were subjected to analysis of $\mathrm{C}_{1}-\mathrm{C}_{5}$ hydrocarbons by gas chromatography (see Data Appendix). Concentrations of $\mathrm{C}_{1}-\mathrm{C}_{5}$ hydrocarbons show a wide range but are often relatively high at all study sites with gas hydrate. Indeed, concentrations of gas are often so high as to indicate that small nodules of gas hydrate were frequently sampled along with the sediment. For example Core $1(0-10 \mathrm{~cm}$ depth) from dive 4404 to GC 233 shows a headspace gas composition of $992,393 \mathrm{ppm}$. In the Gulf slope, any value $>10 \mathrm{ppm}$ is considered to be anomalous.

Isotopic data suggest methane is the most rapidly oxidized free hydrocarbon gas in hydrate-associated sediment. Much of the free vent methane in sediment samples has been bacterially oxidized and replaced by biogenic methane via methanogenesis. For example, the $\delta^{13} \mathrm{C}$ of methane of vent gas from GC 232 varies between $-42.8 \%$ o to 43.0\% . In contrast, the $\delta^{13} \mathrm{C}$ of free methane gas from hydrate-associated sediment of GC 232 collected during dives 4401 and $4403 / 4404$ varies between $-57.5 \%$ to $-85.9 \%$ in sediment samples. Isotopically-light $\mathrm{CO}_{2}$ is present is the same samples, as light as 44.3\%o. The best explanation for the relatively light methane in sediment at GC 232 is bacterial reduction of $\mathrm{CO}_{2}$ (methanogenesis) to form biogenic methane that mixes with residual thermogenic vent methane. Thus, the biogeochemistry of methane at gas hydrate sites appears extremely complex with multiple sources and bacterial processes that affect isotopic properties of the methane.

Samples of free gas show selective preservation of ethane whereas propane, butanes and some pentanes are greatly decreased in abundance, which is indicative of rapid bacterial oxidation of free hydrocarbon gases in sediment (Sassen et al., 2004).

\section{BIODEGRADATION OF CRUDE OIL}

Gas chromatography is an objective method to estimate the concentration of crude oil hydrocarbons in hydrate-associated sediment and to characterize the degree of crude oil alteration by biodegradation. The $\mathrm{C}_{15}+$ gas chromatography of sediment samples from the gas hydrate sites of GC 234, GC 232, and MC 118 are similar in terms of gross characteristics and all show anomalous concentrations of crude oil (see Data Appendix). The thermogenic gas hydrate sites are thus unambiguously associated with crude oil which probably also plays a role as a carbon source for chemosynthetic communities and provides some variable fraction of the carbonate-carbon for authigenic carbonate rock.

All samples of hydrate-associated sediment show highly anomalous concentrations of crude oil, which vents with gas from the deep subsurface to the study sites. The mean 
concentration of crude oil (UCM) in random sediment samples from across the Gulf slope is $<10$ ppm (Sassen et al., 1999a). However, most sediment samples from the gas hydrate sites of GC 234, GC 232, and MC 118 smelled of crude oil, and in many cases free oil was visible filling fractures in sediment as the samples were preserved at sea. One section from a push-core collected on GC 234 (JSL dive 4407) was characterized by visible oil-staining and total UCM concentration is $57,885 \mathrm{ppm}$. To better communicate this concentration, nearly $6 \%$ of the dry sediment is oil by weight. Similarly, a core from GC 232 (JSL dive 4401) was visibly oily and the concentration of UCM is 25,729 ppm, about $2.5 \%$ of the core by weight. The MC 118 site is similar with much oil staining. One push core from MC 118 (JSL dive 4415) contained 23,048 ppm of UCM, about $2.3 \%$ of total sediment weight.

The saturated hydrocarbons of crude oil from hydrate-associated sediment from GC 234, GC 232, and MC 118 are all highly biodegraded, which is generally characteristic of gas hydrate sites with chemosynthetic communities across the Gulf of Mexico slope. The original $n$-alkanes and isoprenoids present in unaltered crude oil from the sub-surface have been destroyed, leaving an elevated chromatographic baseline called the Unresolved Complex Mixture (UCM). An example of highly biodegraded oil is shown in Figure 11.

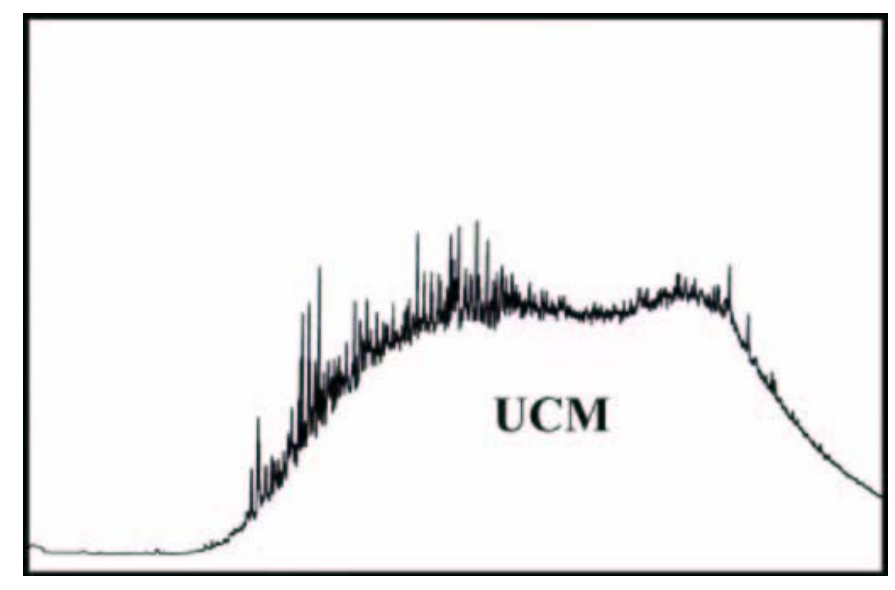

Figure 11. Typical $\mathrm{C}_{15+}$ gas chromatogram of crude oil from GC 234 showing advanced alteration from microbial oxidation.

\section{ISOTOPIC PROPERTIES OF AUTHIGENIC CARBONATE ROCK}

Microbial oxidation of crude oil is associated with bacterial sulfate reduction in hydrateassociated sediment from GC 234, GC 232, and MC 118. The most important products of these linked bacterial processes are $\mathrm{CO}_{2}$ from hydrocarbon oxidation and $\mathrm{H}_{2} \mathrm{~S}$ from bacterial sulfate reduction. The effect of bacterial sulfate reduction is to shift alkalinity in sediment pore water, favoring precipitation of authigenic carbonate rock (e.g. Roberts and Aharon, 1994; Roberts and Carney, 1997; Aharon and Fu, 2000). Authigenic carbonate rock precipitates rapidly, forming massive pavements, slabs, crusts, nodules and cements. The process occurs within mud, not in the water column, but the carbonate rock often encloses the skeletal material from the numerous species of bivalves such as mussels and clams that are abundant in hydrate-associated chemosynthetic communities. 
Visual examination of authigenic carbonate rock from hydrate-associated sediment often shows disarticulated bivalves and shell hash which complicates interpretation of $\delta^{13} \mathrm{C}$ results (see Data Appendix). This is because the carbon from hydrocarbon oxidation in authigenic carbonate rock is strongly depleted in ${ }^{13} \mathrm{C}$ whereas the carbon skeletal material from bivalves and other calcareous organisms is characterized by $\delta^{13} \mathrm{C}$ approximating that of normal marine carbonate. Most samples from the present study are a physical mixture of authigenic carbonate and calcareous skeletal material from chemosynthetic communities.

Ten (10) separate samples of authigenic carbonate rock from GC 234 were subjected to multiple determinations of $\delta^{13} \mathrm{C}$ and the results averaged (see Data Appendix). The range of $\delta^{13} \mathrm{C}$ from GC 234 is from $-18.44 \%$ o to $-27.26 \%$. Six (7) samples from GC 232 showed a range of $\delta^{13} \mathrm{C}$ from $-15.12 \%$ to $-20.15 \%$. The range of $\delta^{13} \mathrm{C}$ values is similar to that measured using three (3) samples collected during JSL dive 4429 at the similar GC 185 gas hydrate site where authigenic carbonate rock is also abundant. The range of carbonate $\delta^{13} \mathrm{C}$ at GC 185 is $-22.38 \%$ to $-22.73 \%$.

In contrast, the $\delta^{13} \mathrm{C}$ values of three (3) samples of authigenic carbonate rock from the GC 233 Brine Pool site from JSL dives later in 2002 is from $-35.02 \%$ to $-32.65 \%$, values that are only marginally lighter than at the gas hydrate sites where thermogenic methane and crude oil dominate as carbon sources to authigenic carbonate rock. At GC 233, biogenic methane $\left(\delta^{13} \mathrm{C}=\sim-60 \%\right.$ ) vents to the water column and is the main source of hydrocarbon-carbon at the site. Thus, there is only a tenuous relationship between hydrocarbon types and $\delta^{13} \mathrm{C}$ of carbonate carbon of authigenic carbonate rock other than to note that they are strongly depleted in ${ }^{13} \mathrm{C}$ relative to normal marine carbonate-carbon. There are multiple sources including methane, other hydrocarbon gases, oil, and skeletal material from bivalves and other organisms.

\section{SITES LACKING GAS HYDRATE}

The 2002 JSL cruise allowed characterization and collection of samples from a number of sites on the Gulf of Mexico slope at which no gas hydrate has been found. It appears that a combination of factors may prevent the crystallization of gas hydrate. Contrary to a superficial interpretation of thermodynamics and the phase rule, free gas is abundant within the GHSZ of the Gulf. This is most obvious when a push-core is acquired or a rock is turned over using the mechanical arm of the JSL research submarine. Mechanical disturbance of the sediment frequently allows release of copious gas bubbles to the water column where it may immediately form "hydrate snow" that slowly floats upwards in the water column. The question to be asked is why does free gas exist within the GHSZ even though excess water is present?

Although the VK 826 and MC 885 sites display obvious geophysical anomalies, no gas hydrate is present. The apparent lack of gas hydrate at the VK 826 and MC 885 sites may be partially attributed to insufficient hydrocarbon gas flux but the carbonate-rich sites are too shallow and warm for structure I or II gas hydrate to be stable. In addition, 
brines are present at both sites that are likely to retard gas hydrate crystallization. The MC 709 is a major geophysical anomaly and may or may not contain any gas hydrate although there are areas with intermediate hydrocarbon flux within the GHSZ. The main reason for the lack of abundant gas hydrate at MC 709 may be brine poisoning. Additional research to explain why gas hydrate fails to crystallize in abundance at sites such MC 709 is justified. As noted earlier, $>160$ gas seeps in the Gulf of Mexico appear to lack gas hydrate (Sassen et al., 2002) so the question is worth answering.

\section{CONCLUSIONS: IMPLICATIONS TO GAS HYDRATE STABILITY AND MONITORING}

It has been uncritically generalized that decomposition of gas hydrate is an important process in nature at this point in geologic time. However, there is no geochemical evidence to support that contention in the Gulf of Mexico. If gas is observed venting from over buried gas hydrate, it is sometimes simply assumed that the vent gas is from decomposition of gas hydrate. However, there are few if any proven examples of geologically significant vent gas derived from gas hydrate decomposition. None are proven in the Gulf of Mexico slope.

Vent gas is better interpreted as the starting material that gives rise to large accumulations of gas hydrate that are at present slowly increasing in size (Sassen et al., 2001b, c). Free gas is constantly migrating from depth and venting to the water column at all studied gas hydrate sites. The end-member gas is either thermogenic $\mathrm{C}_{1}$ to $\mathrm{C}_{5}$ hydrocarbons with oil (Sassen et al., 2001b) or is fossil biogenic methane (Sassen et al., 2003). Thermogenic gas hydrate (structure II) contains $\mathrm{C}_{1}-\mathrm{C}_{5}$ hydrocarbon gases and generally occurs at water depths $>500$ meters under typical conditions in the Gulf. Under typical conditions in the Gulf slope, methane hydrate (structure I) occurs widely at water depths $>650$ meters.

It should be emphasized that the composition of vent gas from all sites thus far studied in the Gulf of Mexico slope is consistent with gas hydrate stability and slow accumulation in sediments over recent geologic time (Sassen et al., 2001e). If gas decomposition of gas hydrate is important, then vent gas would show a molecular distribution enriched in structure II gas hydrate-forming hydrocarbon gases. Vent gas from decomposition of structure II hydrate would show enrichment in ethane, propane, and isobutane. This molecular distribution is not observed, providing strong evidence that gas hydrate is not decomposing at a significant rate at typical gas hydrate sites thus far studied in the Gulf of Mexico slope. Instead, there is evidence at GC 185 that vent gas is preferentially "stripped" of hydrate-forming molecules (ethane, propane, isobutane) during migration to gas hydrate sites (Sassen et al., 2001b). Thus, the molecular properties of vent gas are consistent with a great subsurface thickness of gas hydrate beneath the sea floor sites.

The geochemistry of vent gas supports models of the sub-surface depth and morphology of individual gas hydrate accumulations in the Gulf (Milkov and Sassen, 2003). The single largest gas hydrate accumulation in the Gulf of Mexico slope may be beneath the 
shallow structure II gas hydrate so widespread in Atwater Valley 425 at $~ 1,930$ meters water depth. The thickness of the gas hydrate vein-fillings in mud at the AT 425 site is suggested to be $>1$ kilometer. It is suggested that future study of gas hydrate as a potential alternative source of energy concentrate on the sites predicted to have the largest subsurface volumes of hydrocarbon gas that is concentrated as gas hydrate. Some decomposition of exposed or extremely shallow gas hydrate occurs but deeply buried gas hydrate is thought to be stable in the Gulf of Mexico slope over long spans of time. The models of gas hydrate stability in the Gulf of Mexico by Milkov et al. (2000) are widely accepted. There is an effect on hydrate stability predicted from changes in sea water temperature. It should be emphasized that the sediment has insulating properties, and changes of temperature in the water column are rapidly attenuated with depth in sediment (Milkov et al, 2000).

Models suggest that gas hydrate is unstable only when exposed at the sea floor or at depths in sediment of less $\sim 2$ meters in sediment (Fig. 12). Field observations are consistent with the model that exposed or shallow gas hydrate is affected by changes in the temperature of sea water. The irregular surface of the sea floor at gas hydrate sites is partly the result of sediment deformation caused by cyclical gas hydrate crystallization and decomposition in only a thin layer nearest the sea floor. Thus, seismic evidence of gas hydrate instability is most likely to be observed in the top few meters nearest the sea floor whereas deeper sediment show little or no evidence of change on any short time scale. Moreover, speculations linking gas hydrate decomposition to significant geologic changes such as massive slope failures must be critically examined in the light of geochemical data.

The 2002 JSL study sites with vent gas and outcropping gas hydrate are all similar. The GC 234, GC 232, and MC 118 sites are representative of all other discoveries of gas hydrate thus far made at the sea floor of the Gulf of Mexico slope. Subsurface migration over shallow salt and along faults focuses gas migration to specific sites at the sea floor that are connected to great depth in the sediment section. The known sea floor gas hydrate sites are each characterized by excess hydrocarbon gas that vents from the sea floor and enters the water column. Oil may coat the interiors of gas bubbles contributing to the natural oil slicks observed on the sea surface in association with many gas hydrate sites (Sassen et al., 2001b). The flux of vent gas at hydrate sites is extremely anomalous and can be detected by $38 \mathrm{kHz}$ imagery of the sea floor and water column. A combination of shallow seismic data and $38 \mathrm{kHz}$ imagery may prove to be the most effective tool for gas hydrate exploration in the Gulf of Mexico slope.

Lastly, many seep and vent sites with abundant hydrocarbon gases lack gas hydrate in the Gulf of Mexico slope even though they are in the Gas Hydrate Stability Zone. Seismic amplitudes merely indicate seeps or artifacts that resemble seeps. Although VK 826 and MC 885 display obvious geophysical anomalies, no gas hydrate is present. The apparent lack of gas hydrate at the VK 826 and MC 885 sites may be partially attributed to insufficient hydrocarbon gas flux but the carbonate-rich sites are too shallow and warm for structure I or II gas hydrate to be stable. In addition, brines are present at both sites 
that are likely to retard gas hydrate crystallization. The MC 709 is a major geophysical anomaly and may or may not contain any gas hydrate although there are areas with intermediate hydrocarbon flux within the GHSZ. The main reason for the lack of abundant gas hydrate at MC 709 appears to be brine poisoning.

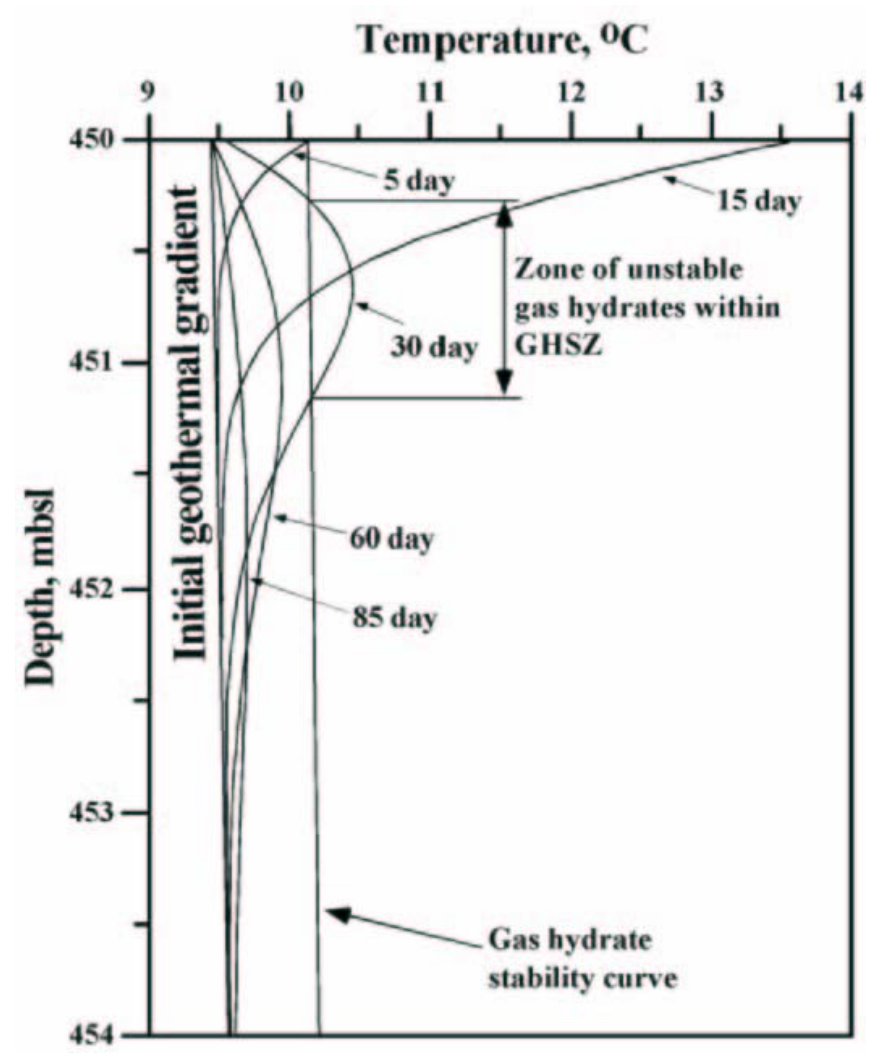

Figure 12. Gas hydrate stability curve showing that, at $450 \mathrm{~m}$ water depth, a warm eddy current may only cause thin-skin decomposition of gas hydrate. Exposed or shallow gas hydrate may be affected, but deeper gas hydrate ( $>2 \mathrm{~m}$ in sediment) is thought to be largely insulated from transient temperature excursions. Diagram from Milkov et al. (2000).

\section{REFERENCES}

Aharon, P., and B. Fu (2000) Microbial sulfate reduction rates and sulfur and oxygen isotope fractionations at oil and gas seeps in deepwater Gulf of Mexico. Geochimica et Cosmochimica Acta 64, 233-246.

Fisher, C.R., I.R. MacDonald, R. Sassen, C.M. Young, S.A. Macko, S. Hourdez, R.S. Carney, S. Joye, and E. McMullin (2000) Methane ice worms: Hesiocaeca methanicola colonizing fossil fuel reserves: Naturwissenschaften, 87, 184-187.

$\mathrm{Fu}, \mathrm{B}$. and P. Aharon (1998) Sources of hydrocarbon-rich fluids advecting on the sea floor in the northern Gulf of Mexico. Gulf Coast Association of Geological Societies Transactions 48, 73-82. 
Galloway, W.E., P.E. Ganey-Curry., X. Li, and R.T. Buffler (2000). Cenozoic depositional history of the Gulf of Mexico Basin. American Association of Petroleum Geologists Bulletin 84: 1743-1774.

Kvenvolden, K.A. (1993) Gas hydrates - geological perspective and global change. Reviews of Geophysics, 31. 173-187.

Kvenvolden, K.A. (1995) A review of the geochemistry of methane in natural gas hydrate. Organic Geochemistry, 23. 997-1008.

MacDonald, I.R., G.S. Boland, J.S., Baker, J.M., Brooks, M.C. Kennicutt, II and R.R. Bidigare (1989) Gulf of Mexico hydrocarbon seep communities II. Spatial distribution of seep organisms and hydrocarbons at Bush Hill. Marine Biology, 101. 235-247.

MacDonald, I.R., J.F. Reilly Jr., S.E. Best, R. Venkataramaiah, R. Sassen, J. Amos and N.L. Guinasso, Jr., (1996). A remote-sensing inventory of active oil seeps and chemosynthetic communities in the northern Gulf of Mexico. In: D. Schumacher and M.A. Abrams (Editors), Hydrocarbon migration and its near-surface expression. AAPG Memoir 66, pp. 27-37.

Milkov, A.V. and R. Sassen (2003) Preliminary assessment of resources and economic potential of individual gas hydrate accumulations in the Gulf of Mexico continental slope: Marine and Petroleum Geology. 20, 111-128.

Milkov, A.V. and R. Sassen (2002) Economic geology of offshore gas hydrate accumulations and provinces. Marine and Petroleum Geology 19: 1-11.

Milkov, A.V. and R. Sassen, (2001) Estimate of gas hydrate resource, northwestern Gulf of Mexico continental slope. Marine Geology, 179: 71-83.

Milkov, A.V., and R. Sassen (2000) Thickness of the gas hydrate stability zone, Gulf of Mexico continental slope: Marine and Petroleum Geology, 17, 981-991.

Milkov, A.V., R. Sassen, I. Novikova, and E. Mikhailov (2000) Gas hydrates at minimum stability water depths in the Gulf of Mexico: Significance to geohazard assessment:

Transactions Gulf Coast Association of Geological Societies.50, 217-224.

Roberts, H.H., and R. S. Carney (1997) Evidence of episodic fluid, gas, and sediment venting on the northern Gulf of Mexico slope. Economic Geology 92, 863-879.

Roberts, H.H. and P. Aharon, (1994) Hydrocarbon-derived buildups of the northern Gulf of Mexico: A review of submersible investigations. Geo-Marine Letters, 14: 135-148.

Roberts, H.H. and R. Carney, R. (1997) Evidence of episodic fluid, gas, and sediment venting on the northern Gulf of Mexico slope. Economic Geology, 92: 863-879.

Salvador, A. (1987). Late Triassic-Jurassic paleogeography and origin of Gulf of Mexico 
basin. American Association of Petroleum Geologists Bulletin 71: 419-451.

Sassen, R., H. H., Roberts, R. Carney, A. V. Milkov, D. A. DeFreitas, B. Lanoil, and C. Zhang (2004) Free hydrocarbon gas, gas hydrate, and authigenic minerals in chemosynthetic communities of the northern Gulf of Mexico continental slope: relation to microbial processes: Chemical Geology v. 205, Issues 3-4, p. 195-217.

Sassen, R., A.V. Milkov, E. Ozgul, H.H. Roberts, J. Hunt, M.A. Beeunas, J. Chanton, S.T. Sweet, and D. A. DeFreitas (2003) Gas venting and subsurface charge in the Green Canyon area, Gulf of Mexico continental slope: evidence of a deep bacterial methane source? Organic Geochemistry. 34, 1555-1464.

Sassen, R., A.V. Milkov, D.A. DeFreitas, and S.T. Sweet (2002) Molecular and isotopic properties of high-flux gas seeps, northwestern Gulf of Mexico: AAPG Annual Meeting, Abstracts, v. 11, p. A. 155.

Sassen, R., S.T. Sweet, A.V. Milkov, D.A. DeFreitas, and M.C. Kennicutt II (2001a) Thermogenic vent gas and gas hydrate in the Gulf of Mexico slope: Is gas hydrate decomposition significant? : Geology, v. 29, p. 107-110.

Sassen, R., S. L. Losh, L. Cathles III, H. H. Roberts, J. K. Whelan, A.V. Milkov, S. T. Sweet, and D.A. DeFreitas (2001b) Massive vein-filling gas hydrate: relation to ongoing gas migration from the deep subsurface in the Gulf of Mexico: Marine and Petroleum Geology, v. 18, p. 551-560.

Sassen, R., S.T. Sweet, A.V. Milkov, D.A. DeFreitas and M.C. Kennicutt II (2001c) Stability of thermogenic gas hydrate in the Gulf of Mexico: constraints on models of climate change. In: C.K. Paull and W.P. Dillon (Editors), Natural Gas Hydrates: Occurrence, Distribution, and Detection. American Geophysical Union, Washington D.C., pp.131-143.

Sassen, R., S.T. Sweet, D.A. DeFreitas, J.A. Morelos, and A.V. Milkov (2001d). Gas hydrate and crude oil from the Mississippi Fan Foldbelt, downdip Gulf of Mexico salt basin: significance to petroleum system. Organic Geochemistry 32:999-1008.

Sassen, R., S.T. Sweet, A.V. Milkov, D.A. DeFreitas, and M.C. Kennicutt II (2001e). Thermogenic vent gas and gas hydrate in the Gulf of Mexico slope: Is gas hydrate decomposition significant? Geology 29: 107-110.

Sassen, R., S.T. Sweet, A.V. Milkov, D.A. DeFreitas, G.G. Salata, and E.C. McDade, E (1999a) Geology and geochemistry of gas hydrates, central Gulf of Mexico continental slope. Gulf Coast Association of Geological Societies Transactions 49: 462-468. Sassen, R., S. Joye, S.T. Sweet, D.A. DeFreitas, A.V. Milkov, and I.R. MacDonald (1999b). Thermogenic gas hydrates and hydrocarbon gases in complex chemosynthetic communities, Gulf of Mexico continental slope. Organic Geochemistry 30: 485-497. 
Sassen, R. and I.R. MacDonald (1997) Hydrocarbons of experimental and natural gas hydrates, Gulf of Mexico continental slope. Organic Geochemistry 26, 289-293.

Sassen, R., I.R. MacDonald, A.G. Requejo, N.L. Guinasso, Jr, M.C. Kennicutt II, S.T. Sweet, and J.M. Brooks (1994) Organic geochemistry of sediments from chemosynthetic communities, Gulf of Mexico slope: Geo-Marine Letters 14, 110-119.

Sassen, R., H.H. Roberts, P.A. Aharon, A., J. Larkin, E.W. Chinn and R. Carney (1993) Chemosynthetic bacterial mats at cold hydrocarbon seeps, Gulf of Mexico continental slope. Organic Geochemistry 20: 77-89.

Sloan, E.D., 1998. Clathrate hydrates of natural gases, (2nd edition). New York, Dekker, $705 \mathrm{p}$.

Valentine, D.L. and W.S. Reeburgh (2000) New perspectives on anaerobic methane oxidation. Environmental Microbiology, 2 (5). 477-484.

Worrall, D.M. and Snelson, S. (1989) Evolution of the northern Gulf of Mexico with emphasis on Cenozoic growth faulting and the role of salt: In A.W. Bally and E.R. Palmer (Editors), The geology of North America - An overview: Boulder, Colorado, Geological Society of America, v. A, 97-138.

Zhang, C.L., Y. Li, J.D. Wall, J.D., L. Larsen, R. Sassen, Y. Huang, Y. Wang, A Peacock, D.C. White, J. Horita,. and D.R. Cole (2002) Lipid and carbon isotopic evidence of methane-oxidizing and sulfate-reducing bacteria in association with gas hydrates from the Gulf of Mexico. Geology 30 (3), 239-242.

\section{BIBLIOGRAPHY}

Brooks, J. M., M.C. Kennicutt II, R.R. Fay, T.J. McDonald, and R. Sassen (1984) Thermogenic gas hydrates in the Gulf of Mexico. Science 225: 409-411.

Brooks, J.M., H.B. Cox, W.B. Bryant, M.C. Kennicutt II, R.G. Mann, and T.J. McDonald, T.J. (1986) Association of gas hydrates and oil seepage in the Gulf of Mexico. Organic Geochemistry 10: 221-234.

Carney, R.S. (1994) Consideration of the oasis analogy for chemosynthetic communities at Gulf of Mexico hydrocarbon vents. Geo-Marine Letters 14: 149-159.

Cook, D. and P. D’Onfro (1991) Jolliet Field thrust structure and stratigraphy, Green Canyon Block 184, offshore Louisiana. Gulf Coast Association of Geological Societies Transactions 41, 100-121.

Coleman, D. D., J.B. Risatti, and M. Schoel (1981) Fractionation of carbon and hydrogen isotopes by methane oxidizing bacteria. Geochimica et Cosmochimica Acta 45, 10331037. 
Fisher, C.R., I. Urcuyo, M.A. Simpkins, and E. Nix (1997) Life in the slow lane: growth and longevity of cold-seep vestimentiferans. Marine Ecology 18, 83-94.

Julian, D., F. Gaill, E. Wood, A.J. Arp, A.J., and C.R. Fisher (1999) Roots as a site of hydrogen sulfide uptake in the hydrocarbon seep vestimentiferan Lammellibrachia sp. Journal of Experimental Biology 202, 2245-2257.

Ginsburg, G.D. and V.A. Soloviev, (1998) Submarine gas hydrates. St. Petersburg, VNIIOkeangeologia, $216 \mathrm{p}$.

Kennicutt, M.C., II, J.M. Brooks, and G.J. Denoux (1988) Leakage of deep, reservoired petroleum to the near surface of the Gulf of Mexico continental slope. Marine Chemistry $24,39-59$.

Kvenvolden, K.A., 1999. Potential effects of gas hydrate on human welfare: Proceedings National Academy of Sciences, USA. 96, 3420-3426.

Lanoil, B. R. Sassen, M.T. LaDuc, S.T., Sweetand K.H. Nealson (2001) Bacteria and Archaea physically associated with Gulf of Mexico gas hydrates. Applied and Environmental Microbiology 67, 5143-5153.

MacDonald, I.R., N.L. Guinasso, Jr., R. Sassen, J.M. Brooks, L. Lee, and K.T. Scott, (1994) Gas hydrate that breaches the sea floor on the continental slope of the Gulf of Mexico. Geology 22, 699-702.

Ripmeester, J.A., J.S. Tse, J.S., C.I. Ratliffe, C.I. and B.M. Powell (1987) A new clathrate structure. Nature 325, 135-136.

Roberts, H.H., W.J. Wiseman, Jr., J. Hooper, and G.D. Humphrey (1999) Surficial gas hydrates of the Louisiana continental slope - Initial results of direct observations and in situ data collection. Offshore Technology Conference, Houston, Texas, OTC 10770, 259272.

Roberts, H.H., R. Sassen, and A.V. Milkov (2001) Seafloor expression of fluid and gas expulsion from deep petroleum systems, continental slope of the northern Gulf of Mexico. Gulf Coast Society SEPM Foundation 21 st Annual Research Conference, pp. 407-421.

Sassen, R., J.M. Brooks, I.R. MacDonald, M.C. Kennicutt II, N.L. Guinasso, Jr., and A.G. Requejo (1994) Association of oil seeps and chemosynthetic communities with oil discoveries, upper continental slope, Gulf of Mexico. Gulf Coast Association of Geological Societies Transactions 44, 349-355.

Sassen, R., I.R. MacDonald, N.L. Guinasso, Jr., S. Joye, A.G. Requejo, S.T. Sweet, J.. Alcala-Herrera, D.A. DeFreitas,. and D.R. Schink (1998) Bacterial methane oxidation in sea floor gas hydrate. Significance to life in extreme environments: Geology 26, 289-293. 
Sassen, R., S.T. Sweet, D.A. DeFreitas, and A.V. Milkov (2000) Exclusion of isopentane during crystallization of structure II gas hydrate in sea floor sediment, Gulf of Mexico. Organic Geochemistry 31, 1257-1262.

Udachin, K.A. and J.R. Ripmeester (1999) A complex clathrate showing bimodal guest hydration. Nature, 397, 420-423.

\section{ACRONYMS AND ABBREVIATIONS}

$\mathrm{C}_{1}=$ methane

$\mathrm{C}_{2}=$ ethane

$\mathrm{C}_{3}=$ propane

$\mathrm{C}_{4}=$ butanes

$\mathrm{C}_{5}=$ pentanes

$\mathrm{CO}_{2}=$ carbon dioxide

$\mathrm{H}_{2} \mathrm{~S}=$ hydrogen sulfide

$\mathrm{PDB}=$ PeeDee Belemnite standard used in carbon isotope measurements

$\mathrm{JSL}=$ Johnson Sea Link research submersible

GHSZ = Gas Hydrate Stability Zone

$\mathrm{AT}=$ Atwater Valley protraction area of Gulf of Mexico

$\mathrm{GC}=$ Green Canyon $=$

$\mathrm{VK}=$ Viosca Knoll

$\mathrm{MC}=$ Mississippi Canyon

\section{LIST OF GRAPHICS}

Figure 1. Map showing locations of main study sites of the Gulf slope with gas hydrate (GC 234, GC 232, and MC 118) within a region of has hydrate occurrences, seeps with chemosynthetic communities, and subsurface accumulations of oil and gas. Other study sites used as control sites without gas hydrate are also shown (MC 709, MC 885, VK 826). Hydrate map modified from Sassen et al. (2001a) and sites of chemosynthetic communities from MacDonald et al. (1996).

Figure 2. A conceptualized north-south cross-section of central Gulf slope from the shelf to the abyssal plain is shown. Numerous geologically young basins formed by salt withdrawal pierce the slope salt thrust. The drainage areas of recently mature Mesozoic source rocks are deeply buried beneath the allochthonous salt. Fluids migrate upward through holes in the salt thrust (arrows). Within basins, salt and related faults provide conduits for vertical migration of fluids to reservoirs and to the seafloor. Hydrocarbon vents and seeps are focused by the structure near the rims of salt withdrawal basins. Modified from Milkov and Sassen (2001).

Figure 3. Features of a typical gas hydrate mound ( $\sim 2 \mathrm{~m}$ across) with gas vents and nearby chemosynthetic community of tubeworms (Sassen et al.,1999b).

Figure 4. Photographs of tube worms and seep mussels from chemosynthetic 
communities are shown.

Figure 5. Summary diagram of the microbially-mediated carbon and sulfur cycles associated with hydrate-related chemosynthetic communities. These basic biogeochemical processes are generalized to occur at many other hydrocarbon vent, seep, gas hydrate, and chemosynthetic community sites globally.

Figure 6. Photograph of structure II gas hydrate on a freshly exposed fault surface at GC 234. Massive vein-fillings of gas hydrate are separated by deformed hemipelagic mud, and the base of the exposure is under cut by flow of free gas. Note scale bar. Photograph by C.F. Fisher.

Figure 7. The $38 \mathrm{kHz}$ image shows rapid gas venting to the water column from the GC 234 site.

Figure 8 . The $38 \mathrm{kHz}$ imagery shows gas venting to the water column from the sea floor at GC 232.

Figure 9. The $38 \mathrm{kHz}$ imagery of MC 885 with no gas vents resolved.

Figure 10. Summary diagrams of molecular and isotopic data from GC 234. Data from Sassen et al. 2004.

Figure 11. Typical $\mathrm{C}_{15}+$ gas chromatogram of crude oil from GC 234 showing advanced alteration from microbial oxidation.

Figure 12. Gas hydrate stability curve showing that, at $450 \mathrm{~m}$ water depth, a warm eddy current may only cause thin-skin decomposition of gas hydrate. Exposed or shallow gas hydrate may be affected, but deeper gas hydrate ( $>2 \mathrm{~m}$ in sediment) is thought to be largely insulated from transient temperature excursions. Diagram from Milkov et al. (2000). 
Appendix 


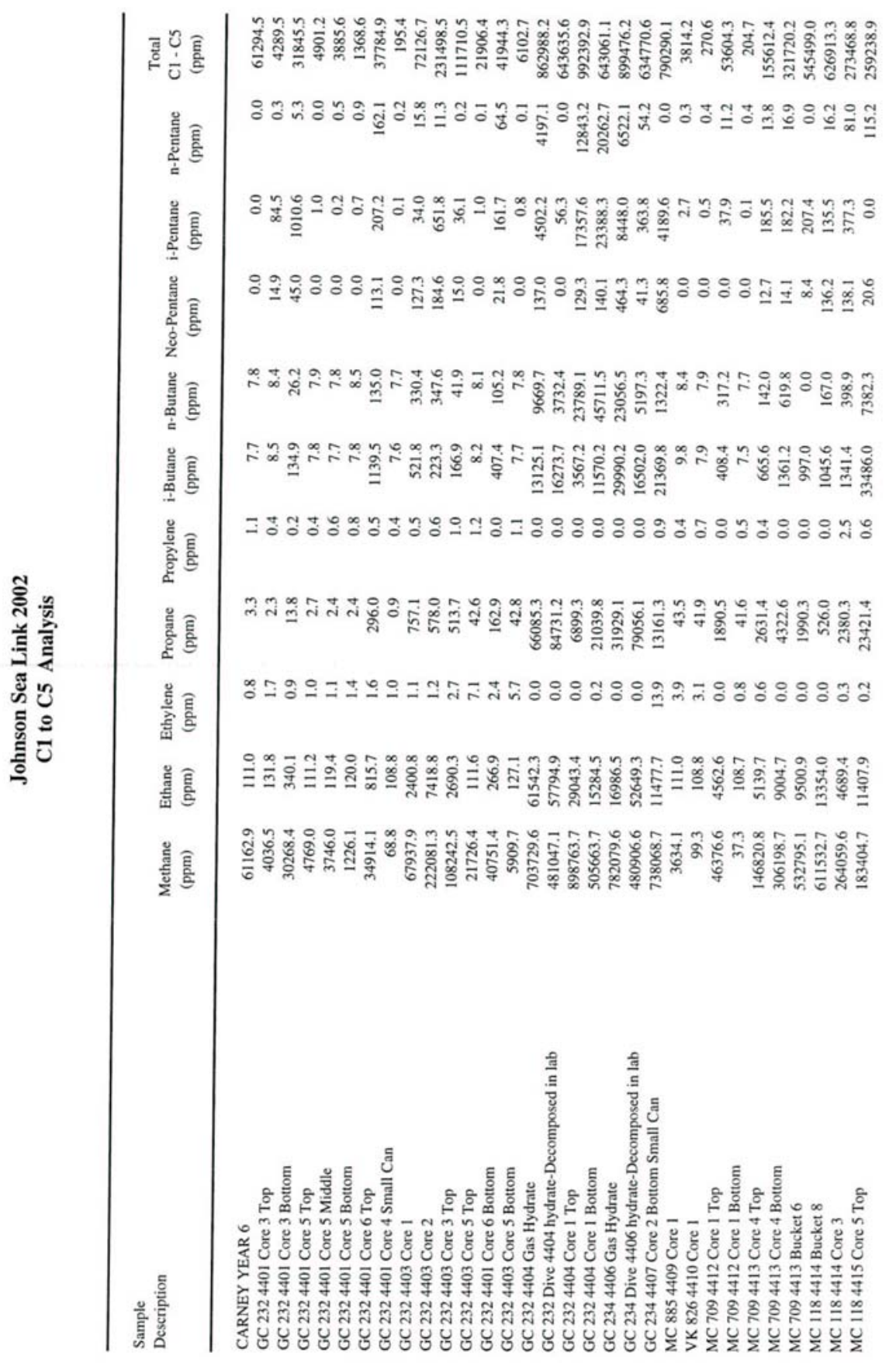




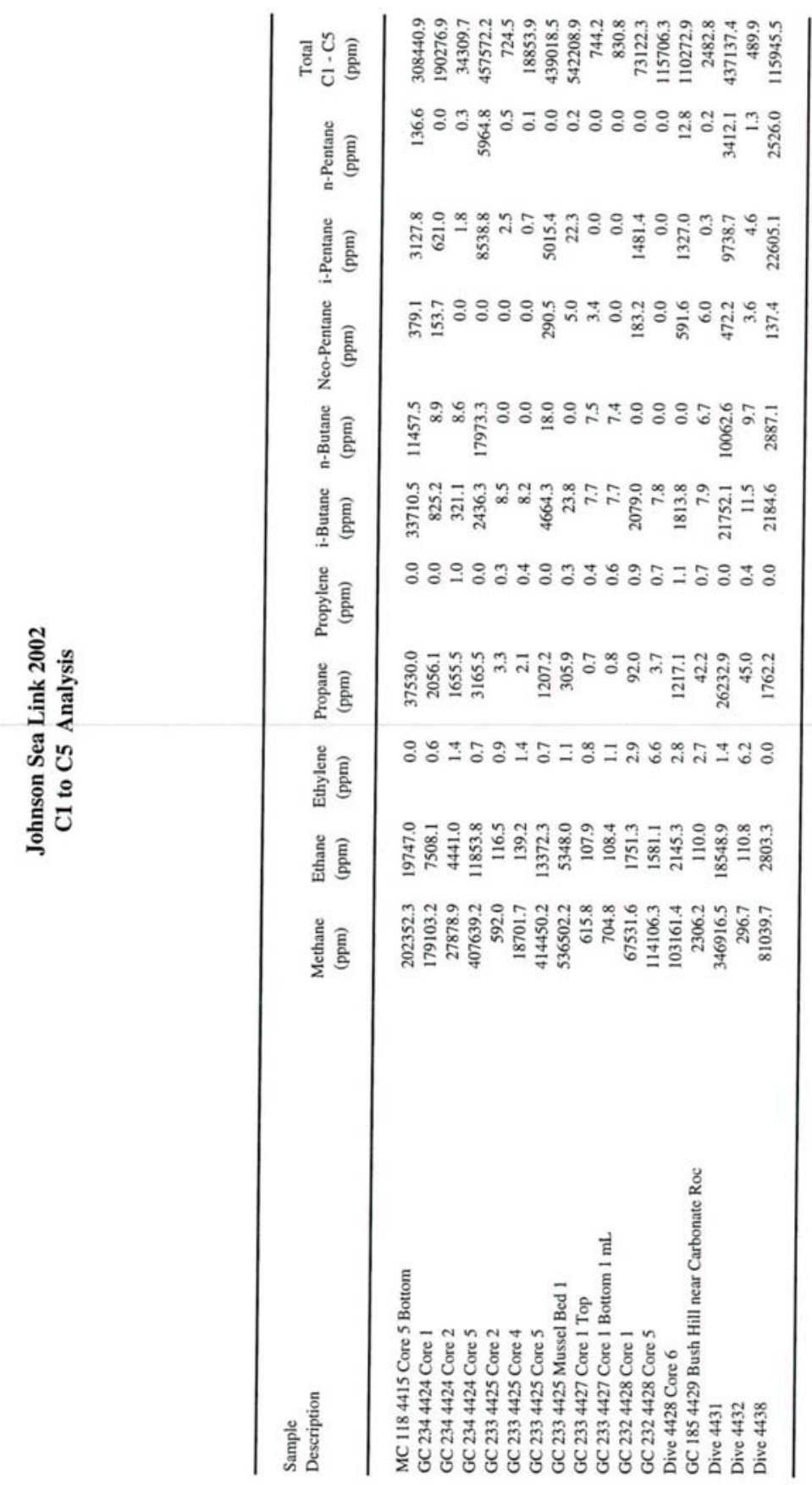




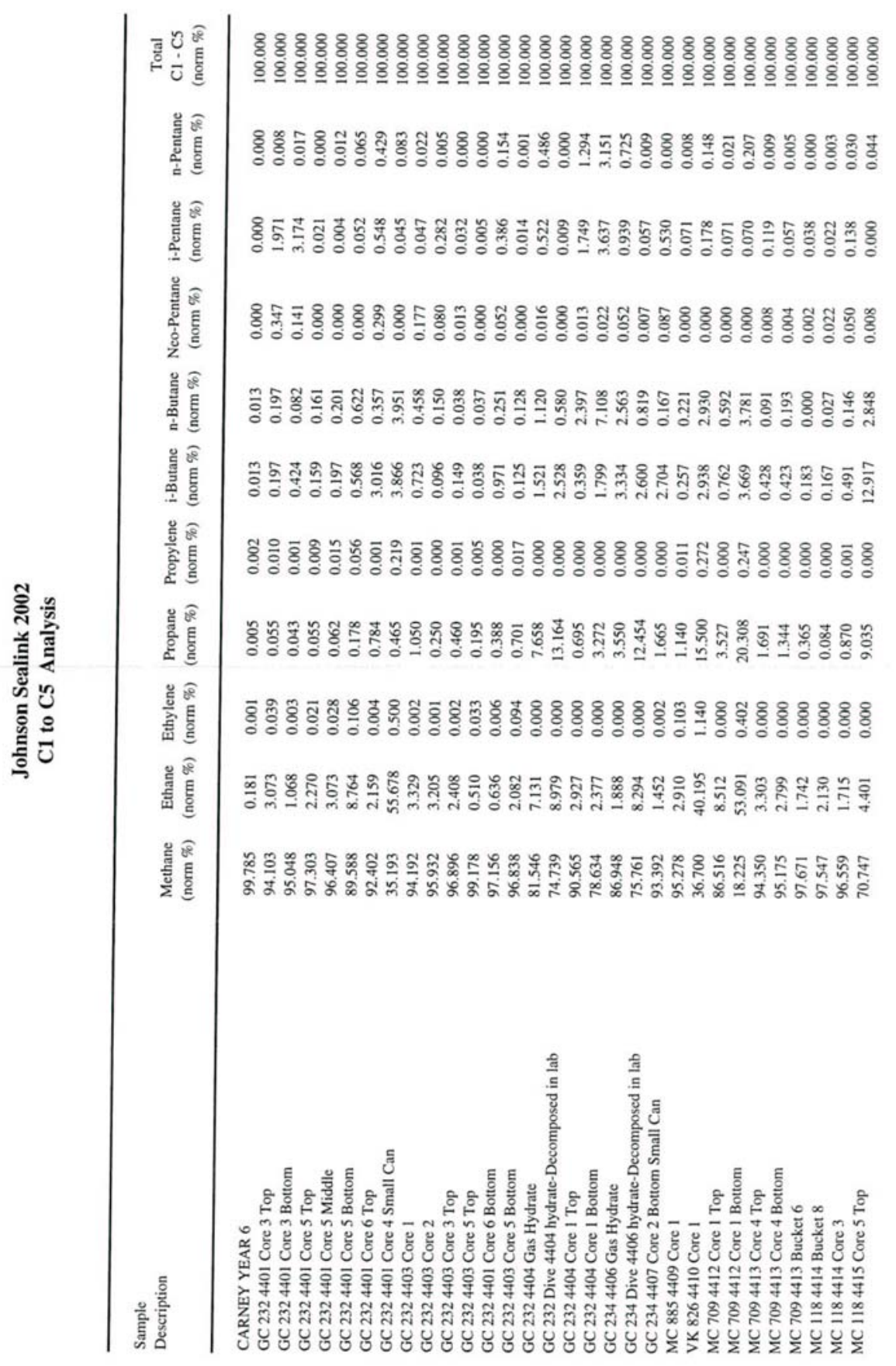




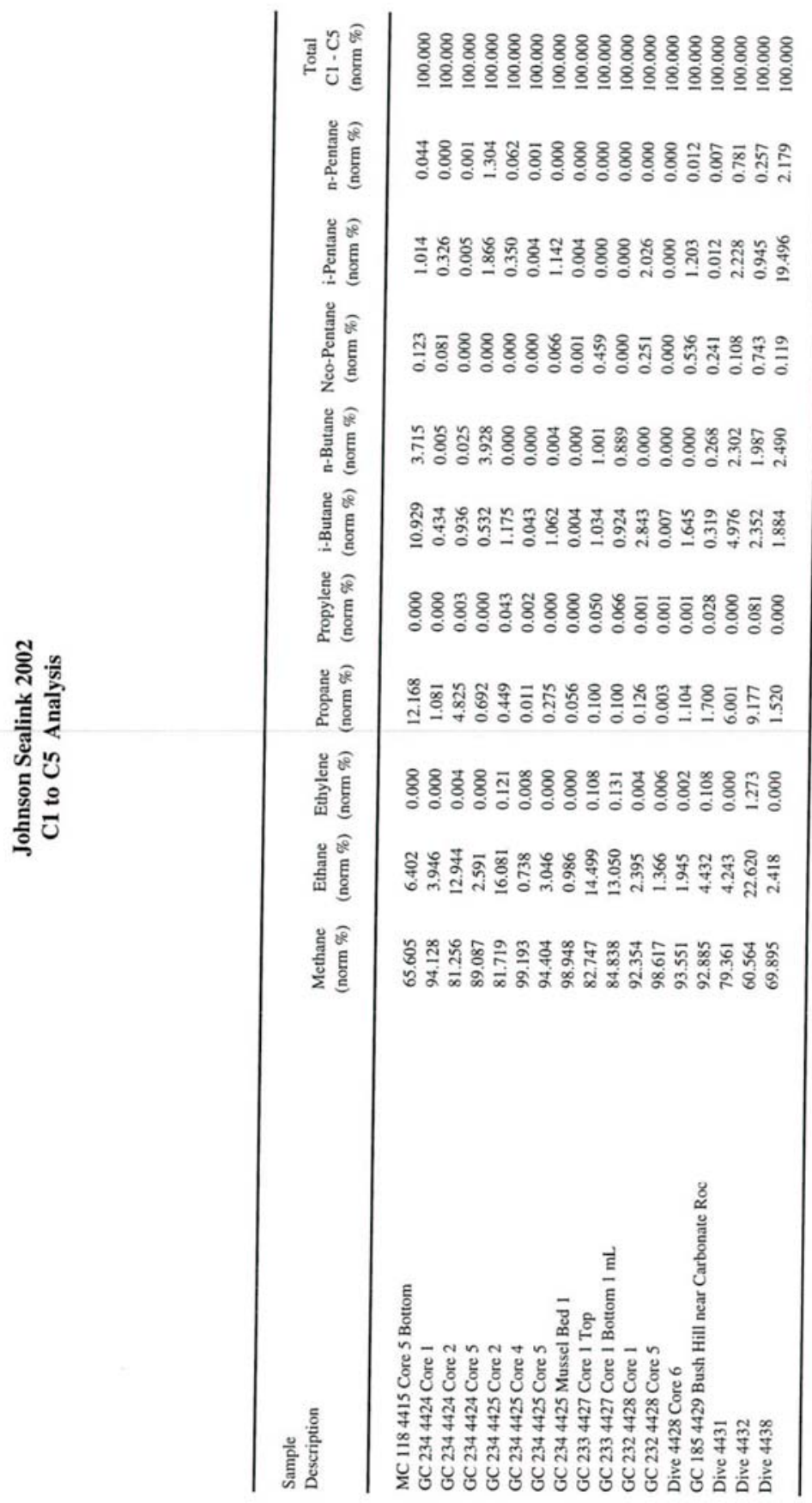




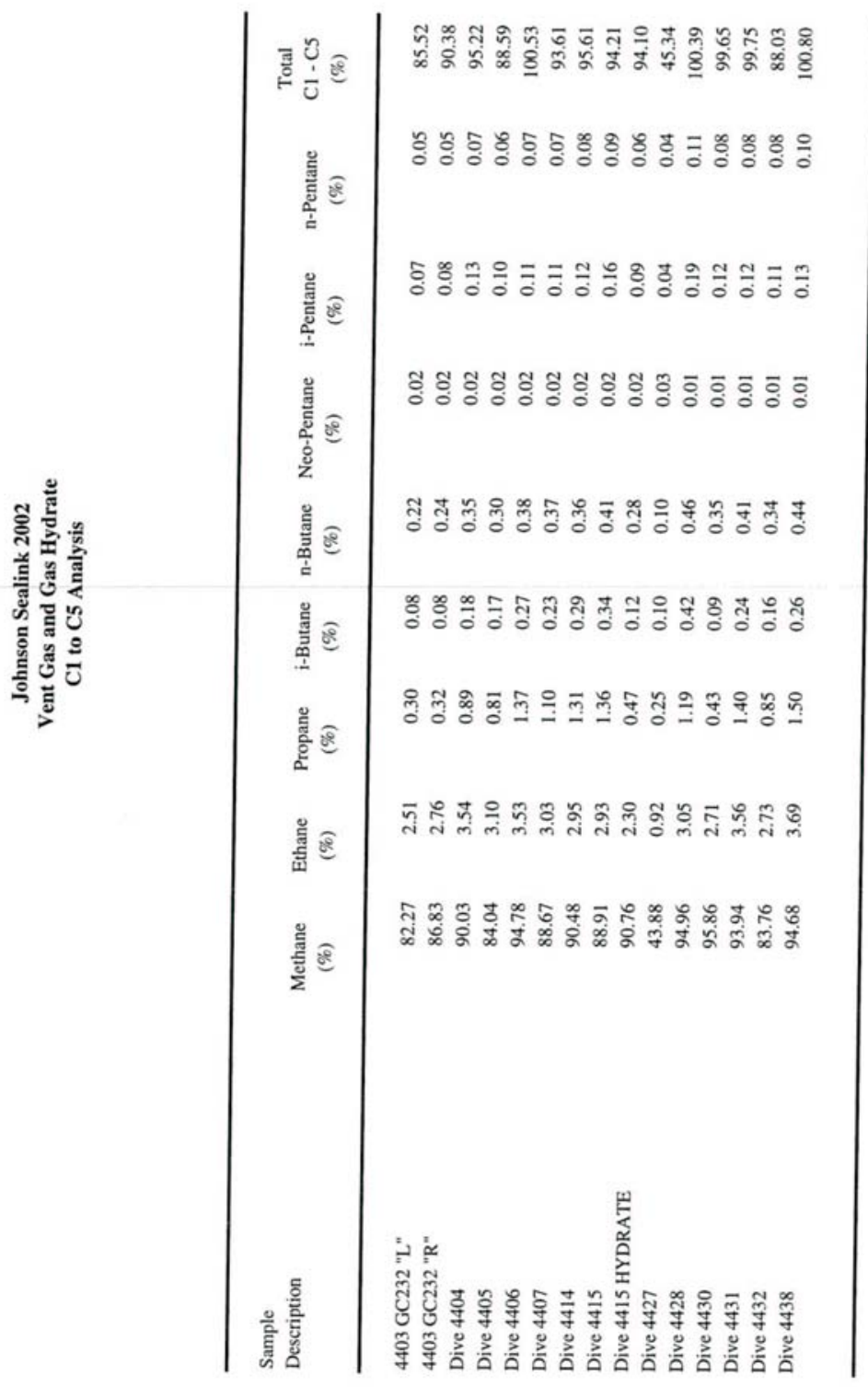




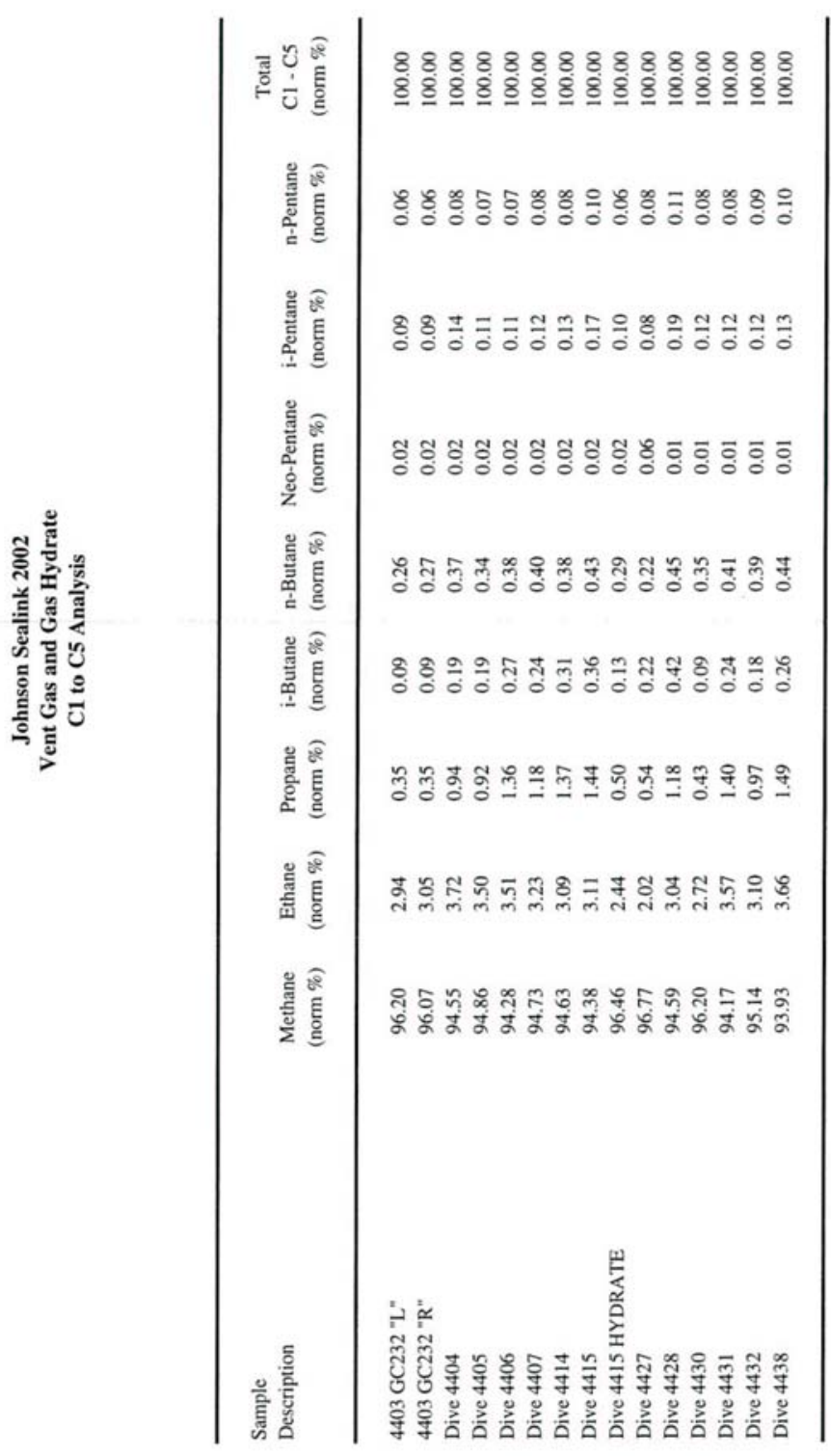




\begin{tabular}{|c|c|c|c|c|c|c|c|}
\hline Dive & Core & Position & $\begin{array}{l}\text { Injection, } \\
\text { micro-L }\end{array}$ & Split ratio & $\mathrm{Cl}$ & $\mathrm{CO} 2$ & Comments \\
\hline & & & & & & & \\
\hline 4401 & 3. & Bottom & 250 & $1: 10$ & -57.28 & -35.87 & \\
\hline 4401 & 5 & Middle & 250 & $1: 10$ & not enough gas & not enough gas & \\
\hline 4401 & 5 & Middle & 1000 & $1: 10$ & -88.02 & not enough gas & \\
\hline 4401 & 6 & Top & 250 & $1: 10$ & too much gas & -39.59 & \\
\hline 4401 & 6 & Top & 50 & $1: 10$ & too much gas & -39.65 & \\
\hline 4401 & 6 & Top & 25 & $1 ; 100$ & -57.51 & not enough gas & \\
\hline 4401 & 6 & & 250 & $1: 10$ & -85.93 & -39.06 & \\
\hline 4403 & 1 & & 250 & $1: 10$ & -46.25 & -13.31 & \\
\hline 4403 & 3 & Top & 250 & $1: 10$ & -59.97 & -31.4 & ethane: -23.89 \\
\hline 4403 & 5 & Top & 250 & $1: 10$ & -82.06 & -41.21 & \\
\hline 4403 & 5 & Bottom & 200 & $1: 10$ & -92.1 & -44.34 & \\
\hline 4409 & 1 & & 250 & $1: 10$ & -64.56 & -43.57 & \\
\hline 4412 & 1 . & Top & 250 & $1: 10$ & -56.62 & -31.96 & \\
\hline 4424 & 2 & & 200 & $1: 10$ & -64.46 & -24.87 & \\
\hline 4425 & 2 & & 250 & $1: 10$ & not enough gas & not enough gas & \\
\hline 4425 & 2 & & 500 & $1: 10$ & not enough gas & -37.14 & \\
\hline 4425 & 2 & & 1000 & $1: 10$ & -86.68 & -36.87 & \\
\hline 4425 & 4 & & 250 & $1: 10$ & -54.68 & -28.87 & \\
\hline 4425 & Mussel bed & & 1000 & $1: 10$ & too much gas & too much gas & \\
\hline 4425 & Mussel bed & & 500 & $1: 10$ & too much gas & too much gas & \\
\hline 4425 & Mussel bed & & 250 & $1: 10$ & -72.45 & -46.47 & \\
\hline 4425 & Mussel bed & & 250 & $1: 10$ & -71.42 & -46.99 & \\
\hline 4427 & 1 & Top & 100 & $1: 10$ & not enough gas & -20.17 & \\
\hline 4427 & 1 & Top & 1000 & $1: 10$ & -103.08 & -20.37 & \\
\hline 4427 & 11 & Bottom & 250 & $1: 10$ & not enough gas & -13.88 & \\
\hline 4427 & 11 & Bottom & 1000 & $1: 10$ & -123.15 & -15.17 & \\
\hline 4428 & 5 & & 250 & $1: 10$ & -55.71 & -38.79 & \\
\hline 4429 & & & 250 & $1: 10$ & not enough gas & -27.89 & \\
\hline 4429 & & & 1000 & $1: 10$ & -95.71 & -28.53 & \\
\hline Carney & & & 250 & $1: 10$ & -89.64 & too much gas & $\mathrm{CO} 2 \gg \mathrm{Cl}$ \\
\hline Carney & & & 100 & $1: 10$ & -88.94 & too much gas & $\mathrm{CO} 2 \gg \mathrm{Cl}$ \\
\hline Carney & & & 30 & $1: 10$ & -89.24 & too much gas & $\mathrm{CO} 2 \gg \mathrm{Cl}$ \\
\hline Carney & & & 25 & $1 ; 100$ & not enough gas & -17.42 & $\mathrm{CO} 2 \gg \mathrm{Cl}$ \\
\hline
\end{tabular}




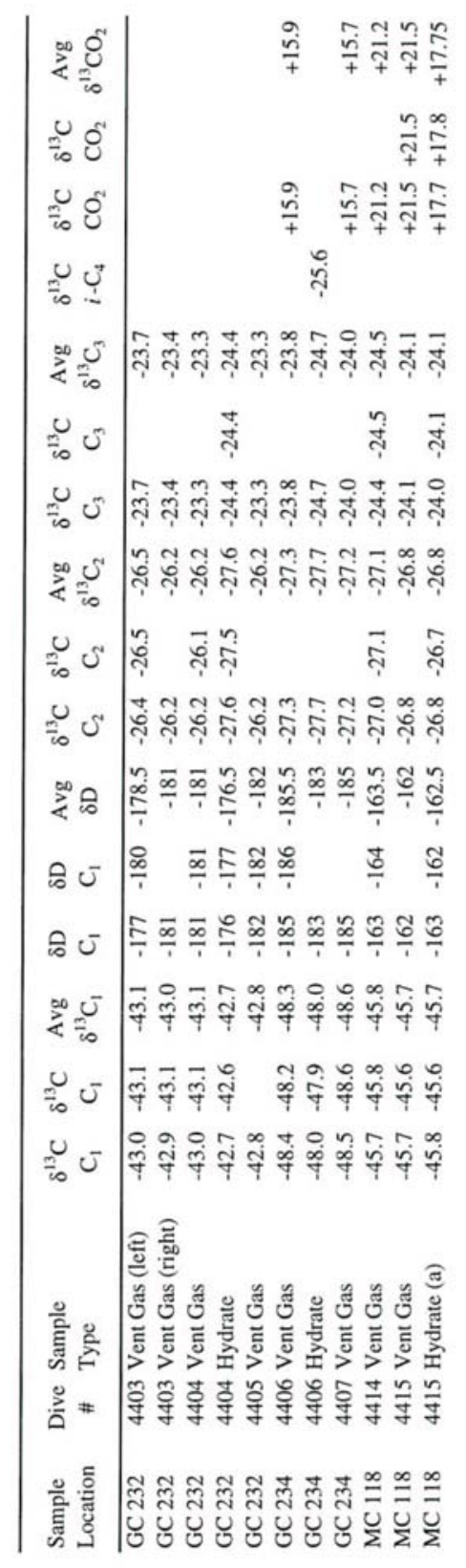


JSL Sealink 2002

Gas Chromatographic Data

\begin{tabular}{|c|c|c|c|c|}
\hline File \# & Description & $\begin{array}{c}\mathbf{U C M}<23 \\
(\mathrm{ppm})\end{array}$ & $\begin{array}{c}\text { UCM }>23 \\
(\mathrm{ppm})\end{array}$ & $\begin{array}{c}\text { Total UCM } \\
(\mathrm{ppm})\end{array}$ \\
\hline L52820 & GC 232, Dive 4401 & 4174 & 4832 & 9006 \\
\hline L.52821 & GC 233, Dive 4425 & 2725 & 4592 & 7317 \\
\hline L52822 & GC 185, Dive 4429 & 2269 & 6491 & 8761 \\
\hline LS2823 & GC 234, Dive 4434 & 389 & 860 & 1249 \\
\hline LS2824 & GC 234, Dive 4435 & 554 & 1071 & 1625 \\
\hline L52825 & GC 234, Dive 4436 & 410 & 785 & 1195 \\
\hline L.52826 & GC 232, Dive 4437 & 912 & 1696 & 2608 \\
\hline L52827 & GC 232, Dive 4403 & 1480 & 2999 & 4479 \\
\hline L52828 & MC 709, Dive 4412 & 280 & 164 & 445 \\
\hline L52829 & MC 118, Dive 4414 & 3847 & 3624 & 7471 \\
\hline L52830 & CARNEY YEAR 6 & 9321 & 3660 & 12982 \\
\hline L52831 & GC 2324401 Core 3 Top & 6761 & 8372 & 15133 \\
\hline L52832 & GC 2324401 Core 3 Bottom & 6860 & 11351 & 18211 \\
\hline L52833 & GC 2324401 Core 5 Top & 4932 & 7483 & 12415 \\
\hline LS2834 & GC 2324401 Core 5 Middle & 9560 & 11125 & 20684 \\
\hline L.52835 & GC 2324401 Core 5 Bottom & 11798 & 13931 & 25729 \\
\hline L52836 & GC 2324401 Core 6 Top & 7490 & 9184 & 16674 \\
\hline LS2837 & GC 2324401 Core 4 Small & 3184 & 6192 & 9375 \\
\hline LS2838 & GC 2324403 Core 1 & 1836 & 4940 & 6776 \\
\hline L52839 & GC 2324403 Core 2 & 8370 & 8371 & 16741 \\
\hline L52840 & GC 2324403 Core 3 Top & 3355 & 6098 & 9453 \\
\hline L52841 & GC 2324403 Core 5 Top & 4541 & 7626 & 12166 \\
\hline LS2842 & GC 2324401 Core 6 Bottom & 7627 & 13454 & 21081 \\
\hline L.52843 & GC 2324403 Core 5 Bottom & 11227 & 14889 & 26117 \\
\hline L52844 & GC 2324404 Gas Hydrate & 1772 & 2694 & 4465 \\
\hline L52845 & GC 2324404 Core I Top & 5363 & 19838 & 25201 \\
\hline L.52846 & GC 2324404 Core I Bottom & 7267 & 8551 & 15819 \\
\hline LS2847 & GC 2344406 Gas Hydrate & 7917 & 10739 & 18656 \\
\hline L52848 & GC 2344407 Core 2 Bottom & 12282 & 45573 & 57855 \\
\hline LS2849 & MC 8854409 Core 1 & 14 & 31 & 46 \\
\hline L52850 & VK 8264410 Core 1 & 71 & 64 & 136 \\
\hline L52851 & MC 7094412 Core I Top & 491 & 1383 & 1874 \\
\hline L52852 & MC 7094412 Core I Bottom & 46 & 359 & 404 \\
\hline LS2853 & MC 7094413 Core 4 Top & 43 & 163 & 206 \\
\hline L52854 & MC 7094413 Core 4 Bottom & 28 & 71 & 99 \\
\hline L52855 & MC 7094413 Bucket 6 & 133 & 352 & 485 \\
\hline LS2856 & MC 1184414 Bucket 8 & 3115 & 6404 & 9519 \\
\hline L52857 & MC 1184414 Core 3 & 10230 & 8591 & 18820 \\
\hline LS2858 & MC 1184415 Core 5 Top & 11068 & 11979 & 23048 \\
\hline L52859 & MC 1184415 Core 5 Bottom & 5056 & 9916 & 14972 \\
\hline L52860 & GC 2344424 Core 1 & 4910 & 7705 & 12616 \\
\hline L52861 & GC 2344424 Core 2 & 7591 & 1644 & 9235 \\
\hline L52862 & GC 2344424 Core 5 & 14987 & 17334 & 32321 \\
\hline L52863 & GC 2334425 Core 2 & 3019 & 8663 & 11682 \\
\hline 152864 & GC 2334425 Core 4 & 3349 & 13034 & 16383 \\
\hline LS2865 & BLANK & 13 & 20 & 33 \\
\hline L52866 & GC 2334425 Mussel Bed I & 1116 & 3956 & 5072 \\
\hline L52867 & GC 2334427 Core 1 Top & 166 & 326 & 492 \\
\hline L52868 & GC 2334427 Core 1 Bottom & 175 & 338 & 513 \\
\hline L52869 & GC 2324428 Core 1 & 11727 & 11491 & 23218 \\
\hline LS2870 & GC 2324428 Core 5 & 3544 & 5332 & 8876 \\
\hline LS2871 & Dive 4428 Core 6 & 8906 & 8018 & 16924 \\
\hline LS2871 & Dive 4428 Core 6 & 8133 & 6836 & 14969 \\
\hline L52872 & GC 1854429 Bush Hill nea & 3373 & 8516 & 11890 \\
\hline L52873 & Dive 4431 & 9106 & 10576 & 19682 \\
\hline LS2874 & Dive 4432 & 2503 & 2986 & 5489 \\
\hline LS2875 & Dive 4438 & 1350 & 3142 & 4491 \\
\hline
\end{tabular}




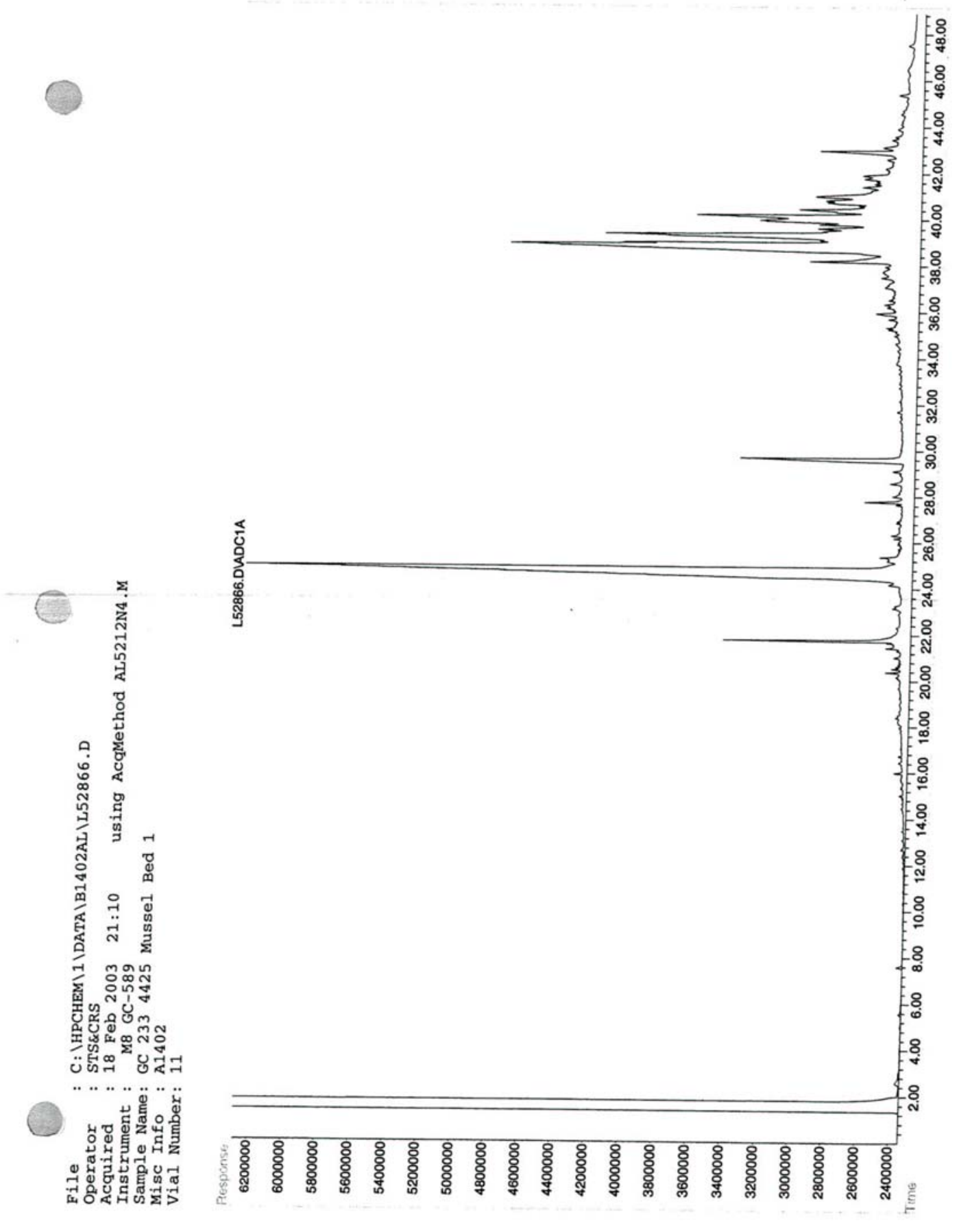




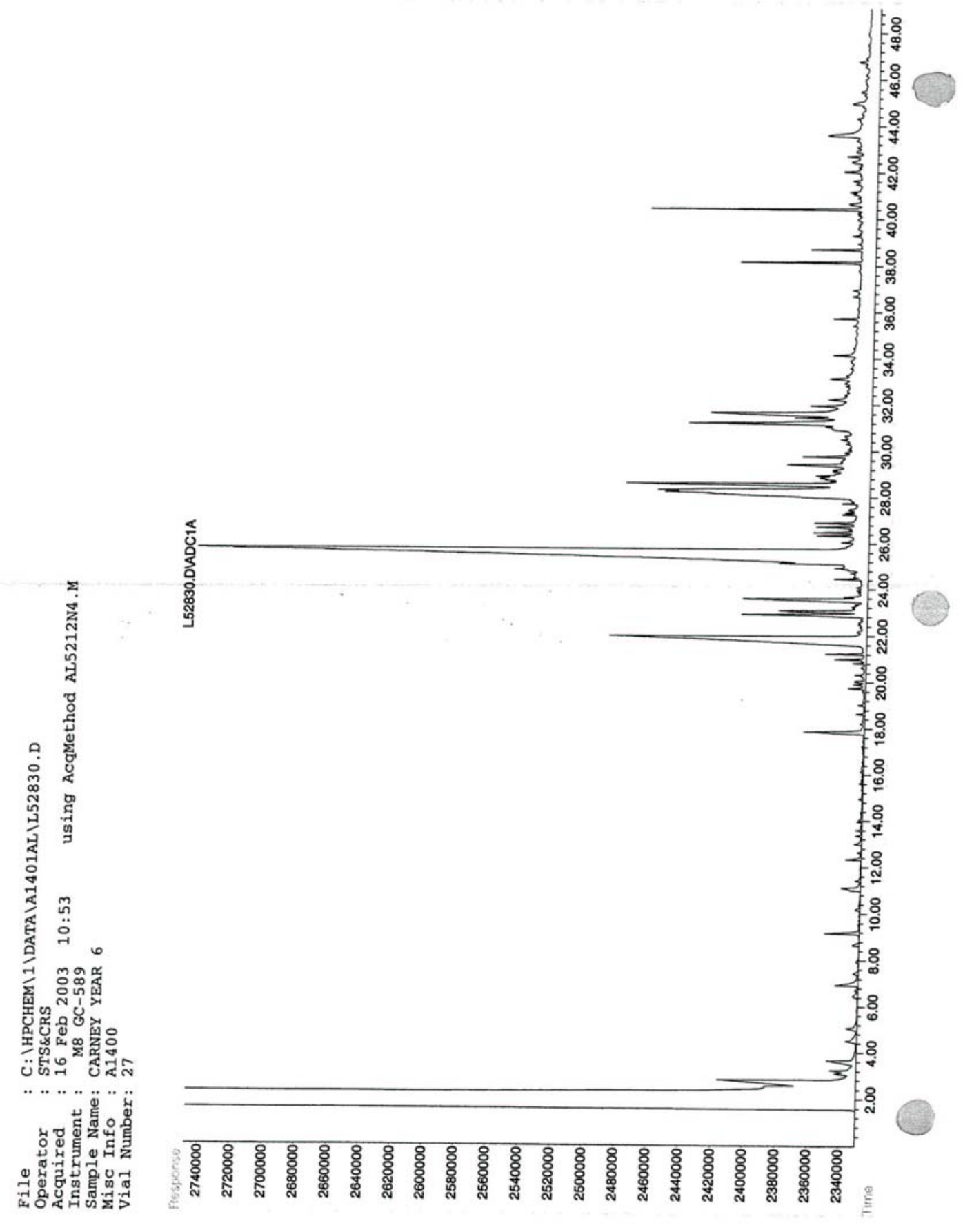




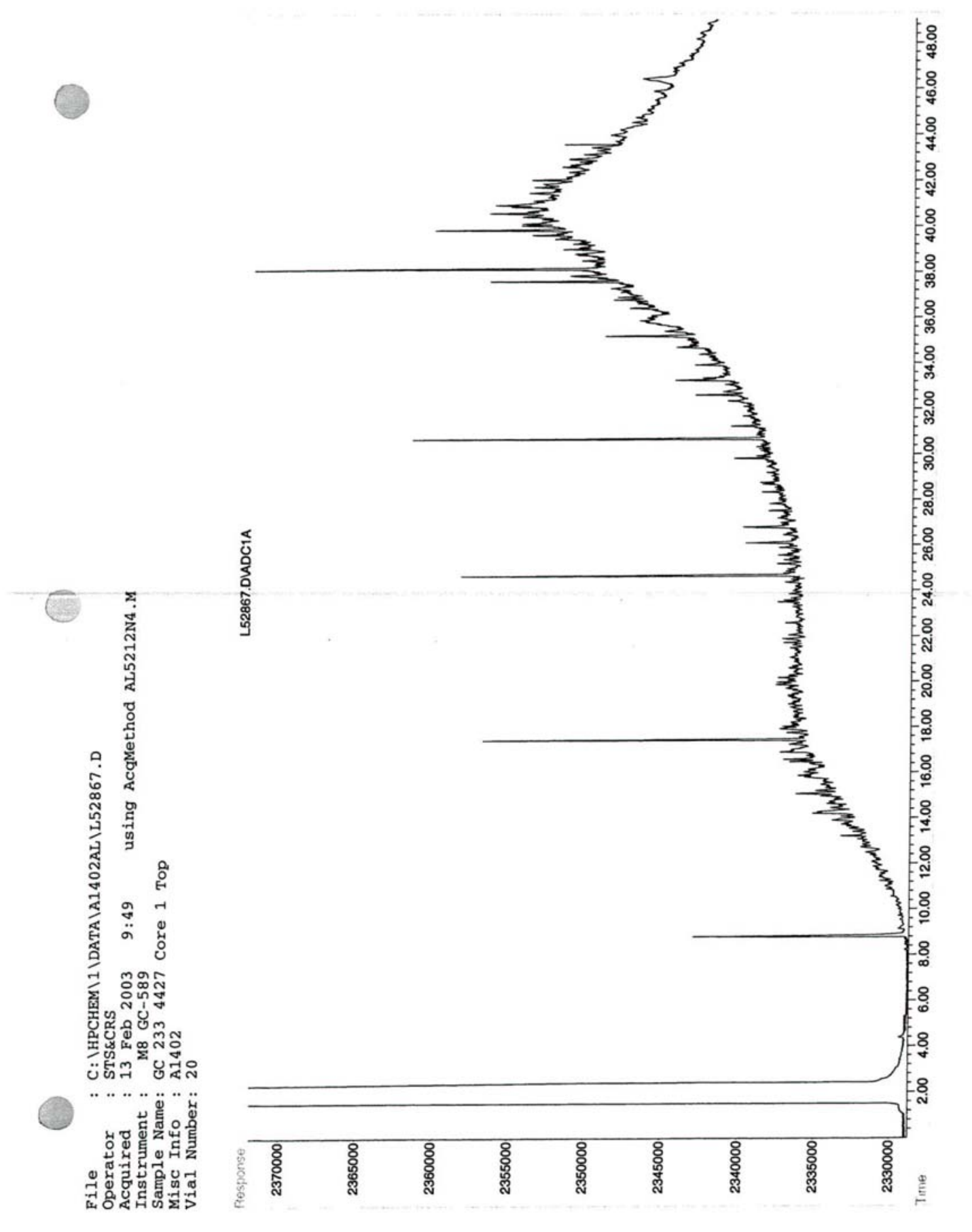




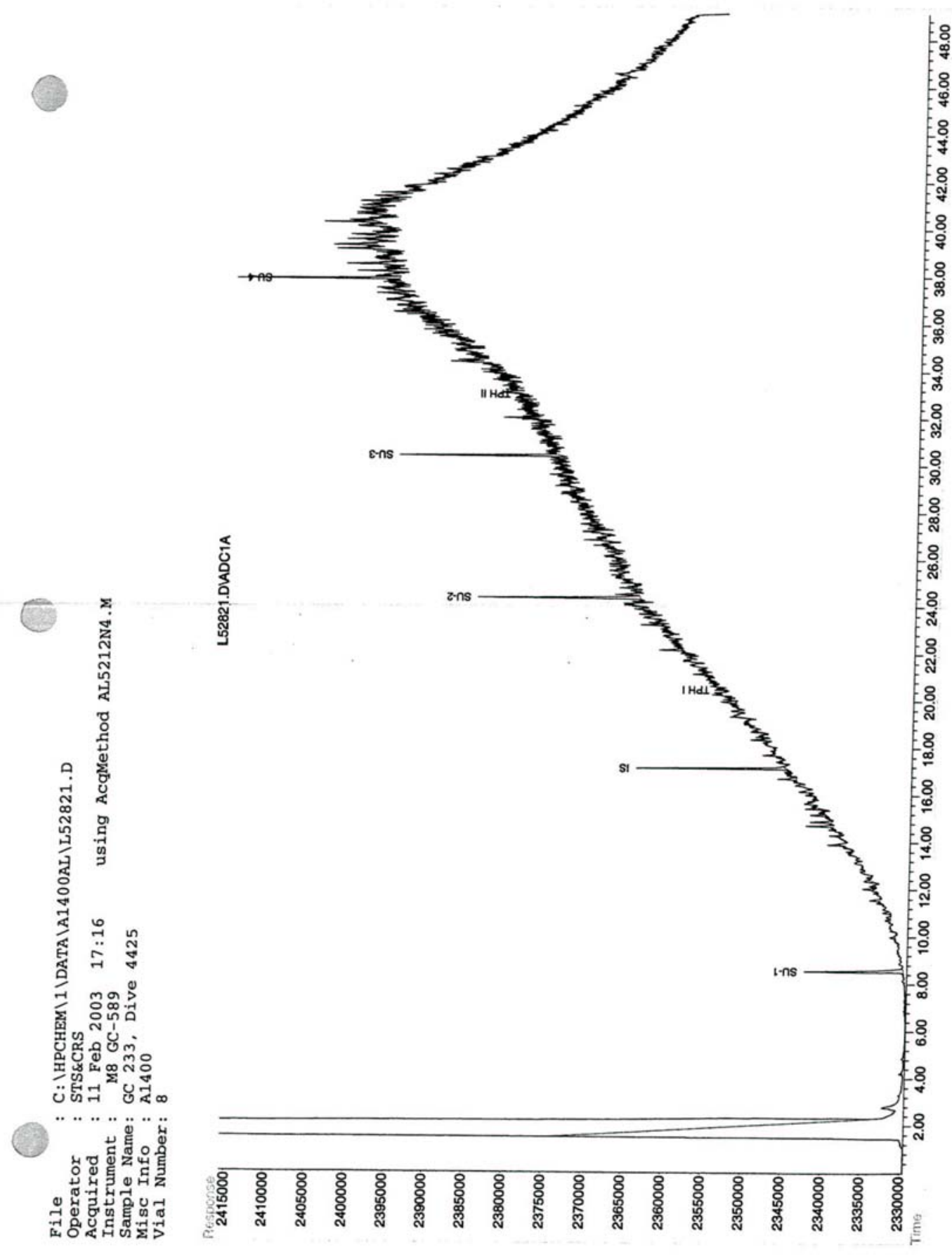




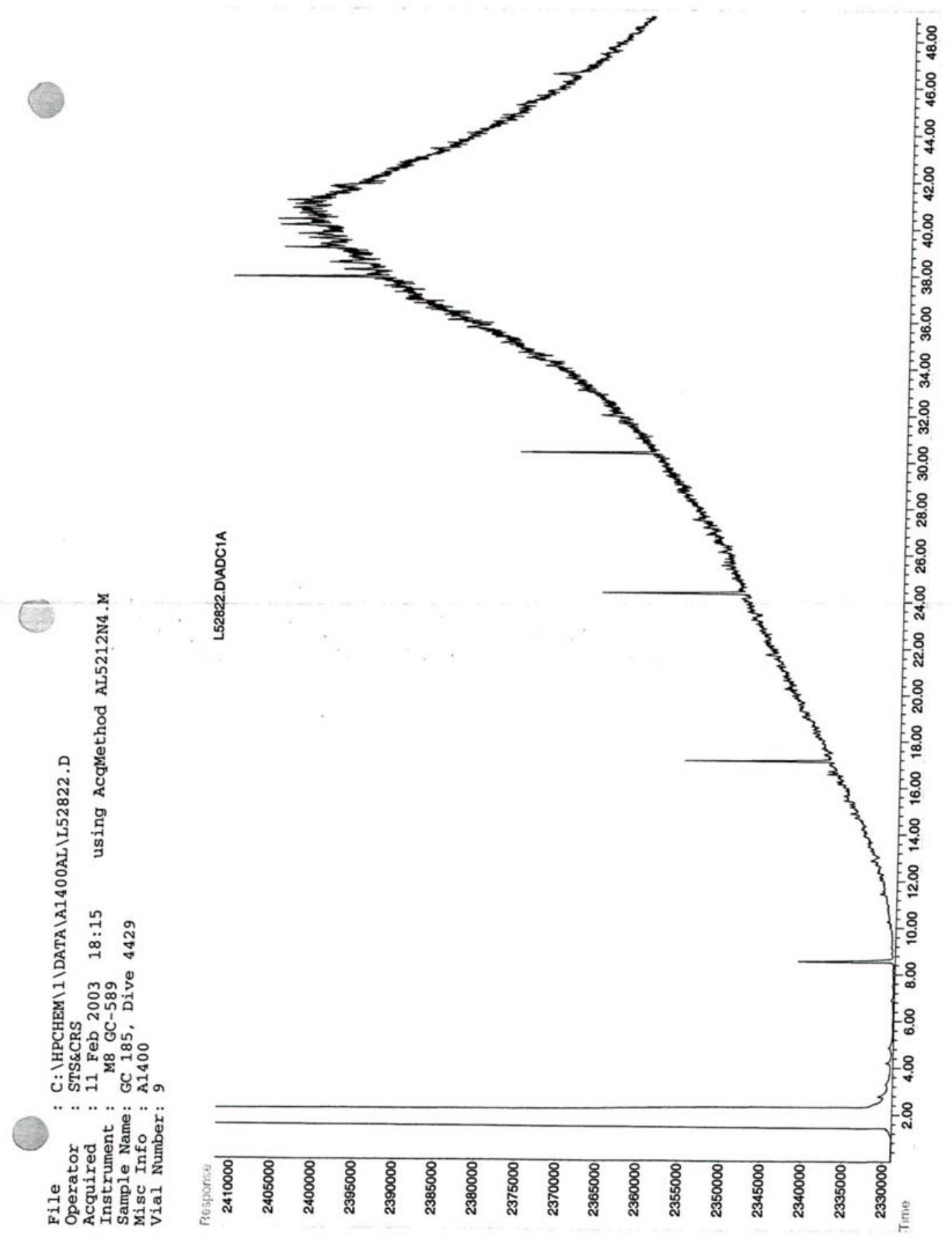




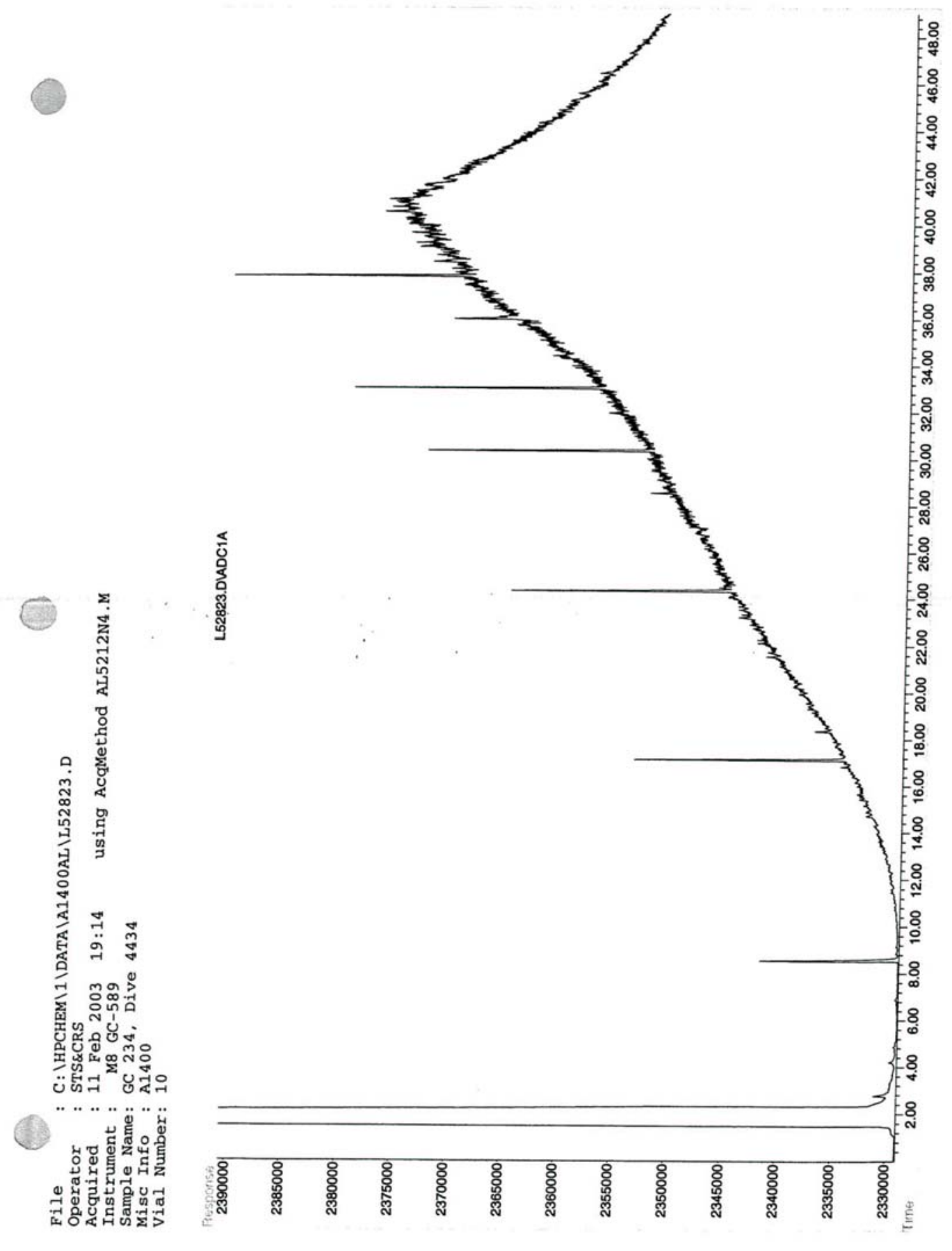




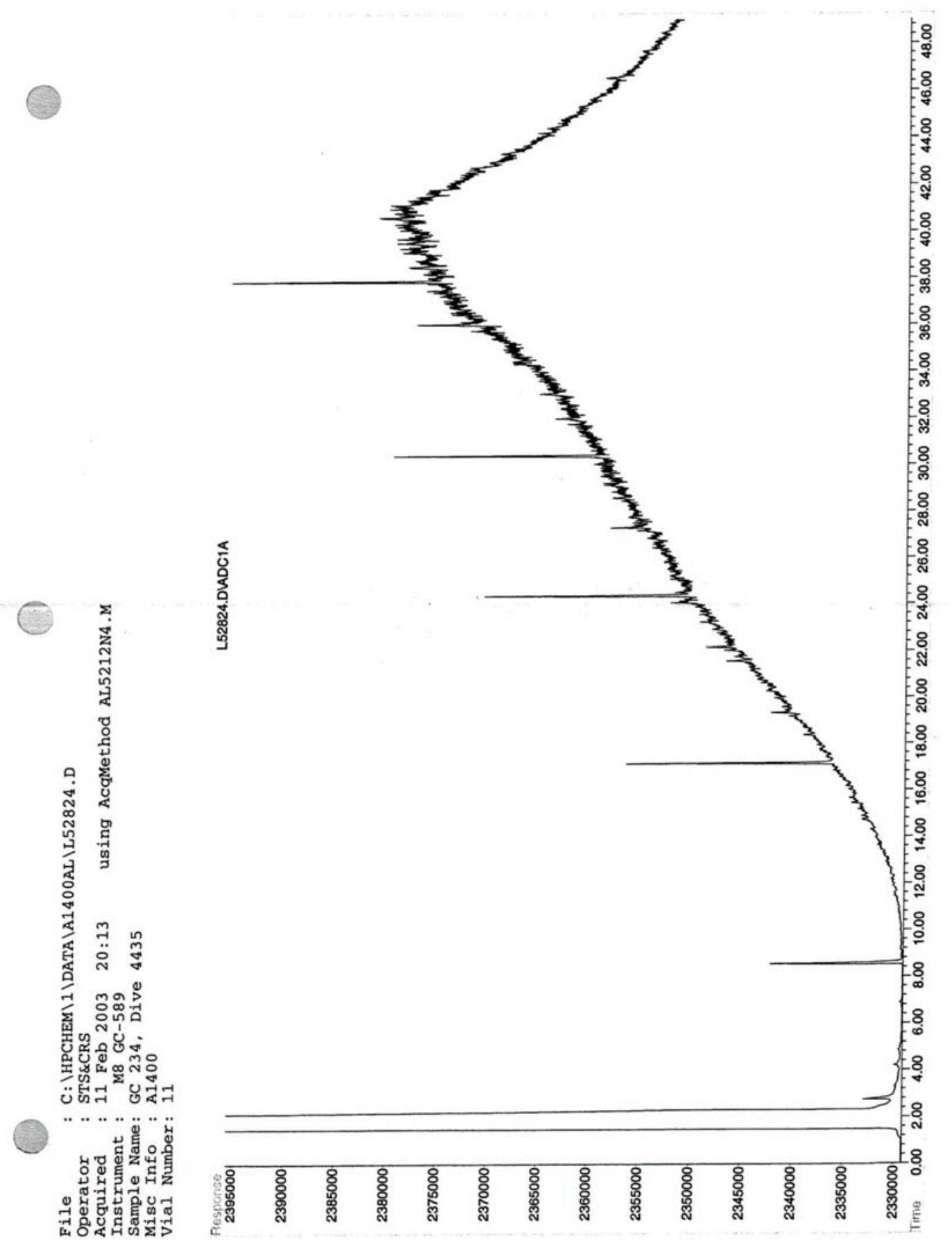




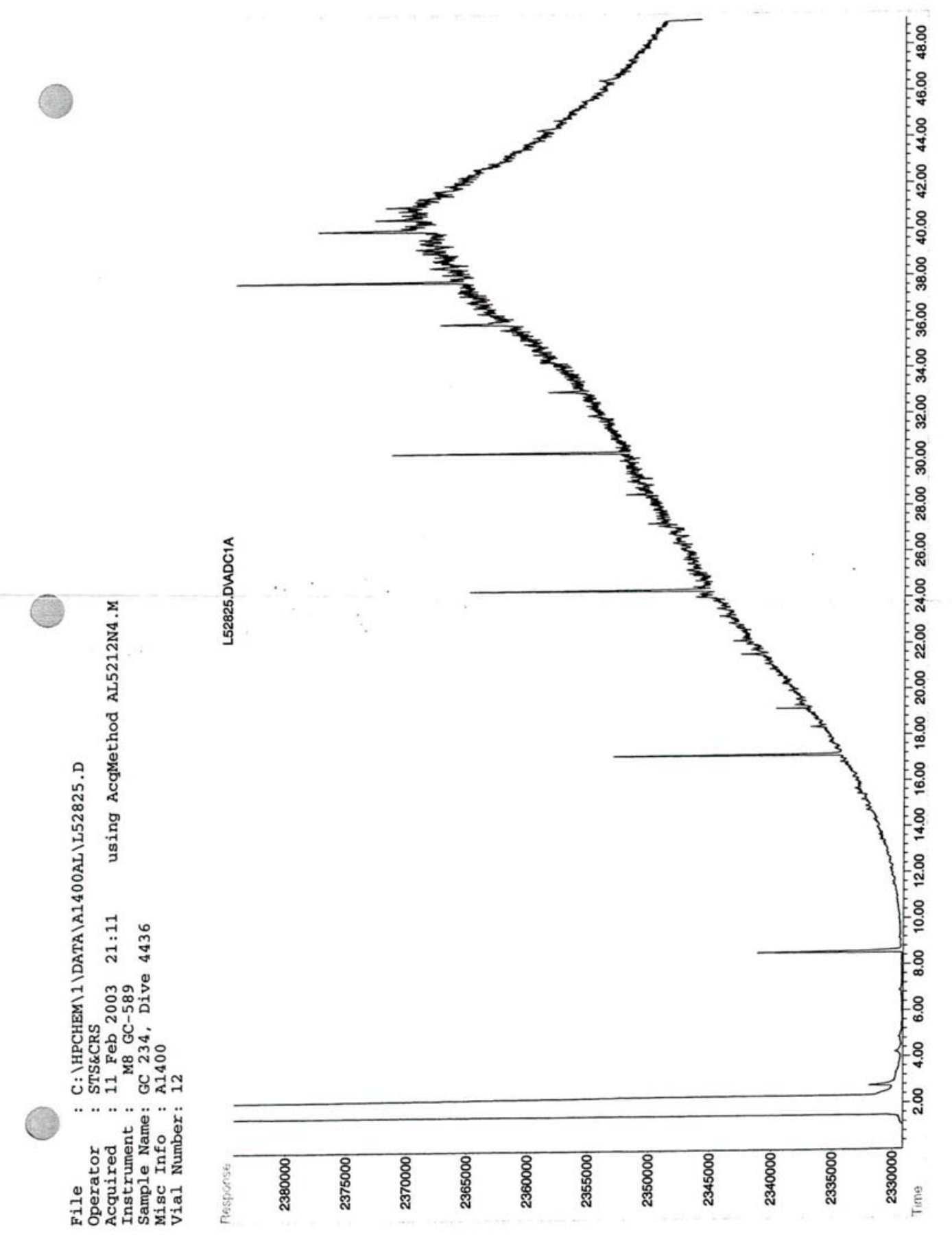




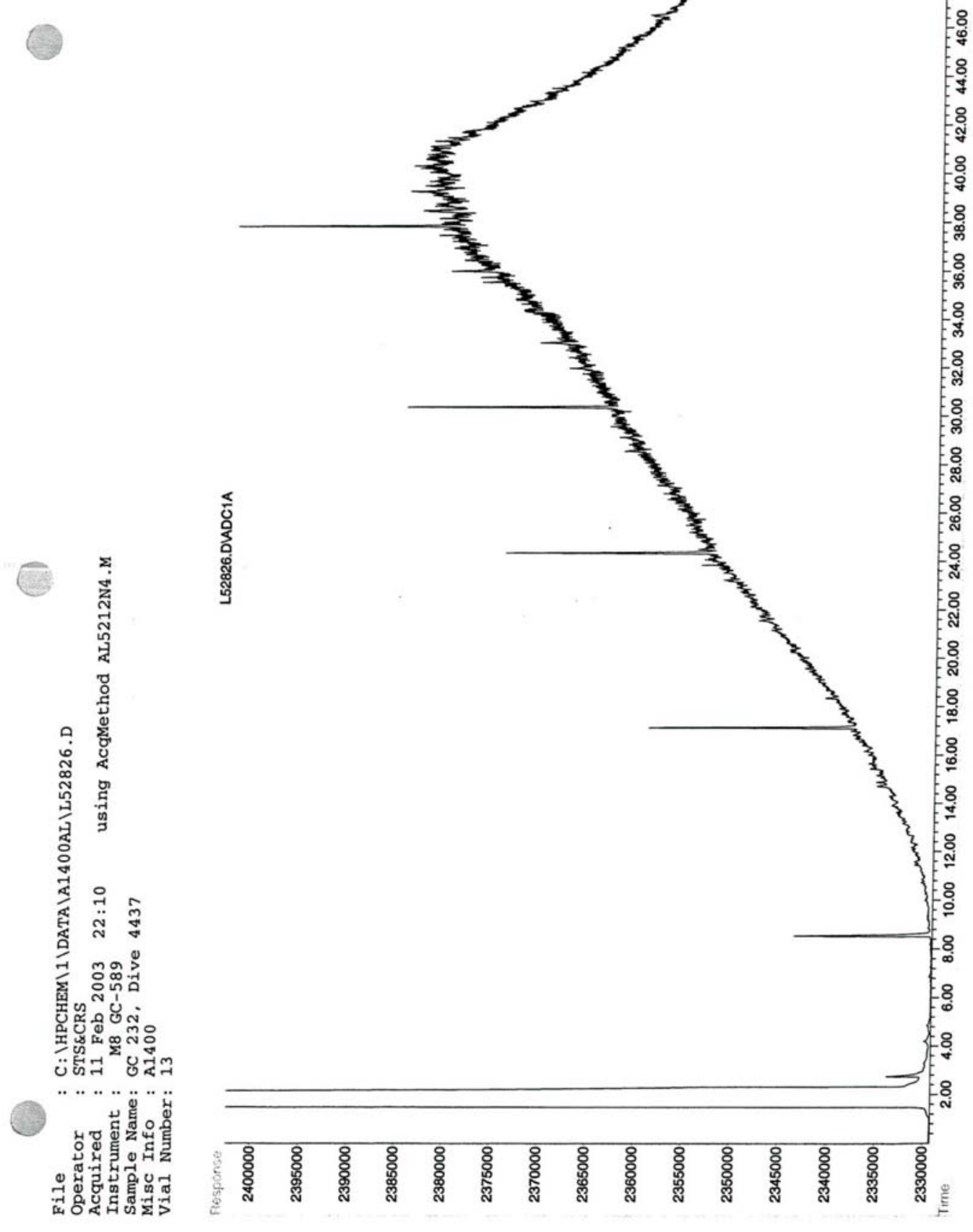




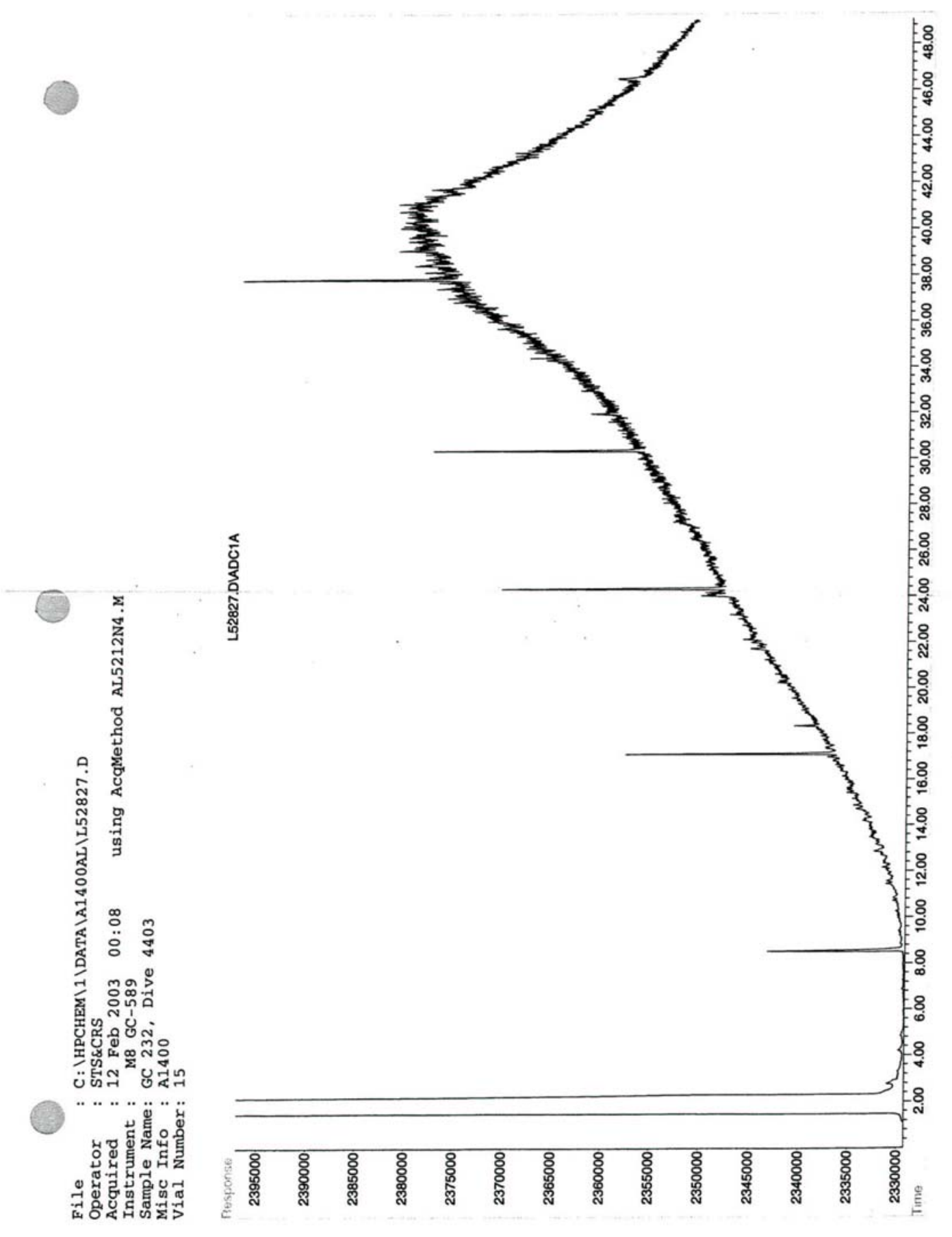




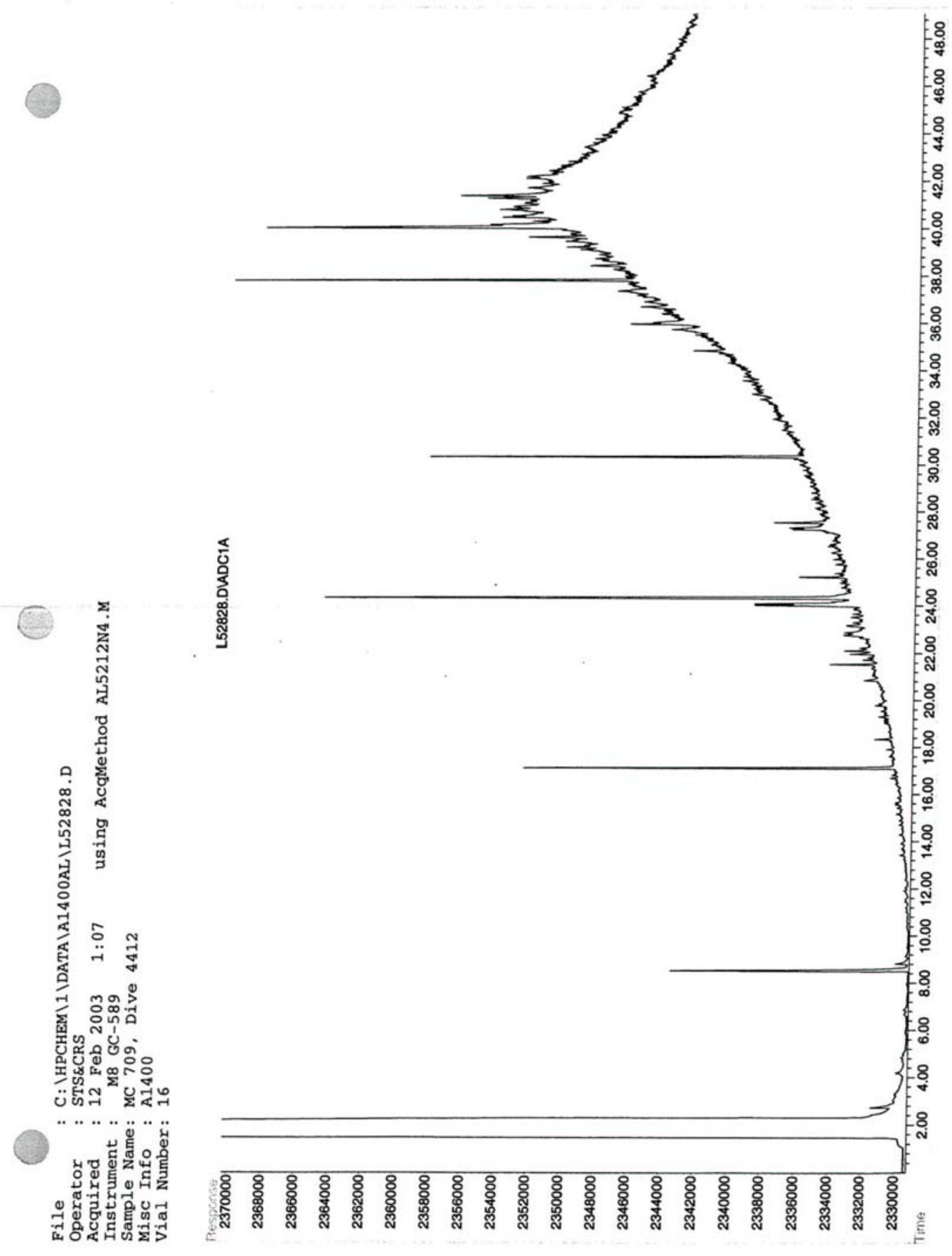




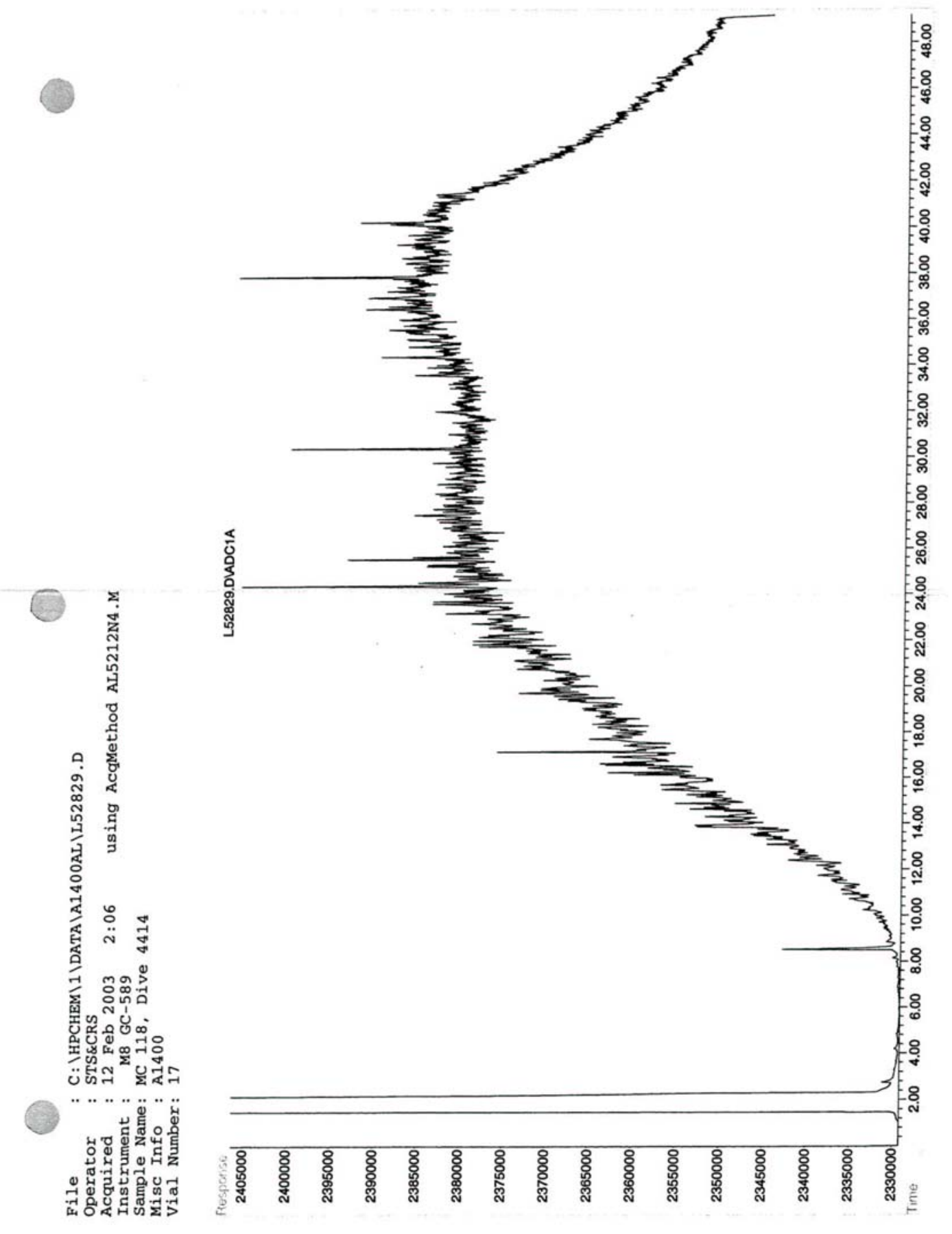




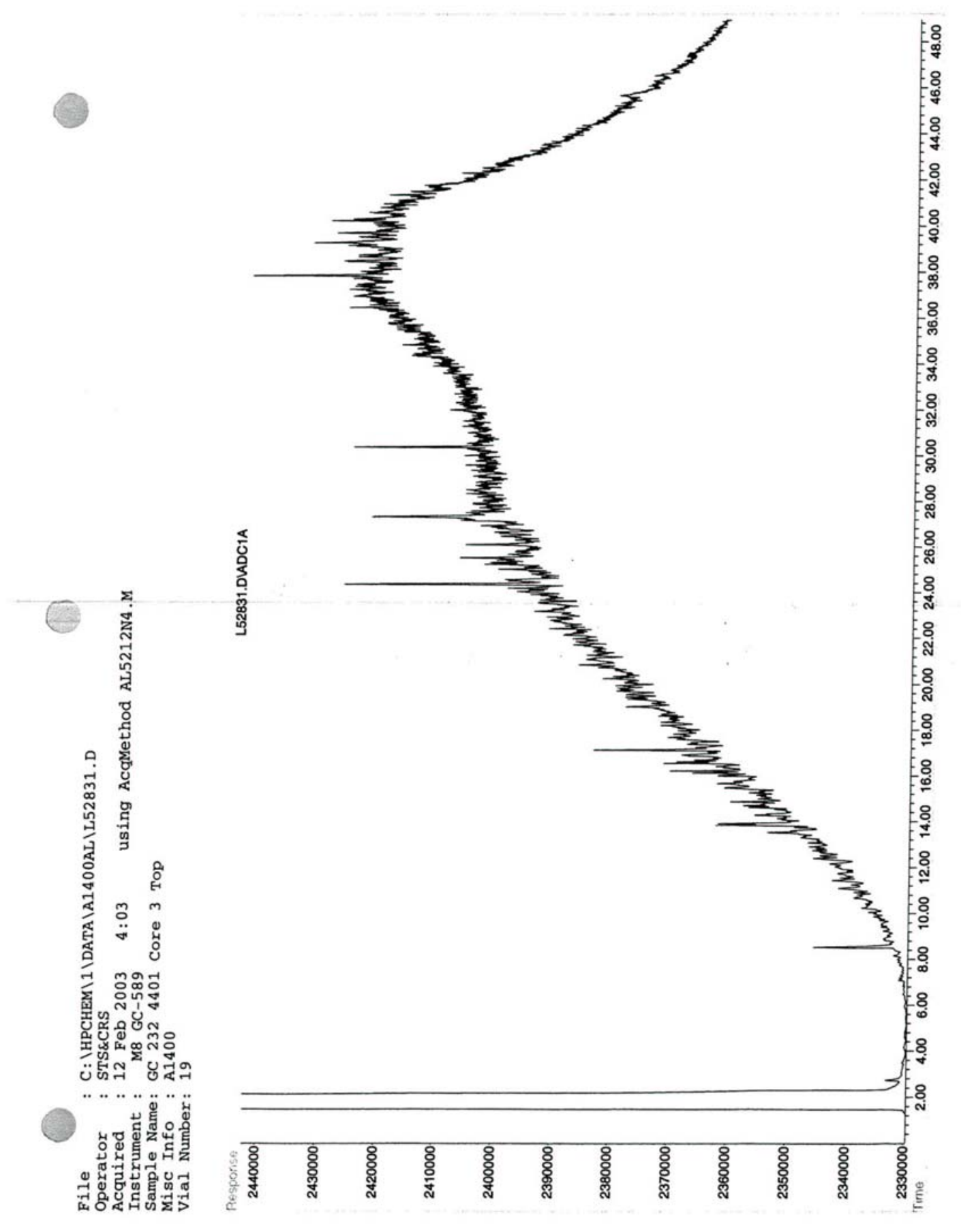




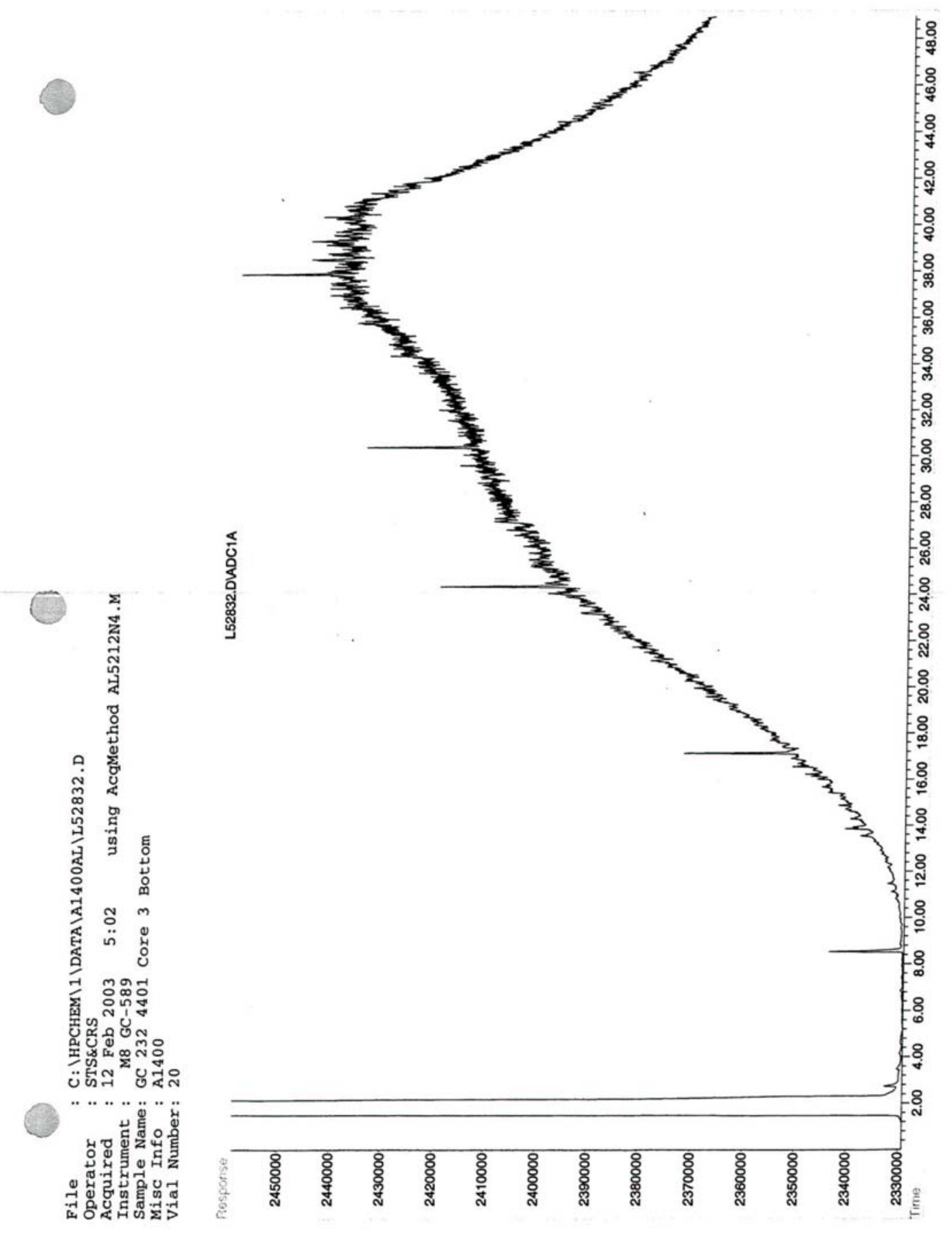




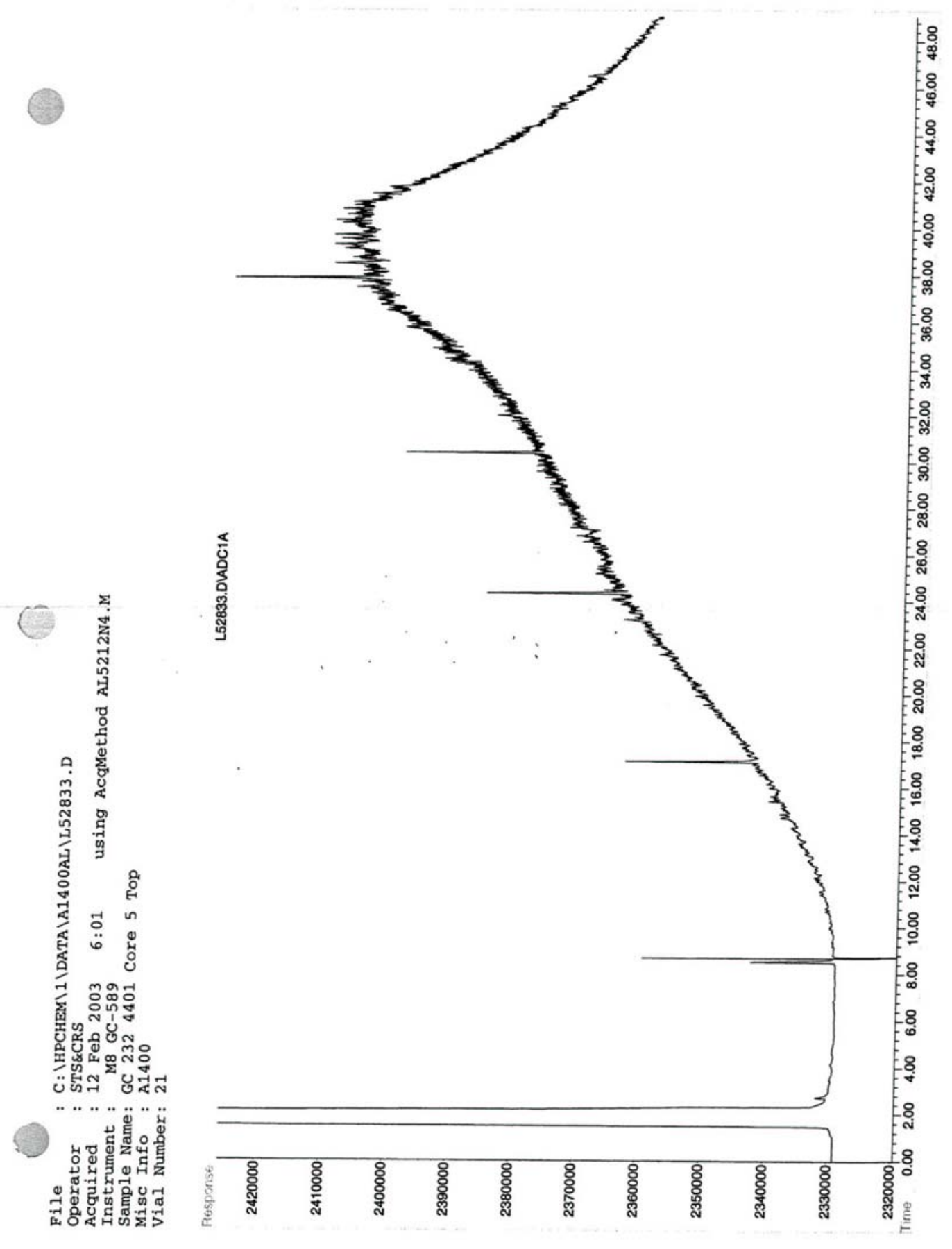




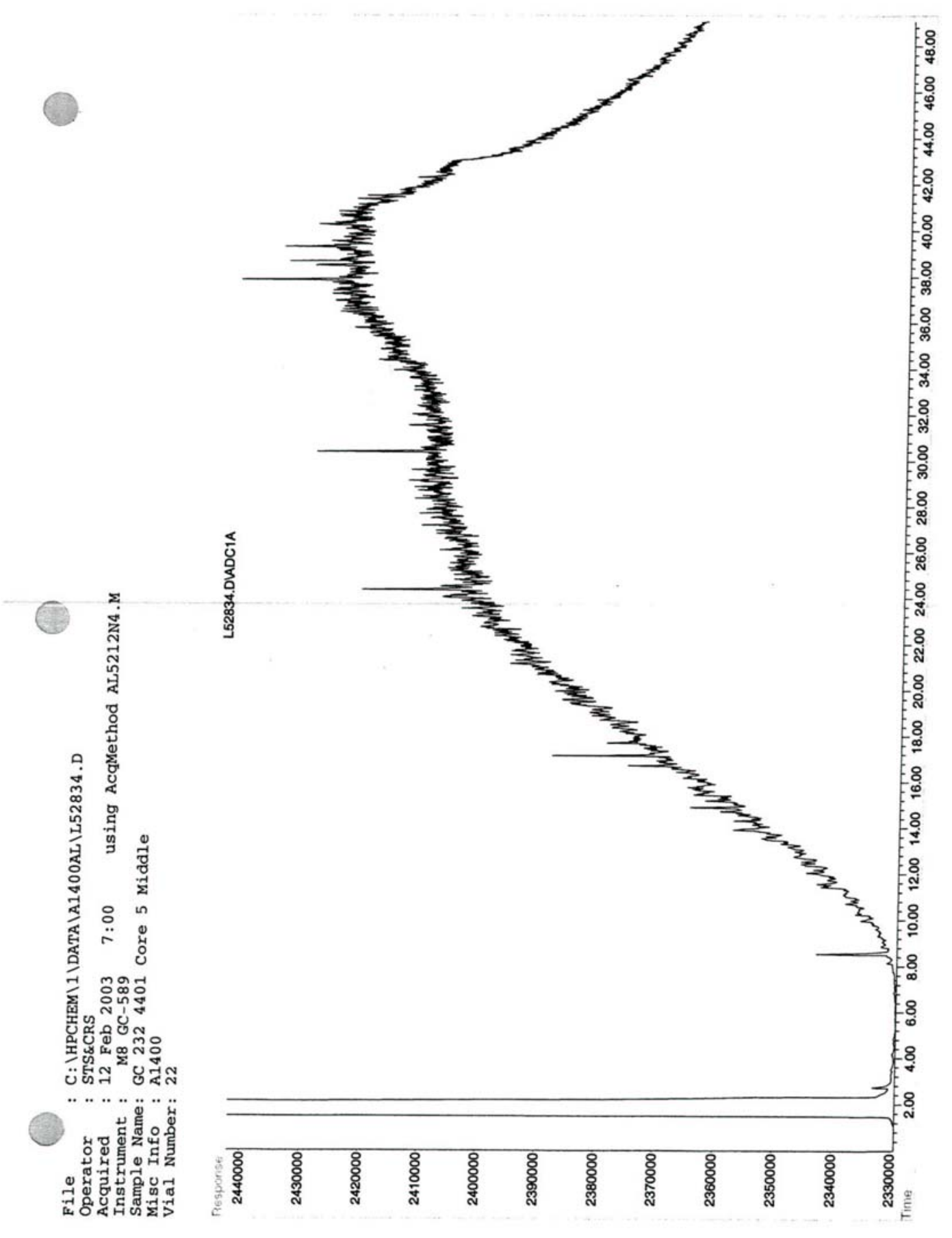




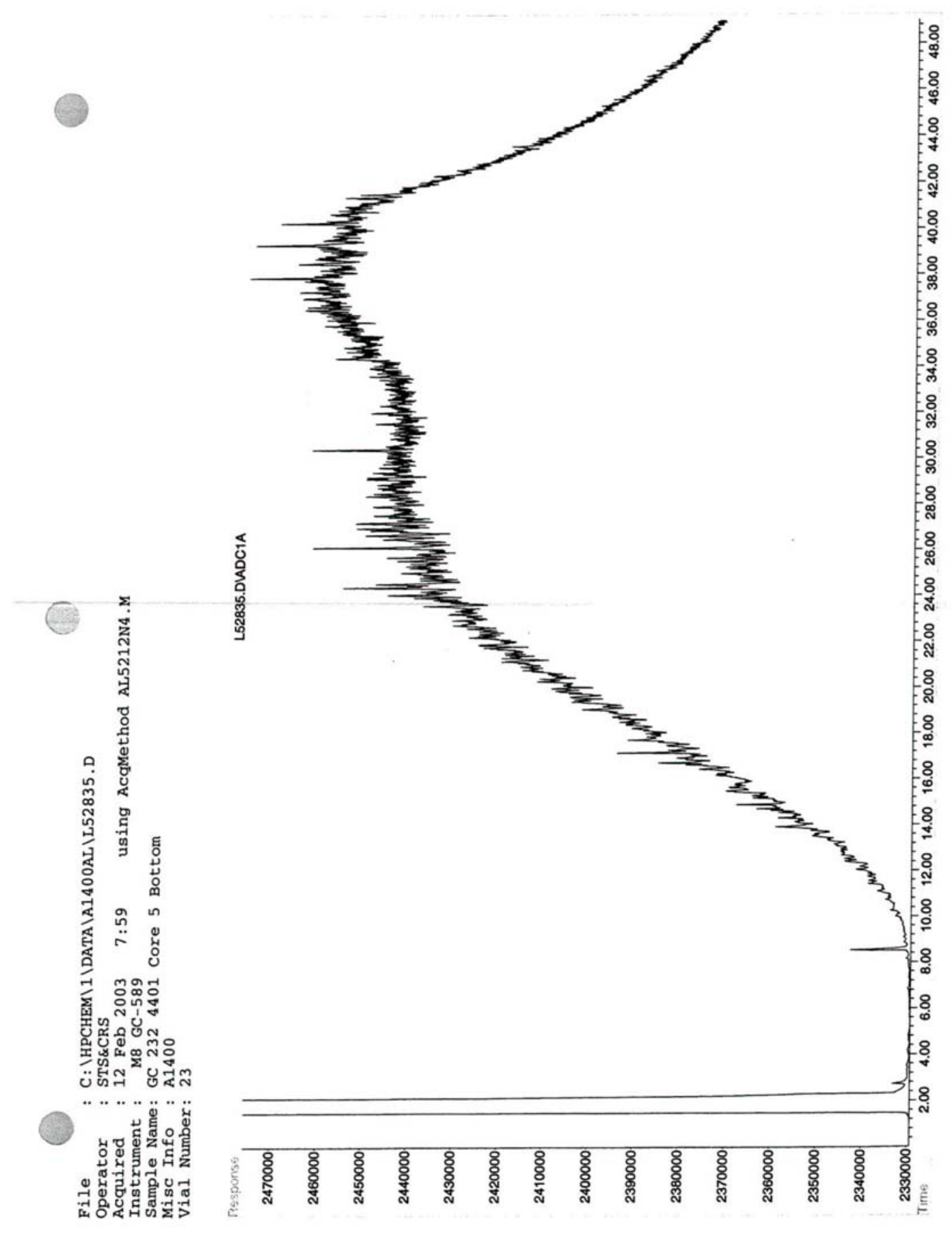




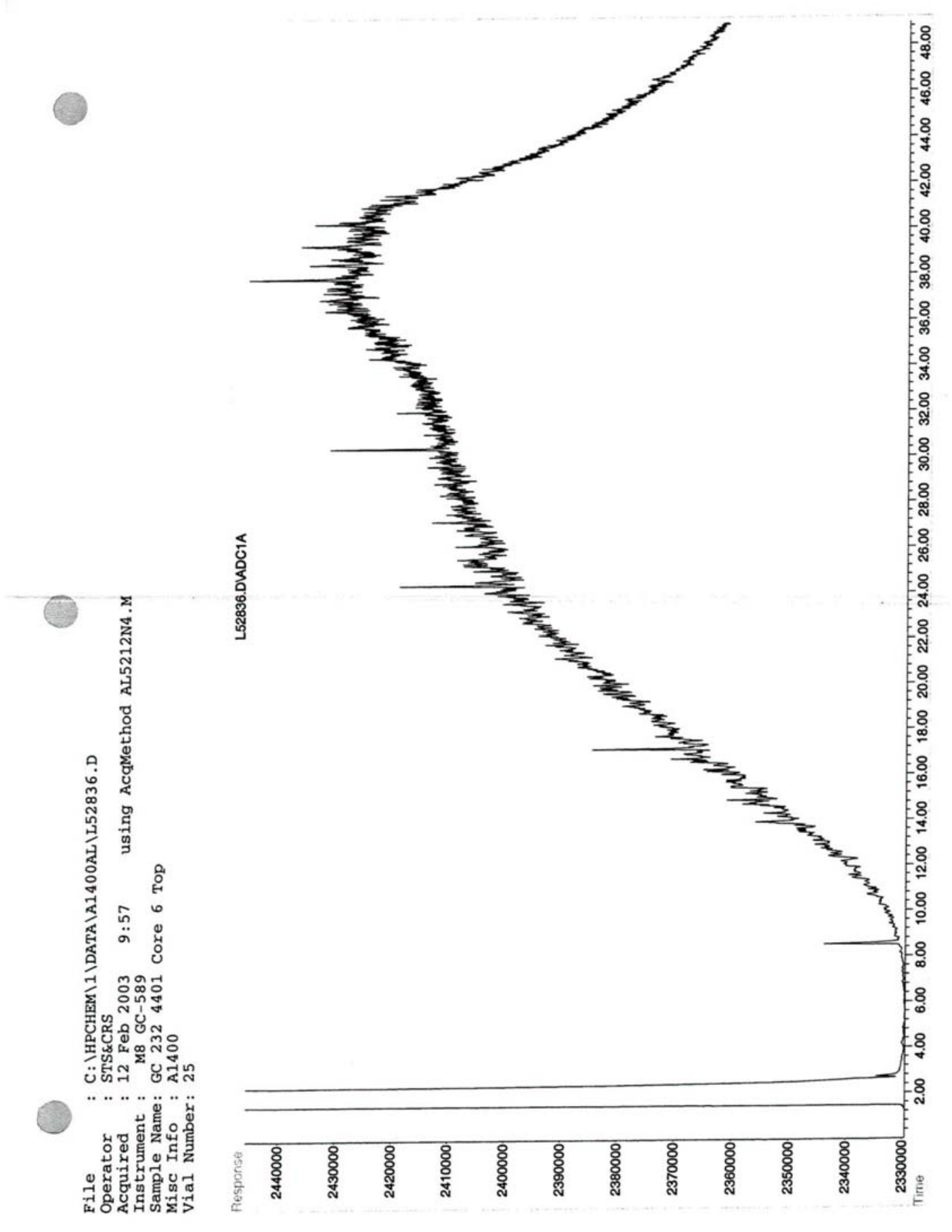




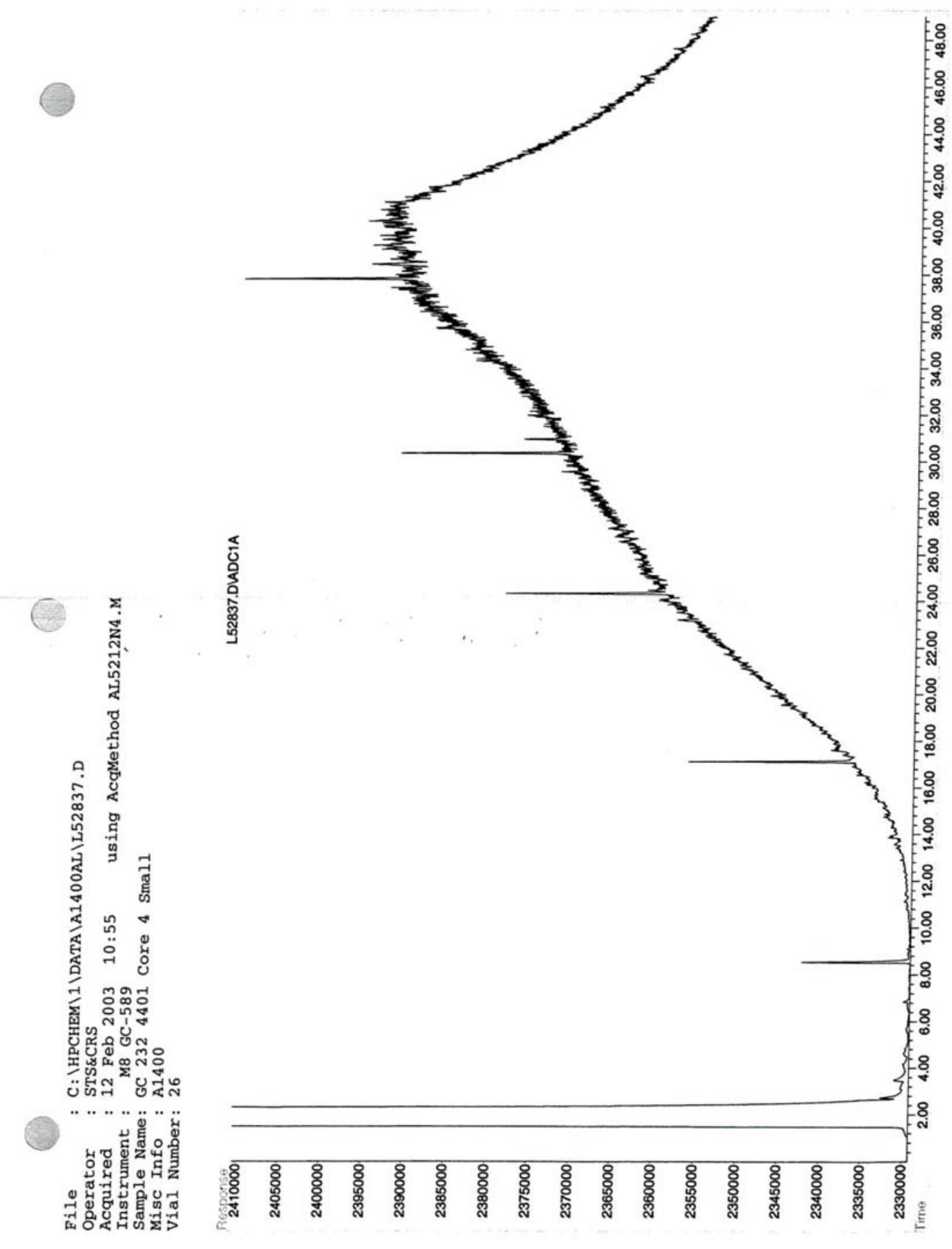




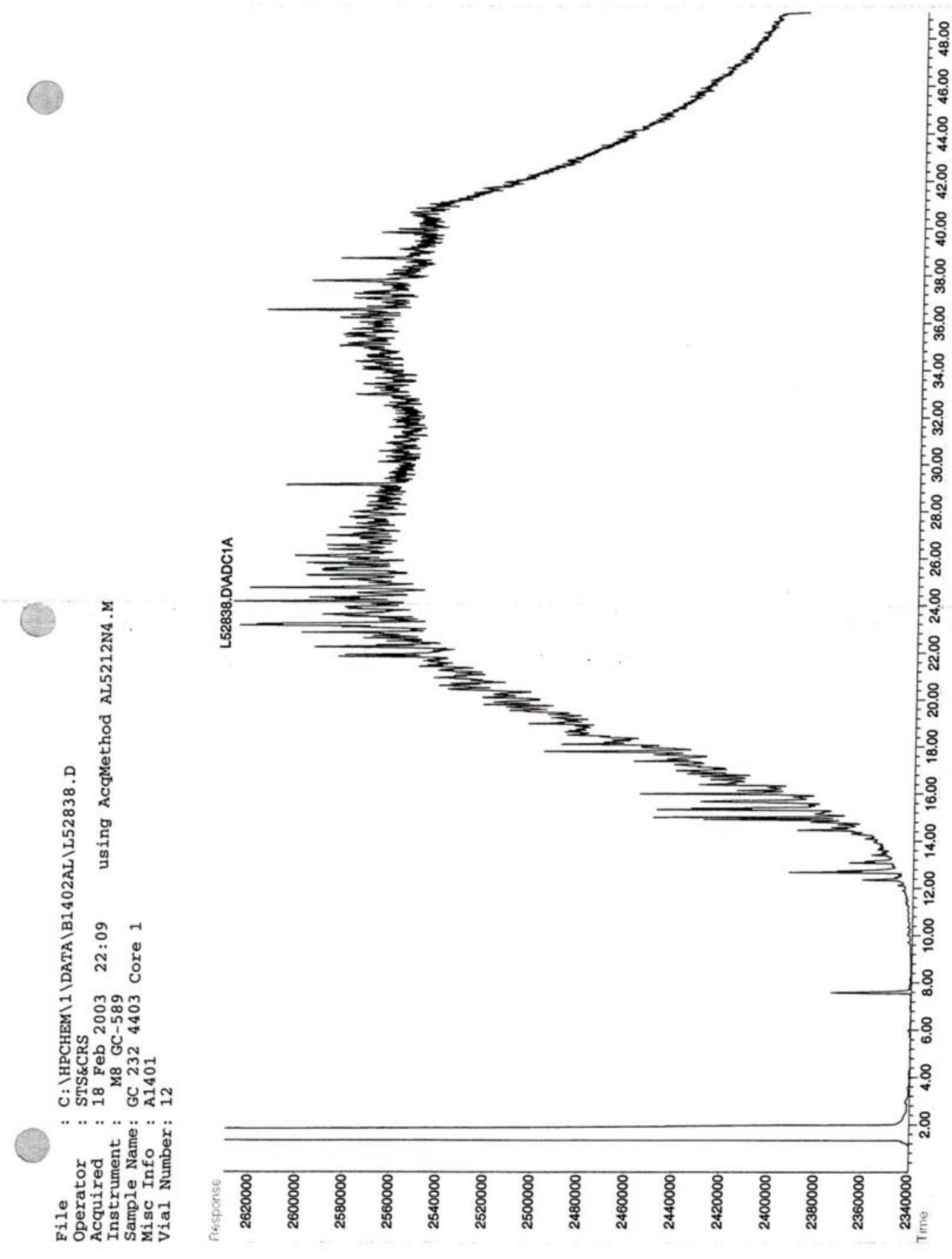




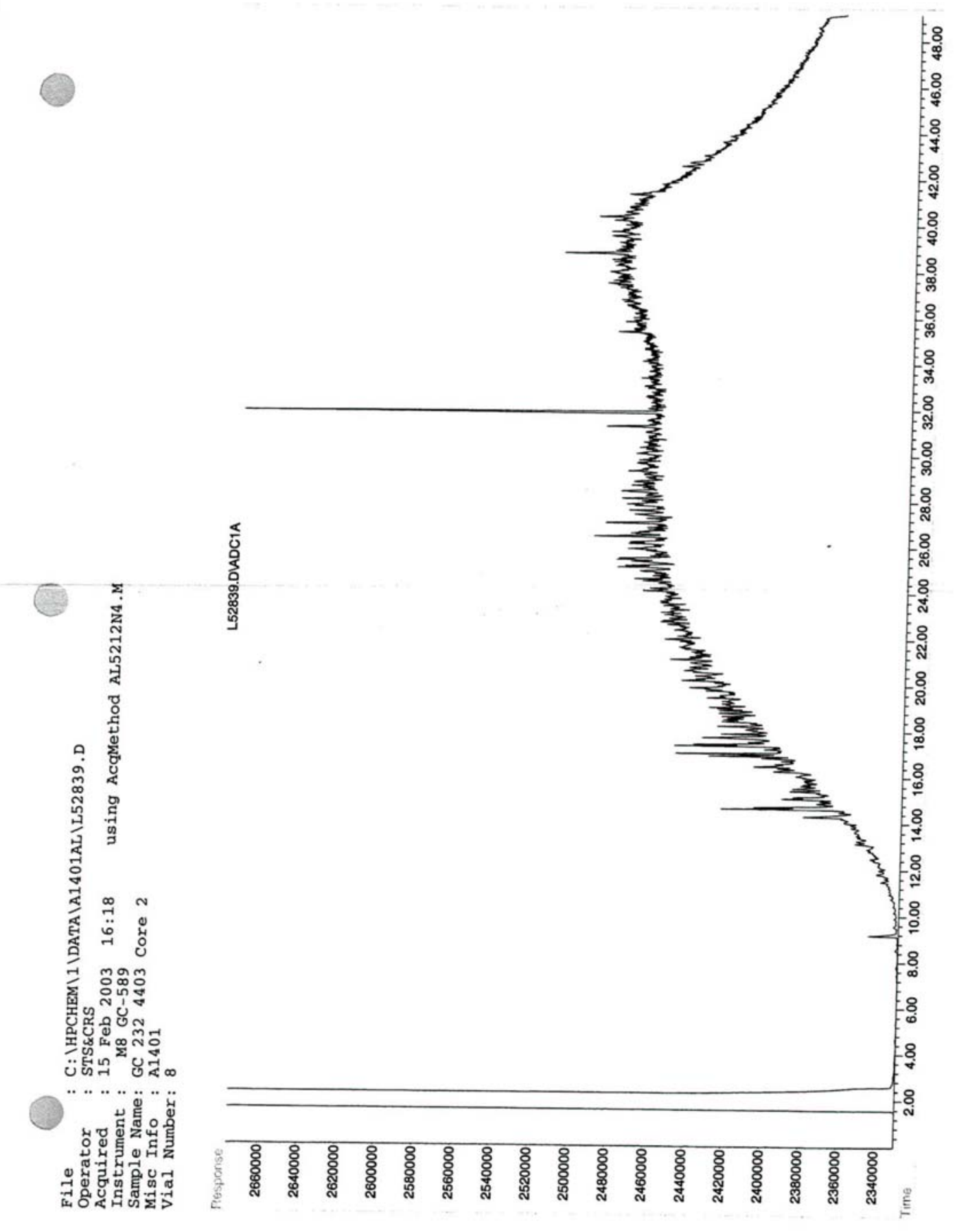




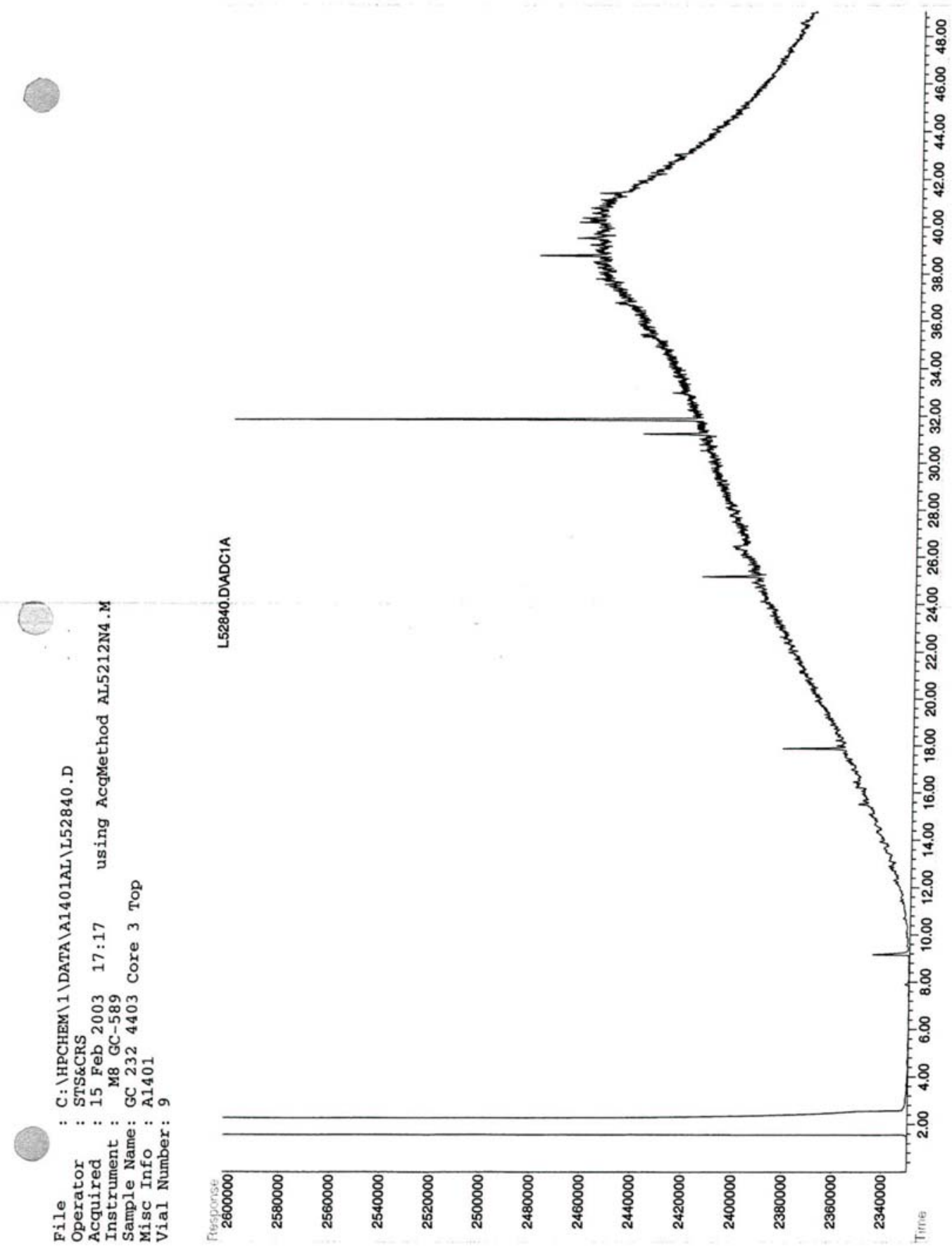




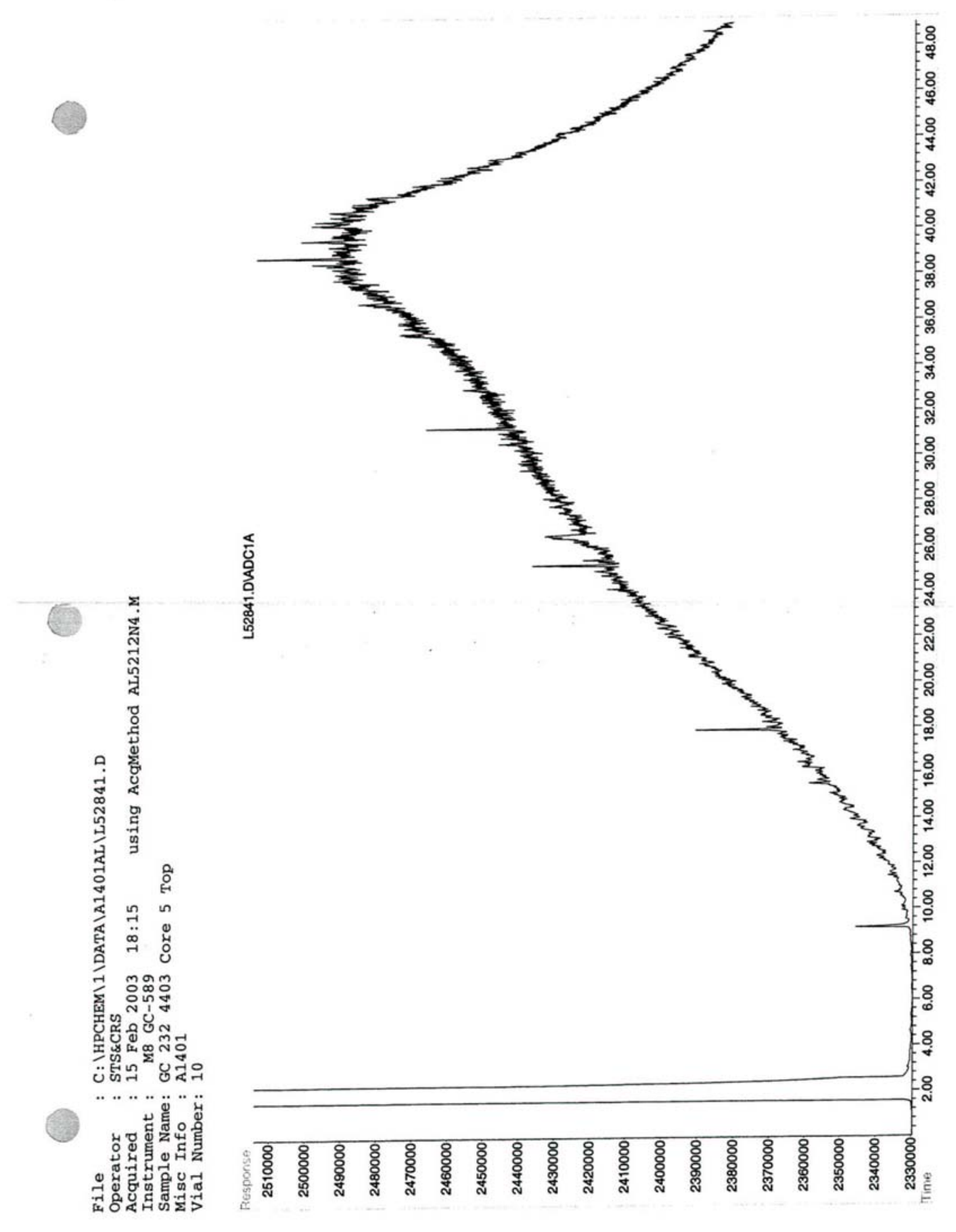




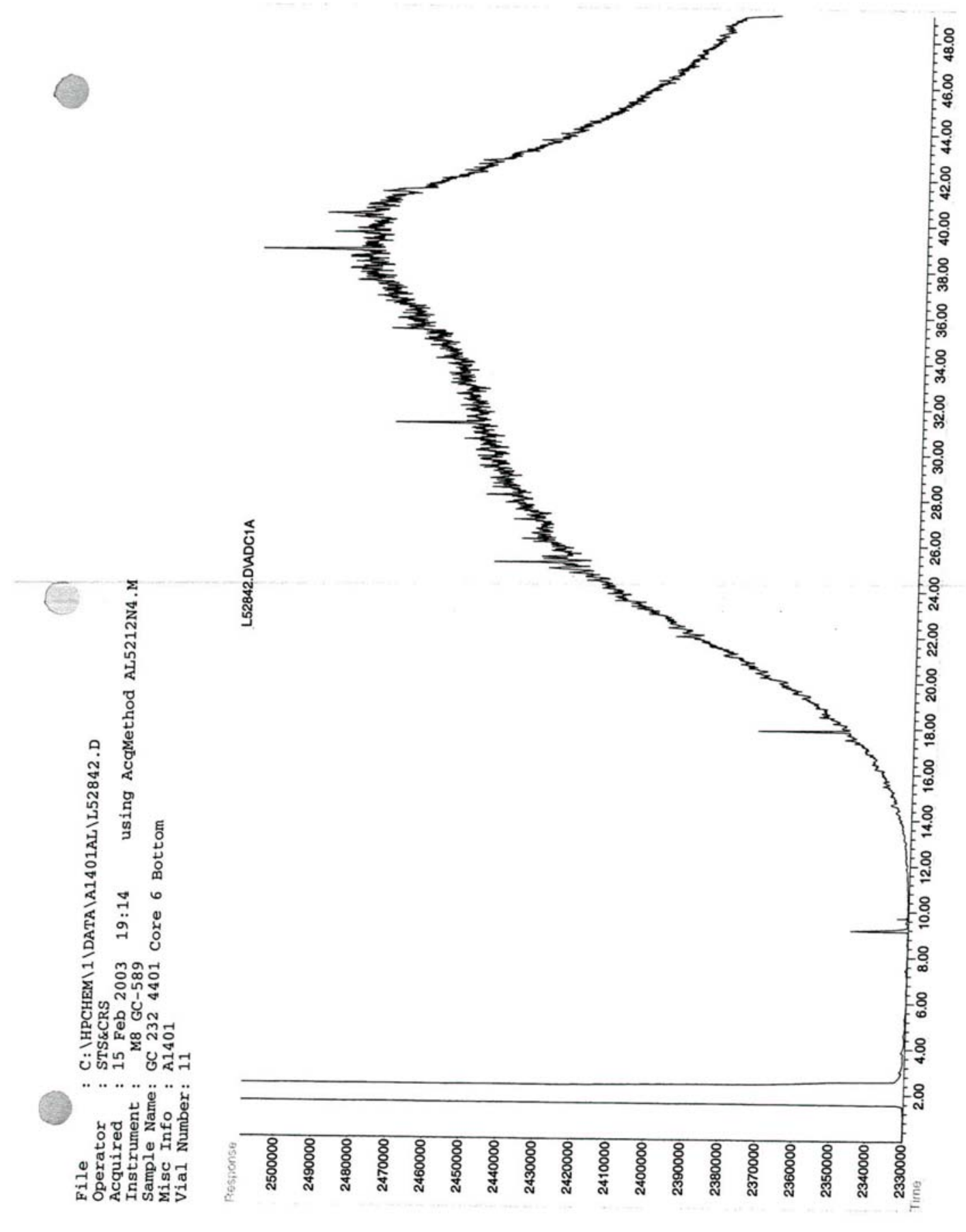




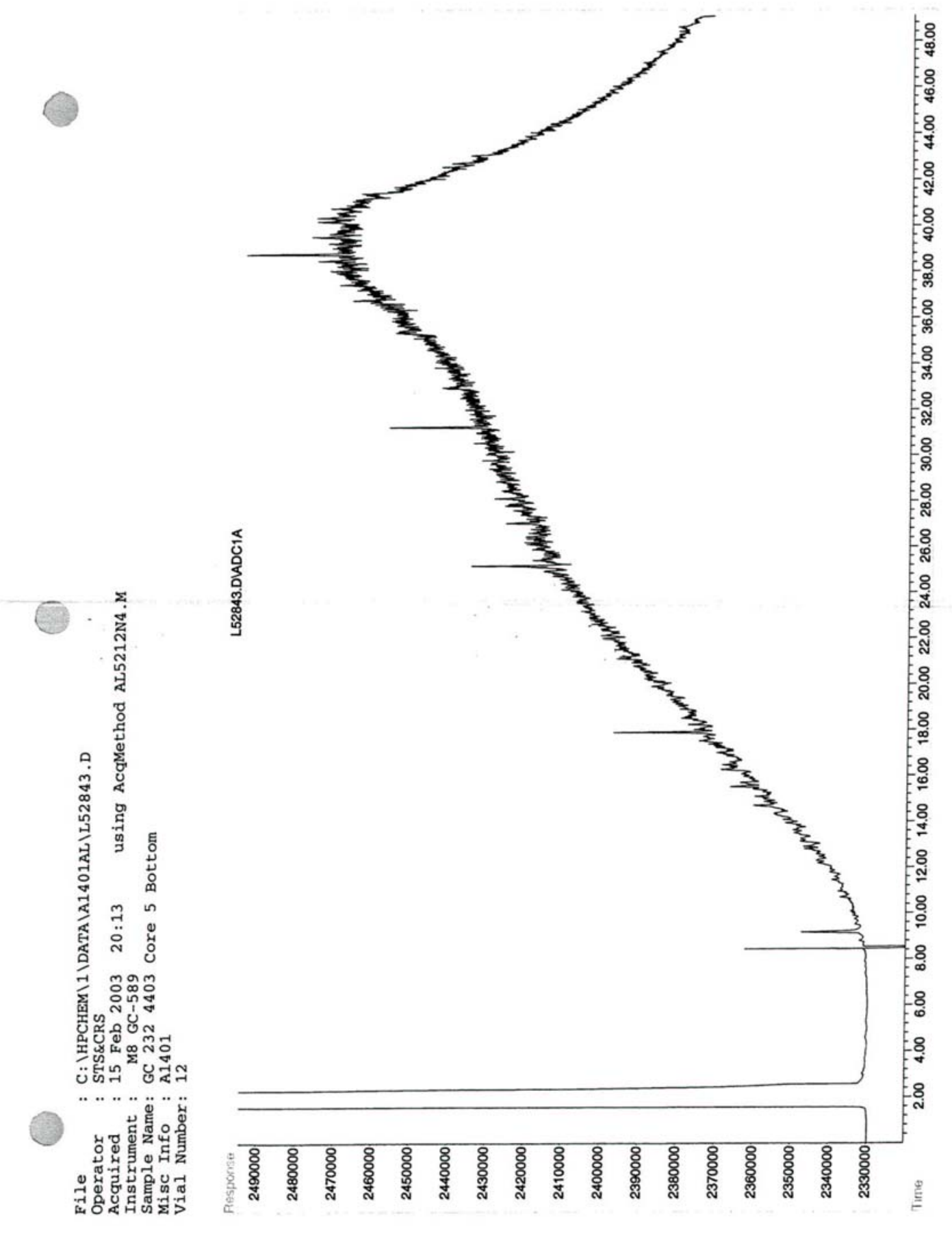




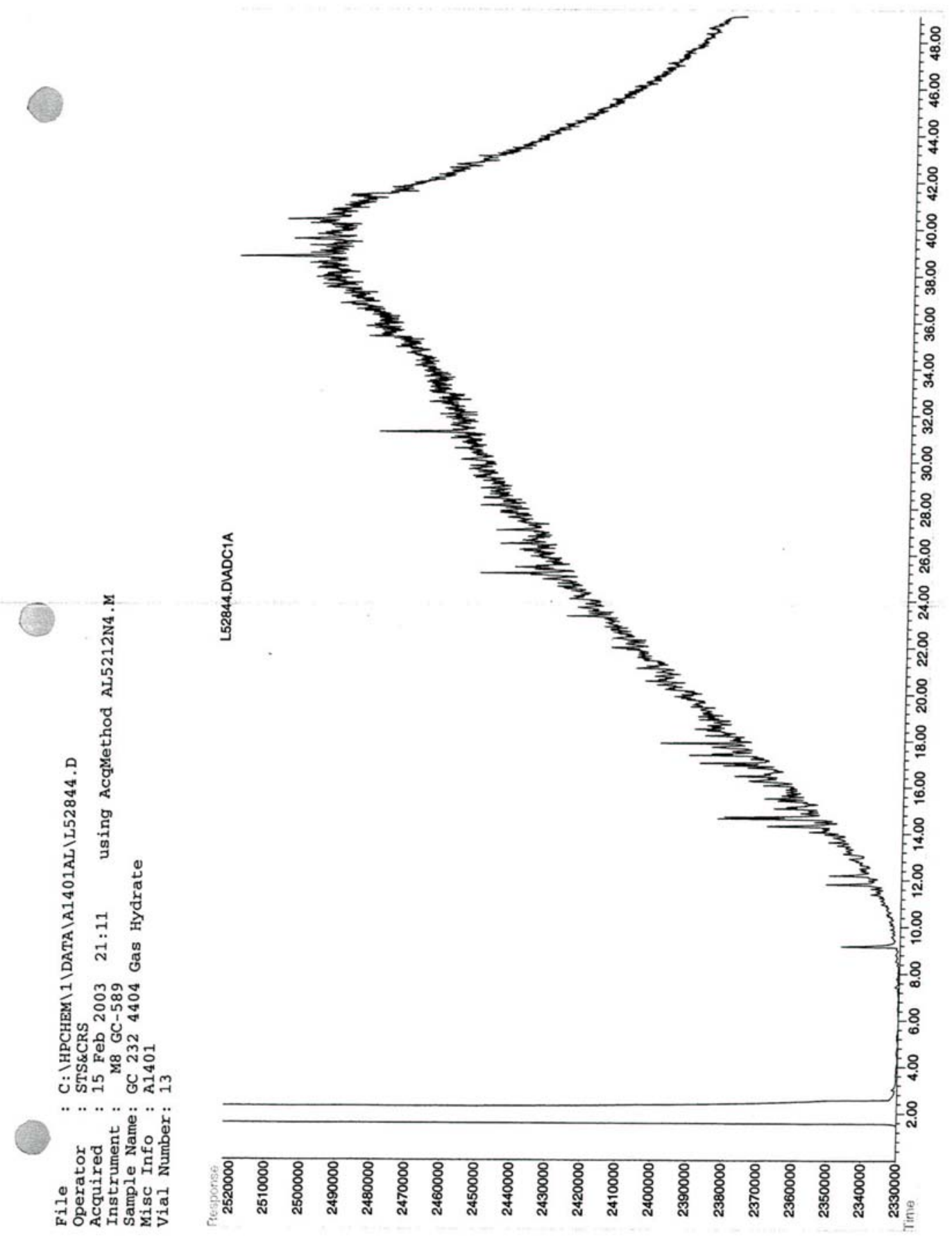




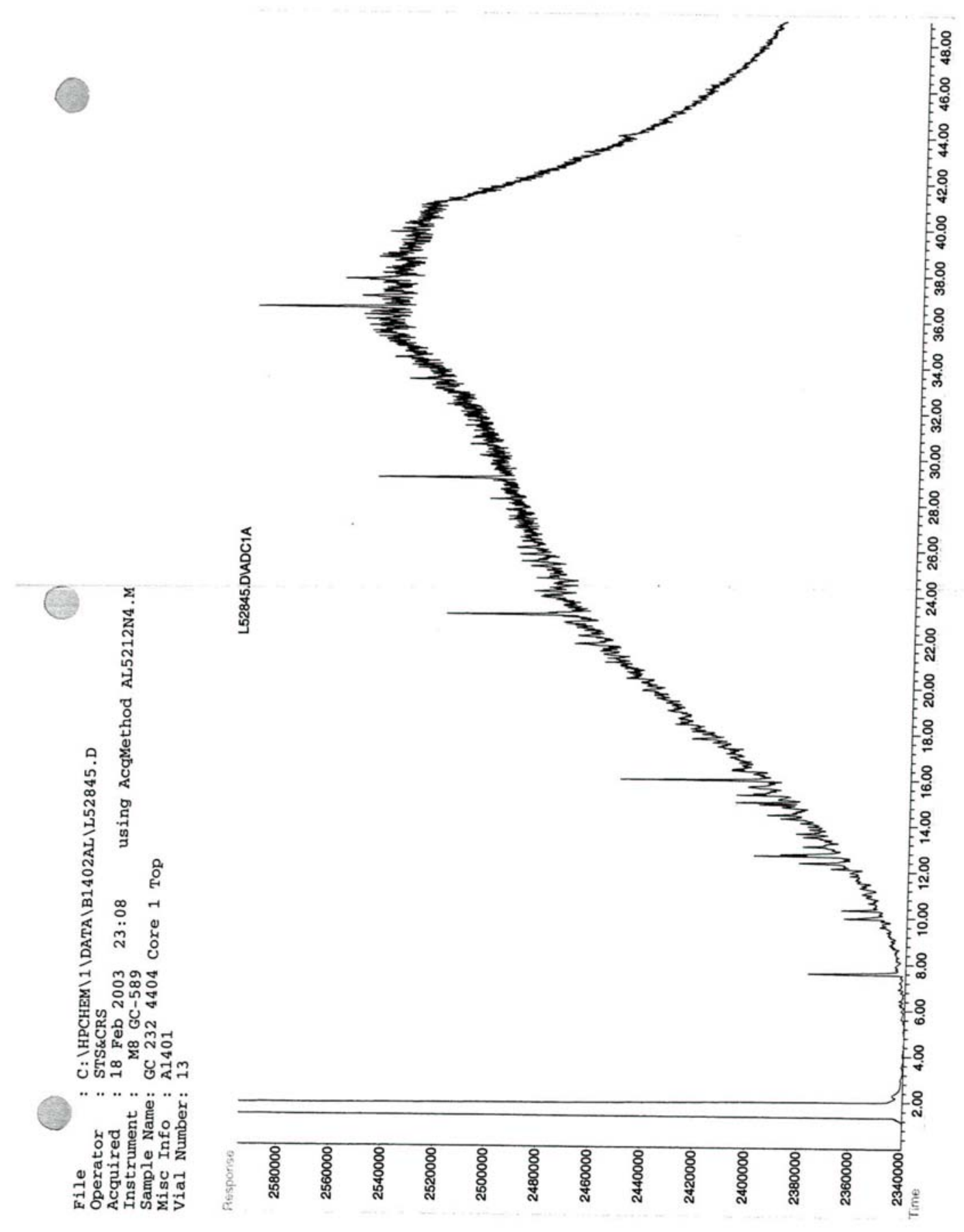




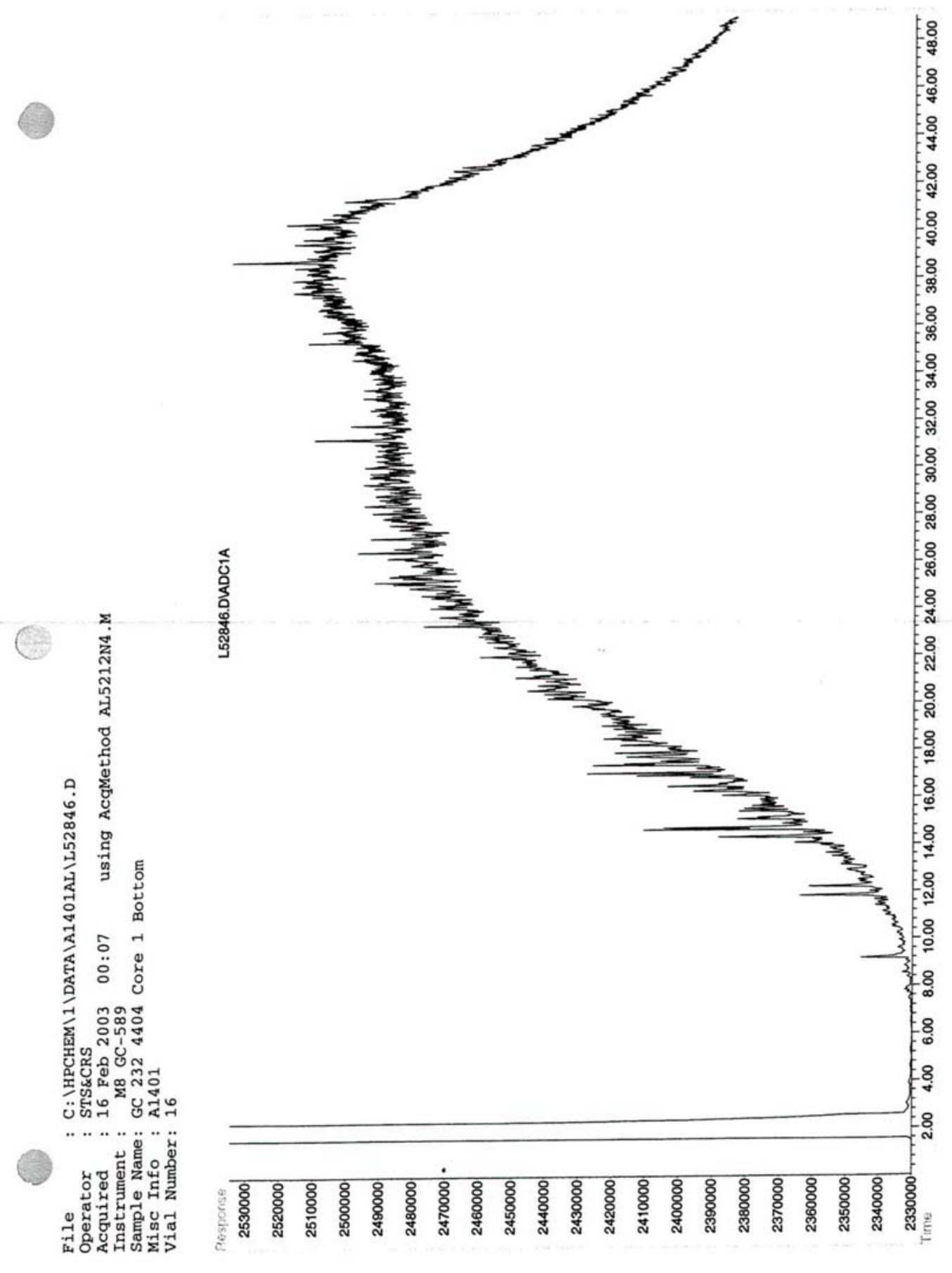




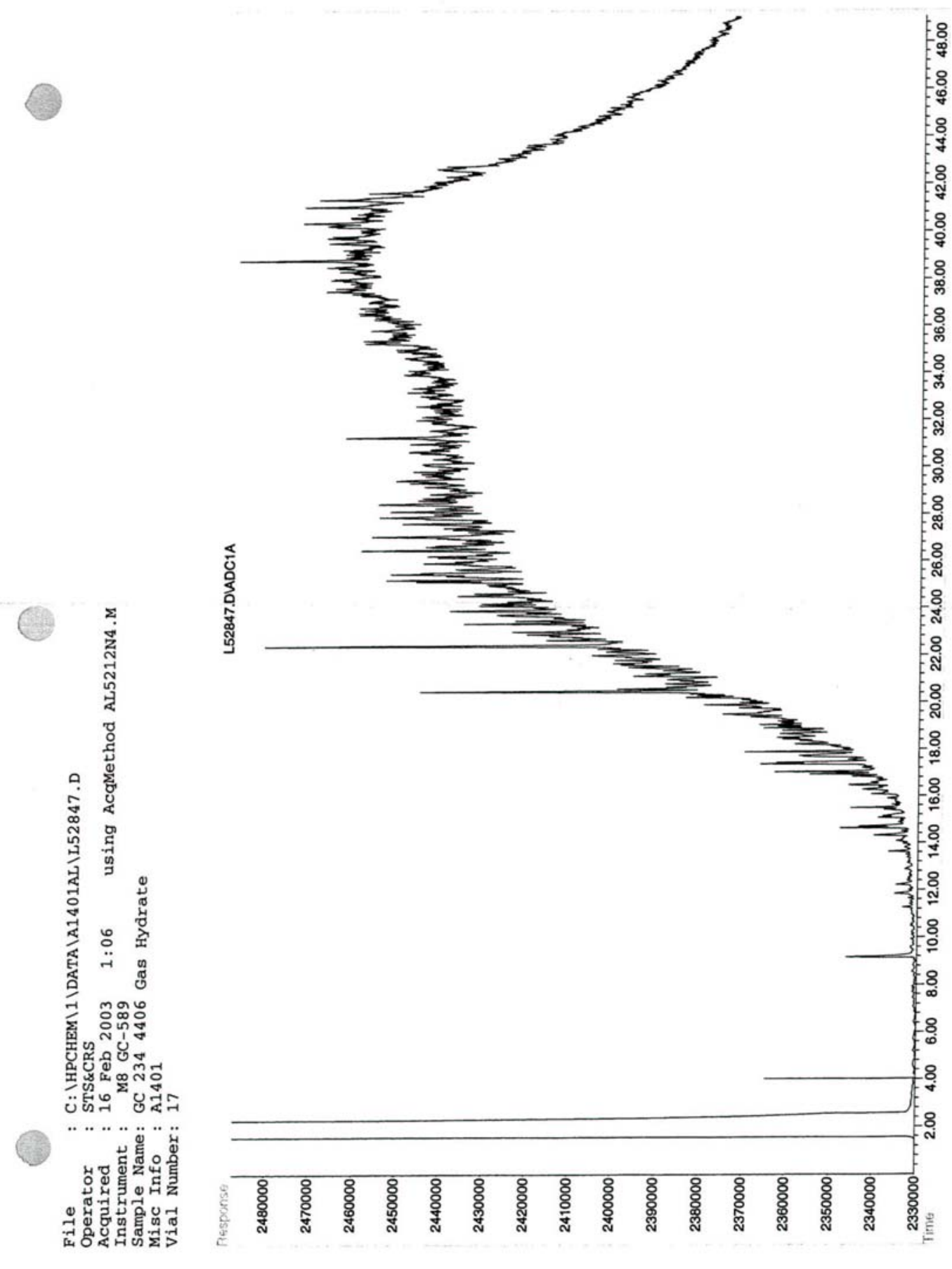




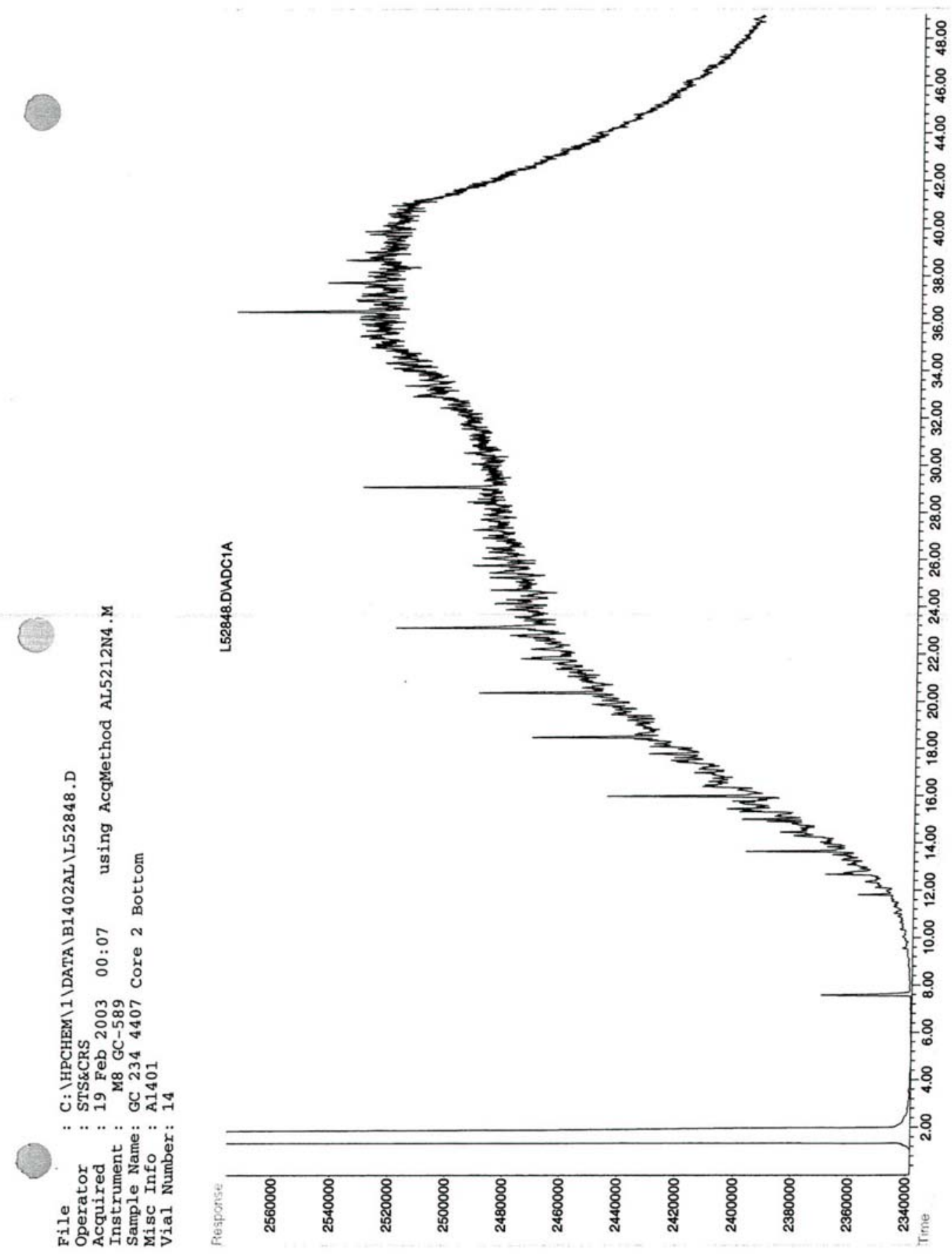




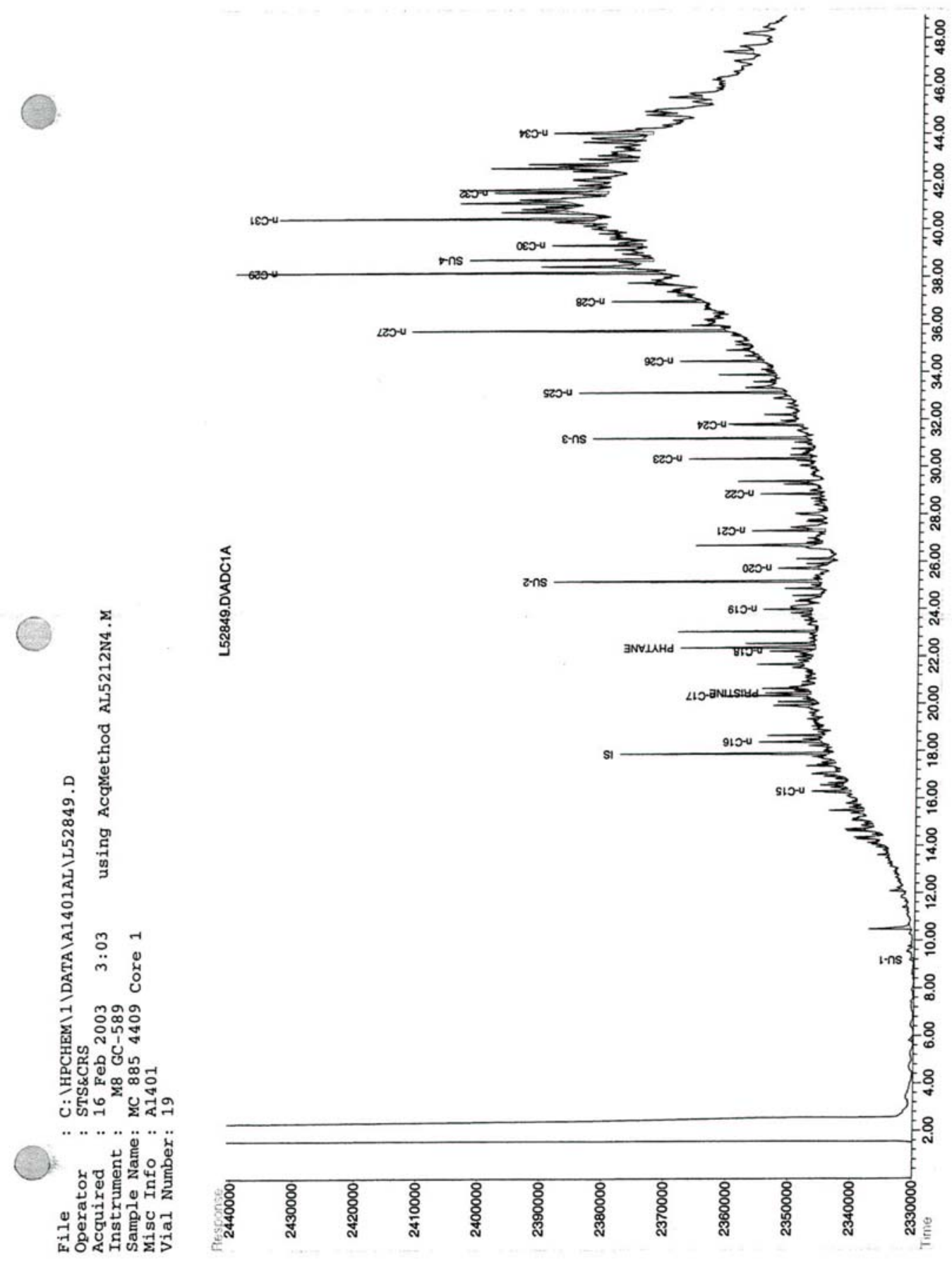




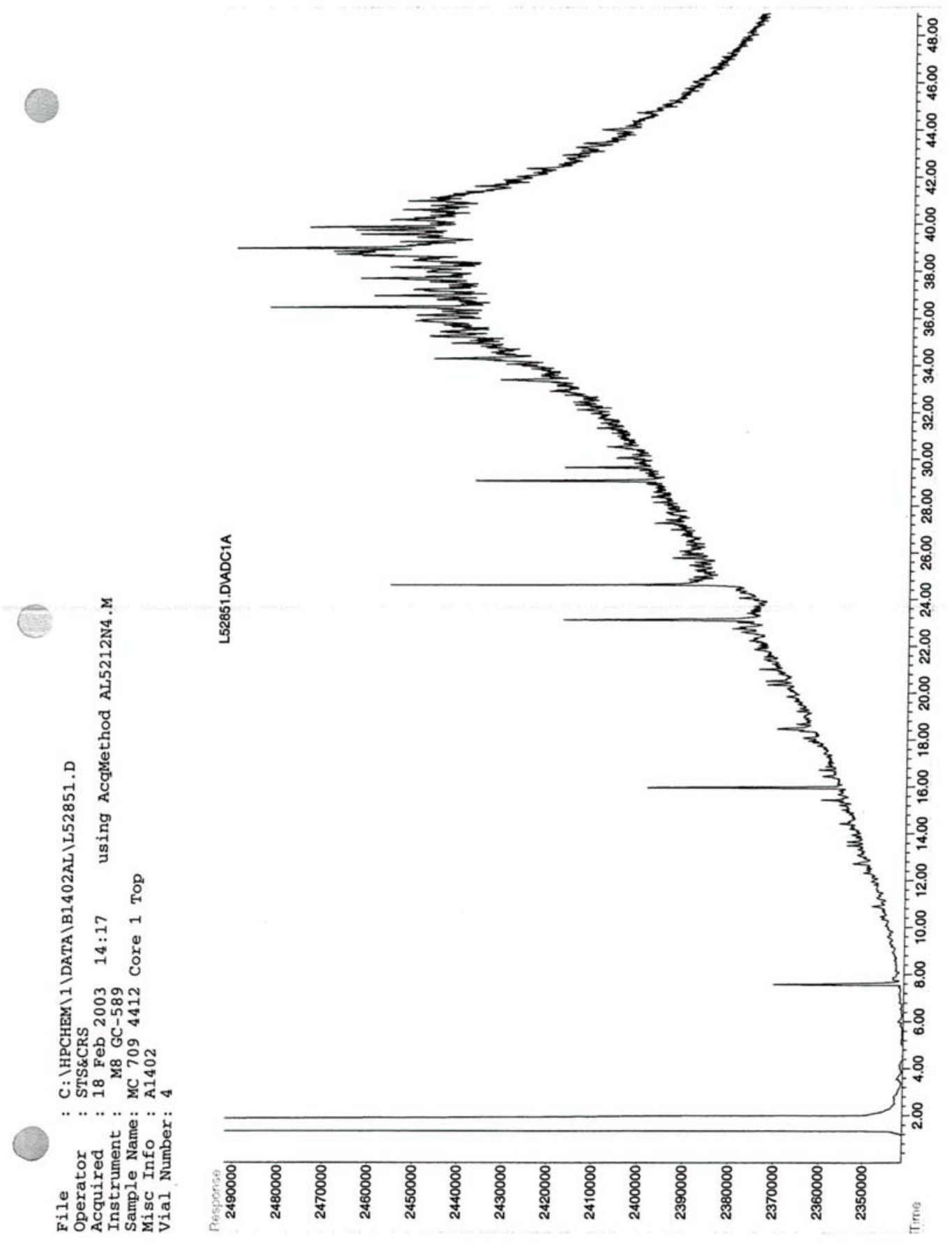




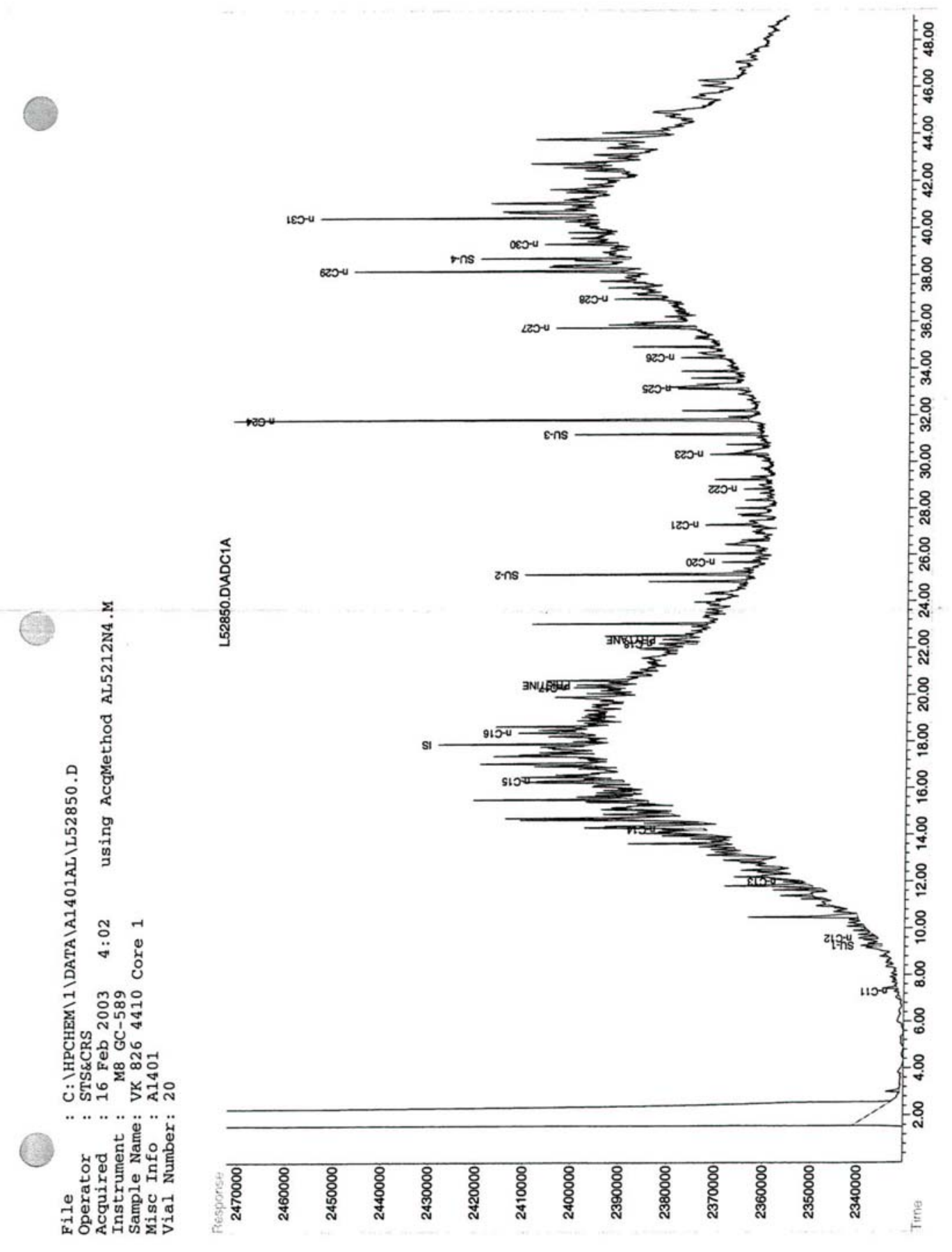




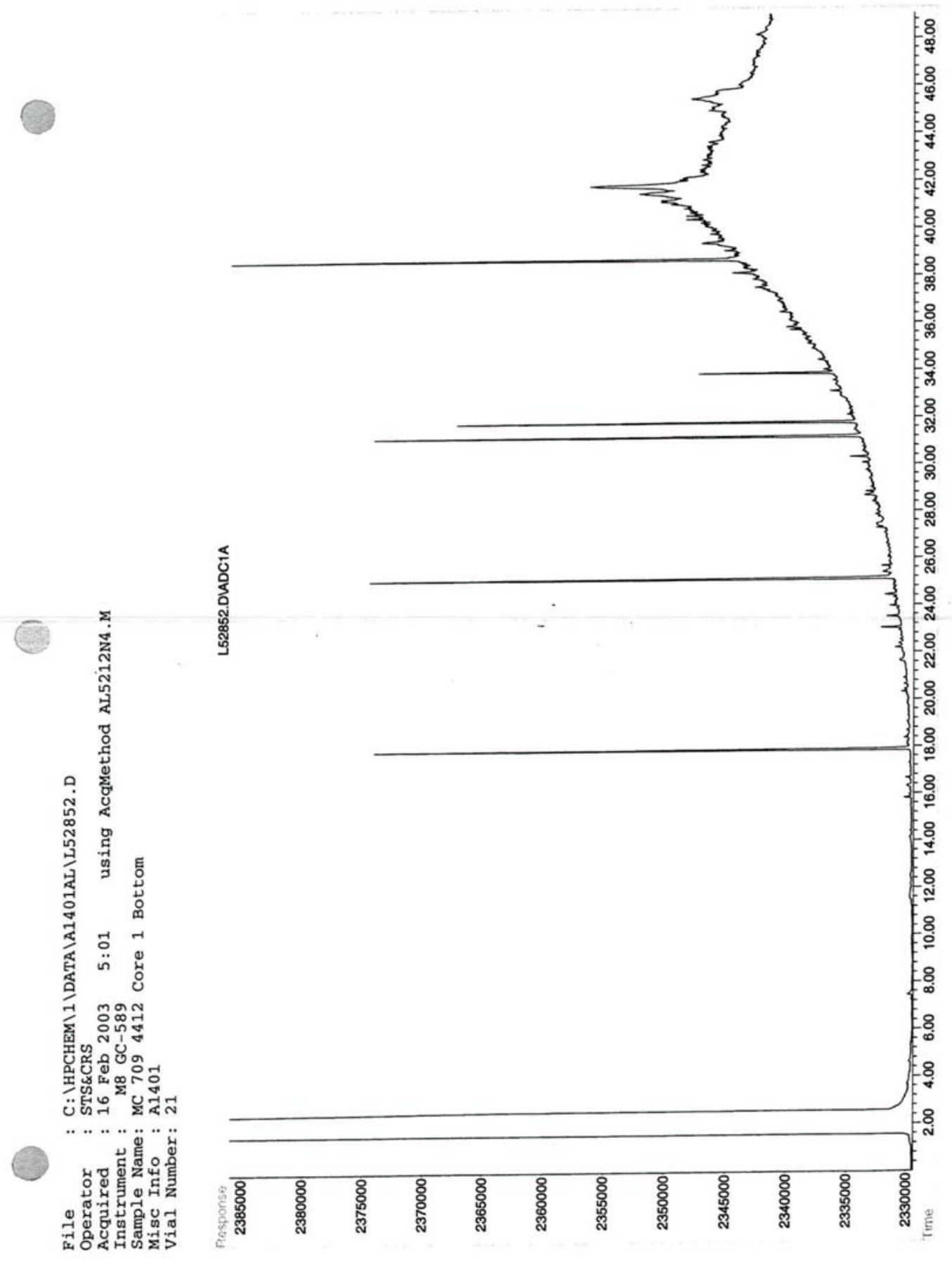




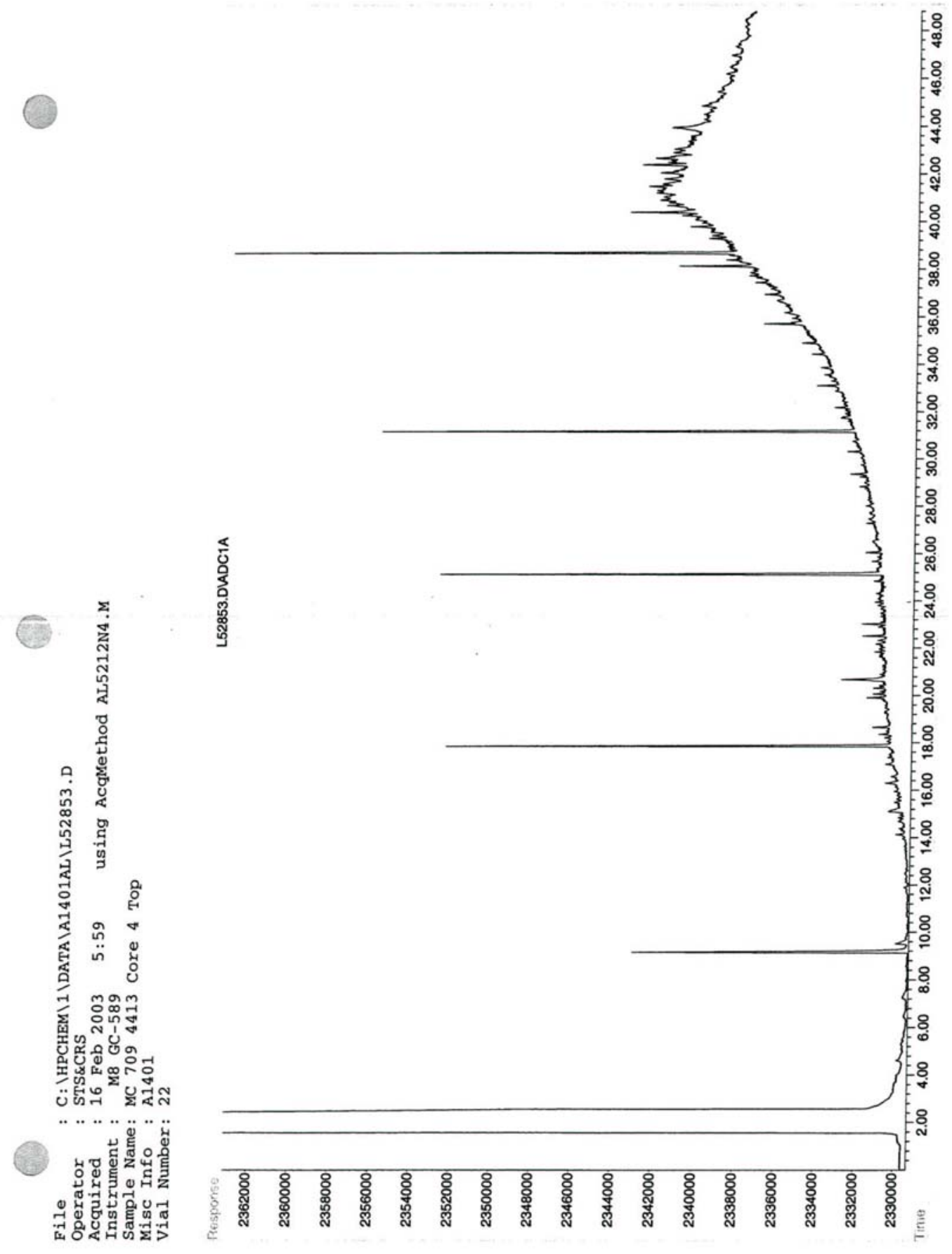




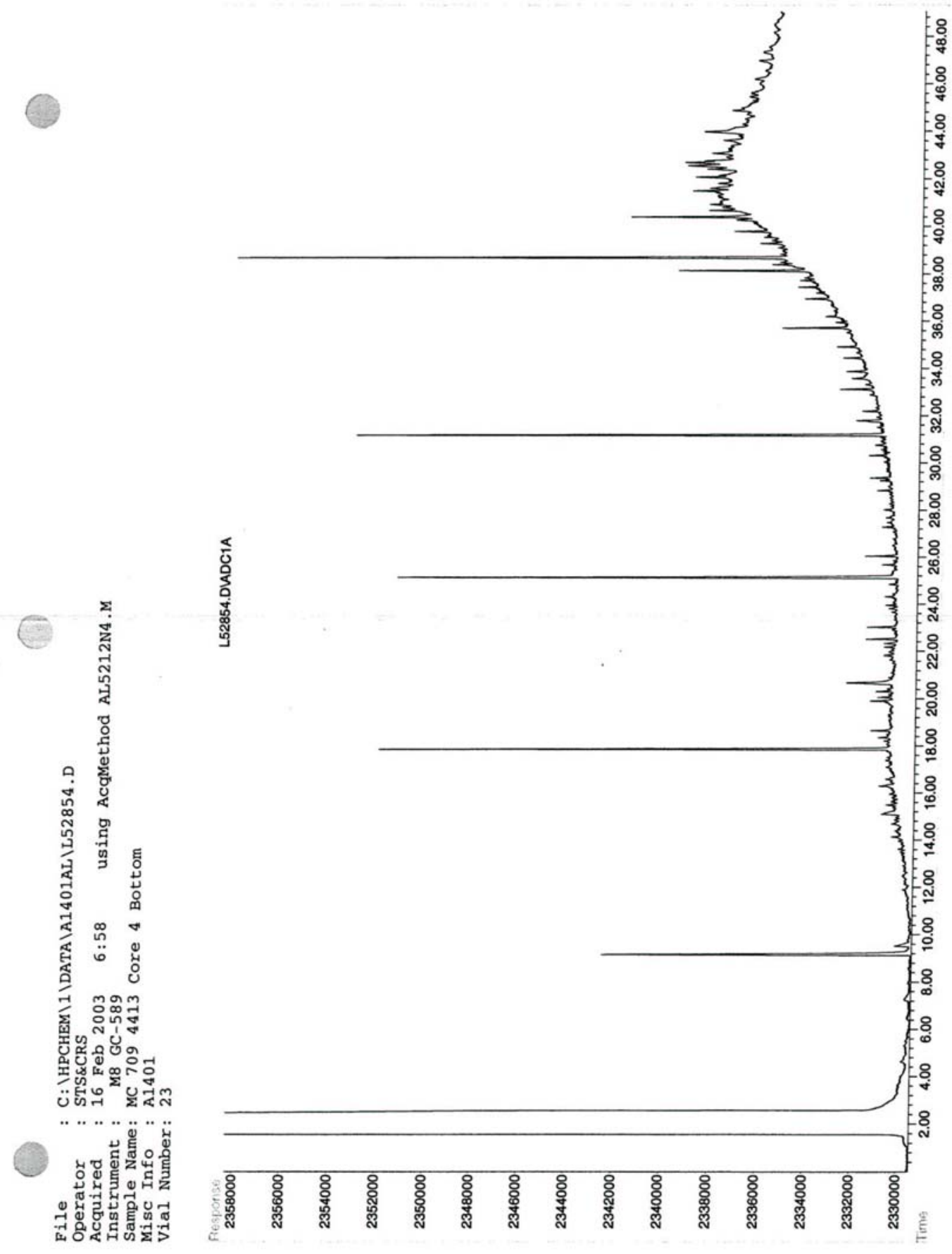




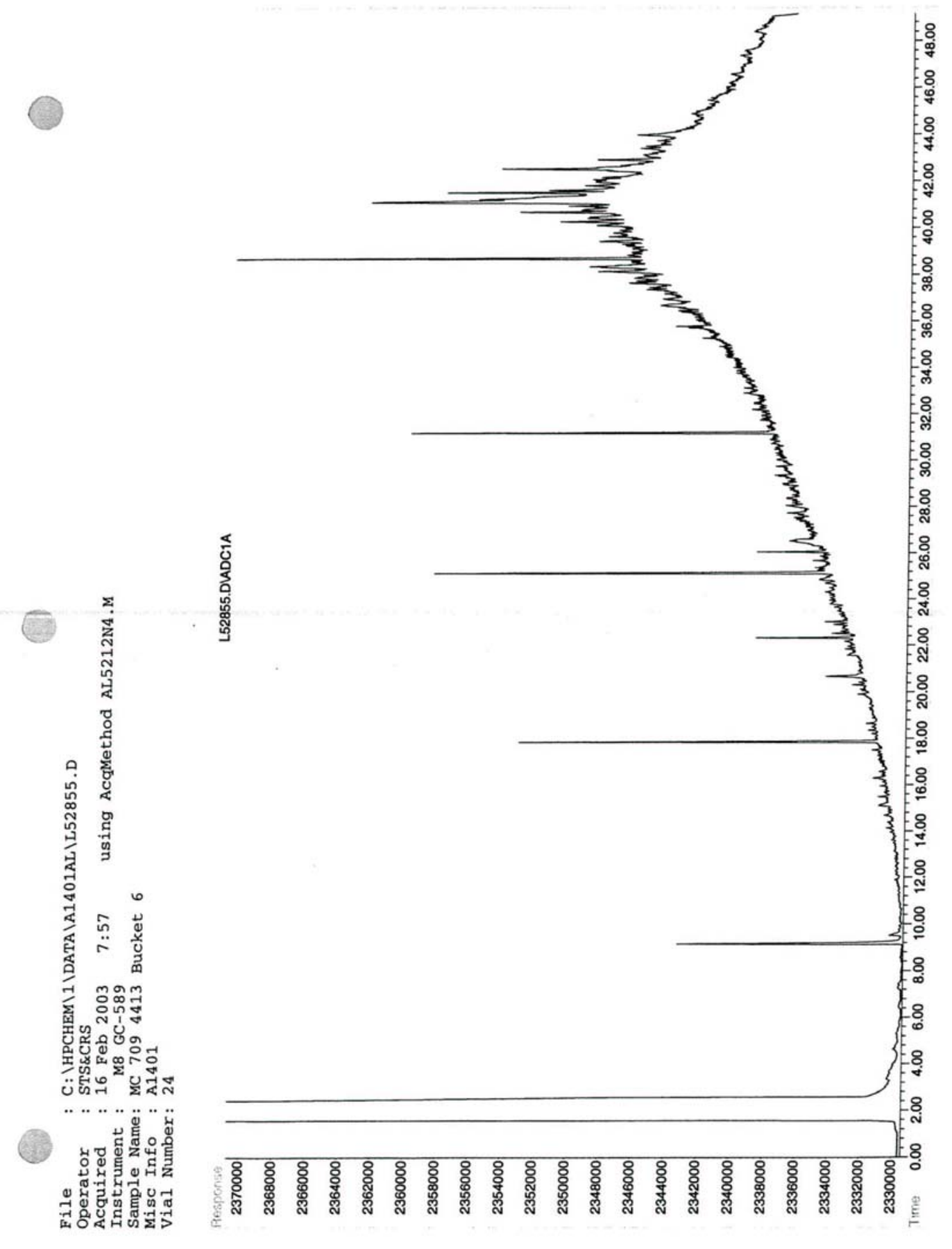




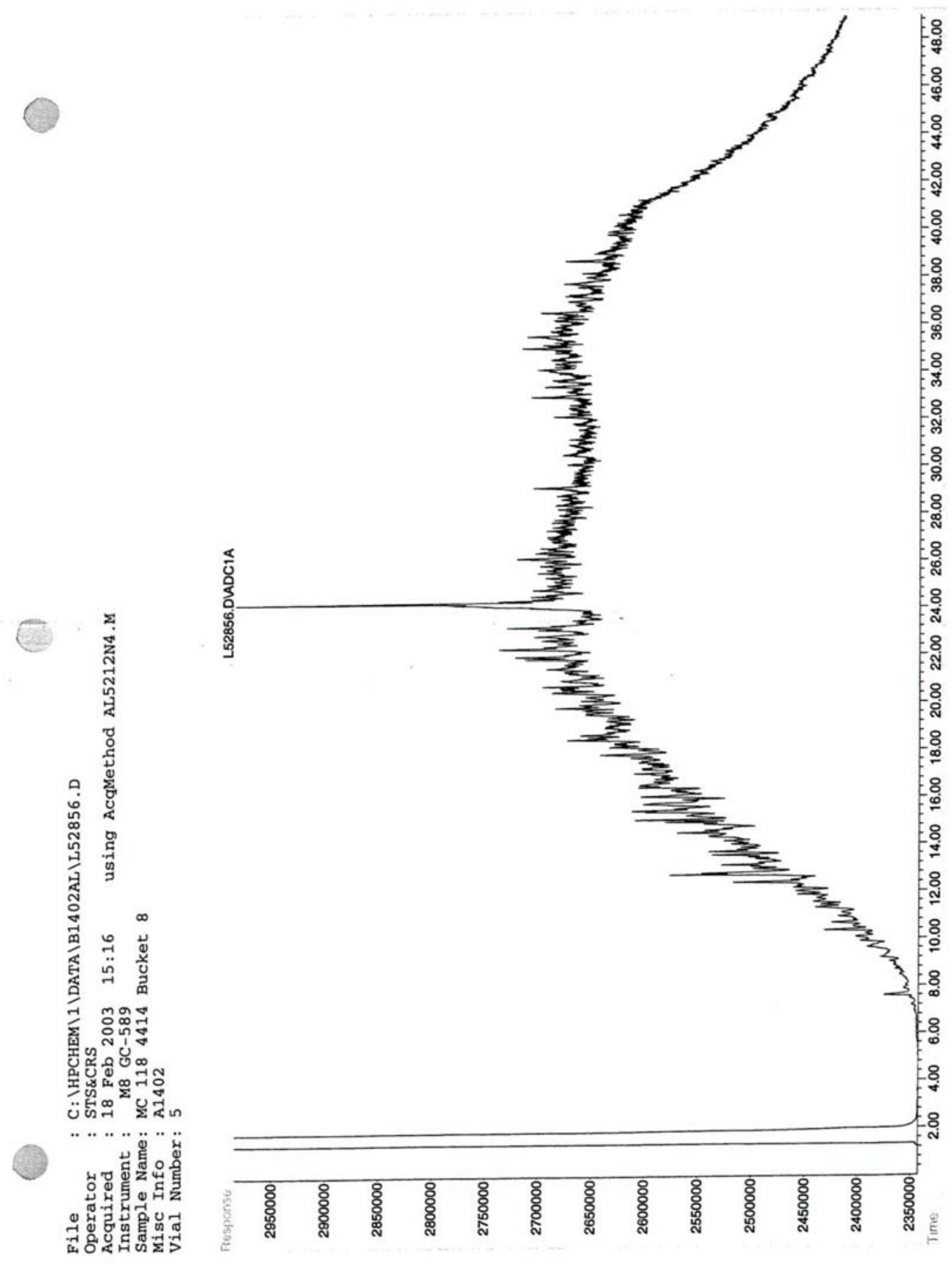




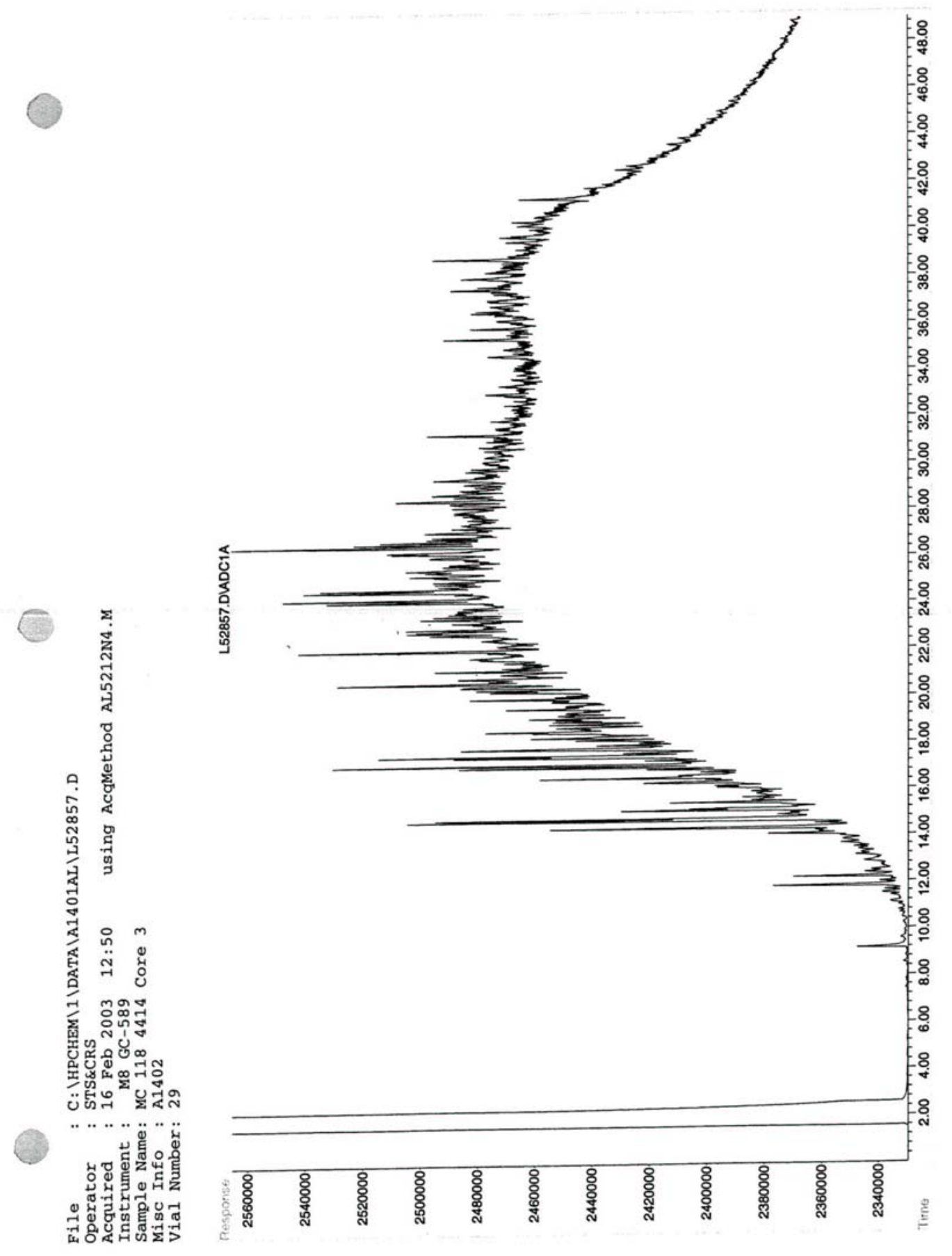




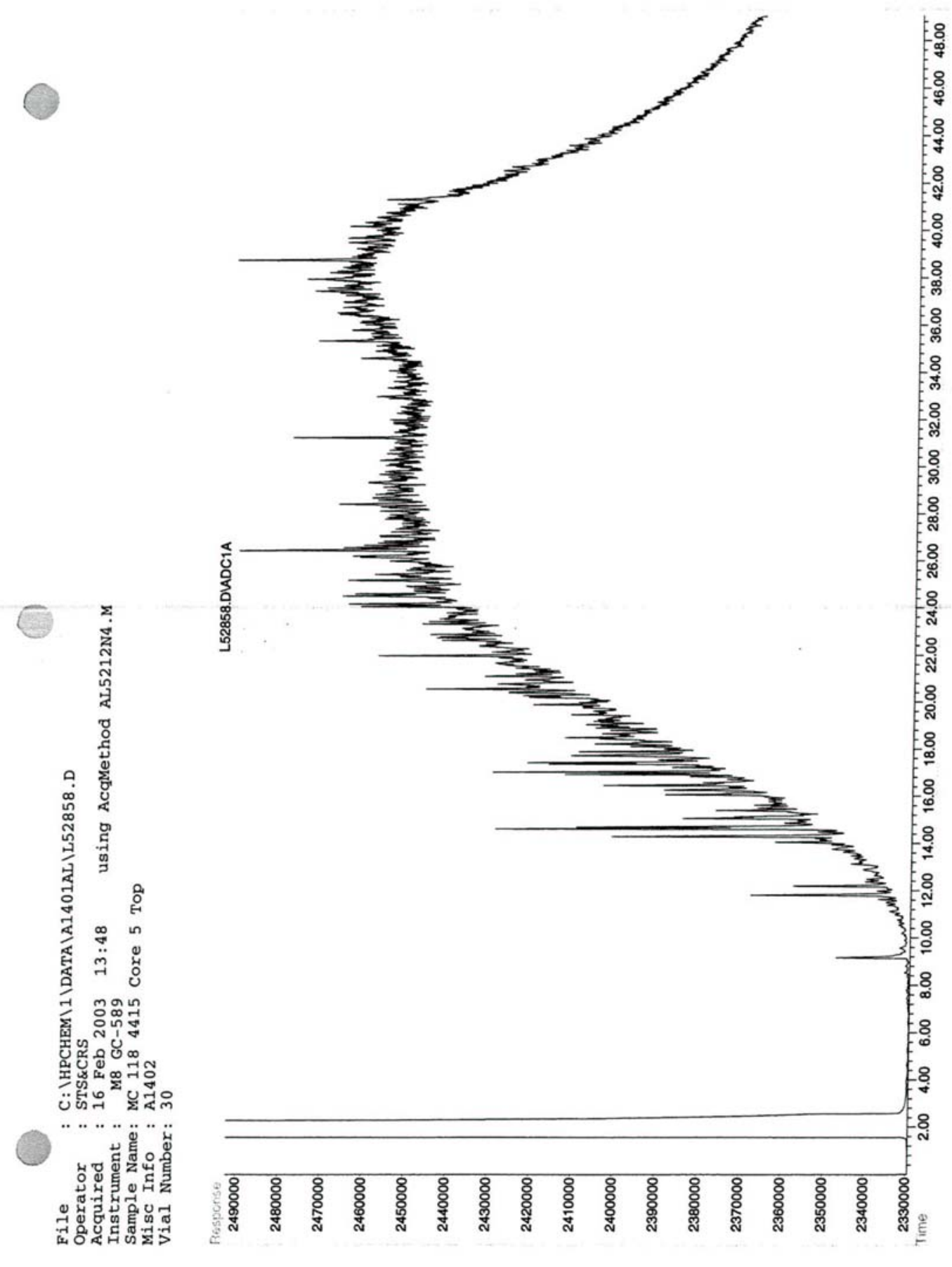




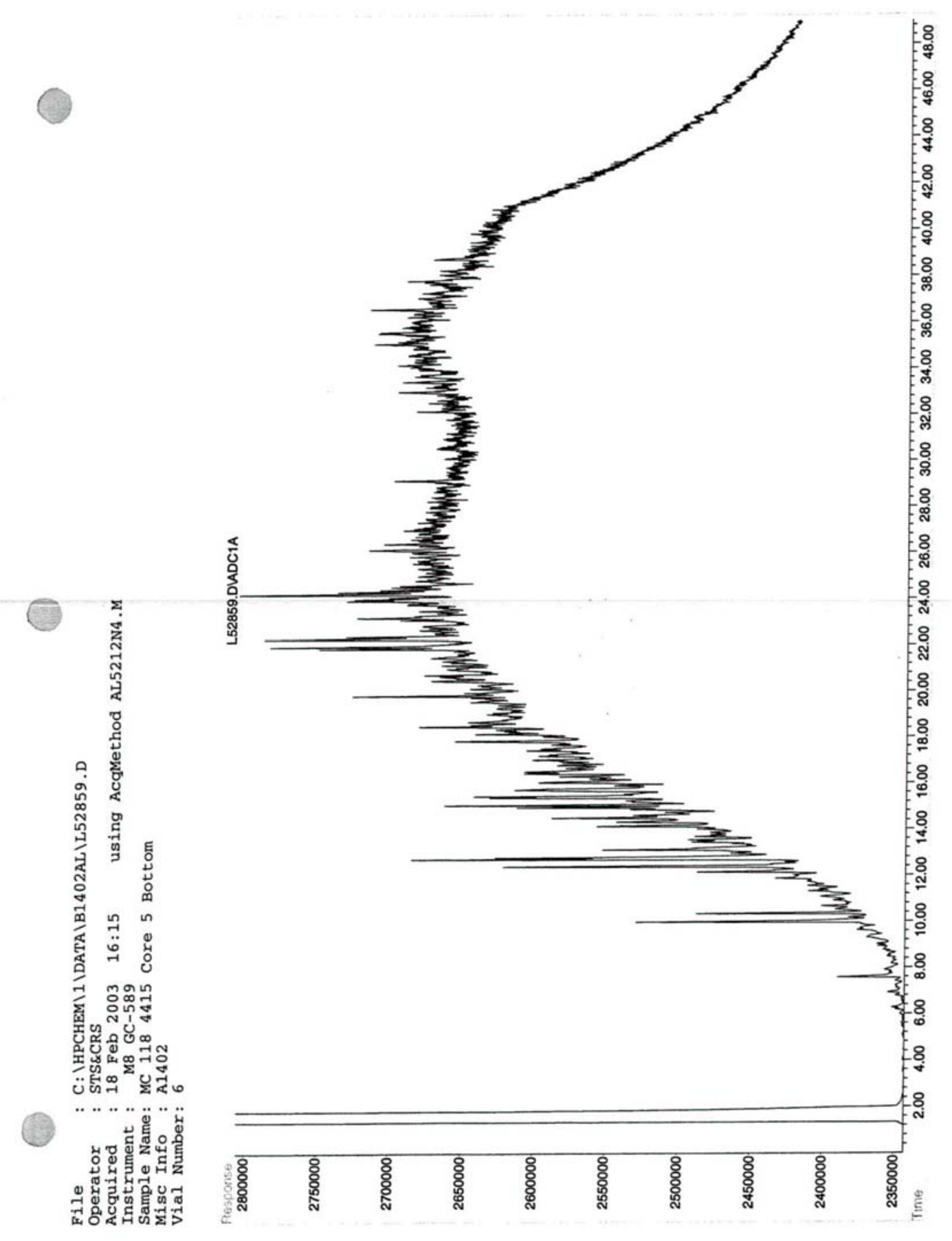




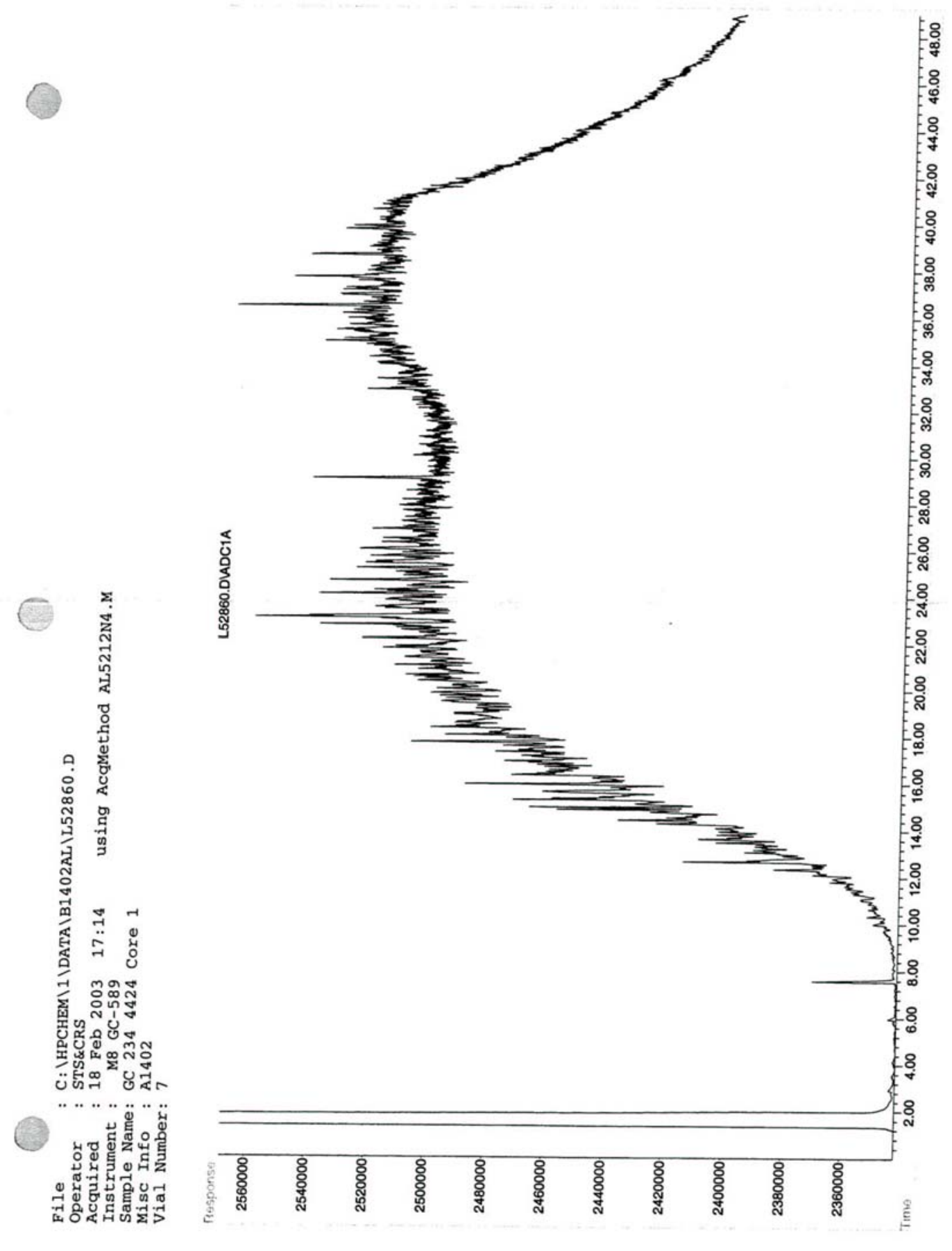




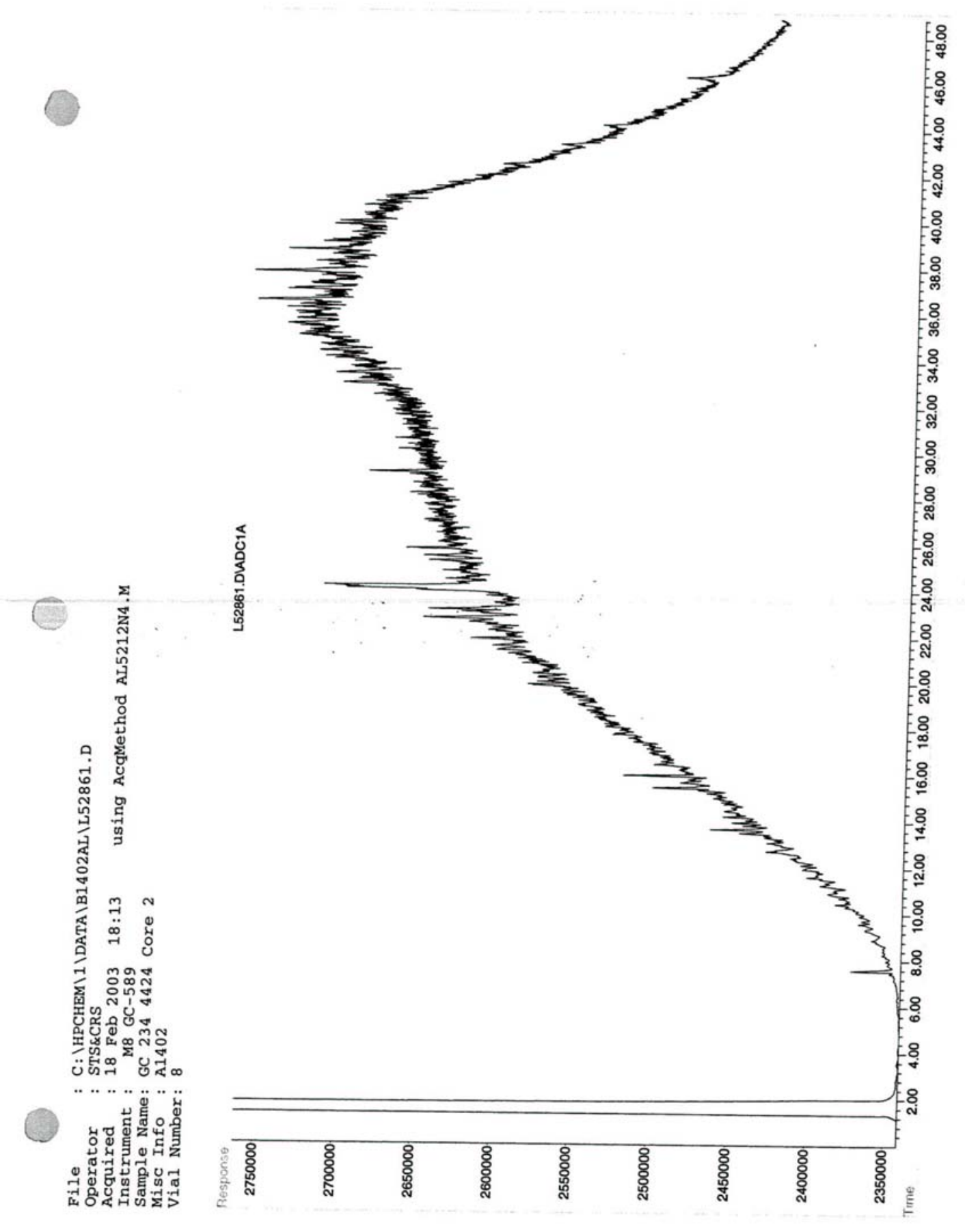




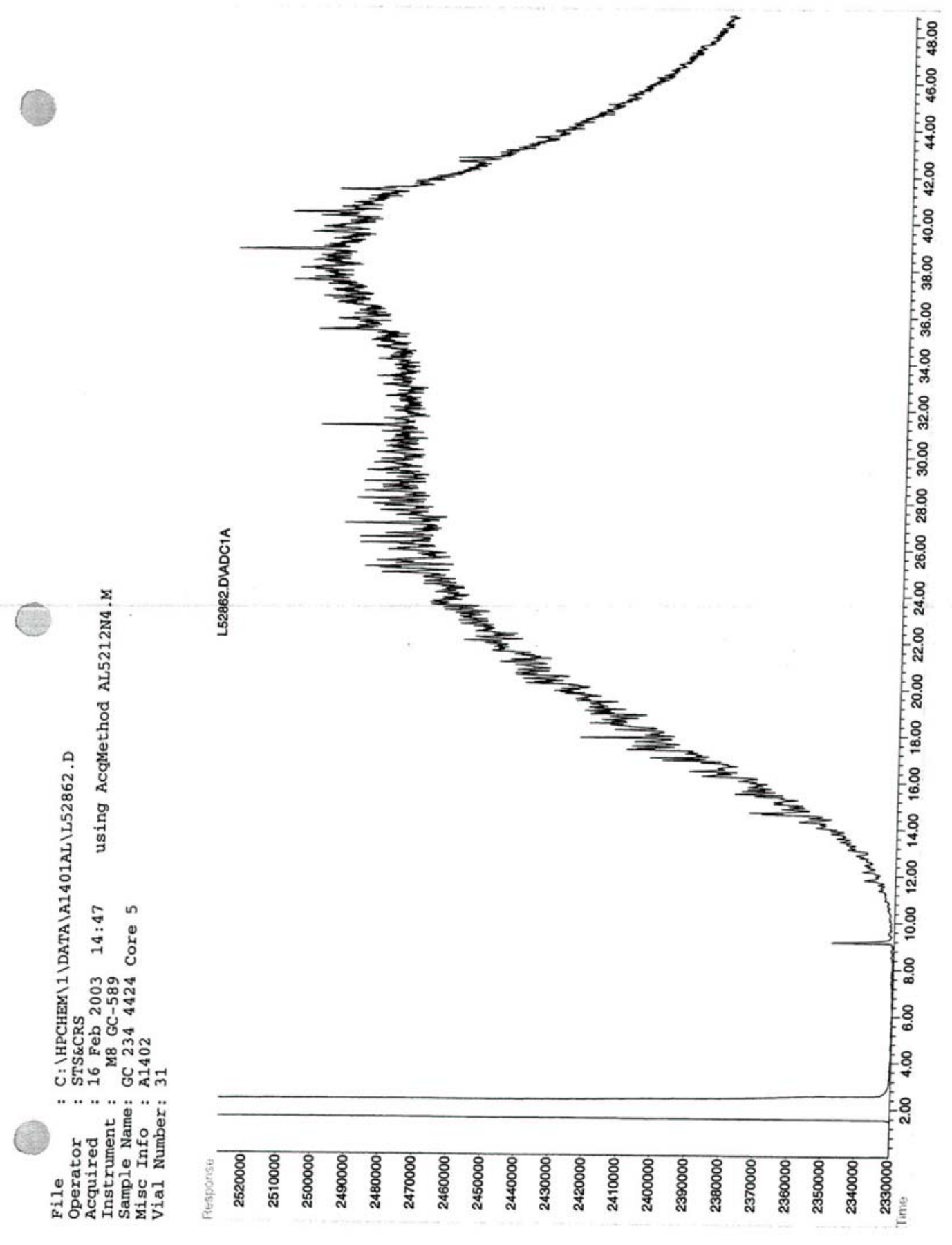




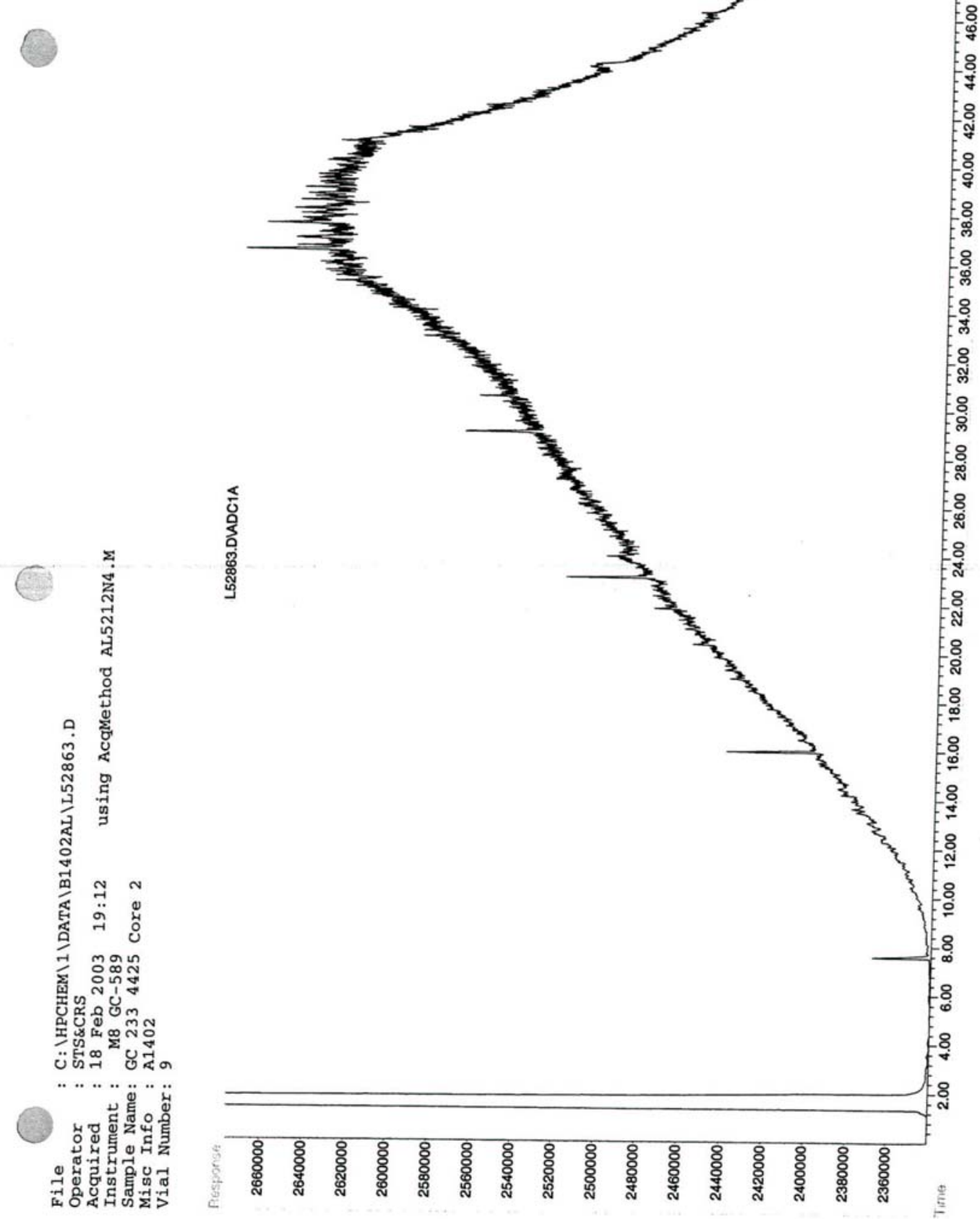




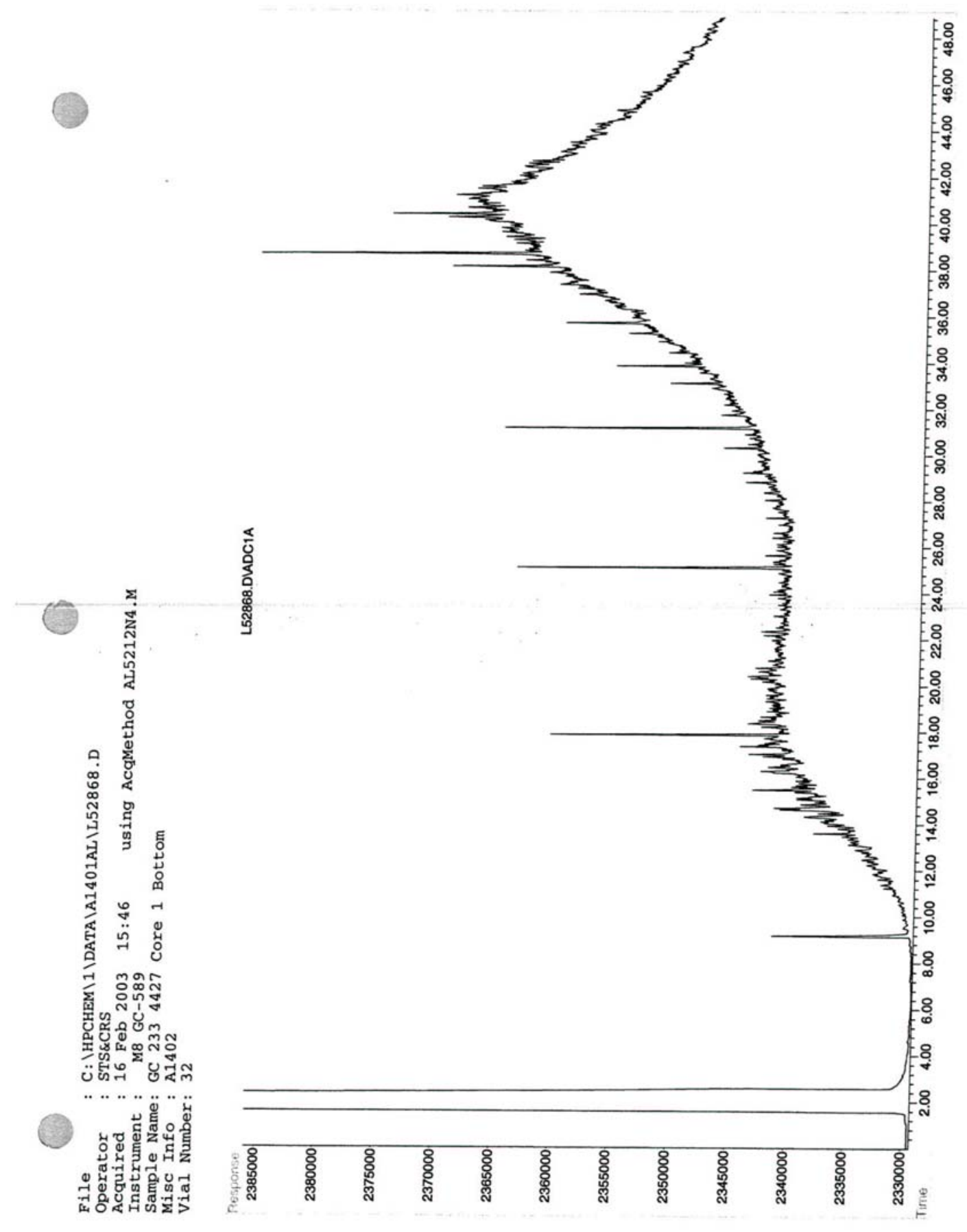




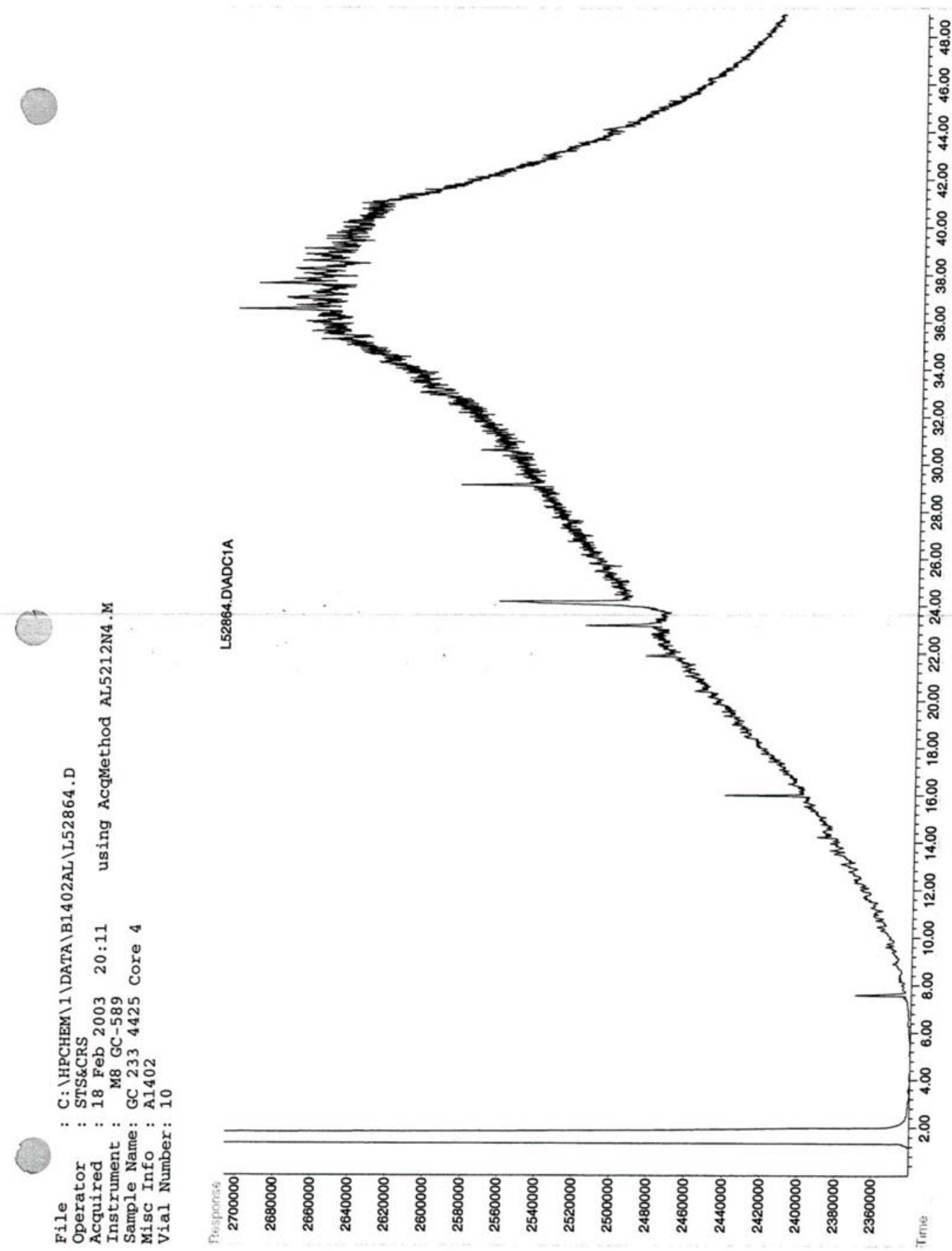




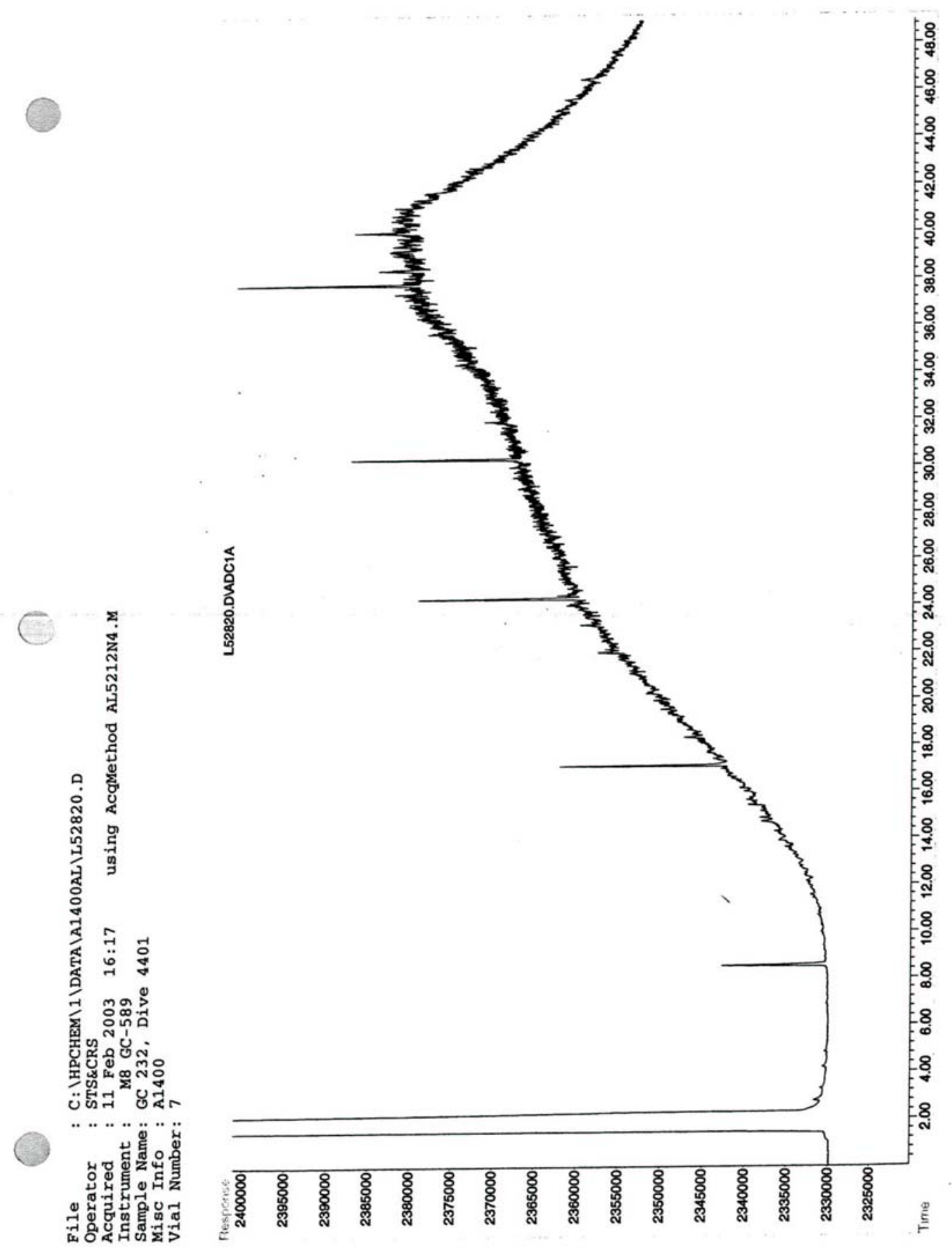




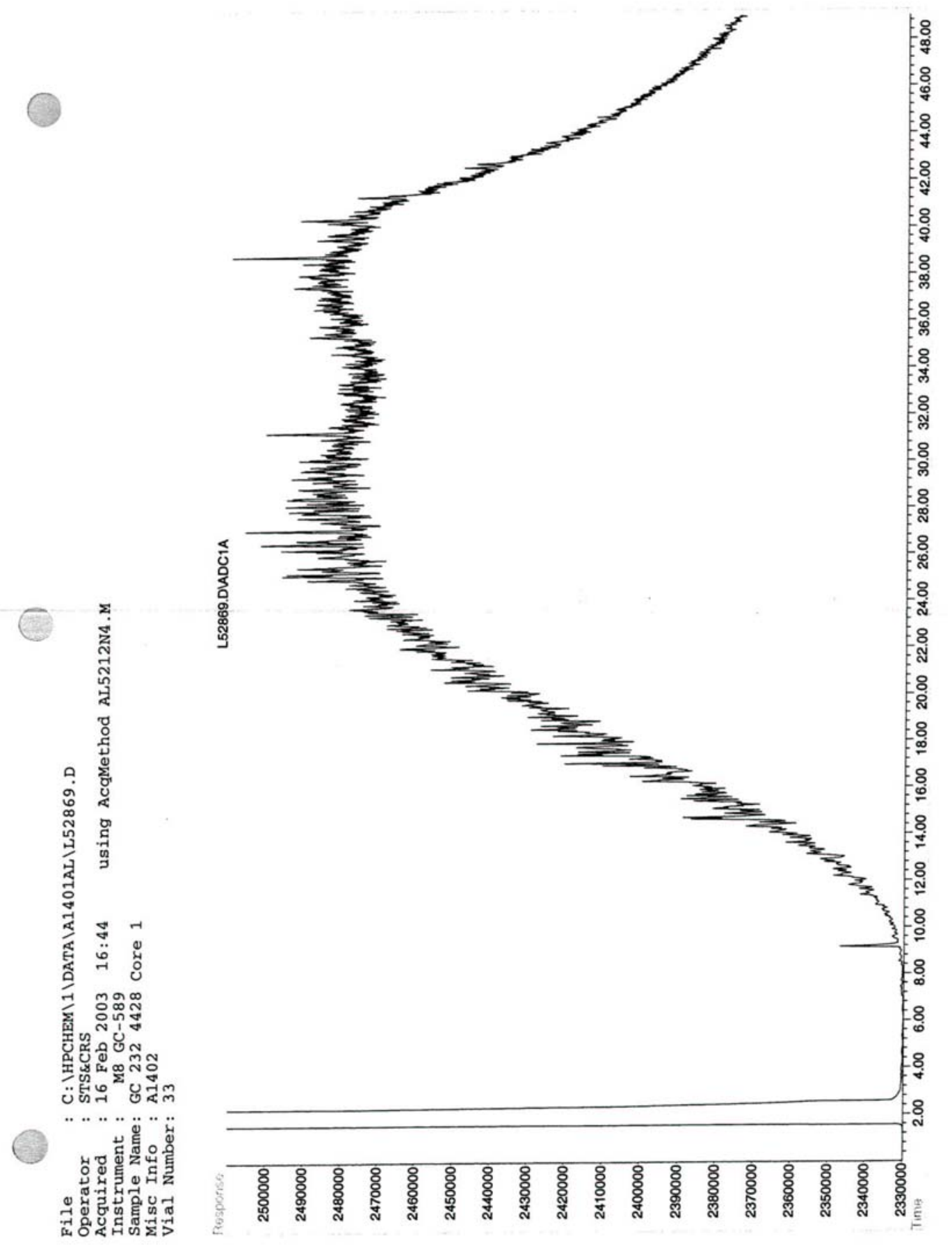




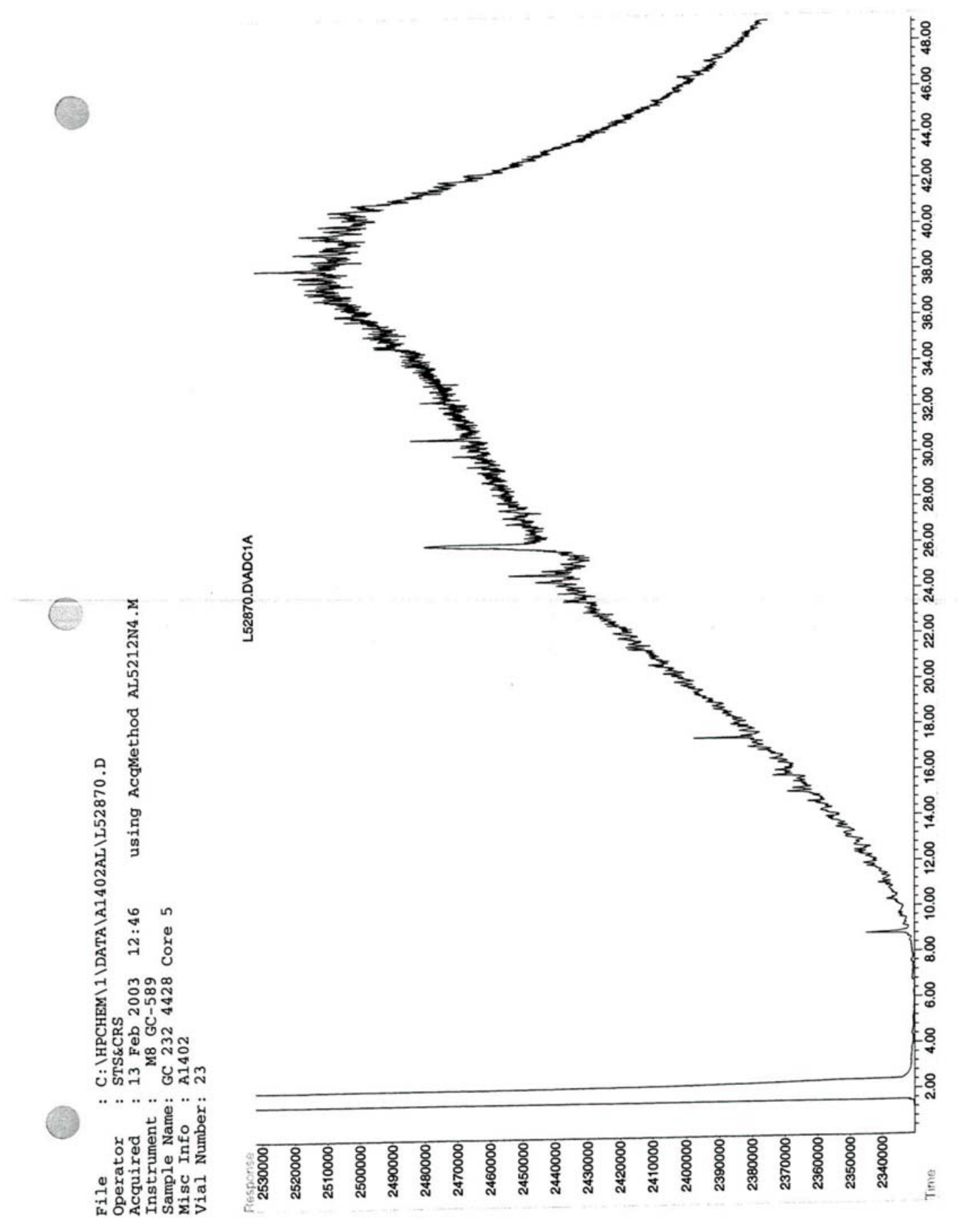




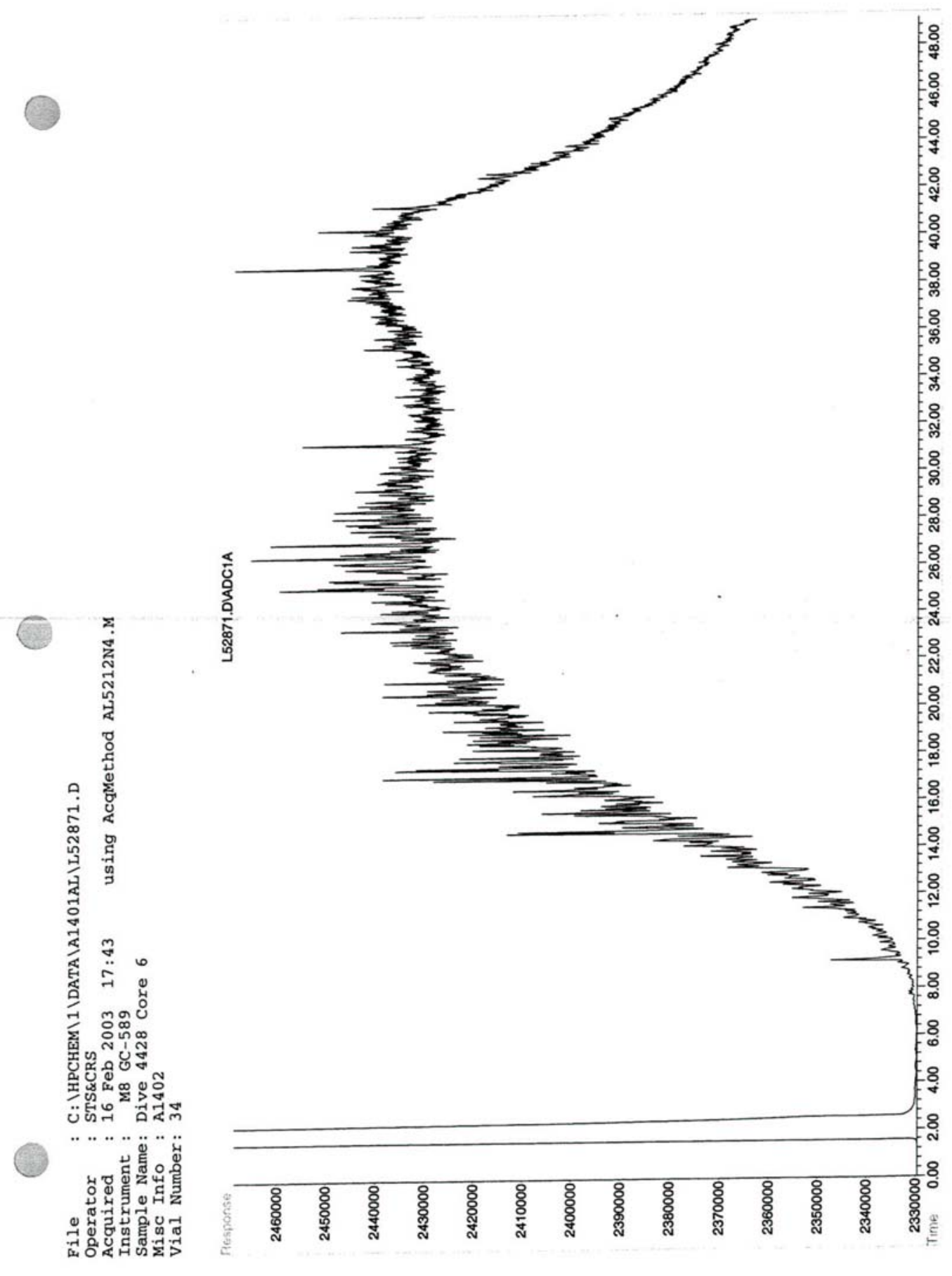




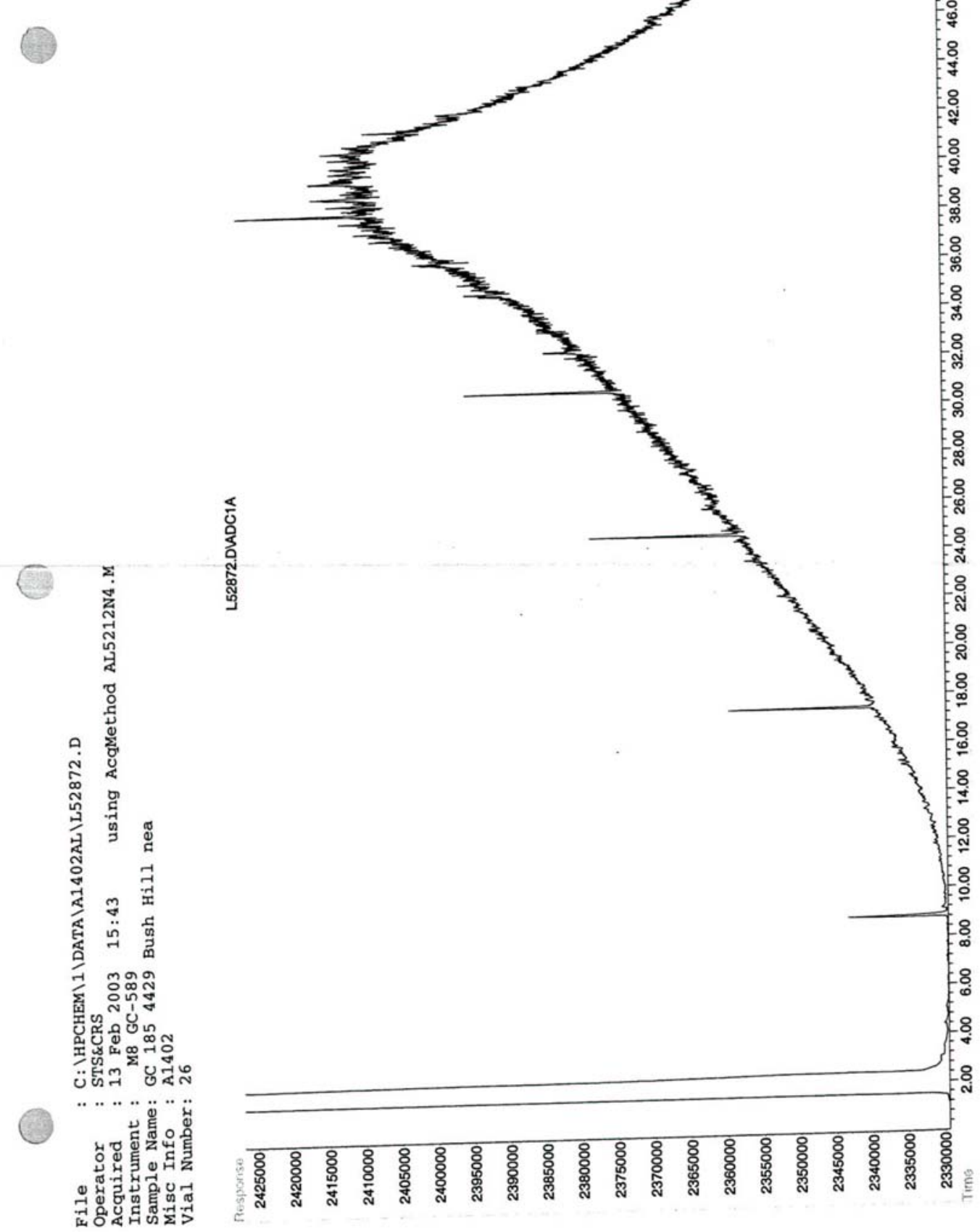




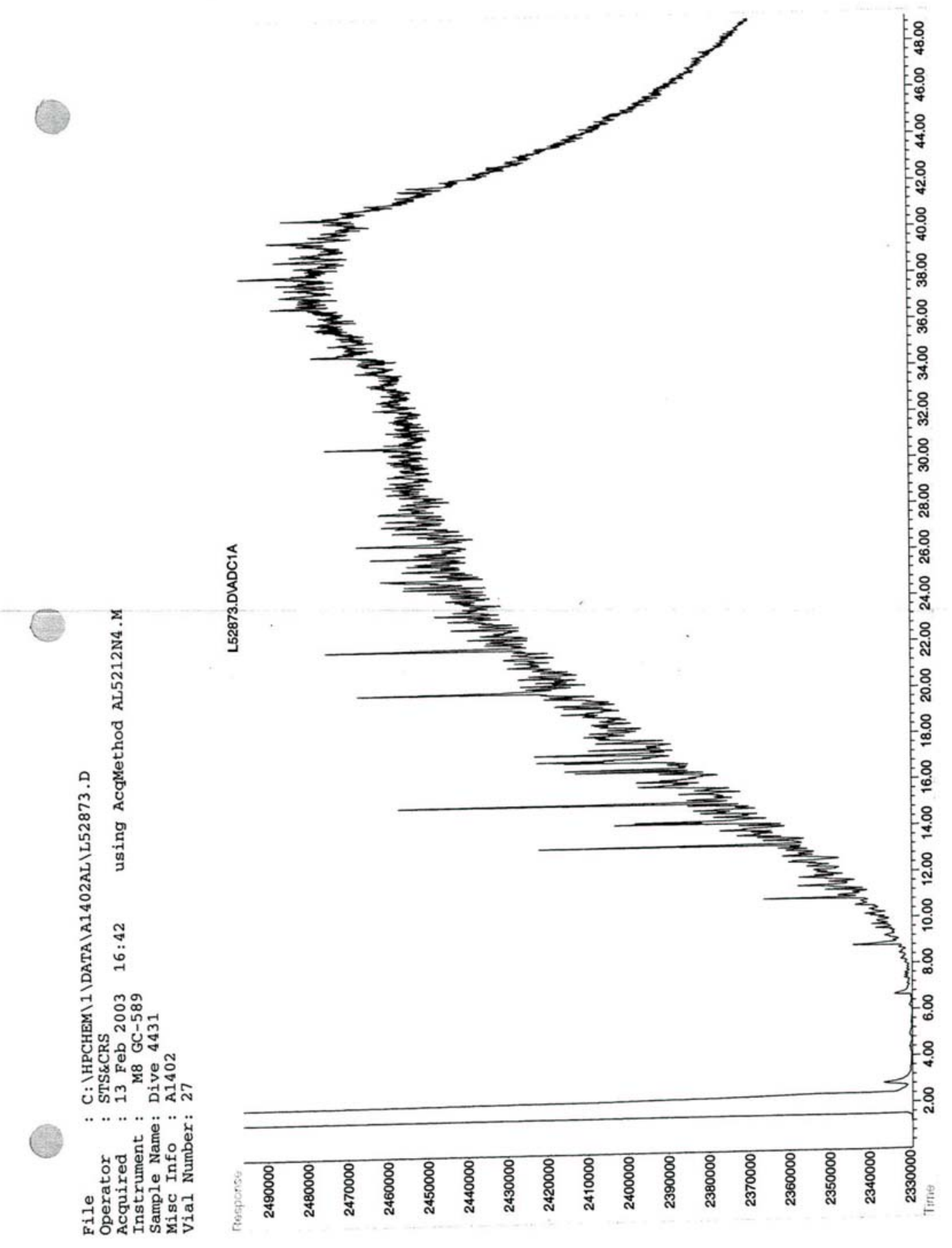




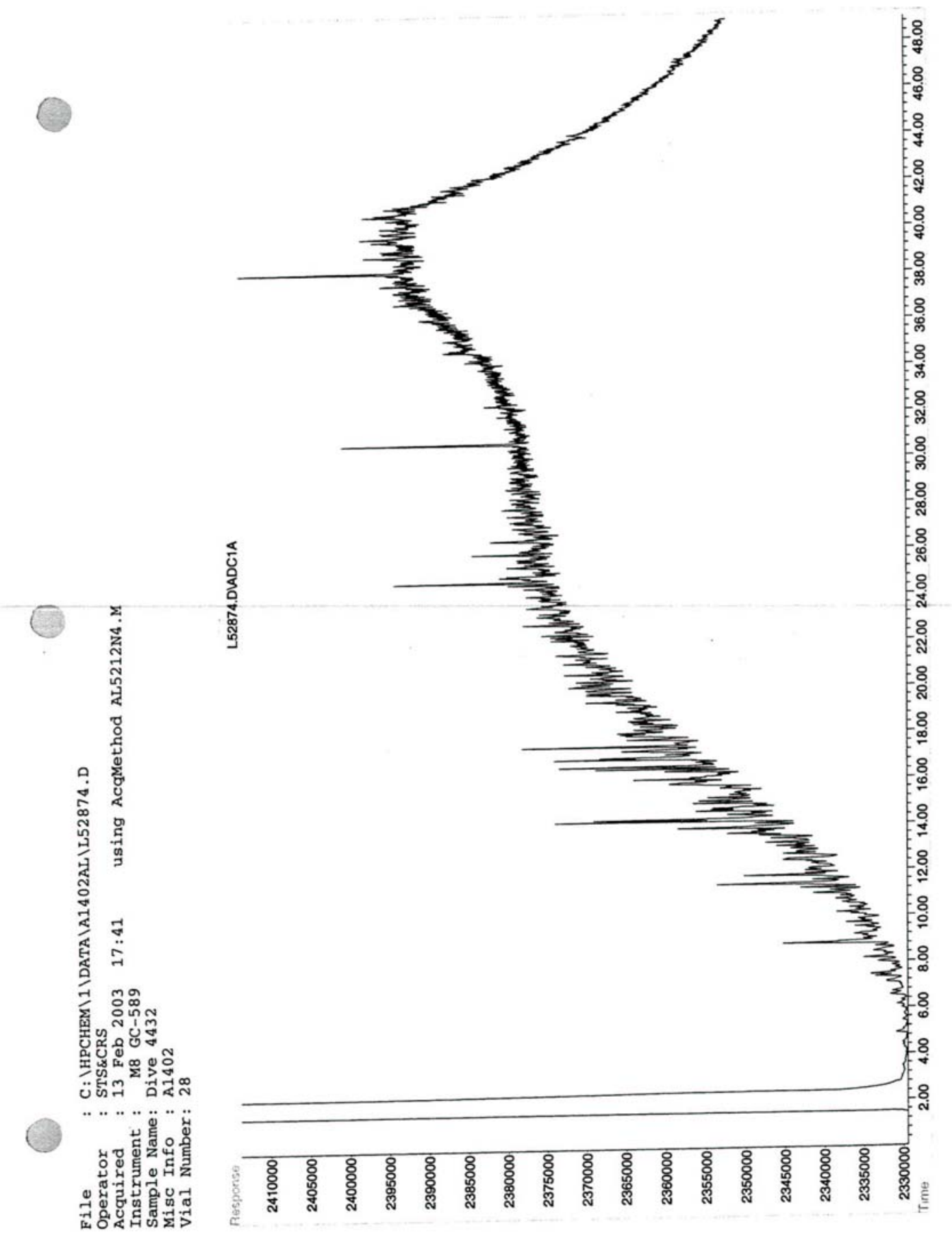




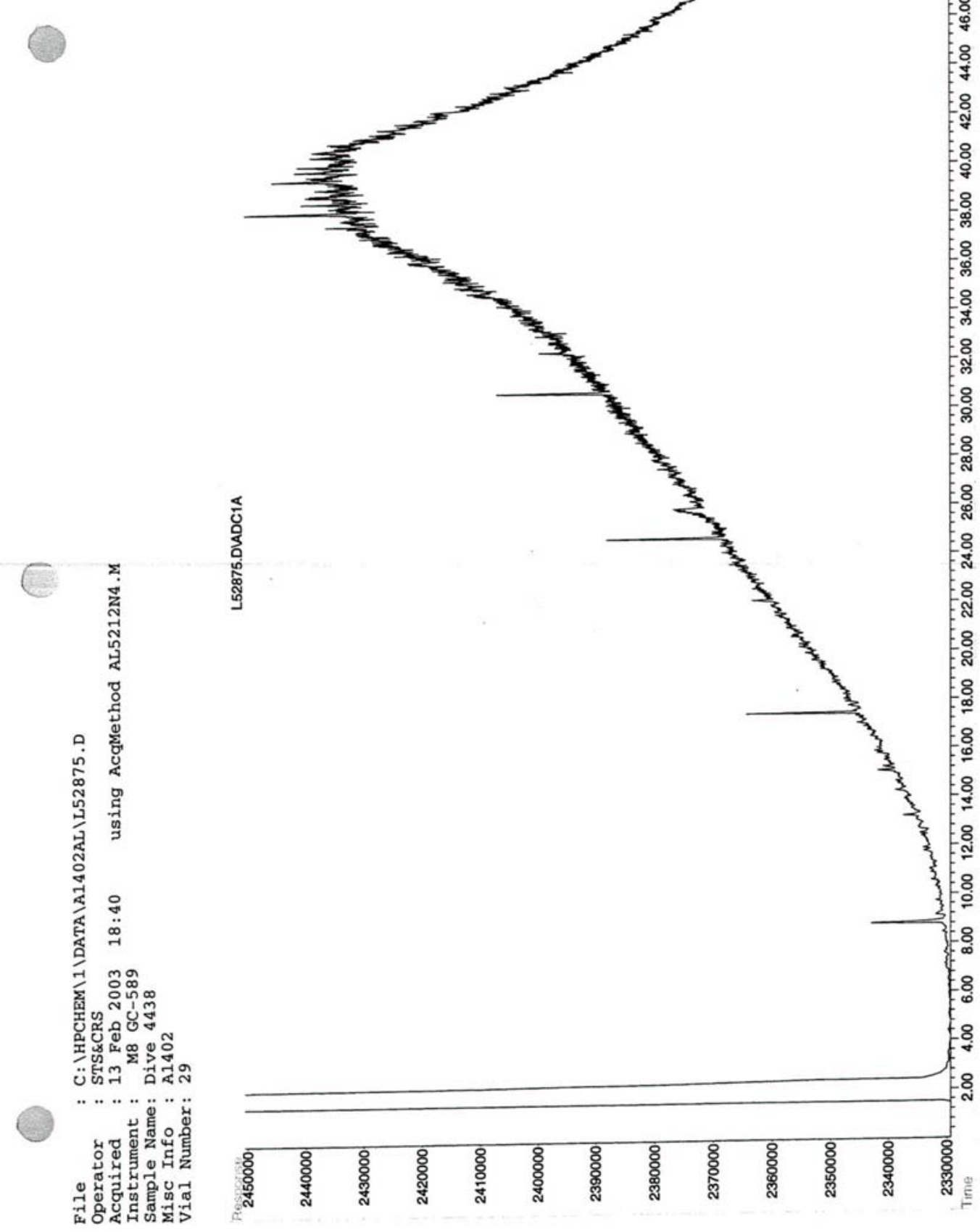




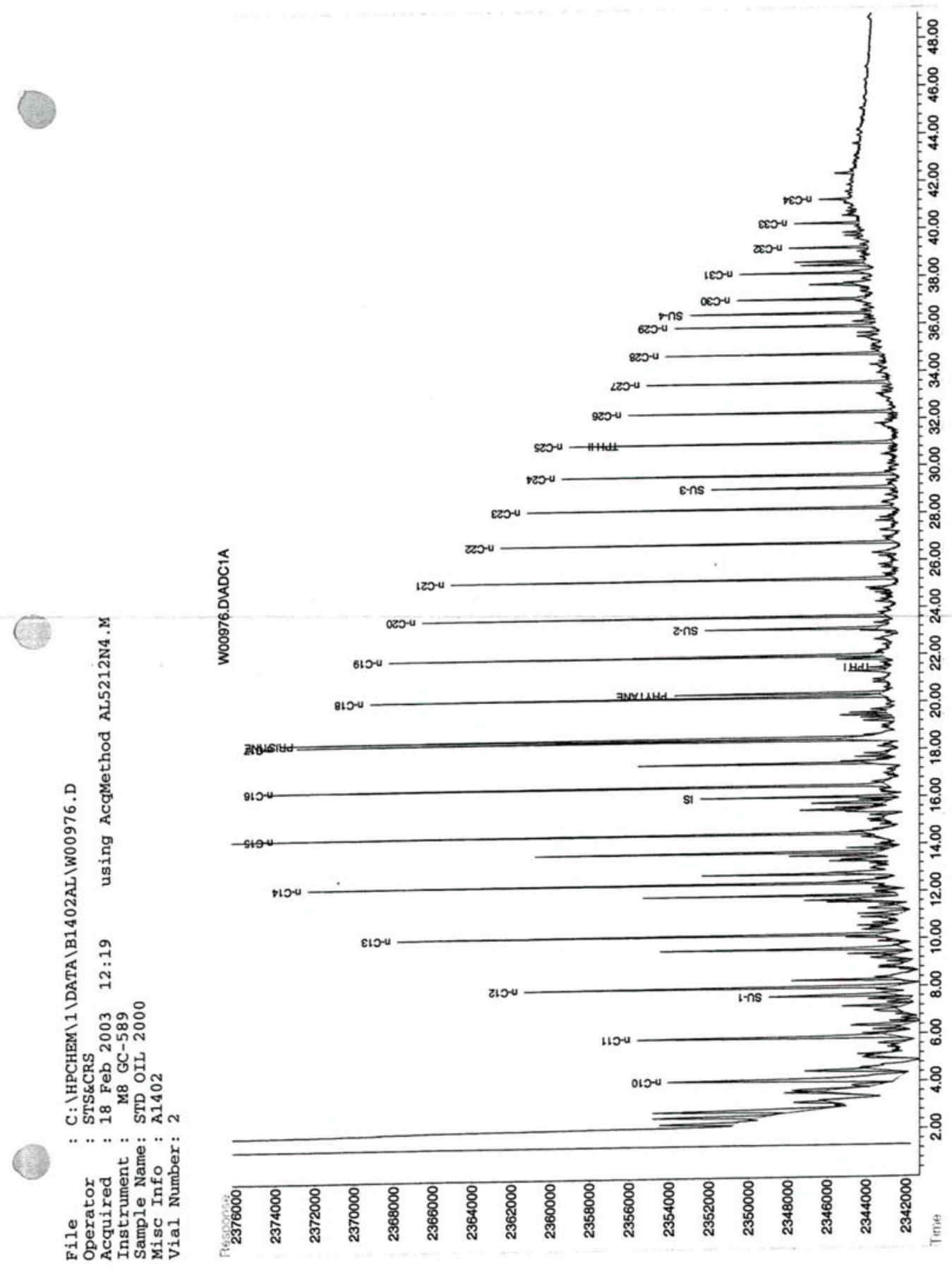




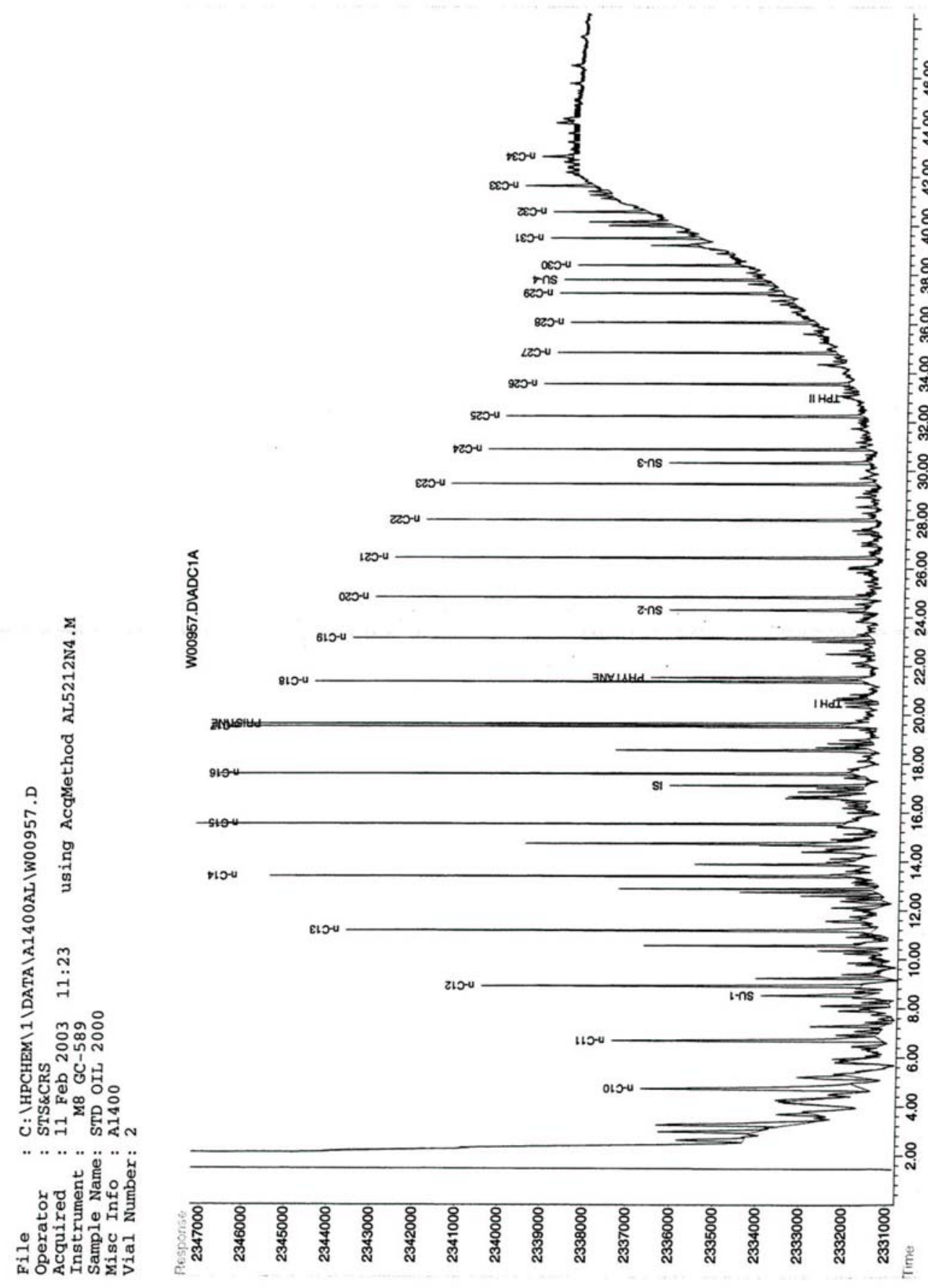




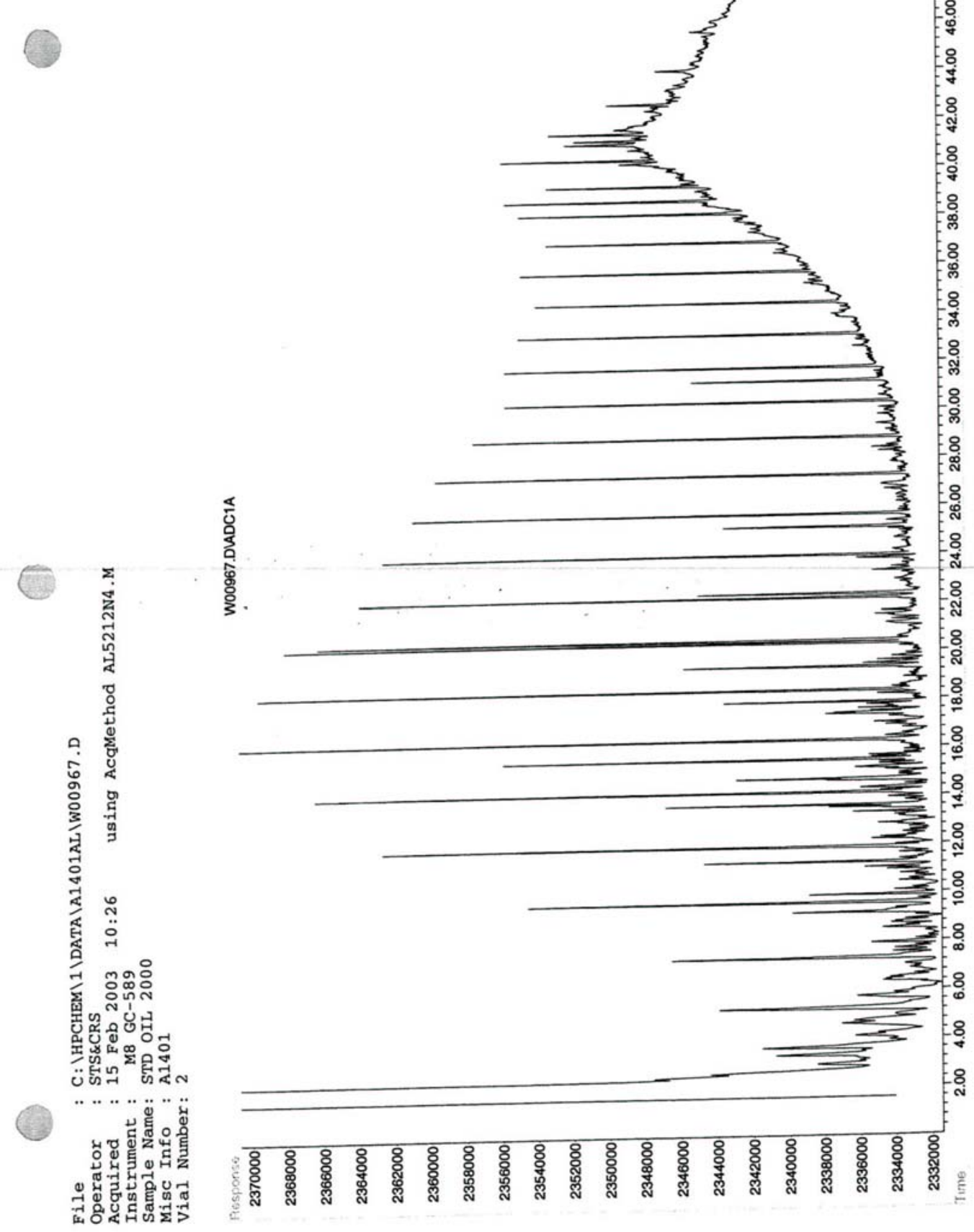


Isotope Data for Carbonate Samples

\begin{tabular}{|c|c|c|c|c|c|c|c|c|c|}
\hline $\begin{array}{l}\text { Sample } \\
\text { Location }\end{array}$ & Dive \# & $\delta^{13} \mathrm{C}$ & $\delta^{13} \mathrm{C}$ & $\delta^{13} \mathrm{C}$ & $\begin{array}{r}\text { Average } \\
\delta^{13} \mathrm{C}\end{array}$ & $\delta^{18} \mathrm{O}$ & $\delta^{18} \mathrm{O}$ & $\delta^{18} \mathrm{O}$ & $\begin{array}{r}\text { Average } \\
\delta^{18} \mathrm{O}\end{array}$ \\
\hline$\overline{\text { GC } 234}$ & 4434 & -26.30 & -26.73 & & -26.52 & 2.89 & 3.07 & & 2.98 \\
\hline MC 709 & 4412 & -26.61 & -27.06 & & -26.84 & 3.88 & 3.80 & & 3.84 \\
\hline MC 709 & 4413 & -14.67 & -12.59 & & -13.63 & 3.40 & 3.36 & & 3.38 \\
\hline MC 709 & 4413 & -29.33 & -30.36 & -28.64 & -29.44 & 3.71 & 3.55 & 3.49 & 3.58 \\
\hline MC 709 & 4413 & -27.33 & -28.08 & & -27.71 & 4.33 & 4.08 & & 4.21 \\
\hline MC 709 & 4413 & -29.73 & -27.96 & & -28.85 & 4.33 & 4.28 & & 4.31 \\
\hline MC 709 & 4413 & -34.29 & -34.78 & & -34.54 & 3.94 & 3.91 & & 3.93 \\
\hline MC 709 & 4413 & -27.36 & -26.10 & -26.61 & -26.69 & 4.30 & 4.49 & 3.95 & 4.25 \\
\hline GC 232 & 4401 & -16.02 & -15.39 & & -15.71 & 3.13 & 3.24 & & 3.19 \\
\hline GC 232 & 4401 & -16.42 & -17.12 & & -16.77 & 3.57 & 3.59 & & 3.58 \\
\hline GC 232 & 4401 & -15.30 & -14.94 & & -15.12 & 3.50 & 3.42 & & 3.46 \\
\hline GC 233 & 4425 & -32.46 & -32.72 & & -32.59 & 4.29 & 4.23 & & 4.26 \\
\hline GC 233 & 4425 & -32.64 & -32.24 & -33.06 & -32.65 & 4.22 & 4.30 & 4.15 & 4.22 \\
\hline GC 233 & 4425 & -35.31 & -34.72 & & -35.02 & 3.98 & 3.71 & & 3.85 \\
\hline GC 185 & 4429 & -22.58 & -22.18 & & -22.38 & 3.34 & 3.32 & & 3.33 \\
\hline GC 185 & 4429 & -22.42 & -23.03 & & -22.73 & 3.20 & 3.29 & & 3.25 \\
\hline GC 185 & 4429 & -22.19 & -22.56 & & -22.38 & 3.20 & 3.42 & & 3.31 \\
\hline GC 234 & 4434 & -27.09 & -26.77 & -26.94 & -26.93 & 3.27 & 3.39 & 3.40 & 3.35 \\
\hline GC 234 & 4434 & -27.26 & -26.81 & & -27.04 & 3.24 & 3.48 & & 3.36 \\
\hline GC 234 & 4434 & -26.64 & -26.68 & & -26.66 & 3.26 & 3.50 & & 3.38 \\
\hline GC 234 & 4435 & -24.18 & -25.94 & & -25.06 & 3.97 & 4.20 & & 4.09 \\
\hline GC 234 & 4435 & -21.13 & -21.50 & & -21.32 & 3.94 & 4.26 & & 4.10 \\
\hline GC 234 & 4435 & -18.53 & -19.34 & -18.44 & -18.77 & 3.91 & 4.08 & 4.35 & 4.11 \\
\hline GC 234 & 4436 & -21.62 & -21.87 & & -21.75 & 4.09 & 4.15 & & 4.12 \\
\hline GC 234 & 4436 & -20.82 & -20.58 & & -20.70 & 4.27 & 3.99 & & 4.13 \\
\hline GC 234 & 4436 & -21.81 & -21.83 & & -21.82 & 3.94 & 3.79 & & 3.87 \\
\hline GC 232 & 4437 & -19.49 & -19.12 & & -19.31 & 3.78 & 3.58 & & 3.68 \\
\hline GC 232 & 4437 & -20.63 & -20.16 & -19.66 & -20.15 & 3.49 & 3.48 & 3.64 & 3.54 \\
\hline GC 232 & 4437 & -19.09 & -18.24 & & -18.67 & 3.55 & 3.62 & & 3.59 \\
\hline
\end{tabular}




\section{Dive Log}




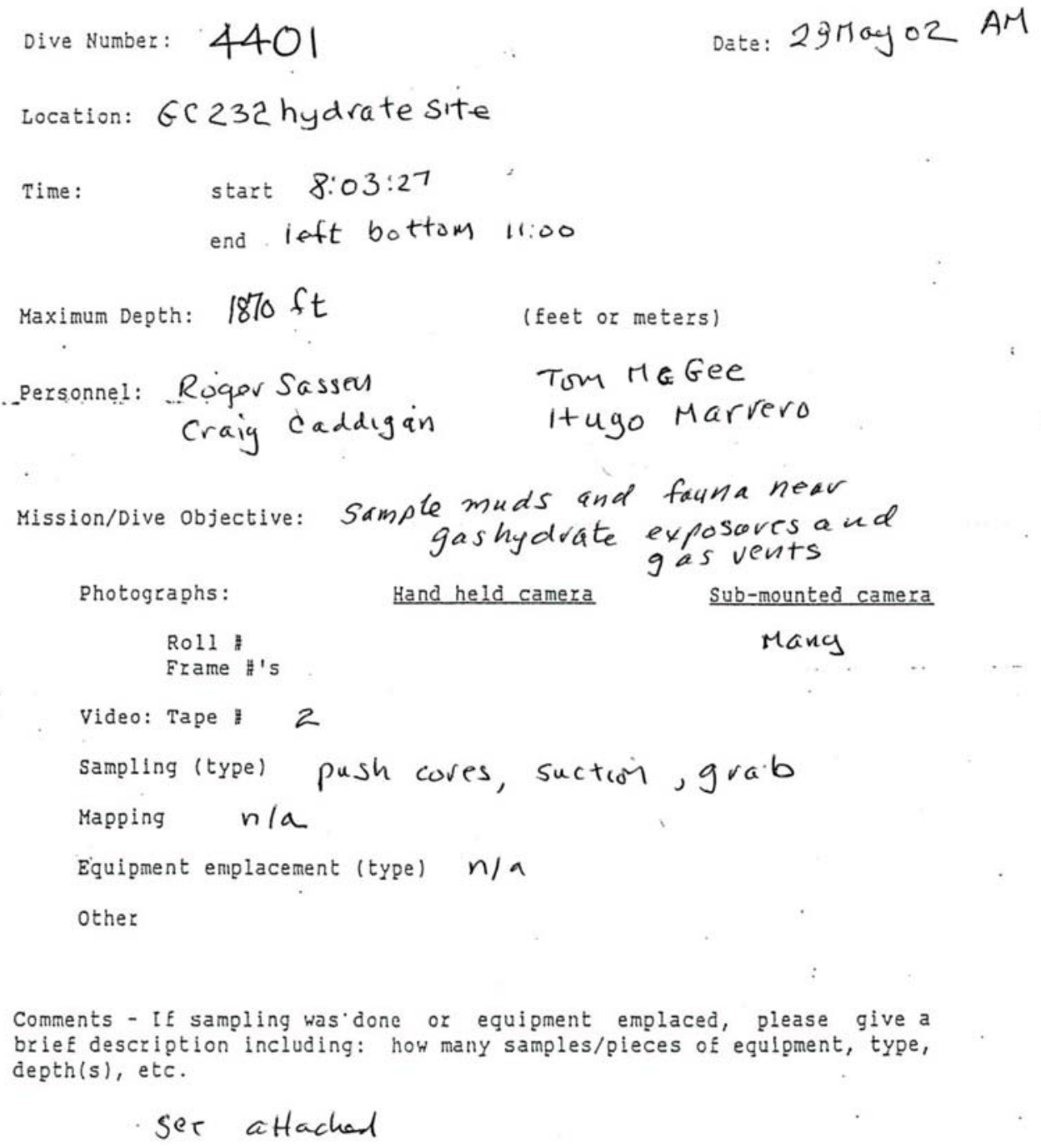


Dive 4401 GC232

(2)

1. Acquired water samples every $200 \mathrm{ft}$ from surface to bottom for Jeff Chandm.

$$
\begin{gathered}
\text { bottom }=1870.1 \mathrm{ft}, \quad 7.59^{\circ} \mathrm{C}, \quad S_{0} \operatorname{linity}=34.93 \% 50 \\
\text { current } 0.1 \mathrm{knot} \quad \mathrm{FIX} 3
\end{gathered}
$$

2. Collected small intact tube worm bush with roots and attacked carbonate hold fasts, crab $186917.55^{\circ} \mathrm{C} \quad 34.93 \%$ salinity

Archived tube worms, roots, carbonate, crab for molecolor and isotopic analyses. FIx. 4 .

3. crossed irvegalor sea floor. Fix 5. Samples white-gray bacterial mat at 0850 . 0 il and $g$ as bubbles veneered from the sea $f(000$ when disturbed. Core 1 used for geotechnical analysis.

4. Hydrate site \#1. outcropping in hummocky area, Large gas and oil-lwow bubbles when sediment disturbed. Fix 7. used grab to fill Bucket 8 with calcite comments sedcwert. 0907 , Hydrate is white thin vein fillings and massive oilastained orange vein filling with gas vents, Used suction to cringle mud in bucket 10. Calcite Ee-comenter sed/mans

118 
5. 0928. Took 4 push cores in line

(3)

fri hydrate outcrop to tube worm buss

$$
F(x 8,1865 \mathrm{ft}
$$

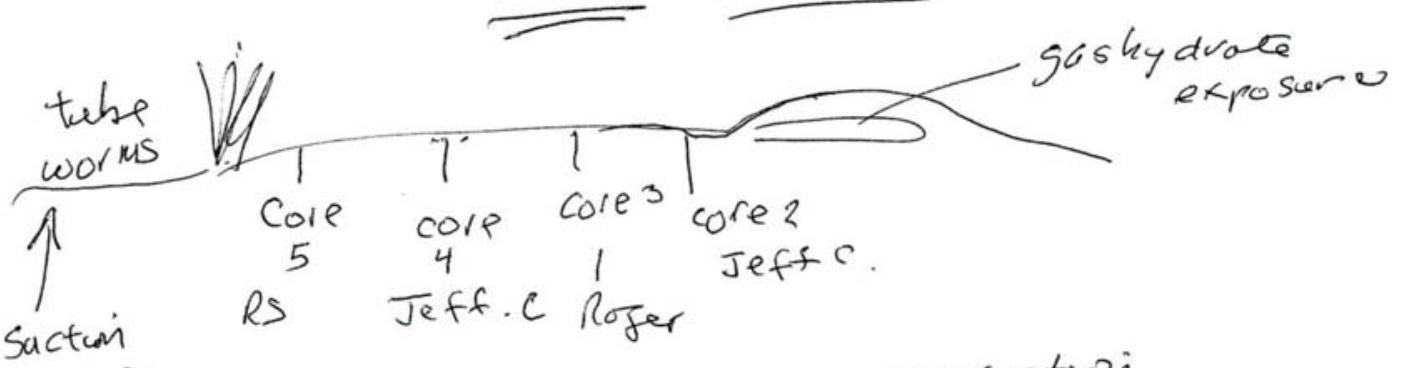

Suction

Sediment

bucket 9

calcite-cemantarl sediment, nodules.

0935
Short core penetration

$220 \mathrm{con}$.

6. Explored. 0950 nearby hydrate site A2

Thin white vein's, thick deformed orange veins, odd carhnoste norletes took Push core \#6. Fix 9 rimimadates cis in front of $\mathrm{hg}$ crate mass.

7 . Passed huge carbonate rock large colones of take worms, odd gas rents Fix 10 8. Souped carbonate slabs $F / x, 11-12$ 9. spited gas hydrate ova n 3, F/X/3 Took core 6 near ligate.

To 6 eft.

119 


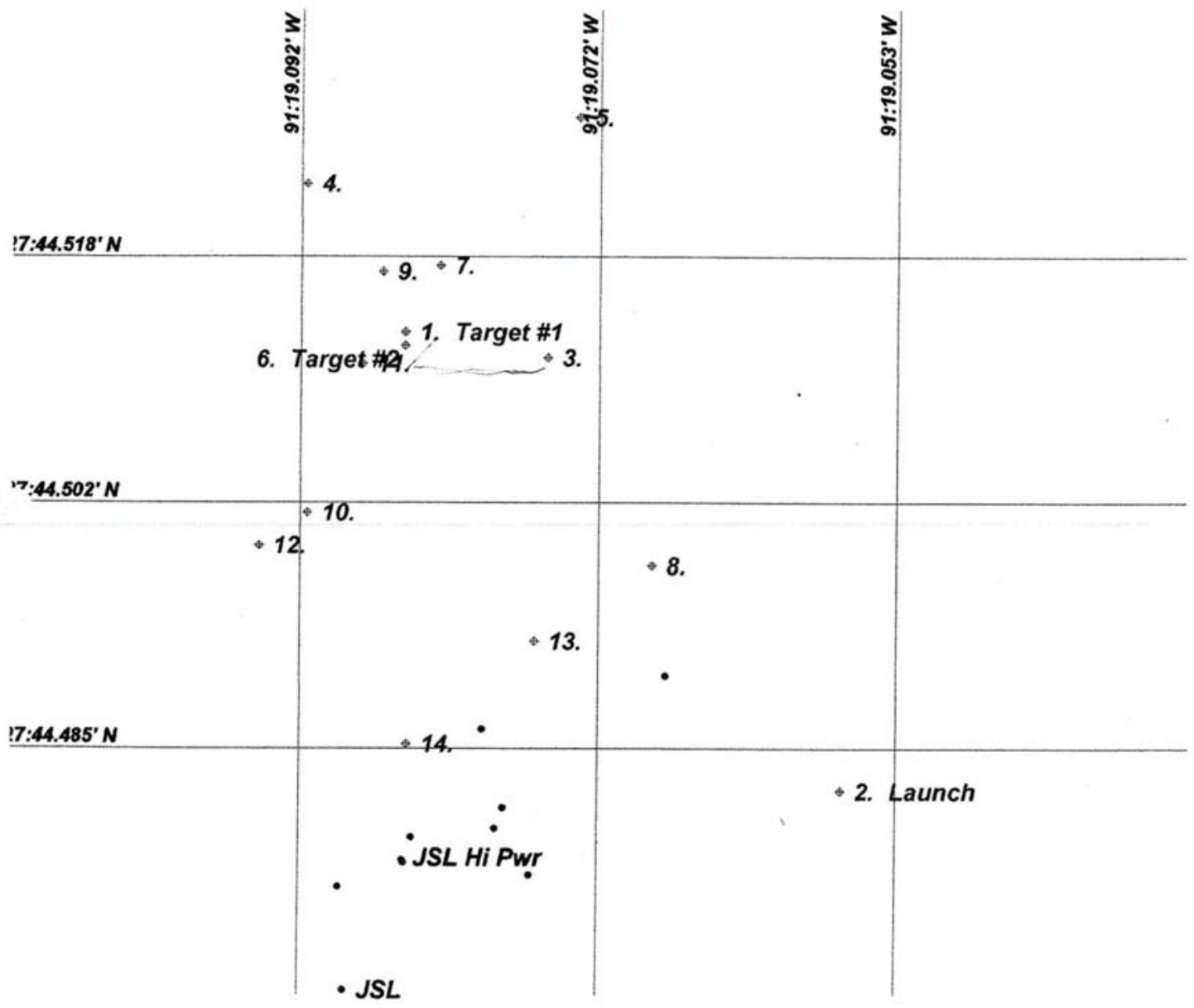


C:IProgram FilesIOREI1-4401

RVSJ 2 \& JSL $1-4401$

1. Ship 7:52:57 N 27:44.5129 W 91:19.0851 oft Target \#1

2. Ship 8:03.27 N 27:44.4825 $W$ 91:19.0562 oft Launch

3. Target [1] 8:31:02 N 27:44.5112 W 91:19.0757 $1870 \mathrm{ft}$ On Bottom -Water Sample

4. Target [1] 8:36:25 N 27:44.5227 W 91:19.0916 $1869 \mathrm{ft}$ Tube Worm Samples

5. Target [1] 8:50:35 N 27:44.5271 W 91:19.0737 $1871 \mathrm{ft}$ Bacterial Sample tube \#1 -

6. User 8:54:59 N 27:44.5120 W 91:19.0851 $1871 \mathrm{ft}$ Target \# 2

7. Target [1] 9:07:30 N 27:44.5173 W 91:19.0828 $1866 \mathrm{ft}$ Exposed Hydrate

- 8. Target [1] 9:28:02 N 27:44.4974 W 91:19.0687 $1865 \mathrm{ft}$ 4-push cores

9. Target [1] 9:58:35 N 27:44.5169 W 91:19.0866 $1865 \mathrm{ft}$ Exposed Hydrate- 6 Push Core

10. Target [1] 10:17:07 N 27:44.5009 W 91:19.0915 $1867 \mathrm{ft}$ Large Area of Tube Worms

11. Target [1] 10:26:35 N 27:44.5108 W 91:19.0879 $1867 \mathrm{ft}$ Small Rocks

12. Target [1] 10:46:10 N 27:44.4987 W 91:19.0947 $1870 \mathrm{ft}$ Exposed Tube Worms \& Rock

13. Target [1] 10:51:30 N 27:44.4924 W 91:19.0765 $1868 \mathrm{ft}$ Large Exposed Hydrate \& Worms

14. Target [1] 11:00:00 N 27:44.4856 W 91:19.0849 $1867 \mathrm{ft}$ Leaving Bottom 


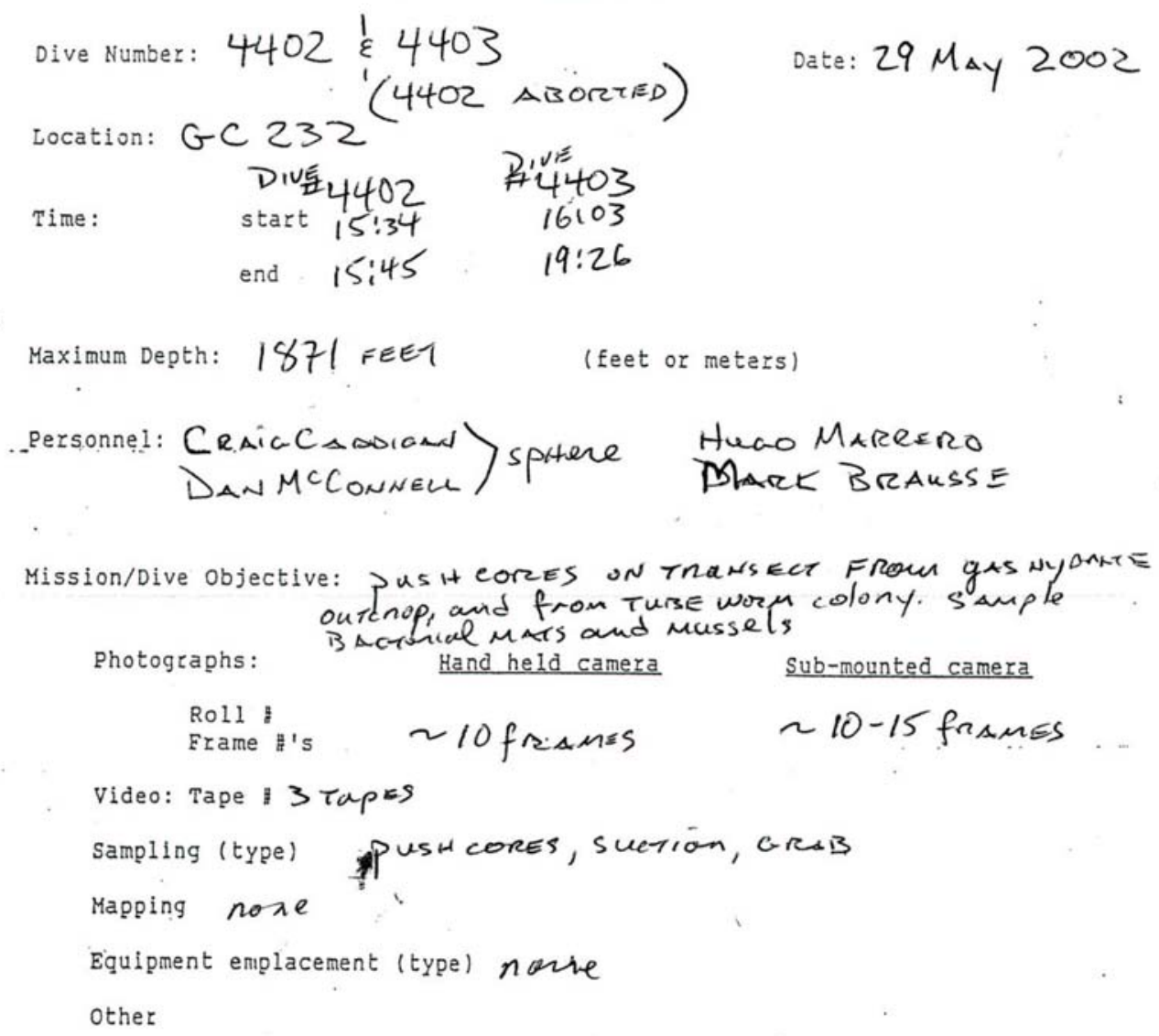


SSL 4402

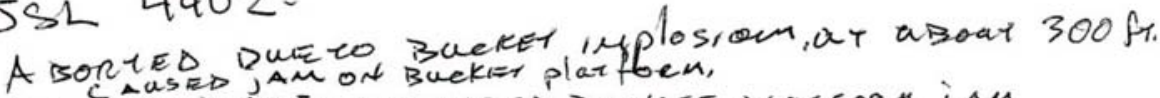

RECOJERED SUS- REPaires BUCKET PLaTCORM jAM

SSL 4403

$$
5 / 29 / 02
$$

Fix (3) BOTHOM 1871'. UNEVON SEA FLOOR, TUBEWORM BUSHES

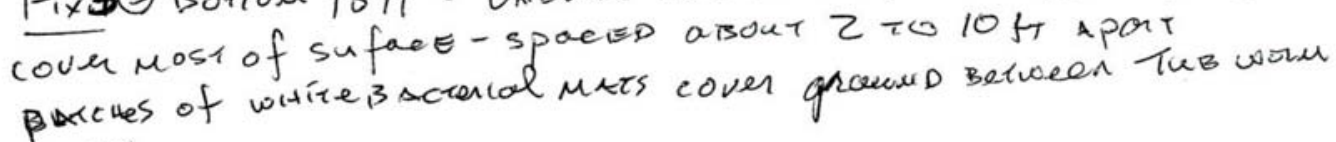

Busters.

Fix 4(4) EXPOSED gAS HYPRNE, LOOKS yellowish. IS AT BASE

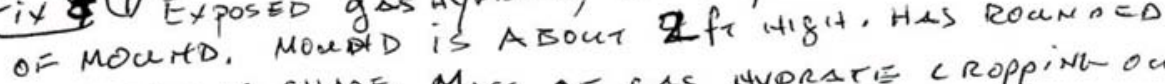
but irregular shape. Mass of gas ihorate croppint-och AT BASE OF MOUMS is a BOUT $1.5 \times 6$ incues.

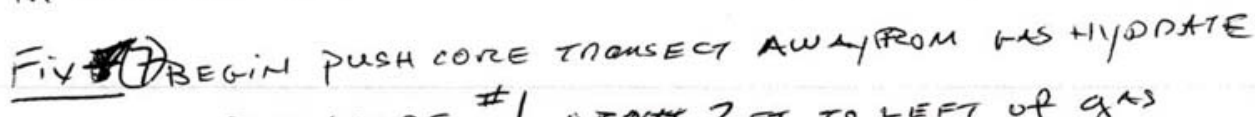
OUTCROP. PUSACORE $\$$ I UEQCT 2 FT TOLEFT of $\mathrm{gAS}$ HyDAATE OUTCROP. PUSH CORE 2 ABQUT 4 FT TO LEF of push cOrE

Fix Bustes of tUBE norus coven a AYPAATE MQUUND- WASHYDATE CROPS OUT aT BASE OF MOUND. ABout 15, pto Right of pushcores: 1, 2 , and 3

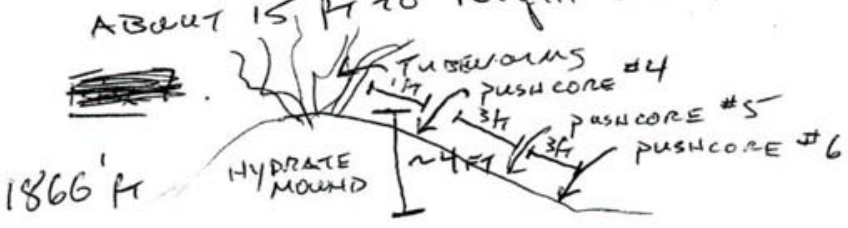

PUSH CORE SAMPLES ON TDANSECT $120^{\circ}$ F ROM TUBE WORM Bust.

Fixg GLS sampce. $1862^{\prime}$ Gas hypate vent appears TO COME FROM HOCE iSt GAS HYPRATE MaSS. BURPED TUSE.

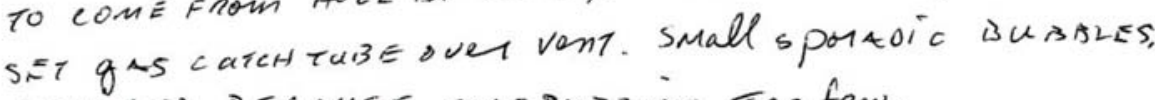
$A B O D=E D$ BEcanse S4S puisices Too few. 


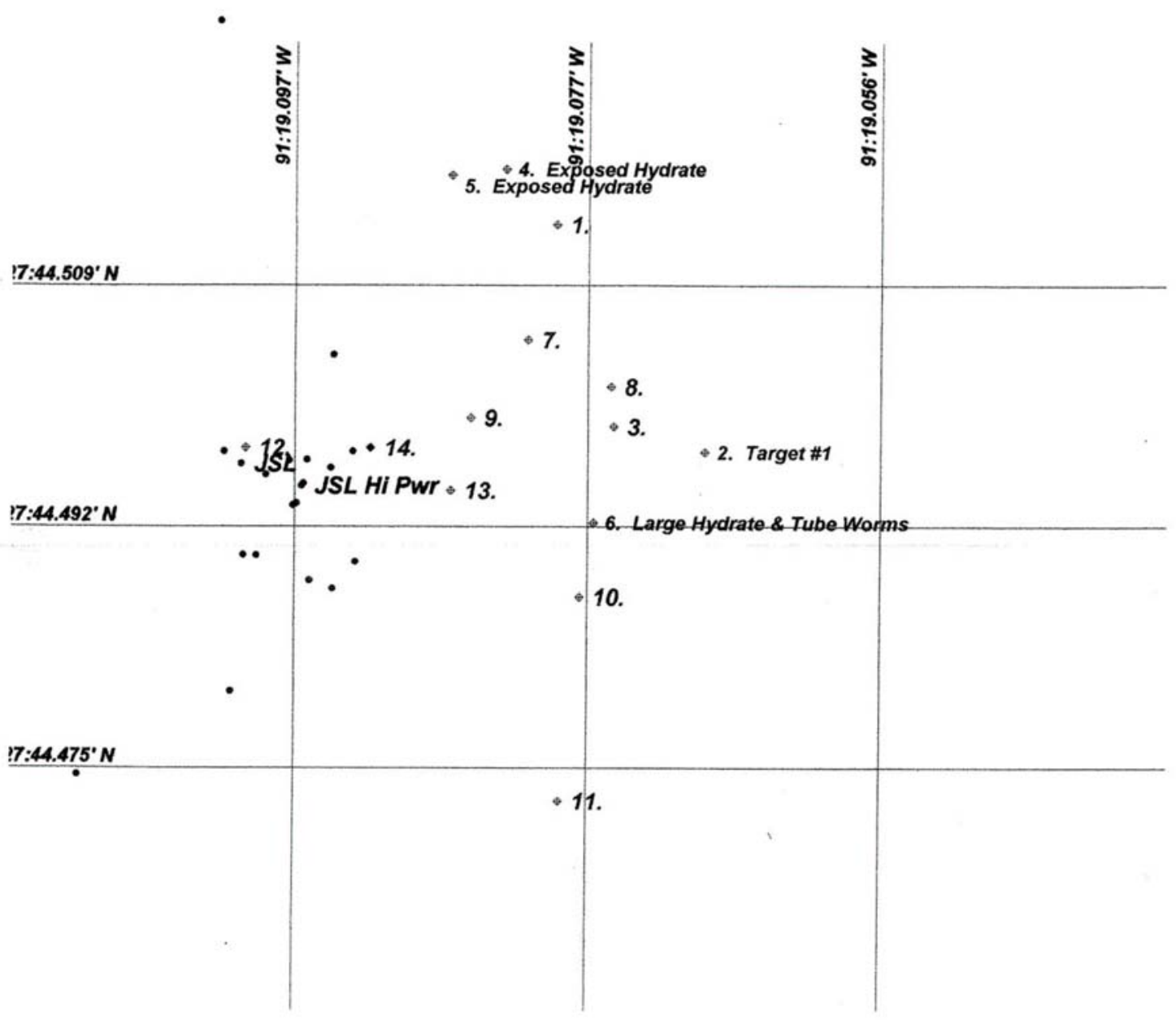


Fix 10. $1866^{\circ}$ sAmpLED WHITE Bscterial mat and

RED Bscterial mat.

BuckET \#10- WHITE BAcrenid mat

Fixil BuckET 12 - REO BActerid mat

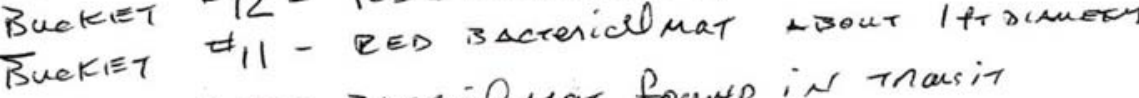

Fix II is RED BSctial mat fow
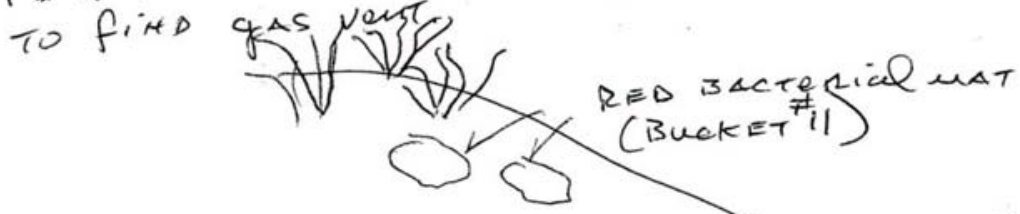

In ensusit to gas vent Gouad durinc. dive 440

Flew over oas ityprate uass Fomad durite 4401

Lookint aroutd for vents,

Fixiz $1867^{\prime}$

GRABSED MUSELS - PUTIN BUCKET 2

(SOME MAY tave spilles over to BUCKE, \#9)

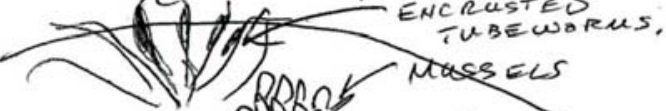

GRABBED EnCRUSTIED TUEEwarus PUT IN DASKET.

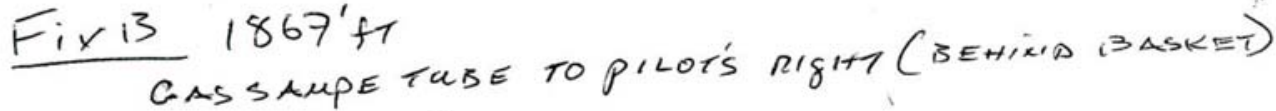

WhS FIIST SAMPLE.

Found suall vent at Bsse of tuBE warm colony.

GAS HYPRATE CTOPPING OUT hT SEAFLOOR, FILLE TUISE Witt OLS SAMPLE. FILLED SecontD GASSAMPLE FROM SAME UEAT. THIS Second SOMPLE IA TUBE tO PGOT'S LEET. MUSSELR gROWIAC AT BASE OF OUTCROPPINA GAS NYDRATE. TABE wOrMg Bustes ALL ARQuxld arEA.

Fix 14 Lesuixig Borrom.

Gas observed comitia from arl pust cores especially from past cores 1,2 , and 3 . 
C:IProgram FilesIORE11-4403_trk

\section{RVSJ 2 \& JSL Hi PWr}

1. Ship 16:03:13 N 27:44.5134 W 91:19.0792 oft Launch

2. User 16:06:29 N 27:44.4974 W 91:19.0687 oft Target \#1

3. Target [1] 16:30:41 N 27:44.4992 W 91:19.0751 $1871 \mathrm{ft}$ On Bottom

4. Target [1] 9:07:30 N 27:44.5173 W 91:19.0828 $1866 \mathrm{ft}$ Exposed Hydrate

5. Target [1] 9:58:35 N 27:44.5169 W 91:19.0866 $1865 \mathrm{ft}$ Exposed Hydrate * 6 Push Core

6. Target [1] 10:51:30 N 27:44.4924 W 91:19.0765 1868 ft Large Exposed Hydrate \& Worms

7. Target [1] 16:59:46 N 27:44.5053 W 91:19.0812 $1865 \mathrm{ft}$ Punch Core \#1,2,3

8. Target [1] 17:09:22 N 27:44.5020 W 91:19.0753 $1865 \mathrm{ft}$ Punch Cores \#4,5,6

9. Target [1] 17:22:18 N 27:44.4998 W 91:19.0852 $1862 \mathrm{ft}$ Gas Sample

10. Target [1] 17:33:22 N 27:44.4872 W 91:19.0775 $1866 \mathrm{ft}$ Bacterial Mat-grab sample

11. Target [1] 17:54:01 N 27:44.4728 W 91:19.0789 $1870 \mathrm{ft}$ Suction Sample Bacterial Mat

12. Target [1] 18:18:57 N 27:44.4977 W 91:19.1010 $1867 \mathrm{ft}$ grabbing mussels and tubeworm

13. Target [1] 18:42:09 N 27:44.4947 W 91:19.0866 $1867 \mathrm{ft}$ Gas sample

14. Target [1] 19:03:29 N 27:44.4977 W 91:19.0922 $1867 \mathrm{ft}$ Leaving Bottom 


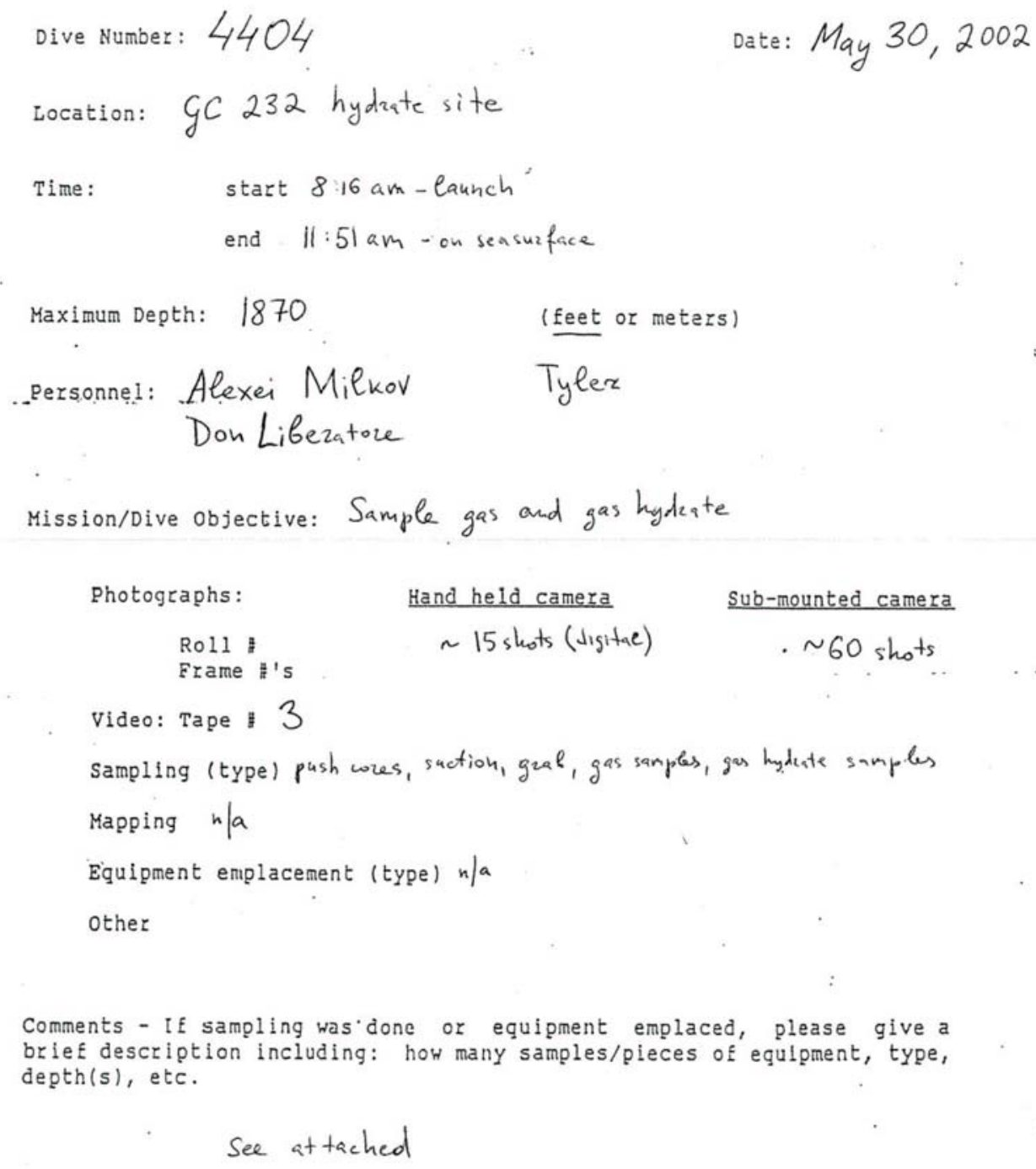




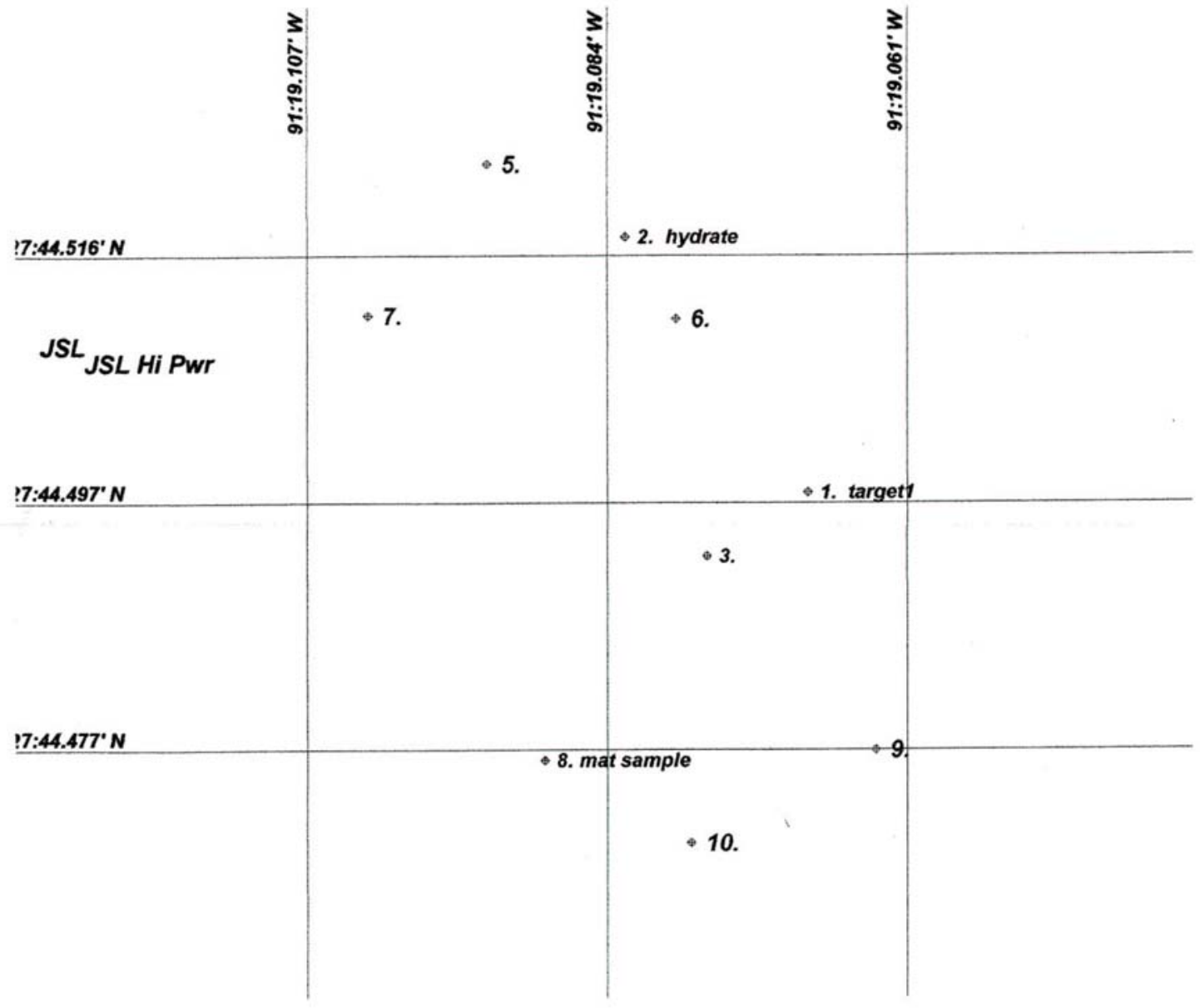


C:IProgram FilesIOREl1-4404_trk

RVSJ 2 \& JSL $1-4404$

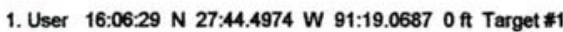

2. Target [1] 9:07:30 N 27:44.5173 W 91:19.0828 1866 ft Exposed Hydrate

3. Target [1] 10:51:30 N 27:44.4924 W 91:19.0765 $1868 \mathrm{ft}$ Large Exposed Hydrate \& Worms

4. Ship 8:18:21 N 27:44.4904 W 91:19.0068 oft launch

5. Target [1] 8:47:05 N 27:44.5230 W 91:19.0936 $1866 \mathrm{ft}$ on bottom

6. Target [1] 8:57:08 N 27:44.5109 W 91:19.0789 oft shooting photos

7. Target [1] 10:22:50 N 27:44.5112 W 91:19.1028 oft Push core

8. Target [1] 10:50:50 N 27:44.4765 W 91:19.0891 oft sample orange mat

9. Target [1] 10:56:46 N 27:44.4773 W 91:19.0634 $1865 \mathrm{ft}$ Grab Sample

10. Target [1] 11:08:46 N 27:44.4701 W 91:19.0777 $1866 \mathrm{ft}$ push core and sample tube worm 
Dive 4404 gC 232

(2)

1) Collected water samples for Jeff every 200 feet from surface to bottom. At seafloor: $8: 47 \mathrm{am}, T=7.4^{\circ} \mathrm{C}, D=1866.4, S=34.94$

2) 8:50 went through a mound $\sim 2$ m high covened with individure bush hills

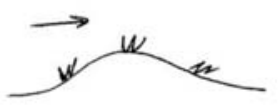

3) Fix $\neq 6$ Exposed gashydute (gh) mound $\sim 0.5 \mathrm{mlong}, 0.4 \mathrm{~m}$ wide. Sas bable trains coning feom 3 or 4 individual small verts. Gas hadrate is range on the expose suaface (it's white on fresh surface). Sh is locited inside"

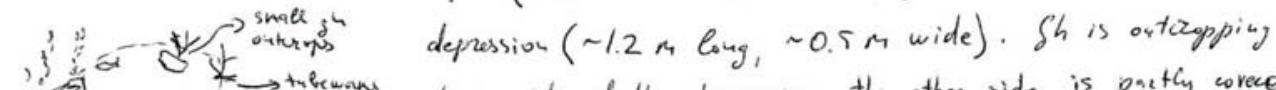

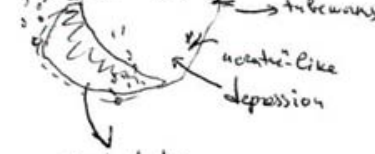
at one side of the depression, the other side is partly corece with sediment and partly exposed. The prelivinary inteapetation is that a hapf of the risinal mound broven and floatec gas bydintc away (Fis.i)

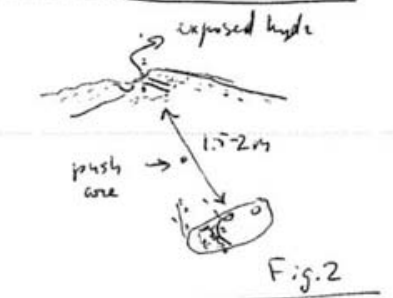
Another gas hydrete out aop is $\sim 1.5-2 \mathrm{~m}$ away (Fig. 2). Sas is coning out from the sccoud outap tow, but is mach less abundant and episodic. The preliminny inteypetation is that the entice rea repuesents a "hydrate" sidge ( $20 \mathrm{~m}$ long, $5 \mathrm{~m}$ wide, hat the dimensions are had to identify form the $54 \mathrm{l}$ ).

4) 9:05 Taking gas sarples $D=1866.4, T=7.39, S=34.92$ Gas bubbles an zelatively small ( $0.5 \mathrm{~cm})$, do not change in size when go up ( $-2 \mathrm{~m}$ vic continuous flow, no oil dryplets. When we took a first somple, we buke tome hydr and it wat up. The fiest sample was durped ( ueverse the tube) because we bid not andy hydrate inside the tube. Sot a new gas sample in presimally clesn tabe. Sas bubbles appear not wounded, but zether oval ( $\supset$ ). Gas sample was finally cotlea at $a: 20 \mathrm{am}$, the tube ( $3.5 \mathrm{indian}$ ) his a gas/woter interface right below the white cap $(\sim 2$ in high gas column $)$. Gas kydrate mond hes some tubewouns inside, but nut much, and only individunus, not bushes. Pilot noticed some oil droplets coning out the sedimert,

5) 9:30 Took -20 still skots of the mand with bubbles.

6) a:33 Start getting a hydrate scruple. Took a layge piece ( $50 \mathrm{cr}$ ?) in the arm (nochipper), put it in the samplere, one pien of the gh want doun into 
the sampler. Tryed to put in second piece, but eost it, the piece floated away. used the chipper to push the first piece inside. Tryed to put the lead on, did not snccead first tine. Cleaned the sirface of the samplere (purp, zeverse pump (wast)). Tryed to put the leed on again- succersfae. Sample is taken at 10:05.

7) 10:20 To0k a pish core right between two gh mounds in a bycession. The we went ont

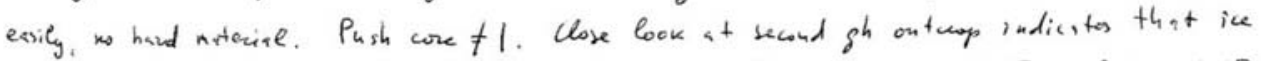
worss an present inside the gh. We took $~ 12$ pictures. Ife warns are 0.5 in long, 10-15 species in orage gas hydiate. We sucked some ice wans into bucket $\neq 5$ (saction sample) at 10:15 am Pash cue $\neq 1$ is taken ketween two antcoops $t g^{4}$, next to small individupe tubewoums. Oill and gas vent yp from the hole made by the push core. Bubbling stoped when we got the core up. It was Fix $\neq 7$

8) 10:35 Going to exploue acound the mound. See white bacterial mits and individual talemom bashes. Worms do not live on mats, only on dean buwn serfloos. WW bactingt Wi't

3) 10:40 Found a bittle ( 20 cm arross) ounge (ud, wose) Gacterial mat. Took 10 shots Thick mat (severale min). Collected suction sargple in Backet $\neq 7$ (Fix $\neq 8$ ) Dopth 1867.3

10) 10:55 Depth $=1865$ Took 3 grab samples nuxt to tubeworn bush. Fix $\neq 9$ Mnishe Bucket $\neq 10$ is right next to the TW bush

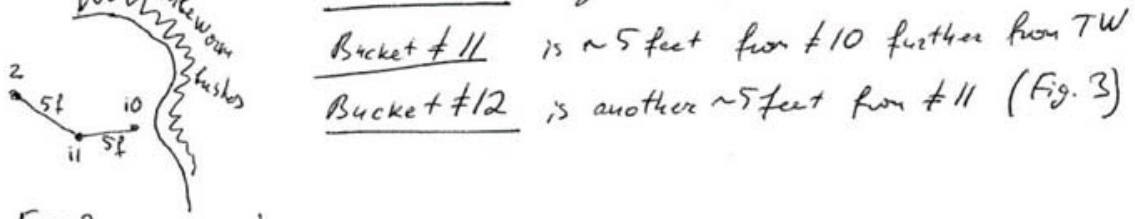

Fig. 3

11) 11:08 Depth 1866, Fix 10, Taking pish wre for geotechnicre and lysis between tavo TW hills Pushure $\neq 2$ is taken near TWs, poor slow penetcation, $W W \quad$ PCf2 Wprastly thece is something hand in sediments (Fix $\neq 10$ ) a mupled TW Fig. 4

12) $11: 15$ Took tabevers next to the pash wre ( $2 / m$ apant), pest them in the foont bucket (Fij. 4)

13) 11:30 Goin up. Gas is wring out push core $\neq 1$

14) $11: 51$-Seasurfice!!! 


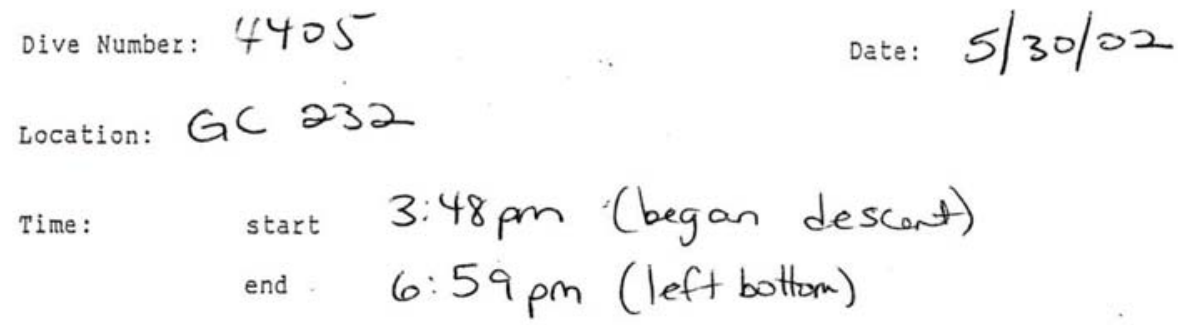

(2)

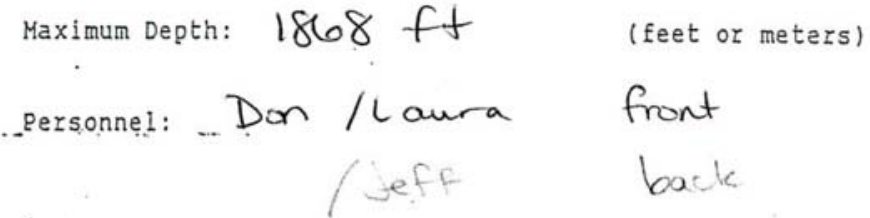


During Descent@3:48 pm.

Saw jelly at $300 \mathrm{ft}$ tap, a shark about $2 \mathrm{ft}$ long was seen rear bottom.

Saw bottom $1840 \mathrm{ft}$

Hydrate mound visited (at 1.e posed was hydrate outcropping very near (we could see it from the sub) the very near (we could see it from the
outcropping wive 10 worms were found on dive
4405 .

Found spot to set probe in mud. Deployed probe it sank to the bottom of tubing $\sim 2 \mathrm{~cm}$ above the pure lip. Values triggered at 4:33 pm. nt $5: 03$, aus put back in quiver closed $5: 03$ pom. Don used the side of the scooper to close 20 values. Need to make tubing longer so the probe can be turned.

7) took $\sim 20 \mathrm{~min}$ to close values.

Core \#4 taken nexteto probe - not much there Core \#3 taken $~ 2 \mathrm{ft}$ from probe - nice size core.

At 5:40 pm, gas sample was taken above hydrate outcropping. While we were sitting on bottornlooking at bubble streams, new ones kept forming - it was quite a site... how does one get a gas flux here?! Secured gas sampler at 5:50 pm.

Put rock sitting right next to hydrate mound in bucket \#2. It looks like light grey w/ lots of holes -maybe lithification around tube worms - Harry R. said.

133 
While sampling for rock, we bumped the hydrate outcrop + it moved -very unstable. gas foil streams coming from all over. Some constant flow, some sporadic others had lith bits of oil coming out.

Core \#2 taken in hydrate crevas. Actually cored the hydrate! We didn't want this though. Shale out hydrate + cored about 2 feet off hyde. mound. Nice core.

Had some time to kill -let's get some bacterial mats! found some white mats, took scoop + put in bucket \#4.

Found a rock outcropping + took a chunk (Fix) off - bucket \#6 Also took scoop of seeds peart to rock outcrop bic lots of shells. -bucket \#8.

Found large rock + put in basket - it had shells, tube worms + maybe some coral on it.

(ix) Bucket $\# 10$ was filled $w /$ orange bacterial mat found on way to see clam beds.

Dive ended at 6:59.jm@1867 ft. fix 10 white ascending, core \#2 lubed like mad, core $\$ 3$ began to degas at the surface + the probe didn't bubble -it looked great!

( see back for map.

134 


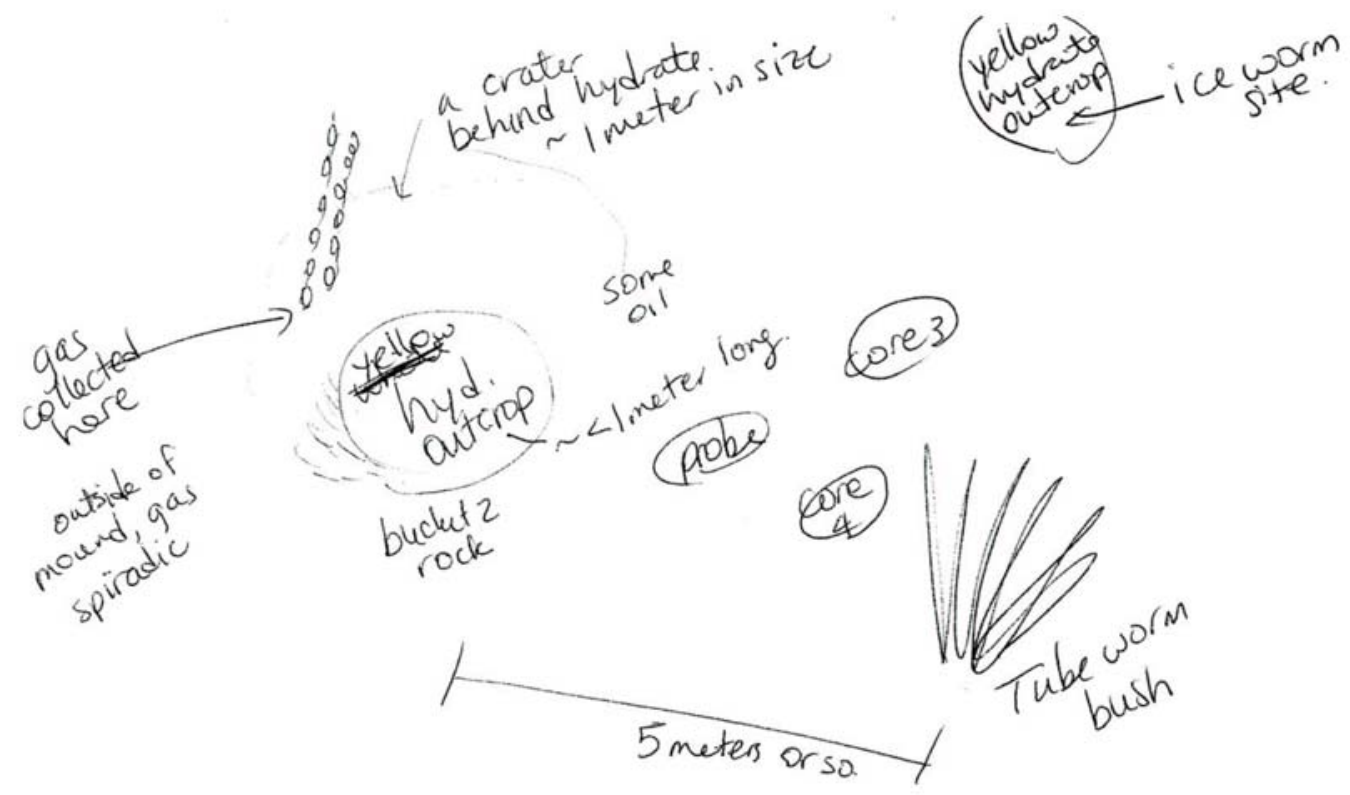




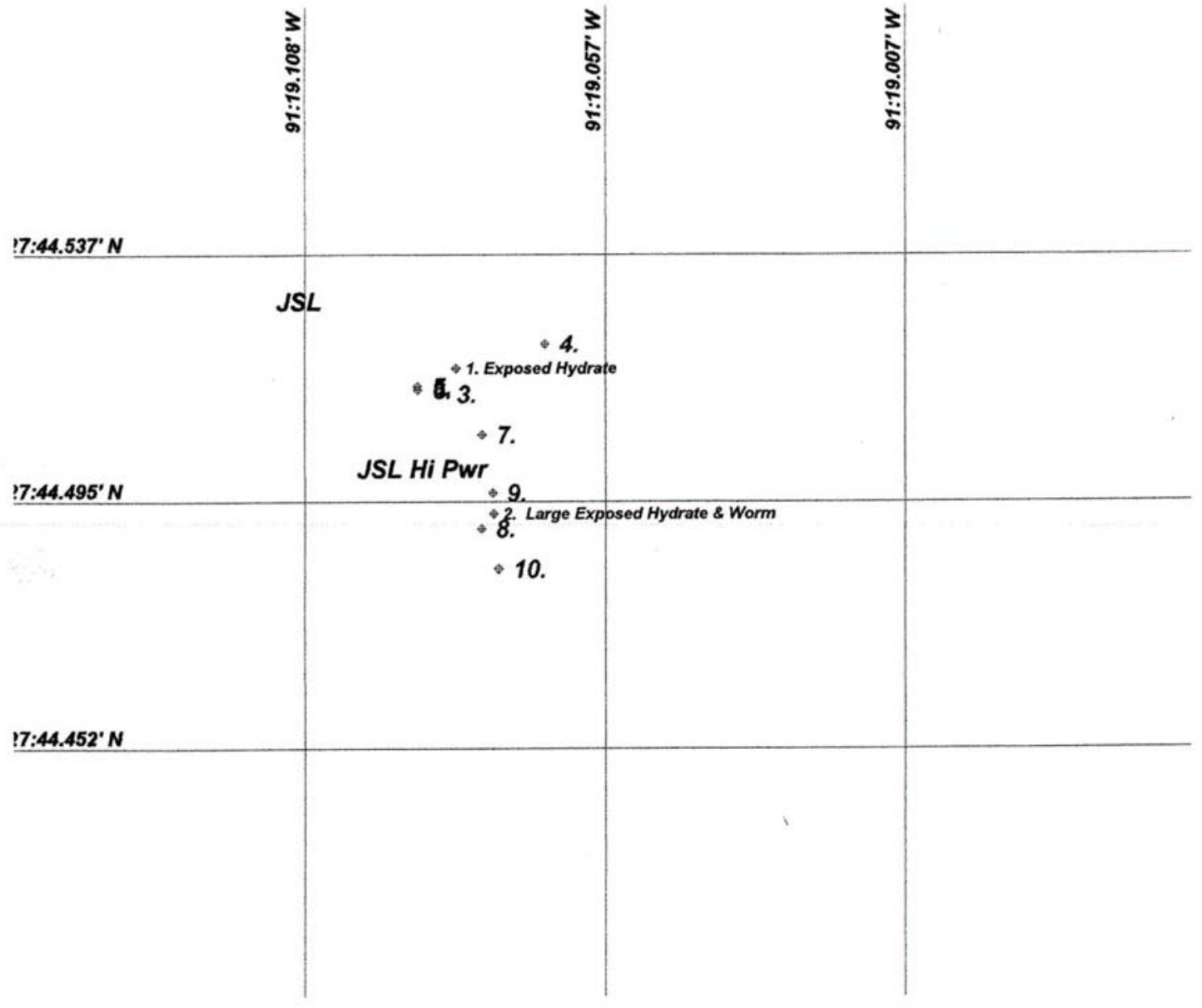


C:IProgram FilesIOREl1-4405

RVSJ 2 \& JSL $1-4405$

1. Target [1] 9:07:30 N 27:44.5173 W 91:19.0828 $1866 \mathrm{ft}$ Exposed Hydrate

2. Target [1] 10:51:30 N 27:44.4924 W 91:19.0765 $1868 \mathrm{ft}$ Large Exposed Hydrate \& Worms

3. User 15:49:23 N 27:44.5129 W 91:19.0851 $1864 \mathrm{ft}$ Target 1

4. Ship 15:49:56 N 27:44.5214 W 91:19.0677 $1855 \mathrm{ft}$ Launch

5. Target [1] 16:21:01 N 27:44.5142 W 91:19.0893 1862 ft On Bottom

6. Target [1] 16:26:23 N 27:44.5136 W 91:19.0893 $1867 \mathrm{ft}$ Deploy water probe

7. Target [1] 17:18:48 N 27:44.5059 W 91:19.0784 $1867 \mathrm{ft}$ Punch core

-8. Target [1] 18:31:17 N 27:44.4899 W 91:19.0785 $1864 \mathrm{ft}$ scoop and rock sample

9. Target [1] 18:44:12 N 27:44.4960 W 91:19.0766 $1864 \mathrm{ft}$ sample orange mat

10. Target [1] 18:55:08 N 27:44.4830 W 91:19.0756 $1867 \mathrm{ft}$ leaving bottom 
Dive Number: 4406

Date: 31 May 02

Location: GC 234

Time: start 8:O AM.

end $11: 48$ Surfece

Maximum Depth:

(feet or meters)

personnel: Rógor Sassen

Kelly Reeler

Dan Boggess Augo Marvero

Mission/Dive objective: hydrate, gas, sedimort

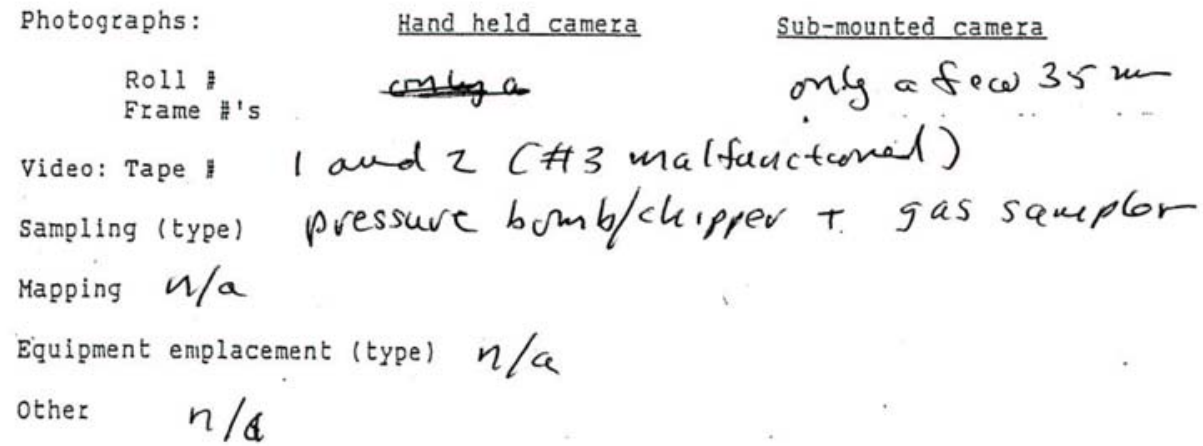

$$
\text { see next sheets }
$$


C:IProgram FilesIOREI1-4406

Dive \#4406 Green Canyon \#243 $234 \quad 3 /$ kng 02

1. Ship 8:01:17 N 27:44.7543 W 91:13.3406 oft Launch

2. User 8:12:56 N 27:44.7700 W 91:13.3400 $1746 \mathrm{ft}$ Target 1

3. Target [1] 8:32:30 N 27:44.7422 W 91:13.4194 oft Bottom Report

4. Target [1] $8: 37: 50$ N 27:44.7766 W 91:13.3613 oft Org. Mat Sample

5. Target [1] 9:02:07 N 27:44.7587 W 91:13.3101 oft Gas Sample \& Hydrate

6. Target [2] 11:18:56 N 27:44.7448 W 91:13.3201 oft Leaving Bottom 1777ft.

7. Target [1] 11:44:48 N 27:44.7797 W 91:13.4166 oft Surfaced 


$$
\begin{gathered}
\text { GC234 Dive } 4406 \\
31 \text { mog or }
\end{gathered}
$$

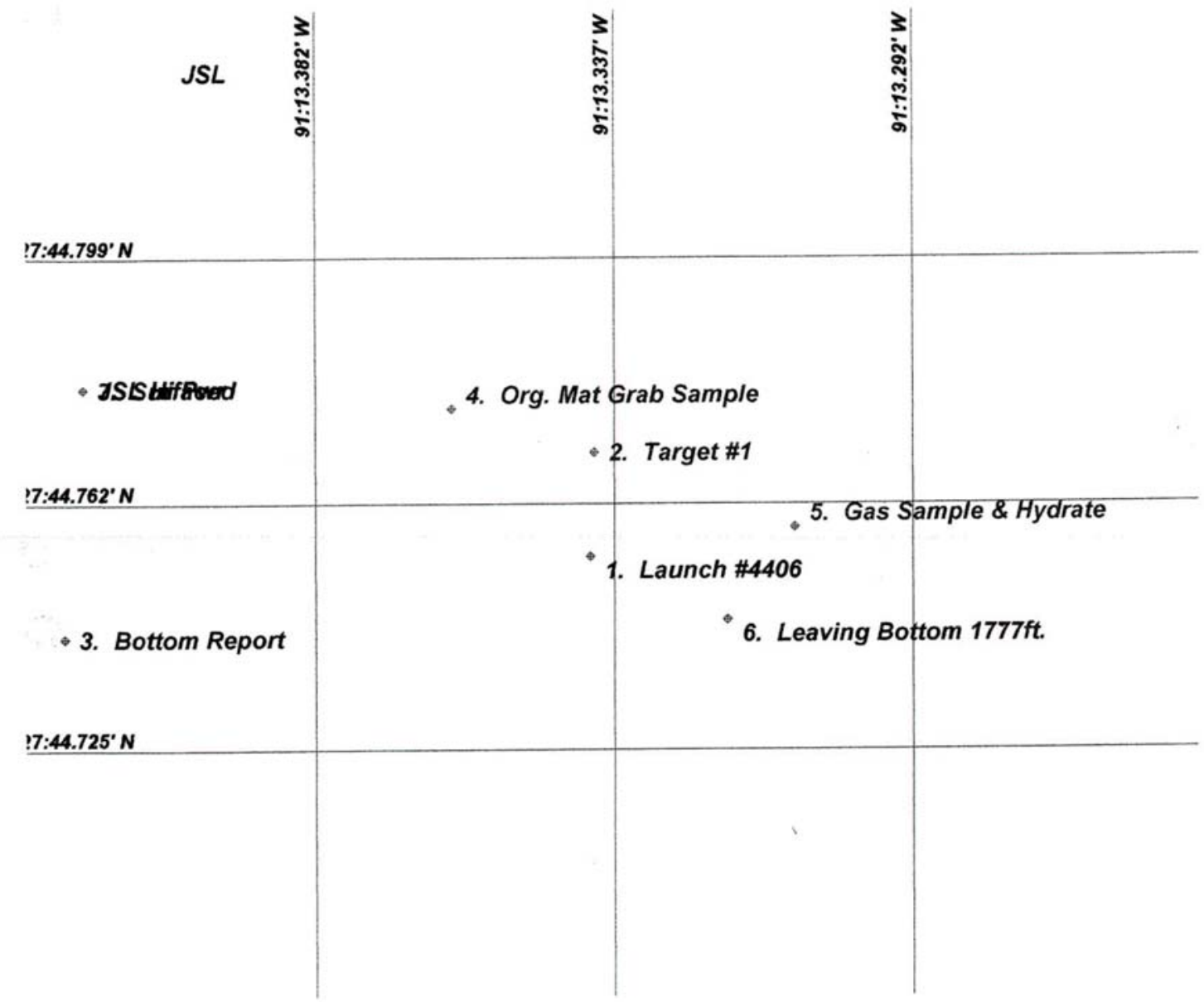




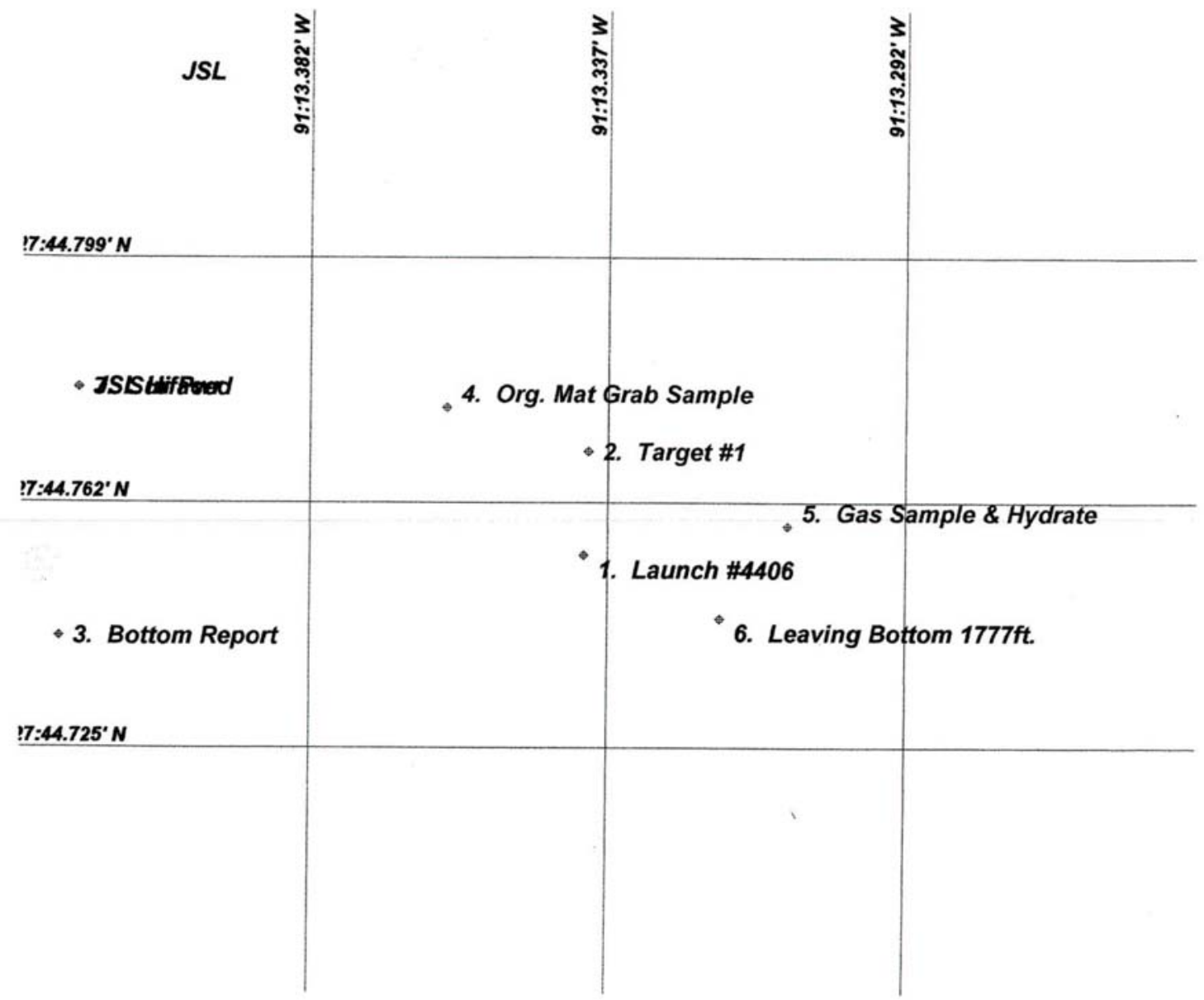




\section{C:IProgram FilesIORE11-4406}

\section{Dive \#4406 Green Canyon \#243}

1. Ship 8:01:17 N 27:44.7543 W 91:13.3406 oft Launch

2. User 8:12:56 N 27:44.7700 W 91:13.3400 $1746 \mathrm{ft}$ Target 1

3. Target [1] 8:32:30 N 27:44.7422 W 91:13.4194 oft Bottom Report

4. Target [1] $8: 37: 50$ N 27:44.7766 W 91:13.3613 oft Org. Mat Sample

5. Target [1] 9:02:07 N 27:44.7587 W 91:13.3101 oft Gas Sample \& Hydrate

6. Target [2] 11:18:56 N 27:44.7448 W 91:13.3201 oft Leaving Bottom 1777ft.

7. Target [1] 11:44:48 N 27:44.7797 W 91:13.4166 oft Surfaced 
4406 GC234 Recon dive

11 took water samples for J. Chationon way down and on bottom at $1714 \mathrm{ft}$.

A observed gorgoncans, carbonate rock, small white mats, burrows, tracks, oil 0830 photographs.

3. Grab sample orange mot at $0835-0844$ Bucket 8 - 1762 ft.

4. main hydrate site at $0850,1778 \mathrm{ft}$ 5 . Acquired ventgas sample with $0.1 \mathrm{~m}$ of bottom. Grew gas hydrate in the. 6. After great struggle, obtained multiple sample o of gas lydute from outcrop. see Henry's no to book for precise site description.

7 found ice worm" with hydrate + "unknown" worm species.

DS 31 major

143 


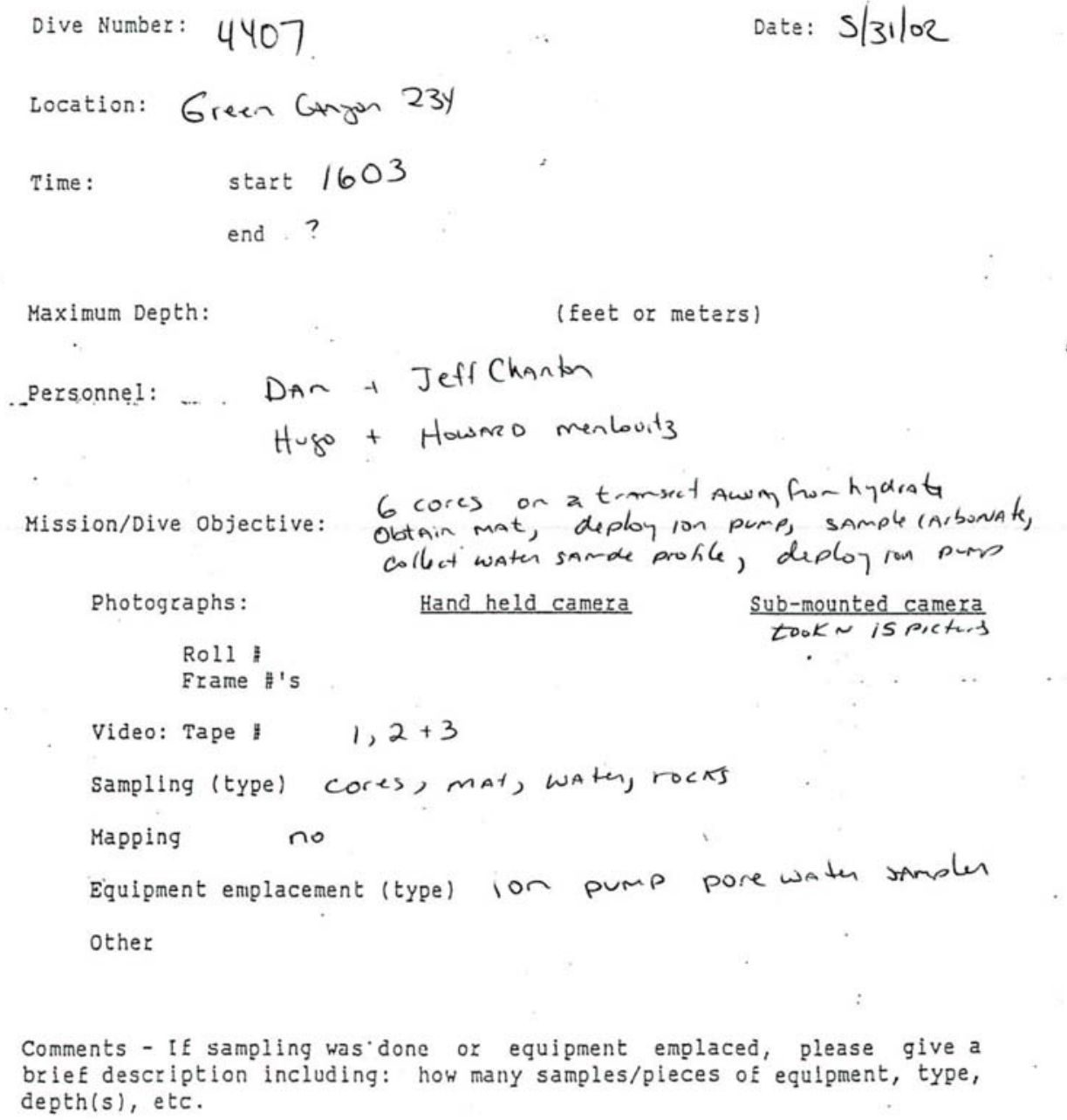

$$
\text { su next Pagl }
$$


$F i x+11$

$16^{38}$ mat samoling orang mat to BAG $\$ 9$ cont

$$
\begin{aligned}
& \frac{16^{38} \text { mat samoling orang mat to BAG } \# 9}{\text { samples stuck - wouldat opent }} \\
& \frac{16^{58} \mathrm{~F} \times \mathrm{a}^{2}}{\text { secmed to work very well }} \\
& \text { seveal shil fiames of mat thaken wath } \\
& \text { subs camen (still cameu) } \\
& 1722 \text { fix } 13 \text { just off to left of hydeth mound } \\
& \text { Marker "RI" on botlom } \\
& \text { collected tube cores } \# 1+2 \text {. } 10 \mathrm{~cm} \text { rencer of } \\
& \text { sediment ores rock or hycente ice }
\end{aligned}
$$
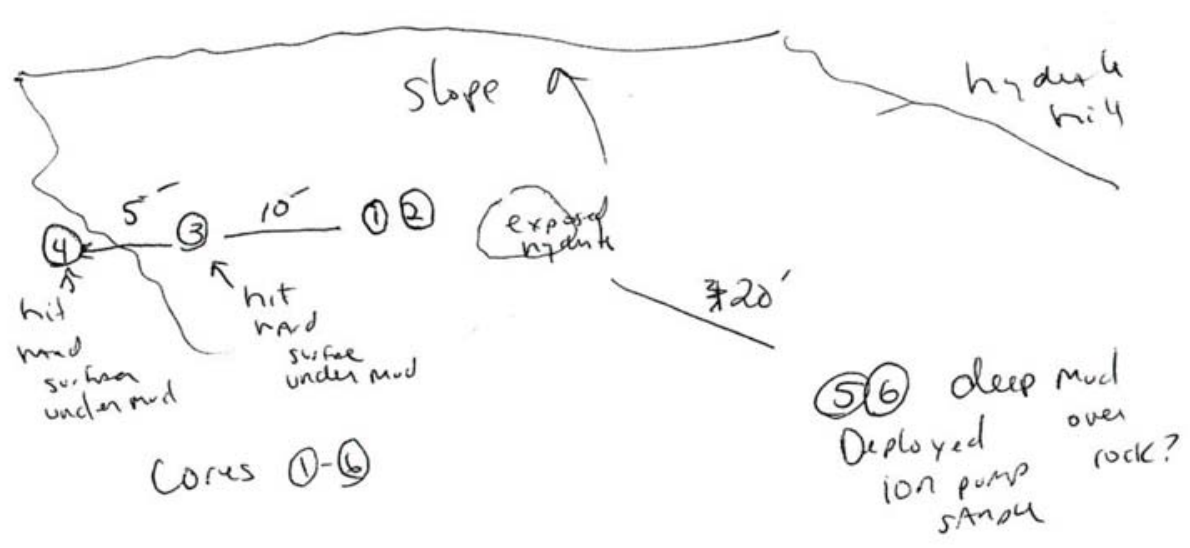

over 


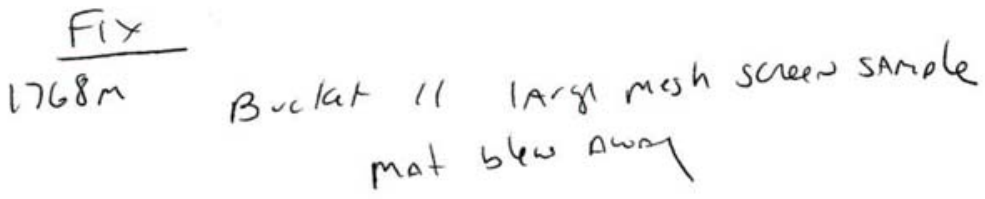

$$
\begin{aligned}
& 1768 \mathrm{ft} \text { - Rock sample from sparst tube worer arest } \\
& 18^{27} \mathrm{pr} \text { to basket (Bij Rock) } \\
& 18^{30} \text { AnothertRy at mat - No go } \\
& 1761 \text { ft } \frac{\text { Fix }}{H \| \text { Gasket more orangment }} \\
& \text { lots glots of flooting oil. }
\end{aligned}
$$


ORE Interenational. IPS: WayPoint Report

C:IProgram FilesIORE11-4407

Dive \# 4407 Green Canyon \# 234

1. Ship 16:06:17 N 27:44.7614 W 91:13.3365 oft Launch \$4407

2. User 17:01:07 N 27:44.7906 W 91:13.3467 oft Bottom

3. User 17:01:47 N 27:44.7700 W 91:13.3400 oft Target \#1

4. Target [1] 17:02:35 N 27:44.7882 W 91:13.3426 oft Red Mat Sample 1759ft.

5. Target [1] 17:21:31 N 27:44.7531 W 91:13.3119 $1777 \mathrm{ft}$ punch core near hydrate

6. Target [1] 17:50:23 N 27:44.7563 W 91:13.3078 $1784 \mathrm{ft}$ punch core \#5

7. Target [1] 18:27:11 N 27:44.7723 W 91:13.3104 Oft Rock Sample

8. Target [1] 18:59:34 N 27:44.7341 W 91:13.3003 Oft Leaving Bottom 


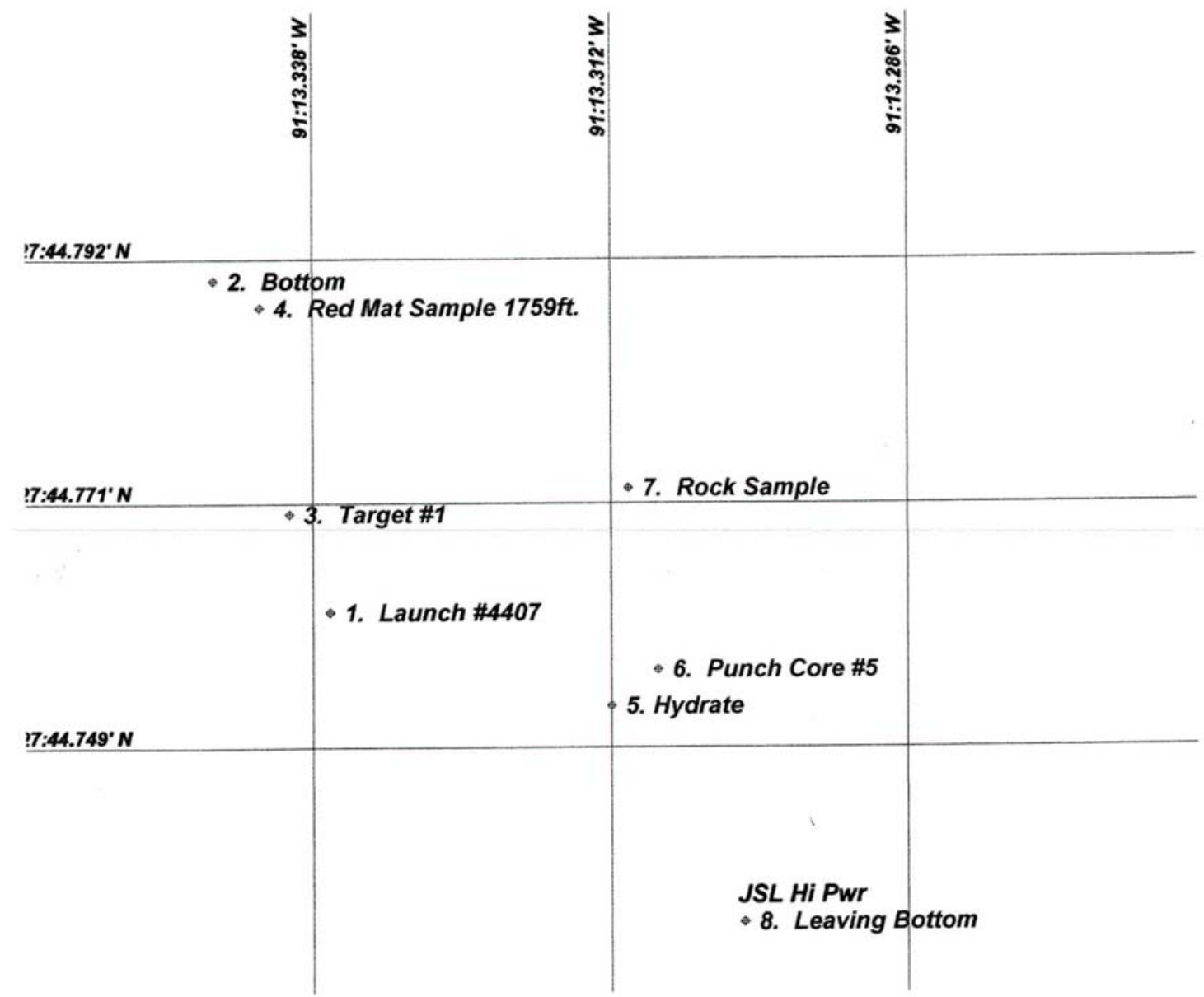

JSL 


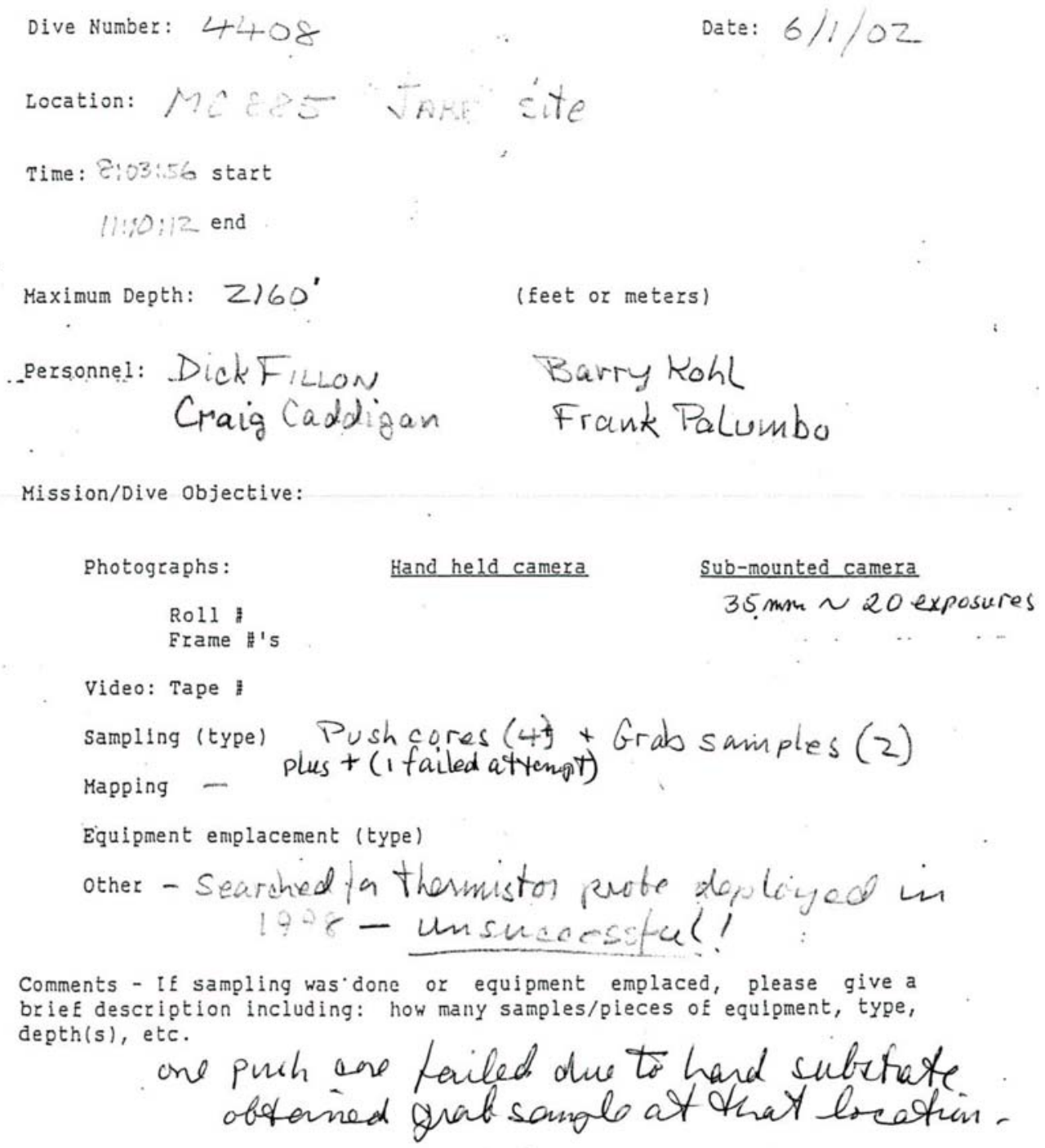


08:32h on bottom - WD $=2160 \mathrm{ft}$. - soft bunwed bottom with 0.4 knot current furm $030^{\circ}$

08:34h Fix \#7 (ON BOTTOM)

$08: 47 \mathrm{~h}$ puch core \#1 (Thbe \#6) - full penctiation

08:55h samo bottom type 2124ft. - neurcounse $020^{\circ}$

rave starfich, brittle star, smuall octorses - 08:56h.

08:58h changed counse to concet lateral ahifi

$08: 59 \mathrm{~h}$ dijital camera shots.

09:00h sub-35mn camera shote

* 09:18h firitgorgonions wobitte stars seanbottom still flat - but gonsonions surgest 09:17h FIX\#8, shallawly undertain by hard sulstiote. 09:21h $\rightarrow$ push core \#2 (Thbe\#5) 2079 ft.

09:25gonganians@zo>>tt.onsimojdip

09:27 chintip ant of dip - $35 \mathrm{~mm}$ shots.

09:30Calyptogena shell hash@2055ft.

croserip serios of sndges topred wf forgavians

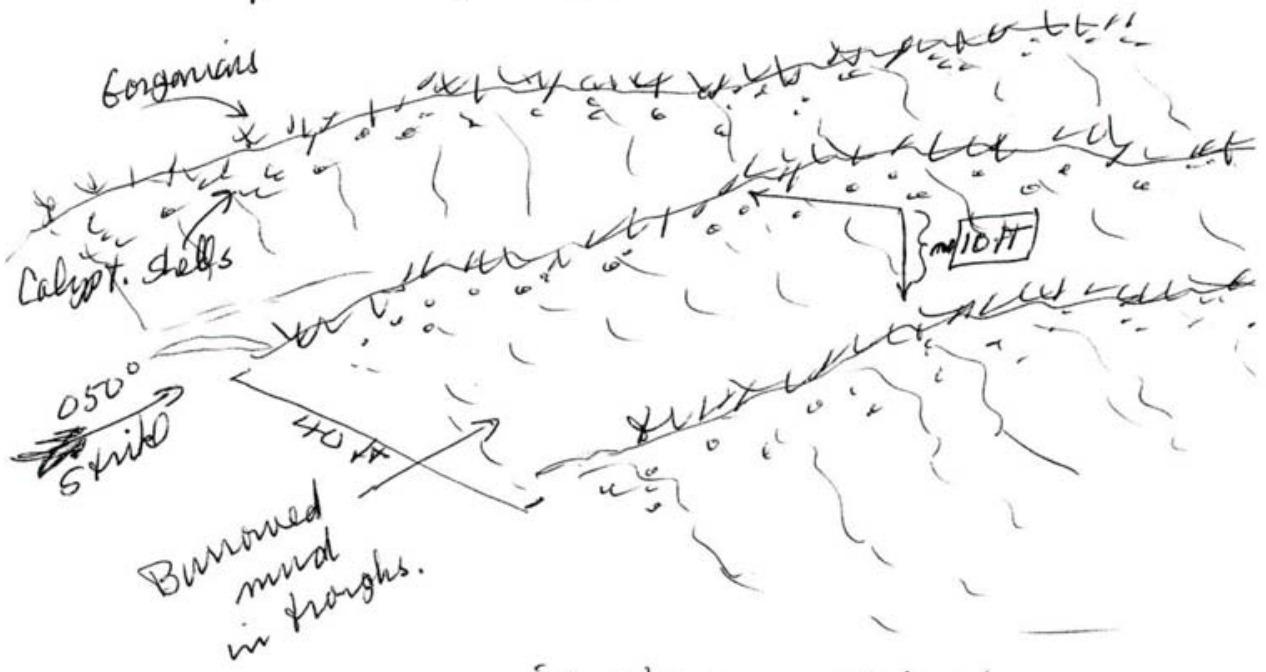

09:39h changed vialeotape-put in tope 2. 
$09: 53$ Fix \#9 pushcre \#3 (Tube \#4)

09:45 - Steep-sided monnd w/ thke wanns + Acesta - onterosing casfrinate.

$09: 47$ - bearing chango. \& $025^{\circ}$

(a) $2045 \mathrm{At}$.

$\rightarrow$ push con 09:55h-aborted diee to lack I penetration cansed by hard substiate. 09:58-Grat songle \#1 (buckot\#4)@2038tt.

Consustent shell hash (Calyptosena) 10:00 h undervay afth grat sample 10:04h Acesta attachedt small rock outerga

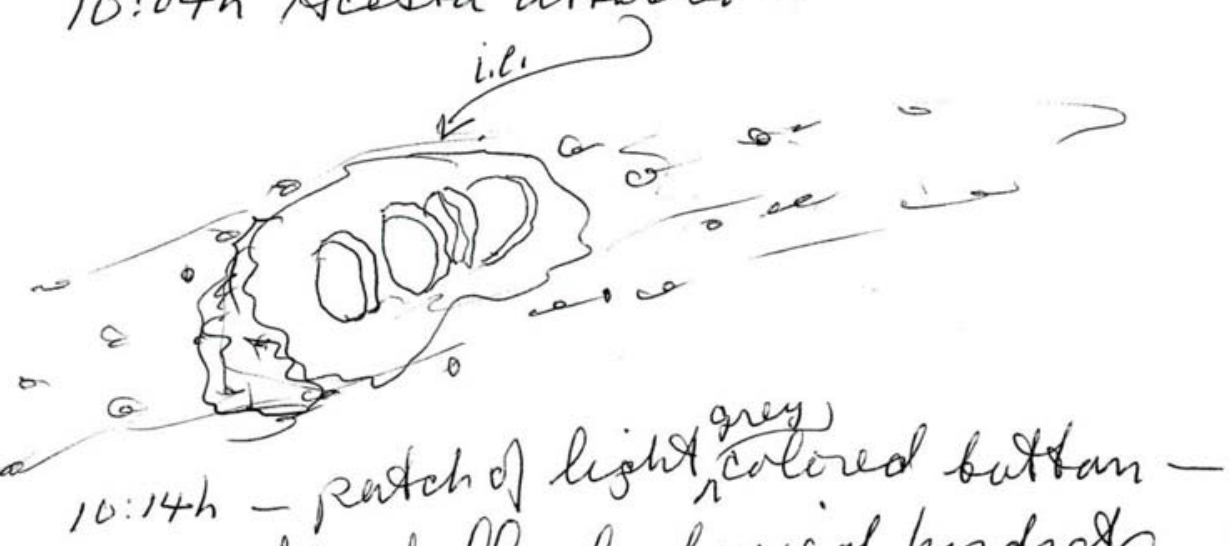

possible shallowly buried hisatrato (4) 2066 H. $-26 f+3 f t$ 10:15h - Leatuless mud botton w/ pock mank hinisis - divital camera shots.

151 
10: 24:5sh rix"/0 coourr (Tube\#4) frell penetation

@10:25:23h@2065.377.6.8 $\mathrm{C}$.

$10: 29 \mathrm{~h}$ underwey an new comse $150^{\circ}$ still shell hash dritituted patchily ver bottom.

10:27h $35 \mathrm{~mm}$ photos

10:33h shlll hash out starbeard post

10:34h Reare pately Gorgenians

10:37 h Peesta an fuble worms

10:40h Gorganaino m rock, shllhash of large gapnip Caly ptagenas - dead bist in growthposition-@2045kt.

10:45h Featurleds bottm

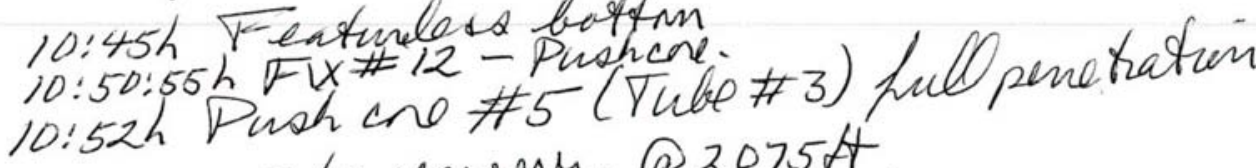

2/3recovery.2075ft.

10:52h feotmless bottan 40\% burrowed

$10: 55^{2}$ underway done noith $\left(000^{\circ}\right)$

11:01h midbottom wffurrows@207ift.

1:09h featureless mud bottrm - Grab cample \#2

(Buoted \#6)

1): 10:12h Fix\#/3

Nill h lave bottom.

152 
ORE Interenational. IPS: WayPoint Report

\section{C:IProgram FilesIOREl1-4408_trk}

\section{RVSJ 2 \& JSL I Dive 4408}

1. User 7:23:48 N 28:04.0830 W 89:43.3170 $2140 \mathrm{ft} \mathrm{SOL}$

2. User 7:24:10 N 28:04.0062 W 89:43.1667 2140 ft WP 1

3. User 7:24:41 N 28:03.9670 W 89:42.7500 2140 ft WP 2

4. User 7:25:01 N 28:03.9500 W 89:42.5170 2140 ft WP 3

5. User 7:25.23 N 28:03.7000 W 89:42.4000 $2140 \mathrm{ft}$ EOL

6. User 8:03:56 N 28:04.0497 W 89:43.2765 oft Launch

7. User 8:34:00 N 28:04.0403 W 89:43.3787 2160 ft Bottom fix

8. Target [1] 9:17:51 N 28:03.9712 W 89:43.0429 2080 ft Long push core PC2

9. Target [1] 9:53:19 N 28:03.9611 W 89:42.7557 2037 ft Long push core PC3 (abonted) GS I

10. Target [1] 10:24:55 N 28:03.9564 W 89:42.5319 $2066 \mathrm{ft}$ Push core $P_{C} 4$

11. User 10:47:45 N 28:03.7699 W 89:42.3825 oft Change hdg to 200 deg

ix 12. Target [1] 10:50:55 N 28:03.6664 W 89:42.4005 $2075 \mathrm{ft}$ Push core \#3 PC 5

13. User 11:10:12 N 28:03.7227 W 89:42.4090 2063 ft Grab sample-leaving bottom as 2 


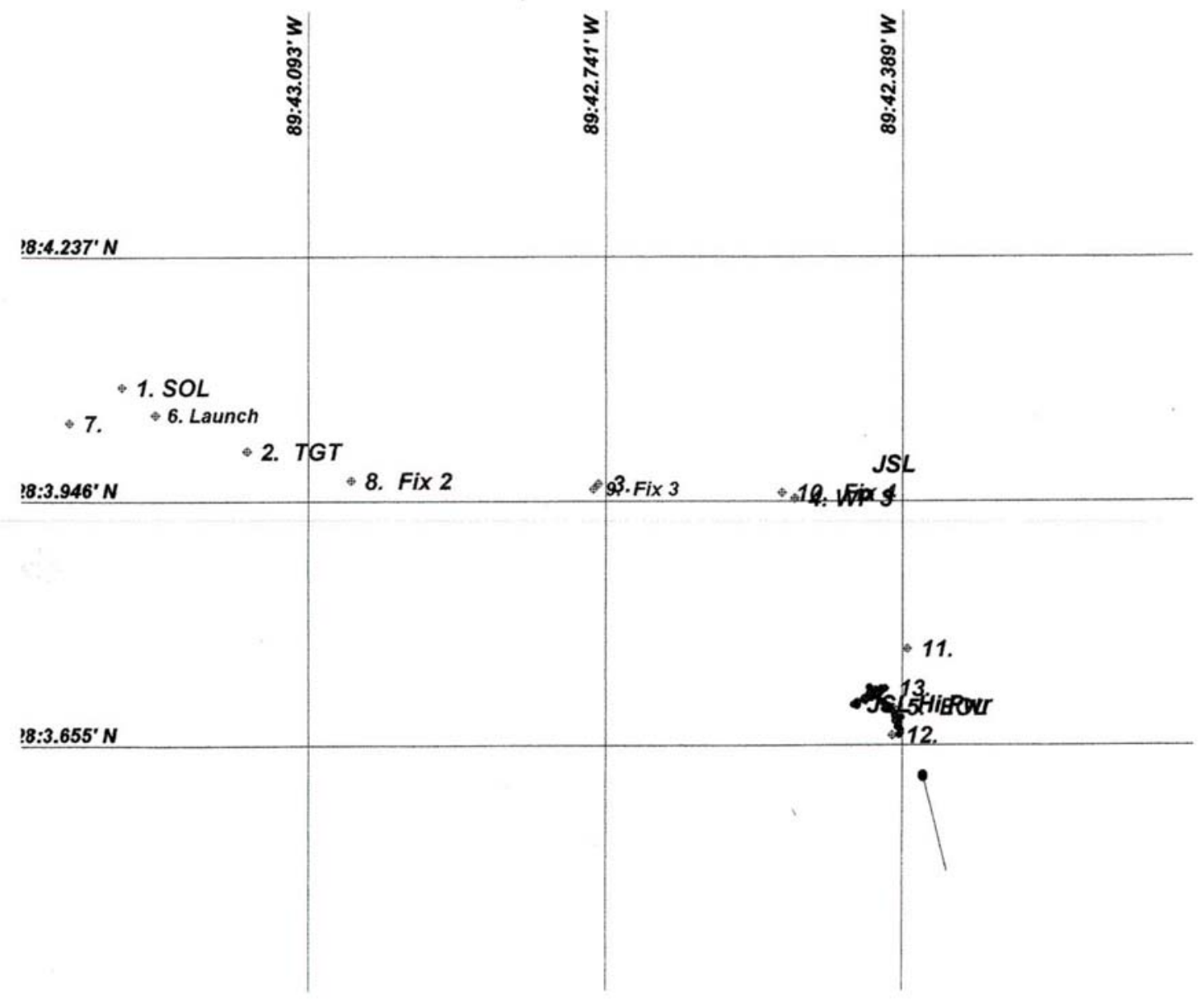

है.

\& 
TSL DIKE 4409

Date: $6 / 1 / 102$

Fonwand: Woolsey

Time

Discription

Aft: Hagles

1514

Commense Lire

1608

on bottom, dpt. -2087.5', t- $6.82^{\circ}$

sel. 348 \%

Bottom conds - Mud, Allat to undulating, bioturbated, surface witl bumows and mounds, 10 to 20

per sq. moter: Type $A$

1611

Commense Trarense $<-060^{\circ}$

1614

cC-150', Aptzo88, T 6.82

Bottom-Type A

1618

$<150^{\circ}$, Apt. 2068, Bottom type A

1623

$<155^{\circ}$, dpt. $2070^{\circ}, t 6.81^{\circ}$, Bottom type $A$

1624

Botfom - type A with lone small rack (auth, carb.)

with gorganian; dpt. 2064', $6.82^{\circ}$

1630

Bottom - typo A with numerous canbonat rocks,

(.15 X.5 m, long axis) with attached gorgonians!

Iype B - Naw bottom type desiznation

sample stop"1 samples: 1) 'arsengonian witt hold fast rack, 2) large a ccesta clam (with bissel a Hachment to base of rack - tom lose in 5ompling), 3) Push core in mud, 3 1640 Depert stop 11 , rexeme traverse; bottom, tpoe $A$ 1644 Bottom type $A$ with patches of clom shell hash, no visable live clams; Aft. $2067^{\prime}$

$1646 \quad 2<220^{\circ}$; Bottom type $A+$ shall hesh 


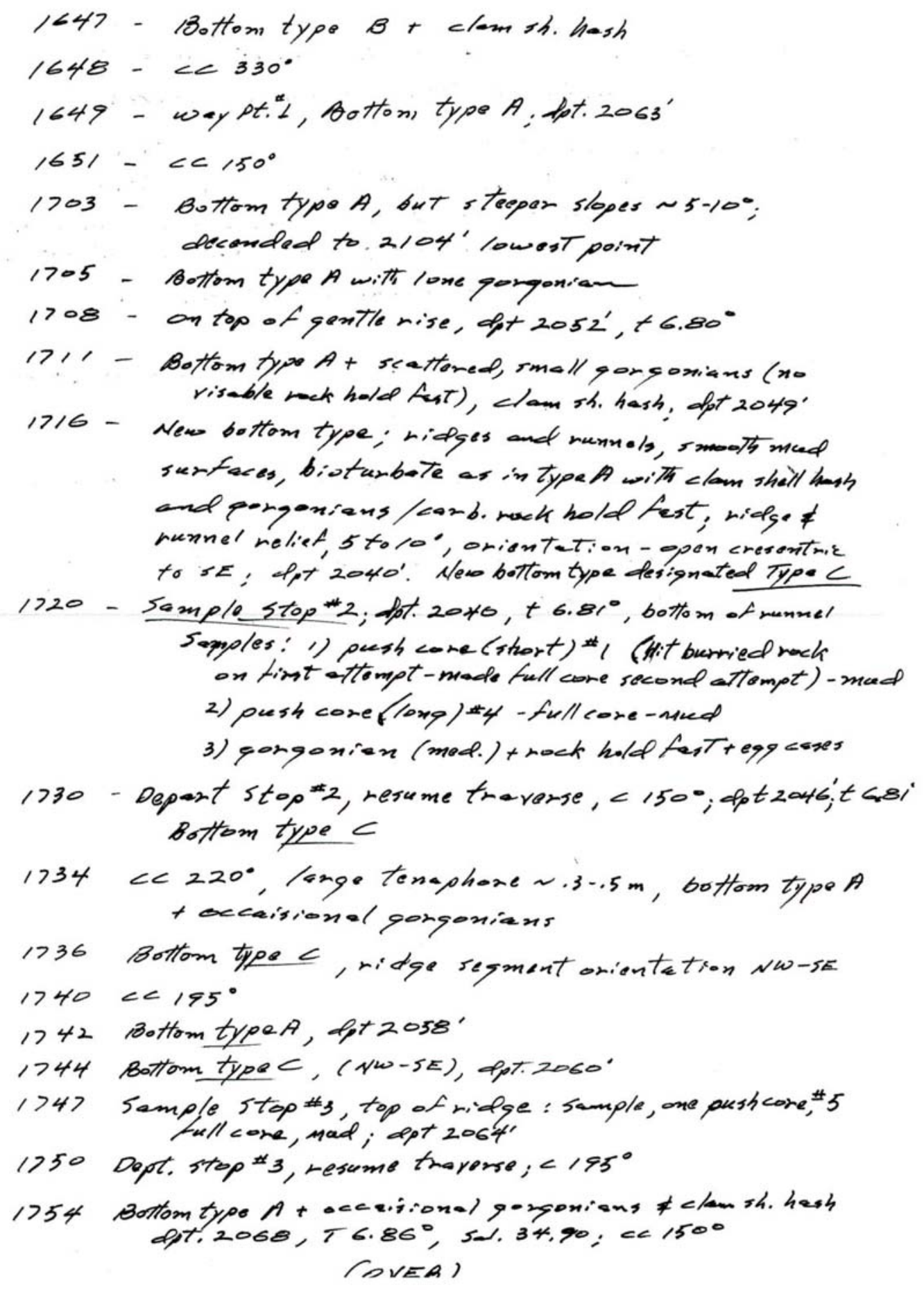



$1758100710 \mathrm{~m}$ type $\mathrm{C}$; Adrised in vicinity of Thamister station, commance seswel
1804 sample stopt4; dpt. $2061.3^{\prime}$ - Top at tidge somples. ") push cone *6 (long) - first - Hempt encountered nock below mud rerffue - Leloctap

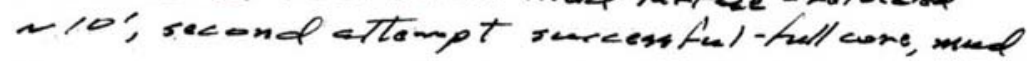

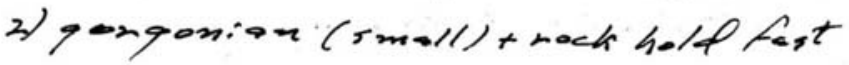

1812 Sompling operation complete, Lesume seareh
Lor Hermisten, $2.280^{\circ}$ 1805. Found Thermister on ridgetop, dpt 2061,

1831 Thermister Probe sotely onboend, departing bottom: Are complete 
C:IProgram FilesIOREI1-4409_trk_wpt

RVSJ 2 \& JSL 1 dive 4409, $1928 \mathrm{ft}$ squares

1. User $12: 47: 10 \mathrm{~N} 28: 04.7000 \mathrm{~W} 89: 42.83302100 \mathrm{ft}$ SOL 2. User 12:47:30 N 28:04.4170 W 89:42.8000 2100 ft WP 1 3. User 12:47:56 N 28:03.9670 W 89:42.7500 2100 ft WP 2 4. User $12: 48: 19 \mathrm{~N} \mathrm{28:03.7061} \mathrm{W} 89: 42.86642100 \mathrm{ft}$ WP 3

5. User 12:48:40 N 28:03.5670 W 89:43.0500 2100 ft EOL

6. Target [1] 15:40:05 N 28:04.5120 W 89:43.3480 oft Launch

7. Target [1] 16:08:44 N 28:04.6908 W 89:42.8598 2087 ft On Bottom

8. Target [1] 16:23:54 N 28:04.5735 W 89:42.7919 $2068 \mathrm{ft}$ Bottom Small Outcroppings

9. Target [1] 16:29:21 N 28:04.4861 W 89:42.7767 $2066 \mathrm{ft}$ Gorgonians \& Punch Core \&3

10. Target [1] 17:27:28 N 28:03.9825 W 89:42.7463 oft Push core \#1 \& \# \& rock

11. Target [1] 17:36:03 N 28:03.9060 W 89:42.7587 $2052 \mathrm{ft}$ Gorgonians \& shell hash

12. Target [1] 17:40:11 N 28:03.8602 W 89:42.8101 oft New hdg 195 deg

13. Target [1] 17:46:05 N 28:03.7866 W 89:42.8436 2064 ft Push core \#5

14. Target [1] 17:53:20 N 28:03.7607 W 89:42.8731 2064 ft Change hdg to $170 \mathrm{deg}$

15. Target [1] 17:55:36 N 28:03.7231 W 89:42.8782 oft Change hdg to 160 deg

16. Target [1] 18:05:22 N 28:03.7116 W 89:42.8518 $2061 \mathrm{ft}$ Tjed push core \#6

17. Target [1] 18.23:59 N 28:03.6917 W 89:42.8765 $2052 \mathrm{ft}$ Thermistor <

18. Target [1] 18:28:39 N 28:03.6863 W 89:42.8649 oft Leaving Bottom 


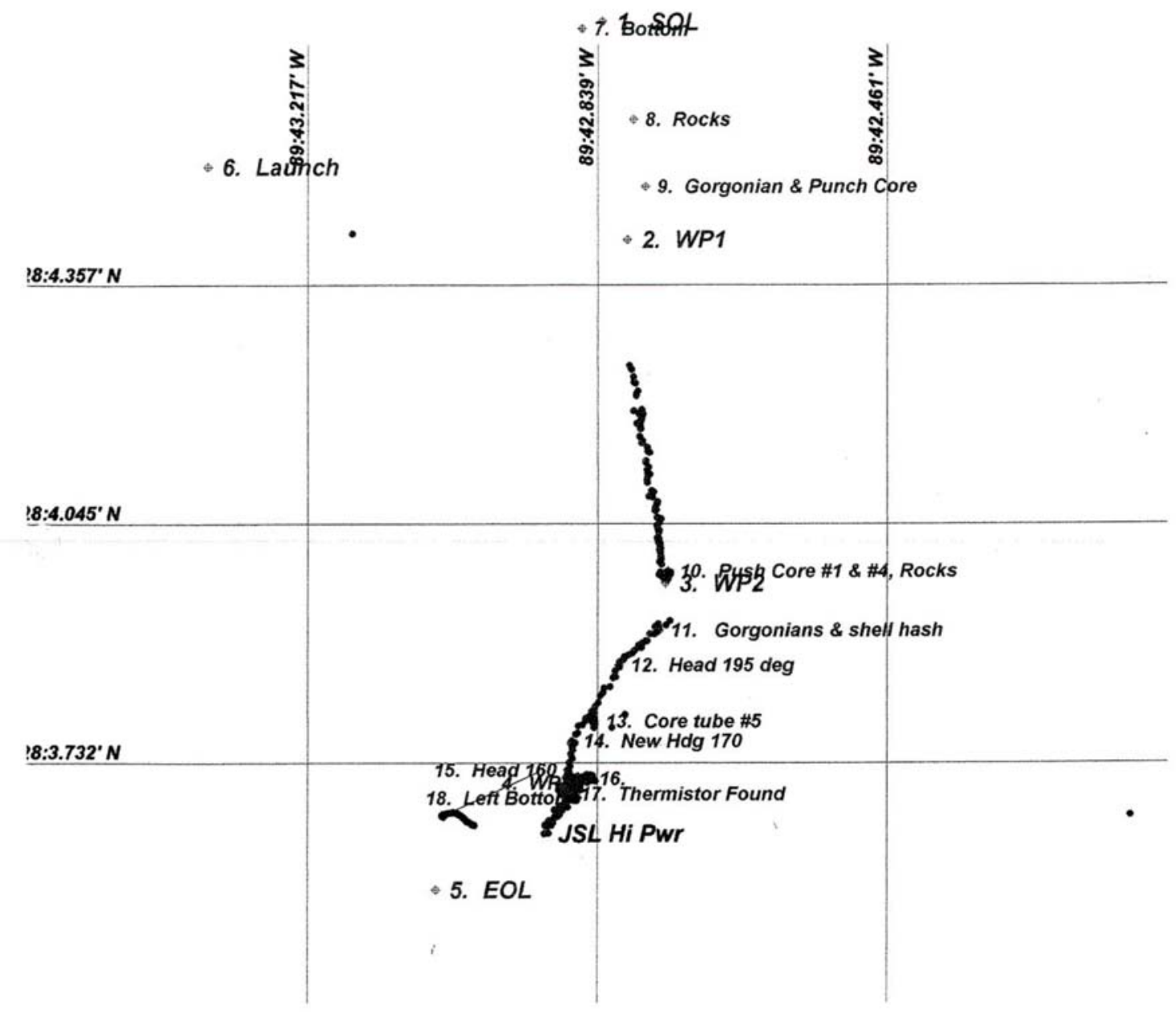


ORE Interenational. IPS: WayPoint Report

C:IProgram FilesIOREl1-4409_trk_wpt

RVSJ 2 \& JSL 1 dive 4409, $1899 \mathrm{ft}$ squares

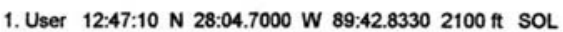

2. User 12:47:30 N 28:04.4170 W 89:42.8000 2100 ft WP 1

3. User $12: 47: 56 \mathrm{~N}$ 28:03.9670 W 89:42.7500 2100 ft WP 2

4. User 12:48:19 N 28:03.7061 W 89:42.8664 2100 ft WP 3

5. User 12:48:40 N 28:03.5670 W 89:43.0500 2100 ft EOL

6. Target [1] 15:40:05 N 28:04.5120 W 89:43.3480 oft Launch

7. Target[1] 16:08:44 N 28:04.6908 W 89:42.8598 2087 ft On Bottom

8. Target [1] 16:23:54 N 28:04.5735 W 89:42.7919 $2068 \mathrm{ft}$ Bottom Small Outcroppings

9. Target [1] 16:29:21 N 28:04.4861 W 89:42.7767 $2066 \mathrm{ft}$ Gorgonians \& Punch Core

10. Target [1] 17:27.28 N 28:03.9825 W 89:42.7463 oft Push core \#1 \& \# \& rock

11. Target [1] 17:36:03 N 28:03.9060 W 89:42.7587 $2052 \mathrm{ft}$ Gorgonians \& shell hash

12. Target [1] 17:40:11 N 28:03.8602 W 89:42.8101 oft New hdg 195 deg

13. Target [1] 17:46:05 N 28:03.7866 W 89:42.8436 2064 ft Push core $\$ 5$

14. Target [1] 17:53:20 N 28:03.7607 W 89:42.8731 $2064 \mathrm{ft}$ Change hdg to $170 \mathrm{deg}$

15. Target [1] 17:55:36 N 28:03.7231 W 89:42.8782 oft Change hdg to 160 deg

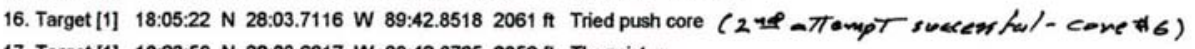

17. Target [1] 18:23:59 N 28:03.6917 W 89:42.8765 $2052 \mathrm{ft}$ Thermistor

18. Target [1] 18:28:39 N 28:03.6863 W 89:42.8649 of Leaving Bottom 


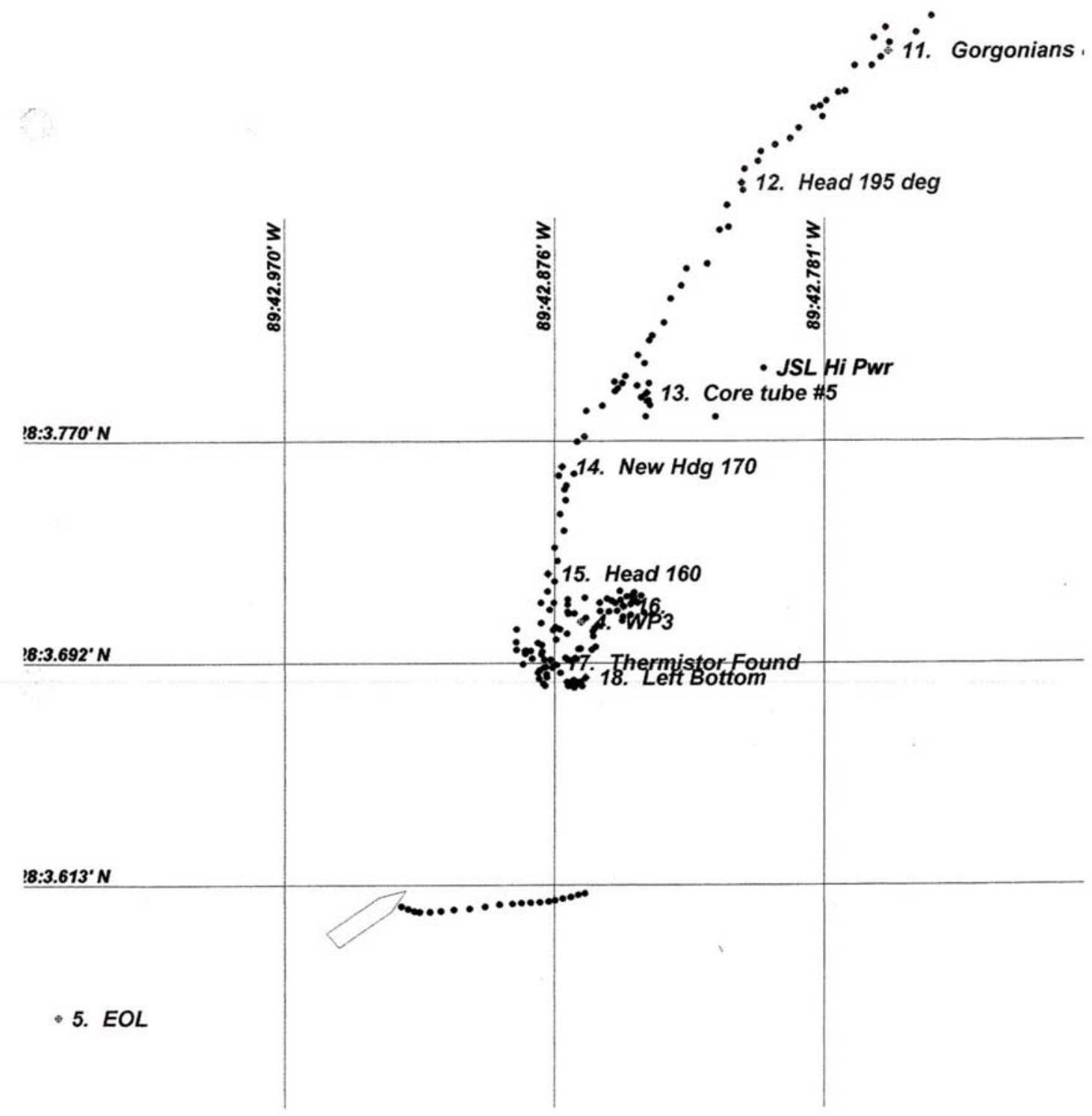




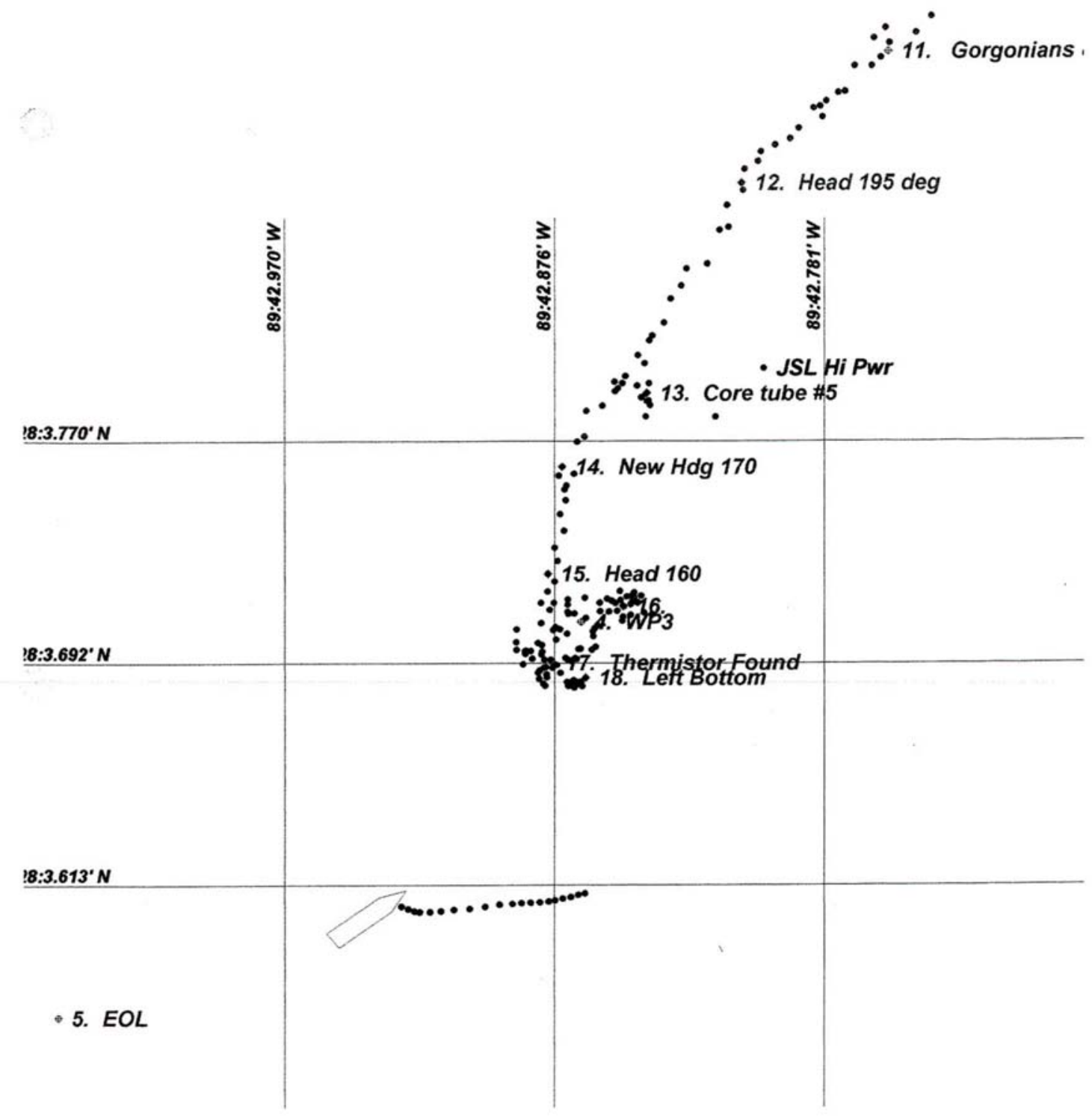




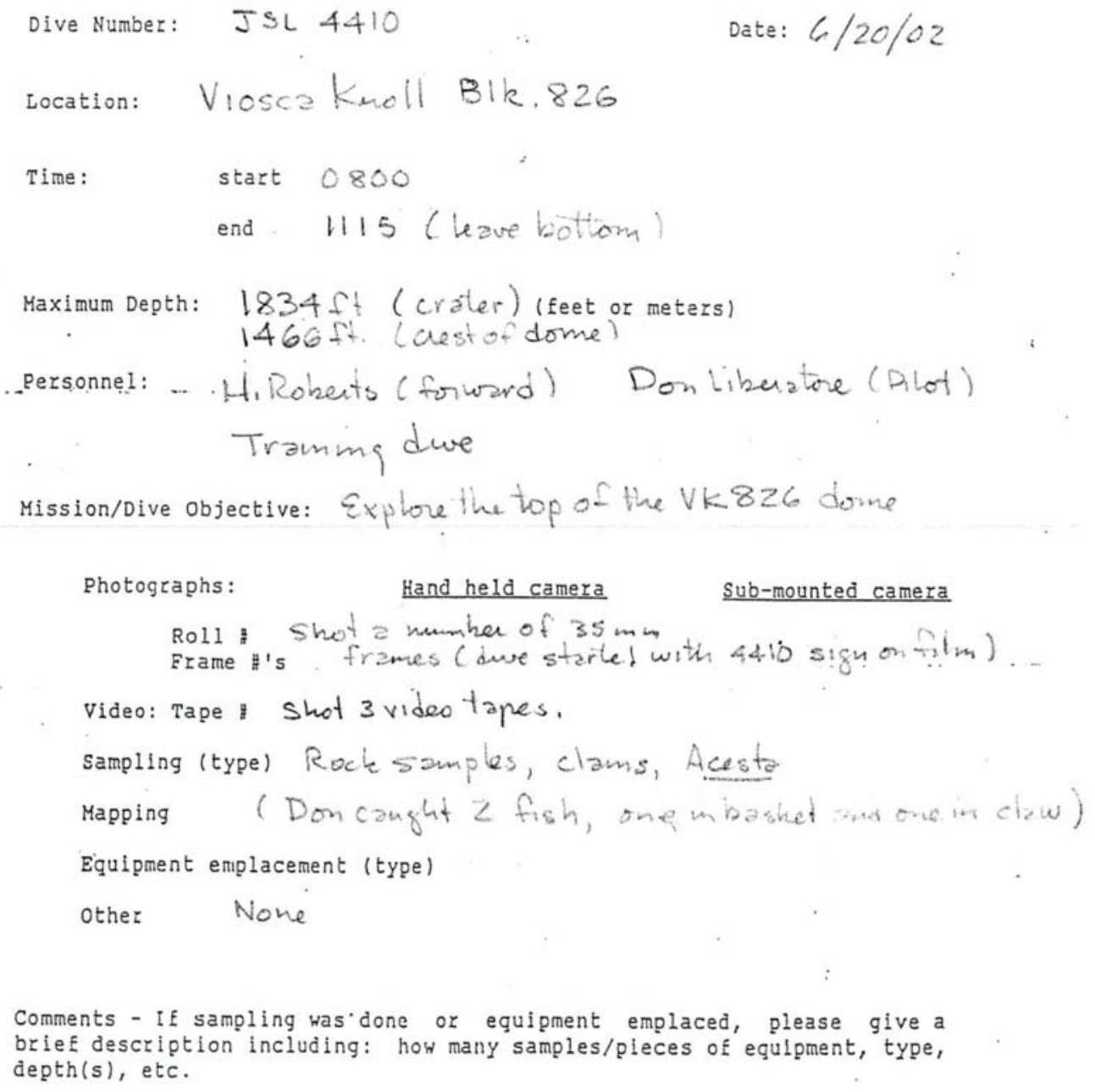




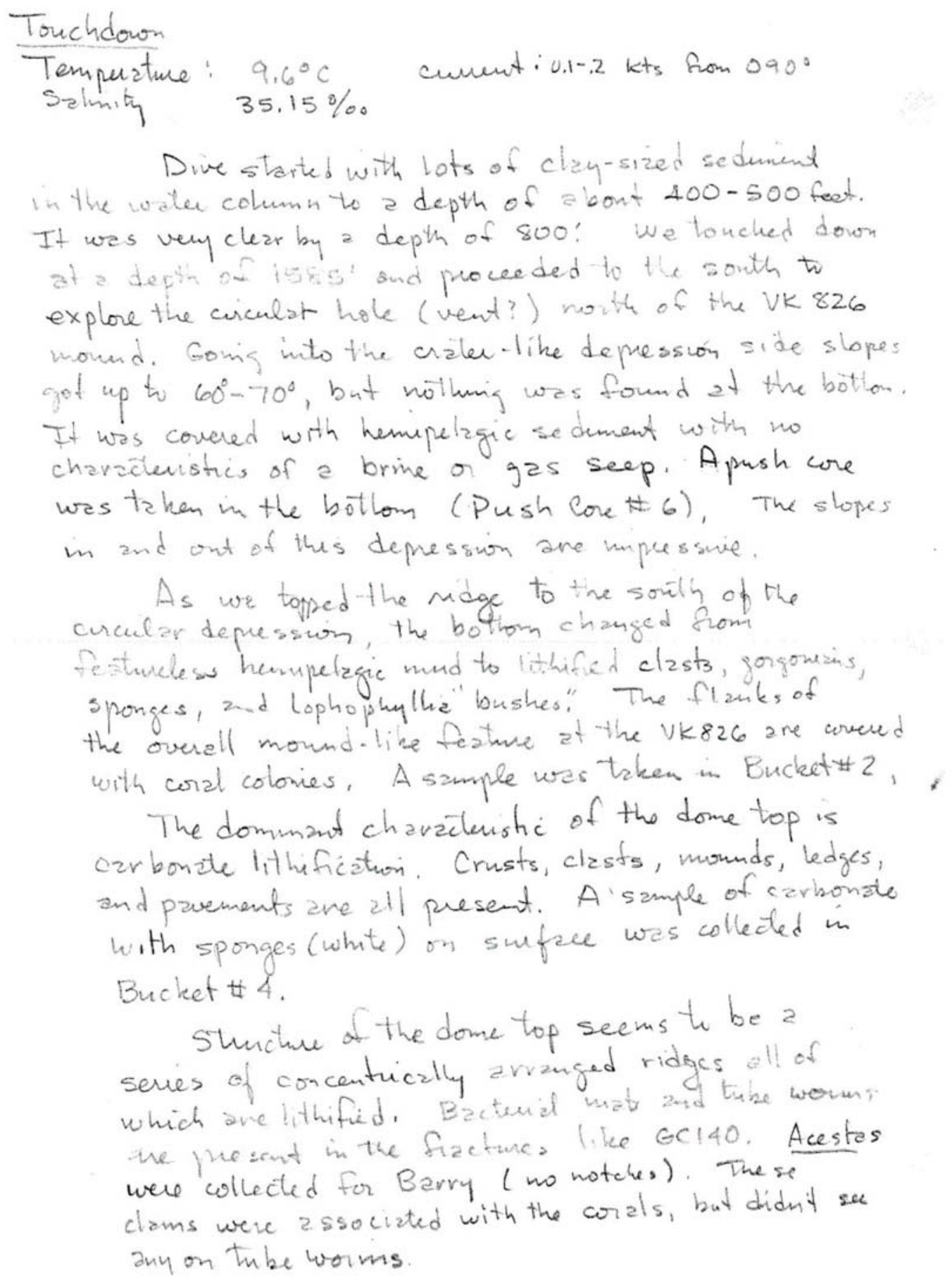




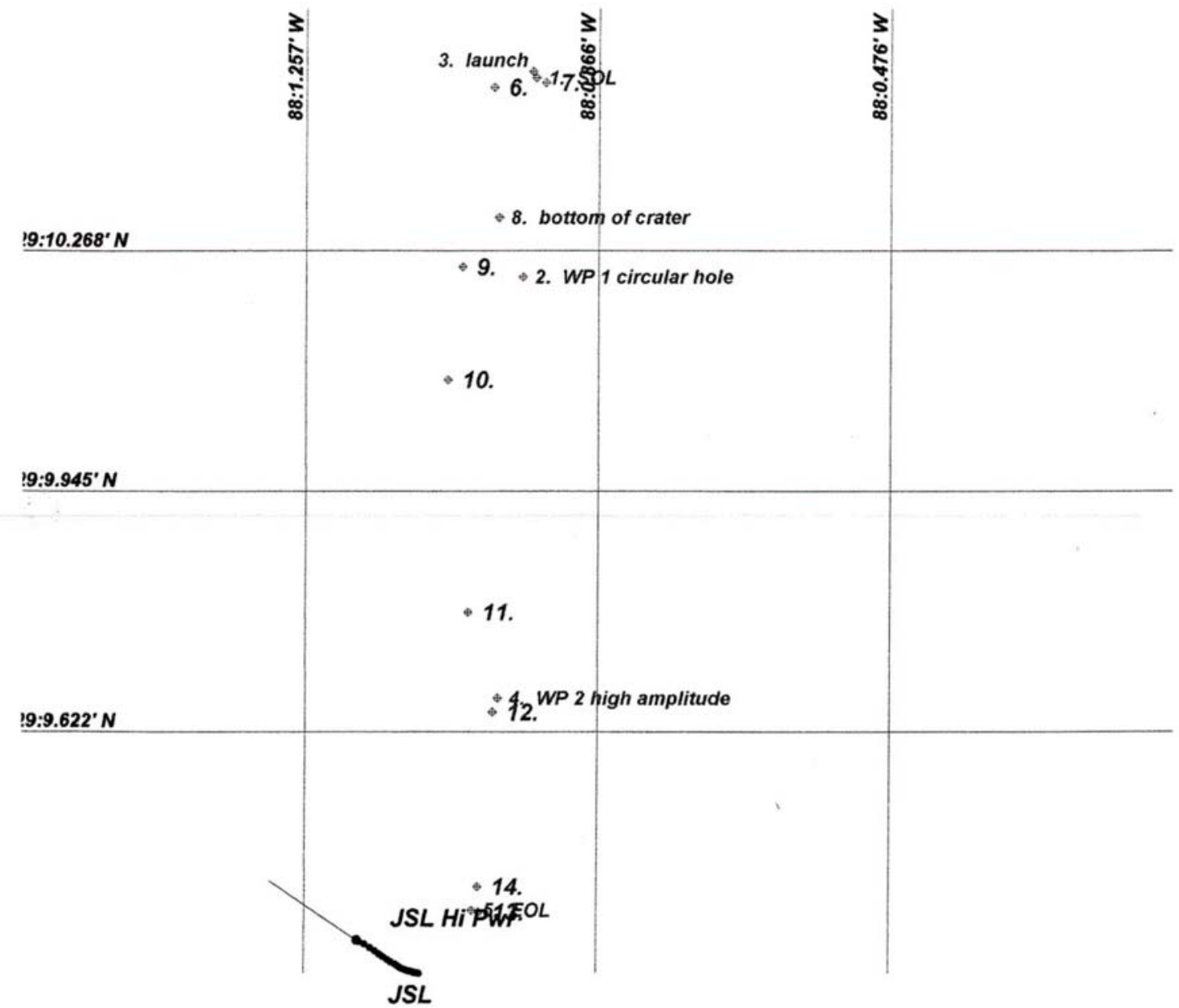


ORE Interenational. IPS: WayPoint Report

C:IProgram FilesIOREI1-4410

RVSJ 2 \& JSL $1-4410$

1. Ship 7:56:27 N 29:10.5000 W 88:00.9500 oft sol

2. User 7:59:28 N 29:10.2330 W 88:00.9670 oft WP 1 Circular hole

3. Ship 8:00:41 N 29:10.5088 W 88:00.9544 oft Launch

4. User 8:08:01 N 29:09.6670 W 88:01.0000 oft WP 2 High Amplitude

5. User 8:08:32 N 29:09.3830 W 88:01.0330 Oft EOL

6. Target [1] 8:25:11 N 29:10.4874 W 88:01.0064 $1581 \mathrm{ft}$ On Bottom

7. Target[1] 8:30:29 N 29:10.4937 W 88:00.9372 $1581 \mathrm{ft}$ Sub starts Xsect

8. Target [1] 8:58:15 N 29:10.3128 W 88:00.9993 $1832 \mathrm{ft}$ Bottom of Crater

9. Target [1] 9:25:52 N 29:10.2464 W 88:01.0481 $1637 \mathrm{ft}$ Top of Crater

10. Target [1] 9:38:48 N 29:10.0951 W 88:01.0672 $1573 \mathrm{ft}$ Ridge

11. Target [1] 9:56:59 N 29:09.7827 W 88:01.0395 $1532 \mathrm{ft}$ coral

12. Target [1] 10:15:32 N 29:09.6482 W 88:01.0060 $1466 \mathrm{ft}$ rock sample

13. Target [1] $10: 59: 19 \mathrm{~N} 29: 09.3803 \mathrm{~W} 88: 01.02391643 \mathrm{ft}$ sampling clams

14. Target [1] 11:15:16 N 29:09.4147 W 88:01.0255 1565 ft Leaving Bottom 


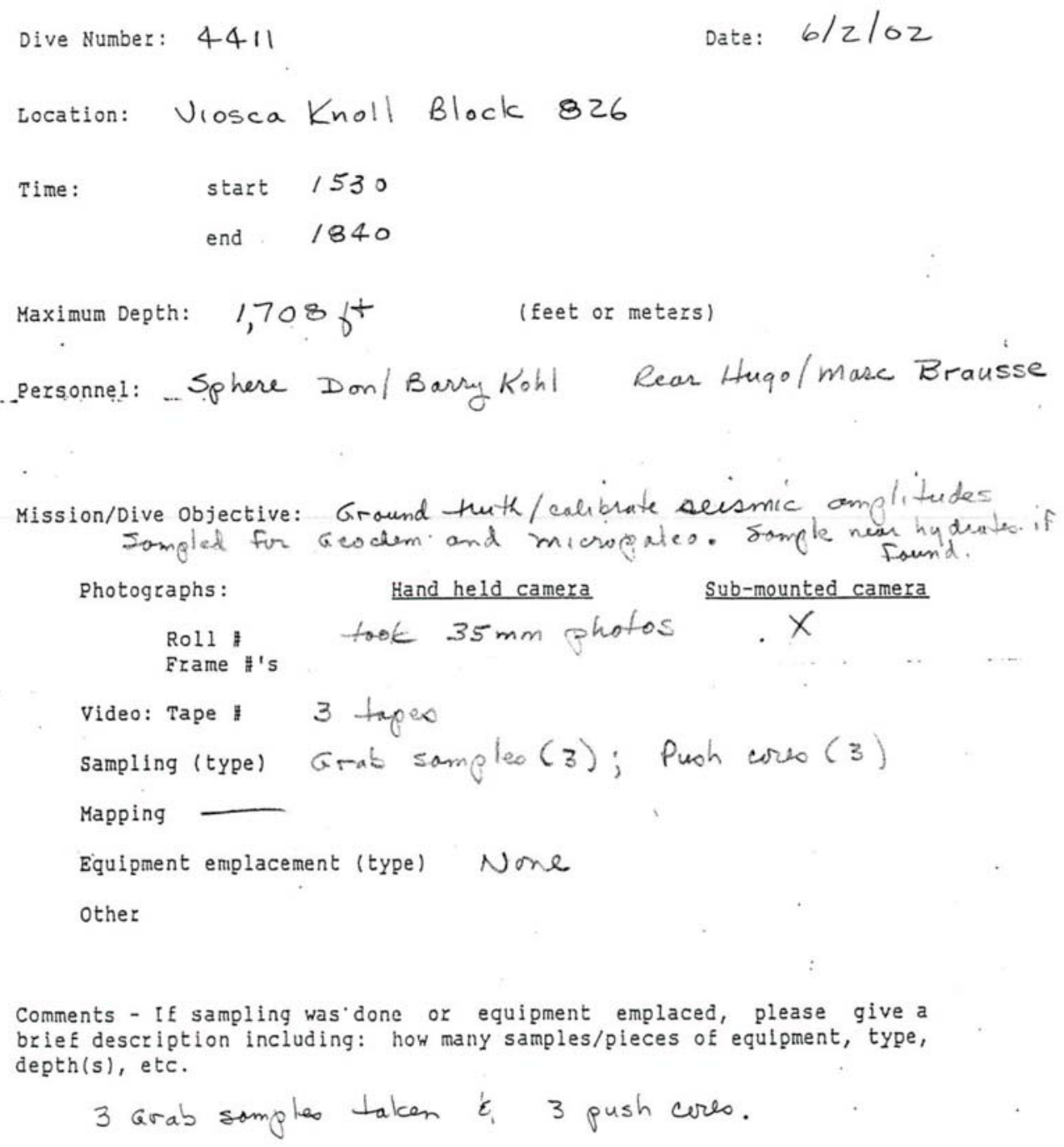


Fix 6 on bottom, mud with small burrows, eepth $1708.4^{\prime}$ 1600 hes took 2 pushare. (at ame luation)

$$
\begin{aligned}
& \left.P C 1-4411 \text { (tube }{ }^{2}+\right)^{2} \text { shot are } \\
& \left.P C 2-4411 \text { (tube }{ }^{2} 2\right) \text { long ore }
\end{aligned}
$$

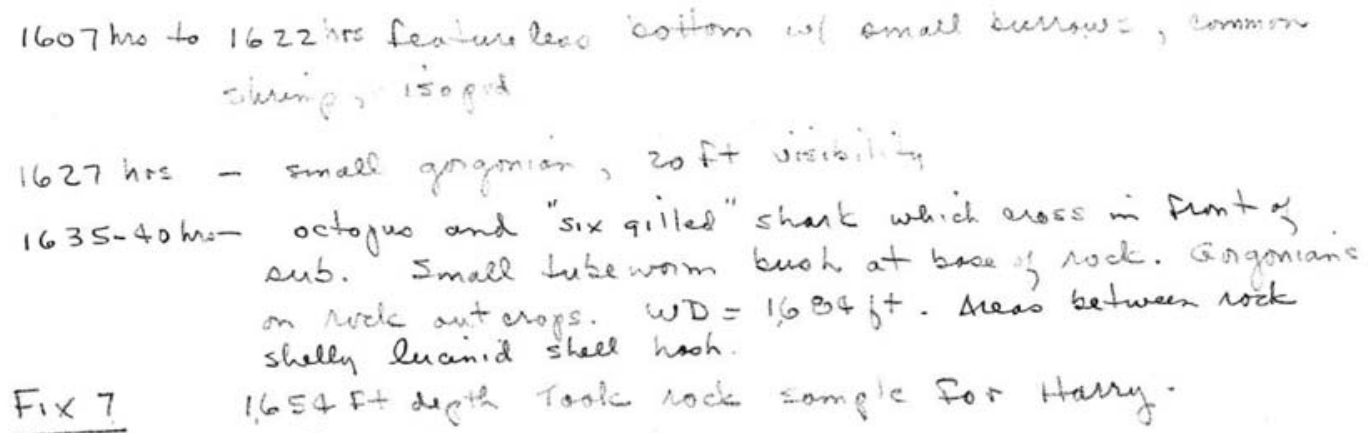

1645 hrs

$$
R_{1}-4411
$$

Fix 8 1,560 Ft Cubmate writh gorgonions and beack earal. $\frac{\text { Fix } 8}{1657 \mathrm{~ms}}$ mix of seatteral carbmate and dead sheel (luanid)

FIX9 1,85 WD Large slabs of carbonate approrimately 1 ift thi 1710 hrs Polygonal in shape with 4-6" cracks betwean slabs Coral thickets (Lophelia), some tube worms goorly developed. Detwied Jides of site showing vertical relatumshy of carbinate, shell and tubeurorms. One tubewarm with blue spinge.

$1715-1730$ hrs Featureless botton with shells civered by mud. ( water depth at 1717 hrs wos 1466 ; 1721 hrs $=1451$ (approx. top of dime)

Fix 10 $\omega D=1480^{\circ}$

1730 hrs Took grat sample to determine compositem of sedement GSI-4411 in bucleet 9 Battery box mpression at $W D=1494.5$ from greverws
transect $(4410)$ 
Fix 11 Pushcre PC3-4411 taken at bottom of furrow 1750 hrs between ridges $(60 \%$ recovery $) . \omega D=1,517^{\prime}$ Bottom featureless with soft mud. w/ cmmon unchins and various soft bedieil muertebrates

Fix 12 Grab somple GS2-4411 (bucket" 10 ). Water septh 1640 ',
1801 hrs sompled in fursow between high areas. 1814 hro - gring up rise

$F 1 \times 13$ $1835 \mathrm{hmo}$ $W D=1,702^{\prime}$ arab somple $G S 3-4411$ (bucket $t 11$ ). Soft
sediment with burrsus. Very fine clay.

FIX 14 Leaving botton. $\omega D=1,702^{\prime}$ 1840 hrs

Generce Comments/Notes:

- no evidence of gos hyderios, gassy sediment, mud volcahys

- tubecsorm bushes, that were seen at some oreas, were ouly poorly developed'

- slopes ove entive area semed to be retatively stap "for submarine slopes

- strong currents

- sheles appear to be deed, no currat fauma-achivity, no veinting $(\rightarrow$ dieting areech) 
$6 / 2 / 02 \quad 3$ ot 5

$V K 826$

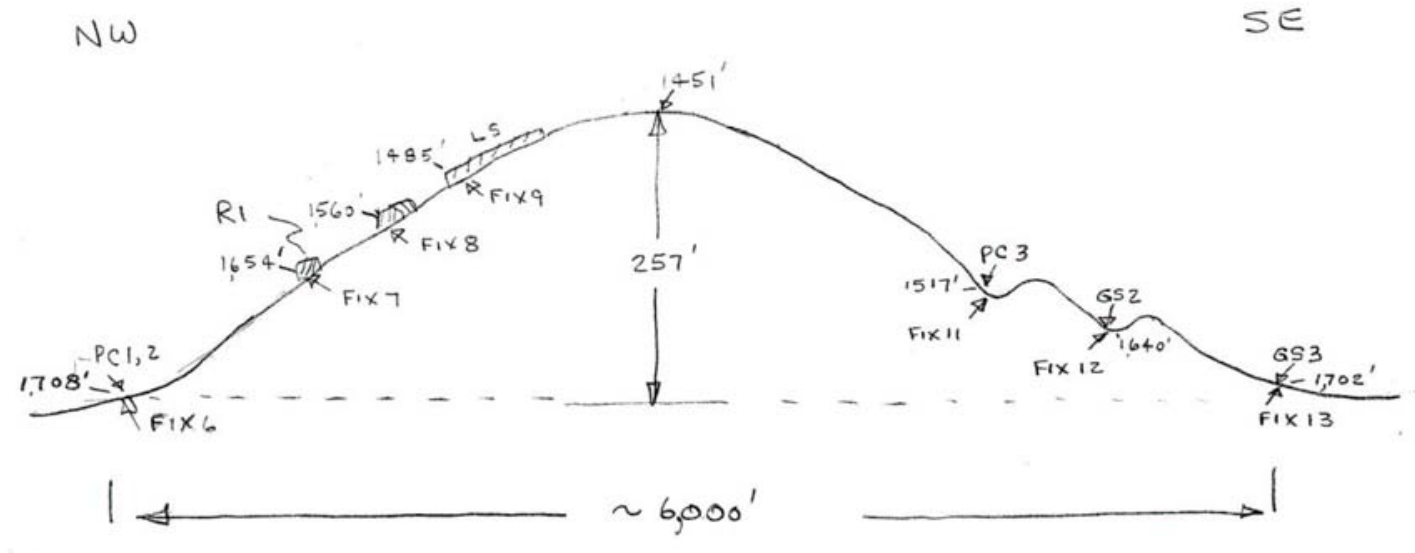

Diagram of traverse across VK 826. 
ORE Interenational. IPS: WayPoint Report

RVSJ 2 \& JSL $1-4411$

1. Ship 13:47:28 N 29:09.7670 W 88:01.9170 oft SOL

2. User 13:50:04 N 29:09.7330 W 88:01.6670 oft WP 1 anomaly 1

3. User 13:51:42 N 29:09.6170 W 88:01.0330 oft WP 2 center

4. User 13:52:21 N 29:09.4670 W 88:00.1330 oft EOL.

5. User 15:32:00 N 29:09.7557 W 88:01.8910 oft Launch

6. Target [1] 16:00:36 N 29:09.7528 W 88:01.9246 1708 ft On Bottom

7. Target [1] 16:46:07 N 29:09.6671 W 88:01.3772 $1654 \mathrm{ft}$ Rock Sample

8. Target [1] 16:57:11 N 29:09.6497 W 88:01.2705 $1560 \mathrm{ft}$ Large Black Carbonate \& Coral

9. Target [1] 17:10:31 N 29:09.6290 W 88:01.1307 1485 ft Taking Video

10. Target [1] 17:30:44 N 29:09.6108 W 88:00.9784 $1480 \mathrm{ft}$ grab sample

11. User 17:50:41 N 29:09.6006 W 88:00.8949 $1517 \mathrm{ft}$ Push Core 3

12. Target [1] 18:01:15 N 29:09.5929 W 88:00.7415 $1640 \mathrm{ft}$ Grab Sample

13. Target [1] 18:35:02 N 29:09.5233 W 88:00.3467 $1702 \mathrm{ft}$ Grab Sample

14. Target [1] 18:40:54 N 29:09.5257 W 88:00.3417 $1702 \mathrm{ft}$ Leaving Bottom 


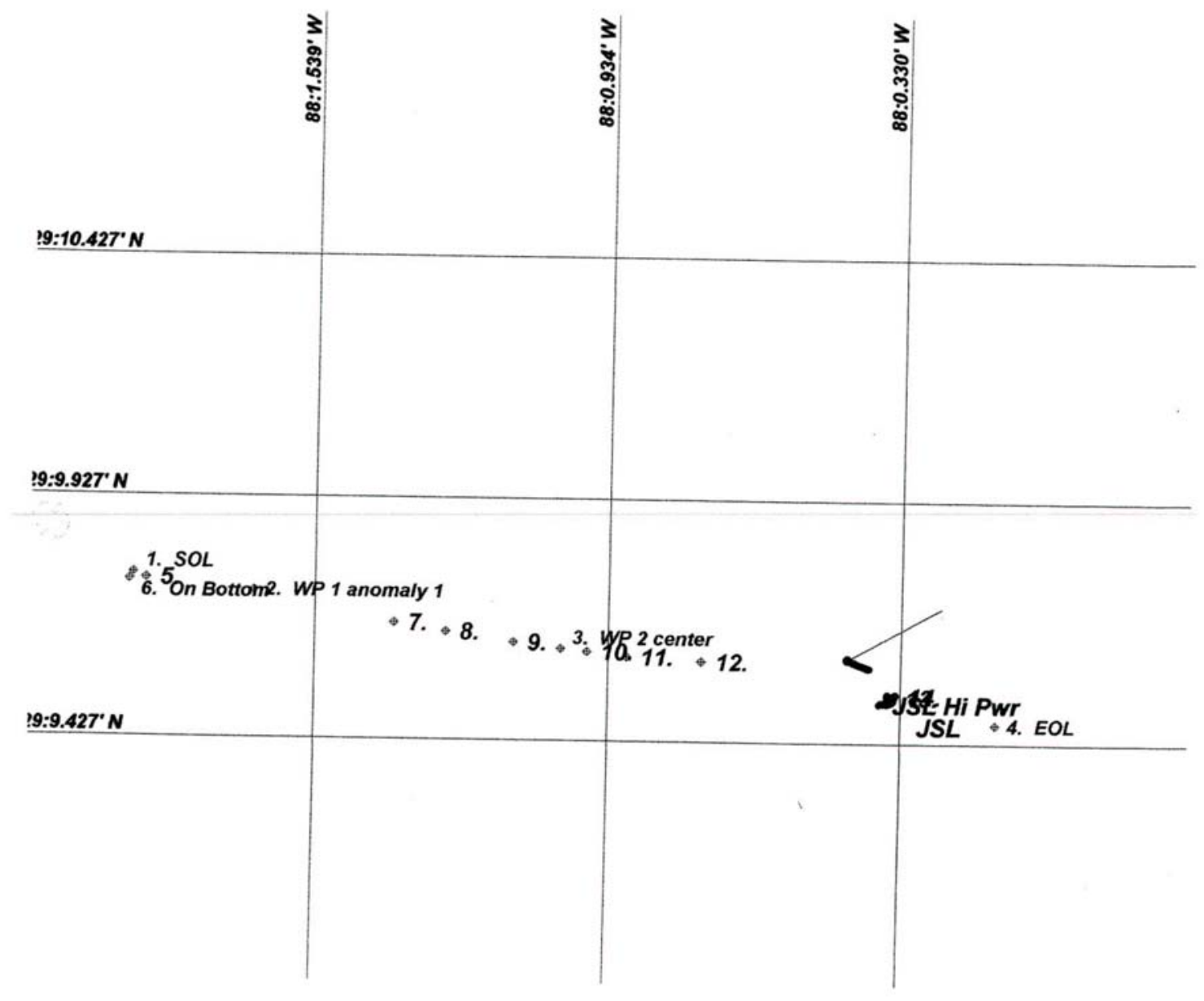

$11 / 4$ mi

173 


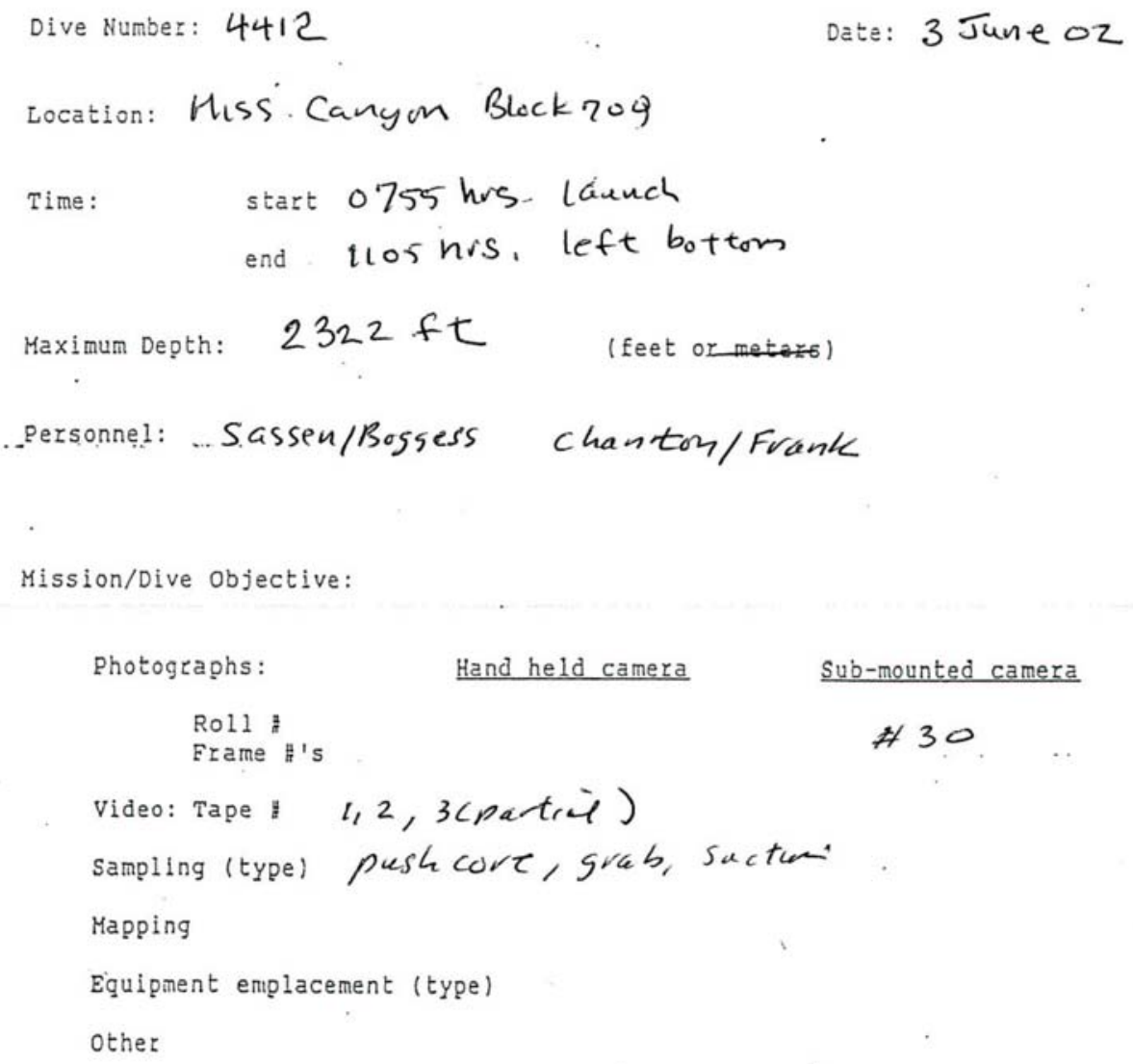




\section{$4412 / M C 709$ RS}

1. acquired water samples on the way down, and on tottom. Time $208306.85^{\circ} \mathrm{C}, 35.15$ salenity.

8. observed brown burrowed mud, shell frogmouts, and low visibility fran suspended sedunest.

3. r 0836 observed caubrute rock, quite 150 ated.

4. 2orts shellhesh, starfish, smad white to gros baccerial mats, scatfered.

8. -0844 started seenis large shallow dopression's (craters) up to $10^{-5}$ of meters across. Mang nolothurcans.

6. Long transit of burvowed brow mud, an clay. Isolated contrins. Fon core for geotech at $2223 \mathrm{ft}$, $7.35^{\circ} \mathrm{C}, 34.90$ salinity. took water sample \#288.

7. 0910. used core\#2 for geotech in typical brown burrowed rud. Gord penetuation anl vecovery, Most of sedment was fine-grached/9roy.

80918 More brown muse, isoloted tiny mats, perhaps diffuse sas seepage

o) 0920 Used grab to sample mussels and selivint M upper outside rimi of shallow corter. Flx.

10. Found black rivulet originating bucket 12 . small orifice upslerelog20) Brine rivulet has been incised into brown mud. Mussels on edges of incised chankal, Used Core ffl for geocheas. Short recovery (n $10 \mathrm{~cm}$ ) $6.87 \% \mathrm{C}, 34.90 \mathrm{Sal} . \mathrm{y} 2226 \mathrm{ft}$ depts. Wos and oil entered watar calumn in minor volumes. Black sedimat. canned. 


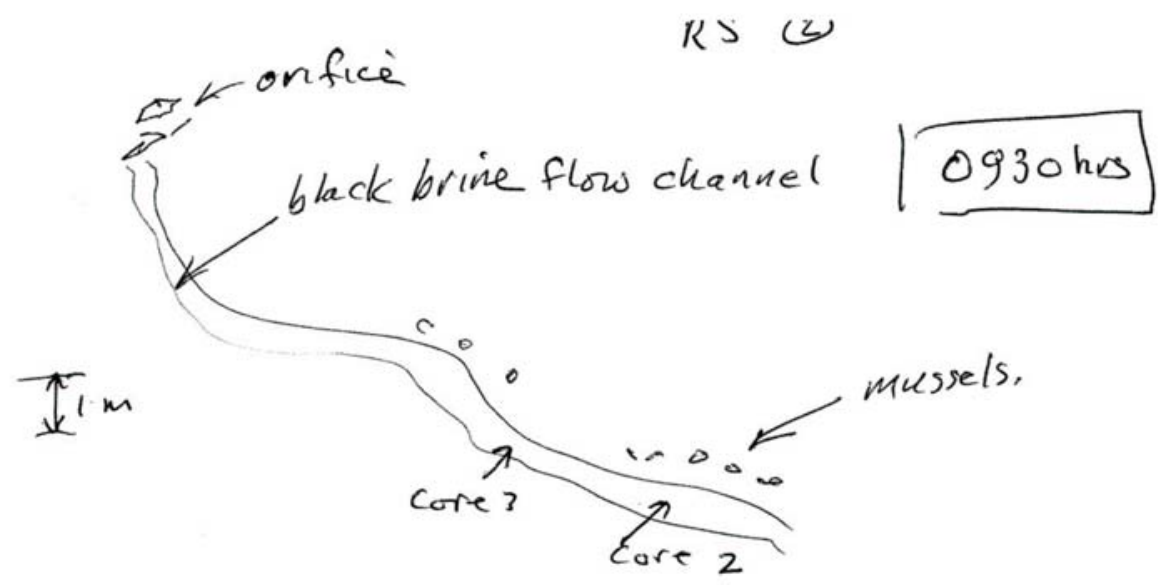

Il. acquired core 3 for chanter. Short penetrates in floor of brine channel. Black sedum is. No bubbles (093 0hrs).

12. Cong stretches a brow surfaced burrowed mud, poor visibility, many shallow rear circular dormant craters

131020 hrs. Observed nice circular hole, $30 \mathrm{~m}$ across at $2280 \mathrm{ft}$. Still murky.

14. 1040. Sow smaller craters, area largely flat. 2286 it $6.5^{\circ} \mathrm{C}$

15: 1045 max depth of $2322 \mathrm{ft}$.

16. 1055 Acquired core \#4 for geotech purposes. Representative of largo aces good penetration and recorry.

$$
2329 \mathrm{ft} 6.3^{\circ} \mathrm{C} 34.88 \mathrm{sal} \text {. }
$$

17. left bottom at 1105 his.

176 
C:IProgram FilesIORE11-4412_trk_trk

RVSJ 2 \& JSL $1-4412$

1. User $8: 46: 09 \mathrm{~N} 28: 13.8670 \mathrm{~W} 89: 42.46702262 \mathrm{ft}$ SOL

2. User 8:48:34 N 28:13.6670 W 89:42.2170 2262 ft WP1

3. Ship 8:49:14 N 28:13.8469 W 89:42.4109 $2254 \mathrm{ft}$ Mud volcano

4. User 8:50:41 N 28:13.8633 W 89:42.4378 $2262 \mathrm{ft}$ Bottom report

5. User 9:01:09 N 28:13.8670 W 89:42.4670 $2262 \mathrm{ft} \mathrm{SOL}$

6. Target [1] 9:02:54 N 28:13.7296 W 89:42.3210 $2223 \mathrm{ft}$ Punch core 1

7. User 9:57:25 N 28:13.5330 W 89:42.0500 2262 ft WP 2

8. User 9:58:03 N 28:13.3170 W 89:41.7830 2262 ft WP 3

9. User 9:58:20 N 28:13.0670 W 89:41.4670 $2262 \mathrm{ft}$ EOL

10. User 9:38:00 N 28:13.6011 W 89:42.1661 $2232 \mathrm{ft}$ mussets and mats

11. User 9:53:00 N 28:13.6050 W 89:42.1433 $2225 \mathrm{ft}$ mussels and clams

12. Target [1] 11:01:11 N 28:13.0739 W 89:41.4570 2329 ft punch core 


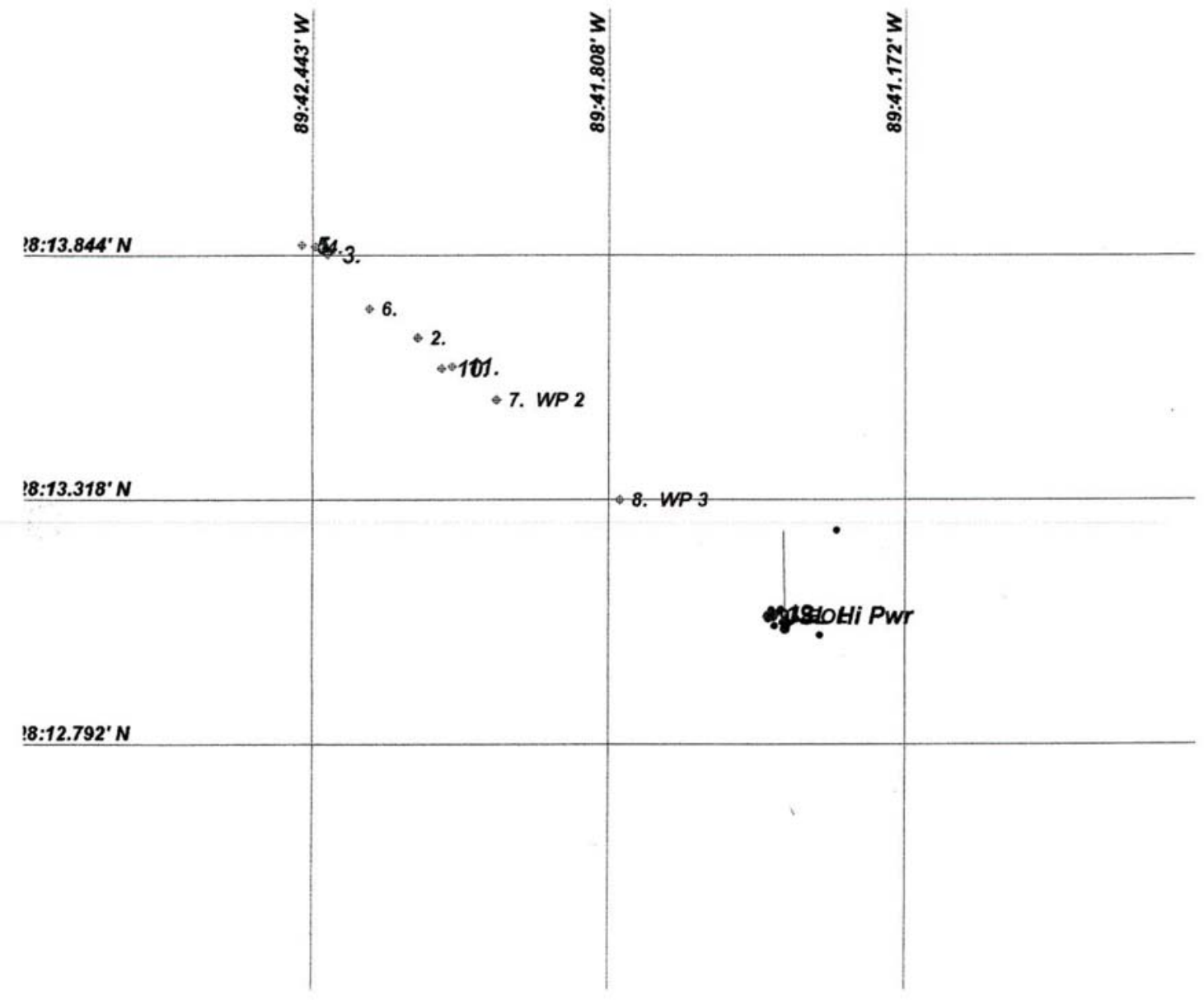




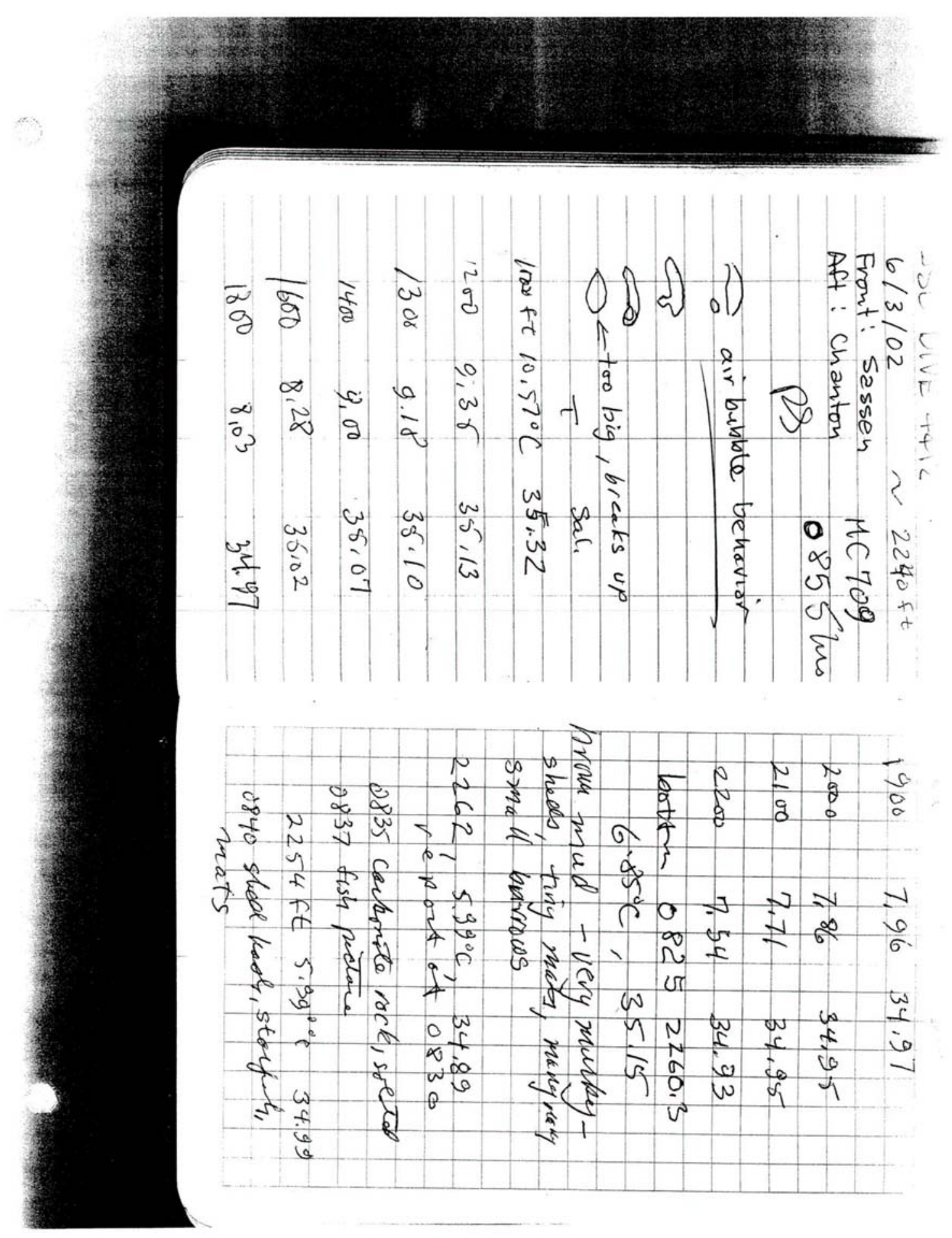




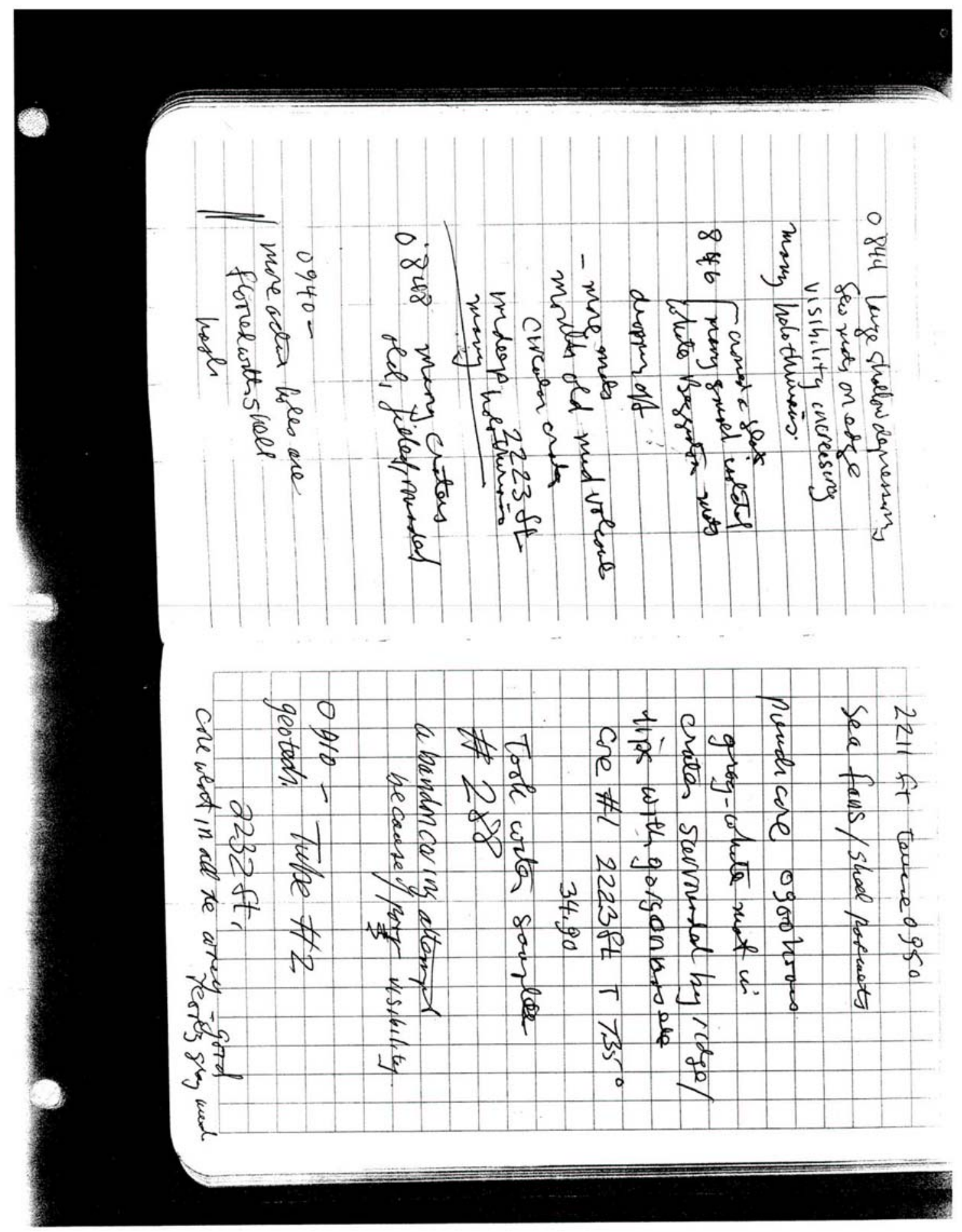




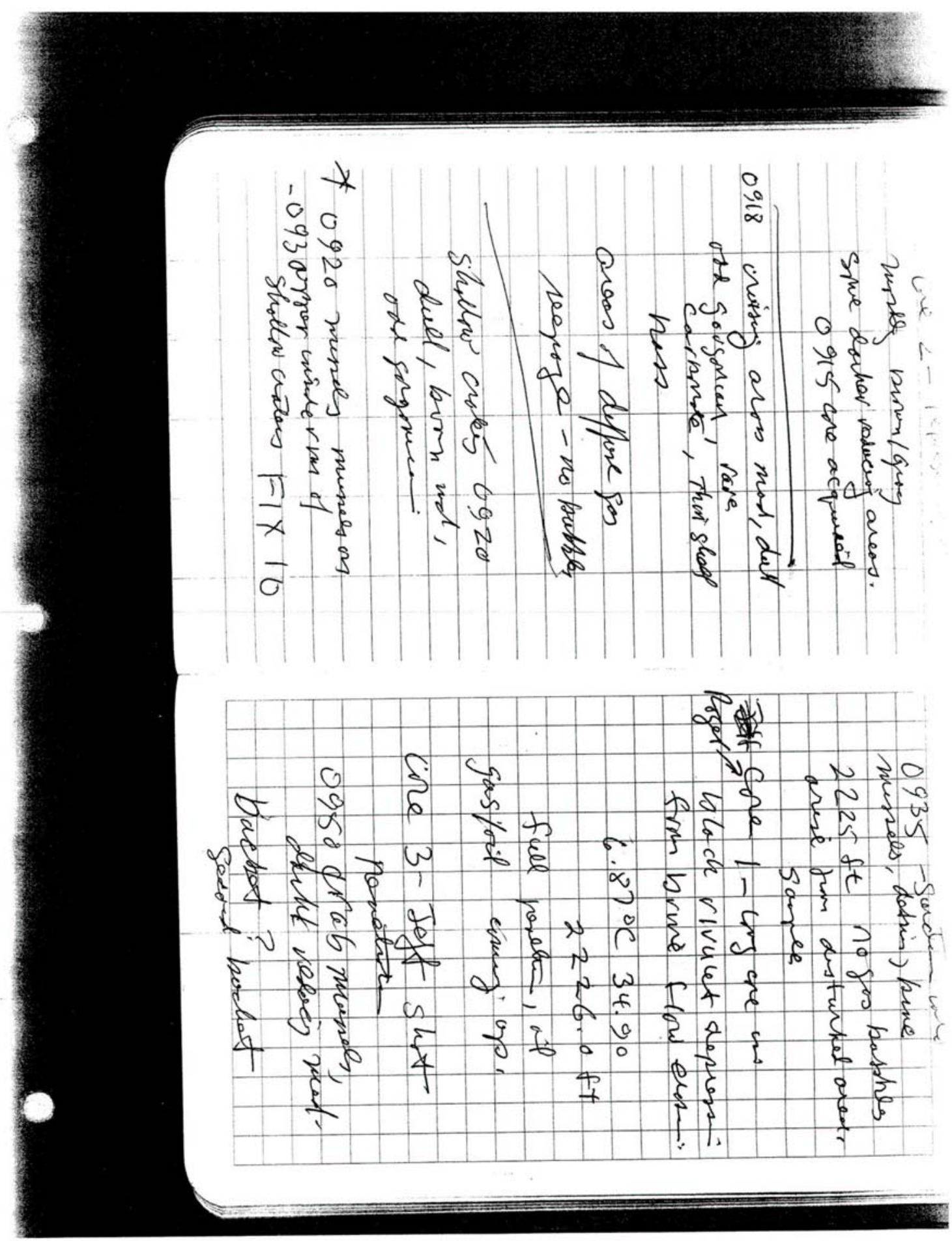




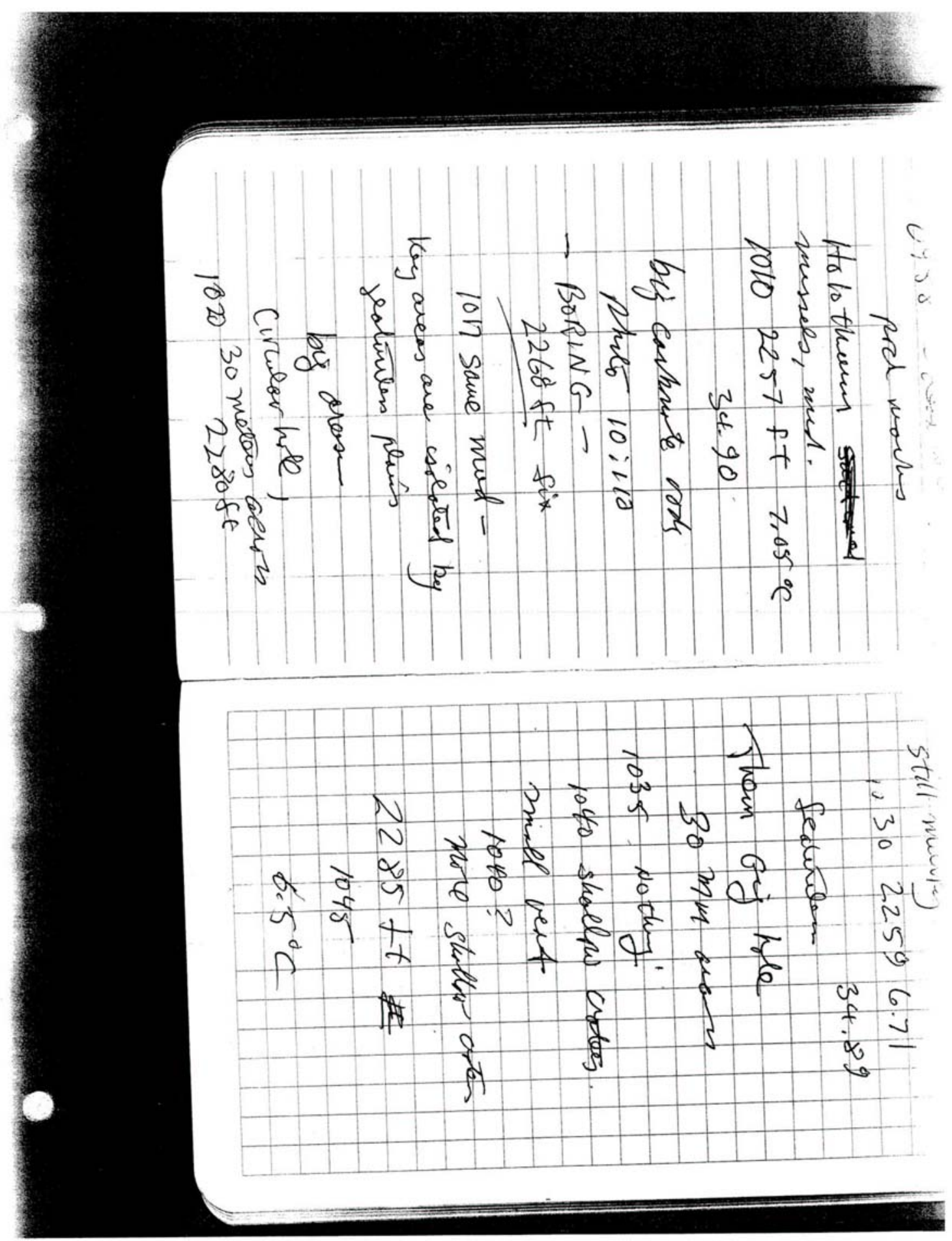




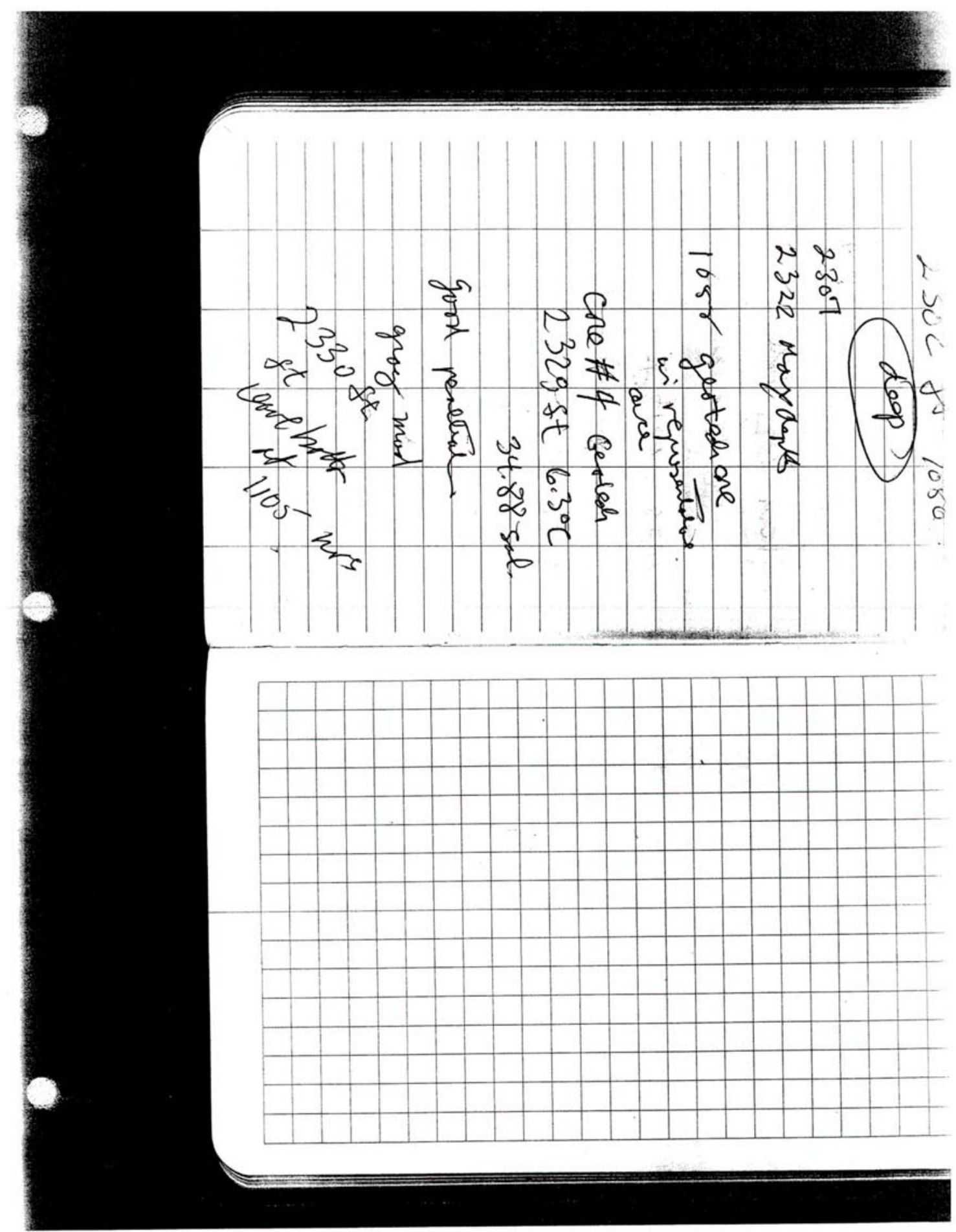




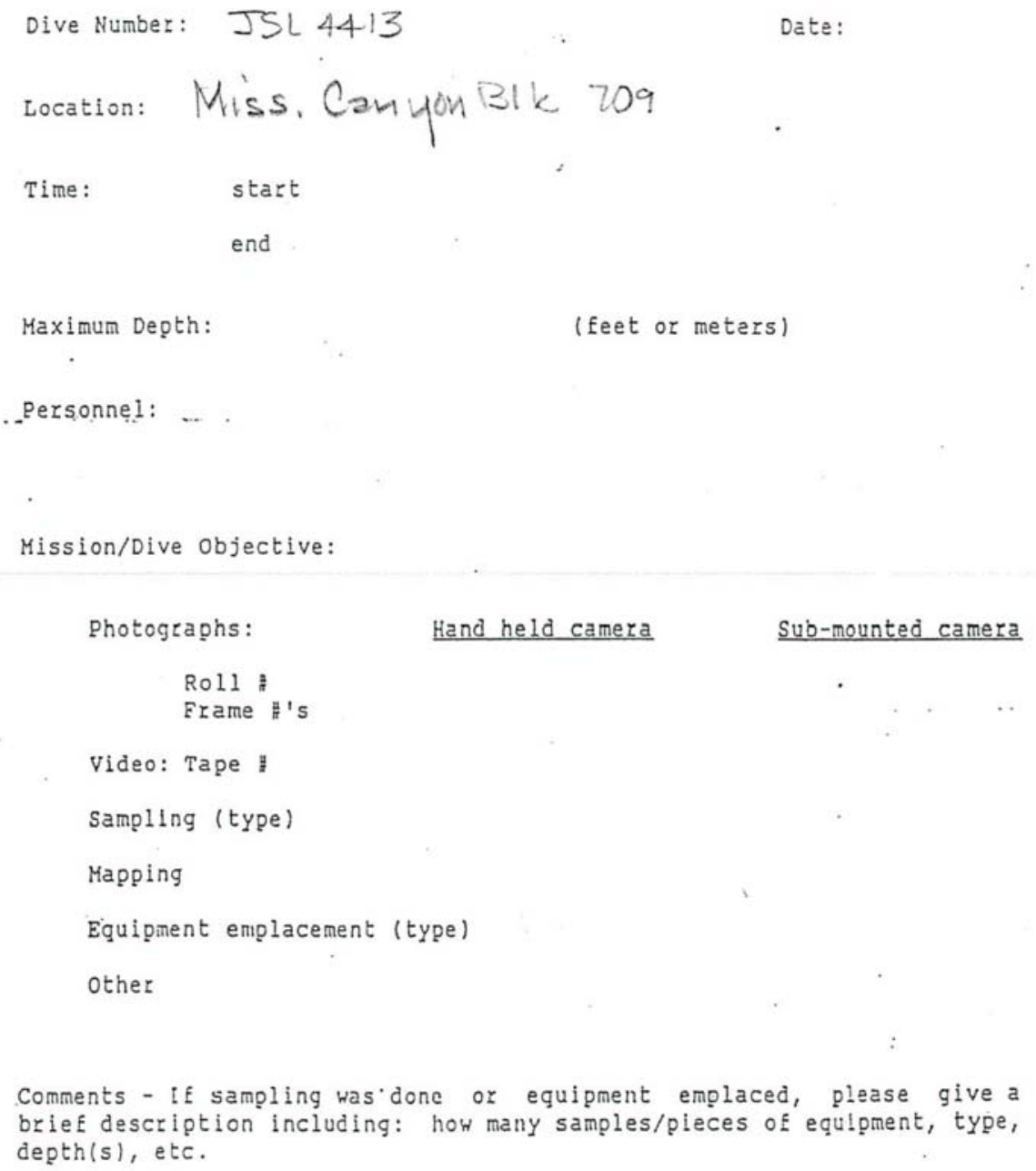


ORE Interenational. IPS: W

ORE Interenational. IPS: W

RVSJ 2 \& JSL 1 dive 4413

1. User $15: 32: 21 \mathrm{~N} 28: 13.6670$ ।

2. Ship $15: 38: 52 \mathrm{~N} 28: 13.6669$ ।

3. Target [1] $16: 16: 07 \mathrm{~N} 28: 13.6$

4. Target [1] 16:30:00 N 28:13.61

5. User 17:41:08 N 28:13.6400 ।

6. Target [1] 17:57:02 N 28:13.6]

7. Target [1] 18:08:50 N 28:13.6.

8. Target [1] 18:24:31 N 28:13.6:

9. Target [1] 18:47:35 N 28:13.6

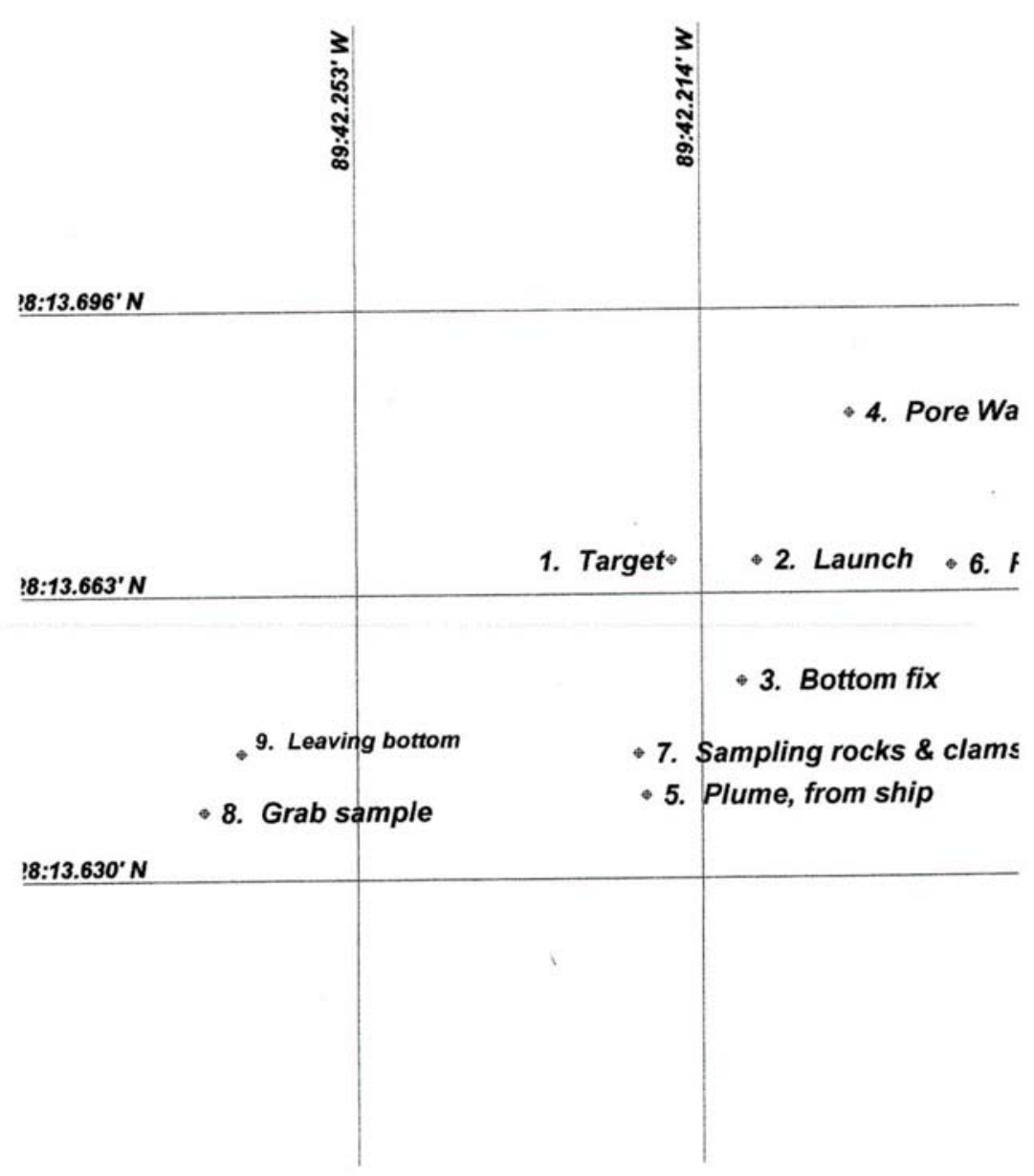




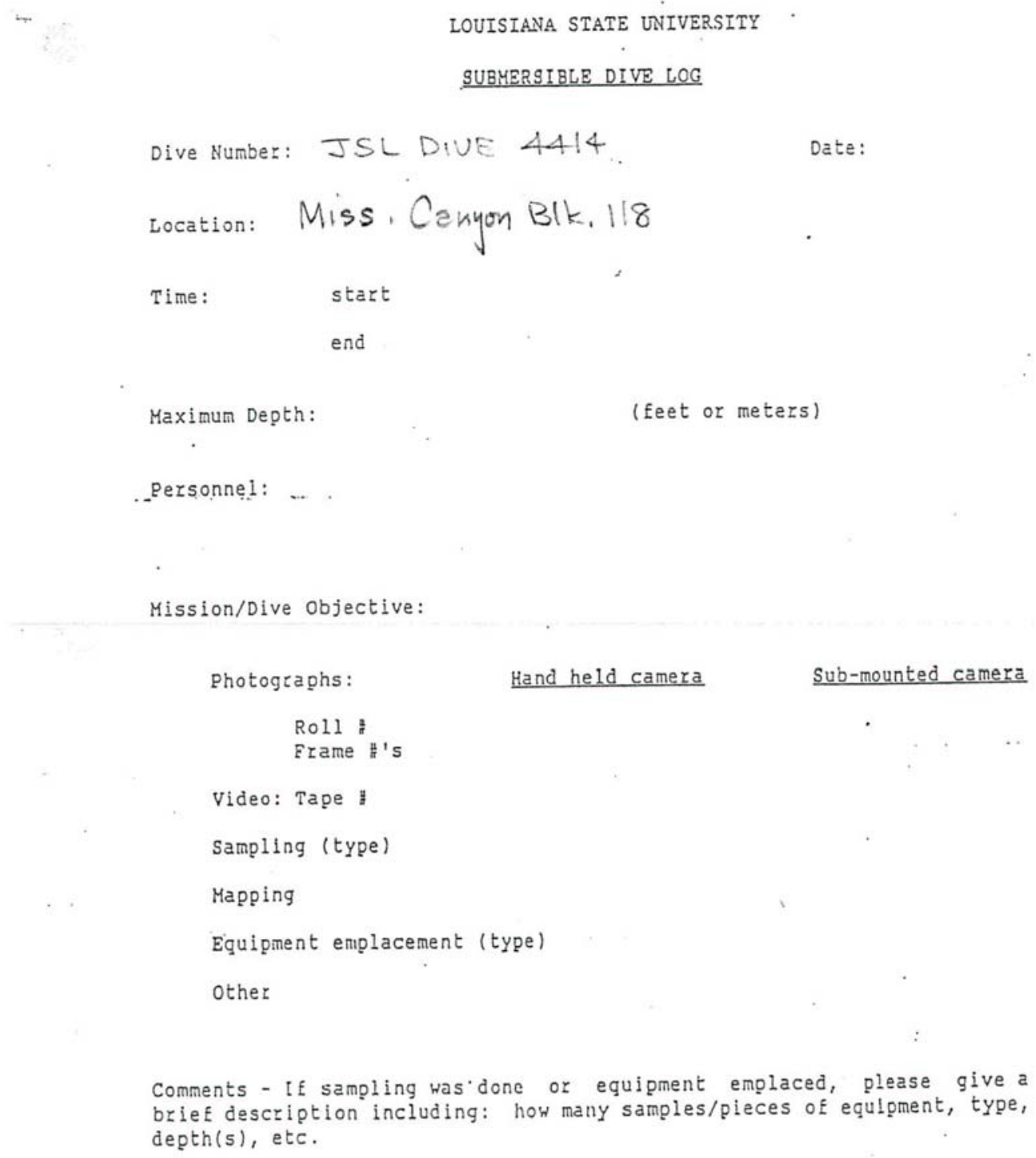




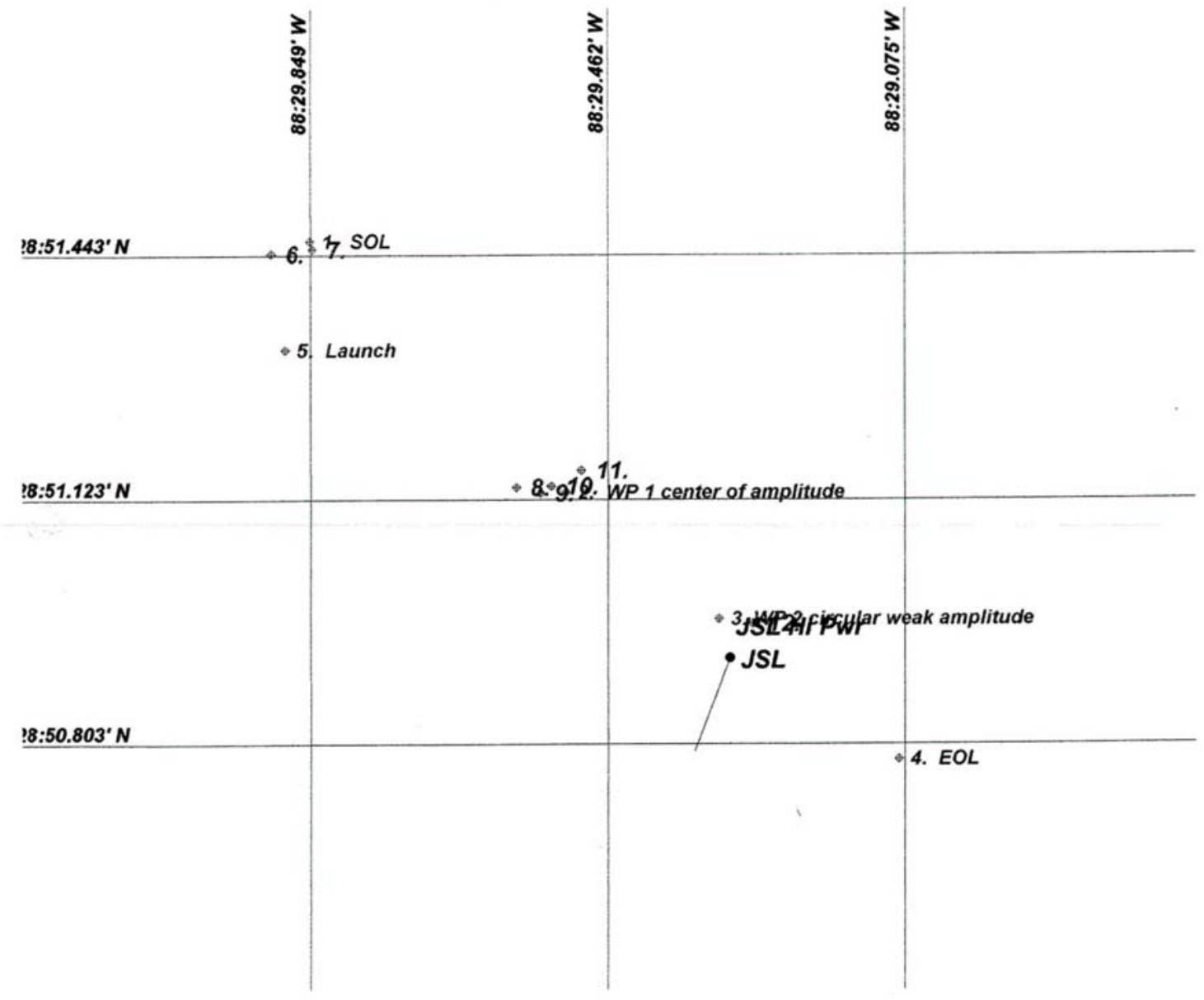




\section{C:IProgram FilesIOREl1-4414}

RVSJ 2 \& JSL. 1-4414

1. User 7:52:55 N 28:51.4624 W 88:29.8500 Oft SOL

2. User 7:54:08 N 28:51.1330 W 88:29.5170 $0 \mathrm{ft}$ WP 1 center of amplitude

3. User 7:55:20 N 28:50.9670 W 88:29.3170 Oft WP 2 circular weak amp.

4. User 7:55:52 N 28:50.7830 W 88:29.0830 Oft EOL

5. Ship 7:58:20 N 28:51.3198 W 88:29.8819 Oft Launch

6. Target [1] 8:39:50 N 28:51.4457 W 88:29.9005 $2869 \mathrm{ft}$ On Bottom

7. Target [1] 8:46:35 N 28:51.4516 W 88:29.8469 $2873 \mathrm{ft}$ Start of Xsect

8. Target [1] 9:16:11 N 28:51.1394 W 88:29.5820 $2898 \mathrm{ft}$ Grab Sample

9. Target[1] 9:30:35 N 28:51.1319 W 88:29.5502 $2912 \mathrm{ft}$ punch core

10. Target [1] 9:38.26 N 28:51.1409 W 88:29.5361 $2915 \mathrm{ft}$ gas seep \& hydrate

11. Target [1] 10:14:03 N 28:51.1615 W 88:29.4967 $2898 \mathrm{ft}$ Push core thru bacterial matt

12. Target [2] 10:49:07 N 28:50.9608 W 88:29.2729 $2970 \mathrm{ft}$ Leaving Bottom 


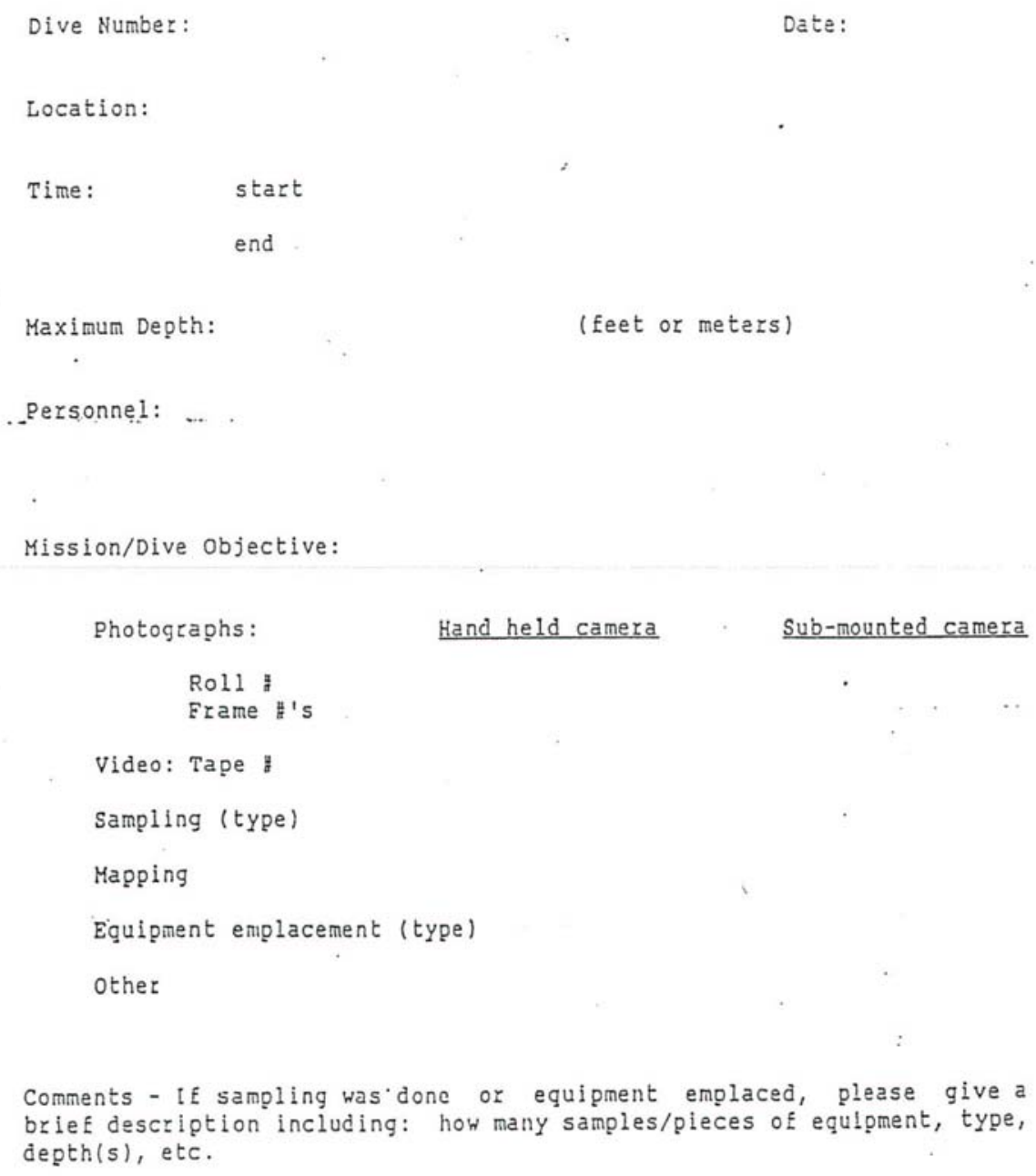




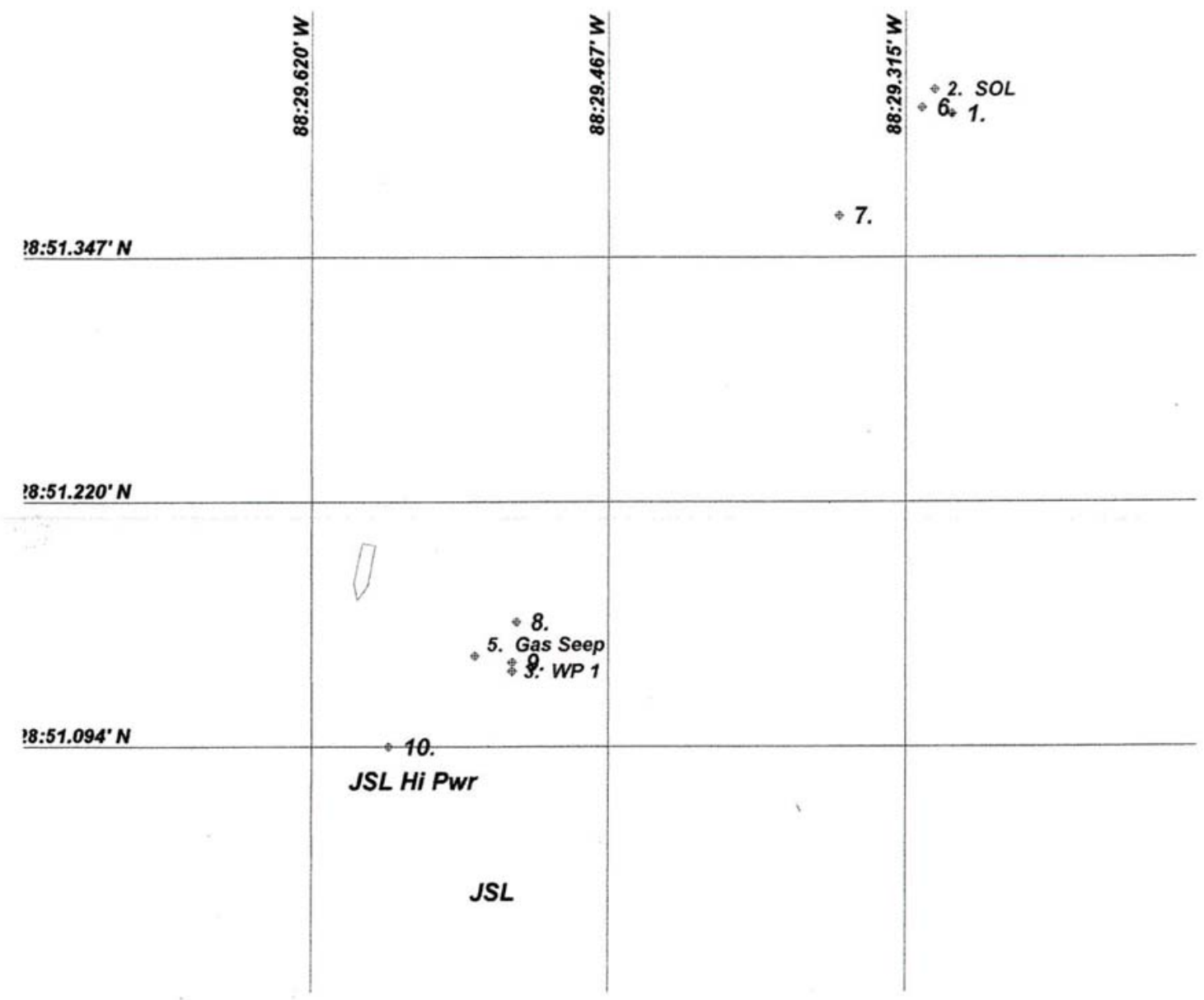


C:IProgram FilesIORE11-4415

RVSJ 2 \& JSL1 -4415

1. Ship 15:29:19 N 28:51.4204 W 88:29.2909 oft Launch

2. User 15:32:35 N 28:51.4330 W 88:29.3000 oft SOL

3. User 15:33:39 N 28:51.1330 W 88:29.5170 oft WP 1

4. User 15:56:20 N 28:50.7670 W 88:29.7830 oft EOL.

5. User 16:04:10 N 28:51.1409 W 88.29.5361 of Gas Seep

6. Target [1] 16:09:07 N 28:51.4235 W 88:29.3064 2907 ft On Bottom

7. Target [1] 16:17:32 N 28:51.3682 W 88:29.3491 2903 ft Grab Sample

8. Target [1] 16:40:52 N 28:51.1585 W 88:29.5148 $2895 \mathrm{ft}$ Crater

9. Target [1] 16:44:51 N 28:51.1377 W 88:29.5170 $2908 \mathrm{ft}$ Alot of gas \& oll

10. Target [1] 18:30:00 N 28:51.0942 W 88:29.5805 2926 ft Push Cores / Leaving Bottom 


\section{Communications Log \\ Johnson Sea Link Submersible 2002 Cruise}

\section{Friday, May 17:}

Load at Gulfport, cold front blew through. Stayed at the dock Sat. and Sun.

Sunday, May 19:

Depart Gulfport and steamed overnight to be on station at VK 826 .

\section{Monday, May 20:}

Too rough to dive. By 2:00pm, decided to return to Gulfport to repair lower deck A/C unit and ship's freezer. Departed Gulfport at 11:30 pm for VK 826.

\section{Tuesday, May 21:}

Hove to all day on VK 826. Still too rough to dive.

\section{Wednesday, May 22:}

Hove to all day. Decided to abort the site and head west in search of better weather. Ran west overnight.

\section{Thursday, May 23:}

Hove to at MC 885 all day.

\section{Friday, May 24:}

\section{885, Dive 4392}

Crew: Front, Craig Caddigan, Mike Smith Rear, Frank Lombardo, Susan Childs

Purpose: Survey traverse and find and recover thermistor. 
Attempted to dive, but had to abort due to weather. Hove to all day, and decided to run west to GC 185 (Bush Hill). Arrived at Bush Hill, attempted to trip acoustic release on current meter array. Did not release.

\section{Saturday, May 25:}

\section{GC 185, Dive 4393}

Crew: Front, Craig Caddigan, Harry Roberts

Rear, Frank Lombardo, Eric Guilbeau

Purpose: Free current meter, collect bubbleometers, deploy rabbit chow.

7:58 Boom out

8:01 Launch

8:02 Permission to dive, problem with a contact in the sub. Depth $250 \mathrm{ft}$.

8:08 Resurface, recovered sub. Water in a breaker tripped it.

8:13 Recovered sub and began repairs.

\section{GC 185, Dive 4394}

Same crew.

Same purpose.

9:23 Boom out

9:25 Launch

9:26 Permission to dive. Launch at 27-46.8031N 91-30.3743W

9:30 Returning to surface. An alarm was getting louder.

9:36 Surface, repaired sub.

\section{GC 185, Dive 4394}

Same crew, same purpose.

15:56 Launch, permission to dive $27-46.8652 \mathrm{~N} 91-30.4087 \mathrm{~W}$

16:09 Depth $1000 \mathrm{ft}$.

16:19 Near bottom, mooring $20 \mathrm{ft}$. in front of sub.

16:22 On bottom. Depth $1793 \mathrm{ft}$., $0.1 \mathrm{kt}$. of current from W, Temp. 7.40

Released mooring. 
GC 185, Dive 4394 (cont.)

16:24 Mooring on the way up.

16:27 Deploy a bucket of rabbit chow at 27-46.0931N 91-30.4081W

16:30 Heading due West

16:32 Current meter array on the surface.

16:34 Deployed bucket \#2 on bearing of 345 (no fix) approx. 27-46.9437N 91-30.4550W.

16:37 Within $50 \mathrm{ft}$. of the numbers, searching for instruments.

16:38 Instruments in sight

16:40 Will deploy bucket \#1 just North of where Harry's gear was, Just down from Junk yard, Depth $1783 \mathrm{ft}$.

16:49 At the first funnel, trying to get the can off the side.

17:00 Can is in the basket, but can't close the lid, will try to fit the funnel on top.

17:10 Funnel onboard, moving away from the time lapse camera.

17:16 Don't know how the funnel will ride. Request permission to leave bottom. Must go back and look at something.

17:20 By Ian McDonald's time lapse camera, looking at thermistors. Will take water samples.

17:23 Depth $1772 \mathrm{ft}$., Permission to leave bottom. 27-46.9763N 91$30.4724 \mathrm{~W}$.

17:47 Done with water samples at $2000 \mathrm{ft}$. Permission to surface.

17:51 On surface.

\section{GC 185, Dive 4395}

$\begin{array}{ll}\text { Crew: } & \text { Front, Craig Caddigan, Harry Roberts } \\ & \text { Rear, Frank Lombardo, Duofu Chen }\end{array}$

Purpose: Retrieve gear and conduct acoustics experiments (night dive)

20:38 Launch, permission to dive, 27-46.9850N 91-30.4715W

20:50 Showing bottom at $1800 \mathrm{ft}$. on ship.

20:53 Range $100 \mathrm{ft}$. bearing 174 degrees to landing site.

20:55 Ship showing bottom at $1775 \mathrm{ft}$.

20:57 Target at $100 \mathrm{ft}$. at 165 degrees.

21:00 Bottom in sight 
GC 185, Dive 4395 (cont.)

21:01 On bottom, $1780 \mathrm{ft}$., Temp. 7.33, visibility 45 to $50 \mathrm{ft} ., 0.1 \mathrm{kt}$. Of current from due North. Next to Ian's cameras, getting video at 27$46.9530 \mathrm{~N}$ 91-30.4178W.

$21: 19$ Got the other funnel on board, will go back and do some video.

21:31 Depth $1763 \mathrm{ft}$., took video, request permission to leave bottom $27-46.9529 \mathrm{~N} 91-30.4865 \mathrm{~W}$

21:55 On surface.

\section{Sunday, May 26}

GC 185, Dive 4396

Crew: Front, Don Liberatore, Harry Roberts Rear, Hugo Marrero, Rex Poling

Purpose: Locate and retrieve tripod and thermistor string, Target mud vent, and shoot stills of the bottom.

07:03 Launch and permission to dive 27-46.0511N 91-30.3480W

07:04 Ship showing bottom at $1858 \mathrm{ft}$.

07:15 Range to target $459 \mathrm{ft}$. at 339 degrees.

07:16 Range $600 \mathrm{ft}$. at 340 degrees.

07:17 Ship showing bottom at $1852 \mathrm{ft}$.

07:19 Ship showing bottom at $1844 \mathrm{ft}$., sub at $1200 \mathrm{ft}$.

07:22 Range $350 \mathrm{ft}$. at 325 degrees, sub at $1400 \mathrm{ft}$.

07:29 Sub depth $1800 \mathrm{ft}$.

07:30 Range $400 \mathrm{ft}$., bearing 345 degrees.

07:31 Bottom in sight.

07:34 Range $150 \mathrm{ft}$., Bearing 350 degrees.

07:36 Range $100 \mathrm{ft}$., Bearing 290 degrees.

07:37 Sighted bacterial mat, probably coming into mud vent site.

07:41 Range $90 \mathrm{ft}$., Bearing 120 degrees. Unable to locate site.

07:46 Range $150 \mathrm{ft}$., Bearing 350 degrees, Unable to locate site.

07:49 Currently searching for site, bottom (from ship) $1862 \mathrm{ft}$.

07:54 Range $250 \mathrm{ft}$., Bearing 040 degrees, searching for site.

07:55 Sub underway at 040 degrees.

07:59 At the mud vent. 
GC 185, Dive 4396 (cont.)

08:02 Found something on mud vent, probably old thermistor. Harry confirms this is the thermistor.

08:03 Sub depth $1876 \mathrm{ft}$.

08:06 See the ADCP, do not see the auger with the thermistor string.

08:09 Sitting at the tripod, Depth $1878 \mathrm{ft}$., Temp. 7.14, Visibility $25 \mathrm{ft}$., Current $0.1 \mathrm{kt}$. at 090 degrees.

08:11 Will move around and look for the auger and come back and retrieve the tripod.

08:24 Unable to locate the auger and thermistor, heading back to retrieve the doppler current meter from tripod.

08:31 Unable to lift instruments, will back off and try again. T-handle at top is broken off (Harry doesn't remember that problem during deployment. At 27-46.1579N 91-30.4132W.

08:40 Unable to dislodge doppler instrument, Regrouping and waiting for sediment to clear.

08:45 Trying to tip the tripod and get the doppler exposed for easier access.

08:58 Tried pulling on it and still unable to dislodge it, waiting for sediment to settle.

09:18 Grabbed the doppler and blew the ballast tanks, failed to dislodge. Standing by.

09:27 Leaving the mud vent site and heading for Bush Hill site.

09:29 Underway due east, depth $1880 \mathrm{ft}$., taking water samples at 27$46.1970 \mathrm{~N}$ 91-30.3672W

09:31 Changed course to north, following mud flow to investigate.

09:33 New vent observed at $1873 \mathrm{ft}$., $27-46.1975 \mathrm{~N}$ 91-30.3589W

09:35 At top of the vent, observing bubble stream. Top at $1875 \mathrm{ft}$.

09:43 Permission to leave bottom at depth $1875 \mathrm{ft}$.

09:44 Leaving bottom at $27-46.2018 \mathrm{~N} 91-30.3587 \mathrm{~W}$

10:08 On surface.

\section{GC 238, Dive 4397}

Crew: Front, Don Liberatore, Mike Smith Rear, Hugo Marrero, Charles Billups

Purpose: Survey Traverse 1 and record observations. If time permits, collect two push cores and 1 carbonate sample. 
GC 238, Dive 4397 (cont.)

13:19 Launch, permission to dive at 27-44.3886N 91-03.0461W. Punch cores are $\mathrm{OK}$.

13:21 Ship showing bottom at $2276 \mathrm{ft}$.

13:24 Depth $295 \mathrm{ft}$., going through murky layer of sediment laden water.

13:36 Range $700 \mathrm{ft}$. Bearing 280 degrees.

13:42 Ship showing bottom at $2325 \mathrm{ft}$.

13:45 Sub at $2000 \mathrm{ft}$. and descending

13:49 Sub at $2200 \mathrm{ft}$., ship shows bottom at $2335 \mathrm{ft}$.

13:52 Bottom in sight.

13:53 On bottom, depth $2343 \mathrm{ft}$., Temp. 6.23, Visibility 35-40 ft., current

$0.1 \mathrm{kt}$. at 300 degrees, $27-44.4453 \mathrm{~N} 91-03.0470 \mathrm{~W}$

13:56 WP1 range $950 \mathrm{ft}$., bearing 140 degrees.

13:58 Underway at 140 degrees.

14:00 Coming up on slight slope at 4 to 5 degrees, on way to WP1.

14:06 Range $250 \mathrm{ft}$., bearing 110 degrees to WP1. Depth $2294 \mathrm{ft}$., Lots of bacterial mats, mounds, shells (mussels and clams). Most shells observed are dead, possibly active area at one time. Seeing chemo biota.

14:08 27-44.3441N 91-03.0025W, traveling to WP1.

14:14 Sending sub to WP2, Range $400 \mathrm{ft}$. bearing 270 degrees.

14:15 More shells, big depressions, smooth bottom, no bacterial mats

present.
14:16 Dan instructs sub to stand by, checking on incorrect bearing of 270 degrees.

$14: 17 \mathrm{WP} 2$ is at $450 \mathrm{ft}$., bearing of 150 degrees.

14:18 Sub at $2276 \mathrm{ft}$., ship showing $2273 \mathrm{ft}$.

14:21 New heading of 175 degrees, depth $2274 \mathrm{ft}$. Big craters with bacterial mats at the base, approximately $3 \mathrm{~m}$ across.

14:23 Changing bottom, more bacterial mats, anemones, mussels.

14:24 Depth $2274 \mathrm{ft}$., anemones only on slope sides.

$14: 27$ New heading of 150 degrees, depth at $2278 \mathrm{ft}$.

14:30 On way to WP3, Range $750 \mathrm{ft}$., bearing 140 degrees.

14:32 Change in bottom, hundreds of starfish, small shallow depressions, occasional hard bottom. Depth $2283 \mathrm{ft}$. 21-44.1256N 91-02.8683W

14:35 Depth $2282 \mathrm{ft}$., "Starfish City".

14:36 Back in larger depression, no starfish.

14:37 Investigating location of bacterial mat for punch core sample. 
GC 238, Dive 4397 (cont.)

14:44 Due east to WP3, Depth $2290 \mathrm{ft}$., taking punch core in bacterial mat at $27-44.0516 \mathrm{~N} 91-02.8310 \mathrm{~W}$

14:49 Soft sediment, push core went in and came out half full, underway due east.

14:50 Range $200 \mathrm{ft}$. to WP3.

14:51 090 degrees, underway due east.

14:52 Crossing over small furrow 1 to $1.5 \mathrm{ft}$. across running N-S.

14:54 Course change, bearing 140 degrees, range $1300 \mathrm{ft}$.

14:56 Depth $2310 \mathrm{ft}$., underway at 140 degrees to EOL.

14:57 Headed downslope

15:02 Depth $2390 \mathrm{ft}$. , changing video tapes, 27-43.9884N 91-02.6707W

15:07 Course change to due S.

15:11 At EOL. Depth $2415 \mathrm{ft}$., bottom is smooth and homogeneous, no mats.

15:16 Shooting video of starfish.

15:17 Depth $2417 \mathrm{ft}$., request permission to leave bottom, 27-

$43.8256 \mathrm{~N} 91-02.6217 \mathrm{~W}$.

15:40 On surface.

\section{GC 238, Dive 4398}

Crew: Front, Don Liberatore, Susan Child

Rear, Hugo Marrero, Bob Hardage

Purpose: Survey and photodocument Traverse 2

19:03 Launch, permission to dive, 27-43.9001N 91-02.8074W

19:08 Going through a layer of murky water at $300 \mathrm{ft}$., looks like sediment in the water column.

19:16 Depth $1000 \mathrm{ft}$.

19:21 Target range $300 \mathrm{ft}$., bearing 220 degrees, getting underway.

19:26 Drop straight down, depth $1770 \mathrm{ft}$.

19:32 Depth $2200 \mathrm{ft}$.

19:37 On bottom, depth $2389 \mathrm{ft} ., 27-43.9072 \mathrm{~N} 91-02.8360 \mathrm{~W}$

19:48 Going upslope

19:50 Course change to 355 degrees, range $350 \mathrm{ft}$.

19:51 Small craters with bacterial mats, depth $2310 \mathrm{ft}$. 
GC 238, Dive 4398 (cont.)

19:57 Bigger craters with some rock, will take sample, also clams and mussels, took push core and grab sample $27-44.0266 \mathrm{~N} 91-$ $02.8252 \mathrm{~W}$

20:11 Completed push core, some hard substrate, will look around for several minutes.

20:15 Came across battery box tracks, get underway at 005 degrees, range $1800 \mathrm{ft}$., depth $2280 \mathrm{ft}$.

20:17 Huge chunk of coral about $4 \mathrm{ft}$. across, will sample 27-44.0772N 91$02.8232 \mathrm{~W}$

20:26 Back underway, small piece of coral in the basket, course 005 degrees, range $1700 \mathrm{ft}$.

$20: 28$ back in big field of starfish

20:31 Two more big mounds of coral, all dead.

$20: 38$ Out of area of depressions and mats, now even seafloor $27-44.2089 \mathrm{~N}$ 91-02.8226W

20:43 Depth $2295 \mathrm{ft}$. Changing video tapes, 27-44.2635N 91-02.7774W

20:44 Feature in the bottom like a tow fish or cable drug across, runs N-S.

20:46 Change course to 330 degrees.

20:56 Waypoint 2, change course to 230 degrees, range $1060 \mathrm{ft}$., overshot WP2, course change 27-44.4021N 91-02.8822W. Depth $2372 \mathrm{ft}$.

21:06 Change course to 230 degrees, $27-44.3691 \mathrm{~N} 91-03.0352 \mathrm{~W}$

21:10 Course change to due S., crossing over battery box tracks again.

21:11 Big depressions with bacterial mats, depth $2294 \mathrm{ft}$., $27-44.3671 \mathrm{~N} 91-$ 03.0629W

21:15 Back on line, course change to 260 degrees, range $1600 \mathrm{ft}$. 27$44.3039 \mathrm{~N} 91-03.0455 \mathrm{~W}$

21:24 Depth $2297 \mathrm{ft}$., shallow depressions with bacterial mats. 27$44.2969 \mathrm{~N} 91-03.1792 \mathrm{~W}$. course change to 240 degrees, range 800 ft., fix $27-44.2701 \mathrm{~N} 91-03.3665 \mathrm{~W}$, depth $2280 \mathrm{ft}$., taking water sample, permission to leave bottom.

$21: 43$ depth $2288 \mathrm{ft}$.

22:12 On surface.

Monday, May 27

GC 257, Dive 4399

Crew: Front, Dan Boggess, Bill Shedd 
GC 257, Dive 4399 (cont.)

Rear, Frank Lombardo, Melissa Lobegeier

Purpose: Survey and photodocument $8200 \mathrm{ft}$. Traverse, depressional seafloor.

07:58 Launch and permission to dive. Ship shows depth of $2887 \mathrm{ft.} 27-$ $42.403 \mathrm{~N}$ 90-07.223W

08:25 Depth $2000 \mathrm{ft}$., ship shows bottom at $2914 \mathrm{ft}$.

08:25 Underway at 271 degrees, range $700 \mathrm{ft}$.

08:32 On the numbers, drop straight down, bottom shows at $2880 \mathrm{ft}$.

08:39 Bottom in sight.

08:42 On bottom, depth $2894 \mathrm{ft}$., Temp 5.42, visibility 35-40 ft., current less than 0.1 kt. from 010 degrees. $27-42.443 \mathrm{~N} 90-07.272 \mathrm{~W}$, bottom soft muddy, small deep slope craters, but mostly soft mud.

08:45 Bearing 050 degrees, range $1400 \mathrm{ft}$.

$08: 48$ Fairly sharp ridge line in sight.

08:48 Fix 27-42.473N 90-07.166W, taking video of ridge and associated bottom.

08:51 Flat spot at depth of $2895 \mathrm{ft}$.

08:53 Depth $2910 \mathrm{ft}$. , steep drop off, terracing down at 60 degree angle. Heading of 050 degrees, ridge lines running perpendicular, 5 to $8 \mathrm{~m}$ wide.

08:55 Leveled off, same soft sediment with some shell hash, depth $2933 \mathrm{ft}$.

08:56 Mussel shell clumps along heading.

08:58 Depth $2928 \mathrm{ft}$., gentle slope.

08:59 Depth $2926 \mathrm{ft}$., small bacterial mat.

09:00 More small bacterial mats.

09:02 Visibility 30-35 ft., turbidit increasing and decreasing, fog-like, possible proximity to mud vent, within $140 \mathrm{ft}$. of WP1.

09:03 Depth $2915 \mathrm{ft}$., very gentle slope, very smooth bottom, no big mounds or large depressions.

09:05 Depth $2911 \mathrm{ft}$., taking punch core sample within $40 \mathrm{ft}$. of WP1, trying to find vent, following flow back to vent, found a big lake, got fathometer trace showing plume in water column, $27-42.609 \mathrm{~N} 90-$ $06.997 \mathrm{~W}$.

09:12 Depth $2910 \mathrm{ft}$., gas coming up, also sediment. Visibility low and slight increase in currents. Bubbles are about a $\mathrm{mm}$ in diameter. 
GC 257, Dive 4399 (cont.)

09:21 Could not tell where flow is originating, no visibility and not enough current.

09:22 Course 055 degrees, range $900 \mathrm{ft}$. to WP2. Back at flow and trying to take samples again, Harry says to take grab sample. Sample in bucket 12. $27-42.609 \mathrm{~N}$ 90-06.997W (sample lost over the side.)

09:30 Have grab sample and underway.

09:32 More flows, smooth, new looking flows, several inches thick. Taking still shots.

09:33 Bill thinks they are at the vent site, $27-42.648 \mathrm{~N} 90-07.006 \mathrm{~W}$, now heading to WP2.

09:42 Pock marks, observing loose scattering of mussels and clams, not much relief.

09:45 Coming up on ridge line, carbonate structures, mussels. $27-42.692 \mathrm{~N}$ $90-06.860 \mathrm{~W}$, within $100 \mathrm{ft}$. of WP2.

09:49 Very steep upslope, within $80 \mathrm{ft}$. of WP2.

09:50 Following slope up, then head on new course of 190 degrees.

09:51 Depth $2938 \mathrm{ft}$., heading to top of slope and took punch core, will send S (190 degrees) at top of slope.

10:01 Depth $2911 \mathrm{ft}$., came up on terrace, seems to be the top, heading 190 degrees, $27-42.716 \mathrm{~N}$ 90-06.797W

10:03 Depth $2925 \mathrm{ft}$., Coming up on drop off of about $25 \mathrm{ft}$.

10:05 Depth $2941 \mathrm{ft}$., Slight drop off, WP2.

10:14 Depth $2934 \mathrm{ft}$., Taking another mat sample (push core), need to go 045 to 050 degrees, get underway, no more samples.

10:17 Depth $2942 \mathrm{ft}$. , Getting underway.

10:19 Coming up on very steep slope, very soft bottom.

10:22 Depth $2919 \mathrm{ft}$., near top of slope (15 degree slope).

10:25 Depth $2912 \mathrm{ft}$., Large depression about $1 \mathrm{~m}$ across, $600 \mathrm{ft}$. due $\mathrm{E}$ from WP2.

10:30 Depth $2898 \mathrm{ft}$., All stop, coming up on a slight slope, changing course to 260 degrees, range $1000 \mathrm{ft}$., $27-42.720 \mathrm{~N} 90-06.691 \mathrm{~W}$

10:32 underway at 260 degrees.

10:34 Depth $2905 \mathrm{ft}$., passing large crater about $3 \mathrm{~m}$ diameter, very round.

10:39 Depth $2925 \mathrm{ft}$., now on downslope, observed small mats along the terrace. Slightly north of previous transect.

10:42 Depth $2938 \mathrm{ft}$., observed carbonate structure, will grab carbonate sample, $27-42.715 \mathrm{~N} 90-06.855 \mathrm{~W}$. Could not get basket to close, had to abandon grab sample of carbonate. 
GC 257, Dive 4399 (cont.)

11:00 Regrouping and plan to get underway at 260 degrees. Observed gorgonians.

11:02 Depth $2923 \mathrm{ft}$., observing mussels and clams.

11:08 Depth $2912 \mathrm{ft}$., observing area of possible old flows, heading 260 degrees, flow is at least $50 \mathrm{ft}$., unable to find the end.

11:10 Reach the edge of the flow field, approximately $120 \mathrm{ft}$. wide.

11:13 Battery getting weak on sub. Will transect at least 5 minutes. Harryprobably $200 \mathrm{ft}$. from site, $500 \mathrm{ft}$. west of WP1.

11:15 Depth $2920 \mathrm{ft}$., Now on mussels and clams, appear to be alive.

11:22 Depth $2930 \mathrm{ft}$., Mounds, about $1.5 \mathrm{~m}$ across, small craters.

11:24 Very soft mounds, like mud vents.

11:26 Depth $2915 \mathrm{ft}$., 10-15 degree upslope.

11:27 70 degree slope about $40 \mathrm{ft}$. tall, instructions to look for carbonates.

11:28 Depth $2873 \mathrm{ft}$., At top of slope, no carbonate. End of transect, leaving bottom. $27-42.607 \mathrm{~N} 90-07.207 \mathrm{~W}$

12:08 On surface.

\section{EW 1001, Dive 4400}

Crew: Front, Dan Boggess, Larry Cathles

Rear, Trainee Jimmy Nelson, Frank Lombardo

Purpose: Run transect, collect carbonates and push cores.

16:34 Launch and permission to dive. 27-57.644N 90-23.477W, Ship shows bottom at $1770 \mathrm{ft}$.

16:40 Bearing 270 degrees, range $450 \mathrm{ft}$., bottom showing at $1775 \mathrm{ft}$.

16:50 Depth $1000 \mathrm{ft}$., bottom showing at $1760 \mathrm{ft}$.

16:55 Bearing 270 degrees, range $150 \mathrm{ft}$.

16:58 Dropping straight down.

17:05 Bottom in sight.

17:07 On bottom, depth $1794 \mathrm{ft}$., temp. 7.73, visibility 35-40 ft., current 0.1 kt. from N. $27-57.686 \mathrm{~N} 90-23.538 \mathrm{~W}$ Light sediment covering bottom.

17:09 Bearing 320 degrees, range $1600 \mathrm{ft}$., getting underway to WP1.

$17: 13$ 45-50 degree slope about $25 \mathrm{ft}$. high, 27-57.731N 90-23.574W, depth $1790 \mathrm{ft}$. 
EW 1001, Dive 4400 (cont.)

17:15 Depth $1740 \mathrm{ft}$., on top of feature.

17:17 Depth 1720 ft., another slope, 55-60 degrees, 27-57.744N 90$23.593 \mathrm{~W}$

17:24 Depth $1693 \mathrm{ft}$., continuing on gentle upslope.

17:26 Depth $1689 \mathrm{ft} .$, Going over beggiatoa mat.

17:27 Bearing 295 degrees, range $325 \mathrm{ft}$.

17:30 Depth $1683 \mathrm{ft}$., going over area with gorgonians, with encrusting sponges and small beggiatoa mats. Took sample of beggiatoa 27 $57.925 \mathrm{~N} 90-23.780 \mathrm{~W}, 300 \mathrm{ft}$. to WP1.

17:39 Bearing 156 degrees, range $350 \mathrm{ft}$. underway to WP1.

17:41 Depth $1675 \mathrm{ft}$., Could be going over gas vent, suspended particles look like they are coming from the east.

17:42 Depth $1675 \mathrm{ft}$., Turning easterly to hunt for origin of suspended sediments.

17:48 Depth $1679 \mathrm{ft}$., Change course to 060 degrees, range $2100 \mathrm{ft}$. to WP1. 27-57.8888N 90-23.7754W

18:01 Depth $1729 \mathrm{ft}$. Noticed gentle downslope.

18:03 Depth $1741 \mathrm{ft}$., Change video tape $27-57.994 \mathrm{~N}$ 90-23.6032W, heading 065 degrees and getting underway.

18:04 Beggiatoa and small tube worms.

18:07 Depth $1758 \mathrm{ft}$. Sampling a beggiatoa mat (bucket sample) 27$58.0014 \mathrm{~N}$ 90-23.5835W, $0.3 \mathrm{kt}$. Current at 090 degrees.

18:18 Sample finished, underway at 070 degrees..

18:22 Depth $1758 \mathrm{ft}$., Going up a 30 degree slope, $750 \mathrm{ft}$. from WP2.

18:27 Observed light sediment plume as approaching a ridge, $650 \mathrm{ft}$. from WP2.

18:29 Depth $1718 \mathrm{ft}$., Top of small ridge and is starting downslope 27$58.0309 \mathrm{~N} 90-23.5211 \mathrm{~W}$, bearing 070 degrees, range $600 \mathrm{ft}$.

18:36 Change course to 030 degrees, at WP2, 27-58.0273N 90-23.4440W.

18:37 Depth $1715 \mathrm{ft}$, heading 030.

18:39 Depth $1710 \mathrm{ft}$., Sub stopped, getting course to WP3.

18:40 Bearing 110 degrees, range $1500 \mathrm{ft}$. to WP3.

18:41 Depth $1704 \mathrm{ft}$., Came up on old mud vent, coming up on slope of mud

volcano.
18:45 Depth $1701 \mathrm{ft}$. , Taking grab sample of mud, same coordinates (active area on fathometer image).

18:49 Good scoop in Bucket \#5, underway on 110 degrees. 
EW 1001, Dive 4400 (cont.)

19:03 Depth $1735 \mathrm{ft}$., Changing tape in video camera, 27-58.0267N 9023.3011W

19:06 heading 110

19:13 Depth $1748 \mathrm{ft}$., Large mat of beggiatoa, white and yellow deposits as well, extensive, goes at least $30 \mathrm{ft}$. to $\mathrm{N}$, new heading 160 degrees, range $180 \mathrm{ft}$.

19:15 Underway heading 160

19:17 Depth $1729 \mathrm{ft}$., New heading of 040 degrees, 27-57.9503N 90$23.1674 \mathrm{~W}$

19:23 Course correction 029 degrees.

19:31 Passed over cable trench perpendicular to sub track.

19:33 Depth $1757 \mathrm{ft}$., Stopped at EOL, 27-58.1166N 90-23.0572W, going over more beggiatoa, no significant relief, soft silty sediment cover over sticky sand. Heading 200 degrees upslope.

19:44 Depth $1726 \mathrm{ft}$., stop, permission to leave bottom.

19:44 Depth $1729 \mathrm{ft}$., leaving bottom, 27-58.0423N 90-23.0430W

18:15 On surface.

\section{Tuesday, May 28}

Port day at Fourchon to change scientific crews.

\section{Wednesday, May 29}

\section{GC 232, Dive 4401}

Crew: Front, Craig Caddigan, Roger Sassen

Rear, Hugo Marrero, Tom McGee

Purpose: Locate, videotape and sample hydrates, take cores in the area.

08:03 Launch and permission to dive, 27-44.4825N 91-19.0562W.

08:28 Bottom in sight.

08:30 On bottom, Depth $1870 \mathrm{ft}$., Temp. 7.6, Visibility 30-40 ft., 0.1 to 0.2

kts. Current, mud bottom with white bacterial mats, taking water sample. $27-44.5112 \mathrm{~N} 91-19.0757 \mathrm{~W}$, within $70 \mathrm{ft}$. of target.

08:34 Heading 310 degrees. 
GC 232, Dive 4401 (cont.)

08:36 Depth $1869 \mathrm{ft}$. , Stopping to take tube worm samples, $27-44.5227 \mathrm{~N}$

91-19.0916W

08:39 Getting back underway.

08:40 Range $80 \mathrm{ft}$., due $\mathrm{S}$

08:42 Course change to 130 degrees.

08:42 Lost main control box that controls camera, arc lamp, pilots box, etc.

Check main connector on back of pilots box.

08:45 Bottom connection loose, tightened it and all came back.

08:48 Depth $1868 \mathrm{ft}$., small tube worm bushes and bacterial mats.

08:50 Setting down to sample bacterial mat, punch core \#1, 27-44.5721N 91-19.0737W.

08:54 Back underway.

08:55 Heading NE of site and searching

08:56 $100 \mathrm{ft}$. N of target

09:06 Good plume, 075 degrees about $200 \mathrm{ft}$. away.

09:07 Good sized piece of exposed hydrate, depth $1866 \mathrm{ft} ., 27-44.5173 \mathrm{~N}$ 91-19.0828W.

09:12 Taking sample, gas escaping from bottom of hydrates, tried to take sample but hundreds of small pieces broke off.

09:23 Took grab sample with tube worms in it.

09:26 Moved left of hydrate, will take 4 punch cores, depth $1865 \mathrm{ft}$., tubes \#2,3,4\&5. 27-44.4974N 90-19.0687W

09:37 Will now do suction sample.

09:58 Another exposed hydrate sample about $15 \mathrm{ft}$. from the other, depth $1865 \mathrm{ft}$., doing last punch core. 27-44.5165N 91-19.0866W. Pin came out of bucket, cannot rotate anymore.

10:03 Finished with push cores and will cruise around and look for more hydrates.

10:11 Hydrates just downhill from landing, did small circle, will get underway at 310 degrees to see what else is there.

10:17 Large area of tubeworms, appears to have exposed carbonate, depth $1867 \mathrm{ft} .27-44.5009 \mathrm{~N} 91-19.0915 \mathrm{~W}$.

10:26 Setting down to take couple of small rocks. $27-44.5108 \mathrm{~N} 91-$ 19.0879W.

10:29 Back underway.

10:46 Fix \#12 27-44.4907N 91-19.0457W, depth 1870 ft., exposed carbonate and tube worms, carbonate sampled. 
GC 232, Dive 4401 (cont.)

10:52 Fix \#13 27-44.4924N 91-19.0765W, depth 1868 ft., exposed gas

hydrates with tube worms and mussels.

10:57 Permission to leave bottom. 27-44.4856N 91-19.0849W.

11:23 On surface.

\section{GC 232, Dive 4402}

Crew: Front, Craig Caddigan, Dan McConnell

Rear, Hugo Marrero, Mark Brausse

Purpose: Push cores, grab samples, and gas samples adjacent to hydrates.

15:34 Launch and permission to dive. 27-44.5190N 91-19.0066W

15:37 Sounded like a bucket imploded at about $150 \mathrm{ft}$.

15:40 Abort dive to repair bucket. Depth $300 \mathrm{ft}$.

15:43 $180 \mathrm{ft}$., permission to surface.

15:45 On surface, repaired imploded bucket.

\section{GC 232, Dive 4403}

Crew: same

Purpose: same

16:03 Launch and Permission to dive. 27-44.5134N 91-19.0792W.

16:21 Bearing 150 degrees, range $400 \mathrm{ft}$., depth now $1000 \mathrm{ft}$.

16:21 Range $100 \mathrm{ft}$., bearing 130 degrees, depth now $1600 \mathrm{ft}$.

16:26 Bottom in sight.

16:30 On bottom, depth $1871 \mathrm{ft}$., Temp. 7.71, Visibility 30-35 ft., current

$0.1 \mathrm{kt}$. from 310 degrees, $27-44.4992 \mathrm{~N} 91-19.0751 \mathrm{~W}$, target $60 \mathrm{ft}$. at 180 degrees.

16:39 Range $150 \mathrm{ft}$., bearing 110 degrees.

16:46 Range $250 \mathrm{ft}$., bearing 340 degrees to $1^{\text {st }}$ hydrate mound.

16:56 At hydrate mound. Will go behind to punch cores with tubes \#1, 2, and 3 .

16:59 Sitting on slope of hydrate mound, will take 3 punch cores in that location. $27-44.5053 \mathrm{~N}$ 91-19.0812W. 
GC 232, Dive 4403 (cont.)

17:02 GPS down.

17:07 Finished with punch cores \#1, 2, and 3 . Will now move over and take the other 3 .

17:08 GPS back on line.

17:08 Will take the other 3 punch cores on back side of tube worm patch. Mud bottom with a little bacteria, will space cores about $3 \mathrm{ft}$. apart.

17:17 Will go to the other side for a gas sample.

17:22 $25 \mathrm{ft}$. from the vent this morning, still bubbling a little. Will try for a gas sample. Depth $1862 \mathrm{ft}$. 27-44.4998N 91-19.0852W

17:30 Abort gas sampling because gas is coming out too slowly.

17:31 Bearing 130 degrees to WP6, large exposed hydrate mound.

17:33 Fix 10, small bacterial mats. Getting 2 grab samples. Depth $1866 \mathrm{ft}$. White and orange bacterial mats.

17:52 Left bacterial mat site to look for gas vent.

17:54 Suction sample of bacterial mat. Depth $1870 \mathrm{ft}$., $21-44.4728 \mathrm{~N} 91-$ 19.0789W.

17:58 Back underway.

18:00 Bearing 010 degrees, range $190 \mathrm{ft}$. to hydrates and mussels.

18:03 Change course to 310 degrees.

18:07 Change course to due N.

18:18 Depth $1867 \mathrm{ft} .$, mussels and tube worms with encrustations, will sample each. $27-44.4977 \mathrm{~N}$ 91-19.1010W.

18:36 On back side of the hydrate mound found this morning. Will try for a gas sample. $27-44.4947 \mathrm{~N} 91-19.0866 \mathrm{~W}$.

18:56 Definitely at the hydrates found this morning, gas coming out but not as much.

19:02 Depth $1867 \mathrm{ft}$., Permission to leave bottom. 27-44.4977N 91$19.0922 \mathrm{~W}$.

19:24 Depth $200 \mathrm{ft}$., permission to surface.

19:26 On surface.

\section{Thursday, May 30}

\section{GC 232, Dive 4404}

Crew: Front, Don Liberatore, Alexi Milkov Rear, Frank Lombardo, Tyler Hodges 
GC232, Dive 4404 (cont.)

Purpose: Collect hydrate samples, cores, grab samples and water samples.

08:16 Launch and permission to dive. 27-44.4904N 91-19.0068W

08:19 Weird caution light on both videos, "Caution C 31 ". Took the tape

out and put it back in and fixed the problem.

08:36 Get underway bearing 330 degrees, range $180 \mathrm{ft}$.

08:40 Change course to 030 degrees.

08:43 All stop, go straight down.

08:45 Bottom in sight.

08:47 On bottom, depth $1866 \mathrm{ft}$., Temp. 7.4, Visibility 30-35 ft., current 0.1

kt. at 035 degrees. $27-44.5230 \mathrm{~N} 91-19.0936 \mathrm{~W}$

08:48 Bearing 140 degrees, range $80 \mathrm{ft}$. to hydrates.

08:50 Sandy area with tube worm bushes, will scout around.

08:53 Coming up on exposed hydrate, will take still shots for a few minutes.

08:56 Hydrates. 27-44.5109N 91-19.0789W

09:01 Seeing fogging in camera (condensation on the lens).

09:03 Emptied air from the gas sampler, going in to collect gas sample.

09:22 Got a gas sample. Will take more still shots.

09:31 Shot still shots all around the mound. Will settle in and try to break a piece of hydrate off to put in pressure bomb.

09:41 Broke off a big piece of hydrate. Stayed in. Waiting for silt to clear to put the lid on.

09:49 Can't get the lid all the way down. Last quarter inch won't go. Will take the lid off and try to vacuum the threads and try again.

10:02 Think they have the lid down, will proceed.

10:10 Moved over to a small depression. Have iceworms in the hydrates. Will attempt to sample ice worms with the suction and take photos.

10:19 Tried to sample iceworms. Broke off a piece and got it in bucket \#5. Don't know if we got a worm or not.

10:21 Getting in position to take push core between 2 hydrate sites. Depth $1865 \mathrm{ft}$. $27-44.5112 \mathrm{~N} 91-19.1028 \mathrm{~W}$.

10:32 Question for Roger: $1^{\text {st }}$ site from yesterday, would you like to break off a big chunk and bring it up in the basket? Leave it alone or try to bring it up? Leave it alone in case we need another sample.

10:35 Bearing 180 degrees, range $100 \mathrm{ft}$. to other hydrate zone. May be able to break off a piece there. 
GC 232, Dive 4404 (cont.)

10:38 Found an orange bacterial mat. Will stop and take a suction sample.

Depth $1867 \mathrm{ft}$., Also took photos and video. $27-44.4765 \mathrm{~N} 91-$ 19.0891W.

10:47 Want push cores or suction samples? Harry - take suction sample, push cores are for Jeff.

10:51 Quite a bit of sponge. Will get another sample. Depth $1865 \mathrm{ft} ., 27-$ 44.4773N 91-19.0634W

10:59 Take $2^{\text {nd }}$ push core by the tube worm bush.

11:08 Will take push core and grab sample of tube worms. Depth $1866 \mathrm{ft}$., 27-44.4701N 91-19.0777W

11:21 Got samples. Now looking for orange bacterial mat for one more suction sample.

11:29 Time to wrap it up.

11:30 Depth $1862 \mathrm{ft}$., permission to leave bottom.

11:50 On surface.

\section{GC 232, Dive 4405}

Crew: Front, Don Liberatore, Laura Laham

Rear, Frank Lombardo, Jeff Chanton

Purpose: Collect pore water samples, and other samples.

15:49 Launch, permission to dive. $130 \mathrm{ft}$. from station, depth showing 1855 ft. $27-44.5214 \mathrm{~N} 91-19.0677 \mathrm{~W}$

16:09 Bearing 210 degrees, range $130 \mathrm{ft}$.

16:15 Bearing 090 degrees, range $280 \mathrm{ft}$. Getting underway to E.

16:17 Bottom in sight.

16:19 Getting close to target. Tube worm bushes in sight.

16:21 On bottom at target site, depth $1862 \mathrm{ft}$. , Temp. 7.77, Visibility $30-35$

ft., current 0 to $0.1 \mathrm{kt}$. at 140 degrees. $27-44.5142 \mathrm{~N} 91-19.0893 \mathrm{~W}$

16:26 At exposed hydrates. Going to deploy the pore water sampler. Fix 6: $27-44.5136 \mathrm{~N}$ 91-19.0893W Depth $1867 \mathrm{ft}$.

16:29 One of the valves is closed, putting it into the bottom.

16:31 Sampler is in the bottom. Seafloor is very soft.

16:33 Got sampler triggered, waiting for 30 minutes.

17:00 Two minutes to go. Then they will take the pore water sampler out of the soft soil. When the sampler is on the sub, will trigger the valves. 
GC 232, Dive 4405 (cont.)

17:02 Pick up probe and sampler.

17:06 Got sampler on sub.

17:18 Closed all valves. Took push core \#4 right next to pore water sampler location. Fix 7 27-44.5059N 91-19.0784W Depth $1867 \mathrm{ft}$.

$17: 29$ Second push core taken next to first one. Moving in to take another sample near bubble stream.

17:41 Collecting a gas sample in the same location as the $2^{\text {nd }}$ push core.

17:50 Grab sample of sediment above the gas hydrate.

18:06 Took push core next to the hydrate mound. Shook the push core to get the sample out.

18:12 Took third push core at the same location (redid the previous one).

18:21 Took scoop sample, moving on bearing 140 degrees, range $90 \mathrm{ft}$. Target location is large exposed hydrate.

18:29 Picked up rock sample at depth of $1864 \mathrm{ft}$., could not find hydrate mound. Range $40 \mathrm{ft}$. to exposed hydrates.

18:31 Picked up scoop sample, Fix 8 27-44.4899N 91-19.0785W, depth $1864 \mathrm{ft}$.

18:41 Sword fish in sight.

18:44 Fix 9 27-44.4960N 91-19.0766W, depth $1864 \mathrm{ft}$., took scoop sample of orange material.

18:52 Still have not found second hydrate mound, time to wrap dive up.

18:56 Permission to leave bottom, depth $1867 \mathrm{ft}$.

19:16 Depth $200 \mathrm{ft}$., permission to surface.

19:18 On surface.

\section{Friday, May 31}

\section{GC 234, Dive 4406}

Crew: Front, Dan Boggess, Roger Sassen

Rear, Hugo Marrero, Kelley Peeler

Purpose: Take hydrate samples, cores, and grab samples.

08:01 Launch and permission to dive. 27-44.7543N 91-13.3406W

08:16 Bottom showing at $1730 \mathrm{ft}$., get underway due $\mathrm{S}$ for $200 \mathrm{ft}$.

08:23 Adjust heading to 200 degrees.

08:25 Change course to 100 degrees. 
GC 234, Dive 4406 (cont.)

08:28 Bottom in sight.

08:31 On bottom, depth $1711 \mathrm{ft}$., Temp. 8.66, visibility 40-45 ft., current 0.1

kt. from 070 degrees. 27-44.7422N 91-13.4194W. Bearing 090

degrees, range $400 \mathrm{ft}$.

08:37 Area with lots of tube worms, will sit down and take grab sample of orange mat, bucket \#8, depth $1762 \mathrm{ft}$. $27-44.7766 \mathrm{~N} 91-13.3613 \mathrm{~W}$

08:45 Done with sample. Underway due E.

08:47 At marker "TX", small float to left, depth $1772 \mathrm{ft}$.

09:00 Moving toward marker "V", depth $1778 \mathrm{ft}$. Think they found hydrate.

09:01 Will try for a gas sample, 27-44.7587N 91-13.3101W.

09:57 took gas sample, getting hydrate sample.

10:36 Dropped chipper off platform, recovering it and still trying to get hydrate sample.

11:02 Still trying to get hydrate sample, got some pieces, depth $1778 \mathrm{ft}$.

11:10 Directed to wrap up operation.

11:17 Permission to leave bottom.

11:18 Left bottom, 27-44.7448N 91-13.3201W

11:43 Depth $200 \mathrm{ft}$. , permission to surface.

11:45 On surface.

\section{GC 234, Dive 4407}

Crew: Front, Dan Boggess, Jeff Chanton Rear, Hugo Marrero, Howard Manlovitz

Purpose: Observe hydrates, take video and stills, take push cores and grab samples.

16:06 Launch and permission to dive. 27-44.7614N 91-13.3365W

$16: 23$ Course change to 050 degrees, range $400 \mathrm{ft}$.

16:25 Stop, drop straight down, bottom showing at $1735 \mathrm{ft}$.

16:31 Bottom in sight.

16:34 On bottom, depth $1756 \mathrm{ft}$., Temp. 8.1, Visibility $35-40 \mathrm{ft}$., current 0 to 0.1 kt. from 130 degrees. Fix $27-44.7906 \mathrm{~N} 91-13.3467 \mathrm{~W}$. Landed in tube worms, no markers, will look around.

16:35 Found Marker "T2" 
GC 234, Dive 4407 (cont.)

16:37 Depth $1763 \mathrm{ft}$., will sample orange mat, will do mesh bag \#9 first, then screen.

16:38 Platform is stuck. Bucket \#9 is the one stuck under the funnel. Will forget that spot and try to find hydrate mound.

16:50 Moving, using gas sampler to push down on lid of bucket. Worked, and platform will rotate again.

16:56 Taking suction sample of red mat into fine mesh bucket, Fix 27$44.7882 \mathrm{~N}$ 91-13.3426W.

17:12 At $1767 \mathrm{ft}$., at marker "A1", also at marker "TX" again. Should be on hydrate.

17:16 Went straight over a tub worm bush at "TX", has flat tile with "12" on it, upslope are 2 floats, perhaps near hydrates.

17:17 Moving in on hydrates now at marker " $B$ ".

17:20 Taking tube \#1 push core. Depth $1777 \mathrm{ft}$., $5 \mathrm{ft}$. from hydrate mound. Fix 27-44.7531N 91-13.3119W.

17:30 Take cores $1 \& 2$ on side of hydrate mound. Take core 3 to left of tube worm bush about $10 \mathrm{ft}$. away.

17:37 Tube core 4 will be taken there also (about $15 \mathrm{ft}$. to west of core 3 location).

17:47 Depth $1784 \mathrm{ft}$., Taking push core 5 downslope from thermistor site. Fix 6: $27-44.7661 \mathrm{~N}$ 91-13.2936W.

18:02 Have Ion pump device deployed, going back to collect mussels.

18:03 Back on mussel bed, same place they took the gas sample this morning. Will take another gas sample.

18:15 Done at hydrate mound, depth $1776 \mathrm{ft}$. Will now go look for rocks for Harry.

18:24 Depth $1768 \mathrm{ft}$., Put one piece of rock in bucket \#12, will try to suction bacterial mat in Bucket 11, which has mesh. Didn't work out.

18:26 Will grab another rock. Fix 27-44.7723N 91-13.3104W. Rock is in the basket, will try for another bacterial mat.

18:29 Depth $1771 \mathrm{ft}$. , Found orange bacterial mat, will attempt to sample.

18:35 Moved over a ridge. Will try to sample another orange bacterial mat.

18:42 Have bacterial mat sampled.

18:57 Depth $1792 \mathrm{ft}$., Request permission to leave bottom (no fix available).

19:15 Permission to surface:

19:15 Surface in sight, surface clear.

19:17 On surface. 


\section{Saturday, June 1}

\section{885, Dive 4408}

\section{Crew: Front, Craig Caddigan, Dick Fillon}

Rear, Frank Palumbo, Barry Kohl

Purpose: Survey preselected traverse, collect still shots, video and samples.

08:01 Launch and permission to dive. Fix 28-04.0497N 89-43.2765W 08:26 Course 085 degrees, range $200 \mathrm{ft}$. Depth showing $1800 \mathrm{ft}$.

08:32 Bottom in sight.

08:34 On bottom. Depth $2160 \mathrm{ft}$., Temp 6.79, visibility 20-30 ft., current 0,4

kt. from 030 degrees. Fix 1: $28-04.0403 \mathrm{~N} 89-43.3787 \mathrm{~W}$. Core

sample 1 .

08:40 Head on course 100 degrees. Bottom is basically mud. 100 degrees

is taking sub diagonally up a gentle slope.

08:50 Depth $2134 \mathrm{ft}$., Bottom is the same.

08:56 New heading of 040 degrees, depth $2117 \mathrm{ft}$., bottom is the same.

08:59 At WP1, All stop, look for thermistor.

09:07 New heading of 100 degrees to WP2.

09:11 Underway at 100 degrees, bottom is the same.

09:15 Depth $2100 \mathrm{ft}$.

09:17 Depth $2080 \mathrm{ft}$., Starting to see rocks with crinoids.

09:19 Took long push core, Fix 2: 28-03.9712N 89-43.0429W Punch core \#5.

09:20 Just lost forward horizontal thruster.

09:26 Coming to small drop off, one big boulder there with coral on it.

Going down, then back up again.

09:28 Area of small rocks and small gorgonians, depth $2065 \mathrm{ft}$.

09:30 Going over a series of ridges, current up to $0.5 \mathrm{kts}$.

09:31 Back over clean mud bottom.

09:31 Lots of shell hash with gorgonians.

09:32 New heading of 070 degrees.

09:39 Change to video tape \#2. 
MC 885, Dive 4408 (cont.)

09:40 Tube worms with clams on top.

09:46 New heading of 035 degrees.

09:47 Underway at 025 degrees.

09:49 Bottom covered with dead clams.

09:52 All stop. Took long punch core. Fix 3: 28-03.9611N 89-42.7557W.

Depth $2037 \mathrm{ft}$. Be advised forward horizontal is coming on by itself.

Punch core only penetrated 2 or 3 inches, took grab sample.

09:59 New course of 080 degrees, range $1000 \mathrm{ft}$.

10:07 Next time clams are seen, take sample.

10:08 Came across fiberglass pole sticking out of bottom.

10:09 Starting to see series of small ridges.

10:11 New heading of 060 degrees.

10:14 New heading of 035 degrees.

$10: 21 \mathrm{WP} 3$ is due $\mathrm{N}$ range $100 \mathrm{ft}$.

10:23 On WP3, depth $2066 \mathrm{ft}$., clean bottom with some shell hash. Took punch core \#4. Fix 4: $28-03.9564 \mathrm{~N}$ 89-42.5319W.

10:28 New heading of 150 degrees to EOL.

10:33 New heading of 120 degrees (current set)

10:38 Changing to video tape \#3.

10:42 Be advised, sub going sideways more than forward.

10:46 New heading of 200 degrees.

10:49 WP due $\mathrm{N}$ about $100 \mathrm{ft}$.

10:50 Stop and take punch core.

10:50 Depth $2575 \mathrm{ft}$., clean mud bottom, punch core \#3, Fix 5: 28-03.664N 89-42.4005W.

10:55 Head back to WP 3, due N.

11:01 Getting swept up slope.

MC 885, Dive 4408 (cont.)

11:02 Go up slope, about 330 degrees.

11:04 At top of slope, depth $2061 \mathrm{ft}$., turning to head back into current.

11:08 Bottom is featureless mud with burrows.

11:09 Will take grab sample. Fix 6: 28-03.7227N 89-42.4090W, Depth

$2063 \mathrm{ft}$.

11:10 Request permission to leave bottom. Total distance covered $6493 \mathrm{ft}$.

11:36 On surface. 


\section{885, Dive 4409}

Crew: Front, Craig Caddigan, Bob Woolsey

Rear, Frank Palumbo, Tyler Hodges

Purpose: Survey traverse 2 and sample, take stills and video.

15:40 Lunch and permission to dive. Fix 1: 28-04.5120N 89-43.3480W

15:57 Start heading on course of 320 degrees.

16:01 New heading of due N, bottom showing at $2060 \mathrm{ft}$.

16:04 New heading of 020 degrees.

16:06 Bottom in sight, new heading of 060 degrees.

16:08 On bottom, depth $2087 \mathrm{ft}$., Temp. 6.82 , visibility $20-25 \mathrm{ft}$., current 0.3

to $0.4 \mathrm{kts}$. at 030 degrees. Fix $2: 28-04.6908 \mathrm{~N} 89-42.8598 \mathrm{~W}$ Same

bottom as this morning, flat muddy bottom. Underway at 060

degrees.

16:13 New heading of 150 , SOL.

16:17 Depth $2068 \mathrm{ft}$., bottom is the same.

16:22 New heading of 155 degrees.

16:23 Came across first outcropping of rocks with gorgonians on them, Fix 3: $28-04.5735 \mathrm{~N}$ 89-42.7919W.

16:26 Bottom featureless again, depth $2063 \mathrm{ft}$.

16:28 Coming into more gorgonians, will sample. Fix 4: 28-04.4861N

89-42.7767W.

16:36 Got sample of small rock with egg sacks on it, will take punch core.

Depth $2055 \mathrm{ft}$.

16:39 Back underway at 155 degrees.

16:44 Depth $2067 \mathrm{ft}$. Crossed small rocks, then barren bottom, starting to see shell hash.

16:45 New heading of 220 degrees, range about $100 \mathrm{ft}$.

16:46 Small rocks with shell hash.

16:48 Near WP1, heading of 150 degrees to WP2.

16:57 Depth $2056 \mathrm{ft}$., Barren bottom, gently rolling up and down maybe 10

ft.

17:01 Came down a slope to $2104 \mathrm{ft}$., now going back up.

17:07 Depth $2056 \mathrm{ft}$. On clean bottom.

17:11 Depth $2049 \mathrm{ft}$. Getting back into shell hash.

17:13 Shell hash getting thicker, also occasionally seeing gorgonians. 
MC 885, Dive 4409 (cont.)

17:16 Shell has thicker, depth $2041 \mathrm{ft}$. Will do 1 long push core and 1 short push core. Fix 5: 28-03.9911N 89-42.7551W

17:22 Punch core 1, punch core 4. Sent to WP3 on bearing of 200 degrees, range $1700 \mathrm{ft}$.

17:32 Small gorgonians, rocks, and shell hash.

17:40 Going slightly upslope. Depth $2057 \mathrm{ft}$. Have now left shell covered bottom and now on burrowed mud.

17:44 Back in shell covered bottom, rocks, and gorgonians. Going back upslope.

17:46 Depth $2064 \mathrm{ft}$. Top of a little ridge, took punch core \#5.

17:49 Have punch core and getting underway bearing 195 degrees.

17:52 Change course to 170 degrees.

17:55 Change course to 160 degrees.

17:57 Change course to 090 degrees.

18:01 Near WP3. Take long push core.

18:03 Setting down, depth $2061 \mathrm{ft}$., sitting atop a silt covered rock

formation. Fix 6: $28-03.7116 \mathrm{~N}$ 89-42.8518W.

18:11 Got core, had to move slightly.

18:11 Proceed approximately $80 \mathrm{ft}$. at 280 degrees.

18:12 Course change, $50 \mathrm{ft}$. due south.

18:15 Course change 110 degrees.

18:23 Looking around for thermistor, heading 110 degrees.

18:24 Found the thermistor. Fix 7: 28-03.6917N 89-42.8765W, Depth

$2052 \mathrm{ft}$., Taking video and will recover thermistor.

18:30 Have thermistor on platform, won't go in the basket.

18:54 Permission to surface.

18:56 Surface in sight, surface clear.

18:57 On surface.

\section{Sunday, June 2}

\section{VK 826, Dive 4410}

Crew: Front, Don Liberatore, Harry Roberts

Rear, Hugo Marrero and trainee Curt Patterson

Purpose: Survey traverse 1, take stills, video and samples. 
VK 826, Dive 4410 (cont.)

08:00 Launch and permission to dive. Fix 1: $29-10.5088 \mathrm{~N} 88-00.9544 \mathrm{~W}$, bottom showing at $1599 \mathrm{ft}$.

08:03 Going through layer of turbid water, coming up on depth of $200 \mathrm{ft}$.

08:14 Get underway at 060 degrees.

08:19 Course change to 080 degrees, range $400 \mathrm{ft}$. Depth showing at 1400

$\mathrm{ft}$.

08:22 Bottom in sight.

08:25 On bottom, depth $1581 \mathrm{ft}$., Temp 9.67, visibility $25-30 \mathrm{ft}$., current 0.2

kts. at 090 degrees. Fix 2: $29-10.4874 \mathrm{~N} 88-00.0064 \mathrm{~W}$. Bottom

flat, soft sediment, and lots of small fish.

08:29 At SOL, new course of 170 degrees, range $1600 \mathrm{ft}$.

08:33 Came over a ridge, now heading down slope.

08:37 Depth $1600 \mathrm{ft}$., Slope increasing, no difference in bottom type.

08:40 Depth $1640 \mathrm{ft}$., Looks like slope is 30 degrees or more.

08:41 Bottom starting to flatten out, same type, mud.

08:42 Continues on down. Will follow. Appears course of 230 degrees.

08:43 Course of 220 degrees, depth $1700 \mathrm{ft}$.

08:45 Request estimated depth of hole. Appears to be 1800 to $1850 \mathrm{ft}$.

deep. At 1728 and still going down.

08:46 Depth $1750 \mathrm{ft}$.

08:48 Still going down, now at $1775 \mathrm{ft}$.

08:56 At $1838 \mathrm{ft}$., not flat but slight slope, waiting for sediment to clear.

Fix 4: $29-10.3128 \mathrm{~N}$ 88-00.9993W

08:59 Bottom looks flat.

09:07 Motored around a bit, depth $1837 \mathrm{ft}$., will take punch core \#6. WP2 is at course of 170 degrees, range $4000 \mathrm{ft}$.

09:15 Found a gorgonian with a clam growing on it.

09:22 Depth $1700 \mathrm{ft}$.

09:26 Depth $1637 \mathrm{ft}$, looks like top of the slope. Fix 5: 29-10.2464N 88-

$01.0481 \mathrm{~W}$. Bottom change, harder with gorgonians, anomenes, etc.

09:27 Down slope slightly, back into soft sediment.

09:31 Working back up a shallow slope.

09:39 Leveled out at $1580 \mathrm{ft}$.

09:40 Ridge with gorgonians and small rock.

09:41 Depth $1573 \mathrm{ft}$., Fix 6: 29-10.0951N 88-01.0672W, back underway at 170 degrees.

09:43 Lost tilt function on video, still have pan. 
VK 826, Dive 4410 (cont.)

09:44 More of hard bottom with gorgonians.

09:47 Going back upslope.

09:50 Course change to 150 degrees, range $1400 \mathrm{ft}$. At the top of a little

hill, depth $1590 \mathrm{ft}$.

09:51 Series of ridges, couple of meters high with shell hash, etc.

09:52 Lot of shell hash, mostly dead stuff.

09:56 Coming across Lophelia coral, depth $1532 \mathrm{ft}$., (photo of fathometer plume) Fix 7: 28-09.7827N 88-01.0395W. Large field of lophelia.

09:58 Depth $1538 \mathrm{ft}$. Will sample coral.

10:02 Back underway on course of 150 degrees.

10:03 Course change to 165 degrees, range $700 \mathrm{ft}$.

10:06 Getting out of coral area and into broken bottom with shell hash.

10:07 Hard bottom, broken material and shell hash.

10:08 Just spotted $1^{\text {st }}$ tube worms, course change to 145 degrees.

10:10 Up a mound of thick coral, carbonate mound.

10:11 Course change to 120 degrees, range $180 \mathrm{ft}$. to WP2.

10:15 New course of 040 degrees, $80 \mathrm{ft}$. Will stop and grab rock sample.

Fix 8: $29-09.6482 \mathrm{~N} 88-01.0060 \mathrm{~W}$. Got rock sample, will continue

on 030 degrees $120 \mathrm{ft}$.

10:24 At WP2, bearing 185 degrees, range $1800 \mathrm{ft}$. to EOL. Depth $1465 \mathrm{ft}$.

10:39 Back into coral field, depth $1529 \mathrm{ft}$., course change to 160 degrees.

10:42 Depth $1546 \mathrm{ft}$., will take scoop sample of clams and little sediment.

10:51 Very rocky bottom, coral and gorgonians.

10:54 Depth $1580 \mathrm{ft}$., down slope at a steep angle.

10:56 At EOL, will mosey around and look for something interesting.

10:58 See a few clams on coral, will take samples. Fix 9: 29-09.3803N 88$01.0239 \mathrm{~W}$, tilt now working again.

11:07 Got 2 clams, heading upslope, depth $1526 \mathrm{ft}$.

11:14 Depth $1565 \mathrm{ft}$., permission to leave bottom. Fix 29-09.4147N 88$01.0255 \mathrm{~W}$.

11:33 Surface in sight, surface clear.

11:34 On surface 


\section{VK 826, Dive 4411}

Crew: Front, Don Liberatore, Barry Kohl

Rear, Hugo Marrero, Mark Brausse

Purpose: Survey traverse 2, take stills, video and samples, get 2 background cores for Jeff.

15:32 Launch and permission to dive. 29-09.7557N 88-01.8910W

15:58 Bottom in sight.

15:58 On bottom, depth $1708 \mathrm{ft}$., Temp. 8.80 , visibility $25-30 \mathrm{ft}$., current 0.1

kt.

16:02 Instructed to take 2 background push cores for Jeff.

16:05 Have push core \#1.

16:08 Have push core \#2. Bearing 100 degrees, range $1400 \mathrm{ft}$. to WP1. Bottom is featureless soft sediment with pock marks. Fix 6: 29$09.7528 \mathrm{~N} 88-01.9246 \mathrm{~W}$

16:21 Course change to 090 degrees, range $400 \mathrm{ft}$. Featureless, small pock marks. (Moderately high amplitude on map)

16:23 Different bottom type, shells, but not hard bottom.

16:24 Depth $1696 \mathrm{ft}$., on hard bottom with gorgonians (corresponds to high amplitude on map). At WP 1, ridge with some tubeworms.

16:26 Course 100 degrees, range $3500 \mathrm{ft}$. to WP2.

16:27 Flat mud bottom, depth $1700 \mathrm{ft}$.

16:31 Huge shark spotted, trying to get video.

16:34 Chasing shark, coming on hard bottom and gorgonians.

16:35 Rock outcrop, depth $1708 \mathrm{ft}$., gorgonians and tubeworms.

16:36 Bigger bushes of tubeworms. Depth $1703 \mathrm{ft}$.

16:37 Over small ridges, rough outcrop.

16:39 Over soft bottom.

16:39 Small patches of hard bottom among flat sand.

16:41 Depth $1684 \mathrm{ft}$., Bottom change upslope and shell hash.

16:43 Crossing isopods, a lot more than before.

16:44 Depth $1661 \mathrm{ft}$., rocky bottom.

16:44 Going to find rock sample.

16:46 Rock sample at depth of $1654 \mathrm{ft}$., Fix 7: 29-09.6671N 88-01.3772W.

16:47 Bearing 100 degrees. Shark following.

$16: 49$ No sign of gas or any hydrate so far.

16:52 Depth $1600 \mathrm{ft}$., Gorgonians, sponges and more hard ground. 
VK 826, Dive 4411 (cont.)

16:53 Slope about 20 degrees, lophilia more dominant.

16:57 Large carbonate structures, depth $1560 \mathrm{ft}$., many gorgonians and black coral. Fix 8: 29-09.6497N 88-01.2705 W.

17:04 Transition to deep soft sediment, depth $1519 \mathrm{ft}$.

17:06 Back in coral area, depth $1487 \mathrm{ft}$.

17:07 Carbonate structure under lophelia bushes, lots of shell hash among boulders.

17:08 Horizontal slabs of carbonate rock (corresponds to high ampltude). $600 \mathrm{ft}$. west on line from WP 2

17:10 Coral, tubeworms, stopping to take video. Fix 9: 29-09.6290N 88$01.1307 \mathrm{~W}$.

17:15 Back underway at 100 degrees.

17:19 Course 090 degrees, 0.4 to 0.5 kts. of current.

17:24 Depth $1453 \mathrm{ft}$., now going downslope.

17:25 Now at WP 2, new course 100 degrees, range $5000 \mathrm{ft}$.

17:27 Softer bottom with shell hash, depth $1474 \mathrm{ft}$.

17:28 Depth $1480 \mathrm{ft}$., going back upslope.

17:29 On top of ridge, now going back downslope, depth $1474 \mathrm{ft}$.

17:30 Depth $1480 \mathrm{ft}$., grab sample. Fix 10: 29-09.6108N 88-00.9787W

17:35 Now have grab sample.

17:37 Back underway.

17:38 Seeing battery track marks, depth $1479 \mathrm{ft}$., 0.6 kts of current at 100 degrees, going against sub.

17:45 Stopping to take push core, Fix $1129-09.6006 \mathrm{~N}$ 88-00.8949W, push core tube \#3. Depth $1517 \mathrm{ft}$.

17:55 Depth $1565 \mathrm{ft}$. , downslope, sharp break downslope.

17:57 Urchins, smooth sediment bottom, depth $1600 \mathrm{ft}$.

18:00 Flat bottom with anemones, depth $1638 \mathrm{ft}$. Stopping for grab sample at depth of $1640 \mathrm{ft}$.

18:17 Heading downslope, depth $1587 \mathrm{ft}$.

18:20 Arrived at bottom of slope, depth $1660 \mathrm{ft}$.

18:32 Bottom a little more local relief.

18:34 Area looks disturbed (perhaps anchor drag). Will take grab sample in flat area at depth of $1702 \mathrm{ft}$.

18:41 Grab sample (Fix 13) acquired, and leaving bottom. 29-09.5233N 88-00.3467W 


\section{Monday, June 3}

\section{709 Dive 4412}

Crew: Front, Dan Boggess, Roger Sassen

Rear, Frank Lombardo, Jeff Chanton

Purpose: Survey Traverse 1, take stills, video and samples, and select site for pore water sample.

07:58 Launch, permission to dive.

08:21 Get underway at 330 degrees.

08:24 Change course to 300 degrees, range $400 \mathrm{ft}$.

08:27 Bottom in sight.

08:31 On bottom, depth $2262 \mathrm{ft}$., Temp. 5.99, visibility $25 \mathrm{ft}$., current $0.1 \mathrm{kt}$. at 220 degrees. Soft brown pock marked mud. New course of 275 degrees to SOL.

08:34 All stop. At SOL, get underway at 140 degrees, range $1800 \mathrm{ft}$. to WP 1. Fix $28-13.8670 \mathrm{~N} 89-42.4670 \mathrm{~W}$.

08:38 Across wide drag marks, $3 \mathrm{ft}$. wide, not very deep.

08:39 Soft mud bottom with pock marks, up shallow slope. Depth $2254 \mathrm{ft}$.

08:41 Light shell hash and bacterial mats.

08:48 Edge of depression, old mud volcano. Has shell hash and bacterial mats. Fix $28-13.8469 \mathrm{~N} 89-42.4109 \mathrm{~W}$.

08:53 Another wide track mark, $1 \mathrm{~m}$ wide, $0.5 \mathrm{~m}$ deep, crossing perpendicular to sub track., back into regular pock marked mud.

08:56 Small terrace about $2 \mathrm{~m}$ wide, another about $10 \mathrm{~m}$. wide, heading down, depth $2213 \mathrm{ft}$.

08:56 Fix (ship) 28-13.82N 89-42.44 W(tracking problems, fix from ship).

09:02 Punch core sample of bacterial mat in tube \#1, Fix 28-13.7296N 89$42.3210 \mathrm{~W}$, depth $2223 \mathrm{ft}$.

09:09 Forget punch core, silted in. no visibility, back underway at 140 degrees.

09:12 Course change, heading 040 degrees (tracking now working). Setting down for core, push core tube \#2. Depth $2232 \mathrm{ft}$., Fix 9: 28$13.6284 \mathrm{~N} 89-42.2415 \mathrm{~W}$

09:17 Getting underway course 035 degrees. 
MC 709, Dive 4412 (cont.)

09:20 New course of 130 degrees, range $1200 \mathrm{ft}$.

09:28 Found small depression, will try to sample mussels and bacterial mat.

Fix 10: 28-13.6011N 89-42.1661W, depth $2232 \mathrm{ft}$.

09:38 Suction sample of clams and mussels, Fix 11: 28-13.6050N 89$42.1433 \mathrm{~W}$, depth $2225 \mathrm{ft}$. (Photo of fathometer - oil seep).

09:53 Two push cores, short push Core (\#3), also taking a carbonate sample. The two initial push cores were long ones, no numbers given. No carbonate sample taken.

09:58 Underway bearing 140 degrees.

10:00 Pockmarks, bacterial mats, and scattered shells (can't get fix).

10:03 Underway at 150 degrees, stopped to sample holothurian, but encouraged to get back underway. (Photo of plume on fathometer).

10:12 At WP 2, Course 125 degrees, range $700 \mathrm{ft}$. to WP 3.

10:18 Going downhill, depth $2269 \mathrm{ft}$., change course to 115 degrees.

10:20 Coming over the edge of a large circular depression about $30 \mathrm{~m}$ in diameter., shell hash and possibly a brine pool, depth $2280 \mathrm{ft}$.

10:28 Change course to 100 degrees.

10:30 At WP 3, new course of 130 degrees, range $2255 \mathrm{ft}$. to EOL.

10:31 Bottom small pock marked fine mud, shallow upslope, depth $2258 \mathrm{ft}$.

10:33 Coming over a big hole with steep sides, $30 \mathrm{~m}$ diameter and $5 \mathrm{~m}$ deep.

10:41 Depression between 2 ridges, now slight upslope of about 15 degrees, every 15 or $20 \mathrm{~m}$ are small depressions with nothing inside.

10:44 Depth $2267 \mathrm{ft}$., small mud vent.

10:49 Course change to 115 degrees.

10:52 Depth $2292 \mathrm{ft}$., been on a shallow downslope.

10:54 Depth $2302 \mathrm{ft}$., on 15 degree downslope. Change course to 090 degrees.

10:56 At bottom of slope, depth $2332 \mathrm{ft}$.

10:58 All stop, geotechnical punch core, depth $2329 \mathrm{ft}$., Fix 28-13.0739N 89-41.4570W.

11:03 Lost up and down on arm.

11:05 Permission to leave bottom.

11:38 On surface. 


\section{709, Dive 4413}

Crew: Front, Dan Boggess, Harry Roberts.

Rear, Frank Lombardo, Laura Laham

Purpose: Find vent site, install and retrieve pore water sampler, survey and take stills, video, grab samples and push cores. Will launch at WP 1 on Traverse 1 and search for vent.

15:38 Launch, permission to dive. Fix 1: $28-13.6669 \mathrm{~N} 89-42.2072 \mathrm{~W}$ 15:43 Murky layer at about $250 \mathrm{ft}$.

15:53 Get underway at 075 degrees.

15:56 Change course to 060 degrees.

15:58 Lot of turbidity in water at $1400 \mathrm{ft}$.

16:00 Change course to 030 degrees.

16:04 Drop straight down.

16:11 Bottom in sight.

16:14 On bottom, depth $2231 \mathrm{ft}$., Temp. 6.85 , visibility $30-35 \mathrm{ft}$., current 0.2

kt. from N, Fix 2: 28-13.6530N 89-42.2089W Landed at brine seeps.

Punch core tube \#4.

16:25 Got punch core, heading of at 030 degrees.

16:29 Haven't found seep site, found tube worms, mussels, depth $2329 \mathrm{ft}$., Deploying pore water sampler.

16:39 Tripped handles, closed $1^{\text {st }}$. Sitting for 28 more minutes.

17:07 30 seconds to go, will move in, pick up pore water sampler and bring back on board. Fix 4: 28-13.6837N 89-42.1965W

17:22 Have sampler back on board, closing valves.

17:34 Got scoop of sediment in bucket \#3, will head off into current (330 degrees), will take punch core sample. (Plume on fathometer, but appears to be sub and not seep, took photo), Fix 28-13.64N 89$42.22 \mathrm{~W}$.

17:41 Heading to plume, $300 \mathrm{ft}$. at 200 degrees.

17:56 Depth $2228 \mathrm{ft}$., stopped to sample carbonate rock, Fix $28-13.6661 \mathrm{~N}$ 89-42.1850W. Did not sample.

18:00 Bearing 225 degrees, range $140 \mathrm{ft}$. to plume.

18:03 On site, will look around.

18:08 Sample rocks and clams, fix 28-13.6448N 89-42.2209W, depth 2230 $\mathrm{ft}$. 
MC 709, Dive 4413 (cont.)

18:24 Will stop and take grab samples of mussels and clams, depth $2232 \mathrm{ft}$., fix $28-13.6531 \mathrm{~N} 89-42.2707 \mathrm{~W}$

18:45 Time to wrap it up, attempting to sample rock.

18:47 Depth $2231 \mathrm{ft}$., request permission to leave bottom, fix $28-13.6540 \mathrm{~N}$ 89-42.2664W

19:13 Permission to surface.

19:15 Surface in sight, surface clear.

19:16 On surface.

\section{Tuesday, June 4}

\section{118, Dive 4414}

Crew: Front, Craig Caddigan, Harry Roberts

Rear, Hugh Marrero, Tyler Hodges

Purpose: Survey Traverse 1, sample.

07:57 Launch, permission to dive. Fix 1: 28-51.3198N 88-29.8819W

08:04 Going through layer of turbid water at $400 \mathrm{ft}$.

08:11 Get underway at 340 degrees.

08:22 Course change to 030 degrees, range $650 \mathrm{ft}$.

08:29 Course change to 040 degrees, range $260 \mathrm{ft}$. Bottom showing at 2200

$\mathrm{ft}$.

08:31 Course change to due $\mathrm{N}$, range $180 \mathrm{ft}$.

08:34 Drop straight down, within $50 \mathrm{ft}$.

08:38 Bottom in sight

08:39 On bottom, depth $2869 \mathrm{ft}$., Temp. 5.74 , visibility 20-25 ft., current 0.3 to $0.4 \mathrm{kts}$. at 050 degrees. Fix $2: 28-51.4457 \mathrm{~N} 88-29.9005 \mathrm{~W}$.

08:40 Get underway on course of 080 degrees, range $300 \mathrm{ft}$. to SOL. Bottom is featureless mud with creature depressions

08:46 At SOL, Depth $2873 \mathrm{ft}$., same bottom, much suspended sediment in water column, get underway at 140 degrees, Fix $3: 28-51.4516 \mathrm{~N} 88$ 29.8469W.

08:55 Depth $2885 \mathrm{ft}$., bottom same.

09:03 Depth $2915 \mathrm{ft}$., down slight slope, visibility worse (15 to $20 \mathrm{ft}$.), course change to 120 degrees, range $1300 \mathrm{ft}$.

09:09 Course change to 100 degrees. 
MC 118, Dive 4414 (cont.)

11:27 Surface in sight, surface clear.

11:27 On surface.

\section{118, Dive 4415}

Crew: Front, Craig Caddigan, Harry Roberts Rear, Hugo Marrero, Howard Manlovitz

Purpose: Will start at EOL and proceed to WP 1 (amplitude), then search for vent site. Won't try for EOL.

15:29 Launch, permission to dive. Fix 1: 28:51.2402N 88-29.2909W

15:45 Get underway at 330 degrees, range $300 \mathrm{ft}$.

15:51 Drop straight down.

16:07 Bottom in sight.

16:08 On bottom, depth $2907 \mathrm{ft}$., Temp. 5.75, visibility 15-20 ft., current 0.2

to $0.3 \mathrm{kts}$. at 080 degrees. Fix $28-51.4325 \mathrm{~N} 88-29.3064 \mathrm{~W}$. Get

underway at 200 degrees, range $2000 \mathrm{ft}$. Lost pan and tilt on camera.

Seeing clams and bacterial mat.

16:14 Got camera back.

16:17 Depth $2903 \mathrm{ft}$., will stop for grab sample. Bottom is plain mud. Fix 28-51.3682N 88-29.3491W. Sample in Bucket \#9.

$16: 22$ Back underway at 200 degrees.

16:25 Back in plain old mud, depth $2908 \mathrm{ft}$.

16:30 Depth $2908 \mathrm{ft}$., Starting to see a few clam shells.

16:38 Depth $2899 \mathrm{ft}$., Some clam shells and small bacterial mats.

16:39 Just passed over site that looks like they worked this morning.

$16: 40$ Starting to see small rocks.

16:40 Depth $2895 \mathrm{ft}$., Fix 28-51.1585N 88-29.5148w, going down into a crater.

16:45 Depth $2908 \mathrm{ft}$., found gas vent with hydrates and a lot of oil. Will take sample. Fix 28-51.1377N 88-29.5170W.

16:59 Got gas sample. Trying to get hydrate sample. Tough to break.

17:04 Repositioned and kicked up a bunch of silt. Will sit a wait for it to

clear.
17:17 Unable to get hydrate sample. Got one small piece in jaws, but couldn't get it into container.

17:23 Lost arm. Shutting down to see if breakers kick back in. 
MC 118, Dive 4414 (cont.)

09:12 Depth $2914 \mathrm{ft}$., bottom same.

09:13 Course change to due E (090).

09:14 Starting to see a few dead shells, starting up slope slightly, depth $2900 \mathrm{ft}$.

09:15 Starting to see a few small rocks, depth $2898 \mathrm{ft}$., will take sample.

Fix 4: $28-51.1394 \mathrm{~N} 88-29.5820 \mathrm{~W}$. Grab sample in bucket \#4.

09:19 Getting back underway at 090 degrees.

09:20 will follow up slope, about 080 degrees.

09:21 Just over a small rise, now dropping back down (photo of plume on fathometer).

09:24 At bottom of the feature, more shell hash.

09:24 Will set down for punch core, depth $2912 \mathrm{ft}$., Fix 5: 28-51.1319N $88-29.5502 \mathrm{~W} .2$ punch cores and 1 grab sample. Oil coming out of bottom of $2^{\text {nd }}$ punch core.

09:37 Found small crater, gas seep with hydrates in the side at depth of $2915 \mathrm{ft}$. Fix 5: 28-51.1409N 88-29.5361W. Will attempt to sample gas. (Photo of fathometer).

09:55 Gas sample successful, also got punch core and small rock, will look around for more hydrates.

09:56 Lost camera, lights out, green light blinking. No pan and tilt.

10:00 Got camera back.

10:02 Depth $2902 \mathrm{ft}$., stop for gorgonian and rock sample.

10:13 Range 200 feet, bearing 200 degrees to middle of anomaly.

10:14 Depth $2898 \mathrm{ft}$., taking push core through bacterial mat. Fix 7: 28$51.1615 \mathrm{~N} 88-29.4769 \mathrm{~W}$.

10:21 Course 130 degrees, range $1500 \mathrm{ft}$. to WP 2 .

10:28 Depth $2922 \mathrm{ft}$., out of shell hash and back onto mud bottom.

10:30 Course change to 120 degrees, range $900 \mathrm{ft}$. depth $2932 \mathrm{ft}$., visibility $15-20 \mathrm{ft}$.

10:36 Course change to 110 degrees, range $400 \mathrm{ft}$.

$10: 38$ Course change to 090 degrees.

$10: 40$ Stopping at $2968 \mathrm{ft}$. to collect sea cucumber.

$10: 41$ Back underway at 070 degrees, range $300 \mathrm{ft}$.

10:43 Depth $2974 \mathrm{ft}$., starting upslope.

10:47 At WP 2, circular weak amplitude, depth $2970 \mathrm{ft}$., fix 28-50.9608N 88-29.2729W

10:48 Permission to leave bottom.

11:25 Permission to surface, depth $175 \mathrm{ft}$. 
MC 118, Dive 4415 (cont.)

17:28 Got arm back.

17:41 Lost arm again, waiting to reset breakers.

18:09 Got lid on and valve closed, will take grab sample and rock sample.

18:13 Course 195 degrees, $100 \mathrm{ft}$. from WP 1, bearing 210 degrees. $2800 \mathrm{ft}$. to EOL, getting underway.

18:17 Depth $2910 \mathrm{ft}$., sea fans and gorgonians.

18:20 Will take 2 punch cores. Time to wrap it up. Depth $2926 \mathrm{ft}$., fix 28-

$51.0942 \mathrm{~N} 88-29.5805 \mathrm{~W}$.

18:29 Permission to leave bottom. 
Task 4: Heat Flow and Temperature Studies at Bush Hill and other Hydrate Sites using the Submersible, Johnson Sea Link

\author{
Final Report
}

Principle Investigators:

James Brooks, TDI-Brooks International, Inc.

Ian MacDonald and Bernie Bernard, Geochemical and Environmental Research Group, Texas A\&M University, College Station, Texas, 77845

DOE Award DE-FC26-00NT40920 


\begin{abstract}
Natural gas hydrates occur on the sea floor of the Gulf of Mexico in outcropping mounds that also contain other minerals, such as carbonates, that have been precipitated by microbial activity. Temperature probes were inserted in one such mound and in the mud nearby by researchers from Texas A\&M University and TDI-Brooks International, Inc. Each probe contained two recording thermistors, one at the top to measure water temperature and one at the bottom to measure temperature in the sea-floor hydrate or mud. Measurements and timelapse photos were taken over about 11 months. Preliminary results of the experiment:

- show no dramatic changes in the size or shape of the mound or in the amount of gas being vented.

- Include mean temperatures of $7.87^{\circ} \mathrm{C}$ in the water and $7.81^{\circ} \mathrm{C}$ in both hydrate and mud.

- Temperatures in hydrate and mud lag behind water temperatures.
\end{abstract}

TABLE OF CONTENTS

TITLE PAGE
ABSTRACT
TABLE OF CONTENTS
GRAPHICAL MATERIALS
INTRODUCTION
EXECUTIVE SUMMARY
EXPERIMENTAL
RESULTS AND DISCUSSION
CONCLUSION
REFERENCES
ACRONYMS/ABBREVIATIONS

\title{
GRAPHICAL MATERIALS
}

Figure 4-1. Temperature in a hydrate mound

Figure 4-2. Temperature measurements

Figure 4-3. Fathometer image of gas seep

Figure 4-4. Device for measuring gas from seeps

Figure 4-5. Temperature and gas activity during passage of Loop Eddy

Figure 4-6. Graph of temperature data from Thermistor Probe \#1

Figure 4-7. Graph of temperature data from Thermistor Probe \#2

Figure 4-8. Short thermistor probe.

Figure 4-9. Long probe prior to deployment on Sea Link.

Figure 4-10. Thermistor probe deployment at GC 234

\section{INTRODUCTION}

Natural gas hydrates occur on the sea floor of the Gulf of Mexico in outcropping mounds that also contain other minerals, such as carbonates, that have been precipitated by microbial activity. Temperature probes inserted into such mounds have been used to collect data that can help define the extent of the hydrate stability zone (HSZ). This study was designed to explore locations and shifts in the HSZ at several sites of documented hydrate occurrence in the northern Gulf of Mexico. Measurements and time-lapse photos were taken over about 11 
months. Further analysis is expected to advance efforts to model and understand the thermal response (i.e. thermal conductivity) of exposed hydrate deposits.

Gas activity also has been observed, acoustically, by depth sounders mounted on surface vessels and by current meters located on the sea floor. Warm-water eddies detach from the principal current in the Gulf of Mexico, the Loop Current, and drift westward along the Louisiana-Texas continental slope causing increases in bottom-water temperature that have been observed to coincide with increases in gas activity.

\section{EXECUTIVE SUMMARY}

Natural gas hydrates occur on the sea floor of the Gulf of Mexico in outcropping mounds that also contain other minerals, such as carbonates, that have been precipitated by microbial activity. Temperature probes were inserted in one such mound (fig.4-1) and in the mud nearby by researchers from Texas A\&M University and TDI-Brooks International, Inc. Each probe contained two recording thermistors, one at the top to measure water temperature and one at the bottom to measure temperature in the sea-floor hydrate or mud. Measurements and timelapse photos were taken over about 11 months. The temperature measurements are presented graphically in fig.4-2. Preliminary results of the experiment are:

1) Photos show no dramatic changes in the size or shape of the mound or in the amount of gas being vented.

2) Mean temperatures were $7.87^{\circ} \mathrm{C}$ in the water and $7.81^{\circ} \mathrm{C}$ in both hydrate and mud.

3 ) Temperatures in hydrate and mud lag behind water temperatures.

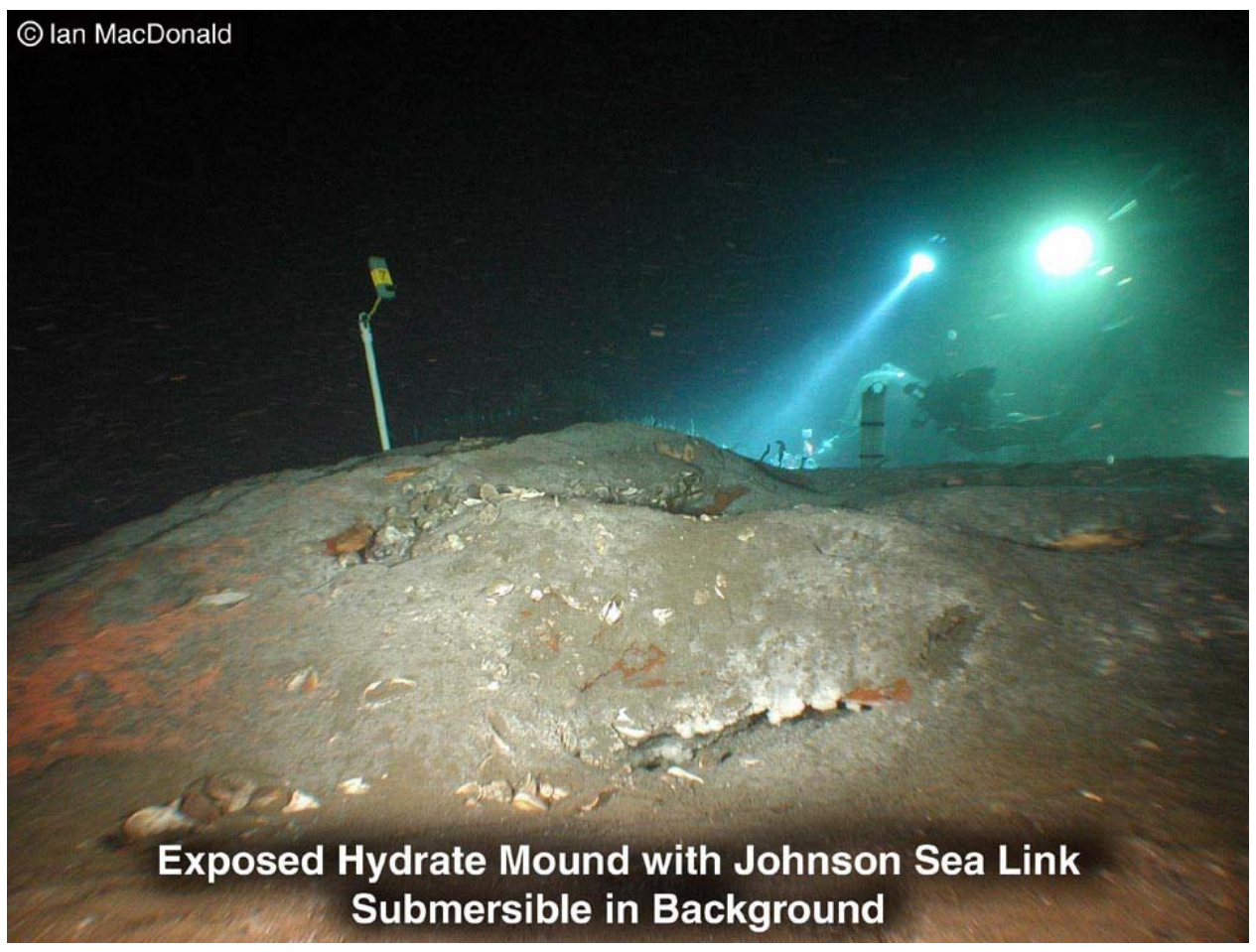

Figure 4-1. Temperature probe in a hydrate mound. 


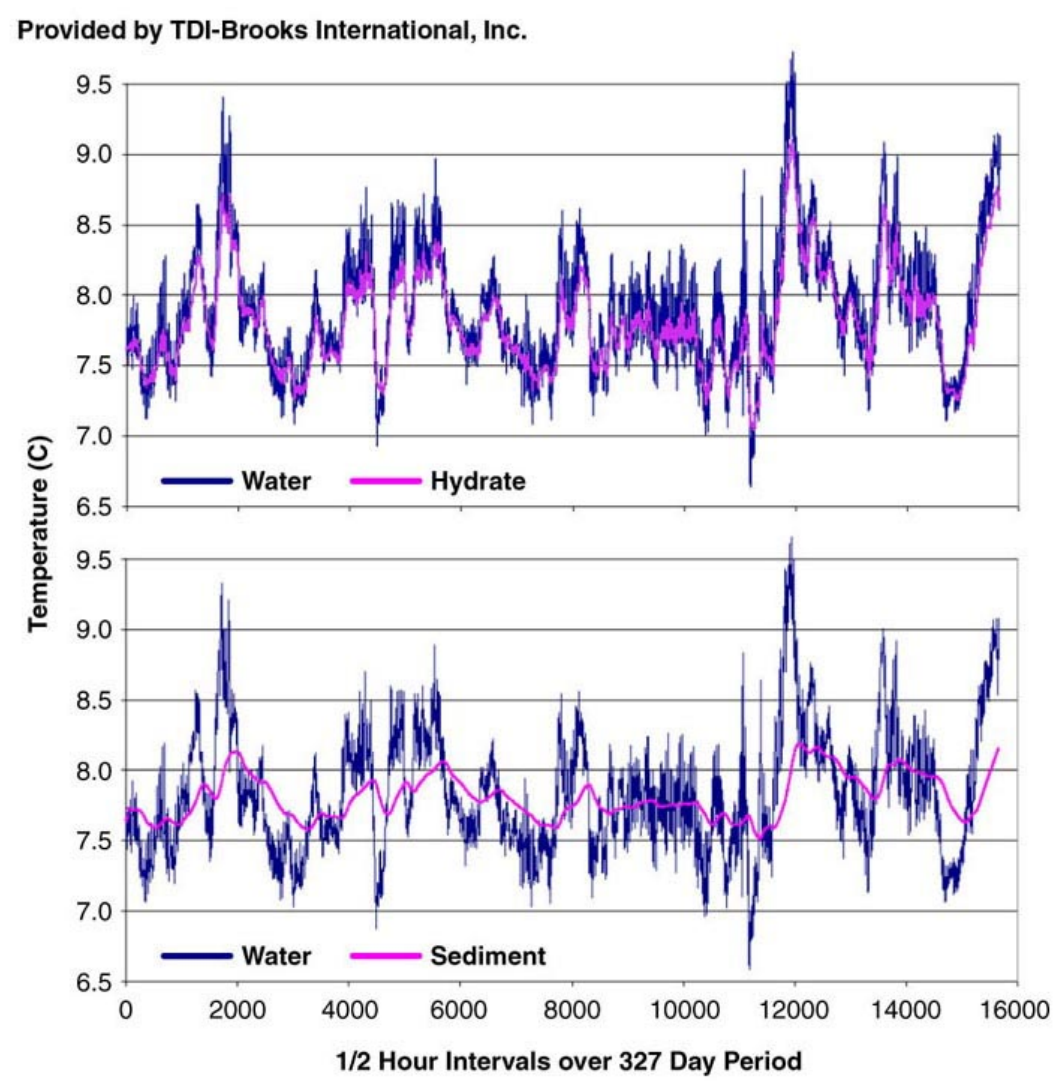

Figure 4-2. Temperature measurements from hydrate mound

Further analysis is expected to advance efforts to model and understand the thermal response (i.e. thermal conductivity) of exposed hydrate deposits.

Gas activity also has been observed acoustically by depth sounders mounted on surface vessels (fig.4-3) and by current meters located on the sea floor (fig.4-4). Warm-water eddies detach from the principal current in the Gulf of Mexico, the Loop Current, and drift westward along the Louisiana-Texas continental slope. These cause increases in bottom-water temperature that have been observed to coincide with increases in gas activity (fig.4-5). 


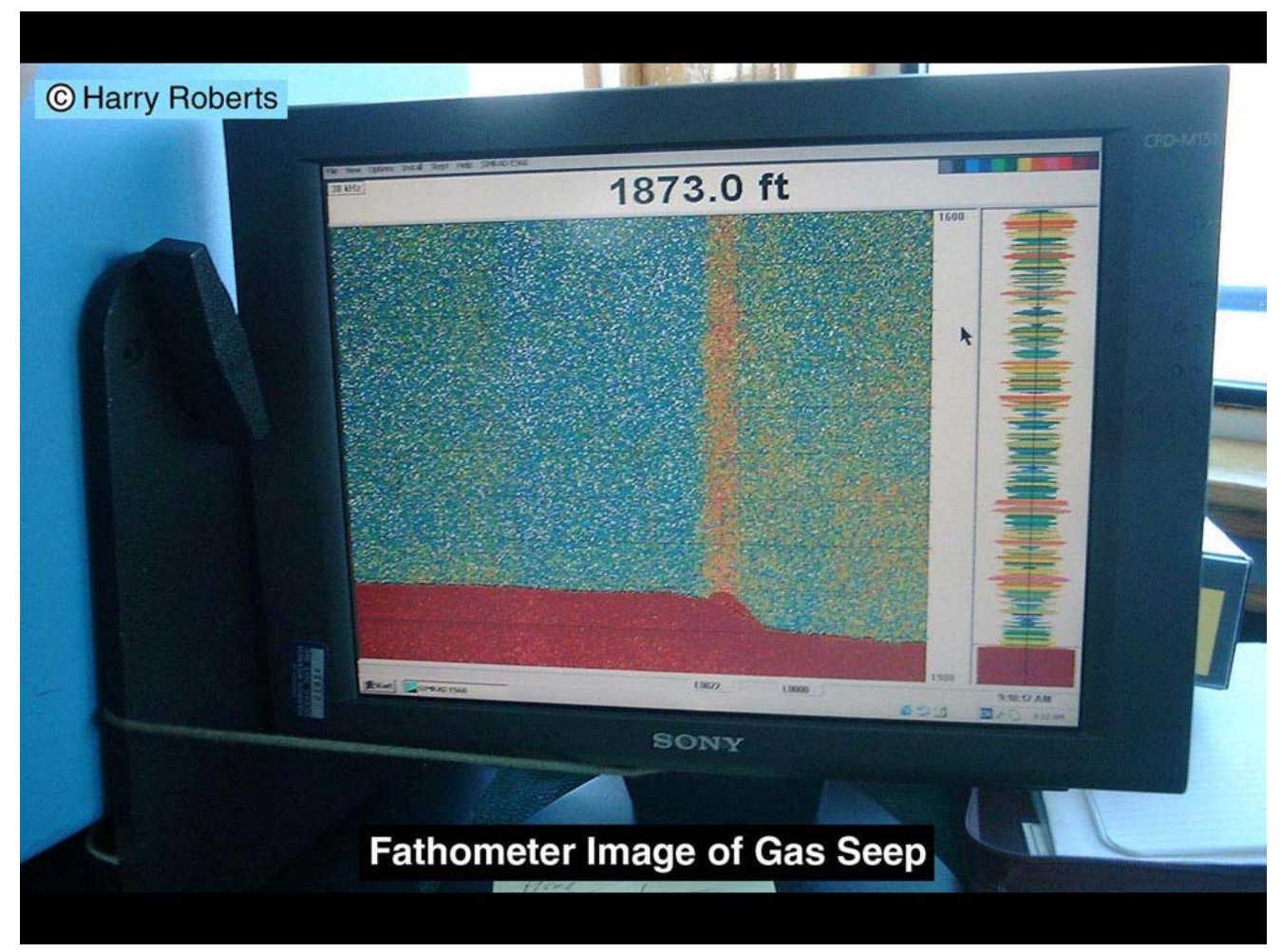

Figure 4-3. Fathometer image of gas seep

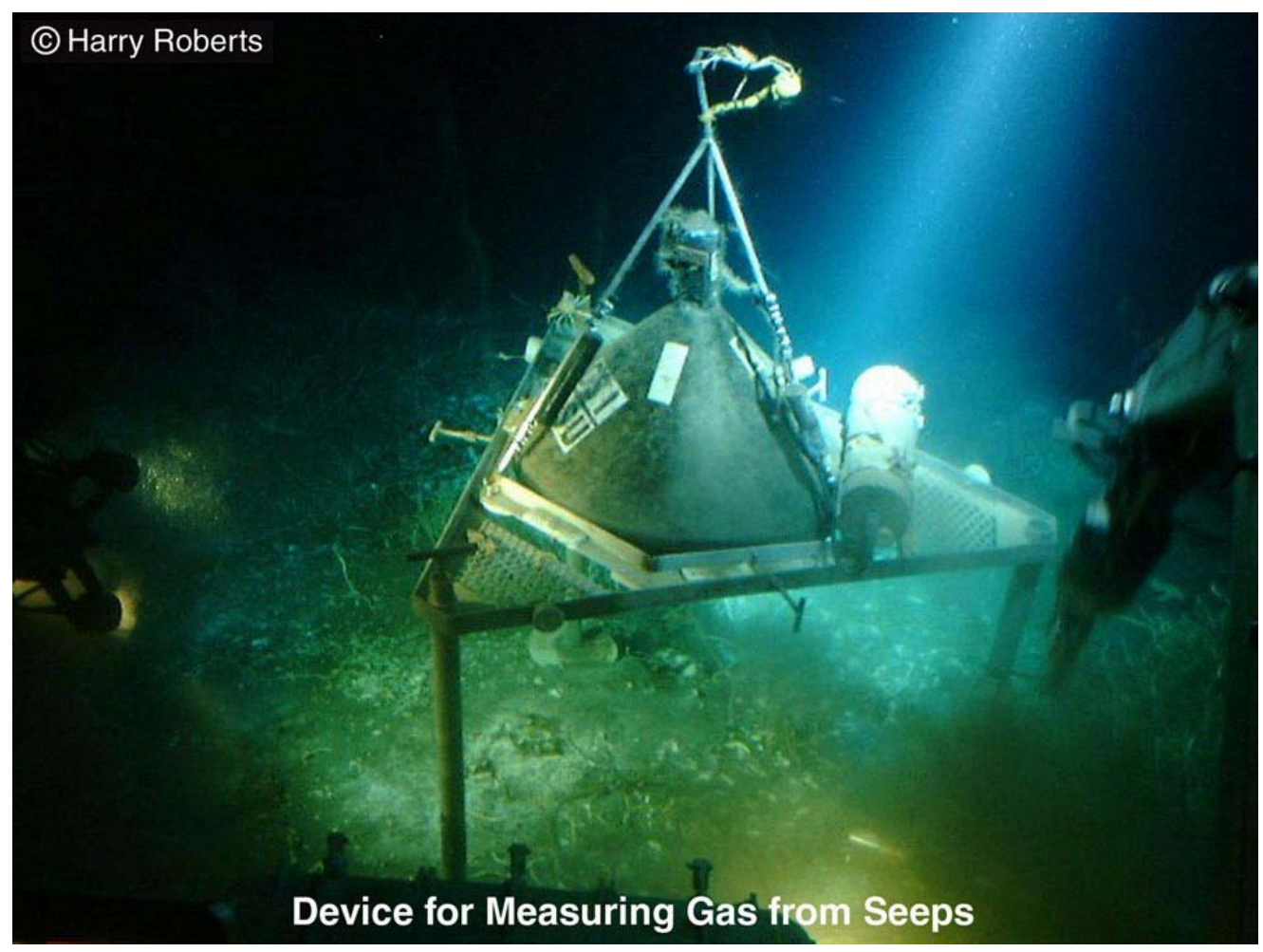

Figure 4-4. Device for measuring gas from seeps 


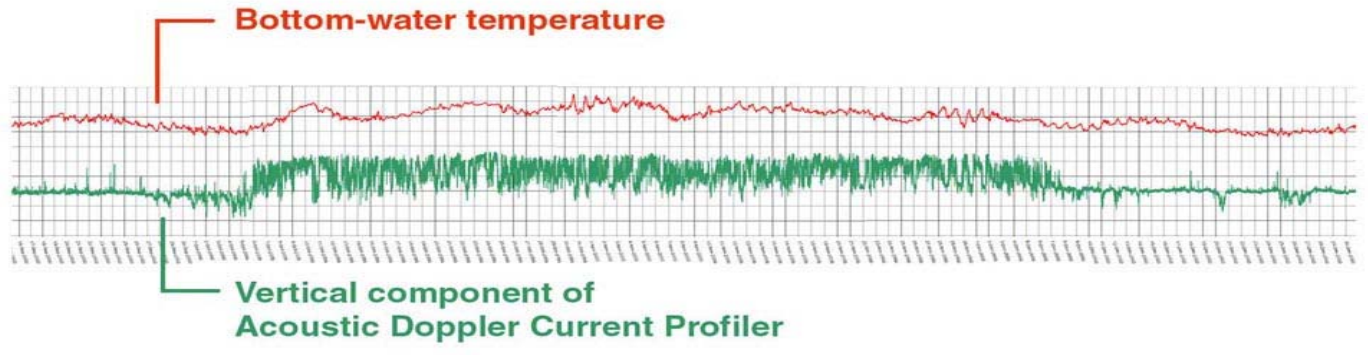

Temperature and Gas Activity During Passage of Loop Eddy (Re: Harry Roberts)

Figure 4-5. Temperature and gas activity During passage of Loop Eddy 


\section{EXPERIMENTAL}

OVERVIEW OF TEMPERATURE LOGGER DEPLOYMENTS/RECOVERIES AT "BUSH HILL” ON 6-14 JUNE CRUISE

Sixteen (16) Antares temperature loggers (thermistors) were previously deployed in July 2001 at "Bush Hill" and at GC-233/234 using the Johnson SEA-LINK during dives funded to Dr. lan MacDonald. Eight (8) temperature loggers were placed in four (4) short PVC probes (each of which could hold two thermistors) which were placed in hydrate or sediment. Two (2) of these hydrate probes were placed on "Bush Hill" and two (2) were placed in GC-233 hydrate areas. The remaining eight (8) were placed in two longer probes and inserted into a brine pool, but only four of these will be recoverable due to a mishap during deployment.

This report describes the recovery of the two (2) sets of probes from "Bush Hill" and the deployment of two new probes during this 6-14 June cruise. Additional probes were recovered and re-deployed on a subsequent SEA-LINK cruise on 1-22 July 2002.

Thermistor Probes 1 and 2 were recovered from Bush Hill (GC-185) during Dive \#4416, and temperature data from Antares data loggers \#33, 34, 41 and 42 was removed and downloaded. 15,693 data points were collected over the period of deployment from 7/16/01 to $6 / 8 / 02$, with a sampling frequency of 30 minutes. Probe 1 had been placed in hydrate, with thermistor \#33 buried 7-cm into a hydrate drill hole and thermistor \#34 exposed to the water (Fig. 4-6). Probe 2 was positioned so that thermistor \#42 was buried in $49 \mathrm{~cm}$ of sediment and thermistor \#41 was exposed to the water (Fig. 4-7).

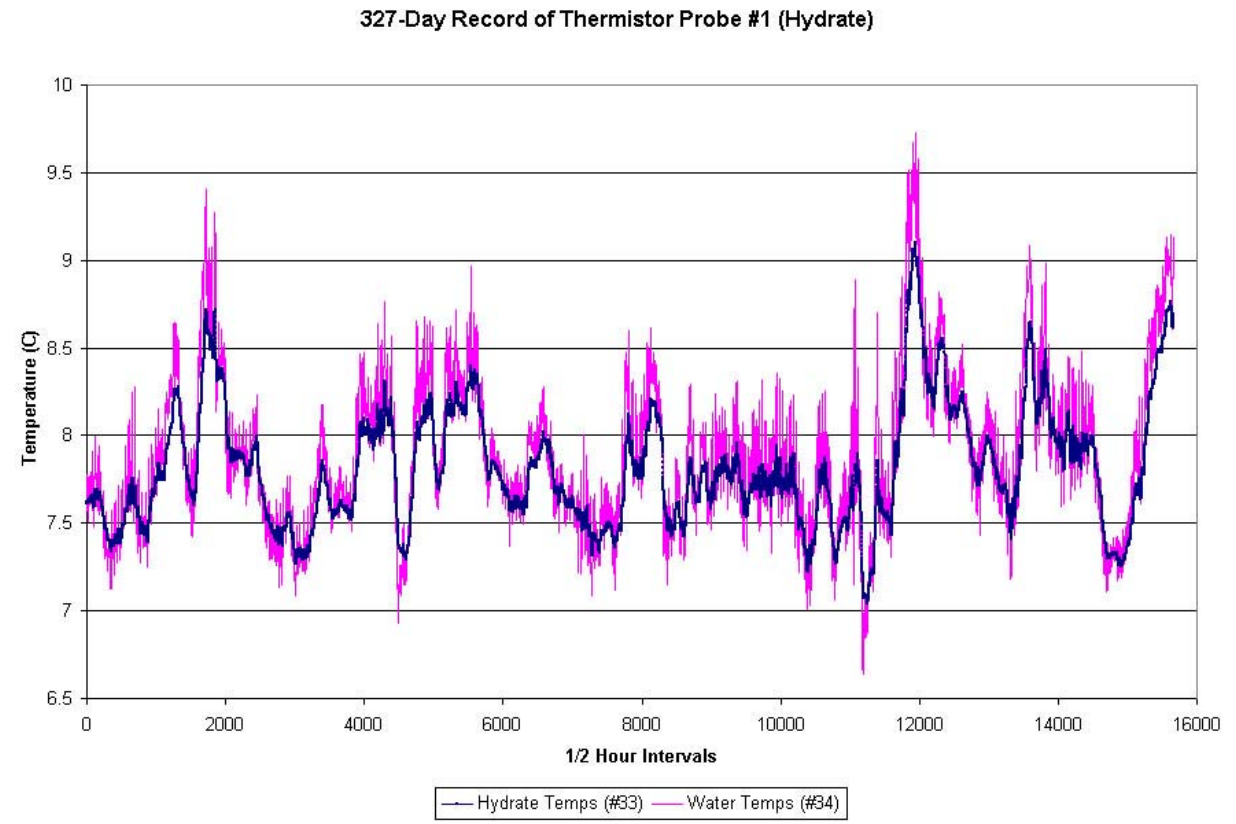

Fig. 4-6. Graph of temperature data from Thermistor Probe 1. 


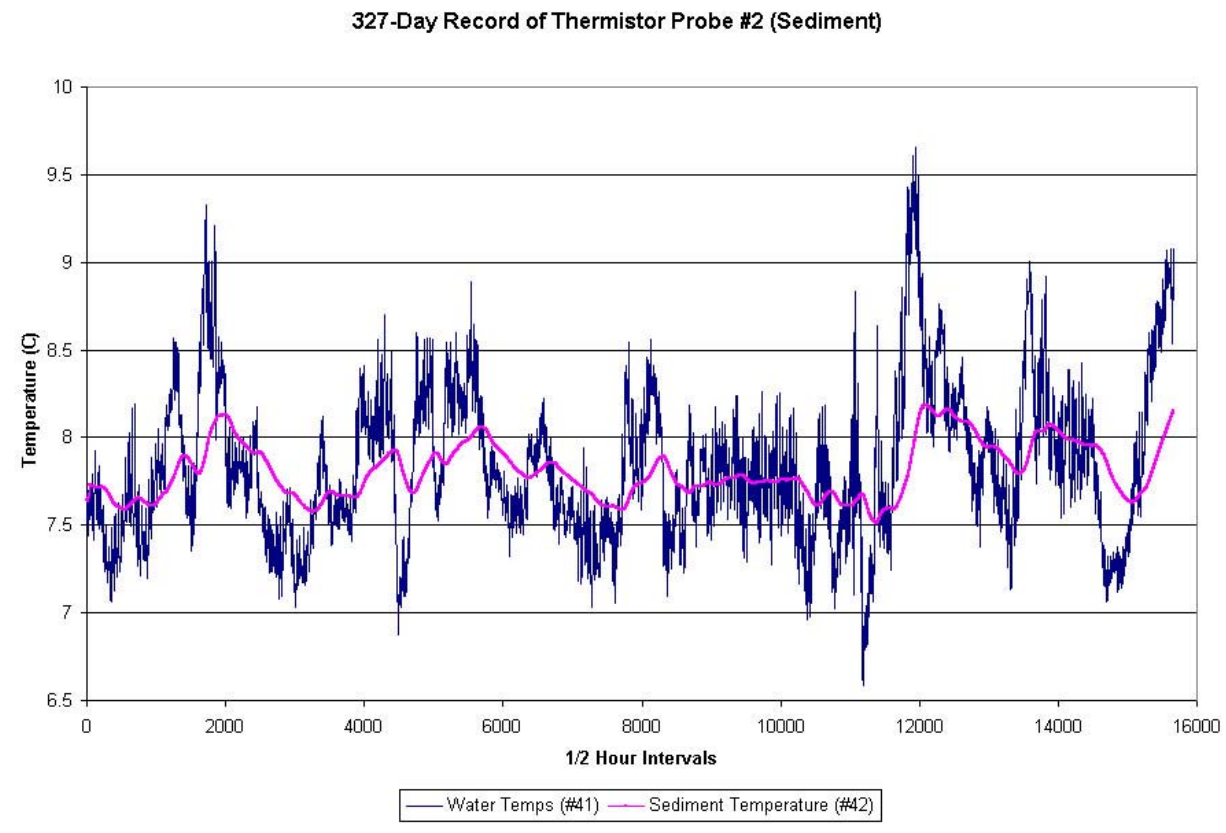

Fig. 4-7. Graph of temperature data from Thermistor Probe 2.

The thermistors were recovered in good condition, except for one which had some corrosion on the case. The batteries were still holding charge, but as they had been running for nearly a full year it was decided that replacing them would be prudent.

Five (5) new data loggers were provided for redeployment during the 6-14 June SEALINK cruise. The thermistors were \#88, 89, 91, 102 and 103. During an inspection of the thermistors and a check of the setup, \#103 stopped working. It was able to record data and be recognized by the download cradle, but after the date/time was reset it suddenly deactivated and was unresponsive after that. The other loggers worked perfectly. They were calibrated during Dive \#4416 by activating them to sample once a second and placing them in a bucket attached to the submersible.

Probes 7 and 8 (of the same design as Probes 1 and 2) were deployed during Dive \#4419 on 6/9/02. Probe 7 contained thermistors \#88 (bottom) and 89 (top), and Probe 8 contained thermistors \#91 (bottom) and 102 (top). The loggers were started at 06:00 on 6/9/02, sampling every 70 seconds for 1,242 hours (the maximum allowed by the internal memory), lasting until 8/1/02 at 00:00. The Johnson SEA-LINK submersible placed the probes in two new holes in the same hydrate mound that Probe 1 had been deployed in previously. Probe 7 was inserted $8 \mathrm{~cm}$ into the drill hole, but Probe 8 was only able to penetrate about $6 \mathrm{~cm}$ into the second hole. Probe 8 was later removed from that hole during Dive \#4421 on 6/11/02 and redeployed $16 \mathrm{~cm}$ deep in sediment adjacent to an active methane and oil bubble stream. These probes will be recovered during the July 2002 SEA-LINK cruise and will provide a short-term, high-frequency sample to accompany the long-term, low frequency data obtained from the 2001 thermistor deployment. 


\section{FIELD REPORT JOHNSON SEALINK CRUISE 2001}

During the JSL 2001 LEXEN cruise we deployed a total of 16 ANTARES High

Resolution Temperature Data Loggers, or thermistors, on the sea floor in the Gulf of Mexico. These are titanium-cased cylindrical temperature sensors, $185 \mathrm{~mm}$ long, $120 \mathrm{~g}$ in weight, with an internal $3 \mathrm{~V} \mathrm{DC}$ battery. The main body of the thermistor is $15 \mathrm{~mm}$ in diameter, and the sensor is at the tip of a $20 \mathrm{~mm}$ long $\times 1 \mathrm{~mm}$ diameter projection, which extends out of the center of one end. They are pressure rated up to 9000 psi and have a measuring range of $20^{\circ} \mathrm{C}$ to $50^{\circ} \mathrm{C}$ ( $-4 \mathrm{~F}$ to $122 \mathrm{~F}$ ), with $+/-0.1^{\circ} \mathrm{C}$ accuracy. The internal memory can hold up to 65000 data points, and the sampling interval is programmable using an ANTARES type 1855 Datalogger Programming Station and ANTARES WinTemp software. The programming station connects to a PC through a RS232 port, using a null modem cable, and holds the thermistor in a springloaded clamp. Galvanic coupling between the thermistor and the station allows both programming and data readout.

All 16 thermistors that were brought on the cruise (numbered 33 through 48) were calibrated by sending them down on Dive \#4306 in bucket \#3 on the submarine. They were activated at 07:00 on 7/4/01, collecting temperature data every 10 seconds for 5 hours. Data was collected on the return of the submarine and placed in an Excel spreadsheet. The data was interpreted using preset calibration files from ANTARES, and analyzed using S-Plus 2000 software. The calibration files for thermistors 45 and 48 were corrupted, so new ones were made using an old calibration file for thermistor 31 as a template. Each thermistor's temperature data was plotted against temperature data from another thermistor using a linear least squares fit, using thermistors that would be deployed together in a probe. A linear regression was then performed to calculate the standard error. The linear coefficients ranged from 0.9923 to 1.021 and the standard error varied from 0.0005 to 0.0017 . The mean linear coefficient was 1.003 , and the mean standard deviation was 0.001. (See attached data)

Each individual thermistor was placed inside a probe constructed of a hollow tube of Schedule 40 PVC. There were six probes constructed, in two configurations: four hydrate/sediment probes which were shorter, containing only two thermistors (Figure 4-8), and two longer brine pool probes which held four thermistors each (Figure 4-9). The short probes were about $55 \mathrm{~cm}$ long, with a $45 \mathrm{~cm}$ shaft and a T-pipe threaded onto the end. One thermistor was in the bottom of the tube, held in by a beveled plug on the bottom and a 1/4" nylon bolt threaded through the pipe above the other end of the thermistor. The tip of the temperature sensor fit into a hole in the plug and projected about $1 \mathrm{~mm}$ beyond the surface of the cap. The thermistor on top was also held up by a bolt, and extended through the T-pipe, with a spacer of plastic tubing between the edge of the thermistor and the small hole in the top, through which the sensor tip protruded about $1 \mathrm{~mm}$. A plastic float covered with a strip of reflective tape was attached to the T-pipe end of the probe with a short length of polypropylene line.

All of the thermistor deployments during the JSL 2001 LEXEN cruise are summarized in Table 1. The short probes were labeled one through four, and deployed on the sea floor. These probes were designed to be pushed into the sediment by the robotic arm of the submarine, or inserted into the drill holes left in the hydrate surface after collecting hydrate samples. This will allow temperature readings to be collected from the interior of the hydrate or sediment, and simultaneous readings to be taken from the ambient seawater. Probes \#3 (containing thermistors 46 and 47) and \#4 (containing thermistors 35 and 36)

were activated at $05: 00$ on $7 / 6 / 01$, sampling every 30 minutes for 32,500 hours (the maximum number of data points). They were deployed at the GC 234 site on 7/6/01 during Dive \#4310. Probe \#3 was wedged about $10 \mathrm{~cm}$ into a hydrate drill hole, while Probe \#4 was thrust about $49 \mathrm{~cm}$ into the sediment, up to the bottom of the T-pipe (see Figure 4-10). 


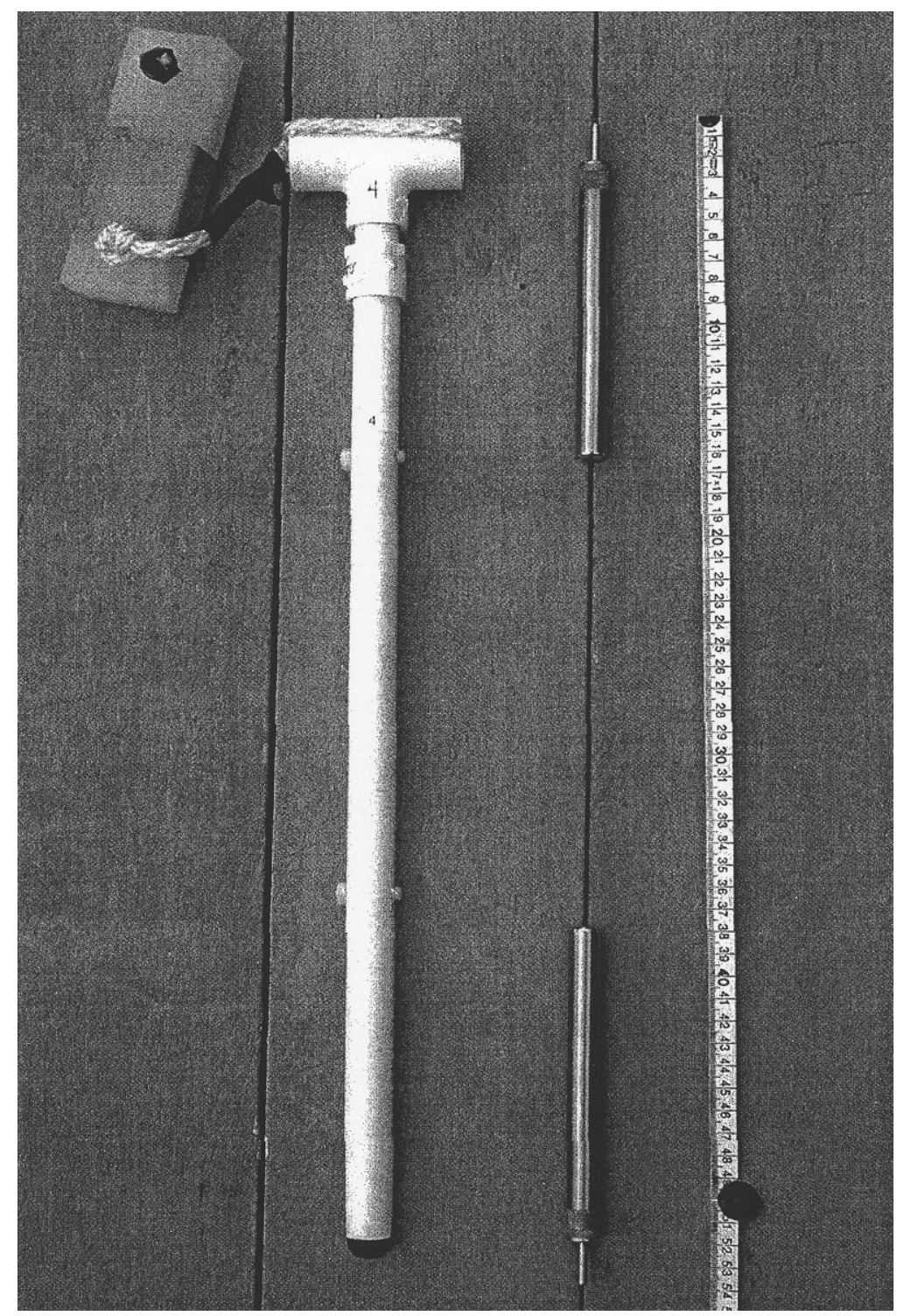

Figure 4-8. Short thermistor probe. From left: Assembled PVC probe, two thermistors showing relative position in probe, and meter scale.

Probes \#1 (containing thermistors 33 and 34) and \#2 (containing thermistors 41 and 42) were activated on $7 / 16 / 01$ at $13: 40$, sampling every 30 minutes for 32,500 hours. Dive \#4325 planted them on the seafloor at Bush Hill, but both thermistors were in hydrate drill holes. Dive \#4327 repositioned Thermistor \#2 so that it was pushed fully into the sediment next to the hydrate mound.

The probes intended for the brine pool were constructed of three individual PVC pipes, joined by slightly larger diameter pipe, which was glued with PVC cement and bolted together. Fully assembled, the probes were about 1.385 meters long. The first long probe constructed, Probe \#5, had thermistor \#45 at the bottom extending through the beveled plug and braced by a nylon bolt. Thermistor \#44 was in the middle section, which was open to the water through a T-connector, which joined that section of PVC pipe to the bottom segment. The top section, 
which had the beveled plug facing upwards, contained thermistor \#43. A plastic spring-loaded handle was clamped to the top section, and a length of rope attached to a yellow plastic float ball on one end and a $25 \mathrm{lb}$. weight attached to the other end, was fed through it. Thermistor \#48 was attached to the float. The weight was screwed to a wooden plate in order to prevent it from sinking into the mud bottom of the brine pool. When the submarine's arm closed on the handle, it could let out more slack on the line and move the probe up and down in relation to the weight. This way Probe \#5 could be deployed in the brine pool at a certain depth and measure the temperature below the brine surface, at the brine/seawater interface, at the ambient water level, and at the level of the float. The thermistors were all activated at 01:20 on $7 / 9 / 01$ and set to record a sample every 30 minutes for 32,500 hours. Probe \#5 was deployed during Dive \#4319 on 7/14/01. Unfortunately, the slope of the brine pool edge was steeper than anticipated: when released, the probe slid below the brine entirely, and is too deeply submerged in the pool to be retrieved.

Probe \#6 was constructed to replace \#5, and it had a similar design. Thermistor \#40 was at the bottom, and thermistor \#38 was in the middle segment, extending into a Tconnector. However, the top section is constructed like the smaller probes, with the beveled plug facing downwards and a T-pipe on top. Thermistor \#37 extended through the plug into another T-connector, and thermistor \#39 extended through the T on top, with a plastic tubing spacer keeping only $1 \mathrm{~mm}$ of the sensor tip protruding beyond the probe surface. Another spring-loaded handle was attached to the top section. In order to prevent this probe from meeting a fate similar to that of \#5, two $25 \mathrm{lb}$. weights were attached to the bottom plate to better anchor it, and a longer rope was strung between the float and the weights. The thermistors were activated at $14: 40$ on $7 / 14 / 01$, sampling every 30 minutes for 32,500 hours. This probe was deployed successfully during Dive \#4321, but it is in a shallow area of the brine pool and thus not recording the full range of temperatures anticipated. Only thermistor \#40 is submerged in the brine.

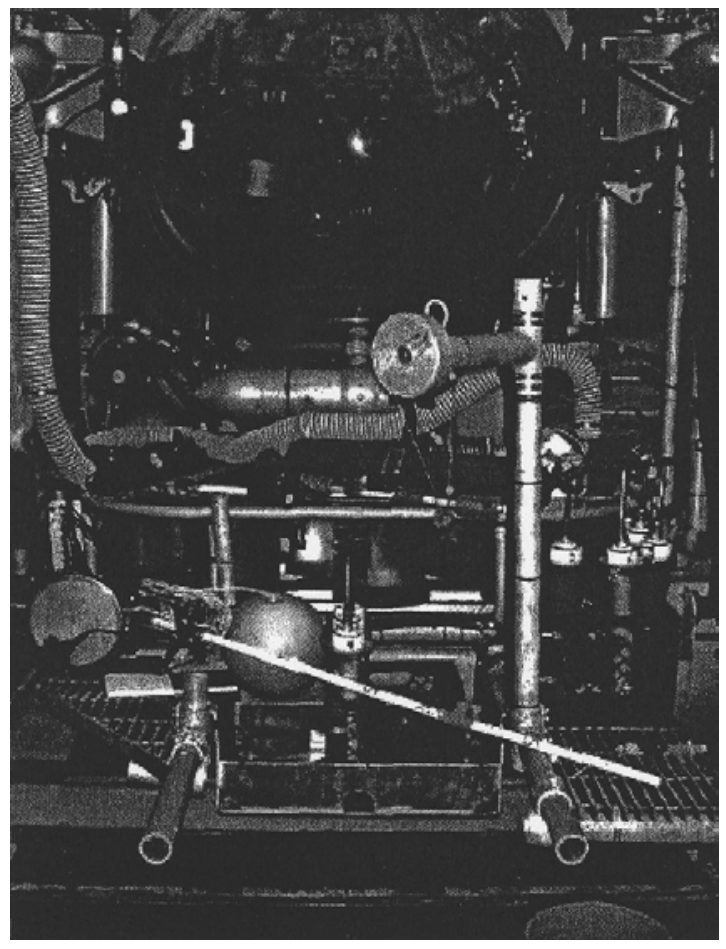

Figure 4-9. Long probe prior to deployment on Sea Link. 


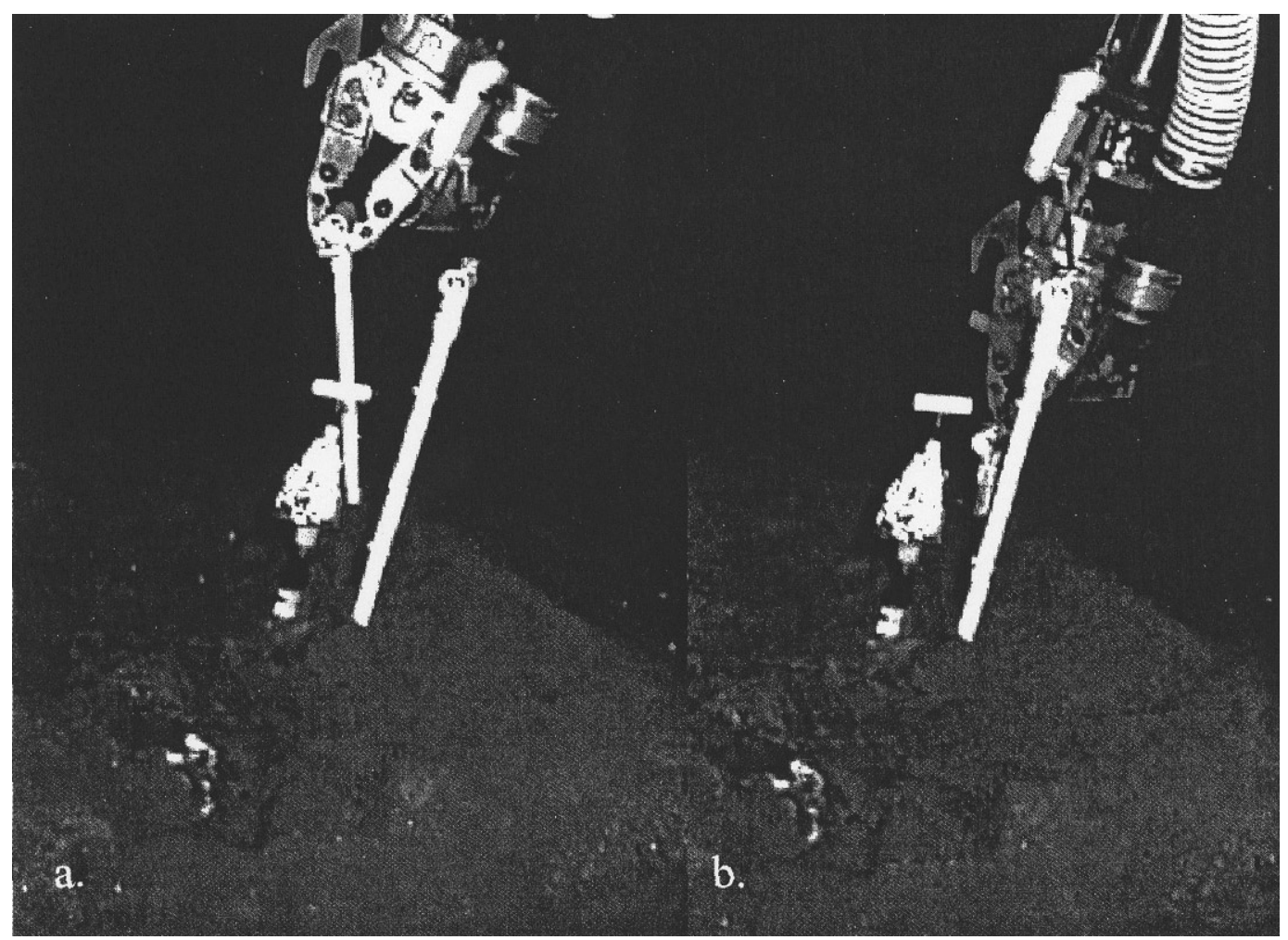

Figure 4-10a. Thermistor probe deployment at GC 234. Probe \#3 is in the foreground, in a hydrate drill hole, Probe \#4 is being pushed into the sediment by the robotic arm. Figure 4-10b shows the final position of Probe \#4. An agar plug is shown in the left foreground. 
Table 1. Summary of thermistors deployed during Johnson Sea Link 2001 Cruise

\begin{tabular}{|c|c|c|c|c|c|c|c|}
\hline $\begin{array}{c}\text { Probe Deployment } \\
\text { Site }\end{array}$ & $\begin{array}{c}\text { Probe } \\
\text { Number }\end{array}$ & $\begin{array}{l}\text { Length of } \\
\text { Probe }\end{array}$ & $\begin{array}{c}\text { Thermistor } \\
\text { Number }\end{array}$ & $\begin{array}{c}\text { Location in } \\
\text { Probe }\end{array}$ & $\begin{array}{c}\text { Date/Time of } \\
\text { Activation }\end{array}$ & \begin{tabular}{|c|} 
Sampling \\
Rate
\end{tabular} & $\begin{array}{c}\text { Dive \#, Date of } \\
\text { Deployment }\end{array}$ \\
\hline Bush Hill, hydrate & 1 & short, $55 \mathrm{~cm}$ & 33 & $\begin{array}{l}\text { Bottom, in } \\
\text { hydrate }\end{array}$ & $7 / 16 / 01,13: 40$ & $30 \mathrm{~min}$. & $\# 4325,7 / 16 / 01$ \\
\hline Bush Hill, hydrate & 1 & short, $55 \mathrm{~cm}$ & 34 & $\begin{array}{c}\text { Top, in } \\
\text { seawater }\end{array}$ & $7 / 16 / 01,13: 40$ & $30 \mathrm{~min}$. & $\# 4325,7 / 16 / 01$ \\
\hline Bush Hill, sediment & 2 & short, $55 \mathrm{~cm}$ & 41 & $\begin{array}{l}\text { Top, in } \\
\text { seawater }\end{array}$ & $7 / 16 / 01,13: 40$ & $30 \mathrm{~min}$. & $\begin{array}{c}\# 4325,7 / 16 / 01, \\
\text { repositioned 7/17/01, } \\
\# 4327 \\
\end{array}$ \\
\hline Bush Hill, sediment & 2 & short, $55 \mathrm{~cm}$ & 42 & $\begin{array}{l}\text { Bottom, in } \\
\text { sediment }\end{array}$ & $7 / 16 / 01,13: 40$ & $30 \mathrm{~min}$. & $\begin{array}{c}\# 4325,7 / 16 / 01, \\
\text { repositioned 7/17/01, } \\
\# 4327\end{array}$ \\
\hline GC 234, hydrate & 3 & short, $55 \mathrm{~cm}$ & 46 & $\begin{array}{c}\text { Top, in } \\
\text { seawater }\end{array}$ & 7/6/01, 05:00 & $30 \mathrm{~min}$. & $\# 4310,7 / 6 / 01$ \\
\hline GC 234, hydrate & 3 & short, $55 \mathrm{~cm}$ & 47 & $\begin{array}{c}\text { Bottom, in } \\
\text { hydrate }\end{array}$ & 7/6/01, 05:00 & $30 \mathrm{~min}$. & $\# 4310,7 / 6 / 01$ \\
\hline GC 234, sediment & 4 & short, $55 \mathrm{~cm}$ & 35 & $\begin{array}{c}\text { Top, in } \\
\text { seawater }\end{array}$ & 7/6/01, 05:00 & $30 \mathrm{~min}$. & $\# 4310,7 / 6 / 01$ \\
\hline GC 234, sediment & 4 & short, $55 \mathrm{~cm}$ & 36 & $\begin{array}{c}\text { Bottom, in } \\
\text { sediment }\end{array}$ & $7 / 6 / 01,05: 00$ & $30 \mathrm{~min}$. & $\# 4310,7 / 6 / 01$ \\
\hline Brine Pool - unrecoverable & 5 & long, $138.5 \mathrm{~cm}$ & 43 & Top & $7 / 9 / 01,01: 20$ & $30 \mathrm{~min}$. & $\# 4319,7 / 14 / 01$ \\
\hline Brine Pool - unrecoverable & 5 & long, $138.5 \mathrm{~cm}$ & 44 & Middle & $7 / 9 / 01,01: 20$ & $30 \mathrm{~min}$. & $\# 4319,7 / 14 / 01$ \\
\hline Brine Pool - unrecoverable & 5 & long, $138.5 \mathrm{~cm}$ & 45 & Bottom & $7 / 9 / 01,01: 20$ & $30 \mathrm{~min}$. & $\# 4319,7 / 14 / 01$ \\
\hline Brine Pool - unrecoverable & 5 & long, $138.5 \mathrm{~cm}$ & 48 & Float & $7 / 9 / 01,01: 20$ & $30 \mathrm{~min}$. & $\# 4319,7 / 14 / 01$ \\
\hline Brine Pool & 6 & long, $138.5 \mathrm{~cm}$ & 37 & Middle-top & $7 / 14 / 01,14: 40$ & $30 \mathrm{~min}$. & $\# 4321,7 / 15 / 01$ \\
\hline Brine Pool & 6 & long, $138.5 \mathrm{~cm}$ & 38 & Middle-bottom & $7 / 14 / 01,14: 40$ & $30 \mathrm{~min}$. & $\# 4321,7 / 15 / 01$ \\
\hline Brine Pool & 6 & long, $138.5 \mathrm{~cm}$ & 39 & $\begin{array}{c}\text { Top, in } \\
\text { seawater }\end{array}$ & $7 / 14 / 01,14: 40$ & $30 \mathrm{~min}$. & $\# 4321,7 / 15 / 01$ \\
\hline Brine Pool & 6 & long, $138.5 \mathrm{~cm}$ & 40 & Bottom, in brine & $7 / 14 / 01,14: 40$ & $30 \mathrm{~min}$. & $\# 4321,7 / 15 / 01$ \\
\hline
\end{tabular}


Probe \#1 - Thermistors 33 and 34

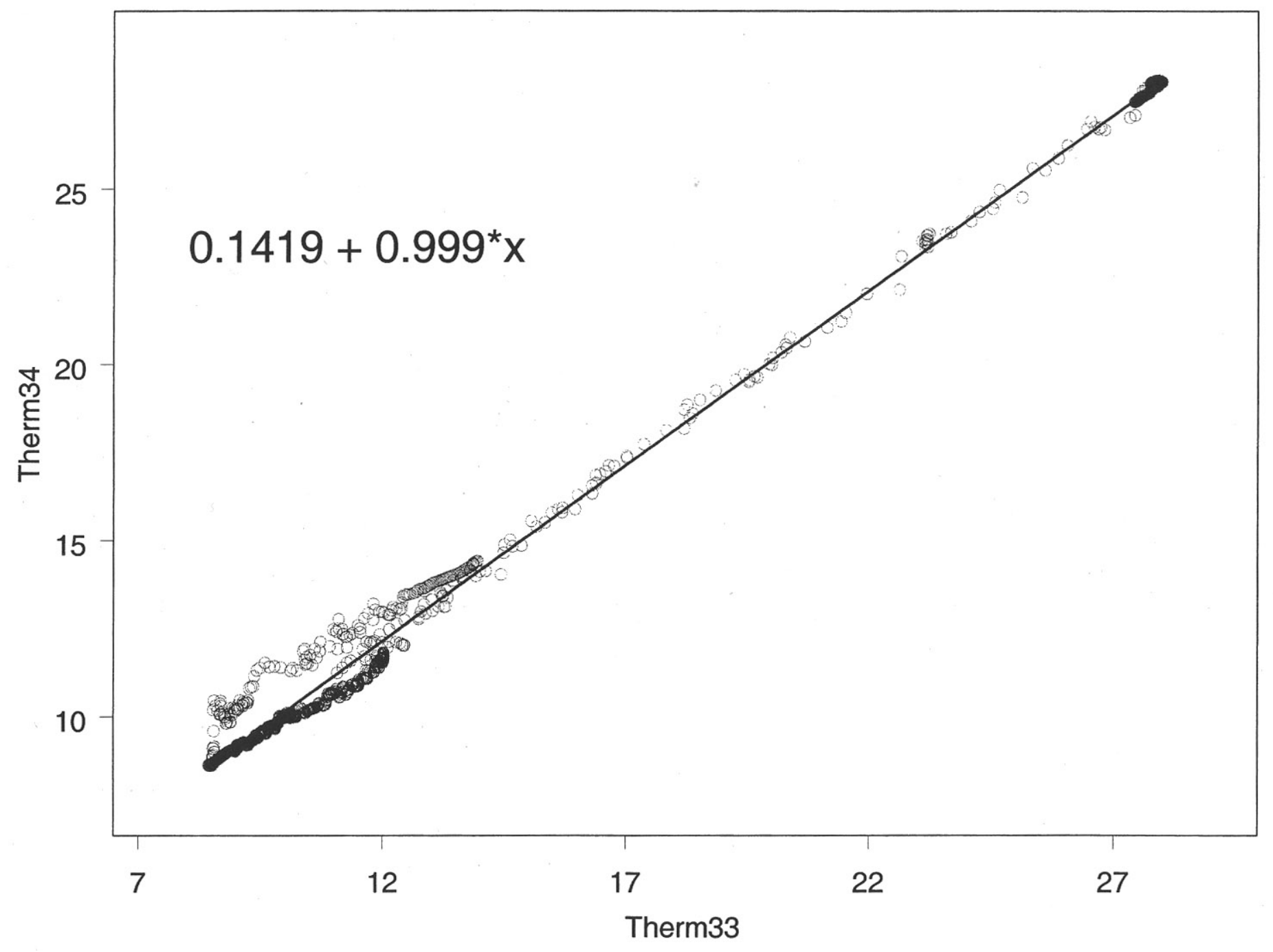


Standard Error - Thermistors 33 and 34

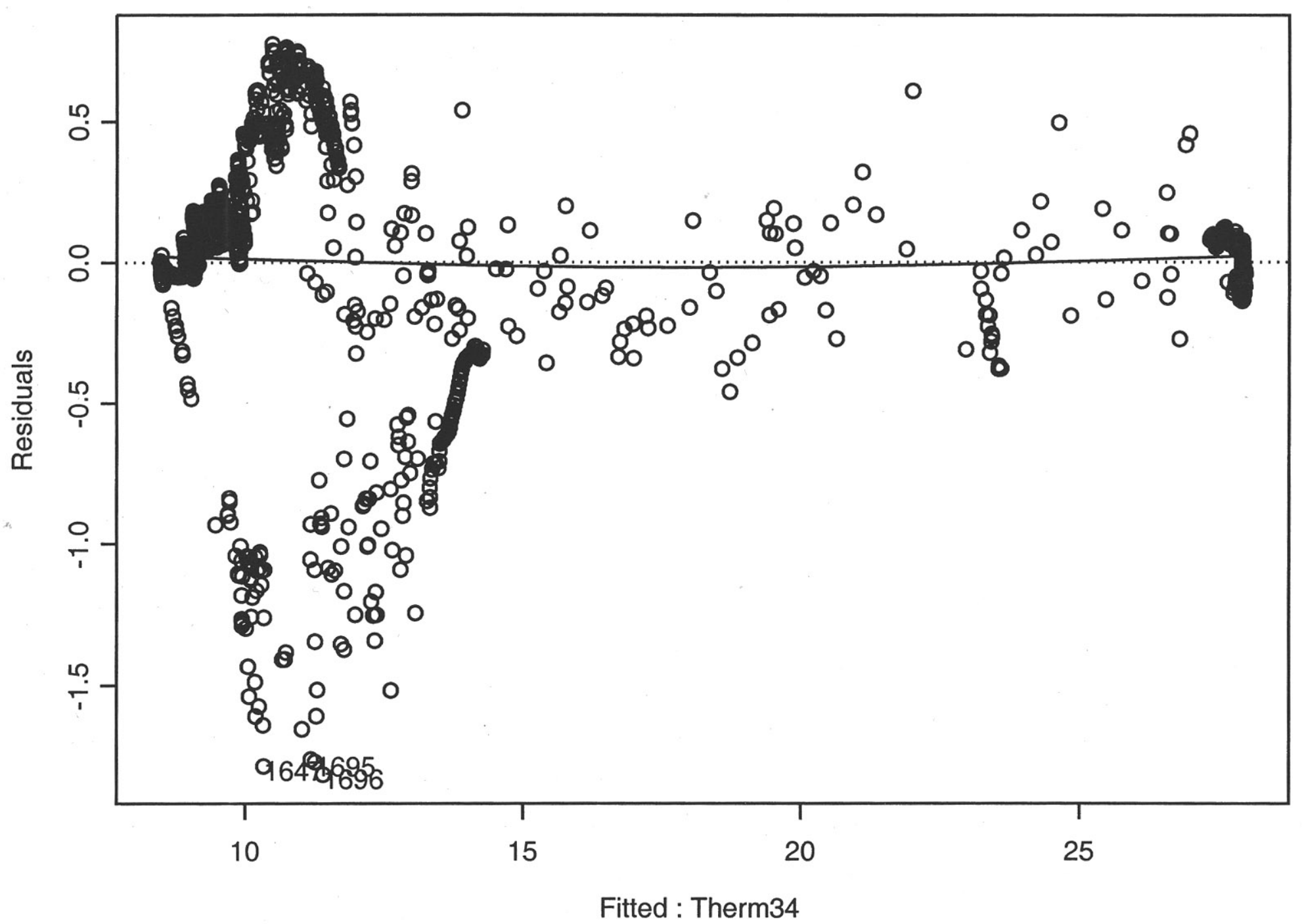


$\star * *$ Linear Model $* * *$

Call: $\operatorname{lm}$ (formula $=$ Therm33 $\sim$ Therm34, data = ThermistorCalibrationData, na. action $=$

na.exclude)

Residuals :

Min 10 Median $3 Q \quad$ Max

$\begin{array}{lllll}-1.814 & -0.04917 & 0.01692 & 0.109 & 0.7734\end{array}$

Coefficients:

$\begin{array}{rrrrr} & \text { Value std. Error } & t \text { value } & \operatorname{Pr}(>|t|) \\ \text { (Intercept) } & -0.1115 & 0.0184 & -6.0583 & 0.0000 \\ \text { Therm34 } & 0.9991 & 0.0010 & 958.4521 & 0.0000\end{array}$

Residual standard error: 0.362 on 1797 degrees of freedom

Multiple R-Squared: 0.998

F-statistic: 918600 on 1 and 1797 degrees of freedom, the p-value is 0

Thermistor $34=0.1419+0.999^{*}$ Thermistor 33 


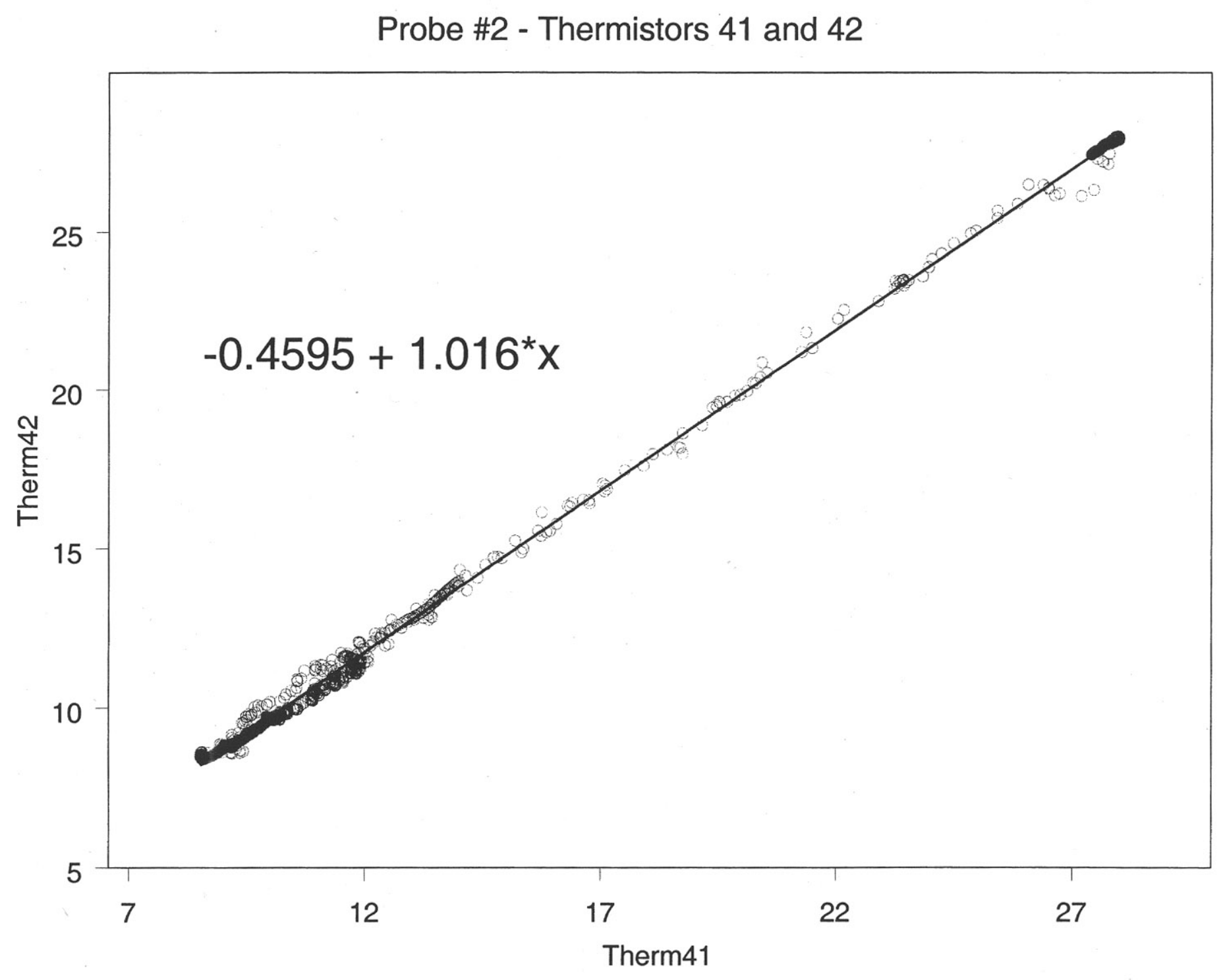


Standard Error - Thermistors 41 and 42

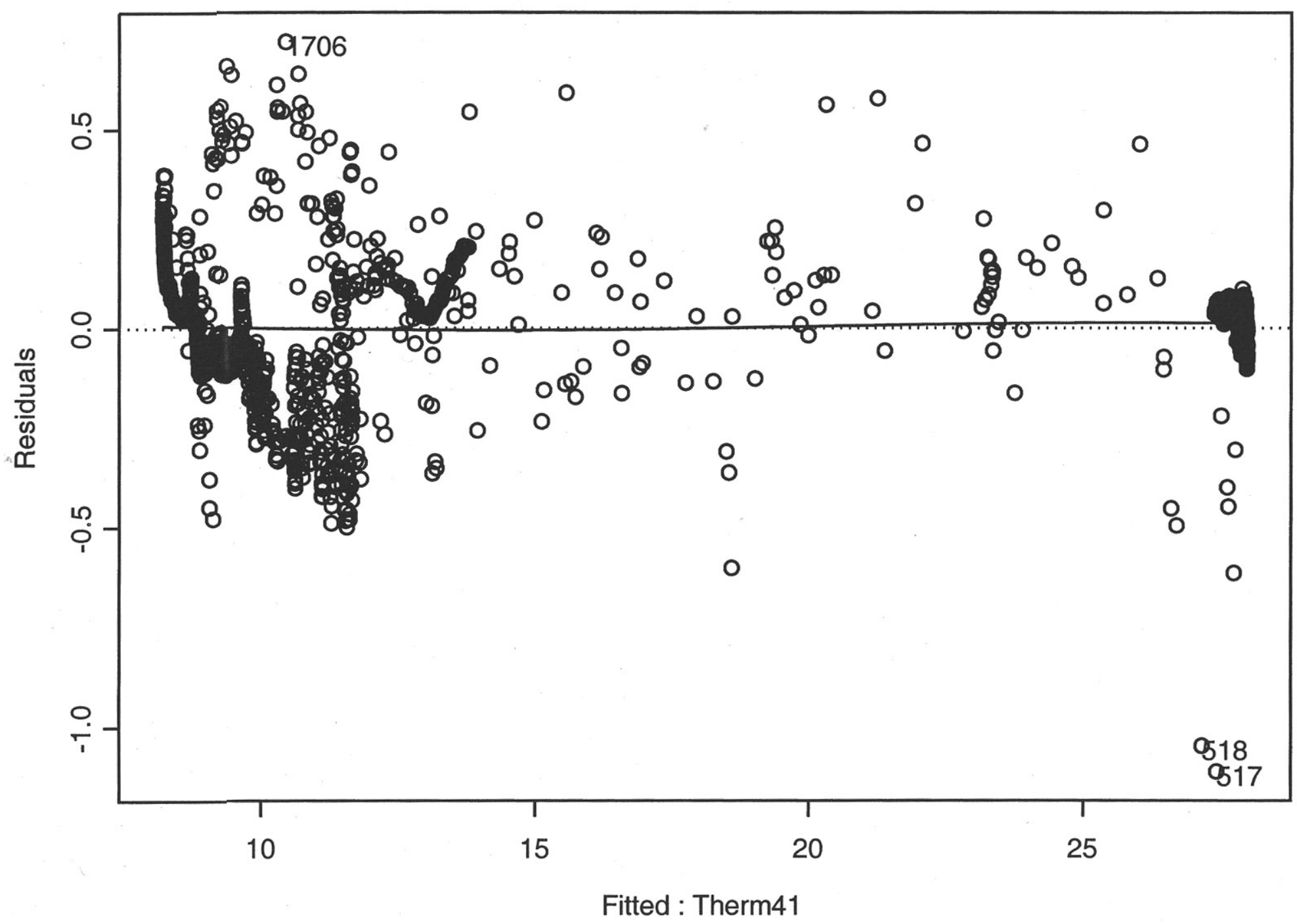


*** Linear Model

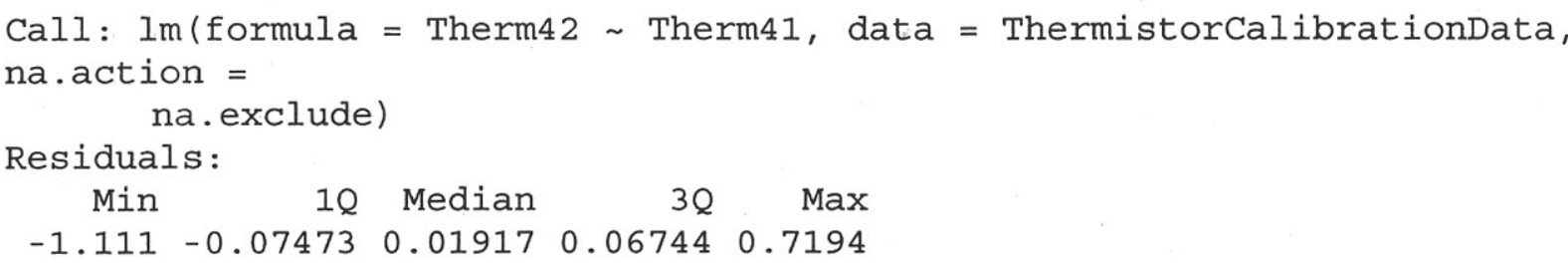

Thermistor $42=-0.4595+1.016^{*}$ Thermistor 41 
Probe \#3 - Thermistors 46 and 47

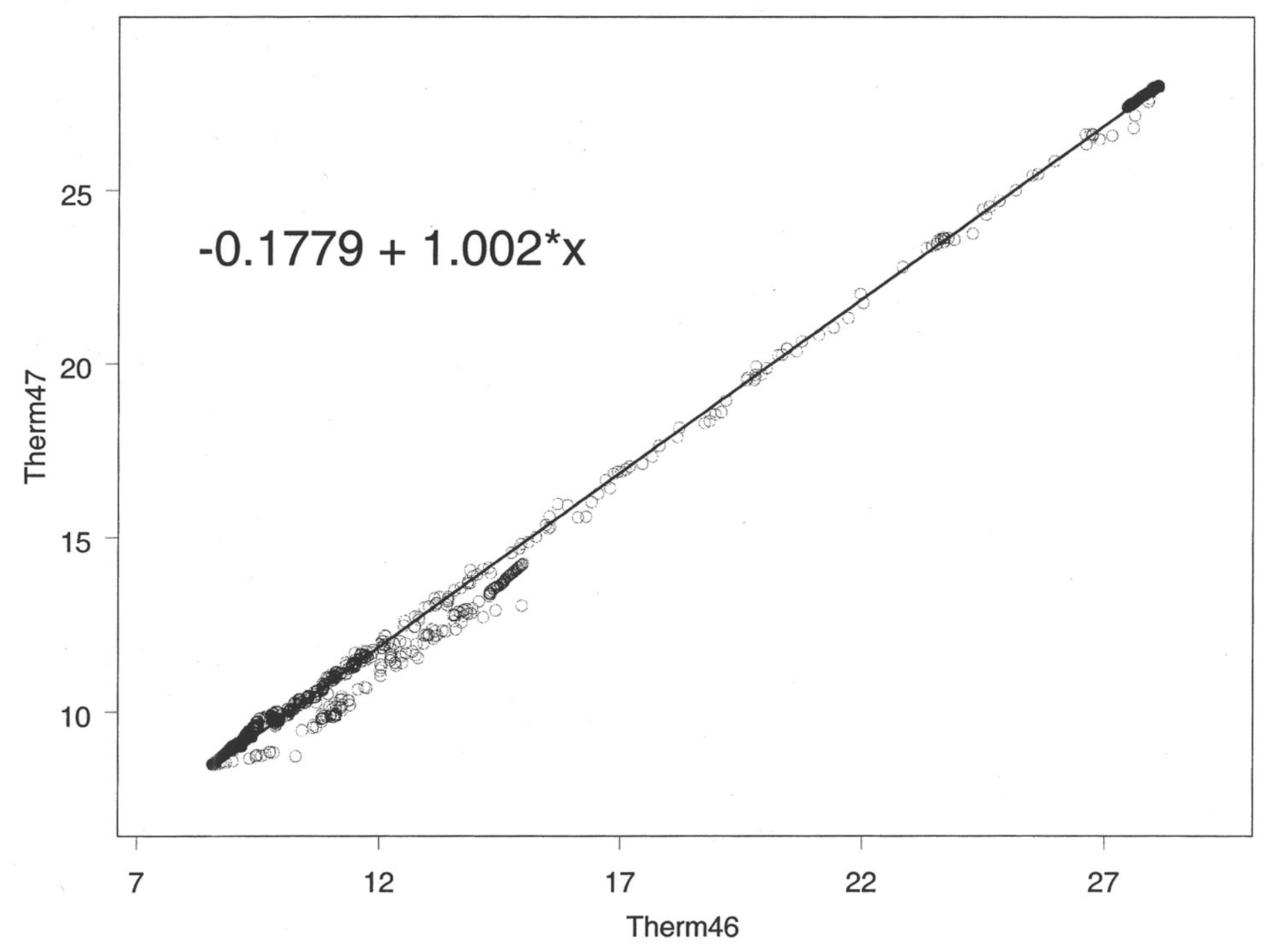


Standard Error - Thermistors 46 and 47

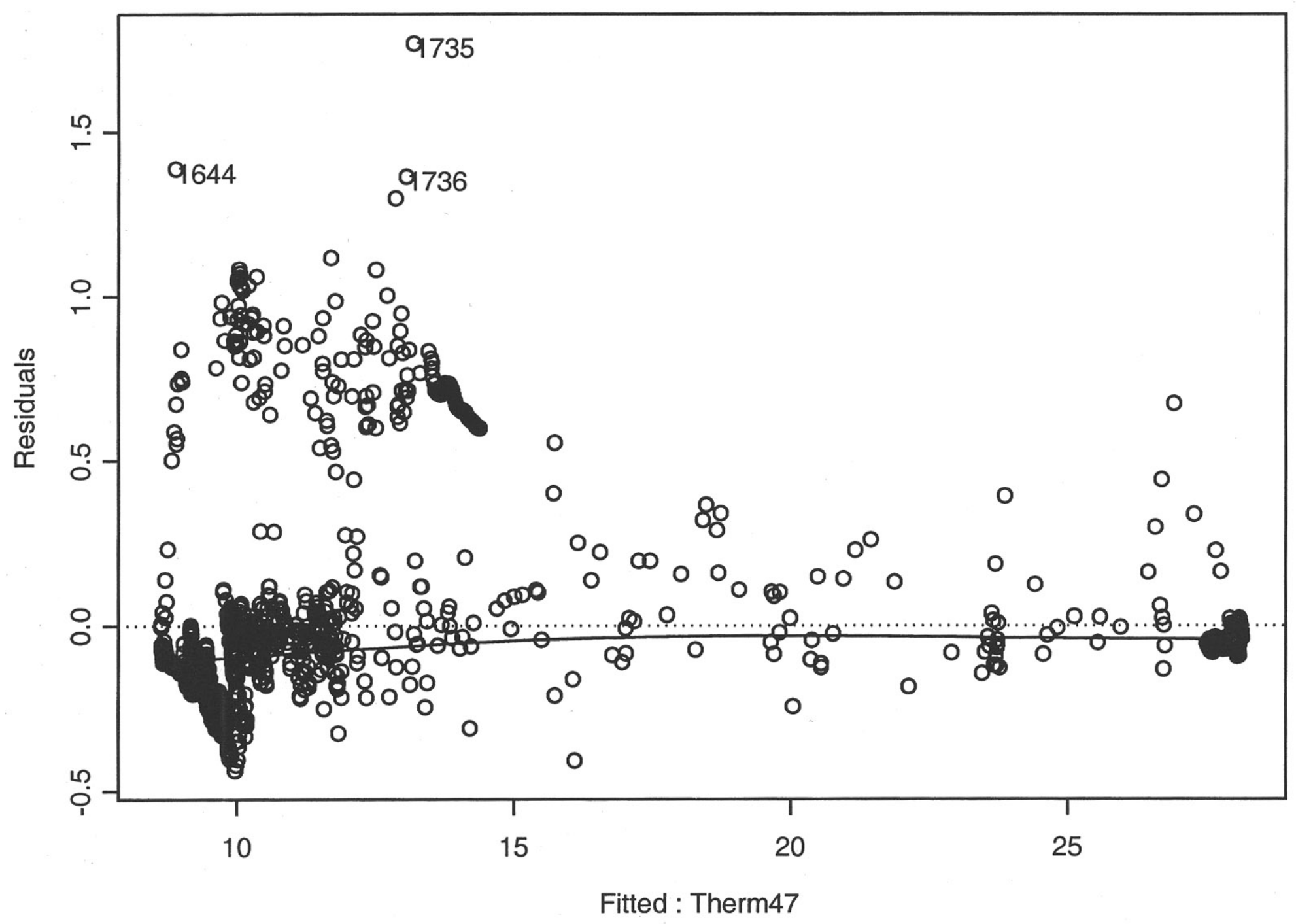




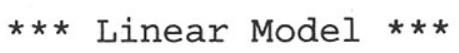

Call: $1 \mathrm{~m}$ (formula = Therm46 $\sim$ Therm47, data = ThermistorCalibrationData, na. action $=$

na. exclude)

Residuals:

Min $1 Q$ Median $30 \quad$ Max

$-0.4374-0.1202-0.05541-0.02351 .768$

Coefficients:

$\begin{array}{rrrrr} & \text { Value std. Error } & t \text { value } & \operatorname{Pr}(>|t|) \\ \text { (Intercept) } & 0.1943 & 0.0136 & 14.2956 & 0.0000 \\ \text { Therm47 } & 0.9966 & 0.0008 & 1287.4252 & 0.0000\end{array}$

Residual standard error: 0.2704 on 1797 degrees of freedom

Multiple R-Squared: 0.9989

F-statistic: 1657000 on 1 and 1797 degrees of freedom, the p-value is 0

Thermistor $47=-0.1779+1.002^{*}$ Thermistor 46 
Probe \#4 - Thermistors 35 and 36

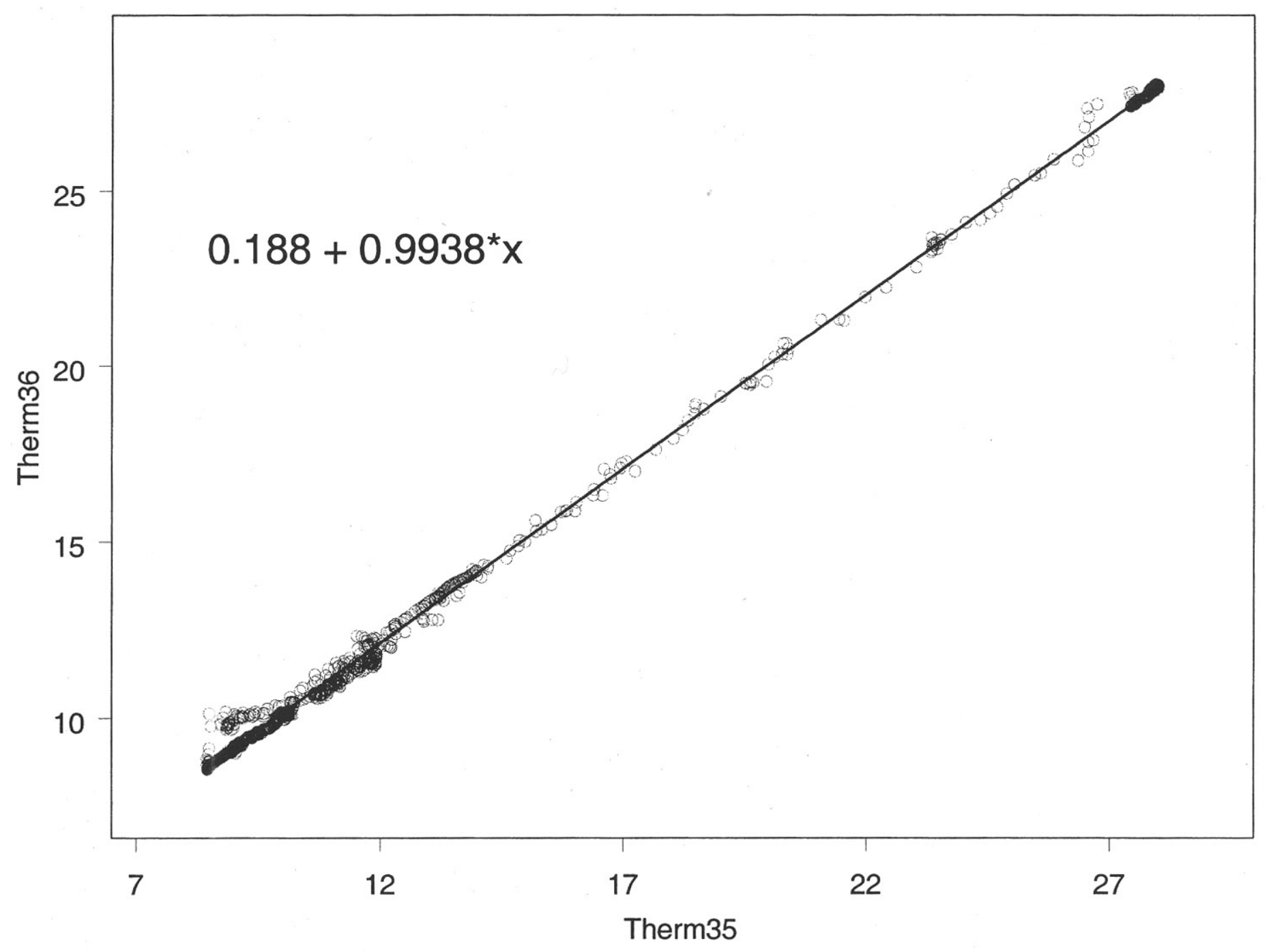


Standard Error - Thermistors 35 and 36

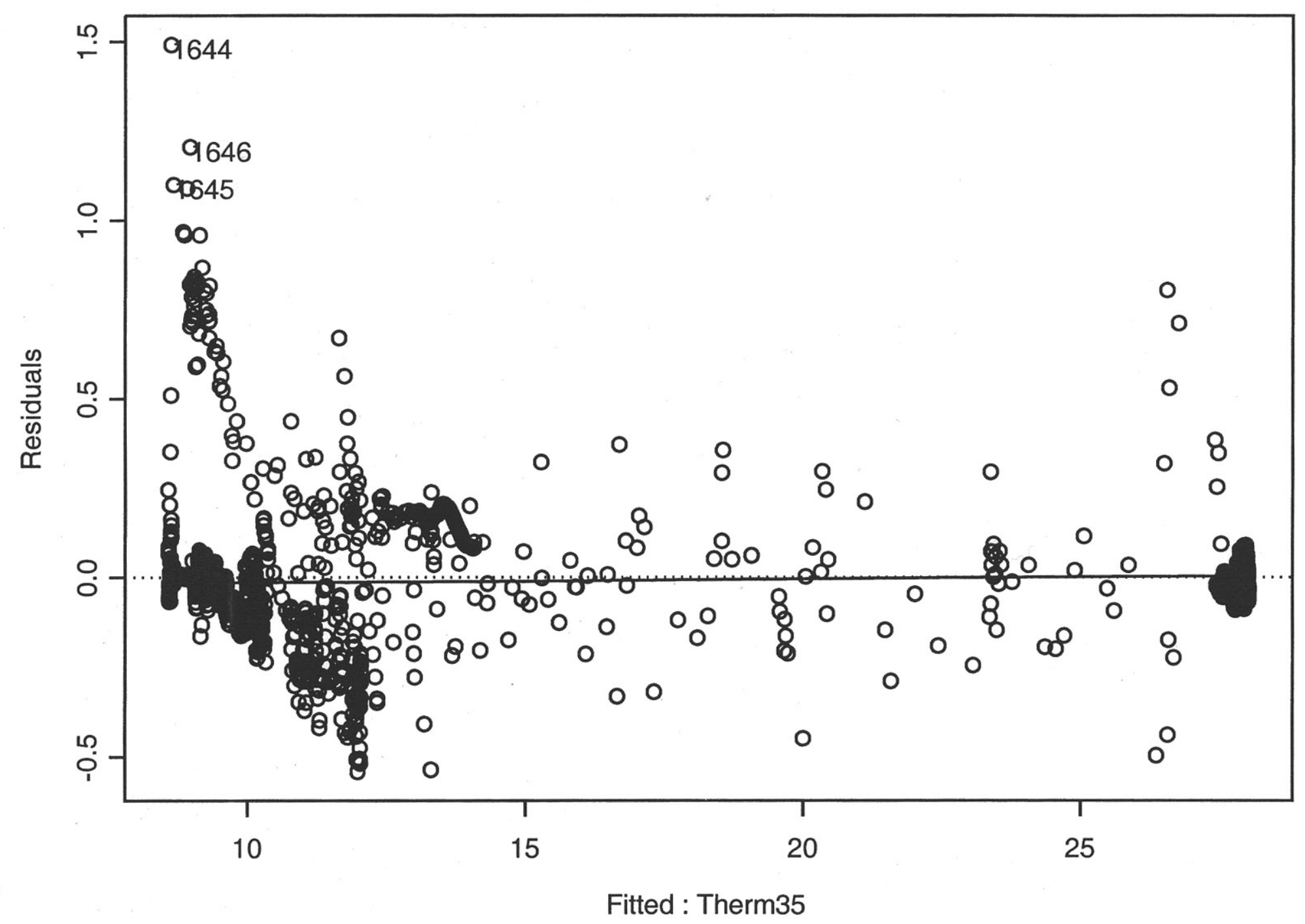


$\star * *$ Linear Model $* * *$

Call: Im(formula = Therm36 Therm35, data = ThermistorCalibrationData, na.action $=$

na.exclude)

Residuals:
Min
1Q
Median
30 Max

$\begin{array}{lllll}-0.5412 & -0.05419 & -0.00005447 & 0.03447 & 1.491\end{array}$

Coefficients :

$\begin{array}{rrrrr} & \text { Value std. Error } & t \text { value } & \operatorname{Pr}(>|t|) \\ \text { (Intercept) } & 0.1880 & 0.0089 & 21.2304 & 0.0000 \\ \text { Therm35 } & 0.9938 & 0.0005 & 1970.2619 & 0.0000\end{array}$

Residual standard error: 0.1759 on 1797 degrees of freedom

Multiple R-Squared: 0.9995

F-statistic: 3882000 on 1 and 1797 degrees of freedom, the p-value is 0

Thermistor $36=0.188+0.9938^{*}$ Thermistor 35 
Probe \#5 - Thermistors 43 and 44 (Lost in Brine Pool)

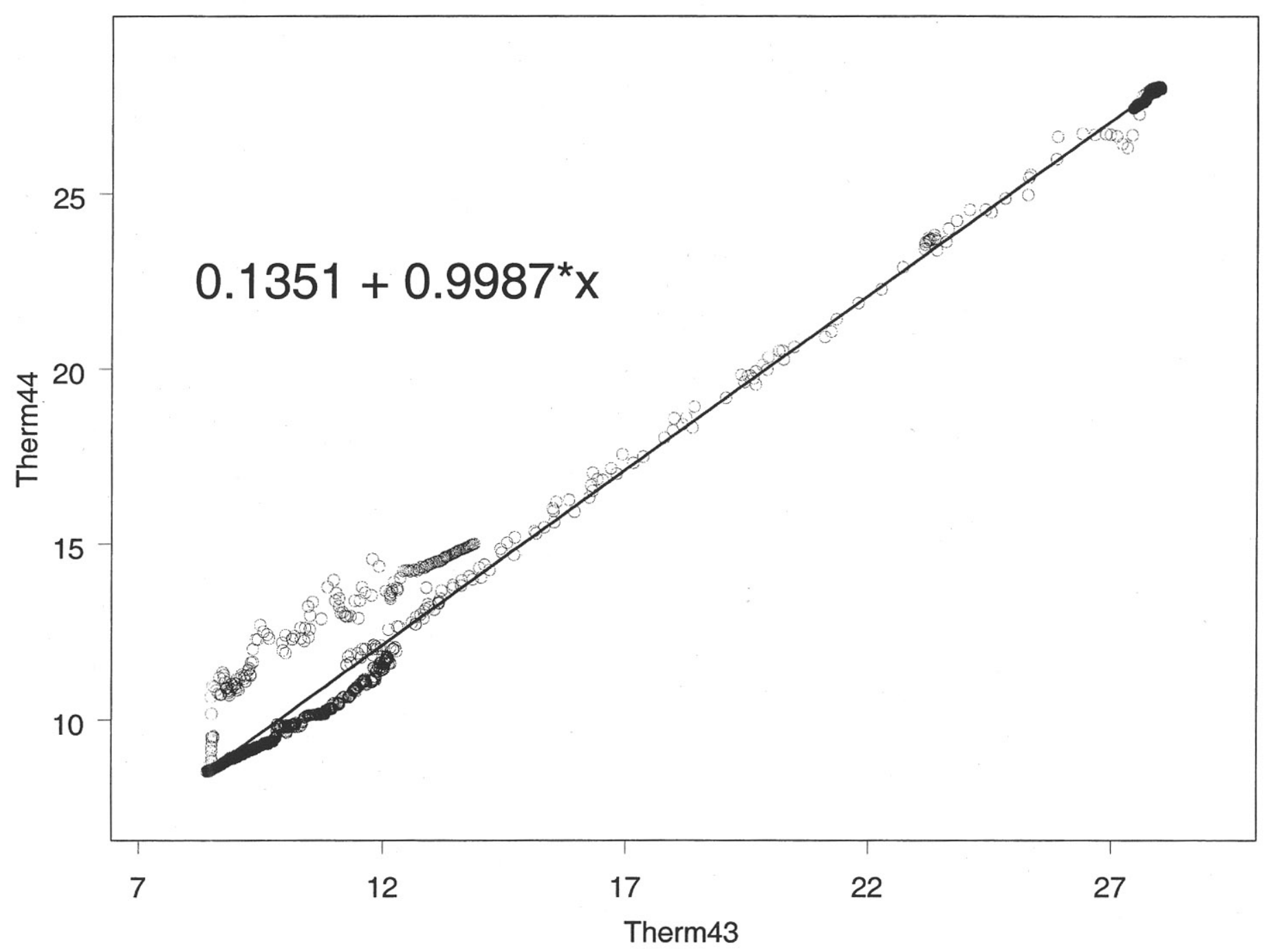


Standard Error - Thermistors 43 and 44

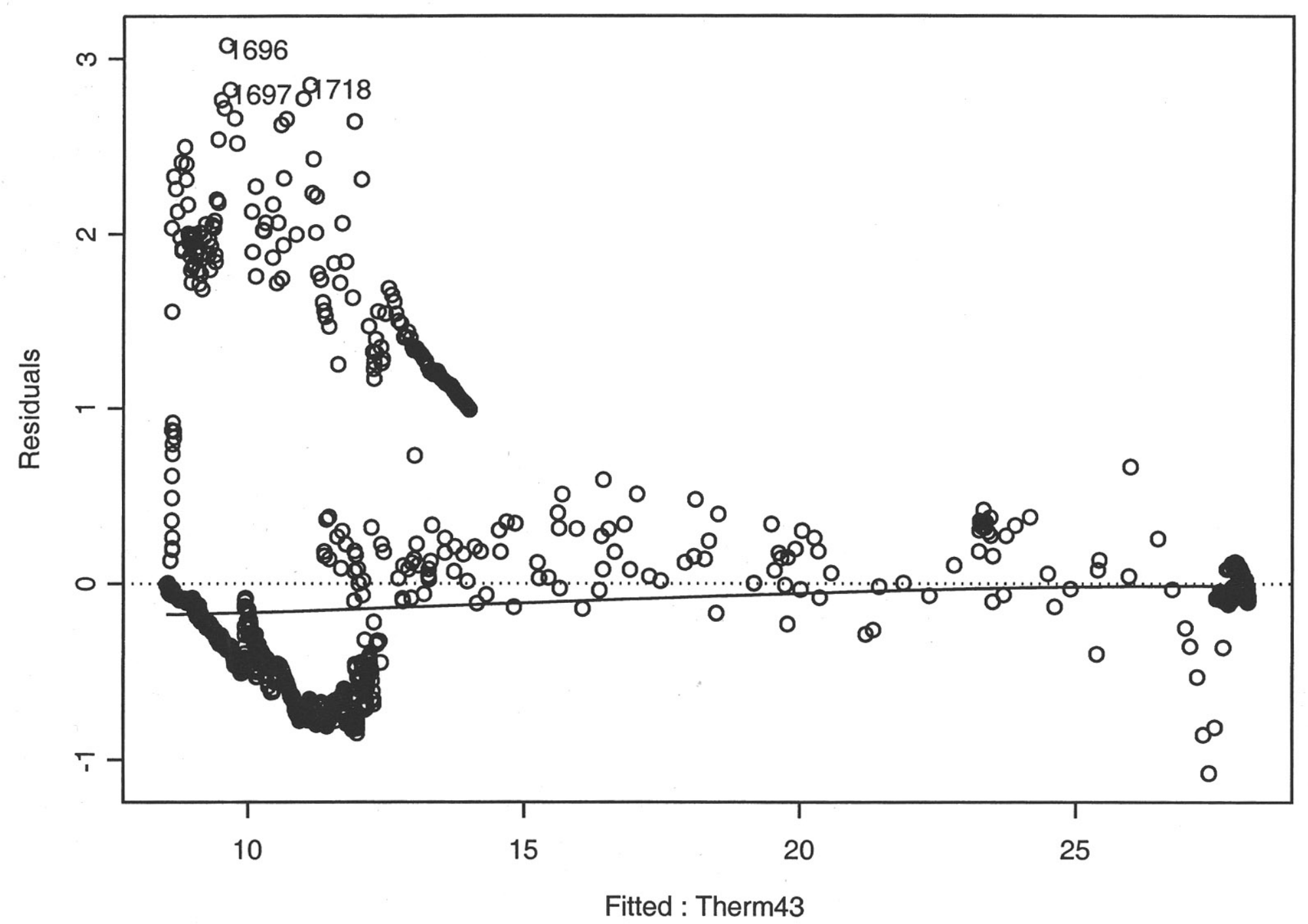


*** Linear Model

Call: $1 \mathrm{~m}$ (formula $=$ Therm44 $\sim$ Therm43, data = ThermistorCalibrationData na.action $=$

na.exclude)

Residuals:
Min
1Q Median
3Q $\quad$ Max

$\begin{array}{lllll}-1.075 & -0.265 & -0.08311 & -0.001328 & 3.077\end{array}$

Coefficients:

$\begin{array}{lrrrr}\text { (Intercept) } & 0.1351 & 0.0304 & 4.4462 & 0.0000\end{array}$

$\begin{array}{lllll}\text { Therm43 } & 0.9987 & 0.0017 & 576.4508 & 0.0000\end{array}$

Residual standard error: 0.6037 on 1797 degrees of freedom

Multiple R-Squared: 0.9946

F-statistic: 332300 on 1 and 1797 degrees of freedom, the p-value is 0

Thermistor $44=0.1351+0.9987^{*}$ Thermistor 43 


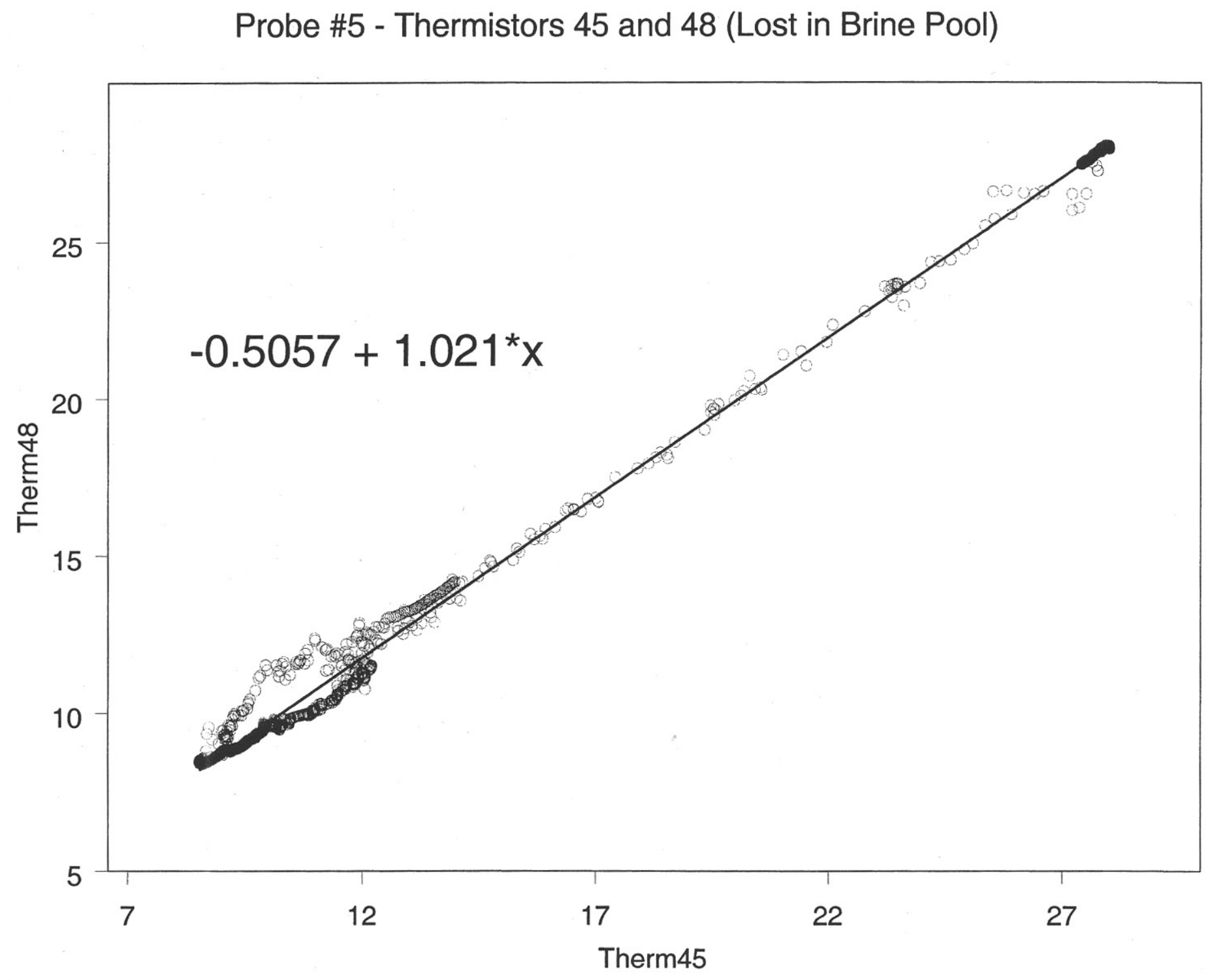


Standard Error - Thermistors 45 and 48

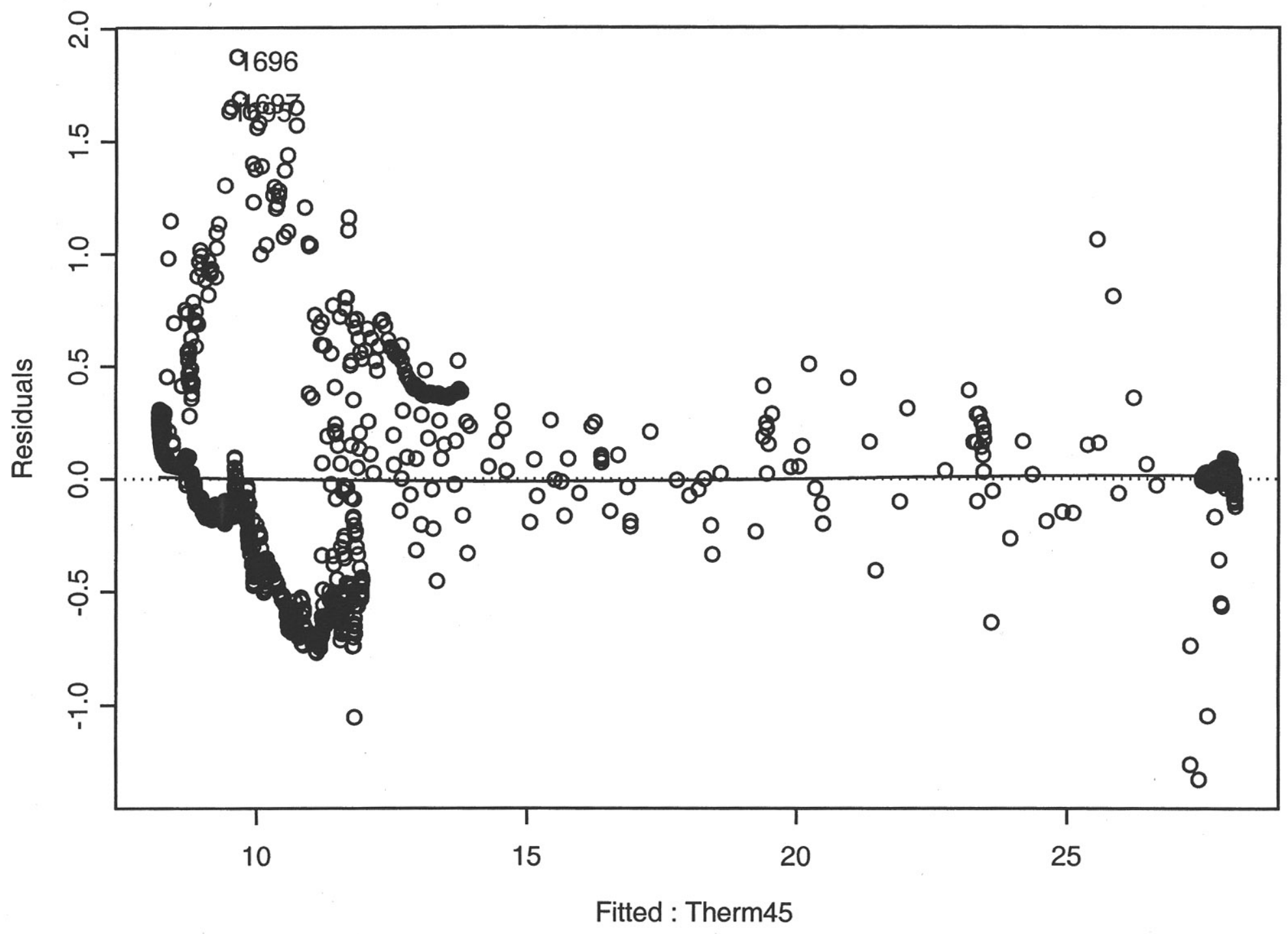




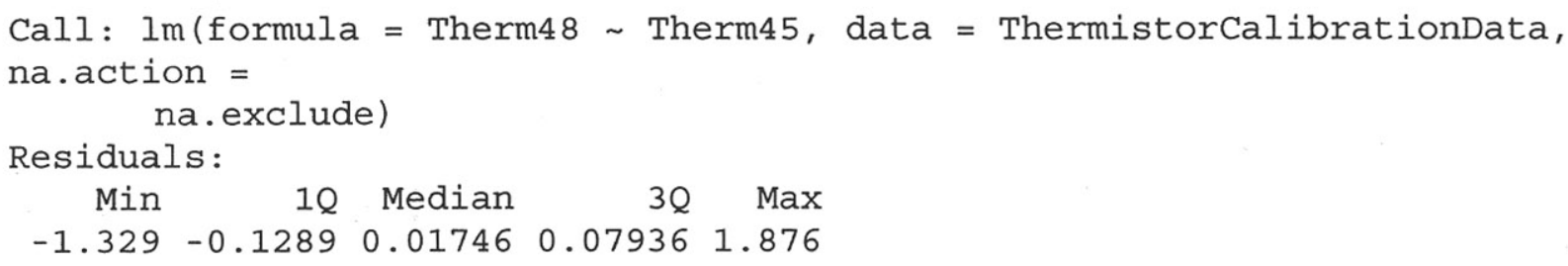

Thermistor $48=-0.5057+1.021^{*}$ Thermistor 45 
Probe \#6 - Thermistors 37 and 38 (Placed in Brine)

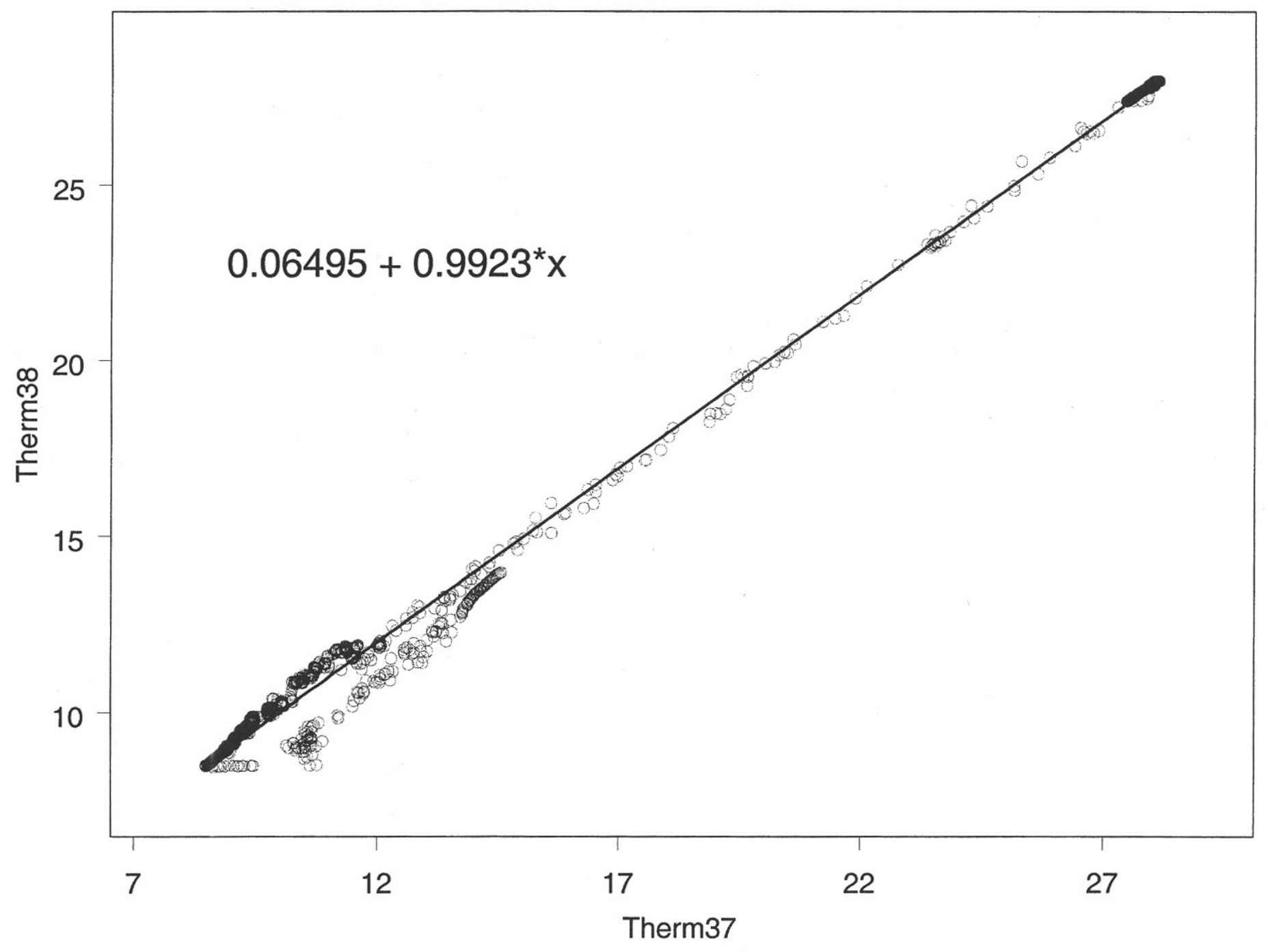


Standard Error - Thermistors 37 and 38

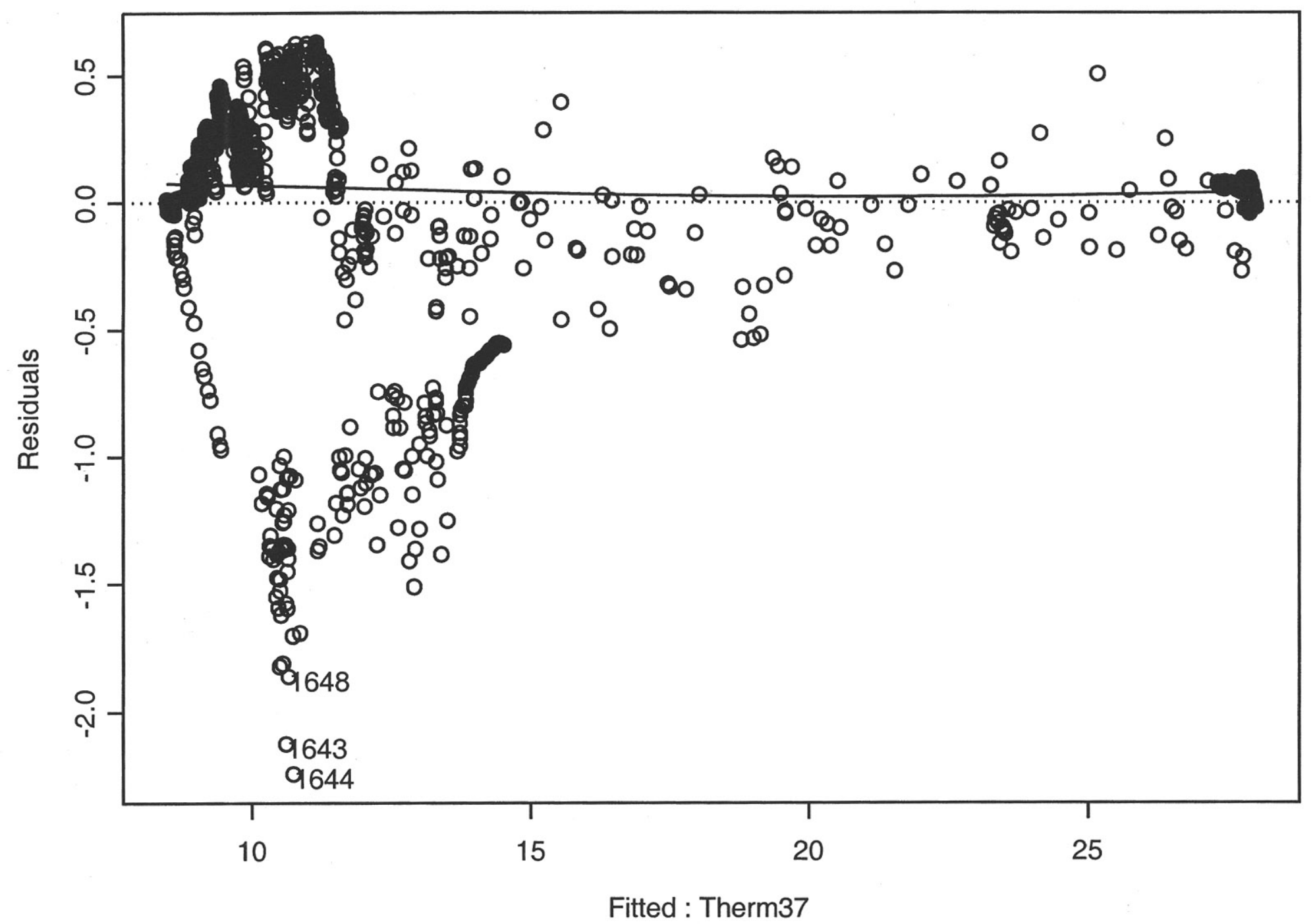


*** Linear Model

Call: Im(formula = Therm38 Therm37, data = ThermistorCalibrationData, na. action $=$

na.exclude)

Residuals :
Min
10 Median
$30 \quad$ Max

$\begin{array}{lllll}-2.242-0.00358 & 0.04901 & 0.1446 & 0.6303\end{array}$

Coefficients:

$\begin{array}{rrrrr} & \text { Value std. Error } & t \text { value } & \operatorname{Pr}(>|t|) \\ \text { (Intercept) } & 0.0650 & 0.0190 & 3.4149 & 0.0007 \\ \text { Therm37 } & 0.9923 & 0.0011 & 919.5900 & 0.0000\end{array}$

Residual standard error: 0.3775 on 1797 degrees of freedom

Multiple R-Squared: 0.9979

F-statistic: 845600 on 1 and 1797 degrees of freedom, the p-value is 0

Thermistor $38=0.06495+0.9923^{*}$ Thermistor 37 
Probe \#6 - Thermistors 39 and 40 (Placed in Brine)

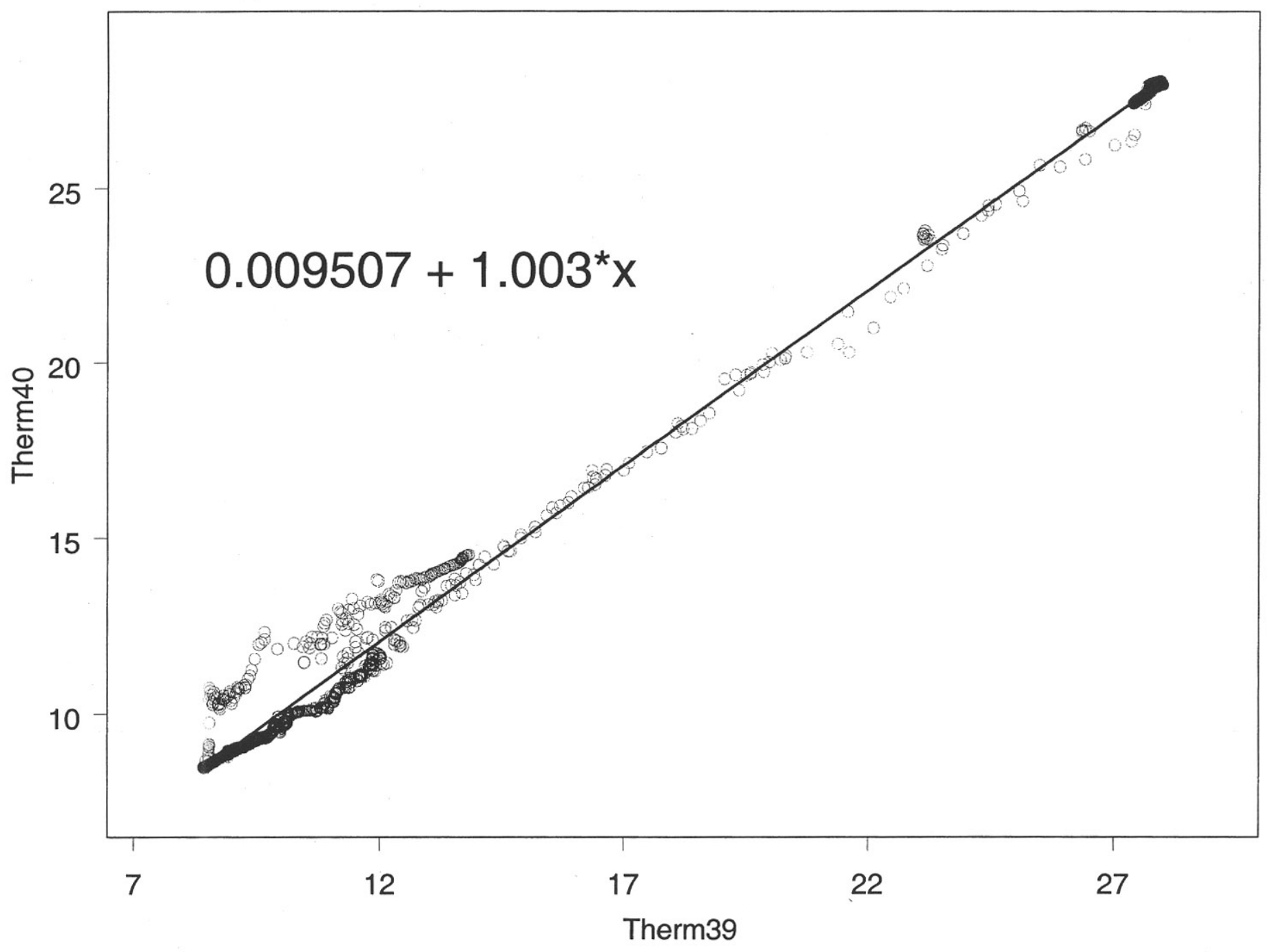


Standard Error - Thermistors 39 and 40

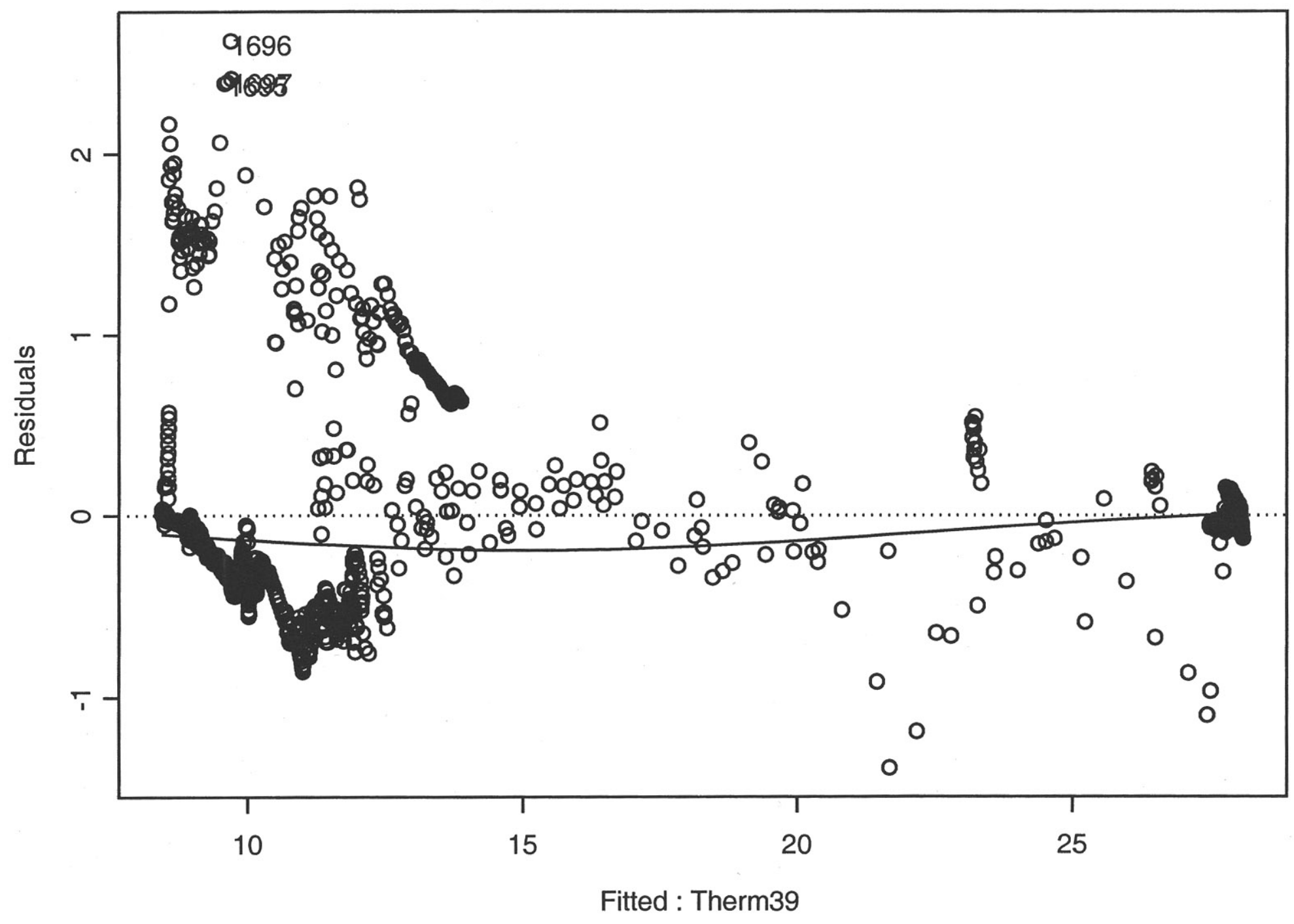


*** Linear Model

Call: $1 \mathrm{~m}$ (formula = Therm40 Therm39, data = ThermistorCalibrationData, na. action $=$

$$
\text { na.exclude) }
$$

Residuals :

$\begin{array}{rrrrr}\text { Min } & 1 Q & \text { Median } & 3 Q & \operatorname{Max} \\ 387 & -0.1983 & -0.04565 & 0.02206 & 2.622\end{array}$

$-1.387-0.1983-0.045650 .022062 .622$

Coefficients :

$\begin{array}{rrrrr} & \text { Value std. Error } & t \text { value } & \operatorname{Pr}(>|t|) \\ \text { (Intercept) } & 0.0095 & 0.0233 & 0.4089 & 0.6827 \\ \text { Therm39 } & 1.0029 & 0.0013 & 754.6330 & 0.0000\end{array}$

Residual standard error: 0.4635 on 1797 degrees of freedom Multiple R-Squared: 0.9969

F-statistic: 569500 on 1 and 1797 degrees of freedom, the p-value is 0

Thermistor $40=0.009507+1.003^{*}$ Thermistor 39 


\title{
Task 5: Gas Hydrate Formation and Decomposition in Ocean Sediments Laboratory Experiments with Hydrated Sediments
}

\author{
Final Report \\ March 2003
}

Principal Investigator: R. E. Rogers

DOE Award DE-FC26-00NT40920

Mississippi State University

Box 9595

Swalm School of Chemical Engineering

Mississippi State, MS 39762 


\begin{abstract}
Gas hydrate induction times and formation rates in seawater-saturated sand/clay packs are catalyzed in the laboratory by a few ppm of biosurfactants. To determine if biosurfactants are indigenous to sediments near Gulf of Mexico gas-hydrates, such sediment samples were analyzed for catalytic effects on hydrate formation. This work addressed some relationships of biosurfactants, sand, and clays with gas hydrate formation rates, induction times, and form (dispersed, nodular, stratified, massive), relating these factors to gas hydrate occurrence in GOM sediments.

Included are two electron microscope views of the tube walls of a tube worm and of a biopolymer on the surface of sand.
\end{abstract}




\title{
TABLE OF CONTENTS
}

\author{
Abstract \\ Table of Contents \\ List of Figures \\ Executive Summary \\ I. Introduction \\ Statement of Theory and Definitions. \\ Biosurfactant Effects on Induction Times \\ Biosurfactant Effects on Formation Rates \\ Experimental Method \\ II. Results and Discussion \\ Electron Microscope Photographs \\ Evaluation of Gulf of Mexico Sediments Near Gas Hydrates.. \\ III. Conclusions \\ Nomenclature \\ References
}

\section{LIST OF FIGURES}

Figure 1 Photomicrograph Emulsan on Sand

Figure 2 Photomicrograph Tube Worm's Tube Wall

Figure 3 Relative Formation Rates with GOM Sediments

Figure 4 Particle Size Distribution Sediment Samples 


\section{EXECUTIVE SUMMARY}

Evaluation was continued of biosurfactant effects on gas hydrate formation.

Although the biopolymer Emulsan does not form micelles, it promotes gas hydrate formation on bentonite surfaces. Emulsan does not promote hydrate formation on sand surfaces. Apparently, the large Emulsan polymer uncoils to spread over the bentonite surface, orienting hydrophilic and hydrophobic groups to associate hydrocarbon gases in nucleation centers on the polymer. A photomicrograph suggests that the orientation is not favorable if Emulsan spreads over a sand surface.

GOM sediment samples from hydrate mounds showed a correlation of hydrate formation rate with particle size of the media. Additional extensive tests are needed to determine if biosurfactants adsorb on GOM sediment surfaces in sufficient quantities to catalyze gas hydrate formation.

GOM sediments, tube worms, and commercially-available biosurfactants were analyzed during the report period to determine effects on seafloor gas hydrates. 


\section{INTRODUCTION}

While searching for a surfactant to retard gas hydrate formation, Bishnoi first reported a catalytic effect of some surfactants on gas hydrate formation (1). Zhong and Rogers (2) first reported not only the catalytic effect of some anionic surfactants on hydrate formation but the additional facilitation of self-packing hydrates on metal surfaces as they form and complete reaction of interstitial water. These properties have been incorporated into a conceptual design of a large gas-storage facility (3), and DOE is currently sponsoring a scale-up of the design for industrial gas storage.

Vysniauskas and Bishnoi developed Equation 1 with data taken from a chilled, hydrocarbon-gas and water, stirred system. The vigorous stirring continually renewed the gaswater interfacial boundary to prevent hydrate barrier films from developing (4).

$$
r=A a_{S} \exp \left(-\Delta E_{a} / R T\right) \exp \left(-a / \Delta T^{b}\right) P^{\gamma}
$$

Significantly, Equation (1) was found to also apply to a non-stirred, anionic-surfactant water solution in which the hydrates migrate and adsorb on the surface of a metal at the watergas interface (2). The migration of forming hydrate crystals clears the water surface of any hydrate film and gives about the same rate of formation as when vigorously stirred. Whether by mechanical stirring or by crystal migration, the reacting surface is kept clear of a barrier film; mass transfer is not a rate-limiting factor in either case.

The results of the synthetic surfactants on the gas storage process suggested a study of the effect of biosurfactants on hydrate formation in sea floors. There, water-borne microbes, in order to feed on the organic matter in ocean-floor sediments, would be expected to produce biosurfactants to make that insoluble matter accessible. Consequently, those biosurfactants could catalyze gas hydrate formation. Results of our investigation address the possibility.

Statement of Theory and Definitions. In the gas storage process, the surfactant's catalytic effect on gas hydrate formation is caused by the formation of surfactant micelles. The sodium dodecyl sulfate (SDS) anionic surfactant used in the gas storage process forms a micelle. The 12carbon alkyl groups form a spherical micelle that solubilizes hydrocarbon gases (5). Water associates around the periphery of the micelle in close proximity to the solubilized gas. Thus, micelles act as nucleation sites for hydrate crystals. The threshold concentration of surfactant to form micelles occurs at the critical micellar concentration (CMC), and this CMC is traditionally measured by surface tension at ambient conditions. Because CMC is difficult to determine by surface tension at hydrate-forming conditions, hydrate induction time has been developed as a precise means of that measurement (2). For a rhamnolipid surface-active agent in distilled water, the CMC was found to decrease by a factor of about 11.2 from ambient conditions to hydrate conditions.

The hydrocarbon-gas-laden micelles are evenly distributed throughout the water. Therefore, crystallization can commence in the water subsurface. The buoyancy of the developing hydrate crystal moves it through the bulk of the free water to the liquid-gas interface, and the affinity of the surfactant to the cold metal surface facilitates movement of surfactant and attached hydrate crystal to adsorb on the metal surface (2).

This interesting laboratory phenomenon with synthetic surfactants could be important in the formation of gas hydrates in ocean sediments, where microbial action is reported to be prolific in the vicinity of gas hydrates in the Gulf of Mexico.

Biosurfactants fall into five classifications: (1) hydroxylated and crosslinked fatty acids, (2) polysaccharide-lipid complexes or polymers, (3) glycolipids, (4) lipoproteins-lipopeptides, and (5) phospholipids $(6 ; 7)$. They are named according to their hydrophilic groups: 
carboxylates, saccharides, glycoproteins, peptides and phosphates (7). The common hydrophobic group is the long-chain fatty acid. Samples that are available commercially from each of the five classifications were used in the experimentation.

Consequently, laboratory tests were made under the subject DOE grant and reported in the previous year's Final Report. A summary of these results is as follows.

Biosurfactant Effects on Induction Times. Surfactin, a lipoprotein-lipopeptide classification, in the presence of bentonite reduced the induction time drastically, shortening the time for hydrate initiation by $71 \%$ from the control in which no biosurfactant was present. Rhamnolipid, a glycolipid, also reduced induction time substantially, decreasing it about $58 \%$ from the bentonite control; the fatty acid exhibited a similar influence. Phospholipids and polysaccharide-lipid complexes (Emulsan and Snomax) reduced the induction times $20 \%$ to $44 \%$ from the bentonite control

With kaolin present instead of bentonite, some different reactions occur. Rhamnolipid has the greatest positive effect, reducing the time by about $66 \%$ over the kaolin control. With fatty acid in the presence of kaolin, no hydrates formed. These results indicate that biosurfactant can determine the residence time required for hydrates to form from a gas migrating through the hydrate zone of the ocean floor, and that the induction time is affected by the biosurfactant and specific sand, bentonite, or kaolin surface present.

Biosurfactant Effects on Formation Rates. Although in the absence of a biosurfactant, kaolin reduces hydrate formation rates compared to when bentonite is present, kaolin further reduces hydrate formation rate in the presence of Surfactin, phospholipids, Snomax, and Emulsan. An exceptional effect was observed in the case of rhamnolipid. A 16-fold increase of rate occurred when rhamnolipid solution was saturating the pack that included kaolin. With bentonite in the pack, the Surfactin biosurfactant increased hydrate formation rate about 4-fold, but kaolinSurfactin interaction suppressed the rate increase.

Our tests also indicate that only small amounts of biosurfactant may be necessary to affect gas hydrate formation in the sea floor. The CMC of rhamnolipid in seawater at hydrate conditions was determined to be only 13 ppm for the $25 \%$ active rhamnolipid sample used in the tests.

Experimental Method. To meet the objectives of the experimental work, it was necessary to compare hydrate formation rates of samples containing the various biosurfactants and to observe visually any specificity the biosurfactants exhibited toward solid surfaces as evidenced by gas hydrate formation appearances.

The experimental apparatus was designed to allow determining induction times and gas-occlusion rates, parameters that define gas hydrate formation rates. Also, the apparatus was designed to allow visual observation of hydrate formation.

Stainless steel test cells were provided with pressure transducer, RTD temperature sensors, and visual access. One means of visual access was a two-inch thick, four-inch diameter quartz viewing port in the top of a test cell. The second visual access came from two sapphire-sealed viewing ports that allowed borescope image capture and fiber-optic light entry. Samples were placed in the test cells, test cells were placed in a cold environment, and temperature-time-pressure recorded with an Omega data-acquisition system.

An induction time for the system was defined as the time to cool the sample from the equilibrium temperature to hydrate initiation. Hydrate initiation was indicated by pressure drop at constant temperature. The equilibrium pressure-temperature curve for the given gas composition was calculated using the CSMHYD program (24). Because beginning pressuretemperature combination before cooldown and rate of cooldown were maintained constant, relative values of induction times for all runs could be made. 
In order to calculate the rate of hydrate formation, the Peng-Robinson equation of state was used to determine moles of gas occluded into hydrates from a known constant cell volume and recorded pressure/temperature. Temperature-pressure-time data were collected every 20 seconds.

Gulf of Mexico seawater was used in all experimental runs. A natural gas of $90.0 \%$ methane, $6.0 \%$ ethane, and $4.0 \%$ propane was used for all runs. Combinations of sand, bentonite or kaolin were used for the porous media.

\section{RESULTS AND DISCUSSION}

Electron Microscope Photographs. Emulsan was found to catalyze gas hydrate formation in porous media, especially in the presence of bentonite. To help understand the mechanism, consider some of the properties of Emulsan, bentonite, and sand that might account for different reactions.

Emulsan is a bioemulsifier originating from the Acinetobacter calcoaceticus oil-degrading bacterium, and falls within the biosurfactant general classification of polysaccharide-lipid complexes. The biopolymer's molecular weight is approximately 980,000 . As a polyanionic heteropolysaccharide bioemulsifier, it is effective in removing and emulsifying oil films $(16 ; 17$; 18; 19). Despite not significantly lowering surface tension, it is a powerful oil-in-water emulsion stabilizer- the large molecule covers the surface of the suspended oil drop to prevent coalescence. Emulsan does not form micelles, however, its separate hydrophobic-hydrophilic moieties could serve to unite the water and the host hydrocarbon to promote hydrate formation.

Sand has a negatively charged surface, whereas the bentonite has positive sodium ions situated at the edge of the clay platelet (a platelet consists of three layers ordered as silicon tetrahedra, aluminum octahedra and silicon tetrahedra), and in the accessible area between bentonite platelets positive sodium ions connect the negatively-charged platelets. One might expect, therefore, that the anionic biosurfactants would attach to the positive sites of bentonite but be repulsed, to some extent, from the sand surfaces. This anticipated result is verified with gas hydrate experiments where hydrates are usually catalyzed on the bentonite surfaces in the presence of biosurfactants.

Although Emulsan does not form micelles, its large molecules help bring the hydrocarbon gases and water to a common hydrate nucleation site. Ordinarily, this longstranded biopolymer might resemble a tangled thread, yet it has been characterized as unfolding to orient hydrophobic groups on an oil-drop surface while positioning its hydrophilic groups into the surrounding water. Probably, the molecule unfolds and spreads on the bentonite surface in a similar manner to facilitate hydrate formation.

In Figure 1 is a photograph from an electron microscope showing the surface of a sand particle after being exposed to an Emulsan-water solution. Seen on the sand surface are some crystals of salt and a fibrous web of a partial coating that is probably Emulsan. It is possible that unfolding and spreading of the Emulsan polymers occur on the sand as well as the bentonite, but the orientation of hydrophobic-hydrophilic groups on the sand is such to not promote hydrate formation.

In Figure 2 is a photograph from an electron microscope showing the surface of a tube worm taken from near a gas hydrate mound in the Gulf of Mexico. Note the fibrous nature of the walls of the tube where one column is broken to reveal a panel-like mass. Hydrates would not form on the tube worm mass in the laboratory.

Evaluation of Gulf of Mexico Sediments Near Gas Hydrates. In the only reported analysis of sediments around or in gas hydrate mounds in the Gulf of Mexico to identify bacteria, the parent microbes that produce Surfactin and rhamnolipid were found by Lanoil, et al, (15). It is 
significant that the biosurfactants of these two bacteria gave the most pronounced effects on gas hydrate formation in the laboratory tests reported here.

Four samples of sediments from gas hydrate fields in the northern Gulf of Mexico were analyzed for any associated catalysis effects on gas hydrates. In each case the dried sediments were mixed with sand to restructure a porous media sample containing $10 \%$ of the sediments. The mixture was reconstituted with seawater from the Gulf of Mexico, and hydrate formation rates calculated from laboratory data.

The results were compared to two control tests where one test included only sand and the second control included the sand saturated with $1000 \mathrm{ppm}$ rhamnolipid in seawater. The relative formation rates are presented in Figure 3. Two of the samples indicated no effect on gas hydrate formation (Samples 4234A and 4236), whereas two samples (4234B and 2904) indicated an increased formation rate. The control with rhamnolipid biosurfactant surpassed all samples with a high formation rate of hydrates. The sediment samples were analyzed for particle size distributions and percentages of sand, silt, and clays. In Figure 4 is shown the percentage in each sediment of particles with sizes $>1 \mathrm{~mm}$. A correlation is suggested by comparing Figures 4 and 3; higher hydrate formation rates occurred with those sediments composed of larger particles.

\section{CONCLUSIONS}

Biosurfactants must fall into one of five classifications. By testing commercially available samples from each of the five classifications, some notable effects on gas hydrate formation in seawater-biosurfactant saturated sand/clay packs resulted. Hydrate induction times and formation rates were generally enhanced by the biosurfactants, and in many cases (especially rhamnolipid and Surfactin) the enhancements were very large. The fatty acid retarded (bentonite) or prevented (kaolin) hydrate formation, the only classification that did so. The biosurfactant effect may occur even at low concentrations; for example, the CMC of rhamnolipid was reduced to $13 \mathrm{ppm}$ at hydrate-forming conditions.

Investigations on GOM sediment samples show a correlation of hydrate formation rate with sediment particle size; higher rates are associated with larger particle sizes.

Analysis of tube worms from around GOM hydrate mounds showed hydrates would not form on the tube surfaces. Photomicrographs of the tube walls showed a very fibrous structure.

Emulsan biopolymer is indicated to spread over bentonite surfaces in such a way as to help concentrate hydrocarbon gases for hydrate nucleation.

\section{NOMENCLATURE}

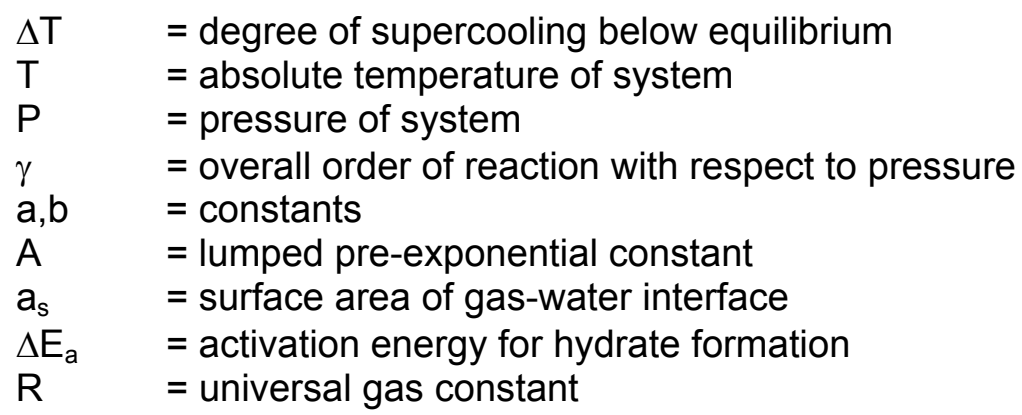




\section{REFERENCES}

1. Kalogerakis, N., Jamaluddin, A.K.M., Dholabhai, P.D., \& Bishnoi, P.R. (1993). "Effect of surfactants on hydrate formation kinetics", SPE 25188, SPE International Symposium on Oilfield Chemistry, New Orleans.

2. Zhong, Y., \& Rogers, R.E. (2000). "Surfactant effects on gas hydrate formation", Chem. Eng. Sci. 55, 4175.

3. Rogers, R.E. (1999). "Natural gas hydrates storage project, phase II. Conceptual design and economic study", Final report. DOE Contract DE-AC26-97FT33203, U.S. Department of Energy.

4. Vysniauskas, A., \& Bishnoi, P.R. (1983). "A kinetic study of methane hydrate formation", Chemical Engineering Science 38, 1061.

5. MacKerell, A.D. (1995). "Molecular dynamics simulation analysis of a sodium dodecyl sulfate micelle in aqueous solution: decreased fluidity of the micelle hydrocarbon interior", J. Phys. Chem. 99, 1846.

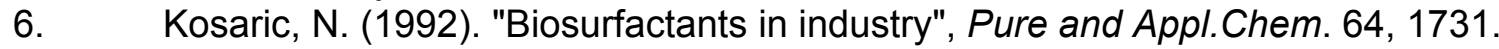

7. $\quad$ Fujii, T. (1998). "Biodetergents", In New Products and Applications in Surfactant Technology, (D.R. Karsa, ed.), Sheffield Academic Press, Sheffield, England. 88.

8. $\quad$ Rosenberg, E. (1986). "Microbial surfactants", CRC Crit. Rev. Biotech 3, 109.

9. Thangamani, S., \& Shreve, G.S. (1994). "Effect of anionic biosurfactant on hexadecane partitioning in multiphase systems", Environ. Sci. Technol. 28, 1993.

10. Herman, D.C., Artiola, J.F. \& Miller, R.M. (1995). "Removal of cadmium, lead, and zinc from soil by a rhamnolipid biosurfactant", Environ. Sci. \& Technol. 29, 2280.

11. Churchill, S.A., Griffin, R.A., Jones, L.P., \& Churchill, P.F. (1995). "Biodegradation and bioremediation", JournalEnviron. Qual. 24, 19.

12. Banat, I.M. (1995). "Biosurfactants production and possible uses in microbial enhanced oil recovery and oil pollution remediation: a review", Bioresource Technology 51, 1.

13. Bai, G., Brusseau, M.L., \& Miller, R.M. (1997). "Influenceof a rhamnolipid biosurfactant on the transport of bacteria through a sandy soil", Appl. Environ. Microbiol. 63, 1866.

14. Champion, J.T., Gilkey, J.C., Lamparski, H.,Retterer, J., \& Miller, R.M. (1995). "Electron microscopy of rhamnolipid (biosurfactant) morphology: effects of $\mathrm{pH}$, cadmium, and octadecane", J. Colloid Interface Sci. 170, 569.

15. Lanoil, B.D., Sassen, R. La Duc, M.T., Sweet, S.T., \& Nealson, K.H. (2001). Appl. Environ. Microbiol. 67, 5143.

16. Jones-Meehan, J., Beard, M., \& Hind, J. (1995). "In-situ maintenance of oil/water separators", Final report CDNSWC/CODE 634, prepared for A. Lardis, Annapolis, MD.

17. Gutnick, D.L. (1987). "The Emulsan polymer: perspectives on a microbial capsule as an industrial product", In Biopolymers, John Wiley \& Sons: New York. S223

18. Cohen, Y., \& Rosenberg, E., eds. (1989). "Microbial mats: physiological ecology of benthic microbial communities", American Society for Microbiology, Washington, D.C.

19. Rosenberg, E. (1993). "Microbial diversity as a source of useful biopolymers", J. Ind. Microbiol. 11, 131.

20. Goodnow, R.A., Harrison, M.D., Morris, J.D., Sweeting, K.B., \& Laduca,R.J. (1990). "Fate of ice nucleation- active Pseudomonas syringae strains in alpine soils and waters and in synthetic snow samples", Appl. Environ. Microbiol. 56, 2223.

21. Amende, B. \& Gordon, J. (1999). Literature and analyses reports on Snomax. York Snow, Inc., Victor, NY. 
22. Nakano, M.M., Marahiel, M.A. \& Zuber,P. (1988). "Identification of a genetic locus required for biosynthesis of the lipopeptide antibiotic Surfactin in Bacillus subtilis", J. Bacteriology 170, 5662.

23. Genzyme Pharmaceuticals (2001). "Synthetic lipids", Product Data Sheet. Cambridge, MA. pharmaceuticals@genzyme.com.

24. Sloan, E.D., Jr., (1990). Natural Hydrates of Natural Gases, Marcel Dekker, Inc., New York, N.Y. 550. 


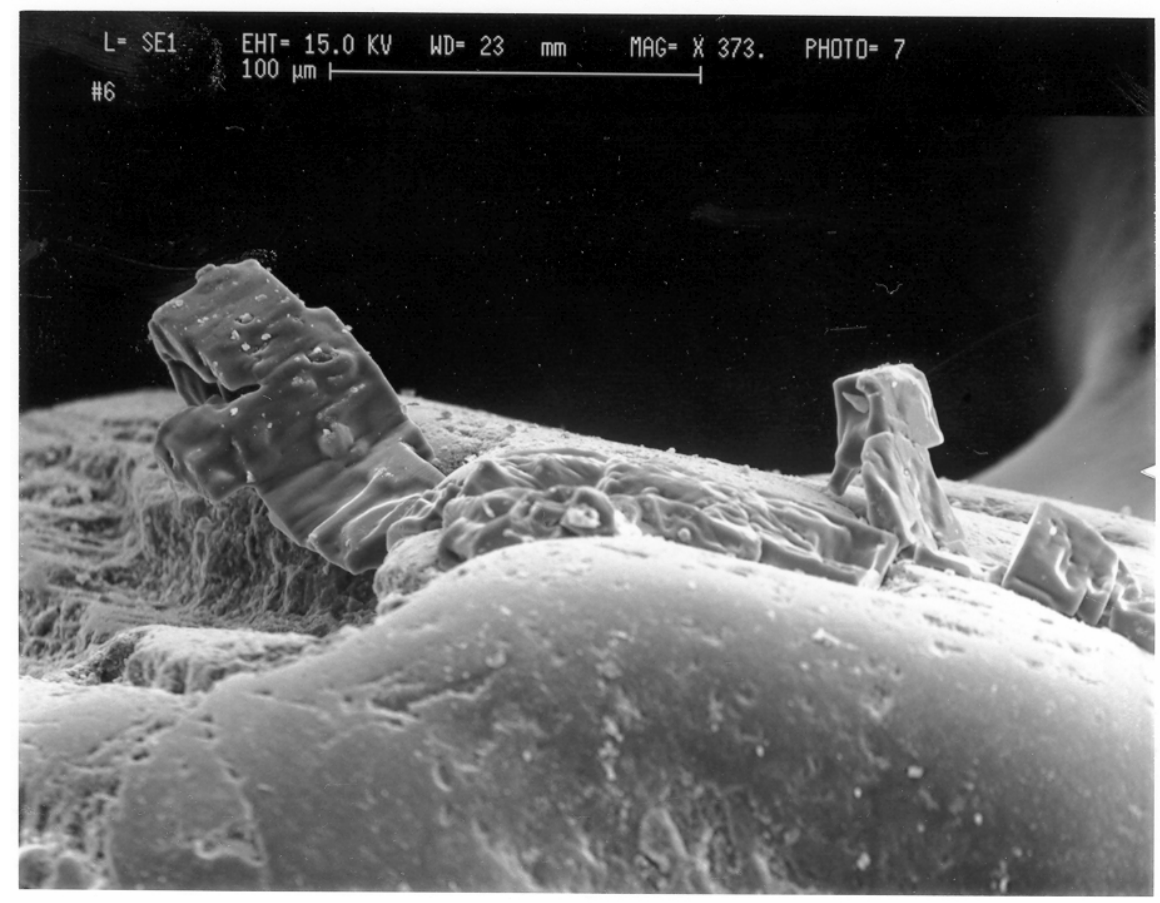

Figure 1. Photomicrograph Emulsan on Sand

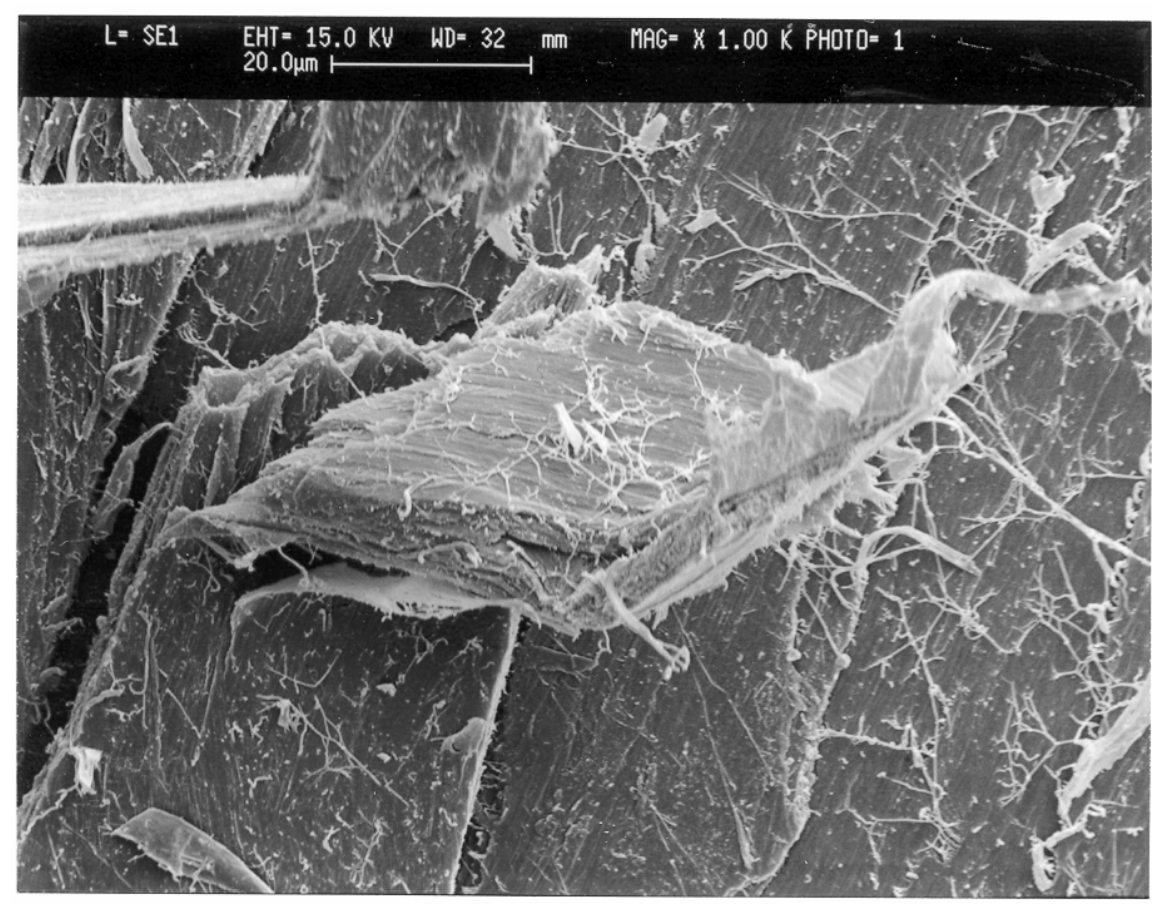

Figure 2. Photomicrograph Tube Worm's Tube Wall 


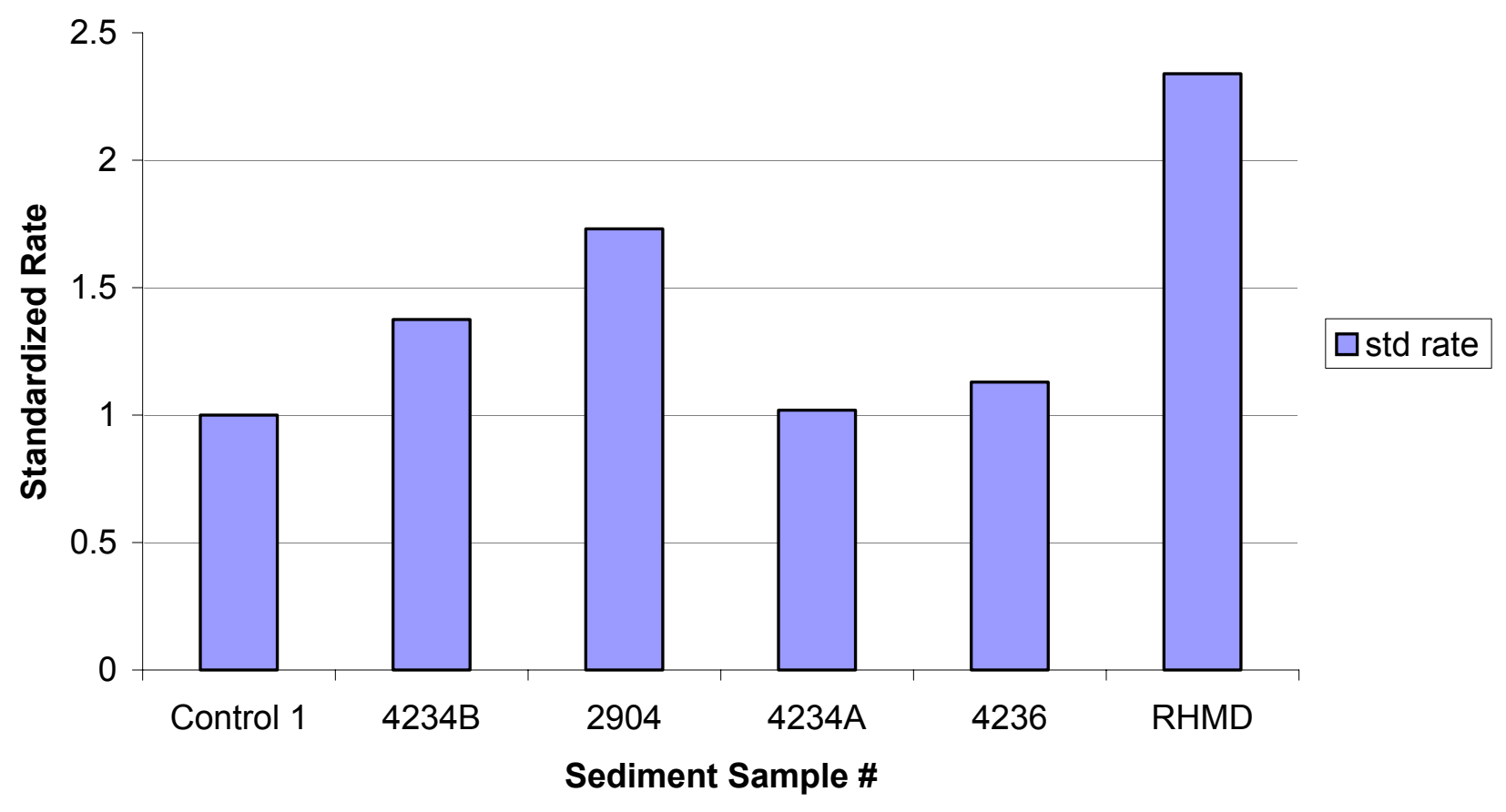

Figure 3. Relative Formation Rates with GOM Sediments.

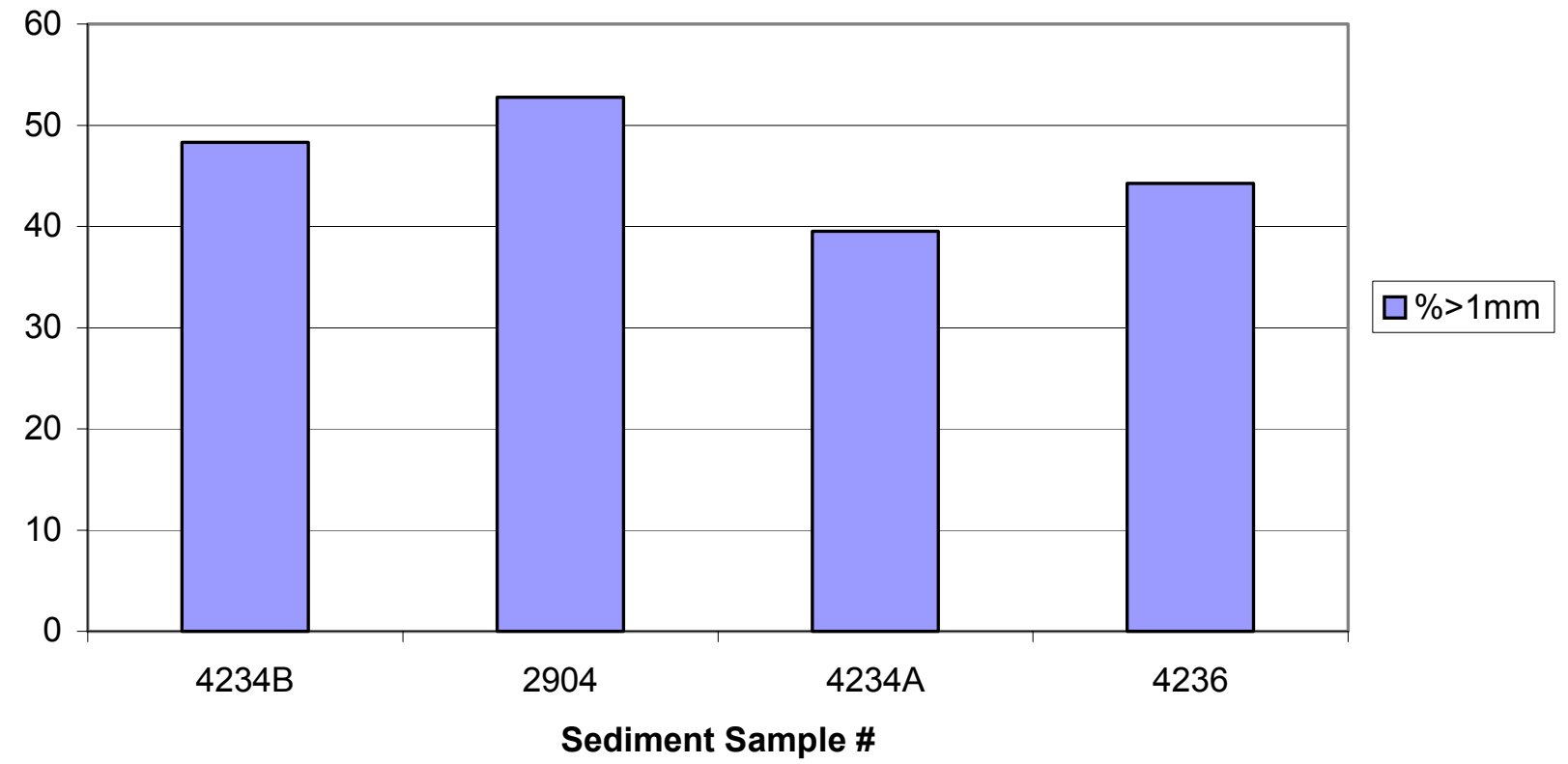

Figure 4. Particle Size Distribution of Sediment Samples. 
Title: Subcontract to University of Mississippi: Gas Hydrates - Organic Geochemistry Type of Report: Final

Reporting Period Start Date: July 1, 2003

Reporting Period End Data: September 30, 2003

Principal Author: Jean Whelan

Date Report Issued: December 30, 2003

DoE Award Number: DE-FC26-00NT40920

Submitting Institution: Woods Hole Oceanographic Instititution, Woods Hole, MA 02543 


\begin{abstract}
Result to date are described in building and deploying an in situ methane sensor to explore for and characterize ocean bottom methane seeps associated with gas hydrates and subsurface petroleum accumulations. A Raman detector has been built and tested and, to date, found not to possess the required sensitivity for this task except in areas of large scale gas venting. Initial tests on the commercially available METs sensor have not been promising to date, although sea trials in March off Victoria B. C. will be used to field test the most recent version of the instrument. Because of these limitations. Work was begun to build an underwater mass spectrometer for this purpose. The advantages of this instrument, the present state of technology, and a possible design are described. Sources of initial funding obtained for this work are described along with proposals still pending. Work has begun on building the MS instrument.

New analytical procedures for characterizing biodegraded seep oils from both the Gulf of Mexico surface seep and hydrate oils and Santa Barbara Coal Oil Point seep oils are described. The most promising technique in two dimensional gas chromatography (2DGC) which rapidly gives quantitative data on individual oil components. This is a very large breakthrough since these oils were previously not very useful for quantitative analyses. These results may allow better coupling of surface seep geochemical data with subsurface reservoir data so that seep geochemistry could become a much better exploration tool than has previously been possible.
\end{abstract}




\section{Table of Contents}

Abstract

List of Figures and Tables

Introduction

Executive summary

Experimental

Results and Discussion

Progress in development of in situ continuous methane sensor

Progress on Raman Sensor and testing of METs sensor

Initiation of development on oceanic in situ mass spec sensor

Measurement of hydrocarbons in biodegraded oils: Gulf of Mexico

Measurement of hydrocarbons in biodegraded oils; Santa Barbara, Coal Oil Point Seep oils

Conclusions

References

List of acronyms and abbreviations

Appendices

I) Progress report submitted to NOAA on development of methane sensor

II) Proposal submitted to Woods Hole Oceanographic Institution for Green Technology Award (funded). A Deep Diving Mass Spectrometer for Ocean Exploration of Gas Seeps and Pollution Monitoring

III) Report: Hydrocarbon distributions from extant petroleum seep sites and associated gas hydrate deposits in sea water and ocean floor sediments from Santa Barbara and Gulf of Mexico, Jean K. Whelan and Lorraine B. Eglinton

\section{Figures and Tables}

Fig 1- Gulf of Mexico hydrate- gas seep overview

Table 1 - Typical methane concentrations in various oceanic seeps and concentration ranges available from various instruments 


\section{Introduction}

Natural oil and gas seep zones associated with seafloor gas hydrate zones in the Gulf of Mexico show very large variations in a number of chemical species associated with venting over very small distances. This natural variability will present major problems for appropriate siting of a monitoring station to follow gas venting as it relates to resource evaluation and hydrate formation and destruction over time. The major accomplishments of our research group in addressing this problem has been two-fold this year:

1) development of a suitable methods for in situ measurements of methane and other light hydrocarbon concentrations issuing from active vents and

2) development of methods for relating biodegraded oils found in hydrates and seeps to both their parent oils and to the degree that they have been affected by various alteration processes, including biodegradation, water washing, and gas washing.

The longer term goal of the project is to integrate these measurements with geophysical data to determine the amounts and compositions of subsurface hydrates and hydrocarbon in reservoirs for use both as an exploration tool and as means of quantitating the amounts of gas either trapped or flowing through the complex sedimentary hydrate-gas flow network (Fig 1).

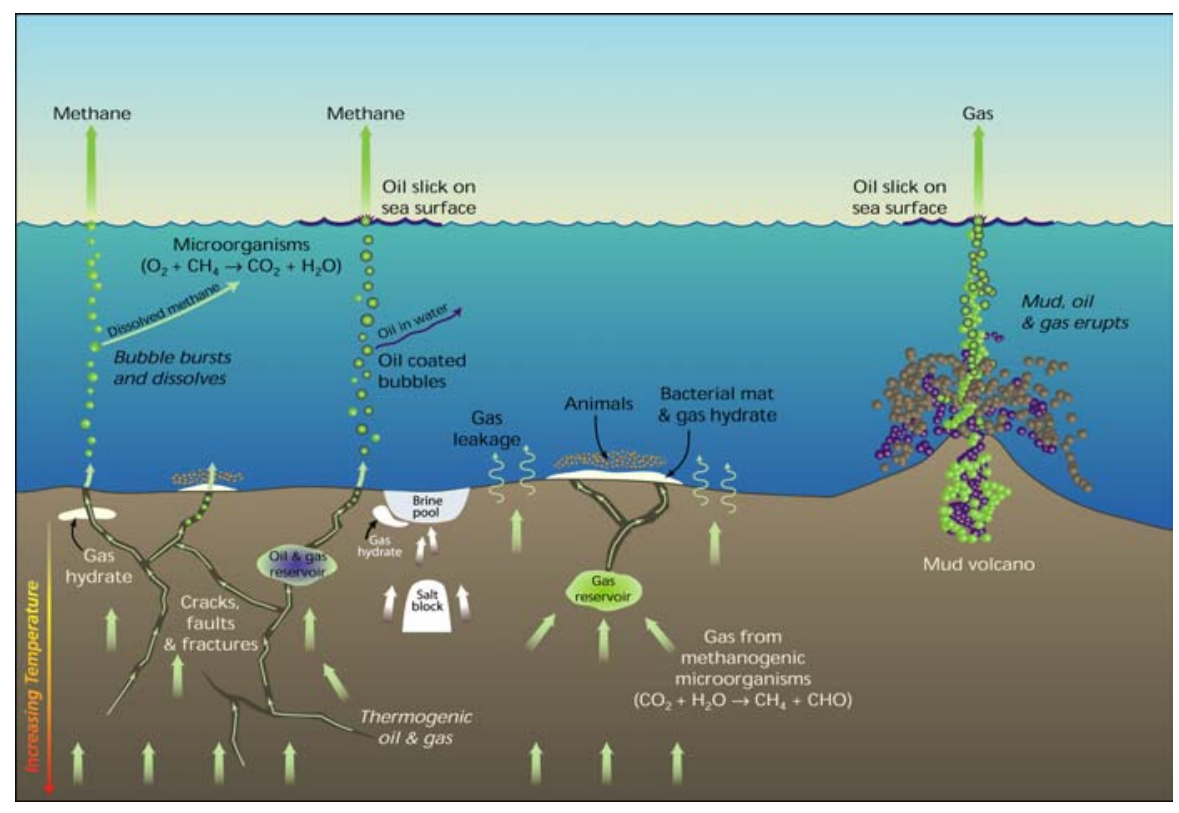

Figure 1: Summary of all gas seep related processes observed in Green Canyon area in the northern Gulf of Mexico (location shown in Fig 1). The processes shown include thermogenic oil and deeper gas generation (to the left) and biogenic methane generation (to the right). Gas from either source migrates upward either rapidly through faults and fractures or more slowly by diffusion through sediments into overlying oil and gas reservoirs. Most of the gas bypasses the reservoirs and continues its upward migration to the sediment water interface where it can form gas (methane) hydrate deposits (shown in white) or can be vented into the overlying water column. If methane concentrations do not reach saturation, the gas in the dissolved phase is largely biodegraded to carbon dioxide in the water column. When methane reaches saturation in sea water, gas bubbles form and migrate upward. Oil can coat the bubbles and be delivered into a sea surface oil slick (see Leifer et al., this volume). If bubbles survive to within $100 \mathrm{~m}$ of the surface, methane can be vented to the atmosphere. Chemosynthetic communities of 
animals, shown with pink dots, tend to congregate on surfaces of gas hydrates and within bacterial mats near surface sediment gas seeps.

The geochemical data to be collected in the initial field study proposed here and from the longer term monitoring station measurements will provide basic information needed to assess the resource potential of seafloor gas hydrates in the Gulf of Mexico. These data address three basic questions: 1) rate of formation and long term stability of the hydrates; 2) their rate of destruction via interaction with the surrounding biological communities which are probably dependent on hydrocarbons as a food source; and 3) rates of hydrate formation and destruction from gas seeping upward from depth and venting into the overlying water column. The specific long term measurements to be carried out, if they prove useful in this initial field work, include changes in salinity, temperature, methane, fluorescence (related to amount of oil and microorganisms), and possibly $\mathrm{pH}$, turbidity, and sulfate. All of these parameters relate directly or indirectly to either gas hydrate stability or to the biogeochemistry of gas hydrates and seeping gas. In addition, discrete water samples must be collected periodically to monitor changes in gas compositions and isotopes.

This initial data and the longer term continuous measurements from the monitoring station will answer several basic questions regarding seafloor gas hydrates in the Gulf of Mexico which are important in assessing their long-term resource potential:

What are the effects of long term venting of gases and fluids from depth on seafloor hydrate formation and destruction? Are these gas hydrates a relatively fixed and reliable resource, which can be produced? Or do they represent only a localized and ephemeral phenomenon dependent on the degree of localized gas flux and the related degrees of microbial cycling of sulfur and carbon?

How much of gas from the hydrates or from the gases venting through them might actually be available as a future potential resource?

How does the very dynamic biogeochemistry and presence of sulfur within and around the hydrate zone affect the resource potential of methane from hydrates? How localized is gas venting from or through hydrates and how is it affected by the biological processes? How does gas venting interact with microbial processes of methane production versus utilization, sulfate reduction versus sulfur oxidation? What other microbial processes are operating and how do they affect methane resource potential from the surface gas hydrates?

Seafloor hydrates recovered during the Aug 2000 cruise had a strong disagreeable odor caused by sulfur compounds. What is the chemical form of the sulfur and does it affect the economic viability of seafloor hydrates as a potential fuel source?

If the upward flow of gas is on-going and hydrates are continuously being formed and destroyed, then seafloor hydrates might be a self-renewing energy source and any disruption caused by their seafloor mining might be minimal.

Can gas hydrates be mined from the seafloor with minimal disruption of the biogeochemistry of animals and organisms dependent on them? 


\section{Executive summary}

Natural oil and gas seep zones associated with seafloor gas hydrate zones in the Gulf of Mexico show very large variations in a number of chemical species associated with venting over very small distances. This natural variability will present major problems for appropriate siting of a monitoring station to follow gas venting as it relates to resource evaluation and hydrate formation and destruction over time. The major accomplishments of our research group in addressing this problem has been two-fold this year:

1) development of a suitable methods for in situ measurements of methane and other light hydrocarbon concentrations issuing from active vents and

2) development of methods for relating biodegraded oils found in hydrates and seeps to both their parent oils and to the degree that they have been affected by various alteration processes, including biodegradation, water washing, and gas washing.

The longer term goal of the project is to integrate these measurements with geophysical data to determine the amounts and compositions of subsurface hydrates and hydrocarbon in reservoirs for use both as an exploration tool and as means of quantitating the amounts of gas either trapped or flowing through the complex sedimentary hydrate-gas flow network

The geochemical data to be collected in the initial field study proposed here and from the longer term monitoring station measurements will provide basic information needed to assess the resource potential of seafloor gas hydrates in the Gulf of Mexico. These data address three basic questions: 1) rate of formation and long term stability of the hydrates; 2) their rate of destruction via interaction with the surrounding biological communities which are probably dependent on hydrocarbons as a food source; and 3) rates of hydrate formation and destruction from gas seeping upward from depth and venting into the overlying water column.

\section{Progress in development o reliable in situ methane sensor:}

Raman Sensor and testing of METs sensor Funding was obtained from NOAA to build a combined METs-Raman methane sensor which would have the wide dynamic range for in situ methane measurements needed to characterize natural ocean floor methane vent features. We proposed to build a dual detection methane sensing system utilizing a Raman spectrometer for monitoring higher methane concentrations near the venting plume (micromolar to millimolar methane concentrations) and a CAPSUMs METS sensor for the lower methane concentrations

further away from the plume. The original concept was that the Raman spectrometer would be used to protect the METS sensor from over saturation by triggering a shut off of the METS detector window whenever high methane concentrations are encountered.

To date, intial tests of the METs sensor have not been promising, although new field trials are planned for Feb 2004 using a newly designed instrument.

Mass Spectral methane detector It came to our attention this year that an underwater mass spectromether might be the ideal instrument for our purposes. Funding and personnel have been obtained to start building and testing such an instrument. Details of the project and initial design of the instrument are described in the results and discussion section and in Appendix 2. 
Experimental - See experimental sections in Appendices I, II, and III

\section{Results and discussion}

\section{Progress in development of in situ continuous methane sensor}

\section{Progress on Raman Sensor and testing of METs sensor}

The main purpose of our research this year is to develop an oceanic in situ methane measurement device which can be used both for exploration and characterization of gas hydrate deposits. At the present time, no system exists which can continuously monitor methane over the very wide dynamic range encountered around ocean floor methane vent features. Existing technologies have, so far, proven to be ill suited to sense and monitor methane dissolved in waters around these vent features.

In this work, with the bulk of the money coming from NOAA we proposed to build a dual detection methane sensing system utilizing a Raman spectrometer for monitoring higher methane concentrations near the venting plume (micromolar to millimolar methane concentrations) and a CAPSUMs METS sensor for the lower methane concentrations further away from the plume. The original concept was that the Raman spectrometer would be used to protect the METS sensor from over saturation by triggering a shut off of the METS detector window whenever high methane concentrations are encountered.

The research to date is described in Appendix 1 which is a annual report submitted to NOAA, who is funding this part of our research, on progress up to September of 2003. The technical data needed to address the scientific problem above are: How methane much and where is methane associated with gas hydrates venting from bottom seeps into the water column? How fast is the venting? What are the temporal and spatial variabilities of methane venting from ocean floor? Data in hand prior to this project show changes in methane concentration of several orders of magnitude ( $\mathrm{UM}$ to $\mathrm{nM}$, Table 1) over small distances (a few meters) in the vicinity of gas hydrates and their associated gas seeps. These initial discrete methane measures showed the need for continuous in-situ profiling of methane by a technique having very large dynamic range and a relatively rapid response time (a few seconds). These constraints ruled out most common methane measurement techniques and required an instrument having a high sensitivity, wide dynamic range, and a rapid response time to handle large spatial and temporal variations expected during long or short time-series monitoring of seeps. 
Table 1: Typical methane concentrations in various water bodies and typical sensitivities of various methane measuring techniques $(2 \mathrm{ppm}=3 \mathrm{nM})$.

\begin{tabular}{|l|l|}
\hline Gulf of Mexico: & Methane concentration \\
\hline Surface waters & $100 \mathrm{ppm}=150 \mathrm{nM}=0.15 \mathrm{uM}$ \\
\hline mid-depth waters & $180-700 \mathrm{ppm}=0.27-1.05 \mathrm{uM}$ \\
\hline Bottom waters- near seep & average: $4-10 \mathrm{uM}$ \\
\hline \multicolumn{1}{|c|}{ - mid water directly over seep } & 0.1 to $0.7 \mathrm{uM}$ \\
\hline pore waters at seep & 0.5 to $40 \mathrm{uM}$ \\
\hline pore waters away from seep & $<0.5 \mathrm{uM}$ \\
\hline methane saturation values, $600 \mathrm{~m}$ & $60 \mathrm{mM}=60,000 \mathrm{uM}$ \\
\hline & Methane Concentration \\
\hline Open Ocean & $>60 \mathrm{mM}=>60,000$ uM \\
\hline Bubbles venting from gas plume & $40 \mathrm{uM}$ \\
\hline A few meters from plume & 10 to $100 \mathrm{nM}=0.01$ to $0.1 \mathrm{uM}$ \\
\hline In plume as it spreads out into ocean & $1-3 \mathrm{nM}$ or less $=0.001$ to $0.003 \mathrm{uM}$ \\
\hline Oceanic background levels & \\
\hline & $1 \mathrm{mM}=1000 \mathrm{uM}$ \\
\hline Sensitivities, methane measuring devices & 25 to $100 \mathrm{uM}$ \\
\hline Current Raman spectrometer & $0.25-1 \mathrm{uM}$ \\
\hline $\begin{array}{l}\text { projected -modified Ramen spectrometer } \\
\text { (minimum modification) }\end{array}$ & all oceanic levels including \\
\hline $\begin{array}{l}\text { Further instrument modification - projected } \\
\text { estimate of maximum sensitivity }\end{array}$ & background \\
\hline $\begin{array}{l}\text { Gas Chromatograph (GC) - FID detector, } \\
\text { surface detection with gas stripping on deck } \\
\text { of ship }\end{array}$ & $5-10 \mathrm{nM}=0.005$ to $0.01 \mathrm{uM}$ \\
\hline $\begin{array}{l}\text { Commercially available METs methane } \\
\text { sensor }\end{array}$ & \\
\hline
\end{tabular}

Our proposed solution was to combine a Raman sensor which has a wide dynamic range and rapid response time, but low sensitivity (10s of uM) with a METS sensor, a commercially available detector utilizing membrane absorption and chemical reaction of methane. Previous researchers have found this instrument to have a low dynamic range and sluggish response time (minutes) but high sensitivity (nM). Raman spectroscopy meets all technical requirements except for sensitivity (existing Welldog instrument: about $100 \mathrm{uM}$; estimated that this can be increased to $100 \mathrm{nM}$ or better with more sophisticated spectrometer)

The commercially available (METS) sensor has smaller dynamic range, but theoretically higher sensitivity. Based on previous work with an instrument borrowed from Bob Lamontagne and co-workers at the Naval Research Laboratory, the METS sensor detects low methane concentrations ( $\mathrm{nM}$ to $\mathrm{uM}$ ) but oversaturates and becomes inoperable at higher methane concentrations. This process is reversible by bringing the METs back on deck and flushing the membrane with nitrogen. Thus, the METs insrument appeared to have a number of limitations for our work. In addition, no reliable laboratory or field calibrations of the instrument were available at the time our work started.

The first task in this project has been to evaluate and test both the Raman and METs sensors in our laboratories to determine for ourselves their suitablity for this project. Our progress to date shows that: 
1) The Raman detector looks very promising and may be much more sensitive that we originally anticipated and

2) The METs sensor doesn't look very promising in work so far. However, the manufacturer of the instrument will be working with us in ship-board testing of a new version of the sensor in February of this year. This will give us a definitive test of the technology as well as a comparison between laboratory and in situ methane measurements. The older METs instruments used so far have not performed very well in these tests.

Progress on Raman Spectometer Initial tests with the existing Welldog instrument indicated that an upgraded Raman may be much more sensitive than we originally predicted and may ultimately produce usable methane signals for concentrations as low as 10 s to 100 s of $\mathrm{nM}$, or almost open ocean background methane levels. The results shown in Figs 1-5 of Appendix 1 were all carried out on the existing Welldog instrument which is much less sensitive than our upgraded spectrometer will be. Work has started on the upgraded Raman spectrometer, but has been delayed because the initial instrument had to be returned to the manufacturer because it did not meet the contracted specifications. We are aiming for a new upgraded (more sensitive) instrument to be ready for its first (shallow water) oceanic tests in Saanich Inlet, B.C. in Feb of 2004 (water depth: 300 m). On that cruise, we plan to compare in situ methane measurements from the Raman and from the METs sensor with laboratory measurements on discrete water samples from the same zone. An ancillary benefit of using the Raman spectrometer in this project is its potential ability to detect and measure other seeprelated organic compounds along with methane possibly including: dimethylsulfide, higher alkanes and aromatic hydrocarbons, alcohols, carboxylic acids, amines, carbon dioxide, etc. Some examples from laboratory analyses to date are shown in Appendix 1.

\section{Initiation of development on oceanic in situ mass spec sensor}

The in situ Raman Spectrometer technology above looks very promising for higher methane concentrations around seeps in active seep areas of the Gulf of Mexico, but may not ever have the sensitivity needed to detect the lower concentrations of methane from deeper water seeps both in the open ocean and in the deep water Gulf of Mexico (range: 100- a few nM). Conversations between Dr Michael Whiticar, myself and Dr. Rich Camilli, who recently received his Ph.D. in Civil Engineering from MIT, made us aware that underwater mass spectrometry might be the best possibility to solve this problem. Recent conversations with scientists at both the University of South Florida (Bob Byrne) and at MIT (Rich Camilli) indicate that the underwater mass spectrometer part of such an instrument is already technologically possible. Both groups have carried out successful intial tests of various types of spectrometers and obtained in situ measurements of pollutant organics in shallow waters (aromatic compounds). Some very preliminary work was also carried out on fixed and hydrocarbon gas analysis in coastal waters to maximum depths of about $100 \mathrm{ft}$. Discussions to date indicate that the main technological problem to going deeper is designing a suitable inlet system. Rich Camilli who built the MIT underwater spectrometer as part of his doctoral thesis is currently applying for a Postdoctoral Scholar award at Woods Hole. (His chances seem very good - he wrote most of the text for the successful Green Technology Award and the pending NOAA proposals described below). In the meantime, he has been appointed as a Guest Investigator at WHOI working in the laboratories of Jean Whelan in the Marine Chemistry and Geochemistry Department and Dr Hanu Singh, a WHOI scientist in the Applied Ocean Physics and Engineering Department. Jean Whelan and Rich Camilli have just received a Green Technology Award from the Woods Hole Oceanographic Institution to build the initial stages of the instrument. Rich Camilli, Jean Whelan, and Mike Whiticar have also applied for funding from the NOAA Ocean Exploration program for this project. A description of the instrument proposed 
and progress so far can be found in Appendix II. We are aiming to carry out initial shallow water sea trials of the instrument at the University of Victoria in the fall of 2004.

Abstract We propose to build and test an in-situ mass spectrometer suitable for continuous monitoring of methane, gases, and low molecular weight organic compounds including pollutants in the ocean and which can operate to at least several hundred meters water depth. This instrument is intended to be a prototype for a future instrument capable of operation at all ocean depths. The scientific motivation for this project is development of a sensitive and versatile in situ monitoring instrument for gases and volatile organic compounds vented from oceanic "cold" seeps associated with methane hydrate deposits. The resulting data will be used along with geophysical data to distinguish between two different models of methane hydrate formation and maintenance - a "stable methane hydrate" model where the hydrate deposit has a relatively long lifetime versus a "steady state" model where methane is continuously streaming upward to form the bottom and is continuously being lost from the top of the hydrate deposit. The mass spectrometer will also serve as an exploration tool for exploration for new thermal and biogenic methane gas seeps in the oceean floor. These can be associated with hydrothermal vent sites and areas of discharge of land-based run-off as well as cold seeps. In situ mass spectrometry would compliment data from our in situ Raman spectrometer (currently being built with NOAA funding-University of Mississippi funding) in characterization of the fluid discharge from all three types of ocean floor vent sites. Alternatively the instrument can be used in shallower waters to unobtrusively monitor a variety of organic compounds in coastal waters impacted by urban runoff, shipping lanes, and point sources of ground water discharge to the ocean.

Description of proposed mass spectrometer (summarized from Appendix II). We propose to develop and deploy a versatile and rugged submersible mass spectrometer, for operation aboard a variety of oceanographic platforms. This new analytical instrument will be capable of real-time, continuous, in-situ, high-resolution measurements of dissolved gases, particularly methane, and volatile organic compounds in the ocean and will be able to operate to at least several hundred meters water depth. Funds from this project will be used as seed money to develop a similar deep water instrument for operation to full ocean depths.

In our laboratory, the instrument will be used to find and monitor methane seeps associated with ocean floor gas hydrate deposits. An abundance of recent evidence has shown that methane venting through small faults and fractures in the seafloor occurs in many locations worldwide and is important to the biology, chemistry, and geology of the ocean. Also, methane is a greenhouse gas. Even though the seafloor gas venting occurs primarily through localized fractures, the volume of methane involved may be substantial enough to contribute to global warming. However, no systematic exploration strategy currently exists for finding these generally very localized methane vents on the ocean floor. Furthermore, it is difficult to obtain reliable measurements of the gas concentrations or fluxes involved because venting tends to be very heterogenous and episodic. Methane concentrations commonly vary over several orders of magnitude within a few meters of the vent; reliable data on amounts as well as molecular and isotopic compositions involved cannot be obtained on samples brought to the surface and allowed to degas. Therefore, it is currently almost impossible to measure quantitatively how the venting methane interacts with the complex biological communities, gas hydrates, and bubble streams flowing around and through the hydrates.

The underwater mass spectrometer will be capable of monitoring oceanic in situ concentrations of both the high methane concentrations near venting plumes (micromolar to tens of millimolar methane concentration) to the lower methane concentrations (as little as 0.01 to $0.02 \mathrm{uM}$ ) typically found in moving away even a few meters away from the plume. The high sensitivity is important for finding new vents and monitoring the effects of methane in moving 
away from the vent site. This work will compliment that currently in progress under a NOAA funded project to build an underwater Raman spectrometer. Laboratory and pressure tank tests so far have shown the Raman instrument to be suitable for the higher but not the lower methane concentrations found at hydrate associated vents. Additional work on this NOAA project has conclusively shown that the commercially available METS sensor possesses a number of technical problems making it unsuitable for this work.

Mass spectrometry is by far the most sensitive and versatile analytical technique available for identifying and quantifying a large variety of organic compounds likely to be found in the ocean in various settings. For some compounds, such as aromatic hydrocarbons typical of petroleum seeps and low molecular weight functionalized organics typical of various types of microorganisms (e.g. methane, acetic acid, carbon dioxide, hydrogen sulfide and alkyl sulfides from anaerobic bacteria in sediments), organic signatures are very diagnostic of the type of chemosynthetic organisms supported by these ocean floor seeps. These ocean floor gas and liquid venting sites have been described in many areas around the world in a number of deep sea environments for which this mass spectrometer is intended, including 1) natural gas and oil seeps ("cold seeps"); 2) hydrothermal vent sites; and 3) areas of discharge of land-based runoff.

Despite the advantages of in-situ mass spectrometry, to date only two submersible mass spectrometer designs have been deployed which are limited to operational depths of less that tens of meters. One of these was designed and built by Rich Camilli who will be involved in this project (Fig 2, Appendix 2). Our instrument is intended to be compact and fully self-contained, permitting continuous in situ measurements aboard platforms including ship-borne rosettes, ROVs such as the Jason vehicle, and various AUVs. It will function as a low power, ruggedized system that can be deployed in harsh environments with a mass range from 1-300AMU, sensitivity of 10-100 ppb for most volatile chemicals, and a time response on the order of tens of seconds.

Measurement of hydrocarbons in biodegraded oils: Gulf of Mexico and Santa Barbara, Coal Oil Point Seep oils

\section{Experimental}

Analysis of Gulf of Mexico seep oils both from waters, sediments and from hydrate melt waters were carried out using the procedures described in Appendix 3. The waters did not contain sufficient hydrocarbon concentrations to warrant further analysis. Results here are for the Gulf of Mexico sediment and hydrate seep oils collected on the Aug 2000 cruise. For comparison to other seep oils where gas hydrates are not involved, three sediment samples and three water samples containing oil globules from seep sites the Santa Barbara Coal Oil Point seep were also analyzed as described in Appendix III.

Univerally, hydrocarbons in seawater samples contained only trace amounts of oil and could not be analyzed except for one Gulf of Mexico sample where trace amounts of monoaromatic steroids could be seen. Because large water volumes would have to be extracted, further analysis of oils in waters was not undertaken.

The sediment and hydrate extracts commonly produced some resolved GC peaks on top of a very large unresolved complex mixture of compounds

\section{Conclusions}

Both Areas Initial results with 2D GC show that this may be the ideal method for characterizing biodegraded seep and reservoir oils. Extensive series of resolvable cyclic and 
branched alkanes are present in both the Gulf of Mexico and Santa Barbara seep oils which remain hidden in the UCM or unresolved complex mixture of most GC methods. This compound class, rather than much higher molecular weight compounds, appear to comprise the bulk of the oil in both areas and were previous unanalyzable. The method also provides quanitative data on the amounts of individual compound present and so my allow us to gain a quantitative measure of the relative extent of various oil alteration processes in both areas, water washing versus gas washing versus biodegradation.

Santa Barbara Based on loss of $n$-alkanes, modification of steranes, an emerging series of 25-norhopanes the Santa Barbara samples have been heavily biodegradation (rank 6) based on the biodegradation rank of Peters and Moldowan (1993). The degree of biodegradation changed little over the sampled area within the sediment extract and the globules. This may indicate rapid replenishment of hydrocarbon material and possibly a high degree of biodegration within the reservoired oils feeding the seep.

Fluids in the Santa Barbara seep site are rich in hydrocarbons consistent with petroleum sourced from the Monterey formation. This correlates with other seep oils found up and down the West coast. The oils are severly biodegraded. Biodegradation may have occurred subsurface in reservoirs or migration conduits cool enough o support biodegradation. Distinguishing biodegradation in reservoirs versus that in the water column requires further investigation but identification of suites of sulfur containing compounds may hold the key

Gulf of Mexico Initial results suggest that the seep oils and underlying reservoir oils are identical in terms of source and maturity, based biomarker and aromatic hydrocarbon analyses. If this observation holds up to further scrutiny, then we will be able to use these seep oils as a proxie for the underlying oils. In contrast, the $\mathrm{n}$ - and branched alkanes are almost completely degraded in the seep oils.

The Gulf of Mexico oils appear to be much less extensively biodegraded than the Santa Barbara oils. 


\section{List of acronyms and abbreviations}

C1-C3 light hydrocarbon methane, ethane, and propane having one to three carbon atoms

13C the isotope of carbon having an atomic weight of 13 mass units. Delta $13 \mathrm{C}$ measurements are expressed as the enrichment of $13 \mathrm{C}$ versus $12 \mathrm{C}$ in comparison to a standard.

DoE Department of Energy

GC gas chromatography

2D GC See figure 8 - The separated material in the initially resolved individual GC peaks is subjected to a 2 nd rapid GC analysis.

GC-IRMS gas chromatography-isotope ration mass spectroscopy

GC185 Green Canyon Block 185 in the Gulf of Mexico - location of seep site on August 2000 cruise

WHOI Woods Hole Oceanographic Institution

CH4 methane

m meters

METs Brand name for commercially available methane sensor

$\mathbf{u M}$ micromolar $=$ micromoles of methane per liter of water

$\mathbf{m M}$ millimolar $=$ thousandths of a mole per liter of water

ft feet

ppm parts per million

MIT Massachusetts Institute of Technology

Raman A technique utilizing the infrared absorption range to identify individual organic compounds

Coal Oil Point location of a persistent oil seep in shallow water in the Santa Barbara Channel

MS mass spectrometer

\section{Appendices}

I) Progress report submitted to NOAA on development of methane sensor

From: Jean Whelan, Woods Hole Oceanographic Institution

Date: September 23, 2003

Associates: John Pope, Well Dog Inc, Laramie, Wy

Mike Whiticar, University of Victoria, B.C., Canada

\section{Background and Introduction}

Gas hydrate deposits are thought to represent one of the largest carbon reservoirs on earth based on interpretations of seismic data (Kvenvolden, 1988, 1993). Understanding the biology and geochemistry as well as the geology of the seafloor surrounding gas hydrate deposits is crucial to understanding where and how these important deposits form as well as 
how they might be exploited commercially. Biogeochemical processes strongly influence rates of both formation and destruction of hydrates (Grabowski et al., 2003).

Gas hydrate are commonly associated with seafloor methane gas venting. It is currently unclear how much of this methane comes from dissociation of the hydrate versus how much is flowing up from depth in a continuous steady state hydrate formation/destruction process.

The purpose of our research is to develop an oceanic in situ methane measurement device which can be used both for exploration and characterization of gas hydrate deposits. At the present time, no system exists which can continuously monitor methane over the very wide dynamic range encountered around ocean floor methane vent features. Existing technologies are ill suited to sense and monitor methane dissolved in waters around these vent features.

In this work, we proposed to build a dual detection methane sensing system utilizing a Raman spectrometer for monitoring higher methane concentrations near the venting plume (micromolar to millimolar methane concentrations) and a CAPSUMs METS sensor for the lower methane concentrations further away from the plume. The original concept was that the Raman spectrometer would be used to protect the METS sensor from over saturation by triggering a shut off of the METS detector window whenever high methane concentrations are encountered.

\section{Progress to date}

The technical data needed to address the scientific problem above are: How methane much and where is methane associated with gas hydrates venting from bottom seeps into the water column? How fast is the venting? What are the temporal and spatial variabilities of methane venting from ocean floor? Data in hand prior to this project show changes in methane concentration of several orders of magnitude ( $\mathrm{uM}$ to $\mathrm{nM}$ ) over small distances (a few meters) in the vicinity of gas hydrates and their associated gas seeps. These initial discrete methane measures showed the need for continuous in-situ profiling of methane by a technique having very large dynamic range and a relatively rapid response time (a few seconds). These constraints ruled out most common methane measurement techniques and required an instrument having a high sensitivity, wide dynamic range, and a rapid response time to handle large spatial and temporal variations expected during long or short time-series monitoring of seeps.

Our proposed solution was to combine a Raman sensor which has a wide dynamic range and rapid response time, but low sensitivity (10s of uM) with a METS sensor, a commercially available detector utilizing membrane absorption and chemical reaction of methane. Previous researchers have found this instrument to have a low dynamic range and sluggish response time (minutes) but high sensitivity (nM). Raman spectroscopy meets all technical requirements except for sensitivity (existing Welldog instrument: about $100 \mathrm{uM}$; estimated that this can be increased to $100 \mathrm{nM}$ or better with more sophisticated spectrometer)

The commercially available (METS) sensor has smaller dynamic range, but theoretically higher sensitivity. Based on previous work with an instrument borrowed from Bob Lamontagne and co-workers at the Naval Research Laboratory, the METS sensor detects low methane concentrations ( $\mathrm{nM}$ to $\mathrm{uM}$ ) but oversaturates and becomes inoperaable at higher methane concentrations. This process is reversible by bringing the METs back on deck and flushing the membrane with nitrogen. Thus, the METs insrument appeared to have a number of limitations for our work. In addition, no reliable laboratory or field calibrations of the instrument were available at the time our work started.

The first task in this project has been to evaluate and test both the Raman and METs sensors in our laboratories to determine for ourselves their suitablity for this project. Our progress to date shows that:

1) The Raman detector looks very promising and may be much more sensitive that we originally anticipated and

2) The METs sensor doesn't look very promising in work so far. 
Progress on Raman Spectometer Initial tests with the existing Welldog instrument indicated that an upgraded Raman may be much more sensitive than we originally predicted and may ultimately produce usable methane signals for concentrations as low as 10 s to 100 s of $\mathrm{nM}$, or almost open ocean background methane levels. The results shown in Figs 1-5 were all carried out on the existing Welldog instrument which is much less sensitive than our upgraded spectrometer will be. Figures 1-3 shows laboratory data for methane dissolved in seawater. Figures 1 shows the methane signal in water and how it responds to progressively lower methane concentrations. Figure 2 shows that the response of the instrument is very linear over a wide concentration range. Comparison of Figures 3 and 4 suggest that it may be possible to utilize the second derivative of the Raman methane signal to minimize the problem of overlap between the methane and the much stronger water peaks which is apparent in Fig 3.

Work has started on the upgraded Raman spectrometer, but has been delayed because the initial instrument had to be returned to the manufacturer because it did not meet the contracted specifications. A new instrument has been ordered. It is expected that the new upgraded (more sensitive) instrument will be ready for its first (shallow water) oceanic tests in Saanich Inlet, B.C. in Nov (water depth: $300 \mathrm{~m}$ ). On that cruise, we plan to compare in situ methane measurements from the Raman with laboratory measurements on discrete water samples from the same zone.

An ancillary benefit of using the Raman spectrometer in this project is its potential ability to detect and measure other seep-related organic compounds along with methane possibly including: dimethylsulfide, higher alkanes and aromatic hydrocarbons, alcohols, carboxylic acids, amines, carbon dioxide, etc. Some examples from laboratory analyses to date are shown in Fig 5

Progress on METs sensor Initial tests of the Naval Research laboratory METs sensor at the University of Victoria by Mike Whiticar and co-workers have not been promising. The METs detects small concentrations of methane. However, there there is little correspondence between the METs readings and the actual concentrations. Also, the recovery of the sensor after introduction of methane is very slow - it took 80 minutes for methane to flush from the membrane in one test.

However, the scientists associated with the French company which makes the instrument believes that these problems are caused by our use of an older instrument. Therefore, Michell Mason, president of the company builds and sells the METS, is traveling to the University of Victory to work with Mike Whiticar on deploying the newer version of the instrument on gas seeps around Vancouver Isand within the next few months. Dr Mason's time and the instrument are donated for this test; we are allocating NOAA funds for reimbursement for his travel.

Exploration of other possibilities - Mass Spectroscopy In the meantime, Jean Whelan and Mike Whiticar are also exploring the feasibility of using an underwater mass spectrometer as an in situ methane sensor. Recent conversations with scientists at both the University of South Florida (Bob Byrne) and at MIT (Rich Camilli) indicate that the underwater mass spectrometer part of such an instrument is already technologically possible. Both groups have carried out successful intial tests of various types of spectrometers and obtained in situ measurements of pollutant organics in shallow waters (aromatic compounds). Some very preliminary work was also carried out on fixed and hydrocarbon gas analysis in coastal waters to maximum depths of about $100 \mathrm{ft}$.

Discussions to date indicate that the main technological problem to going deeper is designing a suitable inlet system. Rich Camilli who built the MIT underwater spectrometer as part of his doctoral thesis is currently applying for a Postdoctoral Scholar award at Woods Hole. If he is successful, he will go to work building a new instrument suitable for deeper water work. 
Jean Whelan and Mike Whiticar are currently seeking funding from the NOAA Ocean Exploration program to support the needed instrument parts for this project.

\section{Future plans:}

A proposal has just been submitted to the DoE gas hydrate program through the University of Mississippi to install in situ water column geochemical methane and CTD sensors on the seafloor northern Gulf of Mexico. Seafloor fluid and gas venting associated with surface and subsurface gas hydrates will be monitored as part of the Gulf of Mexico Research Consortium to use the combined geophysical and geochemical data from the monitoring station are to determine processes controlling hydrate growth and dissociation.

The Woods Hole part of the project is to measure continuous in situ bottom water geochemical data (in situ methane and CTD measurements of salinity, temperature, pressure, fluorescence, and turbidity) to look for changes associated with the gas hydrate. Our overall approach utilizes the observation that most gas hydrates in the Gulf of Mexico and in many other areas world wide are also associated with significant upward gas flow though faults and fractures into overlying sediments, waters, and possibly the atmosphere (e.g., Brooks et al, 1987, Roberts and Carney, 1997, Whelan et al 1998; Kvenvolden and Lorenson, 2001, and Sassen et al, 2001, ).

Two different hypotheses currently exist for the origin of this seeping gas:

1 ) the gas comes from a deeper source, either thermogenic or biogenic, and forms a hydrate deposit whenever high enough pressures and low enough temperatures are present for hydrate formation to occur. In this scenario, the hydrate is viewed as a capacitor in a very dynamic methane flow system (Zatsepina, et al, 1997; Dickens, et al, 1997; Sassen et al, 2001; and Cathles et al, 2002;

2) methane to form hydrates is derived from upward diffusion of local in-situ sediment sources of biogenic methane produced by anaerobic methanogenic bacteria so that hydrate amounts and lifetimes of the methane hydrate deposit can be estimated from in situ porewater profiles of methane and sulfate (as being done by I. MacDonald and M. Kastner in this research using procedures of Xu and Ruppel, 1999, Ruppel, 2000, and Valentine et al., 2001).

In scenario 2, the hydrate and associated seeping gas are primarily a function of the relative rates of hydrate replenishment via upward methane diffusion from the sediments below and hydrate decomposition due to interactions with warmer water column temperatures above. Recent temperature data from the MacDonald/Kastner group on several Gulf of Mexico surface gas hydrate deposits is consistent with scenario 2, but does not explain the vigorous gas evolution through fractures in the hydrates which is commonly observed around these deposits. Furthermore, recent modeling of the GC185 hydrate deposit by Cathles and co-workers at Cornell, based on a thermogenic gas source from underlying sediments, indicates that only 10 percent of the upward flowing gas is trapped in the hydrate while 90 percent is vented to the overlying water column and the atmosphere.

These two scenarios of methane hydrate formation and maintenance are very different; knowing which is correct significantly impacts the amount and concentration of methane ultimately recoverable from any particular hydrate deposit. In the overall DoE UMiss research program, both scenarios are being investigated: scenario 2 by the MacDonald/Kastner group and Scenario 1 by the Whelan/Chanton group. Although more research is needed, our previous work strongly suggests much more heterogeneous and pervasive upward gas flow in the Gulf of Mexico than is available from only the diffusive methane flow (Whelan, 1997, Whelan et al, 1998; Sassen et al. 2001, Cathles, et al., 2002 ; Roberts and Carney, 1997). The seafloor proposed geochemical monitoring combined with the geophysics would provide the quantitative geochemical data needed to distinguish the two scenarios and could be used 
together with the geophysical data to provide a general method for using surface data for detection and characterization underlying gas hydrate deposits.

\section{References}

Brooks, J.M., M.C. Kennicutt II, C.R. Fisher, S.A. Macko, K. Cole, J.J. Childress, R.R. Bidegare, and R.D. Vetter (1987) Deep-sea hydrocarbon seep communities: Evidence for energy and nutritional carbon sources, Science, v. 238, pp 1138-1142.

Cathles, L.M.; Dofu, C.; Roberts, H.H. (2002) Estimation of the hydrate gas content of the Bush Hill vent site, Green Canyon Block 185, Gulf of Mexico, (abstract) AAPG Bull, abstracts of annual meeting in Houston, Tx, March 2002, AAPG Bull, vol. 86.

Dickens, G.R., Castillo, M.M., and Walker. J.C.G., 1997, A blast of gas in the latest Paleocene: simulating first-order effects of massive dissociation of oceanic methane hydrate, Geology, 25:259-262

Grabowski, K., R. Rogers, J.Chanton, J.Whelan, P. Sobecky, 2002. Summary Report - Session II, Biological Influence on Hydrate Formation Stability, Content and Lattice Saturation. International Gas Hydrate Meeting, Washington, DC, November 2002

Kvenvolden, K.A. (1988) Methane hydrate - a major reservoir of carbon in the shallow geosphere? Chem Geol., v.71, pp 41-51.

Kvenvolden, K.A. (1993) Gas Hydrates-Geological perspective and global change. Reviews of Geophysics, v.31, pp 173-187.

Kvenvolden, K.A. and T.D. Lorenson (2001) Attention Turns to Naturally Occurring Methane Seepage (2001), EoS, v. 82, No. 4, p.457

Roberts, H.H. and R.S. Carney (1997) Evidence of episodic fluid, gas, and sediment venting on the Northern Gulf of Mexico Continental Slope. Economic Geology.

Ruppel, Carolyn (2000) Natural gas hydrates in oceanic and permafrost environments, Kluwer Academic Publishers, pp

Sassen, R., S. Losh, L. Cahtles, H. Roberts, J.K. Whelan, A.V. Milkov, S.T. Sweet, and D.A. deFreitas (2001). Massive vein-filling gas hydrate: Relation to ongoing gas migration from the deep subsurface in the Gulf of Mexico. Marine and Petroleum Geology, 18, 551-560.

Valentine, DL. , D.C. Blanton, W.S. Reeburgh and M. Kastner (2001) Water column methane oxidation adjacent to an area of active hydrate dissociation, Eel river Basin. Geochimica Et Cosmochimica Acta, 2001, V 65, N 16, pp. 2633-2640

Whelan, J.K. (1997) The dynamic migration hypothesis, How fast are oil \& gas leaking to the ocean floor and replenishing themselves in some reservoirs? Sea Technology, v.38, pp 1018, September, 1997.

Whelan, J.K., N. Mah, G. Eisheid, R. Chen, X. Wand, H. Roberts, and P Aharon, Gas and Oil Seepage and hydrothermal venting in the ocean bottom - detection by fluorescence. Proceedings of the Marine Technology Society Annual Conference, Nov. 1998, 7 pp:

Xu, W. and C. Ruppel (1999) Predicting the occurrence, distribution, and evolution of methane gas hydrate in porous marine sediments, J. Geophys Res, v.104, pp 5081-5095.

Zatsepina, Olga Y. and Buffett, Bruce A. (1997) Phase equilibrium of gas hydrate; implications for the formation of hydrate in the deep-sea floor, Geophysical Research Letters, v. 24, pp $1567-1570$

\section{Figures}

Figure 1: Spectrum of methane dissolved in water on WellDog's current Raman spectrometer

Figure 2: Dissolved Methane Calibration in Seawater, Existing Welldog Raman spectrometer 
Figure 3: Raman spectra - methane $\mathrm{CH}$ stretch used for quantitation and water bands.

Figure 4: Use of 2nd derivative of $\mathrm{C}-\mathrm{H}$ stretch to increase Raman instrument sensitivity for measurement of methane dissolved in water

Figure 5a: Examples of other chemicals which have been identified and measured with Raman in the laboratory with existing spectrometer

Figure 5b: Raman signature for methanol

Figure 1: Spectrum of methane dissolved in water on WellDog's current Raman spectrometer

\section{Calibration spectra for WellDog@̃ RA-1 spectrometer}

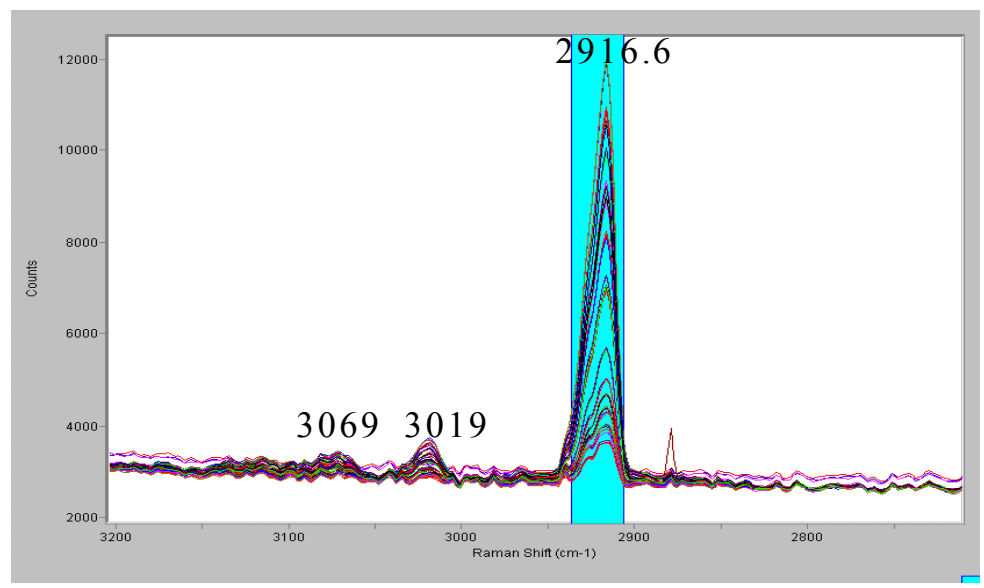

Methane dissolved in water. Each curve represents one measurement WellDog Inc. At a specific methane concentration. 


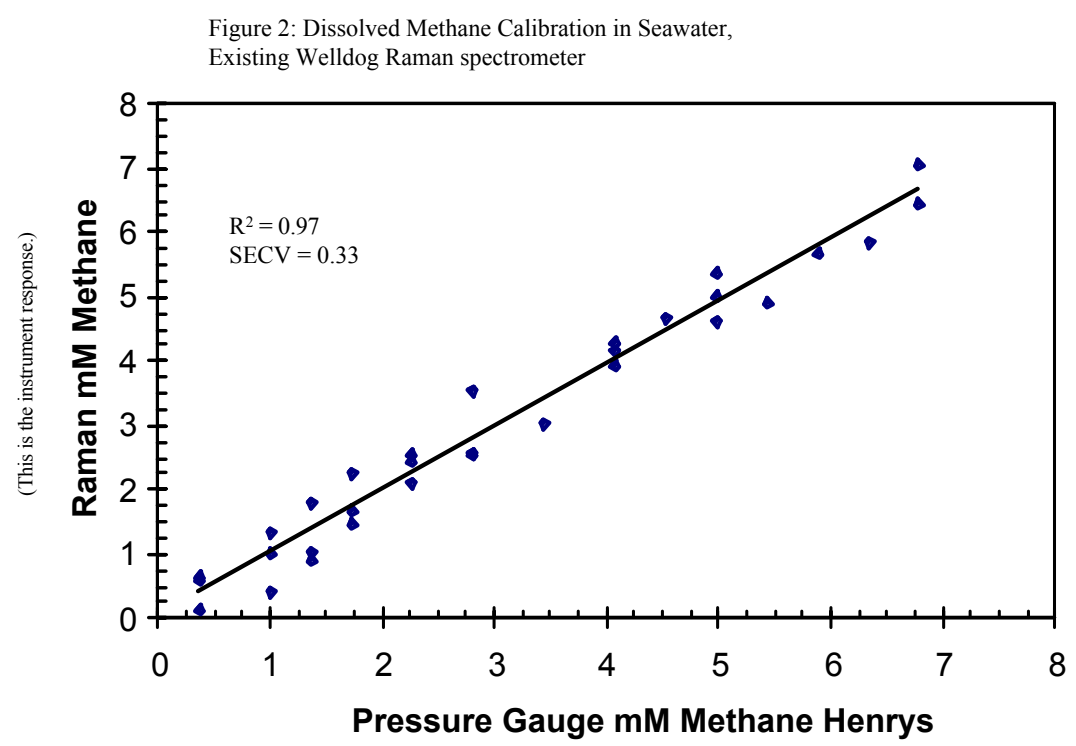

(This is the concentration of methane in the water as determined by the pressure gauge reading and Henrỹ̃ law.)

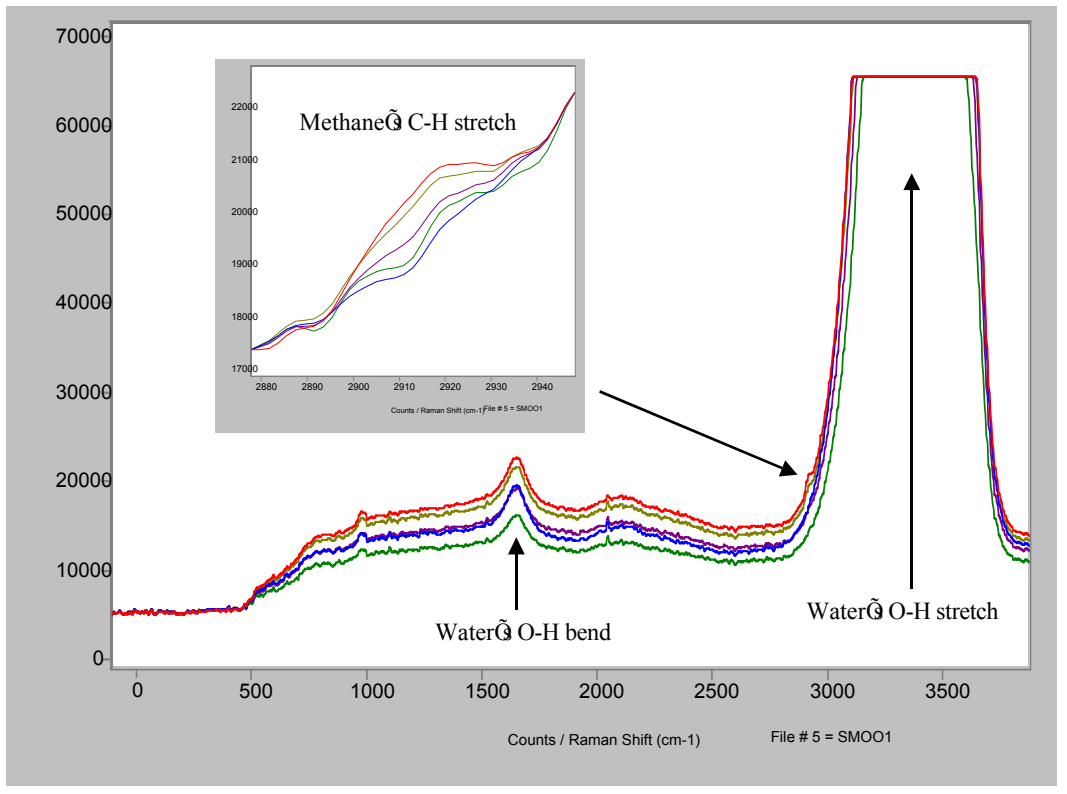

Figure 3: Raman spectra - methane CH stretch used for quantitation and water bands. 


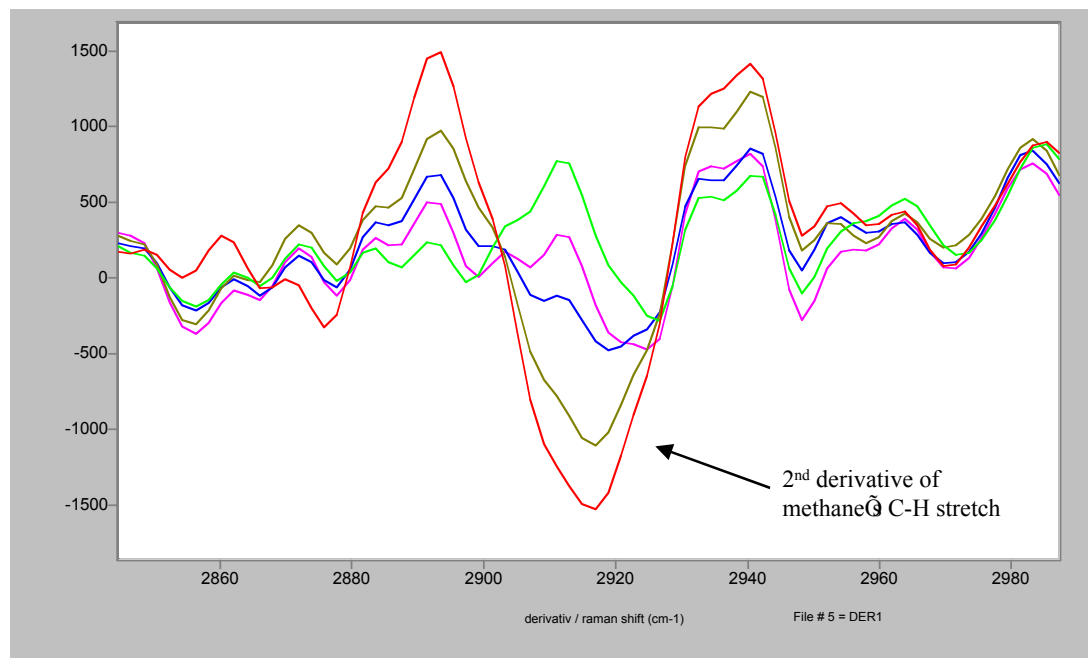

Figure 4: Use of 2nd derivative of C-H stretch to increase Raman instrument sensitivity for measurement of methane dissolved in water

Figure 5a: Examples of other chemicals which have been identified and measured with Raman in the laboratory with existing spectrometer

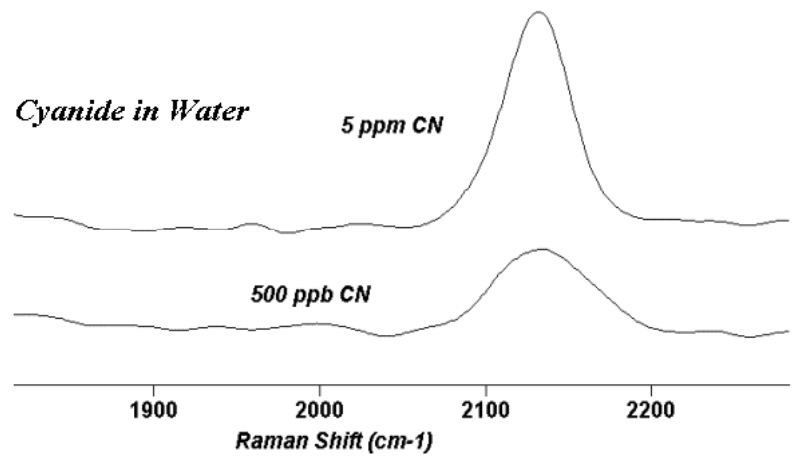


Figure 5b: Raman signature for methanol

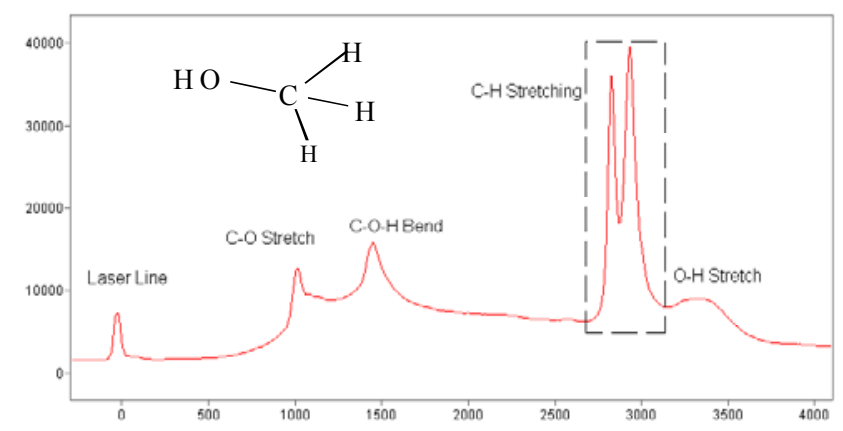

WellDog linc. | $3 / 5 / 2002214034$

\begin{abstract}
Appendix II: Proposal submitted to Woods Hole Oceanographic Institution for Green Technology Award (funded).
\end{abstract}

\title{
A Deep Diving Mass Spectrometer for Ocean Exploration of Gas Seeps and Pollution Monitoring
}

\begin{abstract}
We propose to build and test an in-situ mass spectrometer suitable for continuous monitoring of methane, gases, and low molecular weight organic compounds including pollutants in the ocean and which can operate to at least several hundred meters water depth. This instrument is intended to be a prototype for a future instrument capable of operation at all ocean depths. The scientific motivation for this project is development of a sensitive and versatile in situ monitoring instrument for gases and volatile organic compounds vented from oceanic "cold" seeps associated with methane hydrate deposits. The resulting data will be used along with geophysical data to distinguish between two different models of methane hydrate formation and maintenance - a "stable methane hydrate" model where the hydrate deposit has a relatively long lifetime versus a "steady state" model where methane is continuously streaming upward to form the bottom and is continuously being lost from the top of the hydrate deposit. The mass spectrometer will also serve as an exploration tool for exploration for new thermal and biogenic methane gas seeps in the oceean floor. These can be associated with hydrothermal vent sites and areas of discharge of land-based run-off as well as cold seeps. In situ mass spectrometry would compliment data from our in situ Raman spectrometer (currently being built with NOAA funding-University of Mississippi funding) in characterization of the fluid discharge from all three types of ocean floor vent sites. Alternatively the instrument can be used in shallower waters to unobtrusively monitor a variety of organic compounds in coastal
\end{abstract}


waters impacted by urban runoff, shipping lanes, and point sources of ground water discharge to the ocean.

Description We propose to develop and deploy a versatile and rugged submersible mass spectrometer, for operation aboard a variety of oceanographic platforms. This new analytical instrument will be capable of real-time, continuous, in-situ, high-resolution measurements of dissolved gases, particularly methane, and volatile organic compounds in the ocean and will be able to operate to at least several hundred meters water depth. Funds from this project will be used as seed money to develop a similar deep water instrument for operation to full ocean depths.

In our laboratory, the instrument will be used to find and monitor methane seeps associated with ocean floor gas hydrate deposits. An abundance of recent evidence has shown that methane venting through small faults and fractures in the seafloor occurs in many locations worldwide and is important to the biology, chemistry, and geology of the ocean. Also, methane is a greenhouse gas. Even though the seafloor gas venting occurs primarily through localized fractures, the volume of methane involved may be substantial enough to contribute to global warming. However, no systematic exploration strategy currently exists for finding these generally very localized methane vents on the ocean floor. Furthermore, it is difficult to obtain reliable measurements of the gas concentrations or fluxes involved because venting tends to be very heterogenous and episodic. Methane concentrations commonly vary over several orders of magnitude within a few meters of the vent; reliable data on amounts as well as molecular and isotopic compositions involved cannot be obtained on samples brought to the surface and allowed to degas (Fig 1). Therefore, it is currently almost impossible to measure quantitatively how the venting methane interacts with the complex biological communities, gas hydrates, and bubble streams flowing around and through the hydrates.

The underwater mass spectrometer will be capable of monitoring oceanic in situ concentrations of both the high methane concentrations near venting plumes (micromolar to tens of millimolar methane concentration) to the lower methane concentrations (as little as 0.01 to $0.02 \mathrm{uM}$ ) typically found in moving away even a few meters away from the plume. The high sensitivity is important for finding new vents and monitoring the effects of methane in moving away from the vent site. This work will compliment that currently in progress under a NOAA funded project to build an underwater Raman spectrometer. Laboratory and pressure tank tests so far have shown the Raman instrument to be suitable for the higher but not the lower methane concentrations found at hydrate associated vents. Additional work on this NOAA project has conclusively shown that the commercially available METS sensor possesses a number of technical problems making it unsuitable for this work.

Mass spectrometry is by far the most sensitive and versatile analytical technique available for identifying and quantifying a large variety of organic compounds likely to be found in the ocean in various settings. For some compounds, such as aromatic hydrocarbons typical of petroleum seeps and low molecular weight functionalized organics typical of various types of microorganisms (e.g. methane, acetic acid, carbon dioxide, hydrogen sulfide and alkyl sulfides from anaerobic bacteria in sediments), organic signatures are very diagnostic of the type of chemosynthetic organisms supported by these ocean floor seeps. These ocean floor gas and liquid venting sites have been described in many areas around the world in a number of deep sea environments for which this mass spectrometer is intended, including 1) natural gas and oil seeps ("cold seeps"); 2) hydrothermal vent sites; and 3) areas of discharge of land-based runoff.

Despite the advantages of in-situ mass spectrometry, to date only two submersible mass spectrometer designs have been deployed which are limited to operational depths of less that tens of meters. One of these was designed and built by Rich Camilli who will be involved in this project (Fig 2). Our instrument is intended to be compact and fully self-contained, permitting 
continuous in situ measurements aboard platforms including ship-borne rosettes, ROVs such as the Jason vehicle, and various AUVs. It will function as a low power, ruggedized system that can be deployed in harsh environments with a mass range from 1-300AMU, sensitivity of 10$100 \mathrm{ppb}$ for most volatile chemicals, and a time response on the order of tens of seconds.

The two most challenging aspects of long-term underwater mass spectrometry are: 1)vacuum maintenance and 2) sample introduction. To overcome the limited duration and excessive power consumption of standard ultra-high vacuum pumping strategies, while at the same time avoiding the instability of ion pumps [9], we propose to use a miniaturized molecular drag pump in parallel with an ion pump. Initial calculations indicate that this approach will yield a pumping velocity of approximately $40 \mathrm{l} / \mathrm{s}$ while requiring approximately 15 watts and will probably greatly extend service intervals between ion pump repair and replacement.

The ultimate limiting factor for sending submersible mass spectrometers to full ocean depths is designing a viable inlet system with the required mechanical strength. The analyte introduction system must be exposed to the ambient hydrostatic pressure in order to allow dissolved substances into the analyzer while simultaneously excluding water in order to preserve the near-perfect vacuum within the analyzer region of the instrument. The instrument will be incorporated within a standard cylindrical metal pressure housing and initially a membrane inlet developed specifically for deep deployment. Calculations indicate that this inlet will permit operation to at least several hundred meters depth, thus enabling investigation of many previously inaccessible environments. We will also explore the possibility of incorporating a capillary inlet which would allow operation to much greater depth.

The computational architecture will utilize a low power, small form factor, PC-104 or $\mu-$ controller based embedded computer allowing for real-time data processing. Electronic drift must be minimized for operation over extended timeframes. Furthermore, because the instrument must operate within a dynamic and oftentimes noisy environment, noise filtering techniques need to be incorporated to improve signal to noise ratio. Therefore, a suite of hardware and digital signal processing techniques will be implemented to improve signal quality, including: baseline identification to compensate for electronic artifacts such as detector signal DC offset and baseline drift, bandpass filtering, signal averaging and median filtering to minimize the effects of internal electronic interference, and microphonic vibrational noise.

We intend for the design to be extremely durable, with service intervals on the scale of weeks to months. A modular design will allow for periodic maintenance and component upgrade. For example, the initial analyzer design is a quadrupole type, but can be replaced by more advanced analyzers (i.e. miniature ion trap) as technology progresses. Likewise, variations of the initial inlet system and the embedded computer system will be developed to optimize instrument performance and to make possible real-time autonomous data analysis and adaptive sampling and mapping (5) based on real-time environmental conditions. Adaptive sampling would allow, for example, sample rate to increase whenever high methane concentration gradients were observed. This will require the creation of a type of expert system intelligence that allows the instrument to identify individual ion peaks and to vary its sampling interval in real-time, according to the rate of change of ion signatures; thus helping to avoid the aliasing sometimes associated with uniform temporal or spatial sampling.

Plans for initial deployment: An initial series of deployments are planned for the instrument in areas of known shallow methane seeps (less than $100 \mathrm{~m}$ ), off the coast of British Colombia Canada. These cruises will be conducted in partnership with the Dr Michael Whiticar of University of Victoria in parallel with shipboard methane measurements using standard surface gas stripping and gas chromatograpic procedures. This water column methane data will also be used to calibrate and test the in situ Raman Spectrometer. As development continues, the instrument can be tested in the same way over a deeper water gas hydrate zone (up to $3000 \mathrm{~m}$ ) in the same area and with the same group in cruises to the Cascadia Margin off Oregon and over gas hydrate and seep zones in the Gulf of Mexico which we are currently studying as 
part of a collaborative DoE-UMiss funded project to set up one or more Gulf of Mexico gas hydrate monitoring stations.

Plans for seeking future funding and and to leverage these funds. The Green Technology award would be used primarily to pay for instrument parts and to allow Dr Rich Camilli to rapidly begin building the mass spectrometer. Rich, a recent MIT graduate of the Joint Program, is ready to begin building this instrument as a postdoctoral investigator as soon as funding becomes available. His Ph.D. thesis involved building one of the two existing underwater mass spectrometers. He has already written much of the text for this proposal and for a successful NOAA preproposal to the Ocean Exploration Program. Rich has already written much of the text for the full NOAA proposal which will be submitted later this month. All of this work has been carried out along with dealing with a very serious family medical problem in New York. If funded, the full proposal to the NOAA Ocean Exploration program would provide salary support for Rich to come to Woods Hole as a Postdoctoral Investigator. He is also applying for a WHOI Postdoctoral Scholar award.

Additional funds to extend this work to a system capable of operating at all ocean depths will be sought through the NSF Ocean Technology Program which helped to fund a very successful workshop entitled: "The Next Generation of in situ Biological and Chemical Sensors in the Ocean" at Woods Hole last summer. Several working groups at that workshop indicated the urgent need for an in situ oceanic mass spectrometer. The Green Technology Award funds will also provide a match for the on-going Woods Hole funding from the DoE-Univ of MissWoods Hole grant to install the Gulf of Mexico gas hydrate monitoring station.

\section{References}

5. Smith, C.M., et al. Feature-based concurrent mapping and localization for autonomous underwater vehicles. in Proceedings of IEEE Oceans. 1997.

6. Delaney, J.R. and A.D. Chave, NEPTUNE: A fiber-optic 'telescope' to inner space. Oceanus, 2000. 42(1): p. 10-11.

7. Short, R.T., et al., Underwater mass spectrometers for in situ chemical analysis of the hydrosphere. Journal of the American Society for Mass Spectrometry, 2001. 12(6): p. 676682.

8. Camilli, R., Thesis: Creation and Deployment of the NEREUS Autonomous Underwater Chemical Analyzer and Kemonaut, an Odyssey Class Submarine, in Department of Civil and Environmental Engineering. 2003, Massachusetts Institute of Technology: Cambridge. p. 314.

9. Varian, Ion Pumps- vacuum products from Varian. 2001. 
Appendix III) Report: Hydrocarbon distributions from extant petroleum seep sites and associated gas hydrate deposits in sea water and ocean floor sediments from Santa Barbara and Gulf of Mexico, Jean K. Whelan and Lorraine B. Eglinton

\section{Experimental}

From the Gulf of Mexico, sediment and hydrate seep oils were analyzed together with two sea water samples from the Gulf of Mexico Dive (4208) over a gas hydate ice core , as shown in Figs 1-8.. A number of water samples were also collected which showed no discernable hydrocarbon signal and so are not discussed further. From the Santa Barbara Coal Oil point seep oils, three sediment samples and three water samples containing oil globules were analysed.

Initial screening of the Santa Barbara oil globules was performed on a approximately 10 $\mathrm{mg}$ of oil that was separated from the aqueous sample. Total soluble hydrocarbons were extracted by dissolution in 3 sequential $10 \mathrm{~mL}$ aliquots of dichloromethane. Extracts were reduced by rotary evaporation and further reduced in a stream of nitrogen. The concentrate was transferred with DCM to 150 I glass inserts and followed by gentle evaporated in a stream of nitrogen to near dryness. The fractions were diluted with 100 I DCM and the insert was placed into $1.5 \mathrm{ml}$ autosampler vial capped and submitted for analysis.

Santa Barbara and Gulf of Mexico seawater samples were extracted using conventional liquid/liquid extraction techniques on $2 \mathrm{~L}$ using 3 X $200 \mathrm{~mL}$ Dichloromethane. Samples were concentrated by rotary evaporation. Removal of residual water was performed by passing the samples over sodium sulfate. Final concentration was conducted in a stream of nitrogen.

Quantitative analysis of the Santa Barbara sediment samples was performed on a $10 \mathrm{~g}$ aliquot of homogenized wet sample, spiked with a recovery standard $\left(d_{50} n\right.$-tetracosane and $d_{12}$ 2,6-dimethylnaphthalene), was soxhlet extracted for 48 hours in tandem with a spiked blank. Solvent soluble hydrocarbons were fractioned from the wet sediment using $150 \mathrm{~mL}$ of an azeotrophic mixture of dichloromethane/methanol (93:7 \%o). The total extracts were reduced by rotary evaporation. The extracts were transferred to $4 \mathrm{~mL}$ vials. Total extracts were run through a sodium sulfate funnel for removal of residual water and an activated copper column for removal of elemental sulfur. Micro column chromatography, using $90 \%$ activated silica gel, was used to fractionate the extracts in to saturated and aromatic hydrocarbon pools. The fractions were concentrated using rotary evaporation. The concentrate was transferred with DCM to 150

I glass inserts and followed by gentle evaporated in a stream of nitrogen to near dryness. The fractions were diluted with 100 I DCM and the insert was placed into $1.5 \mathrm{ml}$ autosampler vial and submitted for analysis.

Biological marker distributions were obtained using gas chromatography coupled with mass specific detection using the saturated hydrocarbon fraction. A Hewlett Packard (HP) 6890 gas chromatograph fitted with a Restek ${ }^{\circledR}$ DB-5 MS 60 m glass capillary column and connected directly to a HP 5693 mass specific detector was used. After cool on-column injection the gas chromatograph was programmed from $50^{\circ} \mathrm{C}$ to $325^{\circ} \mathrm{C}$ at a rate of $4^{\circ} \mathrm{C} / \mathrm{min}$ and held at $325^{\circ} \mathrm{C}$ for 20 mins. The mass spectrometer was operated at $70 \mathrm{eV}$ in selected ion monitoring mode. A total of 30 ions characteristics of $n$-alkane, sterane, terpane, aromatic steroid, aromatic hydrocarbons. Full mass spectra $(50-550 \mathrm{amu})$ were recorded on the saturated fraction to identify less common biological markers. Compounds were identified by their key fragmentograms based on relative retention times and comparison with published data (e.g. Mackenzie 1980, 1981). 


\section{Results summary}

\section{Seawater}

Hydrocarbons in seawater samples were below sensitivity levels except for one Gulf of Mexico sample where trace amounts of mono-aromatic steroids could be seen. Future extraction directly onto a solid phase extraction cartridge of several liters of water might improve resolution.

\section{Sediment extract yields}

Santa Barbara Hydrocarbon yields from Soxhlet extracted sediment at the Santa Barbara seep site is $363 \mathrm{mgHC} /$ gdrySed. This decreases to $53 \mathrm{mgHC} / \mathrm{gdrySed}$ within a short distance $(10 \mathrm{~m})$ away from the seep (not shown). This indicates that hydrocarbons may be washed away from the seep site by ocean currents and gas bubbles and/or the seep site is constrained to a somewhat tight fissure. Similar procedure were used for the Gulf of Mexico sediments affected by oil seepage

\section{Aliphatic $\left(\mathbf{C}_{15}+\right)$ hydrocarbons and isoprenoids}

Santa Barbara The Total ion current (Fig. 11) shows an unresolved complex envelope of hydrocarbons with some partially resolved lighter weight hydrocarbons but no resolvable $\mathrm{C}_{15}+$ $n$-alkanes. There are relatively minor amounts of resolved peaks from the TIC from the sample analyzed in scan mode. Even when a diagnostic fragment ion $(\mathrm{m} / \mathrm{z} 57)$ for $n$-alkanes is extracted the samples are devoid of straight chain saturated hydrocarbons. The isoprenoids pristane and phytane are also absent. The lack of $n$-alkanes and the isoprenoids indicates biodegradation has progressed to at least moderate to heavy based on the biodegradation ranking of Peters and Moldowan (1993). The total ion current from the same seep site sample analyzed in SIM mode is dominated by tri- and monoaromatics steroids and hopanes and dibenzothiophenic compounds (Fig. 16).

Gulf of Mexico sites GC 184 \& 234

Similar results were obtained for Gulf of Mexico surface seep oils (Figs 1-3) except that more resolvable GC peaks appear to be present suggesting a lower degree of biodegradation.

\section{Hopanes}

Santa Barbara Hopane distributions are similar for the three Santa Barbara samples but show a decrease in abundance with distance from the seep vent. A typical $\mathrm{m} / \mathrm{z} 191$ fragmentogram for hopanes is shown in Figure 12. The dominant peak in this fragmentogram is the $\mathrm{C}_{29}$. hopane. In unaltered oils the $\mathrm{C}_{30}$. hopane is usually prominent. Another unusual feature is the elevated abundances of $\mathrm{C}_{28}$ and $\mathrm{C}_{29}$ and other tricyclic hydrocarbons together with high abundances of gammacerane and the presence of oleanane. There is also increased amounts of 28,30 bisnorhopane. 25,28,30-trisnorhopane is also observed. This biomarker has been observed in highly anoxic, mostly marine sediments globally (e.g., Grantham et al., 1980; Mello et al., 1988; Requejo et al., 1994). Literature searches indicate that elevated $\mathrm{C}_{28}$ and $\mathrm{C}_{29}$ tricyclic terpanes is a feature of Californian oils ranging from Coal Oil Point seeps to Santa Cruz seeps and the Guaymas basin (this study, Kvenvolden et al.; Simoniet et al.,) indicating a 
common source. Although still open to interpretation, gammacerane in high concentration is often associated with hypersaline depositional conditions (ten Haven et al., 1988; 1989).

The presence of gammacerane, oleanane, elevated 28, 30 bisnorhopane, abundance of sulfur derived hydrocarbons and elevated tricyclic terpanes indicate a source consitstent with those of the Miocene Monterey formation based on published fragmentograms (Peters and Moldowan, 1993). A high abundance of 28,30 bisnorhopane is thought to be due to bacterial mats present in the Monterey formation. The fossilized Thioploca bacterial mats may represent a possible source according to Katz and Elrod (1983).

Trace abundances of $2\left(\mathrm{CH}_{3}\right)$-hopanes and $3\left(\mathrm{CH}_{3}\right)$-hopanes have been tentatively identified from the $\mathrm{m} / \mathrm{z} 205$ from the seep site. Although methylhopanes can derive from a variety of prokaryokes Summons et al., (1996) and Collister et al., 1992 suggest the presence of cyanobacteria and or methylotrophic bacteria to account for homologous series of 2 . and 3. methylhopanes respectively.

Gulf of Mexico The GCMS mass scans for the hopane biomarkers in the GC234 sediment seep samples are shown in Fig 5 . The pattern is almost idential to that found previously for the subsurface GC184 oils, suggesting that biodegradation has affected this fraction only slightly, if at all.

\section{Steranes and aromatic steroids}

Santa Barbara Steranes in the Santa Barbara Seep sediment samples have been affected by biodegradation and do not exhibit a distribution normal to reservoired unaltered petroleum. The high abundance of Mono- and triaromatic steroids indicate conversion of steranes due to thermal maturity and resistance to biodegradation processes (Fig 13). Monoaromatic steroids were also found in trace abundances in a water sample from the Gulf of Mexico.

Gulf of Mexico The normal and aromatic sterane mass scans for the GC 234 seep and hydrate oils are shown in Figs 4 and 6 respectively and are very similar to those of the underlying reservoir oils, consistent with a low degree of biodegradation of this fraction.

\section{Aromatic hydrocarbons}

Gulf of Mexico All of the aromatic hydrocarbon data is available for Gulf of Mexico seep and hydrate melt water oils. For comparison, extensive set of similar data have been measured for all of the underlying Green Canyon oils as part of a previous project.

Santa Barbara In spite of the high degree of biodegradation, the methyl phenanthrenes are well resolved and should provide a reliable maturation markers for these biodegraded oils. A summary of the various classes of compounds analyzed to date is given below.

\section{Methylphenanthrene}

Calculated vitrinite reflectance based on methylphenahtrene index 1 (Radke 19 ) indicates a fluid maturity of around $\mathrm{R}_{\mathrm{c}} 1.00 \%$. Thus, these seep oils are

\section{Naphthalenes}

The extracts are devoid of resolvable naphthalenes indicating removal by biodegradtaion. 


\section{Dibenzothiophene and methyldibenzothiophenes}

$\mathrm{m} / \mathrm{z} 184$ and 198 diagnostic of dibenzothiophenes and methyl dibenzothiophenes are noisy. And contain abundant as yet unknown peaks. Based on the $\mathrm{m} / \mathrm{z} 184$ sulfur containing compounds are consumed at a faster rate since they are present in only trace abundance $10 \mathrm{~m}$ away from the seep site.

\section{Biodegradation}

Based on loss of $n$-alkanes, modification of steranes, an emerging series of 25norhopanes the Santa Barbara samples have been heavily biodegradation (rank 6) based on the biodegradation rank of Peters and Moldowan (1993). The degree of biodegradation changed little over the sampled area within the sediment extract and the globules. This may indicate rapid replenishment of hydrocarbon material.

\section{Conclusions to date}

Fluids in the Santa Barbara seep site are rich in hydrocarbons consistent with petroleum sourced from the Monterey formation. This correlates with other seep oils found up and down the West coast. The oils are severly biodegraded. Biodegradation may have occurred subsurface in reservoirs or migration conduits cool enough o support biodegradation. Distinguishing biodegradation in reservoirs versus that in the water column requires further investigation but identification of suites of sulfur containing compounds may hold the key.

\section{References:}

Grantham , P.J., Posthuma, J., and DeGroot, K. (1980): Variation and significance of the C27 and C28 triterpane content of a North Sea core and various North Sea crude oils. In: Advanbces in organic geochemistry 1979 (A<G Douglas and J.R Maxwell, eds.) Pergamon Press New York P. 29-38

Mello , M. R., Telnaes, N Gaglionone, P.C, Chicarelli, M. I Brassell, S. C., and Maxwell, J. R (1988b): Organic geochemical characterization of depositional paleoenvironments in Brazilian Marginal basins. Organic Geochemistry, Vol. 13, p. 31-46.

A. G. Requejo ${ }^{1}$, R. Sassen ${ }^{1}$, M. C. Kennicutt II ${ }^{1}$, I. Kvedchuk ${ }^{2}$, T. McDonald ${ }^{1}$, G. Denoux ${ }^{1}$, P. Comet ${ }^{1}$ and J. M. Brooks ${ }^{1}$ Geochemistry of oils from the northern Timan-Pechora Basin, Russia Organic Geochemistry, Vol. 23 (3) (1995) pp. 205-222

Kvenvolden, K.A., Hostettler, F.D., Rosenbauer, R.W., Lorenson, T.D., Castle, W.T., Sugarman, S., 2002. Hydrocarbons in recent sediment of the Monterey Bay National Marine Sanctuary. Mar. Geol. 181, p.101-113.

Peters and Moldowan (1993): The Biomarker guide, Prentice Hall

ten Haven, De Leuww, J. W Sinninghe Damste, J.S. Schenck, P. A Palmer, S.E and Zumberge, J. E (1988): Application of biological markers in the recognition of 
palaeohypersaline environments. IN: Lacustrine Petroleum Source Rocks (A.J. Fleet, K. Kelts and M. R. Talbot eds.) Geological Society Special Publication No 40, P.123-130

ten Haven, H. L., Rohmer, M. Rullkotter, J and Bisserret, P (1989): Tetrahymanol, the most likely precursor of gammacerane occurs ubiquitously in marine sediments. Geochimica et Cosmochimica Acta Vol 53., p. 3073-3079.

Collister , J. W. Summons, R.E Lichtfouse, E. L and Hayes J. M (1992): An isotopic biogeochemical study of the Green River oil shale. Org. Geochem. 19, 265-276.

Figure 1: Biodegraded oil recovered from surface seep oil, Green Canyon 234, Gulf of Mexico

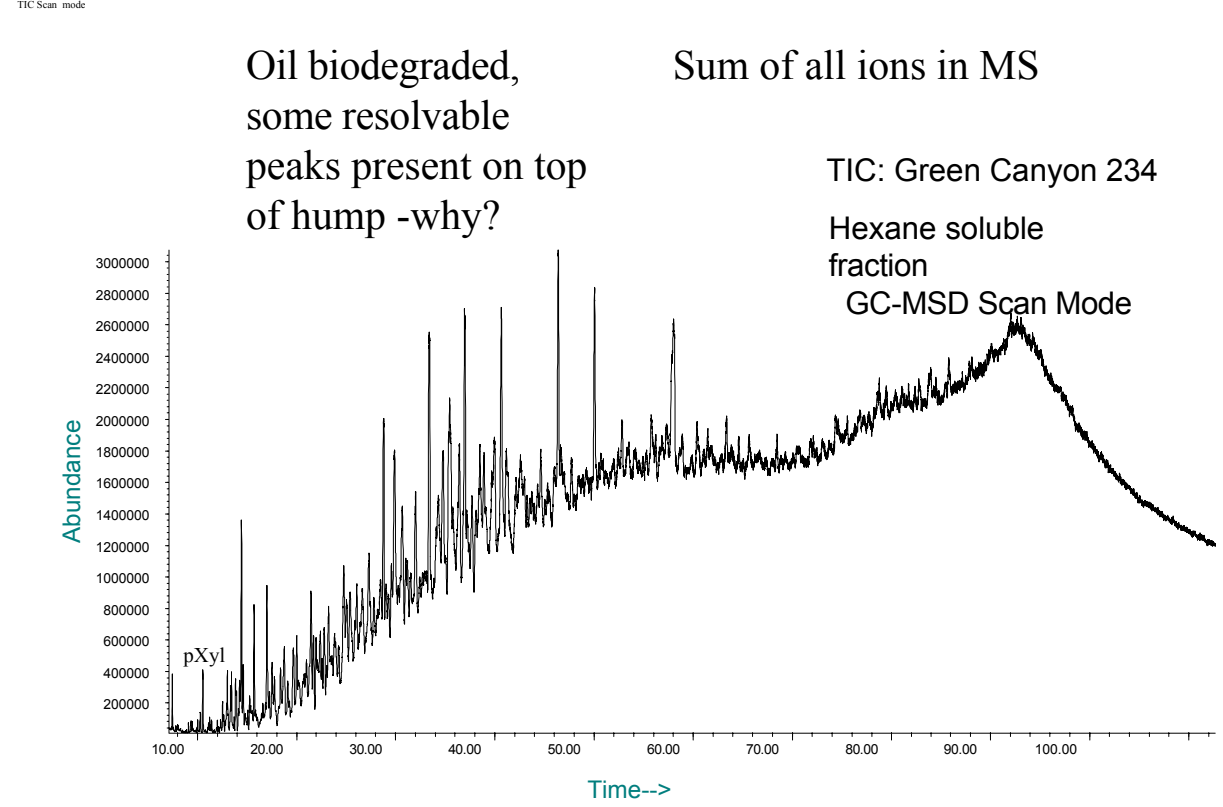

LBE42.D

Figure 2: Same as Figure 1, hexane soluble fraction 
TIC SIM mode

TIC: Green Canyon 234

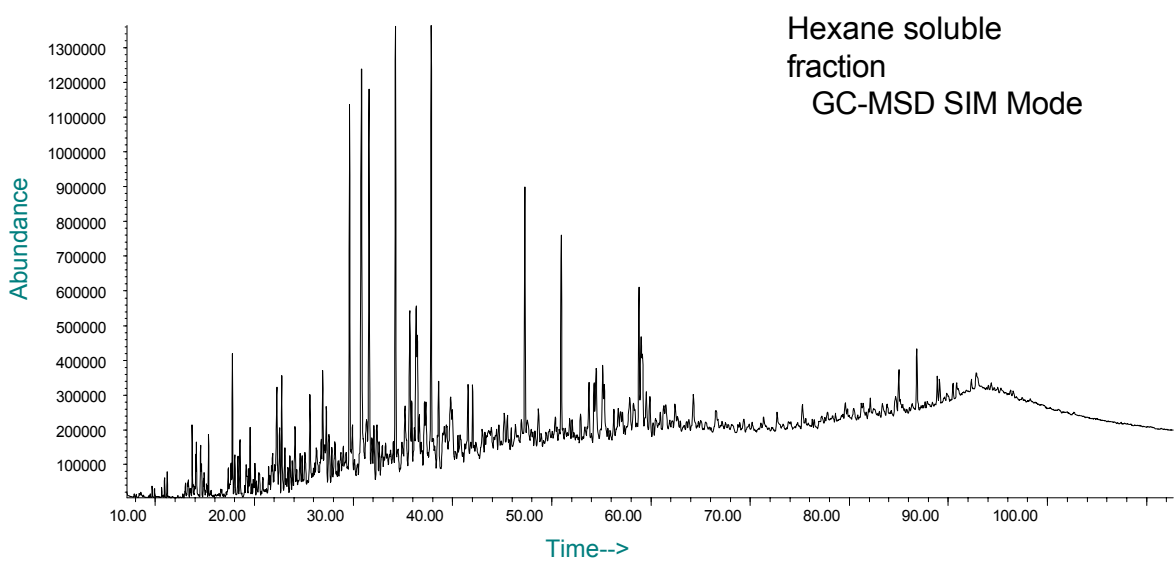

LBE48.D

Figure 3: Same as Figure 1, GCMS scan for alkanes only (mass 57)

57 n-alkanes SIM

Branched, cyclo, \&n-alkanes - impies

biodegradation hasn't progressed very

far
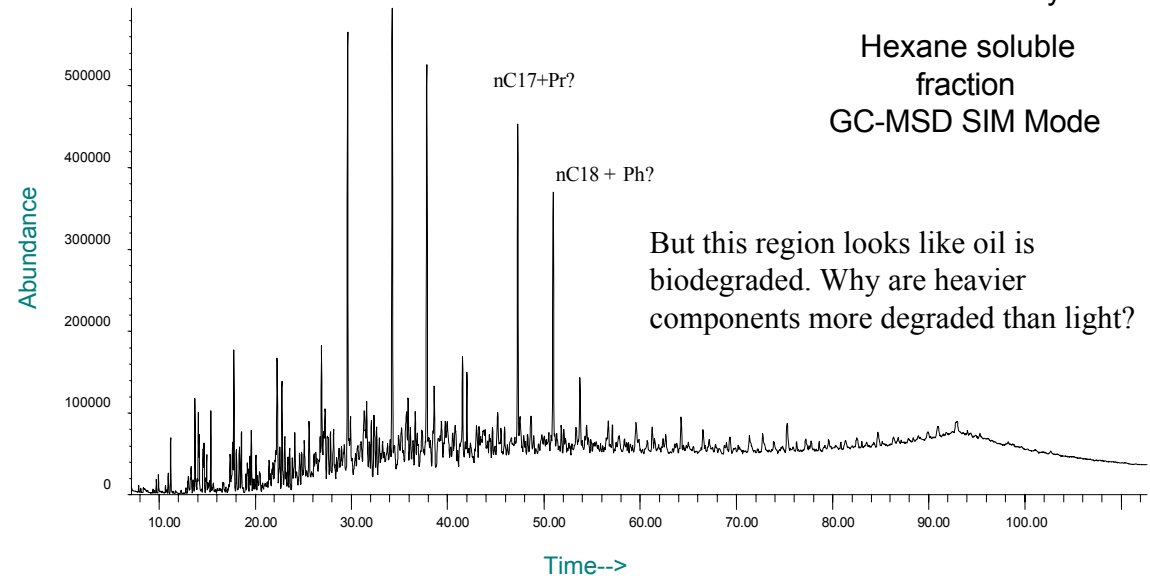

ION 57: Green Canyon 234

Hexane soluble fraction GC-MSD SIM Mode

But this region looks like oil is biodegraded. Why are heavier components more degraded than light?

LBE48.D 
Figure 4: Biomarkers, steranes, from GCMS - GC234 seep oil, hexane extract

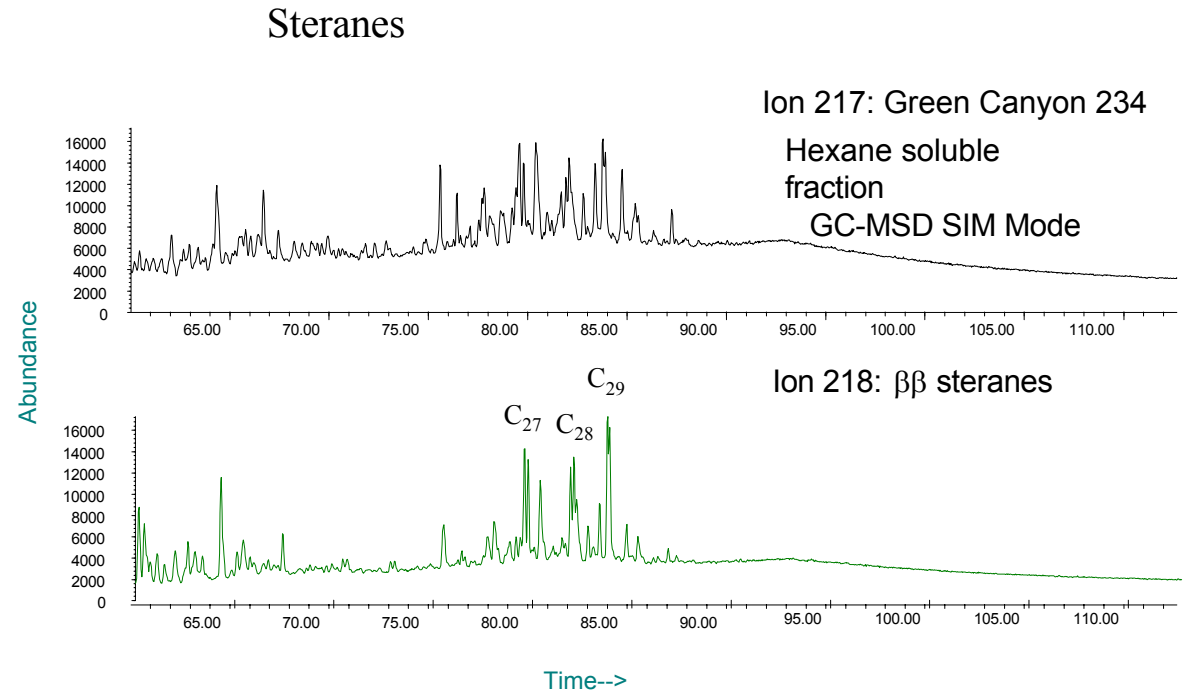

LBE48.D

Figure 5: GCMS data, GC234 seep oil, mass scan showing hopanes in biodegraded seep oil hopanes

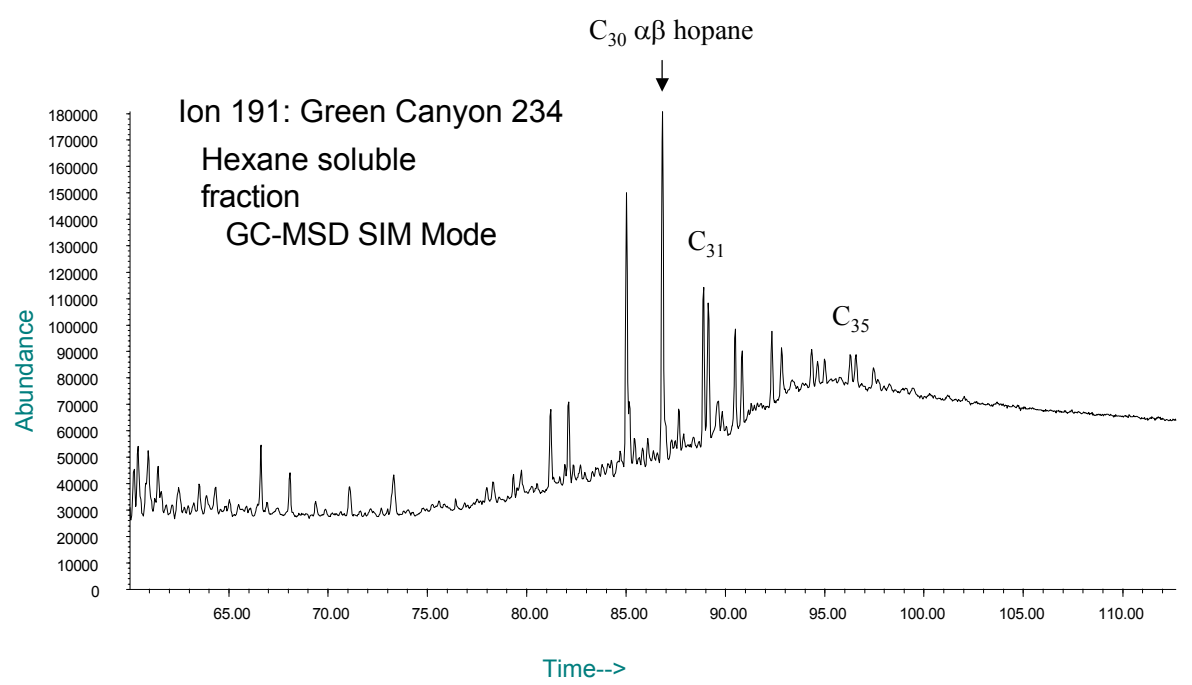


Figure 6: Same as Figure 4, aromatic steranes

Aromatic Steranes

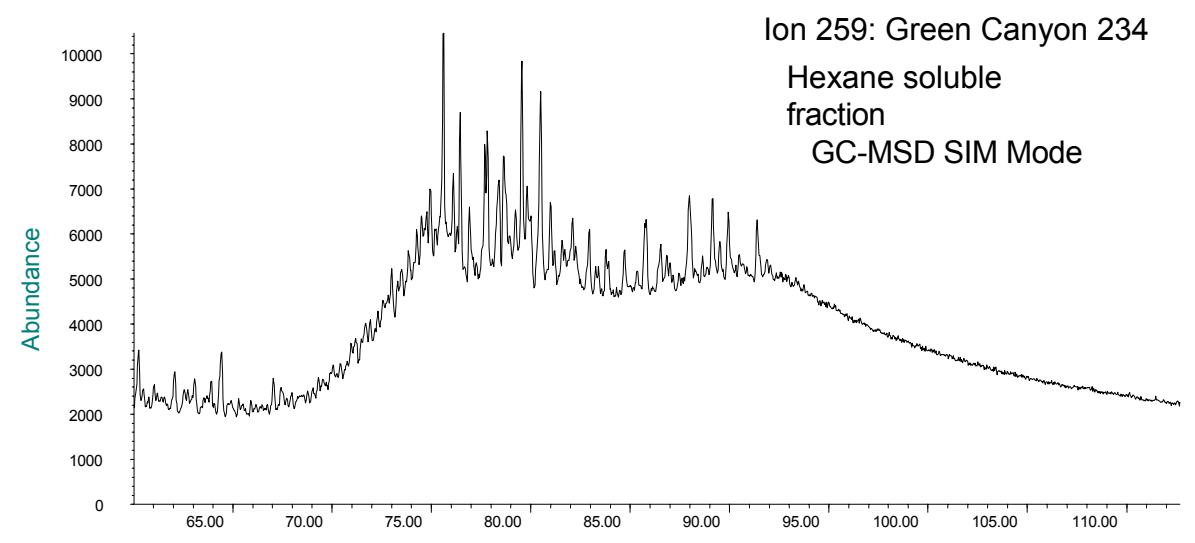

Time-->

Conclude - Biodegradation not extensive enough to affect steranes and hopanes.

LBE48.D

Figure 7: Same as Figure 4, Sulfur containing aromatic compounds

Benzothiophene/C-4 alkylbenzenes

Sulfur containing aromatics

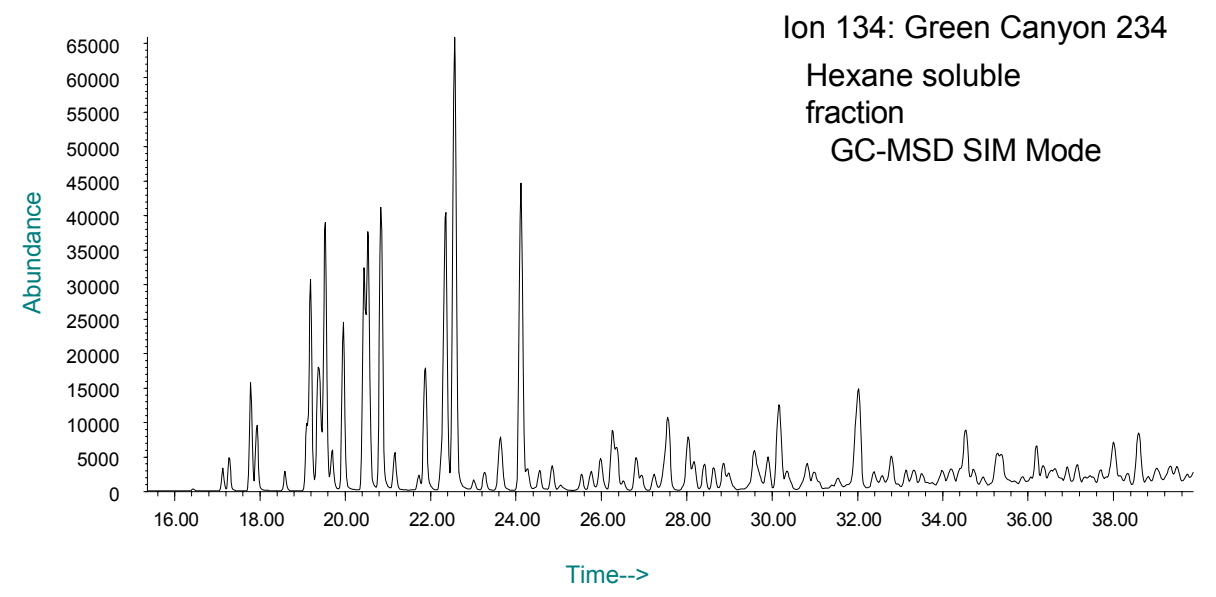

LBE48.D 
Figure 8: 2D GC method for resolving compounds under hump in a biodegraded oil (from C. Reddy)

Further analysis of oil under the hump: 2-D gas chromatography (in process)

- The method:

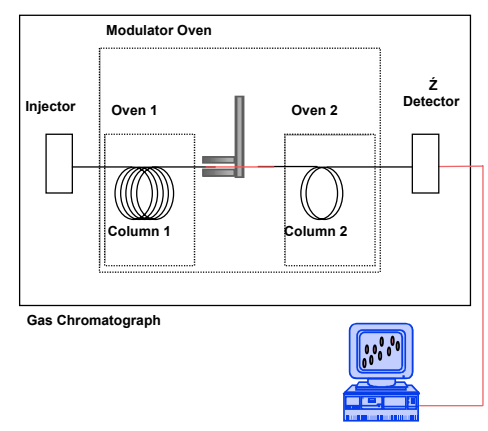

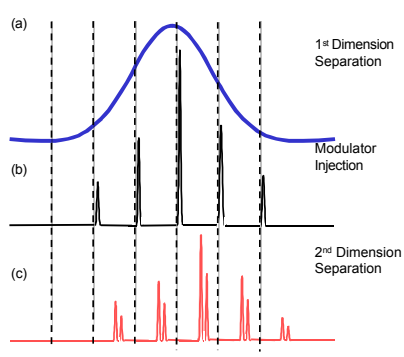

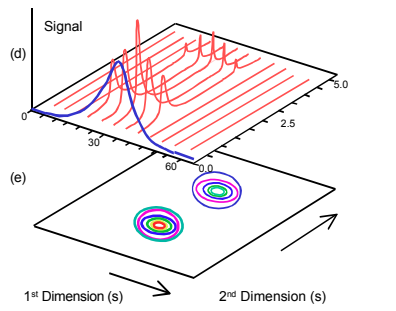

Figure 9: Example of 2D GC technique as applied to oil contaminated sediment and to diesel oil (from Chris Reddy, WHOI)

Two dimensional map from GC-GC showing separation of various compound classes and homologous series in: a) an oil extrated from a contaminated sediment and b) a typical diesel oil. Note the homologous series of n-alkanes at the bottom of each plot. All peaks are identified and quantified using a flame ionization GC detector and a GCMS detector.
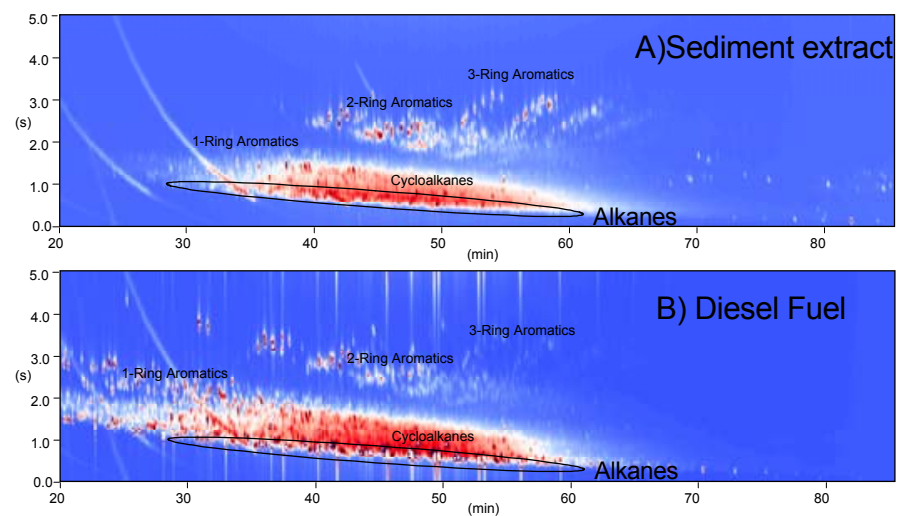
Figure 10: 2D GC, 184 seep oil

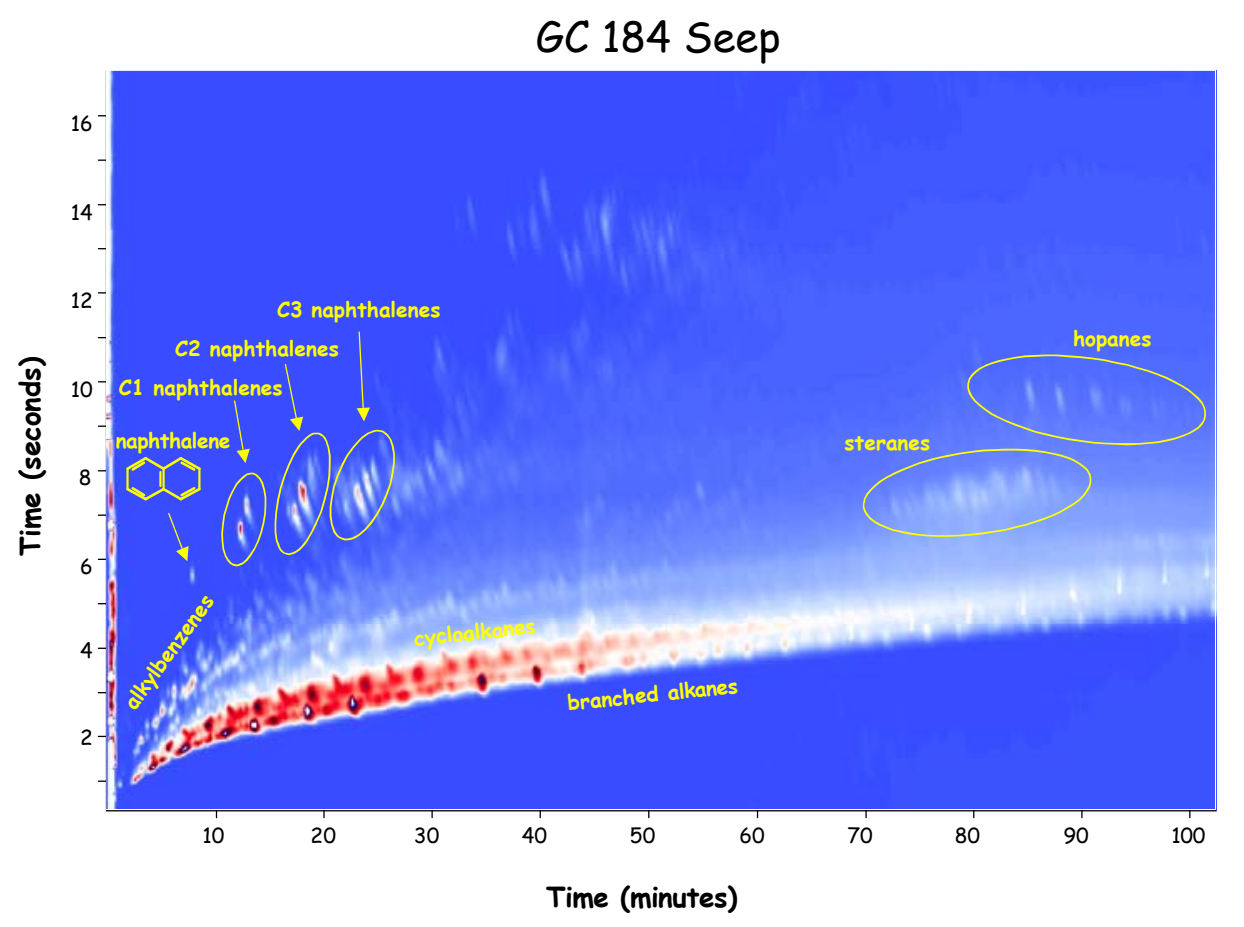

Figure 11: Santa Barbara, Coal Oil Point seep - biodegraded oil, mass scan of biomarker hopanes

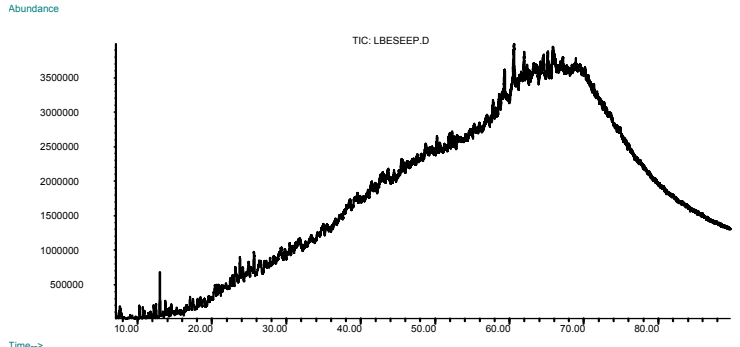


Figure 12: Biomarkers, hopanes, in Santa Barbara Shane tar mound oils

\section{Shane Seep Tar Mound: Ion 191}

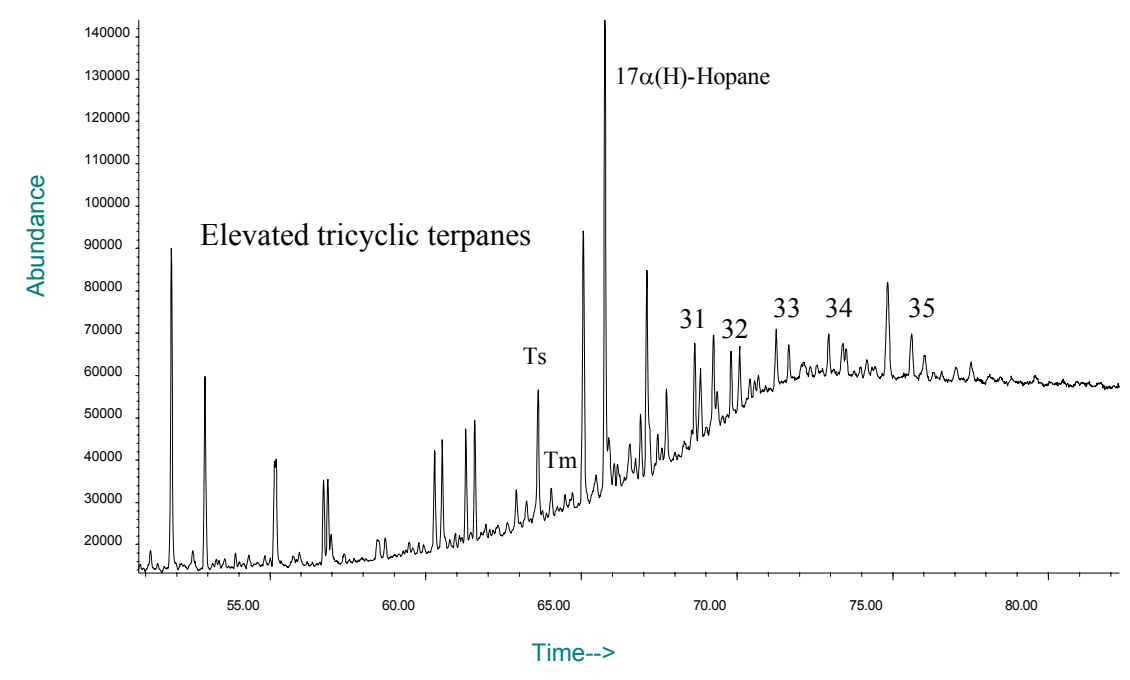

LBESEP7.D

Figure 13: Santa Barbara, Shane Seep tar mounds, aromatic steranes in biodegraded oil

Shane Seep Tar Mound: Ions 253 and 231
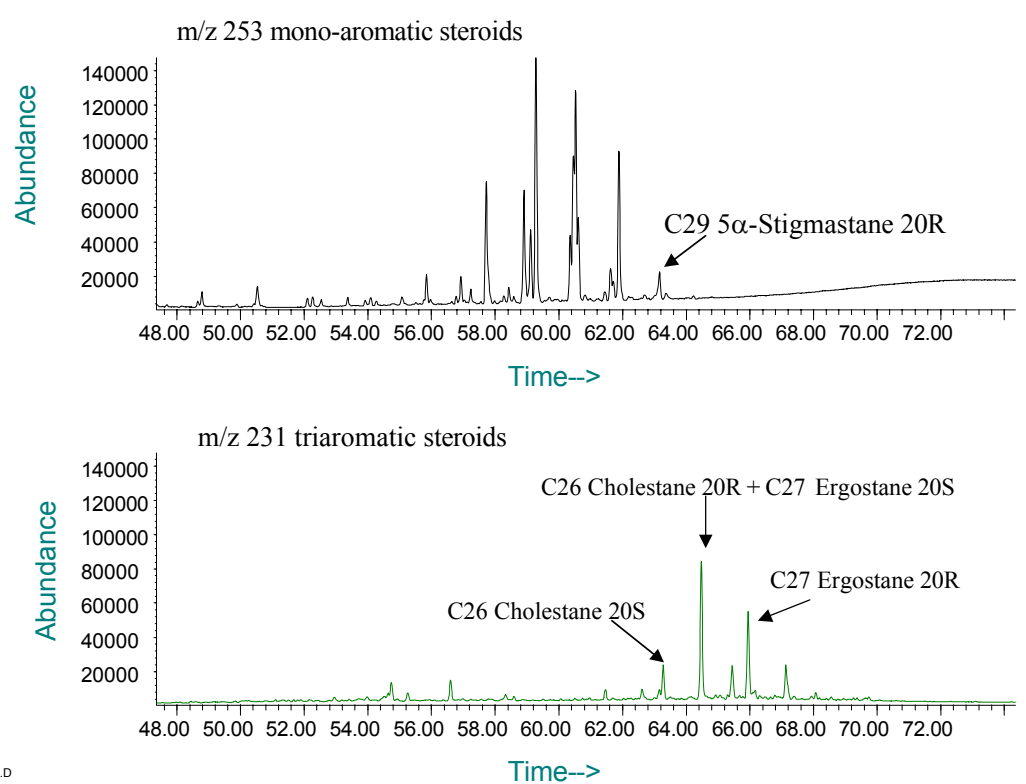
Figure 14: Santa Barbara, Shane Seep Tar mound, methyl phenanthrenes

Shane Seep Tar Mound: Ion 192

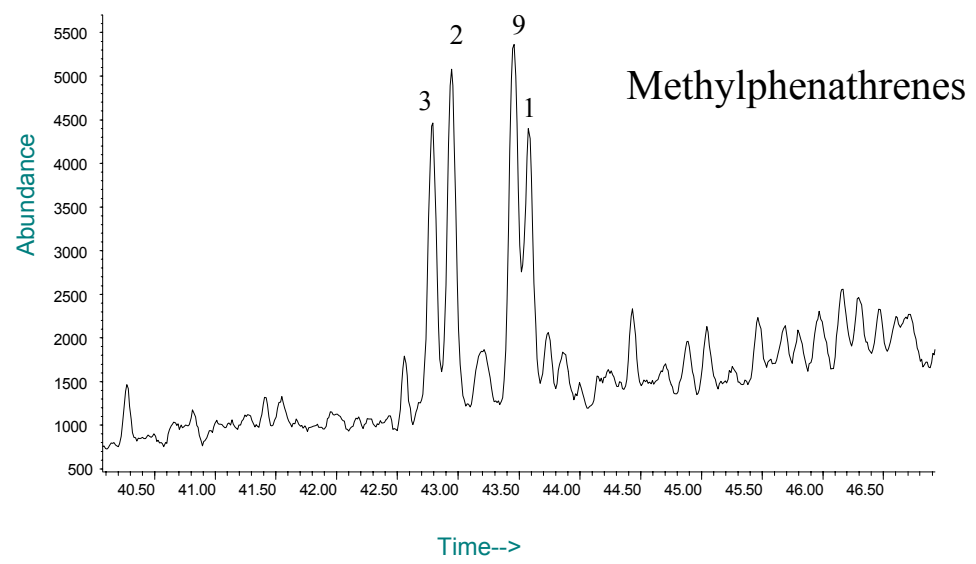

Figure 15: Santa Barbara, Tar mound, total resolvable one dimensional GC peaks in biodegraded oil

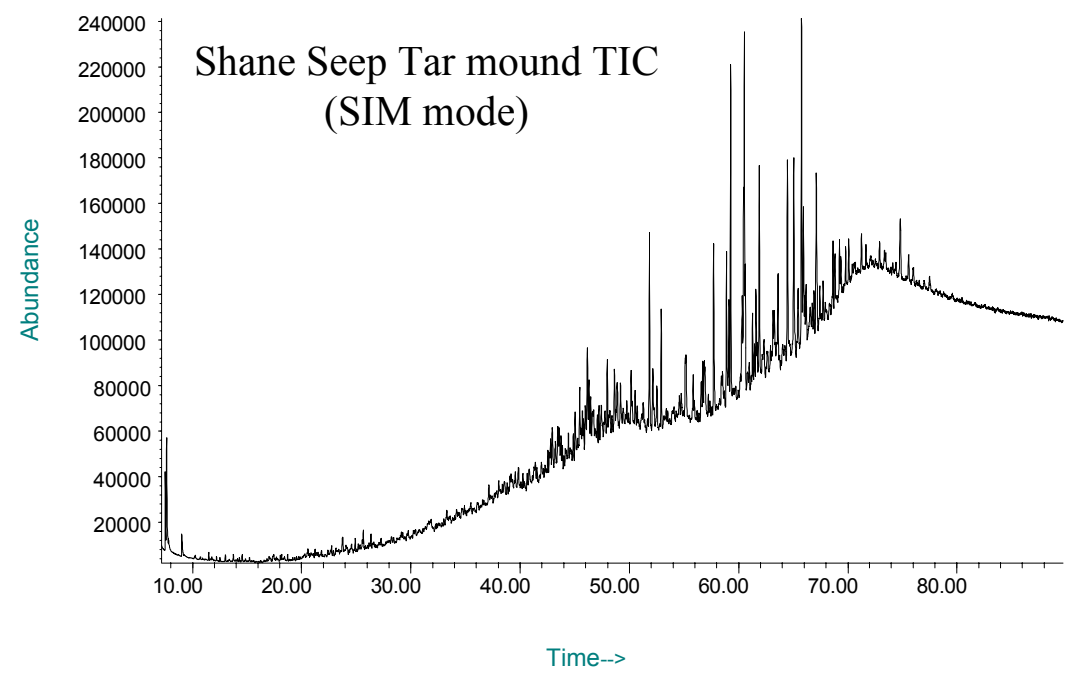


Figure 16: 2D GC - Santa Barbara, Shane seep tar mound

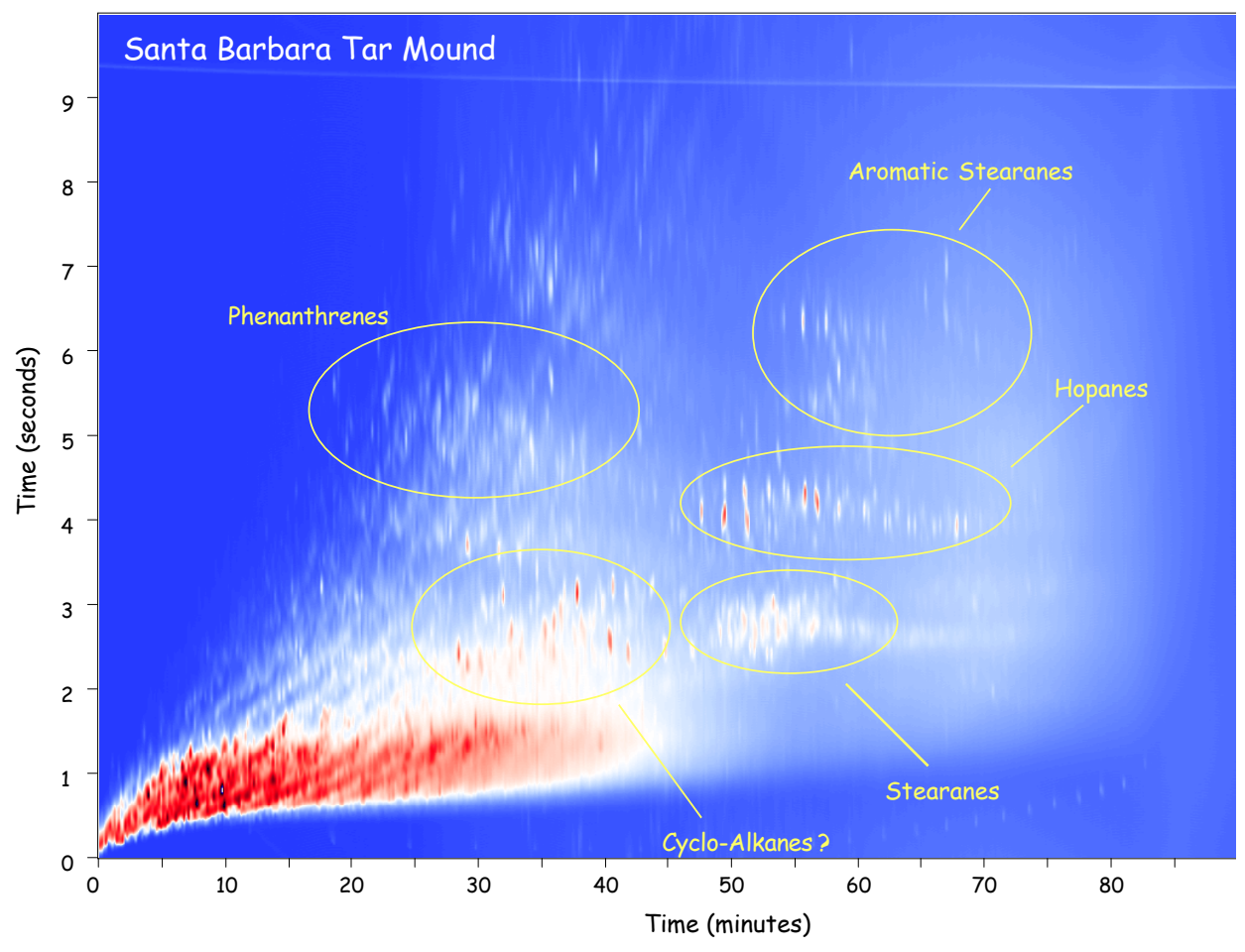


FINAL REPORT

Monitoring biogeochemical processes controlling hydrate formation, decomposition and composition in the Gulf of Mexico

Jeffrey P. Chanton, Department of Oceanography, Florida State University, Tallahassee, Florida 32306-4320. jchanton@mailer.fsu.edu, 850-644-7493.

Christopher S. Martens, Laura L. Lapham, Marine Science Department, University of North. Carolina, Chapel Hill, NC 27599. 919-962-0152. cmartens@email.unc.edu, llapham@email.unc.edu

FINAL Report to the Department of Energy

From the University of Mississippi Gas Hydrate Research Consortium

February 24, 2004 


\section{Abstract/ Executive Summary}

On the global scale, hydrates need to be included in models of how the biosphere functions. A large portion of the earth's reduced carbon is held within the hydrate reservoir and this reservoir may be more dynamic than is currently thought. Understanding the biology and geochemistry of the seafloor surrounding gas hydrate deposits is crucial to understanding where and how hydrates form. Biological processes and geochemistry strongly influence rates of both formation and destruction of hydrates (Figure 1). Biology and geochemistry play a role in determining the type of hydrate formed and, thus, the specific pressure and temperature conditions where hydrate formation can occur in the subsurface. We need biological and geochemical information to constrain rates of formation and destruction of hydrates (Grabowski et al., 2003)

Accurate geochemistry. For this endeavor, in situ concentrations of gases and ions in pore water need to be measured, since samples recovered and brought to the ocean surface without pressurization yield inaccurate results (see below). When measured properly, gas and ion concentrations in pore water can be used to help understand the formation, decomposition and stability of hydrates.

We have developed new tools and techniques for application at the long-term gas hydrate monitoring station. We have constructed an in situ pressurized pore water sampler and built and deployed the first osmosampler in the Gulf of Mexico. Modifications to our in situ porewater probe sampler have allowed the collection and determination of accurate dissolved gas concentration and ${ }^{13} \mathrm{C}$ from the seafloor. Comparisons with pressurized and unpressurized sampling demonstrates the necessity of such a system to collect accurate and meaningful data. Further studies undertaken in summer of 2003 indicate over an order of magnitude greater dissolved gas concentrations with the in situ pressurized sampler relative to traditionally taken samples. The osmosampler will allow long-term data sets to be collected at a single site, and with our design, samples can be recovered at in situ pressures.

We have completed chemical analyses of samples collected on two cruises in the Gulf of Mexico in the summer of 2000 and 2002. Samples from a third cruise from the summer of 2003 are currently being analyzed. These data will be used by Laura Lapham as a portion of her dissertation project. Highlights are described within.

Variability of rates of microbial process is extreme on small spatial scales. Sulfate reduction rates, methane concentrations, total organic carbon, and sulfate depletion all show evidence for extremely high rates of microbial respiration at gas hydrates sites but this respiration is patchy on small scales.

Microbial respiration is not driven only by methane oxidation. There is strong isotopic evidence that oxidation of higher hydrocarbons plays a major role in driving elevated rates of microbial processing in the Gulf of Mexico.

Radiocarbon evidence indicates that hydrocarbon sources are driven by deep reservoirs and there is little evidence for extensive methane production associated with recycling of organic matter fixed on the sea floor via chemosynthetic processes. 


\section{Table of Contents}

List of Figures and Tables

Introduction

Methods

Results and Discussion

Conclusions

Figures

Tables

References

\section{List of Figures}

Figure 1. Conceptual model of methane flux, production and consumption processes that can affect gas hydrate deposits.

Figure 2. In situ porewater probe sampling through a tube worm bush. This figure illustrates the utility of this device in allowing pinpoint sampling of vertical dissolved hydrocarbon sampling on the seafloor.

Figure 3. In situ porewater probe at the base of a gas hydrate mound.

Figure 4. Porewater probe following modification to allow pressurized sample recovery.

Figure 5. Difference in quality of data after modification.

Figure 6. Comparison of methane concentration and isotopic ratio from Probe and traditional cores taken from Cascadia Margin.

Figure 7. Averaged dissolved methane del13C values from the Probe over $50-\mathrm{cm}$ interval.

Figure 8. Example of spatial variability in dissolved gas concentrations.

Figure 9. Example of spatial variability in dissolved sulfate concentrations.

Figure 10. Example of spatial variability in sulfate reduction rates.

Figure 11. Total organic carbon concentrations.

Figure 12. $\delta^{13} \mathrm{C}$ isotopic composition of solid phase organic carbon.

Figure 13. Keeling plot analysis of dissolved $\mathrm{CO}_{2}$ data.

\section{List of Tables}

Table 1. Sites visited from three cruises to the Gulf of Mexico.

Table 2. Calculation of substrates supporting microbial respiration from $\mathrm{CO}_{2}$ isotopic data.

Table 3. $\delta^{3} \mathrm{C}$ isotopic composition of cements.

Table 4. Radiocarbon values of methane. 


\section{Introduction:}

Gas hydrate deposits are important because: a) they represent an enormous potential energy source, b) they present engineering problems for deep water operations, and c) they have been implicated as agents of global climate change, possibly releasing large volumes of methane, a highly efficient green house gas, into the atmosphere thus serving as a positive feedback for warming (Kvenvolden, 1988, Kennet et al., 2003). However, the identification of gas hydrates and estimation of their total volume using remotely sensed seismic data is not straight forward. Little data on seabed methane fluxes from dissociation of gas hydrates exist. Furthermore, we do not know the processes that drive gas hydrate dissociation or the effects of methane release to the water column. Gas hydrate dissociation is an important issue for seafloor stability considerations. We need to develop reliable techniques to relate the quantity of gas hydrate deposits to seismic data and to measure the waxing and waning of gas hydrate reservoirs and the rates of and controls on these processes. The Gulf of Mexico is an ideal gas hydrate study site because these unusual frozen gas/water deposits occur exposed at the seafloor at relatively shallow water depths (Brooks et al., 1984; MacDonald et al., 1994; Sassen et al., 1997, 1999a). Therefore, localized areas of gas hydrate can be identified, gas emission monitored, and water column and pore water chemistry responses to external variables measured.

In this project we have undertaken studies and conducted chemical analysis that will aid in the identification of the sources of the reduced compounds fueling hydrate deposits (e.g. Sassen et al., 1999a,b) and investigated the processes controlling hydrate formation and decomposition so that their current state of stability can be assessed. We have measured rates of sulfur cycling to evaluate the role of hydrates in supporting local benthic and water column communities and developed an approach to evaluate the relative contributions of different substrates driving microbial respiration.

A central focus of our research is "How are spatial and temporal variations in hydrate formation, decomposition and composition related to variations in the mechanisms and rates of carbon and sulfur cycling processes in surrounding sediments?"

Scope of work: The project has three parts:

1. To assess spatial and temporal variations in hydrate stability, we have visited 8 hydratecontaining sites in the Gulf of Mexico (Table 1). We have completed chemical analyses of samples collected on a 12-day cruise in the summer of 2000 in collaboration with Dr. R. Coffin and Dr. K. Grabowski of the Naval Research Lab. Samples have also been analyzed from participation on the consortium cruise in 2002. And finally, significant progress has been made on the completion of sample analysis from the summer 2003 cruise. These analyses are complete and will be used by Laura Lapham as a portion of her dissertation project.

2. Mechanisms and rates of carbon and sulfur cycling processes are assessed by the aforementioned chemical analysis and the development of new tools and techniques for application at the long-term gas hydrate monitoring station. We have developed an in situ pressurized pore water sampler and deployed the first osmosampler in the Gulf of Mexico. As will be shown below, the quality of data retrieved from our pressurized sampler is much better than what we have seen before. Recent studies undertaken in summer of 2003 indicate over an order of magnitude greater dissolved gas concentrations with the in situ pressurized sampler relative to traditionally collected samples. 
3. In the summer of 2003 , we also visited a site similar to the Gulf of Mexico thermogenic gas hydrate sites off Vancouver Island in the Pacific Ocean to further test the device developed in \#2. The in situ pore water probe was used to assess the rate of hydrate dissolution (Flux 9 in Figure 1) and this technique will be used on future dives to the Gulf of Mexico sites.

\section{How does our work fit in with consortium?}

The focus of the consortium is to establish a long term monitoring station, and to follow a gas hydrate deposit over a long time frame using acoustical and geophysical methods. Geochemistry serves as the ground truth for the geophysics. The central issues which our proposal focuses upon are:

1. Patchiness in seepage and hydrate quantity.

2. Exchange of hydrocarbons, monitoring of hydrate decomposition and formation with an eye toward seafloor stability issues.

3. Identification of the sources of reduced compounds fueling the deposits.

Why is patchiness important? The question is related to the hydrate inventory, its formation and its decomposition. We want to know how a $\mathrm{km}^{2}$ of seafloor dominated by hydrates works. Our approach is to deploy tools to quantify hydrate growth and decomposition, the processes that affect the hydrocarbons, and pore water parameters that are affected by them. The monitoring station will sense remotely a limited footprint or spatial area. Our work will determine the variability within that footprint. Our consortium needs to know how the temporal changes we observe at the station are related to spatial variability.

\section{Site descriptions:}

Samples have been collected during three cruises aboard R/V Seward Johnson II in the summers of 2000, 2002, and 2003 at a reference site and eight hydrate sites.

The reference site $\left(27^{\circ} 44.9^{\prime} \mathrm{N}, 91^{\circ} 16.63^{\prime} \mathrm{W}, 535 \mathrm{~m}\right.$ depth) was sampled by collecting a box core over the side of the ship. From the box core, two $5 \mathrm{~cm} \times 30 \mathrm{~cm}$ lexan push cores were taken and immediately stored at in situ temperatures of $7^{\circ} \mathrm{C}$ for later analysis. Sediments were light brown in color and there were no visible disturbances in the cores due to gas expansion or dissociating hydrates. Hydrates are not known to exist in this area.

The hydrate sites were sampled from the submersible Johnson Sea Link. Four of the sites are found in the Green Canyon (GC) Lease Block 185, 234, 233, and 232 and have been described elsewhere (Brooks et al., 1984, Brooks et al., 1985 MacDonald et al., 1994, Roberts et al., 1999; Sassen et al., 1999; Sassen et al., 1998; Sassen et al., 2001; Sassen et al., 1999). Briefly, GC $185\left(27^{\circ} 46.9^{\prime} \mathrm{N}, 9^{\circ} 30.4^{\prime} \mathrm{W}, 550-580 \mathrm{~m}\right.$ depth) and GC 234 (2744.7’ $\mathrm{N}, 9^{\circ} 13.3^{\prime} \mathrm{W}$, $525-560 \mathrm{~m}$ depth) have exposed hydrates at the sediment water interface, chemosynthetic communities (tube worms, methanotrophic mussels, and clams), seeping gas of low molecular weight hydrocarbons (such as methane, ethane, and propane), and oil associated with the surrounding sediments. GC $233\left(27^{\circ} 43.4^{\prime} \mathrm{N}, 91^{\circ} 16.8^{\prime} \mathrm{W}, 640 \mathrm{~m}\right.$ depth) is a brine pool surrounded by a mussel beach. Mississippi Canyon (MC) is east of the GC sites (2851.1409'N, 89²9.5361'W, 2915m water depth).

Mississippi Canyon (MC) 709 and $118\left(28^{\circ} 51.1409^{\prime} \mathrm{N}, 8^{\circ} 29.5361^{\prime} \mathrm{W}, \sim 970-\mathrm{m}\right.$ water depth) were visited in 2002. MC 118 is the easternmost hydrate site of all discoveries in the Gulf of Mexico thus far (Sassen, personal communication). MC 118 has chemosynthetic communities but I am unsure if shallow surfacing hydrates exist. Geochemically, the oil and the 
gas do not look much different than at a well-studied site, MC 852/853 that is thought to contain more gas hydrate accumulations than any other site in the central Gulf of Mexico ((Sassen et al., 1999)).

Viosca Knoll $826\left(29^{\circ} 09.7528^{\prime} \mathrm{N}, 8^{\circ} 01.9246^{\prime} \mathrm{W}, ~ 570-\mathrm{m}\right.$ water depth) and Garden Banks $425\left(27^{\circ} 33.2113^{\prime} \mathrm{N}, 92^{\circ} 32.4463^{\prime} \mathrm{W}, \sim 625\right.$-m water depth) were also visited but information about these sites is still being gathered. An active mud volcano at GC 205 was visited in 2003 that had been discovered by the Navy submersible NR-1 a few years prior. Information on this site is also being gathered.

\section{Methods:}

\section{Sampling methods}

At the hydrate sites, sediment and pore water samples were collected from the submersible platform by two different methods. The first method collected only pore waters by using an in situ pore water extraction device, known as the Probe (Figures 2 and 3). Ideally, for every deployment of the probe, there was a sediment core to compliment. During the 2000 cruise, the probe was not pressure tight so concentrations are a minimum. However, the probe was modified to hold in situ pressures for the 2002 and 2003 cruises (Figure 4). Briefly, the submersible's manipulator arm placed the probe tip into the desired sediment and triggered a hydraulic spring that provided the suction to extract pore water through 10 filtered ports along a $50-\mathrm{cm}$ shaft for 30 minutes. For the 2000 cruise, samples were collected in PVC chambers that were open to the outside pressure thereby allowing expansion of the gas during ascent. These chambers collected about $7 \mathrm{~mL}$ of water. To obtain in situ concentrations, the probe was modified with stainless steel chambers and heavy-duty gas tight valves to hold pressures up to a maximum of 4000 psi. This modification led to significantly improved results. The sample volume stayed the same as the original design. Once the probe was shipboard, pore waters were collected into gas tight syringes and immediately analyzed for gas concentrations using a gas chromatograph. Remaining pore water was preserved by injecting it into evacuated serum vials and frozen upside down for determination of carbon isotopic ratios at the mass spectrometry labs at UNC and FSU.

The second sampling method collected sediment and pore water with traditional Lexan $30 \mathrm{~cm}$ push cores. Once onboard ship, cores were sectioned into 2-3 cm sections and pore waters expressed by pressure filtration. Sub-cores of each section were taken to measure sulfate reduction rates (described below). Pore waters were stored cold in $2 \mathrm{~mL}$ o-ring sealed plastic microcentrifuge tubes. Sediment patties were frozen in plastic bags for determination of total organic carbon concentrations and $\delta^{13} \mathrm{C}$.

\section{Analytical methods}

Sulfate was measured by diluting $100 \mathrm{uL}$ of sample to $10 \mathrm{~mL}$ with eluent and injecting $1 \mathrm{~mL}$ into a Dionex lon Chromatograph. Sample concentrations were determined after running several standards. Dissolved gas samples were analyzed for concentrations of methane, ethane, and propane aboard ship using a Shimadzu Mini-Il gas chromatograph equipped with a poropaq $Q$ column. Dissolved gas samples were also analyzed for carbon isotopic signatures using a gas chromatograph-isotope ratio mass spectrometer (GC-IRMS) with a Hewlett-Packard $5890 \mathrm{GC}$ equipped with a Poroplot $\mathrm{Q}$ column set at $30^{\circ} \mathrm{C}$ and a Finnigan Mat IRMS. Low concentration gases were analyzed by pre-concentrating gas aliquots of $\sim 10-30 \mathrm{~mL}$ in-line with a liquid nitrogen/ethanol slush at approximately $-130^{\circ} \mathrm{C}$ and a column baked at $30^{\circ} \mathrm{C}$ and then introduced into the GC-IRMS system. Microliter volumes of high concentration gases were 
directly injected onto the column to be introduced to the mass spectrometer. Total organic carbon concentrations and isotopes were measured on freeze-dried and ground sediment patties. Approximately $30 \mathrm{mg}$ of sample was introduced by flash combustion into a Carlo Erba Elemental Analyzer and resultant carbon dioxide swept into the same IRMS system as described above. Results are presented in the standard d notation, in \%o.

$\delta^{13} \mathrm{C} \%=[($ Rsam $/$ Rstd $)-1] * 1000$ where $\mathrm{R}={ }^{13} \mathrm{C} /{ }^{12} \mathrm{C}$ and Rsam is the sample and Rstd is PDB standard.

\section{Sulfate reduction rate measurements}

Briefly, triplicate sub-cores were taken from each section, carefully excluding oxygen with silicone-filled stoppers. They were then injected with approximately $40 \mathrm{KBq}{ }^{35} \mathrm{~S}$-sulfate contained in a $10 \mu \mathrm{L}$ volume and incubated at $7^{\circ} \mathrm{C}$ for 24 hours onboard ship. After incubation, sub-cores were extruded into empty $120 \mathrm{~mL}$ glass serum vials and preserved as a slurry in $5 \mathrm{~mL}$ of $1.6 \mathrm{M}$ zinc sulfate and $1 \mathrm{~mL}$ of $0.1 \mathrm{M}$ sodium sulfide carrier solution. These chemicals stabilized sulfide in the form of insoluble zinc sulfide and stopped biological activity. Samples were then stoppered with blue butyl rubber stoppers and frozen for later determination of biologically produced ${ }^{35}$ S-sulfide.

Determination of biologically produced ${ }^{35} \mathrm{~S}$-sulfide was measured by a modification of an active distillation/chromium reduction technique, also described in Albert et al. (1995). In short, samples were flushed with oxygen-free nitrogen and then acidified through the septa with $10 \mathrm{~mL}$ of $2 \mathrm{M} \mathrm{HCl}$ containing $0.5 \mathrm{M} \mathrm{Cr}^{2+}$, prepared previously by reduction of an anoxic solution of $\mathrm{CrCl}_{3}$ solution in a bed of zinc-mercury amalgam bubbled with nitrogen. Upon acidification and heating $\left(4\right.$ minutes at $\left.100^{\circ} \mathrm{C}\right)$, labeled and unlabeled hydrogen sulfide was released from the slurry, carried through a glass-wool aerosol trap, and finally precipitated out in a solution of $6 \mathrm{~mL}$ of $0.5 \mathrm{M}$ zinc acetate in $0.5 \%$ iso-butanol. Trap contents were transferred to scintillation vials and an equal volume of Scintiverse II (Fisher Scientific) for counting on a Beckman LS 6800 scintillation spectrometer was added. Counts were corrected for quench from the counting efficiency of an external standard. Sulfate reduction rates were than calculated using the following equation,

$$
\text { Sulfatereductionrate }(\mathrm{mM} / d)=\frac{\mathrm{H}_{2}{ }^{35} \mathrm{~S} *\left[\mathrm{SO}_{4}{ }^{2-}(\mathrm{mM})\right]^{*} 1.04 * \phi}{{ }^{35} \mathrm{SO}_{4}{ }^{2-}(\text { added }) * \text { incubationtime }(d)}
$$

Where, $\mathrm{H}_{2}{ }^{35} \mathrm{~S}$ is the amount produced during the incubation time, $\mathrm{SO}_{4}{ }^{2-}$ is the concentration of pore water sulfate measured in the cores, 1.04 is the fractionation factor taken into account when bacteria preferentially take up the lighter sulfur isotope rather than the heavier isotope, $\phi$ is the porosity of the sediments, ${ }^{35} \mathrm{SO}_{4}{ }^{2-}$ is the amount of labeled sulfate added to the incubations and incubation time is in days.

\section{Results and Discussion:}

We have successfully developed an in-situ pore water probe to allow collection and recovery of dissolved gas samples without de-pressurization. This improvement led to the observation of significantly greater concentrations and smoother concentration profiles (Figure 5).

Experiments were conducted in 2003 between traditional coring techniques and the Probe to compare dissolved methane concentrations and isotopes (Figure 6). As expected, 
methane concentrations are an order of magnitude lower in the cores than in the Probe. However, there are also slight variations between the methane carbon isotopic composition from the core and Probe pore waters. These variations are being further investigated with the same comparison samples taken from the Gulf of Mexico this past summer 2003.

With the use of the Probe, we were able to look at a spatial survey of methane concentrations and isotopes in the shallow subsurface from eight different locations in the Gulf of Mexico. In the upper $50-\mathrm{cm}$ of the subsurface, methane concentrations increased downcore (Figure 5) and varied widely between sites. For example, we observed great variability at nearby locations at GC-234. Methane concentrations varied by almost two orders of magnitude (Figure 8) between two separate deployments of the Probe taken only several meters from each other. Methane carbon isotopes did not show much variability within a profile (see error bars on Figure 7) but showed some variation over several sites. Most methane showed a thermogenic signature $(-48.36 \pm 5.27)$ while the brine pool at GC 233 was biogenic $(-64.61 \pm 0.10)$ (Figure 7). Variability was also seen in sulfate concentrations and sulfate reduction rates between sites. Sulfate showed greater depletion at site 4217 and almost no depletion, only a few meters away, at site 4218 (Figure 9). Sulfate reduction rates varied by two orders of magnitude, similar to methane concentrations (Figure 10).

One might suspect from perusal of this data that methane concentrations were driving the elevated rates of sulfate reduction at site 4217 relative to site 4218 . However, TOC (total sedimentary organic carbon) was also significantly greater at site 4217 than 4218 (Figure 11). Stable isotope data, indicated that the organic carbon was not methane derived (Figure 12) as it was more like conventional organic matter (low - 20's \%o rather than methane $-48 \%$ ). We hypothesize that microbial degradation of petroleum could also be driving the high rates of microbial respiration observed. To evaluate this hypothesis we have examined the $\delta^{13} \mathrm{C}$ of dissolved porewater $\mathrm{CO}_{2}$ and calculated the excess $\mathrm{CO}_{2}$ over background seawater which is produced by microbial respiration. This approach, called a Keeling plot, plots the reciprocal of the $\mathrm{CO}_{2}$ concentration versus the $\delta^{13} \mathrm{C}$ of dissolved porewater $\mathrm{CO}_{2}$ and allows us to calculate the isotopic signature of the $\mathrm{CO}_{2}$ produced by microbial respiration as the $y$-intercept of the line (Figure 13). The $\delta^{13} \mathrm{C}$ of $\mathrm{CO}_{2}$ produced from microbial respiration is identical to the isotopic composition of the substrates supporting microbial respiration (Lapham et al., 1999; Proctor et al., 2001).

From our Keeling plot approach (Figure 13), we calculate that the $\delta^{13} \mathrm{C}$ of organic carbon driving microbial respiration is $-36.1 \%$, a value intermediate between methane and petroleum hydrocarbons. If we assume that the petroleum compounds are $-29 \%$, and the methane we have measured to be $-49 \%$ with a fractionation effect of 1.012 , we can calculate that the microbial respiration at this site was $24 \%$ supported by methane oxidation while $76 \%$ is supported by oil oxidation (Table 2). Analysis of cements (calcified $\mathrm{CO}_{2}$ ) collected at a variety of gas hydrate sites are consistent with the hypothesis that methane oxidation alone is not driving microbial respiration at gas hydrate sites (Table 3 ) as only 1 site (4408) showed unequivocal evidence for methane oxidation.

In collaboration with Dr. Sassen, of Texas A\&M University, we recently measured the ${ }^{14} \mathrm{C}$ content of vent gases of both biogenic and thermogenic origin (Table 4; Sassen et al., 2003). The thermogenic gas is the first in the table below as indicated by its $\delta^{13} \mathrm{C}$ value of $46.7 \%$. The two biogenic gases have $\delta^{13} \mathrm{C}$ values of $-62 \%$. We tested the hypothesis that the biogenic gases would have some contribution from modern carbon associated with decomposition of recently fixed carbon on the seafloor. Our hypothesis was not consistent with the data. All of the hydrocarbons are of ancient origin. 


\section{Conclusions:}

Preliminary Conclusions from our first years of funding are:

1. Modifications to our in situ porewater probe sampler have allowed the collection and determination of accurate dissolved gas concentration and ${ }^{13} \mathrm{C}$ from the seafloor. Comparisons with pressurized and un-pressurized sampling demonstrates the necessity of such a system to collect accurate and meaningful data. When measured properly, gas and ion concentrations in pore water can be used to help understand the formation, decomposition and stability of hydrates.

2. Variability of rates of microbial process is extreme on small spatial scales. Sulfate reduction rates, methane concentrations, total organic carbon, and sulfate depletion all show evidence for extremely high rates of microbial respiration at gas hydrates sites but this respiration is patchy on small scales.

3. Microbial respiration is not driven only by methane oxidation. There is strong isotopic evidence that oxidation of higher hydrocarbons plays a major role in driving elevated rates of microbial processing.

4. Radiocarbon evidence indicates that hydrocarbon sources are driven by deep reservoirs and there is little evidence for extensive methane production associated with recycling of organic matter fixed on the sea floor via chemosynthetic processes. 
Figures:

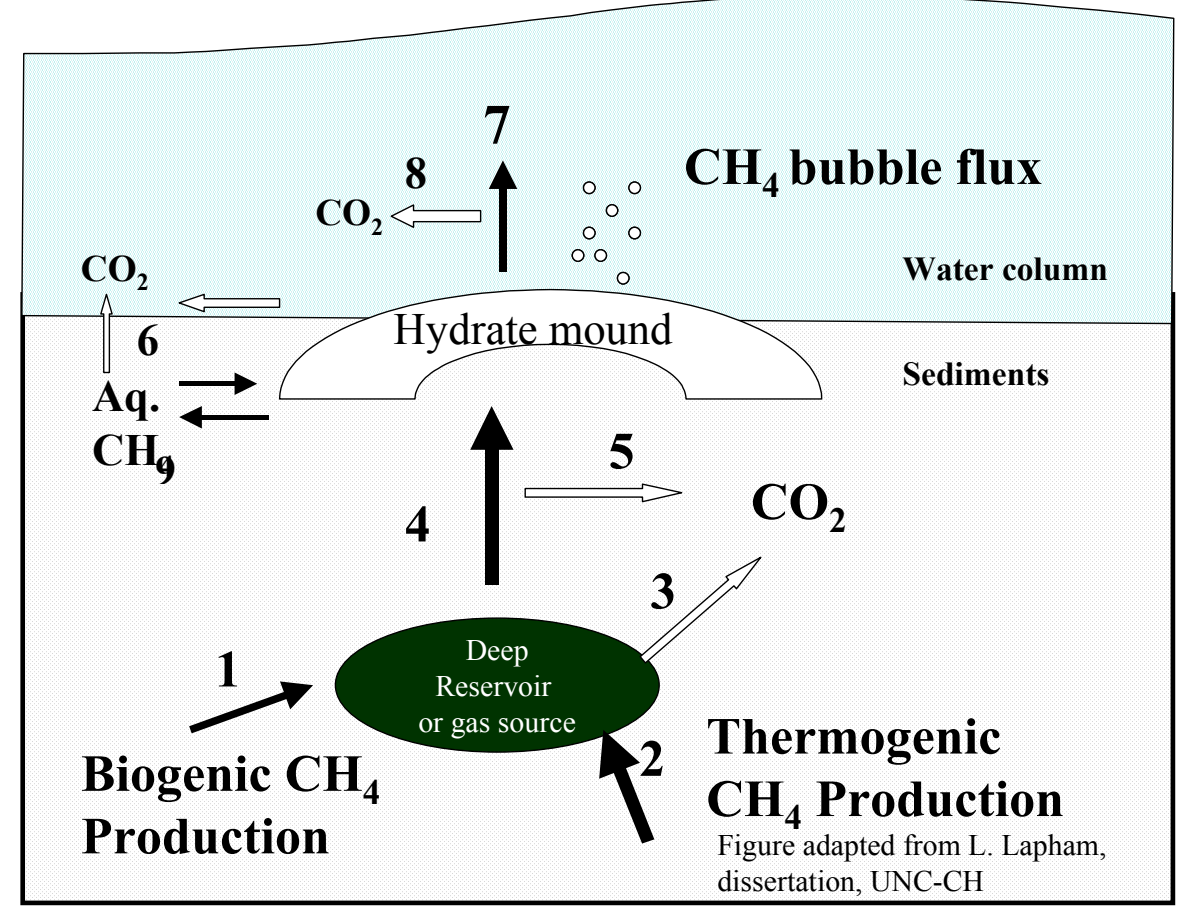

Figure 1 depicts a conceptual model of methane flux, production and consumption that can affect gas hydrate deposits. The deposits considered derive contributions of methane from both biogenic and thermogenic sources. A biogenic hydrate model might simply omit the thermogenic components of this model. Black arrows represent methane flux from a source; open arrows represent microbial methane consumption. Flux 1 is from biogenic methane production, 2 is from thermogenic methane production, 4 represents the flux from a deep reservoir or distributed sources to the surface where it can form hydrates, bubbles or dissolved methane. Flux 7 represents methane flux from either hydrate, pore water, or gas bubbles into the water column where it can enhance secondary production. Fluxes 3, 5, 6 and 8 represent anaerobic and aerobic methane oxidation. Equilibrium and exchange with dissolved methane is also depicted, flux 9. Any bubbles that make it to the top 100-m of the water column can be injected directly into the atmosphere. 


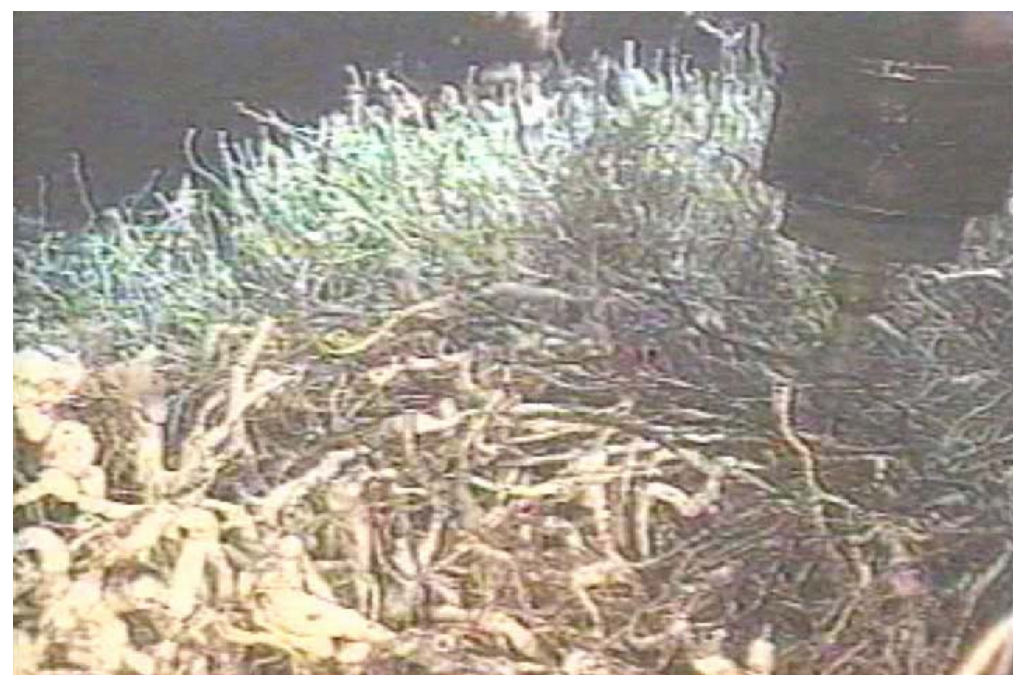

Figure 2. The pore water probe can be utilized to sample a variety of discrete environments. In the picture above, a porewater profile below a tube worm bush is being obtained.

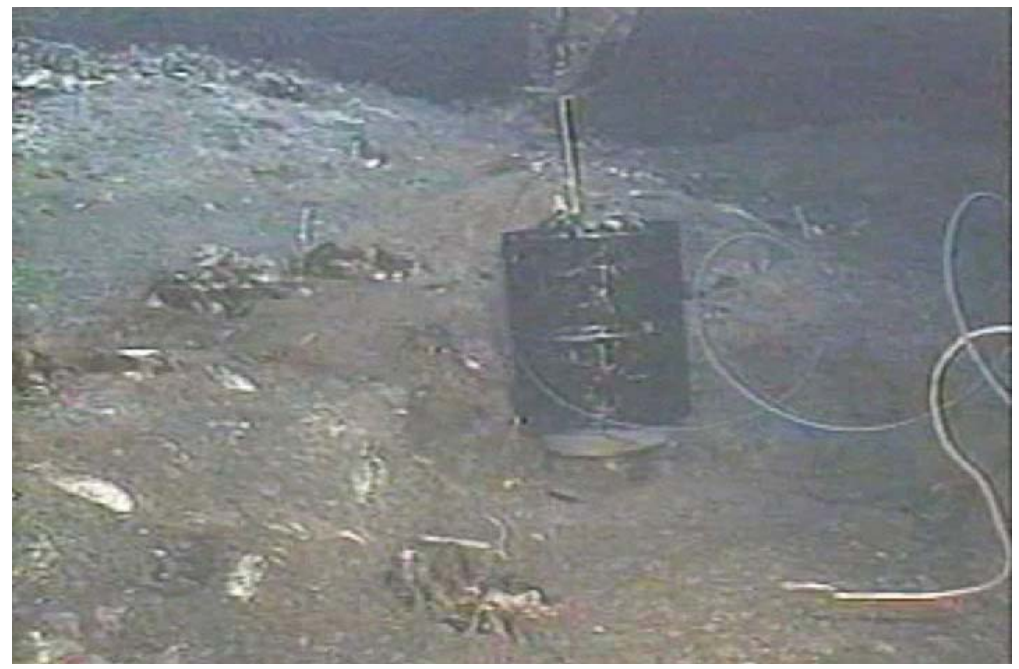

Figure 3. Here the probe is deployed by the arm of the Sea Link to collect a discrete sample immediately at the base of a gas hydrate deposit. 


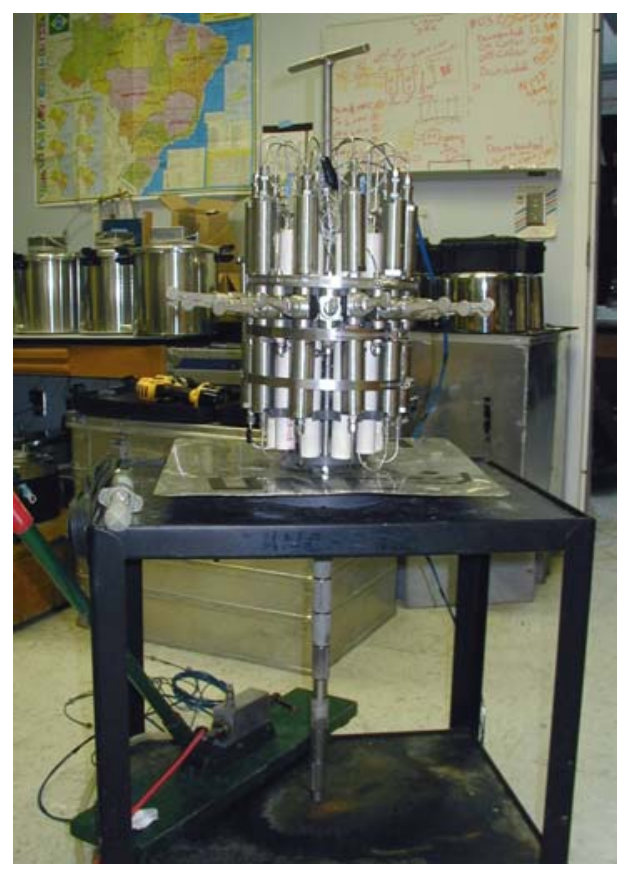

Figure 4. The porewater probe following its redesign to allow collection and retrieval of in situ samples at ambient pressure. The probe is resting on a table with the sampling proboscis extending through the deck of the table.

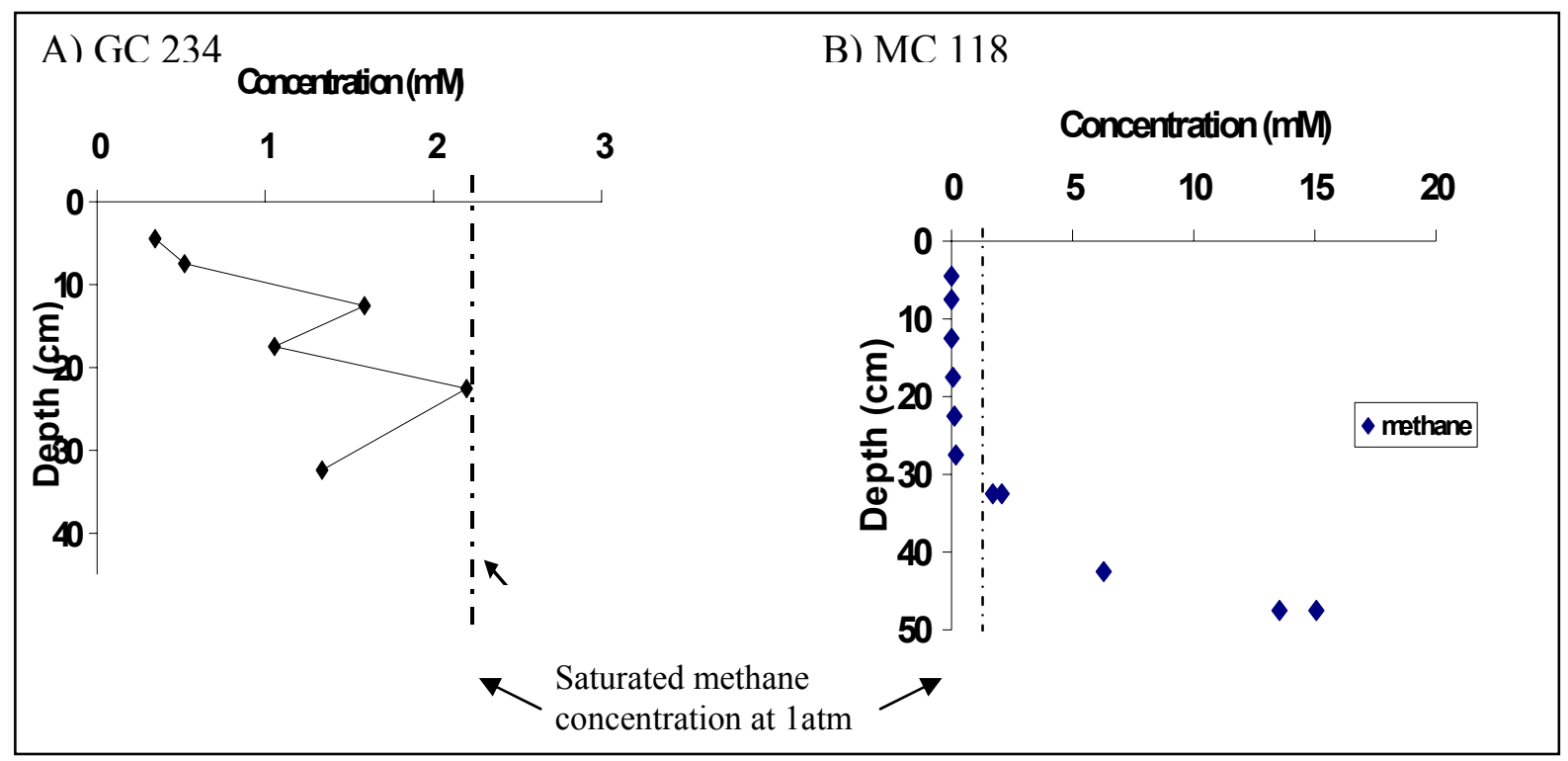

Figure 5. Comparison of in situ pore water gas probe gas analysis on un-pressurized (left) and pressurized (right) pore water sampler. The profile on the left does not exhibit gas concentrations above equilibrium values with 1 atmosphere pressure (dashed line) whereas the right profile does. These results indicate the loss of dissolved gases as the instrument ascended 
to the surface. The saw-tooth nature of the profile also may indicate degassing during assent. The right profile reaches concentrations of $15 \mathrm{mM} \mathrm{CH}_{4}$ and is smooth, exhibiting a convex upward shape consistent with methane oxidation. Modeling exercises will enable us to calculate a rate of methane consumption from this data that would not be possible otherwise. To our knowledge, this profile is the first ever pressurized in situ profile collected from the deep sea.

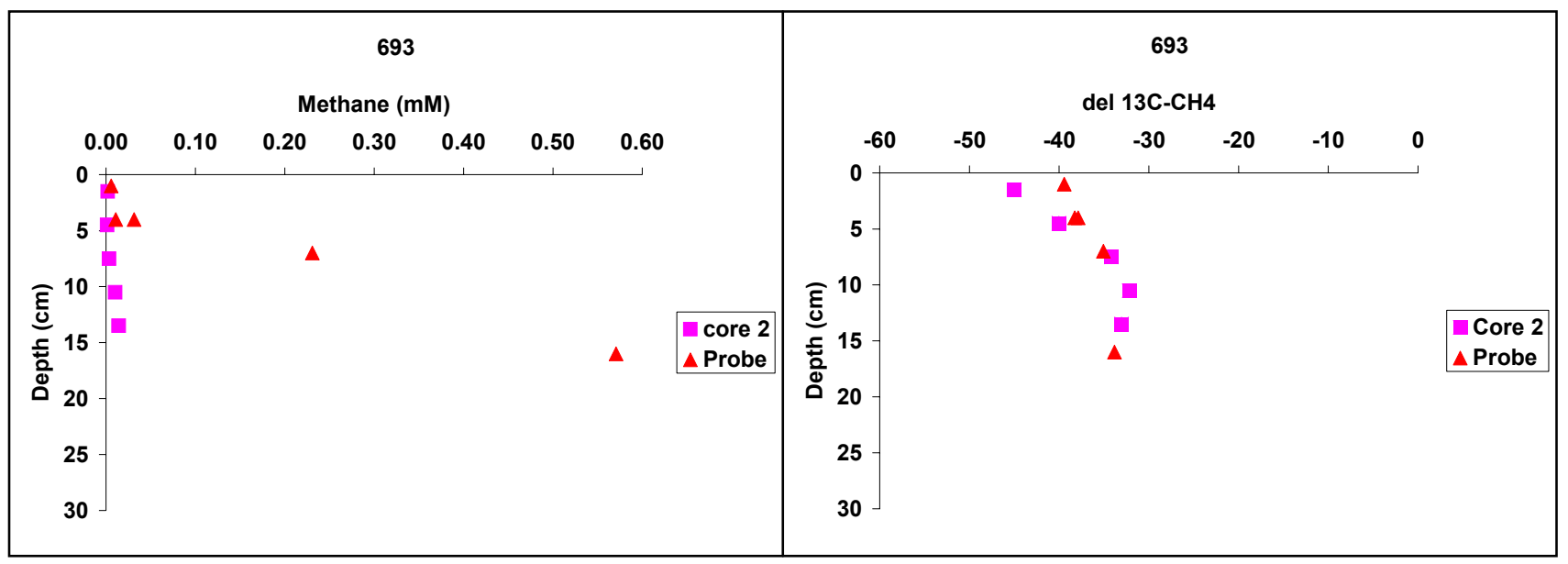

Figure 6. Comparison of methane concentration and isotopic ratio from Probe and traditional cores taken from Cascadia Margin.

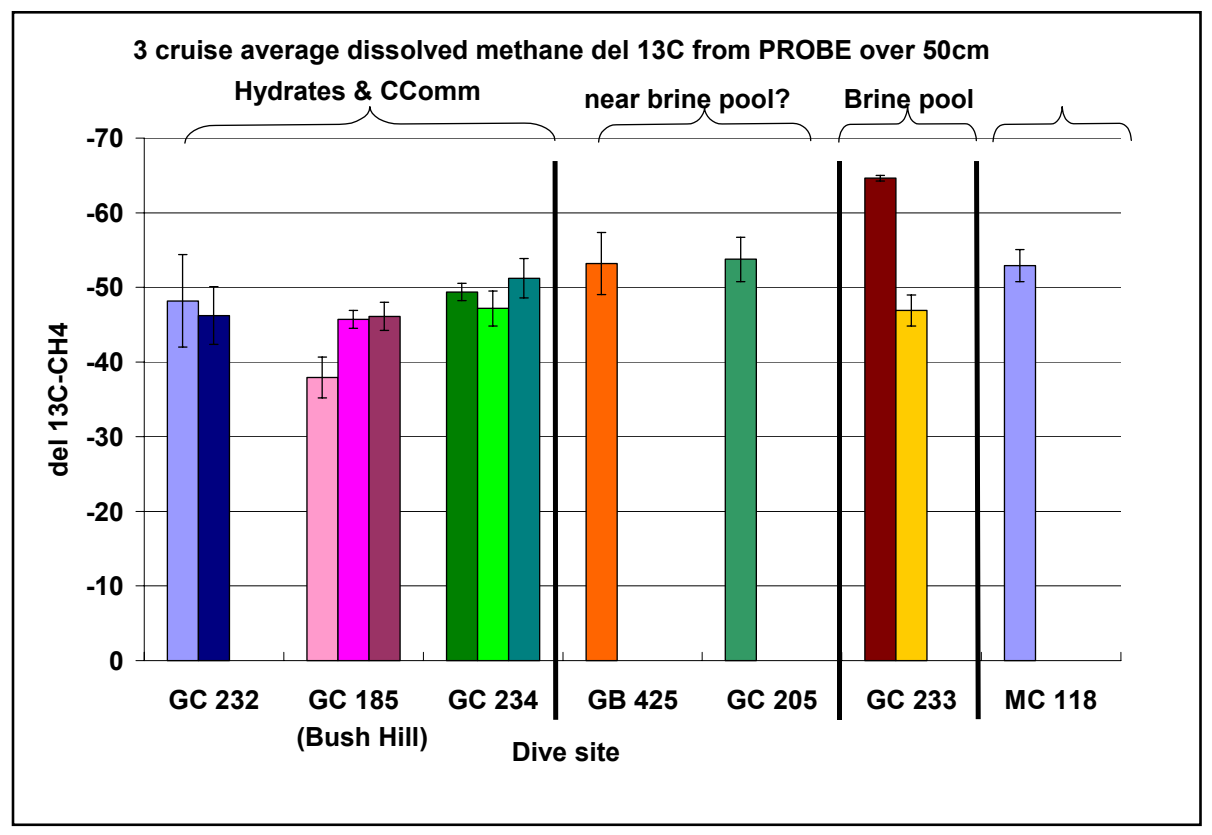

Figure 7. Averaged dissolved methane dell3C values from the Probe over 50-cm interval. Error bars signify 1 standard deviation. 


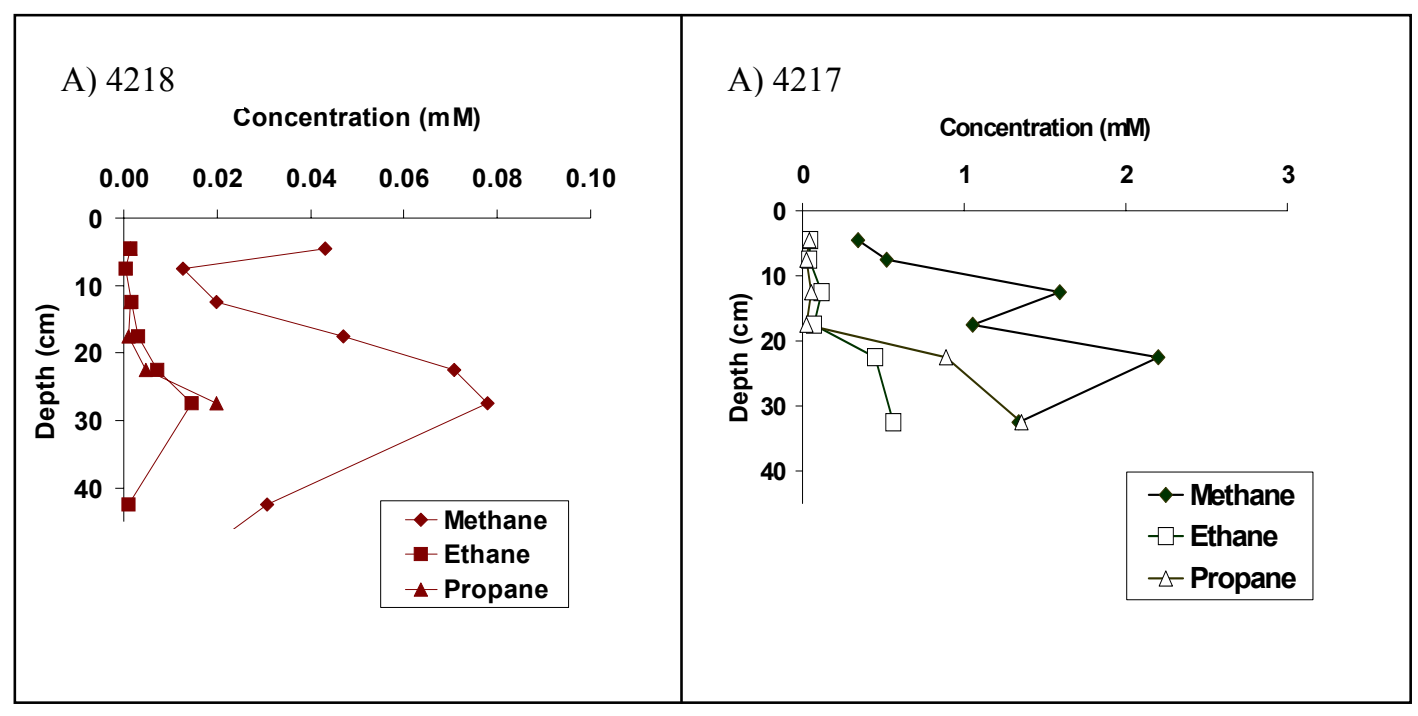

Figure 8. We observed almost two orders of magnitude variability hydrocarbon concentrations in sediments surrounding the gas hydrate deposits. These two sites also showed great variability in sulfate reduction rates (see Figures 9 and 10).

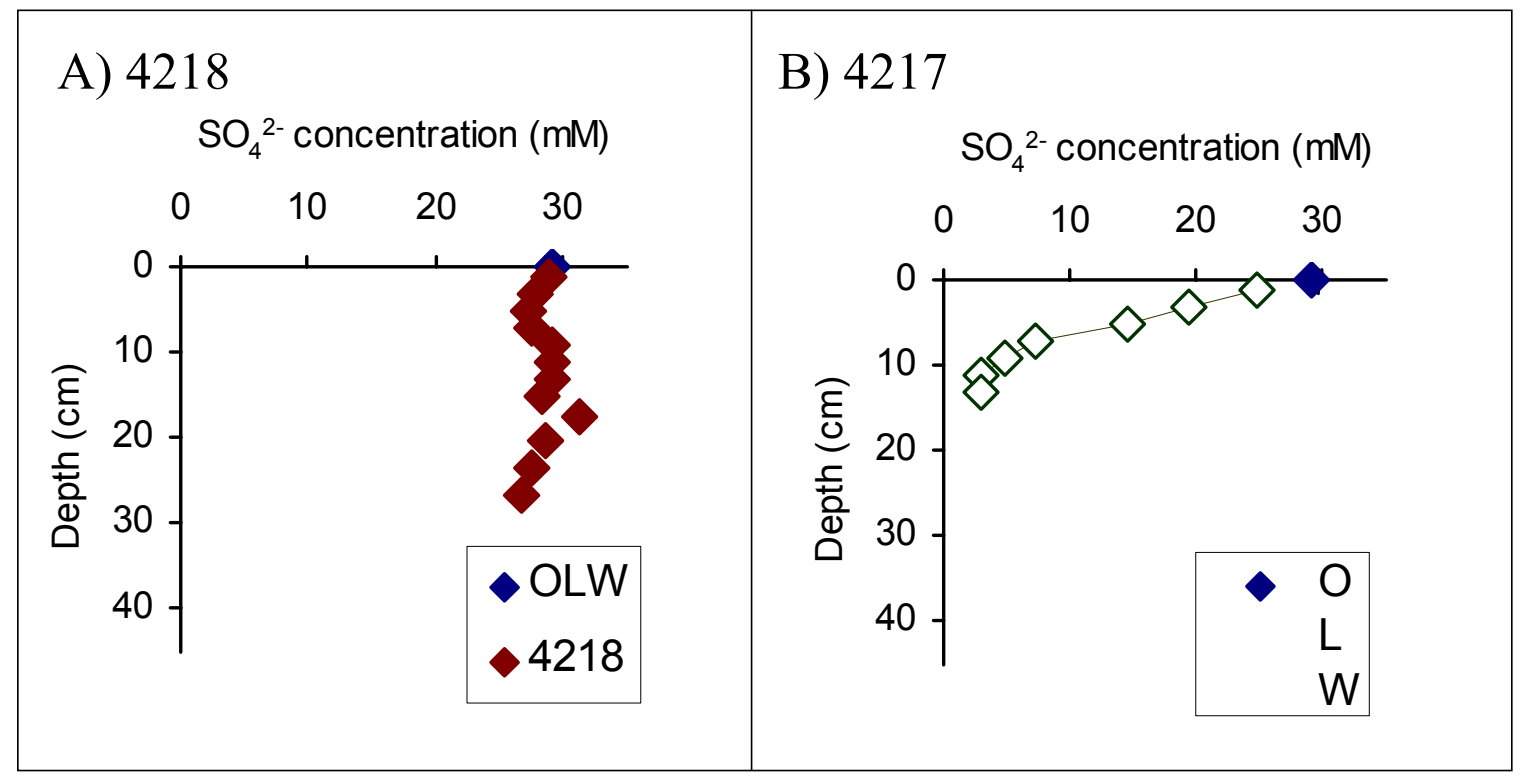

Figure 9. Sulfate depletion is greatest in core 4217, consistent with the higher hydrocarbons measured there and the greater sulfate reduction rates. 


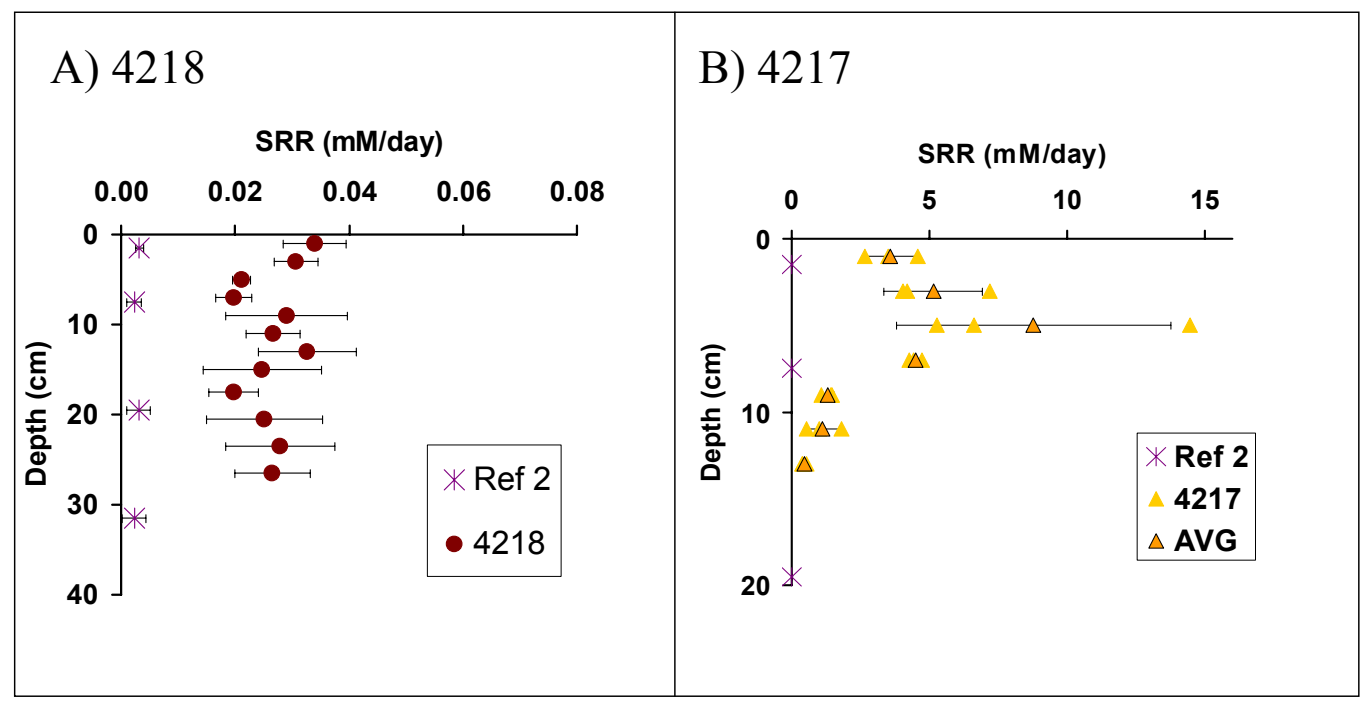

Figure 10. Sulfate reduction rates varied by over two orders of magnitude between the sites. Also shown in the figure are sulfate reduction rates at a reference or background site away from a gas hydrate deposit.

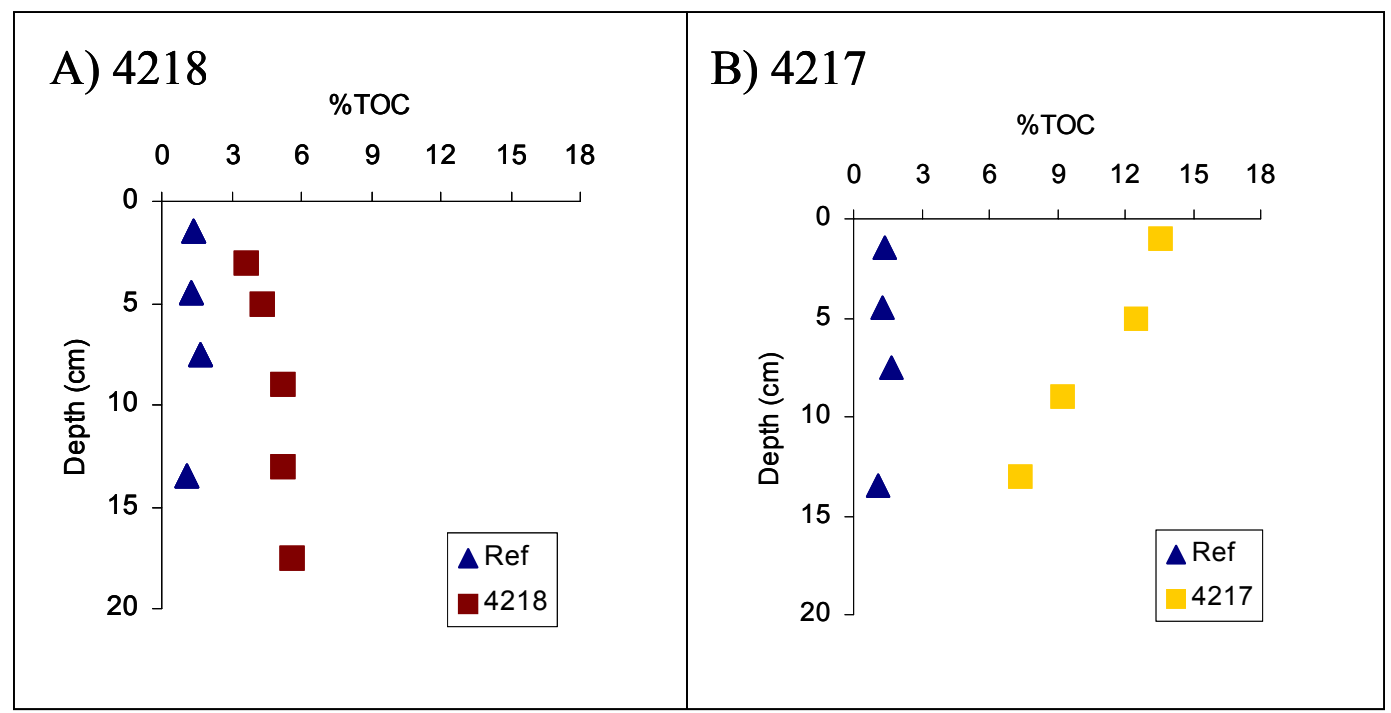

Figure 11. The \% organic carbon at the sites discussed above. Similar to methane concentrations and respiration rates, organic carbon concentration was much greater at site 4217. 


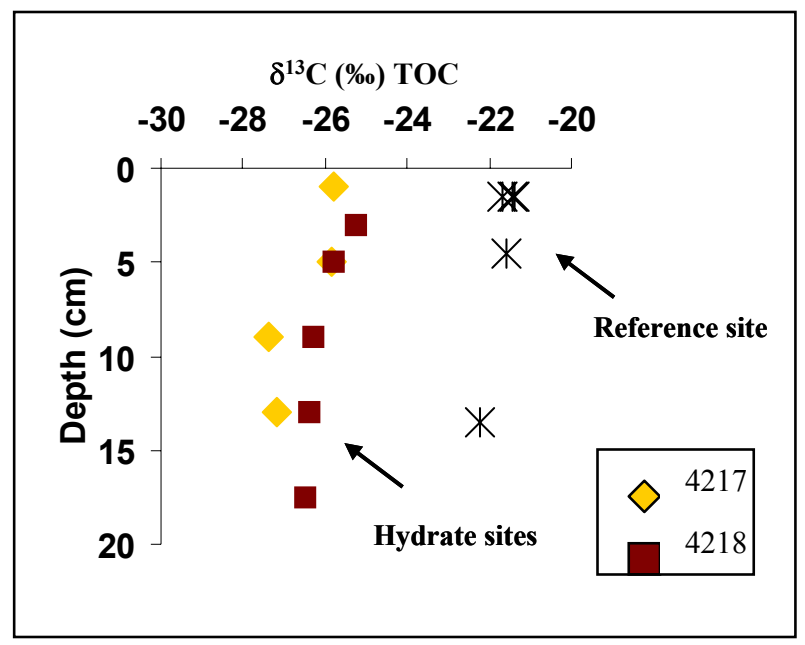

Figure 12. The $\delta^{13} C$ of TOC at the two sites was not different, nor was it clearly derived from methane oxidation. TOC $\delta^{13} C$ was more similar to petroleum values.

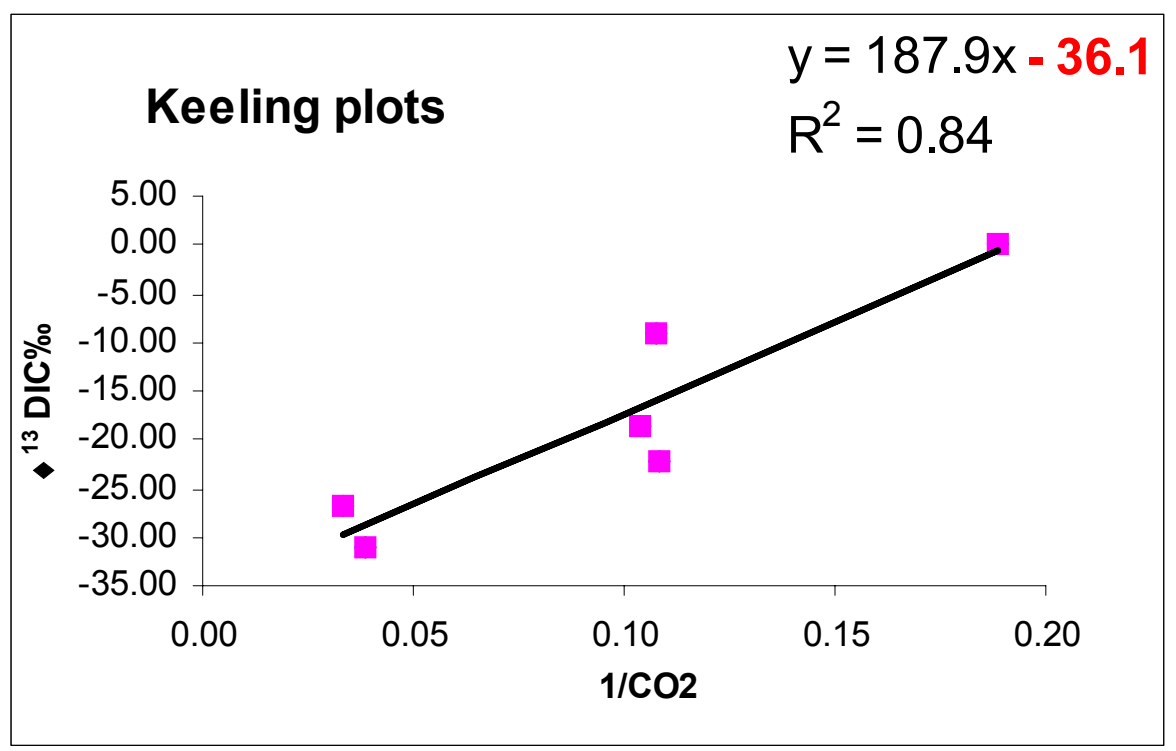

Figure 13. Keeling plot analysis to determine the $\delta^{13} \mathrm{C}$ of excess $\mathrm{CO}_{2}$ in porewater driven by respiration. The intercept of the line fit to the data is the $\delta^{13} \mathrm{Cof}_{\mathrm{CO}}$ in excess over background seawater. This excess $\mathrm{CO}_{2}$ was supplied by microbial respiration, and reflects the isotopic composition of the substrate utilized by the bacteria (Lapham et al., 1999). This value was used in Table 1, above to determine the relative contributions of the hydrocarbons driving microbial respiration at the gas hydrate sites. 
Tables:

\begin{tabular}{|c|c|c|c|c|c|}
\hline Site & $\#$ dives & $\begin{array}{l}\text { Exposed } \\
\text { hydrate }\end{array}$ & $\begin{array}{l}\text { Chemosynthetic } \\
\text { communities }\end{array}$ & \# cores & $\begin{array}{l}\text { \# Probe } \\
\text { deployments }\end{array}$ \\
\hline $\begin{array}{l}\text { GC } 185 \\
\text { Bush Hill }\end{array}$ & 10 & Y & Y & 14 & 3 \\
\hline $\begin{array}{l}\text { GC } 233 \\
\text { Brine Pool }\end{array}$ & 2 & $\mathrm{~N}$ & $\mathrm{Y}$ & 2 & 2 \\
\hline GC 234 & 8 & Y & $?$ & 10 & 3 \\
\hline GC 232 & 4 & Y & Y & 7 & 2 \\
\hline GC 425 & 1 & $?$ & Y & 1 & 1 \\
\hline $\begin{array}{l}\text { GC } 205 \\
\text { mud volcano }\end{array}$ & 1 & $\mathrm{~N}$ & $\mathrm{~N}$ & 2 & 1 \\
\hline \begin{tabular}{|r|} 
Viosca Knoll \\
826 \\
\end{tabular} & 2 & $?$ & $?$ & 3 & 0 \\
\hline $\begin{array}{r}\text { Miss Canyon } \\
118 \\
\end{array}$ & 3 & $?$ & $\mathrm{Y}$ & 3 & 1 \\
\hline
\end{tabular}

Table 1. Sites visited from three cruises to the Gulf of Mexico.

\section{Petroleum or methane oxidation?}

- Signature of added DIC $=-36.1 \%$

- Methane source $=-47(\alpha=1.012) \rightarrow-59 \%$

- Oil source $=-29 \%$

- Mass Balance:

$$
\begin{aligned}
& -59(f)+-29(1-f)=-36.1 \\
\sim & 24 \% \text { methane oxidation } \\
\sim & 76 \% \text { petroleum oxidation }
\end{aligned}
$$

Table 2. Calculation of the relative importance of substrates driving microbial respiration from an isotopic balance. 


\begin{tabular}{|c|c|}
\hline Cement $\mathrm{CaCO}_{3}$ & ${ }^{3} \mathrm{C} \%$ \\
\hline Sample ID & $\delta 13 C$ \\
\hline Bucket 3, 709, Miss. Canyon, 4413 & -23.8 \\
\hline $4405,5 / 30 / 02$, Rock & -20.5 \\
\hline $4401,5 / 29 / 02$, Carbonate & -22.0 \\
\hline 4413, Core 6 Rocks from $6-9 \mathrm{~cm} \mathrm{6/3/02}$ & -26.8 \\
\hline 4403, \#6, $27 \mathrm{~cm}$ carbonate, 5/29/02 & -18.0 \\
\hline GC 232,4403 , core $\# 4$, carbonate at $21 \mathrm{~cm}$ & -14.6 \\
\hline 4401, Core \#2, 5/29/02, carbonate & -18.0 \\
\hline $4405,5 / 30 / 02$ & -22.0 \\
\hline $4408,6 / 1 / 02$ Offshore $65-1$ & -49.7 \\
\hline 4413, 118 Miss. Canyon 6/3/02 & -28.6 \\
\hline Gc $234,4407,5 / 31 / 02$ smaller of 2 rocks & -20.4 \\
\hline 4413, Miss Canyon, Block 3/6/02, 709, Bucket 9 & -28.2 \\
\hline
\end{tabular}

Table 3. $\delta^{13} \mathrm{C} \%$ of carbonate cements recovered from gas hydrate sites. With the exception of the sample highlighted in yellow, none of these samples show unequivocal evidence for methane oxidation. The samples highlighted in pink are 13 C enriched relative to marine organic matter.

\begin{tabular}{|l|c|c|c|c|c|}
\hline Sample & $\delta^{13} \mathrm{C} \% \mathrm{o}$ & AGE yrs & F modern & Fm error & $\Delta^{14} \mathrm{C}$ \\
\hline $4228-3$ & -46.7 & $>52000(1100)$ & 0.001061 & 0.0001 & -998.9 \\
\hline $4232-2$ & -62.8 & $48100(610)$ & 0.00253 & 0.0002 & -997.5 \\
\hline $42291 \mathrm{R}$ & -62.9 & $45700(410)$ & 0.003384 & 0.0002 & -996.6 \\
\hline
\end{tabular}

Table 4. Radiocarbon values of methane (modified from Sassen et al., 2003). F modern stands for fraction modern carbon. 


\section{References}

Aharon, P. and Fu, B. (2000). Microbial sulfate reduction rates and sulfur and oxygen isotope fractionations at oil and gas seeps in deepwater Gulf of Mexico. Geochim. Cosmochim. Acta, 64 (2), 233-246.

Boetius, A., Ravenschlag, K., Schubert, C.J., Rickert, D., Widdel, F., Gieseke, A., Amann, R., Jorgensen, B.B., Witte, U., and Pfannkuche, O. (2000). A marine microbial consortium apparently mediating anaerobic oxidation of methane. Nature 407, 623-626.

Brooks, J.M., Kennicutt II, M.C., Fay, R.R., McDonald, T.J., and Sassen, R. (1984). Thermogenic gas hydrates in the Gulf of Mexico. Science 225, 409-411.

Chanton, J.P., Martens, C.S., Paull, C., and Coston, J.A. 1993. Sulfur isotope and porewater geochemistry of Florida Escarpment Seep Sediments, Geochim. Cosmochim . Acta, 57, 1257-1266.

Chanton, J.P., Martens, C.S., and Paull, C.K. 1991. Control of pore-water chemistry at the base of the Florida Escarpment by processes within the platform. Nature, 349, 229-231.

Cragg B.A., Parkes R.J., Fry J.C., Weightman A.J., Rochelle P.A., and Maxwell J.R. (1996). Bacterial populations and process in sediments containing gas hydrates (ODP Leg 146: Cascadia Margin). Earth and Planetary Science Letters 139, 497-507.

Grabowski, K., R. Rogers, J. Chanton, J. Whelan, P. Sobecky, 2003, Summary Report Session II, Biological Influence on Hydrate Formation, Stability, Content and Lattice Saturation. International Gas Hydrate Meeting, Washington DC, November, 2003.

Kvenvolden K.A. (1988). Methane hydrate-a major reservoir of carbon in the shallow geosphere? Chem. Geol. 71, 41-51.

Lapham, L., L. Proctor and J. Chanton. 1999. Using Respiration Rates and Stable Carbon Isotopes to Monitor the Biodegradation of Orimulsion by Marine Benthic Bacteria, Environmental Science and Technology, 33, 2033-2039.

MacDonald I.R., Boland G.S., Baker J.S., Brooks J.M., Kennicutt II M.C., and Bidigare R.R. (1989). Gulf of Mexico hydrocarbon seep communities. Mar. Biol., 101, 235-247.

MacDonald I.R., Callender W.R., Burke Jr. R.A., McDonald S.J., and Carney R.S. (1990). Fine-scale distribution of methanotrophic mussels at a Louisiana cold seep. Prog. Oceanogr. 24, 15-24.

MacDonald I.R., Reilly II J.F., Guinasso Jr, N.L., Brooks J.M., Carney R.S., Bryant W.A., and Bright T,J. (1990). Chemosynthetic mussels at a brine-filled pockmark in the northern Gulf of Mexico. Science 248, 1096-1099.

MacDonald I. R. N. L. Guinasso, R. Sassen, J. Brooks, L. Lee, K. Scott. (1994) Gas hydrate that breaches the sea floor on the continental slope of the Gulf of Mexico. Geology 22, 699702. 
Martens, C. S. and R. A. Berner, 1977. Interstitial water chemistry of anoxic Long Island Sound sediments. I. Dissolved gases. Limnol. Oceanogr. 22: 10-25.

Martens, C.S., Chanton, J.P., and Paull, C.K. (1991). Fossil Biogenic methane at the Florida Escarpment. Geology, 19, 851-854.

Martens, C.S. (1990) Generation of short chain organic acid anions in hydrothermal altered sediments of Guaymas Basin, Gulf of California. Applied Geochemistry, 5, 71-76.

Martens, C.S., D.B.Albert, and M.J. Alperin. 1999. Stable isotope tracing of methane production and oxidation in the gassy sediments of Eckernforde Bay, German Baltic Sea. Amer. Journal Science (Invited paper for R.A. Berner Special Issue ) 299: 589-610.

Paull, C.K., Chanton, J. P., Neumann, A.C., Coston, J.A., and Martens, C.S. (1992). Indicators of methane derived carbonates and chemosynthetic organic carbon deposits: examples from the Florida Escarpment. Palaios, 7, 361-375.

Paull, C.K., Chanton, J.P., Martens, C.S., Fullagar, P.D., Neumann, A.C., and Coston, J.A. (1991). Seawater circulation through the flank of the Florida platform: evidence and implications. Marine Geology, 102, 265-279.

Paull, C.K., Martens, C.S., Chanton, J.P., Neumann, A.C., Coston J., Jull, A.T., and Toolin, L.J. (1989). Fossil methane carbon in living chemosynthetic organisms and young carbonate cements at the Florida Escarpment. Nature, 342, 166-168

Proctor, L.M., E. Toy, L. Lapham and J. Cherrier and J. Chanton. 2001. Enhancement of orimulsion biodegradation through the addition of natural marine carbon substrates. Environmental Science and Technology, 35, 1420-1424

Sassen R., Roberts H.H., Aharon P., Larkin J., Chinn E.W., and Carney R. (1993). Chemosynthetic bacterial mats at cold hydrocarbon seeps, Gulf of Mexico continental slope. Org. Geochem., 20, 77-89.

Sassen, R. and I.R. MacDonald. (1997). Hydrocarbons of experimental and natural gas hydrates, Gulf of Mexico continental slope. Org. Geochem, 26, 289-293.

Sassen, R., I.R. MacDonald and others. (1998). Bacterial methane oxidation in sea-floor gas hydrate: Significance to life in extreme environments. Geology, 26, 851-854.

Sassen, R., S.T. Sweet, A. Milkov, D. DeFretias, F. Salata, E. McDade. (1999) Geology and geochemistry of gas hydrates, central Gulf of Mexico Continental Slope, Gulf Coast Association of Geological Societies Transactions, vol XLIX, pages 462-468.

Sassen, R. S. Joye, S. Sweet, D. DeFreitas, A. Milkov and I. MacDonald. (1999). Thermogenic gas hydrates and hydrocarbon gases in complex chemosynthetic communities, Gulf of Mexico, continental slope. Organic Geochemistry, 30, 485-497.

Sassen, R. et al., 2003. Gas venting and subsurface change in the Green Canyon area, Gulf of Mexico continental slope: evidence of a deep bacterial methane source? Organic Geochemistry, 34: 1455-1464. 


\title{
Deep Sea Microbes as Sources of Biomedically-Important Natural Products
}

Task 8

Final Report

December 2003

Principal Investigator: Marc Slattery

DOE Award DE-FC26-00NT40920

\author{
University of Mississippi \\ National Center for Natural Products Research \\ University, Mississippi, 38677
}




\begin{abstract}
Extremophile microbes, specifically those from methane hydrate seeps in the Northern Gulf of Mexico, represent a potentially important and novel resource for marine biotechnology. Microbes are known to produce up to one third of all antibiotics on the market today, and deepsea organisms also produce unusual enzymes that operate under extreme pressures and temperatures. The purpose of this project was to isolate strains of microorganisms from the methane hydrate seep sites, to culture these strains in our labs at the University of Mississippi, and to test broth extracts for unusual biomedical activity.
\end{abstract}

\title{
Table of Contents
}

\author{
Abstract \\ Introduction \\ Executive Summary \\ Experimental \\ Results and Discussion \\ Conclusion
}

\section{Introduction}

Recent studies have demonstrated the significance of the marine environment as another resource for the discovery of novel bioactive metabolites (Paul 1992; Attaway \& Zaborsky 1993). The oceans encompass approximately $75 \%$ of the planet's surface and over $90 \%$ of its crust volume, thus conditions represent the extremes of temperature, pressure, and salinity encountered by organisms. To survive, marine organisms have adapted unique metabolic processes, and often metabolites, which are not observed in terrestrial systems (Pietra 199*). Many important biomedical metabolite discoveries have been reported and at least two compounds, Bryostatin 1 and Didemnin B, have advanced to clinical trials as anticancer agents (Flam 1994). Like their terrestrial counterparts, marine microbes (bacteria and fungi) offer the potential of a rich resource of novel chemotherapeutic agents. Nonetheless, relatively few studies have focused on marine microbes; this may be due in part to the complexities involved in the isolation and cultivation of this resource (Fenical 1993; Liberra \& Lindequist 1995), or to misidentified metabolite source organisms (Stierle et al. 1988; Faulkner et al. 1994).

The bacteriocidal properties of seawater were recognized by ZoBell and coworkers approximately 50 yrs ago; they subsequently showed that the diverse bacterial communities produced antimicrobial agents. Nonetheless the first marine bacterial metabolite was only recently isolated from a species of Alteromonas found on blades of the seagrass Thalassia sp. in Puerto Rico (Burkholder et al. 1966). The highly brominated pyrole (1), pentabromopseudiline, exhibited impressive in vitro antibiotic activity (MIC's of 0.0063 to $0.2 \mathrm{ug} / \mathrm{ml}$ ) against Gram-positive bacteria including Mycobacterium tuberculosis but was not active in

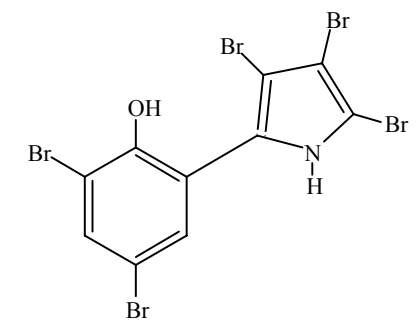


vivo; antitumor properties of this compound have been reported more recently (Laatsch \& Pudleiner 1989).

Historically, terrestrial soil samples have yielded many of the antibiotic producing microorganisms (Robbers et al. 1996). Microbes derived from marine sediment samples, which

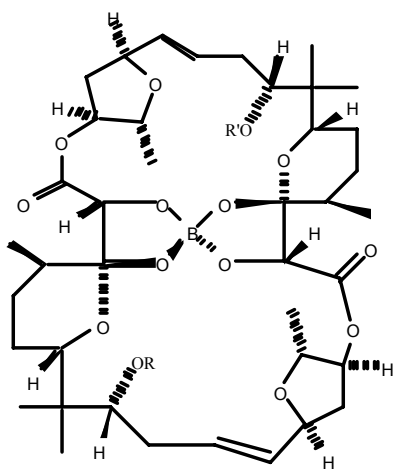
tend to be nutrient-rich, produce a diversity of bioactive compounds as well. For instance, Streptomyces griseus from a coastal sediment sample in Japan produced an unusual group of boron-containing antimicrobials: aplasmomycin A

2: $\mathrm{R}=\mathrm{R}^{\prime}=\mathrm{H}$ 3: $\mathrm{R}=\mathrm{H}, \mathrm{R}^{\prime}=\mathrm{Ac}$ 4: $\mathrm{R}=\mathrm{R}^{\prime}=\mathrm{Ac}$ (2), B (3), and C (4) (Hotta et al. 1980). These compounds were active against Gram-positive bacteria (MIC's of 0.8 to $3.0 \mathrm{~g} / \mathrm{ml}$ ), but were particularly effective in vivo against Plasmodium berghei, the causal agent of malaria in mice. More recently this strain has been crossed with the species $S$. tenjimariensis, which produces a class of compounds that have strong activity against aminoglycoside-resistant microbes, to produce a strain that yields novel indolizine antibiotics (Yamashita et al. 1985).

Marine fungi also offer a potential source of bioactive compounds including alkaloids, polyketides, and terpenes. For example, an undescribed species of Phoma was isolated from the carapace of the crab Chionoecetes opilio which yielded the phomactines A-D; these act as inhibitors of PAF receptor binding (Sugano et al. 1994). The D analog (5) exhibited the lowest IC50 $(=0.12 \mathrm{uM})$ of all conformers. In addition, it was discovered that these compounds don't effect the ADP-,

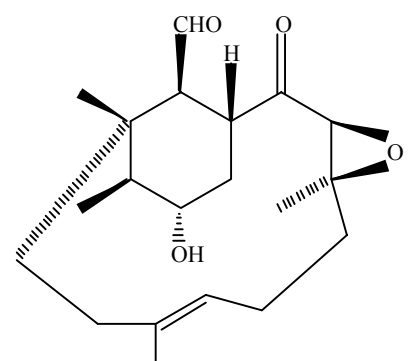
arachidonic acid-, or collagen-induced platelet aggregation indicating phomactines represent a new class of potent and specific PAF antagonists.

Recent evidence has confirmed the importance of microbial biofilms as settlement cues for invertebrate larvae representing diverse taxonomic affinities (Johnson \& Sutton 1994, Keough \& Raimondi 1995, Wieczorek et al. 1995). Included among the species which respond to biofilms are several examples of commercially cultured or harvested marine invertebrates (Cameron \& Hinegardner 1974, Weiner et al 1985, Pearce \& Scheibling 1991, Rodriguez et al 1995, Anderson 1996). Marine biofilms possess physical and chemical characteristics that can influence larval settlement. The presence of bacteria can significantly change the nature of a substrate through alteration of wettability; this can positively or negatively impact various larval settlers (Holmstrom \& Kjelleberg 1994). Likewise, bacterial surface domain characteristics can influence cell-cell and cell-surface interactions. The production of exopolysaccharides might promote settlement: 1) by passive entrapment of the larvae, 2) by direct attraction of larvae, and 3) by amplification of inductive metabolites sequestered within the slime layer (Bonar et al. 1986, Szewzyk et al. 1991). Bacteria-derived inductive chemical cues have proven exceptionally difficult to identify although evidence for their existence appears unequivocal. Unknown chemical "factors" have been isolated from Alteromonas espejiana, Vibrio sp., and Shewanella colwelliana (previously Alteromonas colwelliana and LST) which induce settlement in a hydrozoan, a scyphozoan, and 2 species of oysters respectively (Neumann 1979, Weiner et al. 1989, Leitz \& Wagne 1993). While various neurotransmitters and inorganic compounds can be used by aquaculturists to induce laboratory settlement and metamorphosis of commerciallyimportant marine invertebrates, the compounds are often very costly and use can lead to 
significant developmental aberrations and post-settlement mortality (Hadfield 1984, Pawlik 1990, Slattery 1992). Thus the search for natural inducers of larval settlement is still warranted and requisite for cost-effective hatchery reared resources.

Marine microbes have been notoriously difficult to culture despite evidence for vast numbers and species diversity in seawater (Sieburth 1979). Direct enumeration of seawater samples using fluorochrome stains indicate that as much as $99 \%$ of the bacteria in any given sample are incapable of forming colonies on traditional media formulations (Daley \& Hobbie 1975; Kirchman et al. 1982). These media typically incorporate complex carbon and nitrogen sources at concentrations that are an order of magnitude higher than found in the oligotrophic conditions of the open ocean (sensu Valiela 1984). Moreover, the observations that marine bacterial growth can be inhibited by peptone and some amino acids (Buck 1974; Button et al. 1993), and that many marine bacterial species exhibit physiological adaptations to growth in low nutrient conditions (Roszak \& Colwell 1987; Ostling et al. 1993), indicate that the successful culture of marine microbes will need to be based on specific low nutrient formulations. Recent high recoveries of viable marine bacteria have utilized pre-dilution of microbes and low nutrient formulations (Schut et al. 1993; Jensen et al. 1996).

\section{Executive Summary}

Extremophile microbes, specifically those from methane hydrate seeps in the Northern Gulf of Mexico, represent a potentially important and novel resource for marine biotechnology. Microbes are known to produce up to one third of all antibiotics on the market today, and deep sea organisms also produce unusual enzymes that operate under extreme pressures and temperatures. The purpose of this project was to isolate strains of microorganisms from the methane hydrate seep sites, to culture these strains in our labs at the University of Mississippi, and to test broth extracts for unusual biomedical activity. Our group conducted a broad survey of the microorganisms from the 2002 GOM cruise, and isolated at least 237 strain. During the 2003 GOM cruise, we specifically assessed actinomycetes using selective media and isolated at least 41 strains. Those isolates have been maintained in our culture facility for 3-15 mos (= 1-5 cell line transfers) on specific media developed for this purpose. Approximately $26 \%$ of the isolates were cryophilic and $12 \%$ had unusual hydrocarbon nutrient requirements suggesting they might contain novel enzymes with biotechnological roles (antifreezes and oil spill remediation, respectively). EtOAc broth extracts yielded a biomedical "hit rate", in at least one of our antimicrobial screens, of $6 \%$ compared to an average of $5 \%$ for most of our shallow-water microbial isolate extracts. Preliminary chemical fingerprinting LC/MS data indicate that unusual halogenated compounds may be relatively common amongst these bioactive extracts. Bacteriaphage experiments and further isolation/structure elucidation of the bioactive constituents are ongoing and will form the general outline of a PhD dissertation (T. Hodges; University of Alabama, expected completion 2007).

\section{Experimental}

To maximize species diversity, marine microbes will be collected from multiple cores taken at the GOM methane hydrate seep sites. Sites will be chosen based on eco-physiological variability which can have tremendous influence on phenotypic (=chemical compounds) 
variation (Harvell et al. 1993; Slattery \& Paul in press). For instance, we will collect in the shallow intertidal communities, deeper soft-bottom habitat characteristic of the Gulf Coast and the relatively rare hard-bottom structures, as well as in and outside of run-off plumes (varying in size from the Mississippi River to seasonal streams). Likewise seasonal collections will increase the chances of isolating temporally discrete microbial blooms. It is often difficult to isolate enough microbial cells from seawater samples so these collections will focus only on the benthic microbial fauna. I have had much success in swabbing substrate and/or sediment in

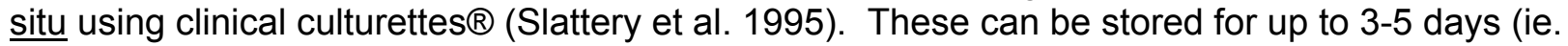
the approximate time of a collection trip) prior to isolation in the laboratory onto marine agar plates. Isolated strains are then cultured in batch marine broth (alternatively, I have utilized Mueller-Hinton and Sabaroud Dextrose successfully for certain microbe species) on a shaker table at approximate ambient conditions. Subsamples of the monocultures are stored frozen ($80^{\circ} \mathrm{C}$ ), lyophilized, and under oil for subsequent identification or re-isolation of bioactive species.

Marine microbes exhibit much variability in doubling times, however it is my experience that a batch culture (approximately $50 \mathrm{ml}$ broth in a $125 \mathrm{ml}$ flask) usually produces detectable quantities of bioactive compounds within 5-8 days. The broth will be extracted in a separatory funnel using 1:1 methylene chloride/methanol and the resulting lipophilic and hydrophilic fractions will be dried under reduced pressure (Okazaki \& Okami 1972). A bioassay guided scheme (see below) will guide subsequent fractionation via column- and high performance liquid chromatography (Still et al. 1978). As the sample becomes progressively purer, NMR can be used to assist in dereplication. Those samples that show the greatest activity and/or potential for development can be re-isolated from fermentation plants (ie., liter+ batch cultures) using the archived microbes and taxonomic identities ascertained with BioLog methodologies. In addition, compound selectivity can be enhanced through the production of novel microbial biotransformation analogs (Clark \& Hufford 1991).

Chemo-attractant/repulsive compounds from the batch microbe cultures will be assessed using standard microalgae and invertebrate larval assays (Rittschof et al. 1984; Rittschof et al. 1988; Slattery 1992; Slattery et al. 1995). It is my experience that Navicula sp., Balanus amphitrite, and Bugula neritina represent significant and common fouling organisms on the Gulf Coast; Crassostrea virginica represents the most common wild mollusc species along the Southeast Gulf Coast and a potential species for aquaculture. The experimental techniques have been previously described; briefly, cultures of either microalgae, barnacles, bryozoans, or oysters are maintained in the laboratory and subsampled at a stage prior to spawning and/or settlement. Diatoms or larvae are introduced to a container with either control nutrients (Guillard's f/2 for diatoms: Guillard 1983) \&/or seawater, or microbe isolated compounds resuspended at natural concentrations in nutrients \&/or seawater to assess chemically-mediated settlement cues. These data will have implications either to aquaculture (enhancement) or fouling prevention (inhibition). Moreover, the compounds will be tested in a standard battery of pharmaceutical assays (tumor, protozoal, microbial, opportunistic infection, malarial) established at the National Center for the Development of Natural Products; these assays will determine the practicality of drug development from marine microbial sources.

\section{Results and Discussion}

Between August and December of 2002, single strains of bacteria and fungi were isolated from the mixed cultures obtained from gas hydrate sites in the Gulf of Mexico. At least 237 strains of microorganisms, including a number of Streptomyces and fungi, were isolated from the 2002 gas hydrate cruise. The isolated microorganisms were stored on media slants at $4^{\circ} \mathrm{C}$. Now that these microorganisms have been isolated, work has been initiated on small scale broth cultures 
and solvent extraction of some of theses microbes. The extracts of these microorganisms will be submitted for biomedical evaluation. We have a number of cellular based biomedical assays at our disposal for the screening of these extracts; these include antibacterial, anticancer, antimalarial, and antifungal bioassays.

\begin{tabular}{|l|l|l|}
\hline & 2002 & 2003 \\
\hline Number of Isolates & 237 & 41 \\
\hline Number of Cryphiles & 58 & 13 \\
\hline $\begin{array}{l}\text { Number of Hydrocarbon } \\
\text { Metabolizers }\end{array}$ & 27 & 6 \\
\hline Number Biomedical Hits & 3 & 13 \\
\hline
\end{tabular}

On the August 2003 Gulf of Mexico cruise, our efforts were focused on marine sediment in the vicinity of gas hydrate mounds. We were particularly focusing on isolation of actinomycete species and organisms capable of degrading hydrocarbon mixtures found in crude oil. A variety of cultivation medias were employed for this purpose. Although some colonies have appeared, minimal work has begun on isolation of individual strains. These organisms are being grown near environmental temperatures, and many reproduce slowly under these conditions. Another experiment conducted on the most recent cruise was the collection of sediment for isolation of bacteriophage capable of infecting bacteria collected at similar sites. We are using bacteria collected from the 2002 cruise as indicator strains for bacteriophage infection. bacteriophage have been shown to have important roles in bacterial ecosystems, and are relatively unexplored from these environments. 


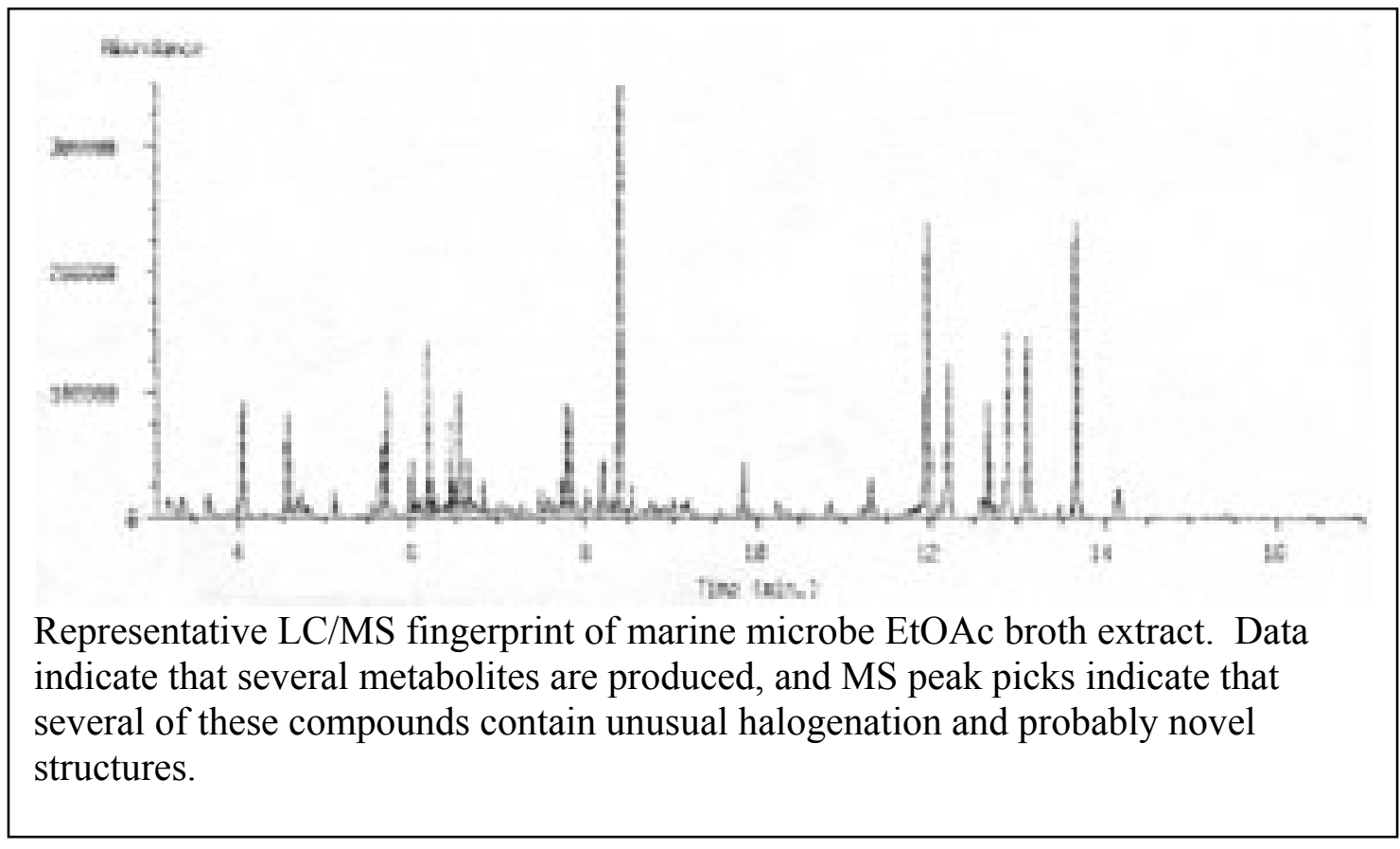

Concl usion

Our group has isolated several hundred extremophile microbe strains, and has developed culture methods to maintain these strains in the laboratory. Many of these strains appear to be cryophilic (i.e., they cannot adapt to laboratory bench temperatures and only survive at $4^{\circ} \mathrm{C}$ ), and several appear to have unique hydrocarbon requirements probably representative of their adaptation to the methane hydrate seep environment. While the degree of novel biomedical activity was modest, it did overlap with activity levels reported previously by our group from shallow coastal (Florida) and coral reef (Bahamas, Belize, Jamaica, Ponape, Guam, Saipan, Hawaii, and Egypt) ecosystems. Moreover, preliminary LC/MS chemical fingerprinting data indicate that the bioactivity may be due to unique natural products with unusual halogenation patterns. Ongoing research by our group will examine the biotechnological potential for enzymes responsible for hydrocarbon utilization, and the importance of bacteriophages to deep sea microbial communities. 


\section{Department of Energy via University of Mississippi Coperative Agreement DE-FC26-00NT40920 \\ Scripps Institution of Oceanography Microbiology and Geochemistry Final Report}

PI: $\quad$ Douglas H. Bartlett, Professor

Marine Biology Research Division

858-534-5233

dbartlett@ucsd.edu

Co-PI: Miriam Kastner, Professor

Geosciences Research Division

858-534-2065

mkastner@ucsd.edu

Scripps Institution of Oceanography

University of California, San Diego

La Jolla, CA 92093 


\begin{abstract}
:
Funds from this award were used for the partial support of two Staff Research Associates, supplies, flux meters and travel to the University of Mississippi, Oxford for planning meetings.

Microbiological analyses were performed on a sediment core obtained near the monitoring station. DNA was extracted from this material and used to isolate and characterize 16S rRNA bacterial gene sequences, from which inferences were obtained regarding the diversity of microorganisms present. The results indicated a remarkable similarity to Bacteria present in other anoxic hydrocarbon seep/hydrate environments examined in other parts of the world. The great majority of the Bacteria belonged to the epsilon- Proteobacteria cold seep group.

Geochemical analyses were performed on pore fluids from sediment push cores at Bush Hill $(\mathrm{BH})$ and on water column samples at and near the main Bush Hill plume. Pore fluid samples from the cores and CTD water samples were analyzed for the depth distributions of dissolved methane, $\mathrm{Cl}, \mathrm{Ca}, \mathrm{Mg}$, alkalinity, sulfide, and sulfate concentrations, and for dissolved inorganic carbon (DIC) and methane $\delta^{13} \mathrm{C}$ values. The spatial and depth distribution of pore fluid solute concentrations provide information on the subsurface hydrology, and on solute fluxes across the sediment-water interface. The pore fluid chemistry indicates regional sulfate reduction and anaerobic methane oxidation (AMO), particularly intense in and near seeps, the intensity is manifested by the spatial distribution of benthis chemosynthetic communities, and in situ methane hydrate formation and authigenic carbonate formation near the sediment-water interface at and in the vicinity of the main $\mathrm{BH}$ plume. In the water column, a significant enrichment in ${ }^{13} \mathrm{C}-\mathrm{CH}_{4}$ along isopycnals away from the plume, from the methane source, is observed, indicative of extensive aerobic methanotrophy in the water column. The $\delta^{3} \mathrm{C}-\mathrm{CH}_{4}$ value range from -41 to $-49 \%$ at Bush Hill.
\end{abstract}




\section{List of Graphical Materials:}

Table 1: Similarity of selected Bush Hill 16S RNA sequences with those present at the National Center for Biotechnology Information.

Figure 1: Pore fluid $\delta^{13} \mathrm{C}-\mathrm{DIC}(\% \circ)$ versus Alkalinity $(\mathrm{mM})$ at Bush Hill and GC234.

Figure 2: Water column methane concentrations at and near the methane hydrate mound plume, at Bush Hill. 


\section{Introduction:}

Microoorganisms are likely to play important roles in the formation, stability and decomposition of gas hydrates and hydrocarbons present in sediment pore fluids. A number of methodologies have been used to characterize the microbial diversity associated with cold seep or gas hydrate environments. These include 16 s rRNA gene sequencing $(3,4,6,9,13,19,21)$, lipid stable isotope analyses $(9,23)$, fluorescent in situ hybridization $(4,20,23-25)$, secondary ion mass spectrometry $(24,25)$, and more recently proteomics (12). An excellent recent review of this topic can be found in a book chapter by Widdel et. al (30).

The results of these studies suggests that anaerobic methane oxidation (AMO) in these settings is frequently accomplished through the activities of methanogen-like archaea belonging to the ANME-1 or ANME-2 groups, in syntrophic association with sulfate-reducing bacteria, typically members of the Desulfosarcinales order. These associations may not always be necessary for AMO as in many cases ANME-1 and ANME-2 archaea are present in monospecific cell aggregates (25). Studies of this biochemical process have been greatly hampered by the lack of cultures of microbes capable of AMO, but laboratory incubation experiments have been performed which support a linkage between AMO and sulfate reduction $(8,22)$.

At this time relatively few studies have been performed on the microbial diversity associated with the hydrates and hydrocarbon seeps present in the Gulf of Mexico. The Gulf of Mexico hydrate and seep environments are distinct from other regions studied to date by the presence of both thermogenic and biogenic methane, as well as a variety of aliphatic and aromatic hydrocarbons (see for example, (28). Lanoil et. al (13) have reported on bacterial and archaeal diversity associated with thermogenic and biogenic gas hydrates taken from the Gulf of Mexico. Their results indicated moderately high bacterial diversity, but low archaeal diversity, with most of the Archaea being closely related to members of the Methanosarcinales. More recently Mills et. al (21) have published on the microbial diversity present on hydrates and associated sediments obtained from two gas hydrate sedimentary systems in the Gulf of Mexico. As with the Lanoil study they also found low archaeal diversity. However, the bacterial diversity in their samples was lower than that of Lanoil. $62 \%$ of their clones were most closely related to the epsilon-Proteobacteria ( $\varepsilon$-Proteobacteria), and delta-Proteobacteira ( $\delta$-Proteobacteria) were also highly abundant.

In this project we have used culture-independent rRNA sequence analyses to assess the bacterial diversity in a sediment sample in the vicinity of the monitoring station. Our results indicate a large fraction of $\varepsilon$-Proteobacteria belonging to a group of clones that has come to be known as the cold seep group (10). We suggest that there is a need for further investigations of the activities of these microbes in anoxic hydrocarbon-bearing sediments.

Seafloor or shallow-buried gas-hydrates, within 10-30 mbsf, have been recovered at Hydrate Ridge and offshore Vancouver, Cascadia, (e.g. Kastner et al., 1998; Suess et al., 1999; Spence et al., 2000; ODP Leg 204 reports). Near seafloor hydrates have also been recovered in the Eel River Basin, northern California (brooks et al. 1991), Okhotsk Sea and Black and Caspian Seas (Kvenvolden et al., 1993). The seafloor occurrence is especially prominent and the Gulf of Mexico, at Bush Hill (e.g. Macdonald et al., 1994). The occurrence of methane hydrate near or at the seafloor indicates active upward advection of methane-rich fluids or of methane gas. The seafloor and shallow buried gas hydrates are the most vulnerable hydrates to global warming. The environmental impact of release of large quantities of methane into the ocean and atmosphere could have important societal and microbiological consequences. 
The Bush Hill hydrate mound site has been studied extensively for some time (e.g. MacDonald et al., 1994; Sassen et al., 1998; Aharon and Fu, 2000; and references therein). However, in situ temperature monitoring, continuous flux measurements, and the impact of the methane advection into the water column have not been documented as yet.

In this project we focused on documenting the present system that maintains the $\mathrm{BH}$ hydrate mound. The main questions we tried to address were: what is the subsurface hydrology, what are the methane and solute fluxes at the hydrate mound, in the vicinity, and at a "background" site; how much of the methane escapes across the seafloor into bottom water; how much of the methane gets oxidized in the water column, thus how much escapes into the atmosphere.

Accordingly, we recovered push cores as close to the mound as possible and in the vicinity and regionally, we analyzed the pore fluids for methane, major and minor component concentrations, and for methane and DIC C-isotope ratios; we analyzed the water column samples for methane concentrations and $\delta^{13} \mathrm{C}$ values, and deployed flux meters, MOSQUITOs (Multiple Orifice sampler and Quantitative Injection Tracer Observer), at critical sites for longterm continuous monitoring seafloor temperature and sampling fluids and gases. The processing of the flux data are still in progress. 


\section{Experimental:}

\section{Sediment Collection:}

Sediments were recovered with push cores near and in the vicinity of the Bush Hill hydrate mound, as well as regionally, to document "background" properties. At each sediment sampling site a separate push core was recovered for pore fluid chemistry, and a third core for dissolved methane concentration analysis. For microbiology, sediment was obtained from push core \#8, obtained during Johnson Sea Link submersible Dive \# 4556 (2003), from a water depth of 1772 feet located at N 27deg 46.953', W 91deg 30.4724'. It was in the footprint of our MOSQUITO \#1 Osmotic Sampler which was deployed in a mussel field just west of the main Bush Hill hydrate mound. The sediment used for molecular biology was from $5-10 \mathrm{~cm}$ below the surface. Authigenic carbonates were as well recovered, but as yet not analyzed.

\section{DNA isolation, PCR amplification, and cloning:}

Total nucleic acids were extracted from $1 \mathrm{~g}$ of sediment using the Ultraclean Soil DNA Isolation Kit (MO BIO Laboratories, Inc.). Modifications to the standard protocol included bead-beating the sediment for 25 seconds at $4.5 \mathrm{~m} / \mathrm{s}$ (Mo Bio Laboratories), following the extraction with a chloroform: isoamyl alcohol (24:1) clean-up, and precipitating the DNA with 3M sodium oxaloacetate and 2 volumes of $95 \%$ ethanol (personal communication, Brian Lanoil). Bacterial 16s rDNA was amplified using primers Eub27F and Eub1492R (7). The PCR conditions used were 40 seconds of denaturation at $94^{\circ} \mathrm{C}, 40$ seconds of annealing at $48^{\circ} \mathrm{C}$, and 2 minutes of elongation at $72{ }^{\circ} \mathrm{C}$ for 25 cycles in an Applied Biosystems GeneAmp PCR System 2700 thermal cycler. Amplification products were cloned into vector pCR2.1 according to the TOPO TA cloning kit instructions (Invitrogen, Carlsbad, Calif).

\section{RFLP analysis and sequencing clone libraries:}

The 16s rDNA inserts were amplified using M13 primers. The polymerase chain reaction conditions used were 40 seconds of denaturation at $94^{\circ} \mathrm{C}, 40$ seconds annealing at $52{ }^{\circ} \mathrm{C}$, and 2 minutes of elongation at $72^{\circ} \mathrm{C}$ for 30 cycles. The product was digested with Hae III restriction endonuclease (New England Biolabs, Beverly, Mass.) at $37^{\circ} \mathrm{C}$ for 2 hours. Banding patterns were grouped according to similarity, and representative members of each pattern group were fully, bidirectionally sequenced using M13 primers by San Diego State University Core Facility.

\section{Pore fluid and water column Analyses:}

Pore fluids were recovered by centrifuging subsections of the push cores at in situ temperature and under anaerobic conditions. The samples were immediately analyzed for alkalinity and sulfide and sulfate concentrations, and different aliquots were individually pretreated, depending on the component, and appropriately stored for shore-based analyses. For most solutes the pore fluids were analyzed by ICP-OES or ICP-MS. Chloride concentrations were determined by titration with $\mathrm{AgNO}_{3}$, and Sulfate concentrations were also determined by IC. The pore fluid samples for methane and other hydrocarbon analyses, as well as and the water column samples were stored in serum bottles poisoned with mercuric chloride. Concentrations of hydrocarbons were measured by gas chromatography and the $\delta^{13} \mathrm{C}-\mathrm{CH}_{4}$-and-DIC were determined by mass spectrometry. The flux meter data in progress are being analyzed for dye concentrations; the degree of the dye dilution is determined by the fluid flow rate. 


\section{Results/Discussion:}

The bacterial diversity present in the Bush Hill sediment sample is presented in Table 1. Restriction fragment length polymorphism analysis indicated 6 dominant clone types. However, sequence analysis of representatives of these clones revealed that $4 / 6$ were derived from closely related members of the $\varepsilon$-Proteobacteria cold seep group (10). The remaining clones reflected affiliation with the genus Holophaga and the order Desulfosarcinales. All of the sequences were obtained from Bacteria closely related to microbes obtained in other cold-seep marine sediments.

The predominance of the $\varepsilon$-Proteobacteria in our sample is quite striking. Related Bacteria are probably best appreciated as members of microbial mats or ectosymbionts of invertebrates in hydrothermal vent environments. For example, similar microbes have been detected in the mucous secretions of the hydrothermal vent polychaete Paralvinella palmiformis (1). Culture-dependent and independent methods have resulted in the identification of similar $\varepsilon$ Proteobacteria from white tubes of the polychaete Alvinella pompejana collected on East Pacific Rise at $13^{\circ} \mathrm{N}$ (2) Cambon-Bonavita et. al; unpublished results). They have also been found in a microbial mat from an active hydrothermal vent system in Hawaii (Moyer, Dobbs and Karl; unpublished results).

Table 1: Similarity of selected Bush Hill 16S RNA sequences with those present at the National Center for Biotechnology Information.

\begin{tabular}{|c|c|c|c|}
\hline Sequence Type & Closest match or clone name & $\%$ identity & $\begin{array}{l}\text { Phylogenetic } \\
\text { affiliation }\end{array}$ \\
\hline $\mathrm{BH} 8$ & $\begin{array}{l}\text { Uncultured hydrocarbon seep clone } \\
\text { (AF154101) }\end{array}$ & $97 \%$ & $\varepsilon$-Proteobacteria \\
\hline $\mathrm{BH} 10$ & $\begin{array}{l}\text { Nankai Trough cold seep clone } \\
\text { (AB013263.1) }\end{array}$ & $96 \%$ & $\varepsilon$-Proteobacteria \\
\hline $\mathrm{BH} 13$ & $\begin{array}{l}\text { Nankai Trough cold seep clone } \\
\text { (AB013263.1) }\end{array}$ & $96 \%$ & $\varepsilon$-Proteobacteria \\
\hline $\mathrm{BH} 23$ & Cariaco Basin anoxic zone (AF224803.1) & $96 \%$ & $\varepsilon$-Proteobacteria \\
\hline $\mathrm{BH} 15$ & $\begin{array}{l}\text { Nankai Trough cold seep clone } \\
\text { (AB013269.1) }\end{array}$ & $97 \%$ & Holophaga \\
\hline $\mathrm{BH} 25$ & $\begin{array}{l}\text { Santa Barbara Basin cold seep clone } \\
\text { (AF354158) }\end{array}$ & $94 \%$ & $\begin{array}{l}\text {-Proteobacteria } \\
\text { (Desulfosarcinales) }\end{array}$ \\
\hline
\end{tabular}

Many cultured vent -Proteobacteria, such as Sulfurimonas autotrophica, grow chemolithoautotrophically with elemental sulfur, sulfide and thiosulfate as sole electron donors and oxygen as electron acceptor, while others are able to grow oxidizing hydrogen and using sulfur, thiosulfate or nitrate as an electron acceptor (2, 11, Takai et al.; unpublished results). The latter physiological mode could be operating within anoxic cold-seep sediments, such as those from which our Bush Hill sample was obtained.

The $\tilde{\varepsilon}$ Proteobacteria cold-seep group has been observed in many clone libraries obtained from seep or probable seep environments. These include the anoxic zone of the Cariaco Basin where Euryarchaeota sequences implicated in anaerobic methane oxidation (AMO) have also been noted (18). The Cariaco Basin is known to be rich in methane (29). Other sites include the Nankai Trough at a depth of $3.8 \mathrm{~km}$ where AMO communities have been found and a model for AMO presented $(14,15)$, various depths and locations within the Japan Trench $(10,15)$, and methane-rich borehole fluid obtained from Ocean Drilling Project borehole 892b off the coast of 
Oregon (3). Many of our clones also display high similarity to sequences derived from a hydrocarbon seep environment not yet described (O'Neill et. al; unpublished results, see accession number AF154101 for example). In the case of one Japan Trench cold seep study it was discovered that shallow sediments of $2-4 \mathrm{~cm}$ contained mostly -Proteobacteria whereas deeper sediments of $8-10$ and $14-16 \mathrm{~cm}$ contained increasing proportions of - Proteobacteria (10).

The $\delta$-Proteobacteria clones are all closely related to the putative AMO syntrophic group, such as those previously described by the DeLong research group in Eel River Basin seep sediments and in the Santa Barbara Basin (23). They are also similar to clones obtained from anoxic sediments from marine salinity meromictic lakes and a coastal meromictic marine basin, Vestfold Hilds, in eastern Antarctica (5). These microbes have been hypothesized to function as sulfate-reducing partners with anaerobic methane-oxidizing Archaea (see for example (30).

The last additional bacterial group observed in our sample belonged to the little-known Holophaga/AcidobacteriumGeothrix phylum and the genus Holophaga. This genus has been detected in diverse $16 \mathrm{~S}$ rRNA gene libraries retrieved from diverse soil, sediment and aquatic environments (17). Little is know about the physiological properties of Holophaga except that its one cultured member, $H$. foetida, is an obligate anaerobe, isolated from a black anoxic freshwater mud sample, and it degrades aromatic compounds to acetate (16). Holophaga clones have previously been detected in cold-seep sediments of the Nankai Trough (14), sediment samples collected at Hornsund off the coast of Spitsbergen, in the Arctic Ocean (26), Eastern Antarctica anoxic sediments (5), and in anaerobic marine sediments enriched with organic carbon (27). Curiously, Holophaga is related to Geopsychrobacter multivorans which is being evaluated for electricity production by as a marine sediment fuel cell (Holmes et. al; unpublished results, see accession number AY579996.1).

The pore fluid data indicate that although sulfate reduction and anaerobic methane oxidation is regionally pervasive, methane (plus other hydrocarbons) upward advection is mostly focused at $\mathrm{BH}$; the intensity and frequency of focused sites increases at and adjacent to the hydrate mound. This is manifested in the very high alkalinity concentrations, $>40 \mathrm{mM}$ at and near the mound, and the low $\delta^{13} \mathrm{C}$-DIC values, shown in Figure 1, a cross plot of $\delta^{13} \mathrm{C}$-DIC (\%o ) versus alkalinity $(\mathrm{mM})$. Most interesting is the observation that unlike at north and south Hydrate Ridge, for example, where $\delta{ }^{13} \mathrm{C}$-DIC values range from -25 to $-49 \%$, the most negative $\delta{ }^{13} \mathrm{C}$-DIC value at $\mathrm{BH}$ (Fig. 1) is -22\%o. This indicates that at BH crude oil serves as the primary electron-donor and metabolic substrate for anaerobic sulfate reduction. Calcium, Mg, and Sr concentration profiles indicate in situ carbonate precipitation, some of the sulfide precipitates as Fe sulfides, and $\mathrm{Cl}$ concentrations indicate in situ methane hydrate formation at and adjacent to the main $\mathrm{BH}$ mound and methane seep. The high chlorinities in the pore fluids are localized in specific horizons of active hydrate formation, are not pervasive throughout the concentration-depth profiles, thus, do not suggest that brines are controlling the stability of the $\mathrm{BH}$ hydrate mound.

Water column methane concentration (Fig. 2) and isotope data show that at the main plume methane concentrations are high, and even in the uppermost 5 meters of the water column seawater is considerably supersaturated (150-200 times) with respect to methane, hence, methane escapes into the atmosphere. The methane flux at this and other plume sites in the Gulf of Mexico is as yet unknown. Away from the plume methane concentrations decrease rapidly (Fig. 2) and the $\delta{ }^{13} \mathrm{C}-\mathrm{CH}_{4}$ values indicate aerobic methanotrophy is widespread, following the reaction: 
$\mathrm{CH}_{4}+2 \mathrm{O}_{2} \rightarrow \mathrm{CO}_{2}+\mathrm{H}_{2} \mathrm{O}$

In addition to depleting dissolved oxygen concentrations, this reaction effects the $\delta{ }^{13} \mathrm{C}$-DIC values of the bottom waters at and near the $\mathrm{BH}$ mound and main plume, where methane concentrations are high, therefore also the $\mathrm{C}$ isotope values of benthic carbonates.

\section{Conclusions:}

All of the microbial phylotypes discovered through this study are related to clones present in other hydrocarbon seep environments. Their presence suggests that active hydrocarbon (in particular methane) consumption is taking place at the study site. Considering the prevalence of

-Proteobacteria cold seep group clones, AMO syntrophic SRB group -Proteobacteria and Holophaga species in many seep environments, including that of Bush Hill, future studies should be directed at their culturing and physiological characterization. Genomic and functional genomic studies of the bacterial components of seep environments is also likely to reveal new insights into microbial influences on hydrocarbon gases and hydrates.

The intense anaerobic sulfate reduction and AMO in the subsurface strongly influence the pore fluid chemical environment, thus the microbiology. Methane and other hydrocarbon fluxes at $\mathrm{BH}$ are highly focused. At and near seeps pore fluid chemistry indicates that in addition to the coupled reactions of anaerobic sulfate reduction and AMO, methane hydrate, authigenic carbonates, and Fe-sulfides precipitate in situ. The pore fluids DIC ${ }^{13} \mathrm{C}$ values indicate that at $\mathrm{BH}$ crude oil serves as the primary electron-donor and metabolic substrate for anaerobic sulfate reduction. Much methane escapes across the sediment-water interface; analysis of the fluxes of methane and other solutes across the seafloor is in progress. In the water column aerobic methanotrophy is suggested by the dissolved methane $C$ isotope values. Where methane fluxes are high this reaction utilizes dissolved oxygen, thus its concentration, and also the ${ }^{13} \mathrm{C}$-DIC values. At and adjacent the main $\mathrm{BH}$ methane plume, methane escapes into the atmosphere. 


\section{List of References:}

1. Aharon, P., and F. Baoshun. 2000. Microbial sulfate reduction rates and sulfur and oxygen isotope fractionations at oil and gas seeps in deepwater Gulf of Mexico. Geochim. Cosmochim. Acta 64:233-246.

2. Alain, K., M. Olagnon, D. Desbruyeres, A. Page, G. Barbier, S. K. Juniper, J. Quellerou, and M. A. Cambon-Bonavita. 2002. Phylogenetic characterization of the bacterial assemblage associated with mucous secretions of the hydrothermal vent polychaete Paralvinella palmiformis. FEMS Micro. Ecol. 42:463-476.

3. Alain, K., M. Zbinden, N. Le Bris, F. Lesongeur, J. Querellou, F. Gaill, and M. A. Cambon-Bonavita. 2004. Early steps in microbial colonization processes at deep-sea hydrothermal vents. Environ. Microbiol. 6:227-241.

4. Bidle, K. A., M. Kastner, and D. H. Bartlett. 1999. A phylogenetic analysis of microbial commuities associated with methane hydrate containing marine fluids and sediments in the Cascadia Margin (ODP site 892b). FEMS Microbiol. Lett. 177:101-108.

5. Boetius, A., K. Ravenschlag, C. J. Schubert, D. Rickert, F. Widdel, A. Gieseke, R. I. Amann, B. B. Jørgensen, U. Witte, and O. Pfannkuche. 2000. A marine microbial consortium apparently mediating anaerbic oxidation of methane. Nature 407:623-26.

6. Bowman, J. P., S. M. Rea, S. A. McCammon, and T. A. McMeekin. 2000. Diversity and community structure within anoxic sediment from marine salinity meromictic lakes and a coastal meromictic marine basin, Vestfold Hilds, Eastern Antarctica. Env. Microbiol. 2:227-237.

7. Brooks, J.M., M.E. Field, and M.C. Kennicut. 1991. Observations of gas hydrates in marine sediments, offshore northern California. Mar. Geol. 96, 103-109.

8. Carson, B., M. Kastner, D. Bartlett, J. Jaeger, H. Jannasch, and Y. Weinstein. 2003. Implications of carbon flux from the Cascadia accretionary prism: results from long-term, in situ measurements at ODP Site 892B. Mar. Geol. 198:159-180.

9. DeLong, E. F. 1992. Archaea in coastal marine environments. Proc. Natl. Acad. Sci. U. S. A. 89:5685-5689.

10. Hansen, L. B., K. Finster, H. Fossing, and N. Iversen. 1998. Anerobic methane oxidation in sulfate depleted sediments: effects of sulfate and molybdate additions. Aquat. Microbiol. Ecol. 14:195-204.

11. Hinrichs, K.-U., J. M. Hayes, S. P. Sylva, P. G. Brewer, and E. F. DeLong. 1999. Methane-consuming archaebacteria in marine sediments. Nature 398:802-805.

12. Inagaki, F., Y. Sakihama, A. Inoue, C. Kato, and K. Horikoshi. 2002. Molecular phylogenetic analyses of reverse-transcribed bacterial rRNA obtained from deep-sea cold seep sediments. Environ. Microbiol. 4:277-286.

13. Inagaki, F., K. Takai, K. I. Hideki, K. H. Nealson, and K. Horikishi. 2003. Sulfurimonas autotrophica gen. nov., sp nov., a novel sulfur-oxidizing epsilonproteobacterium isolated from hydrothermal sediments in the Mid-Okinawa Trough. Int. J. Syst. Evol. Microbiol. 53:1801-1805.

14. Kastner, M., K.A. Kvenvolden, and T. Lorenson. Chemistry, isotopic composition, and origin of methane hydrogen sulfide hydrate at the Cascadia subduction zone. Earth Planet. Sci. Lett., 156:173-183.

15. Kruger, M., A. Meyerdierks, F. O. Glockner, R. Amann, F. Widdel, M. Kube, R. Reinhardt, R. Kahnt, R. Bocher, R. K. Thauer, and S. A. Shima. 2003. conspicuous nickel protein in microbial mats that oxidize methane anaerobically. Nature 426:878-881.

16. Kvenvolden, K.A., G.D. Ginsburg, and V.A. Soloviev. 1993. Worldwide distribution of subaquatic hydrates. Geo-Mar. lett., 13:32-40. 
17. Lanoil, B. D., R. Sassen, M. T. La Duc, S. T. Sweet, and K. H. Nealson. 2001. Bacteria and Archaea physically associated with Gulf of Mexico gas hydrates. Appl. Env. Microbiol. 67:5143-5153.

18. Li, L., J. Guezennec, P. Nichols, P. Henry, M. Yanagibayashi, and C. Kato. 1999. Microbial diversity in Nankai Trough sediments at a depth of 3,843 m. J. Oceanography 55:635-642.

19. Li, L., and C. Kato. 1999. Microbial diversity in the sediments collected from cold-seep areas and from different depths of the Deep-sea., Extremophiles in deep-sea environments., vol. pp.55-89. Springer-Verlag, Tokyo.

20. Liesack, W., F. Bak, J. U. Kreft, and E. Stackebrandt. 1994. Holophaga foetida gen. nov., sp. nov., a new, homoacetogenic bacterium degrading methoxylated aromatic compounds. Arch. Microbiol. 162:85-90.

21. Ludwig, W., S. H. Bauer, M. Bauer, I. Held, G. Kirchhof, R. Schulze, I. Huber, S. Spring, A. Hartmann, and K. H. Schleifer. 1997. Detection and in situ identification of representatives of a widely distributed new bacterial phylum. FEMS Microbiol. Lett., 153:181-190.

22. MacDonald, I.R., N.L. Guinasso Jr., R. Sassen, J.M. Brooks, L.Lee, and K.T. Scott. 1994. Gas hydrate that breaches the sea floor on the continental slope of the Gulf of Mexico. Geology 22:699-702.

23. Madrid, V. M., G. T. Taylor, M. I. Scranton, and A. Y. Chistoserdov. 2001. Phylogenetic diversity of bacterial and archaeal communities in the anoxic zone of the Cariaco Basin. Appl. Env. Microbiol. 67:1663-1674.

24. Marchesi, J. R., A. J. Weightman, B. A. Cragg, R. J. Parkes, and J. C. Fry. 2001. Methanogen and bacterial diversity and distribution in deep gas hydrate sediments from the Cascadia Margin as revealed by $16 \mathrm{~S}$ rRNA moleculae analysis. FEMS Microbiol. Ecol. 34:221-228.

25. Michaelis, W., R. Seifert, K. Nauhaus, T. Treude, V. Thiel, M. Blumenberg, K. Knittel, A. Gieseke, K. Peterknecht, T. Pape, A. Boetius, R. Amann, B. B. Jorgensen, F. Widdel, J. R. Peckmann, N. V. Pimenov, and M. B. Gulin. 2002. Microbial reefs in the Black Sea fueled by anaerobic oxidation of methane. Science 297:1013-1015.

26. Mills, H. J., C. Hodges, K. Wilson, I. R. MacDonald, and P. A. Sobecky. 2003. Microbial diversity in sediments associated with surface-breaching gas hydrate mounds in the Gulf of Mexico. FEMS Micro. Ecol. 46:39-52.

27. Nauhaus, K., A. Boetius, M. Kruger, and F. Widdel. 2002. In vitro demonstration of anaerobic oxidation of methane coupled to sulphate reduction in sediment from a marine gas hydrate area. Env. Microbiol. 4:296-305.

28. Orphan, V. J., K. U. Hinrichs, W. I. Ussler, C. K. Paull, L. T. Taylor, S. P. Sylva, J. M. Hayes, and E. F. DeLong. 2001. Comparative analysis of methane-oxidizing archaea and sulfate-reducing bacteria in anoxic marine sediments. Appl. Environ. Microbiol. 67:1922-1934.

29. Orphan, V. J., C. H. House, K. U. Hinrichs, K. McKeegan, and E. F. DeLong. 2001. Methane-consuming archaea revealed by directly coupled isotopic and phylogenetic analysis. Science 293:484-487.

30. Orphan, V. J., C. H. House, K. U. Hinrichs, K. D. McKeegan, and E. F. DeLong. 2002. Multiple archaeal groups mediate methane oxidation in anoxic cold seep sediments. Proc. Natl. Acad. Sci. U. S. A. 99:7663-7668.

31. Ravenschlag, K., K. Sahm, J. Pernthaler, and R. Amann. 1999. High bacterial diversity in permanently cold marine sediments. Appl. Environ. Microbiol. 65:3982-3989. 
32. Rossello-Mora, R., B. Thamdrup, H. Schafer, R. Weller, and R. Amann. 1999. The response of the microbial community of marine sediments to organic carbon input under anaerobic conditions. Syst. Appl. Microbiol. 22:237-248.

33. Sassen, R., S. Joye, S. T. Sweet, D. A. DeFreitas, A. V. Milkov, and I. R. MacDonald. 1999. Thermogenic gas hydrates and hydrocarbon gases in complex chemosynthetic communities, Gulf of Mexico continental slope. Organic Geochem. 30:485-497.

34. Sassen, R., I.R. MacDonald, N.L. Guinasso, S. Joye, A.G., Requejo, S.T. Sweet, J. Alcala-Herrera, D.A. DeFreitas, D.R. Schink. 1998. Bacterial methane oxidation in sea-floor gas hydrate: significance to life in extreme environments. Geology. 26:851-854.

35. Scranton, M. I. 1988. Temporal variations in the methane content of the Cariaco Trench. Deep Sea Res. 35:1511-1523.

36. Suess, E., M.E. Torres, G. Bohrmann, R.W. Collier, J. Greinert, P. Linke, G. Rehder, A. Trehu, K. Wallmann, G. Winckler, and E. Zuleger. 1999. Gas hydrate destabilization: enhanced dewatering, benthic material turnover and large methane plumes at the Cascadia convergent margin. Earth Planet Sci. Lett., 170:1-15.

37. Widdel, F., A. Boetius, and R. Rabus. 2004. Anaerobic biodegradation of Hydrocarbons including methane. In M. E. A. Dworken (ed.), The Prokaryotes: An Evolving Electronic Resource for the Microbiological Community, 3rd edition, release 3.16, http://link.springer-ny.com/link/service/books/10125/. Springer-Verlag, New York. 
Figure 1.

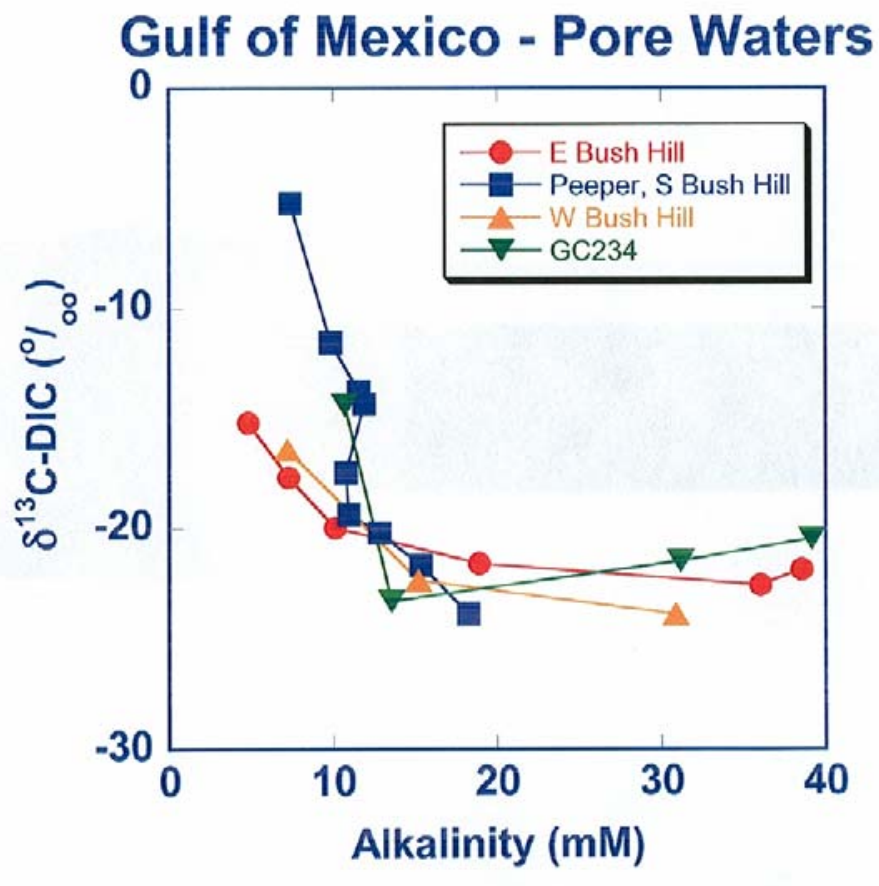


Figure 2.
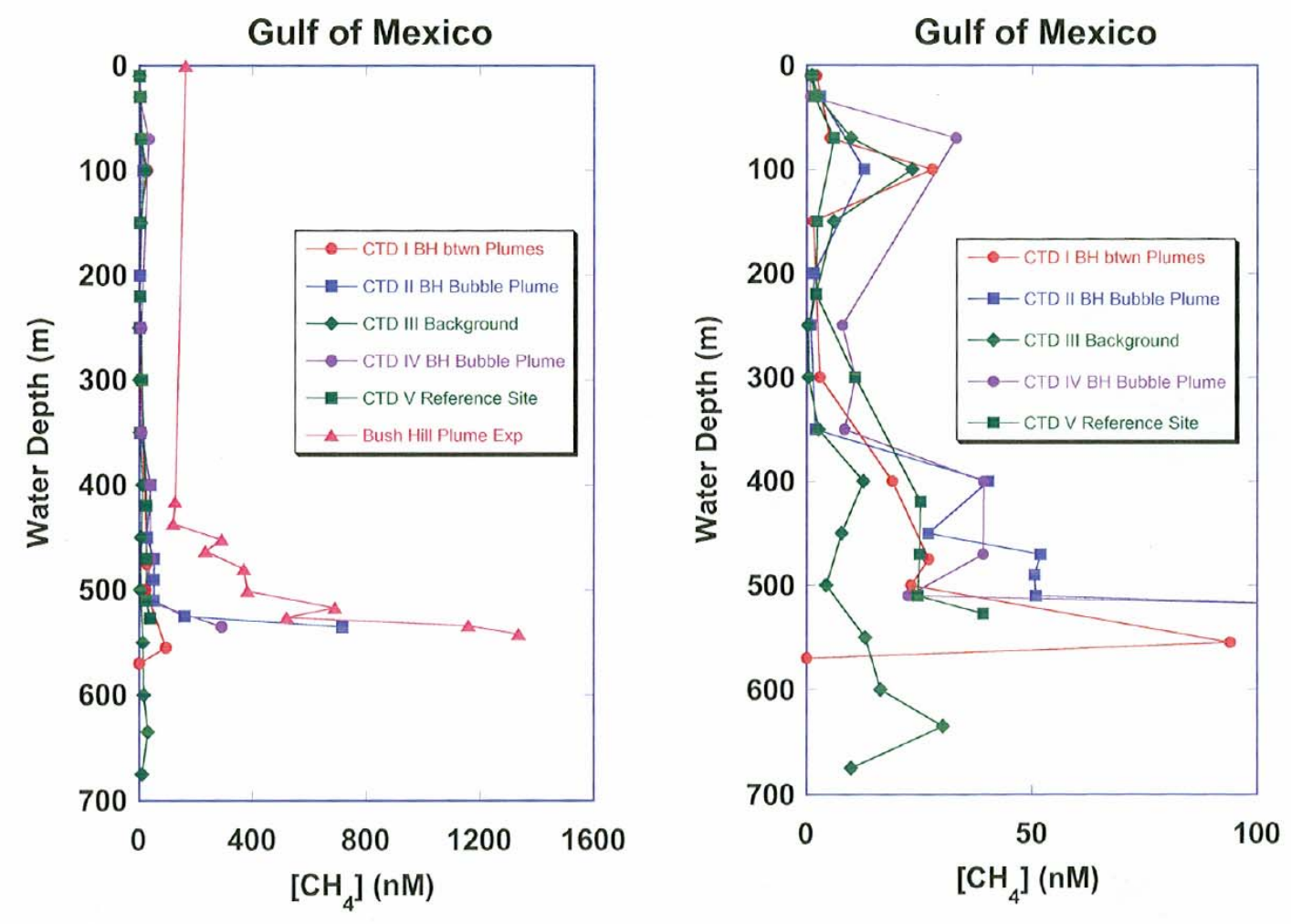


\section{List of Acronyms and Abbreviations:}

AMO: $\quad$ Anaerobic methane oxidation

$\delta: \quad$ delta

$\varepsilon: \quad$ epsilon

rRNA: ribosomal RNA

SRB: $\quad$ sulfate-reducing bacteria

DIC: $\quad$ Dissolved inorganic carbon

MOSQUITO: Multiple Orifice Sampler and Quantitative Injection Tracer Observer

GOM:

$\mathrm{BH}$ :

Gulf of Mexico

Bush Hill 


\title{
Establishing the Parameters Required for Spatial Models of Gas Hydrate Collections
}

\author{
Report of Findings: December 2003
}

DOE Cooperative Agreement DE-FC26-00NT40920

Joel S. Kuszmaul and Emily Strack Department of Geology and Geological Engineering The University of Mississippi

University, MS 38677 


\section{ABSTRACT}

Global observations of gas hydrates provide a basis for characterizing and modeling the spatial continuity of gas hydrate environments. Indicator variography and logistic regression were applied to gas hydrate samples reported in published data sets. Each data set included sites where gas hydrates were observed or where drilling occurred without gas hydrate contact. The spatial distribution of the deposits within two study areas were characterized by preparing multiple experimental indicator variograms with values assigned as one for hydrate presence and zero for hydrate absence. The spatial distribution of gas hydrate deposits on a global scale was modeled using logistic regression. A best-fit model was determined based on the significance of multiple model variables.

An isotropic variogram with an active lag of $180 \mathrm{~km}$ and a uniform interval of $5 \mathrm{~km}$ produced the clearest structure for the Gulf of Mexico study area. The isotropic variogram was fit with an spherical model with a correlation length of $65 \mathrm{~km}$, a sill value of 0.0396 , and a relative nugget of $58.1 \%$. The maximum direction of spatial continuity trends approximately $43^{\circ}$ with the minimum axis at approximately $133^{\circ}$. Sampled sites are correlated at a distance of $35 \mathrm{~km}$ in the direction of maximum continuity and $25 \mathrm{~km}$ in the direction of minimum continuity. The sill value for an anisotropic spherical model was 0.0396 with a relative nugget effect of $63.1 \%$.

An isotropic variogram with an active lag of $180 \mathrm{~km}$ and a uniform interval of $5 \mathrm{~km}$ produced the clearest structure for the Nigerian continental margin study area. The isotropic variogram was fit with a spherical model with a range of $110 \mathrm{~km}$, a sill value of 0.035 , and a relative nugget effect of $3.0 \%$. No significant spatial anisotropy was evident in the Nigerian continental margin for the data set as a whole.

The nugget effect observed in the variogram models of both study areas is likely attributable to our definition of a find and may also be a consequence of the sampling procedures used to establish each data set. The maximum direction of spatial continuity derived from the Gulf of Mexico data set corresponds with the alignment of gas hydrate samples. The alignment suggests an underlying structural control on hydrate formation. Additional analysis, and perhaps additional sampling, is required for investigations of anisotropy in the Gulf of Guinea case. Indicator variograms suggest a spatial relationship does exist between gas hydrate locations as a function of both distance and direction. The stability of the spatial structure suggests a sufficient stationarity of hydrate forming processes that can be used for spatial prediction with stochastic models.

A best-fit logistic regression model for determining the conditional probability of gas hydrate occurrence includes two independent variables, water depth and temperature. The model accurately classifies the presence of gas hydrate at approximately $80 \%$ of the known gas hydrate locations. An improved dataset including all recently discovered gas hydrates as well as more observations where gas hydrates have not been found would serve to further verify the value of a logistic regression model. 


\section{List of Figures}

Figure 1: Isotropic experimental variogram and best-fit model for the Gulf of Mexico

Figure 2: Anisotropic experimental variogram and best-fit model for the Gulf of Mexico

Figure 3: Isotropic experimental variogram and best-fit model for the Gulf of Guinea

Figure 4: Isotropic conditional probability map of gas hydrate occurrences: Gulf of Mexico

Figure 5: Residual plots for logistic models

Figure 6: Predictive ability of the selected logistic regression model

List of Tables

Table 1: Isotropic model results for the Gulf of Mexico and the Gulf of Guinea

Table 2: Anisotropic model results for the Gulf of Mexico

Table 3: Logistic regression model statistics

Table 4: Classification table based on the logistic regression model 


\section{INTRODUCTION}

Environments conducive to natural gas hydrate formation occur worldwide in both polar and marine environments. Globally, gas hydrates have been discovered in nineteen regions, including the continental slopes of the Gulf of Mexico and offshore Nigeria (Kvenvolden, 1993). Research into the occurrence, distribution, and detection of gas hydrate deposits has escalated in the past decade because (1) gas hydrates represent a potential energy source, (2) gas hydrate formation and destabilization alters the geotechnical properties of surrounding sediments potentially causing mass movements and offshore drilling difficulty, and (3) the dissociation of gas hydrate may be a significant factor in global climate change.

The objectives of our study focused on (1) summarizing the controls on hydrate formation in marine sediments, (2) describing how the spatial continuity of gas hydrate deposits change as a function of distance and direction, and (3) determine the best-fit model to describe the relation between gas hydrate occurrences and properties of the surrounding environment. The purpose of this modeling was to characterize the spatial continuity of gas hydrate depositional environments. This characterization should prove valuable in the study of the distribution of gas hydrate deposits and may be valuable in assisting in improving the ability to predict the location of future finds of these deposits.

The analysis begins with isotropic variograms to define the distance parameters that produce the clearest structure. Anisotropic variograms are investigated to determine the directional dependence or pattern in the spatial continuity of the area. Models based on the sample variograms were used in indicator kriging to estimate the conditional probability of finding gas hydrate locations within the study areas.

A logistic regression analysis was used to determine a best-fit model that describes gas hydrate occurrences based on environmental properties associated with known gas hydrate deposits. The logistic model takes account of our binary dependent variable and can be used to calculate the conditional probability of observing gas hydrate at unsampled locations.

\section{CONTROLS ON GAS HYDRATE FORMATION}

Natural gas hydrates form a class of chemical compounds known as clathrates (Kaplan, 1974). Gas hydrates consist of a rigid structure of a low-molecular weight gas (typically methane) surrounded by water molecules. The water molecules form rings joined by hydrogen bonds which host gas molecules, producing a stabilized crystalline structure.

\section{Stability Regime}

Gas hydrate formation depends on a specific pressure-temperature regime. Hydrate deposits form where pressures are relatively high and bottom-temperatures approach $0^{\circ} \mathrm{C}$. Adequate temperatures for hydrate deposition generally range from -8 to $12^{\circ} \mathrm{C}$ (Malone, 1994). The geothermal gradient of a region controls the maximum lower limit of temperatures facilitating hydrate formation. Fluctuations in bottom-water temperatures occur due to seasonal changes, propagation of warm water across slopes, and heat flow into sediments and the sea floor from the subsurface. Pressures conducive to hydrate formation range from approximately 3-60 MPa. The presence of higher molecular weight hydrocarbons serves to stabilize gas hydrates at higher temperatures and/or lower pressures (Brooks et al., 1986).

\section{Gas Hydrate Stability Zone (GHSZ)}

Variables used to predict the hydrate stability zone include bottom-water temperature, geothermal gradient, water depth, and gas availability and composition (Brooks et al., 1986). The sea floor forms the upper limit of the GHSZ in outer continental margin sediments (Kvenvolden and McMenamin., 1980). The intersection of the geothermal gradient and the projected hydrate phase boundary form the base of the GHSZ. Local controls influence the 
actual location of the base of the GHSZ for a given area. In areas where the geothermal gradient is relatively constant, the thickness of the GHSZ increases with increasing water depth (Kvenvolden and McMenamin, 1980). The zone of actual gas hydrate accumulation often differs from the theoretical gas hydrate stability zone. The presence of gas hydrate above a regional phase boundary suggests additional factors other than pressure, temperature, and gas composition influence gas hydrate accumulation (Booth et al., 1996).

\section{Water Depth and Sediment Depth}

Marine bathymetry and sediment thickness also influence gas hydrate deposition. Studies in the Gulf of Mexico report gas hydrate observations in water depths ranging from 300-2400 meters, typically encountered at less than 1000 meters (Malone, 1994). Brooks et al., (1999) report gas hydrate occurrences offshore Nigeria in water depths ranging from 560-770 meters. Bottom-water temperature, water salinity, gas availability, and gas composition control the minimum water depth associated with gas hydrate stability (Brooks et al., 1986). Equilibrium conditions for gas hydrate occur in shallow sediments less than 6 meters below the sea floor. Seasonal changes in temperature may only affect gas hydrate stability in the upper 1-2 meters of sediment (Milkov and Sassen, 2000). Deeper deposits of gas hydrate form approximately 100-1000 meters below the sea floor. However, deposits in the Orca Basin occur at depths as low as 20-40 meters below the sea floor. Shallow marine sediments of continental slopes and abyssal plains typically exhibit a pressure-temperature envelope conducive to gas hydrate formation (Malone, 1994).

\section{Depositional Environment}

Environments associated with natural gas hydrate deposition typically exhibit high sedimentation rates and a continuous supply of methane (Cox, 1983). High rates of sediment supply and accretion promote gas hydrate formation in primarily two ways. First, proper mixtures of sediments containing organic matter can be quickly buried to the pressuretemperature regime required for gas hydrate formation. Second, a high sediment flux supports methane recycling (Cox, 1983).

The methane supply of an environment controls the preservation of natural gas hydrates. Methane gas concentrations must exceed the amount necessary to saturate pore waters in order for gas hydrate to form (Tucholke et al., 1977). Studies identify two sources of methane including microbial and thermogenic origins. Microbial, or biogenic, hydrocarbon gases form through degradation of organic matter in the absence of oxygen and sulfates (Booth et al., 1996 Thermogenic methane forms through catalytic reactions at temperatures exceeding $50^{\circ} \mathrm{C}$ (Kvenvolden and McMenamin, 1980).

\section{Structural Controls}

Geologic features commonly associated with gas hydrate deposits include mass movements, salt deformation, and active normal and growth faults. Mass movements may occur due to gas hydrate decomposition (Malone, 1994). Decomposition occurs based on several factors including fluctuations in seawater temperature, changes in sea level, and variable rates of methane gas concentration. Hydrate decomposition poses safety hazards in drilling and pipeline construction.

Piston cores and marine seismic records establish a strong association between shallow salt, active faulting, and gas hydrate deposits (Sassen et al., 1999). Gas hydrates coincide with diapiric crests and deep faults on flanks of diapirs. Faults resulting from the upward movement of diapirs produce lateral extension and fracturing. These mechanisms provide conduits for gas migration from deeper horizons into the pressure-temperature envelope of gas hydrate formation. Tectonics associated with salt diapirs and natural gas hydrate layers may also produce structural traps for hydrocarbons (Brooks et al., 1986). Sassen et al. (1994) report gas 
hydrate observations along rims of salt-withdrawal basins and over salt ridges. Salt related fracture zones and faults provide pathways for heat flow and warm fluids from the subsurface to the sea floor, altering bottom-water temperatures, and modifying the hydrate stability zone (Booth et al., 1996). The relation between active geologic features and gas hydrate deposits suggests conditions other than regional temperatures and pressures dictate the vertical distribution of hydrate accumulation zones.

\section{Properties of Host Sediments and Gas Hydrate Deposits}

Gas hydrate formation affects diagenetic processes of sediments by producing unique migration pathways, products, and sedimentation rates that allow seeps and oil and gas traps to form. Gas hydrate deposition prevents normal sedimentation and obstructs diffusion and dissolution of ions in interstitial waters. Initial hydrate formation may occur along fractures, faults, and/or within cavities of coarse-grained sediment (Brooks et al. 1986). Previous discoveries show an irregular distribution of gas hydrates in ocean sediments (Brooks et al., 1986). However, limitations on reliable data on the in situ nature of gas hydrates exist due to poor core recovery techniques.

The textures of host sediments present a potential influence on the nature of hydrate occurrences. Booth et al. (1996) describe two general types of gas hydrate occurrences. The first type includes laminae, layers, plates, and mats suggesting crystallization parallel to bedding planes and faults. The second type includes nodules and massive deposits that form large particles capable of deforming bedding planes. Grain sizes observed with hydrate deposits range from coarse to very fine-grained sediments. Examples include medium to coarse-grained volcanic ash, sandy layers, carbonate rubble, silty mudstones, calcareous clay oozes, and high porosity silty sediments (Brooks et al., 1986). Booth et al. (1998) report the following associations between hydrate habit and host sediments: (1) layers or laminae of hydrate deposits are commonly observed in fine sediments, (2) granules and nodules are observed in fine sediments and coarse grain-size sediments, and (3) Hydrates formed within the matrix of sediments as cementation agents are strongly associated with coarser sediments. Biogenic hydrates primarily form crystal and small nodules, whereas thermogenic hydrates form nodules to thick layers to massive mounds (Brooks et al., 1986). Booth et al. (1998) report the porosity of gas hydrate host sediments ranges from $40-75 \%$ with an average of $55 \%$. Thicker accumulations of pure gas hydrate typically occur in higher porosity sediments. Sassen et al. (2001) report outcrops of gas hydrate mounds on the sea floor in the Gulf of Mexico. Research submersible observations establish a strong association between gas hydrate mounds and active vent sites. Gas hydrates rarely develop uniformly in nature or in a solid mass.

Observations on the Louisiana slope in the northern Gulf of Mexico exhibit gas hydrates in many forms including small to medium sized nodules $(0.5-50 \mathrm{~mm})$, interspersed layers $(1-10$ $\mathrm{mm}$ ), and as solid masses (>150 mm) (Brooks et al., 1986). Other observations of hydrate samples contain carbonate concretions approximately $1 \mathrm{~cm}$ in diameter and fine-grained sediments stained with biodegraded crude oil (Cox, 1983). Oil-stained cores typically infer a thermogenic methane origin. However, some biogenic hydrates also contain oil stains (Brooks et al., 1986). Gas hydrates sampled from offshore Nigeria include large nodules $(1-1.5 \mathrm{~cm})$ and thin interspersed layers (Brooks et al., 1999).

\section{ANALYSIS OF SPATIAL CONTINUITY USING INDICATOR VARIOGRAPHY}

\section{Methods}

The spatial distribution of gas hydrate deposits within the two study regions was characterized by preparing indicator variograms. The data sets were compiled from multiple sources and include (1) sites where gas hydrates were either sampled directly or observed and (2) sites drilled without contact with gas hydrates. The data considered for each study area 
included gas hydrate sampled locations reported by Sassen et al. (2001) and Milkov et al. (2000) for the Gulf of Mexico and Brooks et al. (1994) for the Gulf of Guinea. Sampling locations where hydrate was not observed were provided by TDI-Brooks International, Inc. as part of the 1997-2000 Gulf Program for surface geochemical exploration (SGE) and the 19962000 Nigeria Consortium Program. The Gulf SGE Program provided over 1000 core locations spanning the western and central sections of the Gulf of Mexico. The Nigerian Consortium Program provided close to 500 core locations.

The first study area includes a section of the continental margin of the Gulf of Mexico, located offshore southeast United States. The area is approximately 400 by $1000 \mathrm{~km}$. The second study area includes a section of the continental margin of the Gulf of Guinea, located offshore Nigeria, West Africa. The area is approximately 350 by $550 \mathrm{~km}$.

Sampled locations were assigned values based on an indicator function (Isaaks and Srivastava, 1989):

$$
\mathrm{I}(\mathrm{x})=\mathrm{I}\left(\mathrm{V}_{\mathrm{x}} \mid \mathrm{H}_{\mathrm{x}}\right)=\left\{\begin{array}{ll}
1 & \mathrm{~V}_{\mathrm{x}}=\mathrm{H}_{\mathrm{x}} \\
0 & \text { otherwise }
\end{array}\right. \text {. }
$$

An indicator value of one was assigned to locations where gas hydrates were present and a value of zero where gas hydrates were absent. GS+ software (Robertson, 2000) was used to generate both isotropic and anisotropic sample variograms based on the indicator values. Multiple isotropic variograms were examined to establish the appropriate distance parameters in each study area. The lag spacing and distance over which the variance was calculated was adjusted to produce a variogram with clear structure. The lag spacing $(\delta)$ and lag distance $(L)$ satisfied the following conditions:

$$
\begin{aligned}
& \delta>\text { minimum distance between points } \\
& L=\mathrm{K} \delta
\end{aligned}
$$

where $K \delta \approx 1 / 4$ maximum distance between points and $L$ is less the than maximum distance between points. The nugget, sill and range of the isotropic variogram model were adjusted to determine the best-fit model. Variograms with an active lag and a uniform interval ranging from $100-500 \mathrm{~km}$ and $2-20 \mathrm{~km}$, respectively, were used in the comparison process. The isotropic analysis provided a description of the spatial continuity as a function of distance. Model parameters including a regression coefficients $\left(R^{2}\right)$ and residual sums of squares $(R S S)$ calculated by GS+ provided a measure of the model fit to the variogram data.

A contour map of the variogram surface identified the orientation of the anisotropy axes used in modeling the directional trends in the data. The principal axis defined the direction of least variation across the variogram surface, or direction of maximum continuity. Multiple directional variograms based on the principle axis were generated using different offset angles. The structure, fit, and number of pairs for each point of the directional variograms were compared to obtain a suitable offset tolerance. The nugget, sill and correlation length of the anisotropic variogram model were adjusted to determine the best-fit model.

The isotropic variogram model was used to indicator krige unsampled locations on a uniform grid of the Gulf of Mexico study area. Indicator kriging provides a useful non-parametric estimation technique and assumes the distribution is constant across the study area. Indicator kriging calculates the probability of sampling gas hydrate as conditioned by the value, location, and separation distance between neighboring sampled sites and the site to be predicted. The equations used for indicator kriging include (Isaaks and Srivastava, 1989):

$$
\begin{aligned}
& \sum_{j=1}^{N} w_{j} C_{i j}+\mu=C_{i 0} \\
& \sum_{i=1}^{N} w_{i}=1
\end{aligned}
$$


where $w_{i}$ corresponds to the weight assigned to each known point and $C_{i j}$ defines the covariance function between neighboring pairs. The predicted value, or local average of an indicator, was calculated using (Isaaks and Srivastava, 1989):

$$
\hat{I}\left(V_{\left(x_{0}\right)}\right)=\sum_{i=1}^{N} w_{i} I\left(V_{\left(x_{i}\right)}\right)
$$

The predicted indicator value represents the probability of sampling gas hydrate based on a conditional expected value:

$$
\mathrm{E}\left\langle\hat{\mathrm{I}}\left(\mathrm{V}_{\left(\mathrm{x}_{0}\right)} \mid \mathrm{V}_{\left(\mathrm{x}_{\mathrm{i}}\right)}\right)=\mathrm{P}\left(\mathrm{V}_{(\mathrm{x})} \leq \mathrm{V} \mid \mathrm{V}_{\left(\mathrm{x}_{\mathrm{i}}\right)}\right)\right. \text {. }
$$

\section{Results and Discussion}

The isotropic variogram with an active lag of $180 \mathrm{~km}$ and a uniform interval of $5 \mathrm{~km}$ produced the clearest structure for the Gulf of Mexico study area (Figure 1). The isotropic variogram was fit with a spherical model with a correlation length of $65 \mathrm{~km}$, a sill value of 0.0396 , and a relative nugget of $58.1 \%$ (Table 1). The maximum direction of spatial continuity trends approximately $43^{\circ}$ with the minimum axis at approximately $133^{\circ}$. Directional variograms along the maximum and minimum anisotropic axes with an offset tolerance of $10^{\circ}$ were fit with a spherical model (Figure 2). Sampled sites are correlated at a distance of $35 \mathrm{~km}$ in the direction of maximum continuity and $25 \mathrm{~km}$ in the direction of minimum continuity. The sill value for the anisotropic spherical model was 0.0396 with a relative nugget effect of $63.1 \%$ (Table 2 ).

The isotropic variogram with an active lag of $180 \mathrm{~km}$ and a uniform interval of $5 \mathrm{~km}$ produced the clearest structure for the Gulf of Guinea study area (Figure 3). The isotropic variogram was fit with a spherical model with a range of $110 \mathrm{~km}$, a sill value of 0.035 , and a relative nugget effect of $2.9 \%$ (Table 1). No significant spatial anisotropy was evident in the Nigerian continental margin for the data set as a whole.

A contour map of the conditional probability provides a summary of the spatial continuity of gas hydrate deposits derived from the indicator kriging process. Figure 4 is a conditional probability map for the Gulf of Mexico and represents the likelihood of sampling gas hydrates within the area. To include geometric constraints associated with the shore line, soft data points were assigned a value of zero at locations with water depths too shallow to allow hydrate formation. Core locations with their corresponding indicator values are shown for reference. Isotropic variograms for both study areas show a strong variance structure. The increased variance observed above the sample variance and the slightly erratic nature of the points beyond the correlation length suggest a regional trend is present in both data sets. Gas hydrate forming processes appear similar in both environments when comparing spatial structure. The correlation length is shorter in the Gulf of Mexico than in the Gulf of Guinea, suggesting gas hydrate deposits are correlated over a shorter distance in the Gulf of Mexico. 


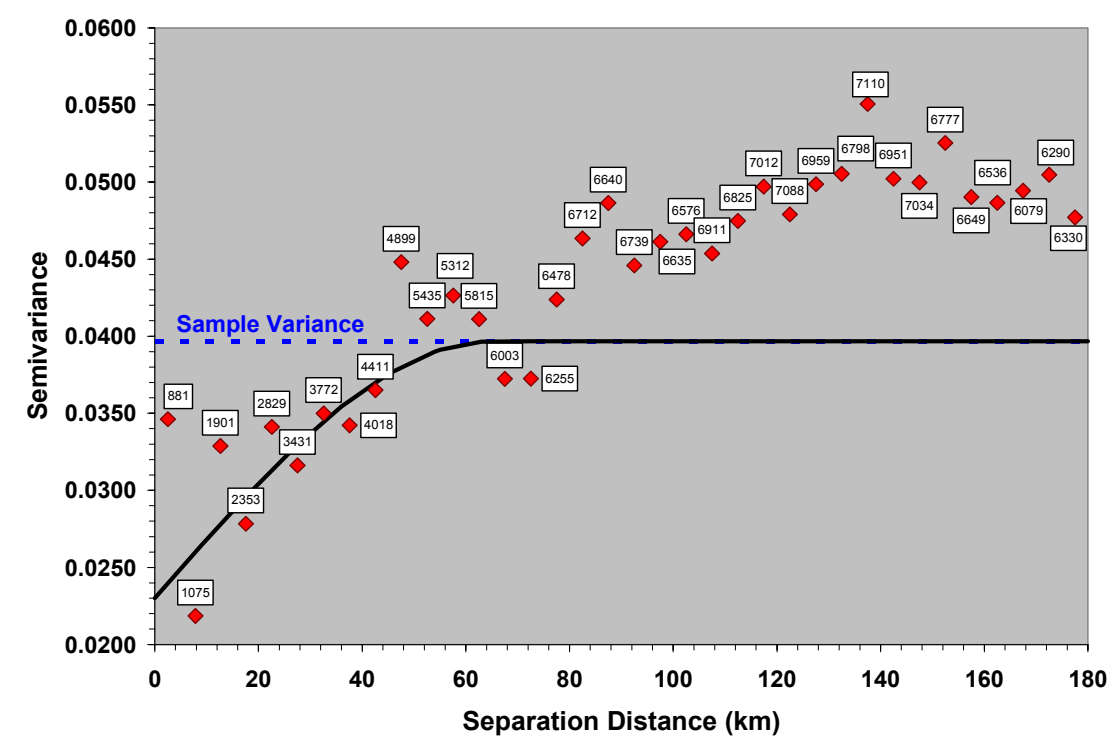

Figure 1

Isotropic experimental variogram and best-fit model for the Gulf of Mexico

Table 1

\begin{tabular}{|l|c|c|}
\hline \multicolumn{3}{|c|}{ Isotropic Model Results } \\
\hline Site & $\begin{array}{c}\text { Gulf of } \\
\text { Mexico }\end{array}$ & $\begin{array}{c}\text { Gulf of } \\
\text { Guinea }\end{array}$ \\
\hline Model & spherical & spherical \\
\hline $\begin{array}{l}\text { Lag } \\
\text { Distance } \\
\text { (km) }\end{array}$ & 180 & 180 \\
\hline $\begin{array}{l}\text { Lag Class } \\
\text { Interval } \\
\text { (km) }\end{array}$ & 5 & 5 \\
\hline $\begin{array}{l}\text { Correlation } \\
\text { Length } \\
\text { (km) }\end{array}$ & 65 & 110 \\
\hline Nugget & 0.023 & 0.001 \\
\hline Sill & 0.0396 & 0.034 \\
\hline$R^{2}$ & 0.637 & 0.747 \\
\hline$R S S$ & 0.001938 & 0.002200 \\
\hline
\end{tabular}

\begin{tabular}{|l|c|}
\multicolumn{2}{c}{ Table 2 } \\
\begin{tabular}{|l|}
\multicolumn{1}{|c|}{ Anisotropic } \\
Variogram Results
\end{tabular} \\
\hline Site & $\begin{array}{c}\text { Gulf of } \\
\text { Mexico }\end{array}$ \\
\hline Model & spherical \\
\hline $\begin{array}{l}\text { Max } \\
\text { Continuity } \\
\text { Direction }\end{array}$ & $43^{\circ}$ \\
\hline $\begin{array}{l}\text { Min } \\
\text { Continuity } \\
\text { Direction }\end{array}$ & $133^{\circ}$ \\
\hline $\begin{array}{l}\text { Lag } \\
\text { Distance } \\
\text { (km) }\end{array}$ & 180 \\
\hline $\begin{array}{l}\text { Lag Class } \\
\text { Interval } \\
\text { (km) }\end{array}$ & 5 \\
\hline $\begin{array}{l}\text { Major Axis } \\
\text { Correlation } \\
\text { Length } \\
(\mathrm{km})\end{array}$ & 35 \\
\hline $\begin{array}{l}\text { Minor Axis } \\
\text { Correlation } \\
\text { Length } \\
\text { (km) }\end{array}$ & 25 \\
\hline \begin{tabular}{l} 
Nugget \\
\hline Sill
\end{tabular} & 0.025 \\
\hline
\end{tabular}




\begin{tabular}{|l|c|}
$R^{2}$ & 0.314 \\
\hline$R S S$ & 0.0262 \\
\hline
\end{tabular}
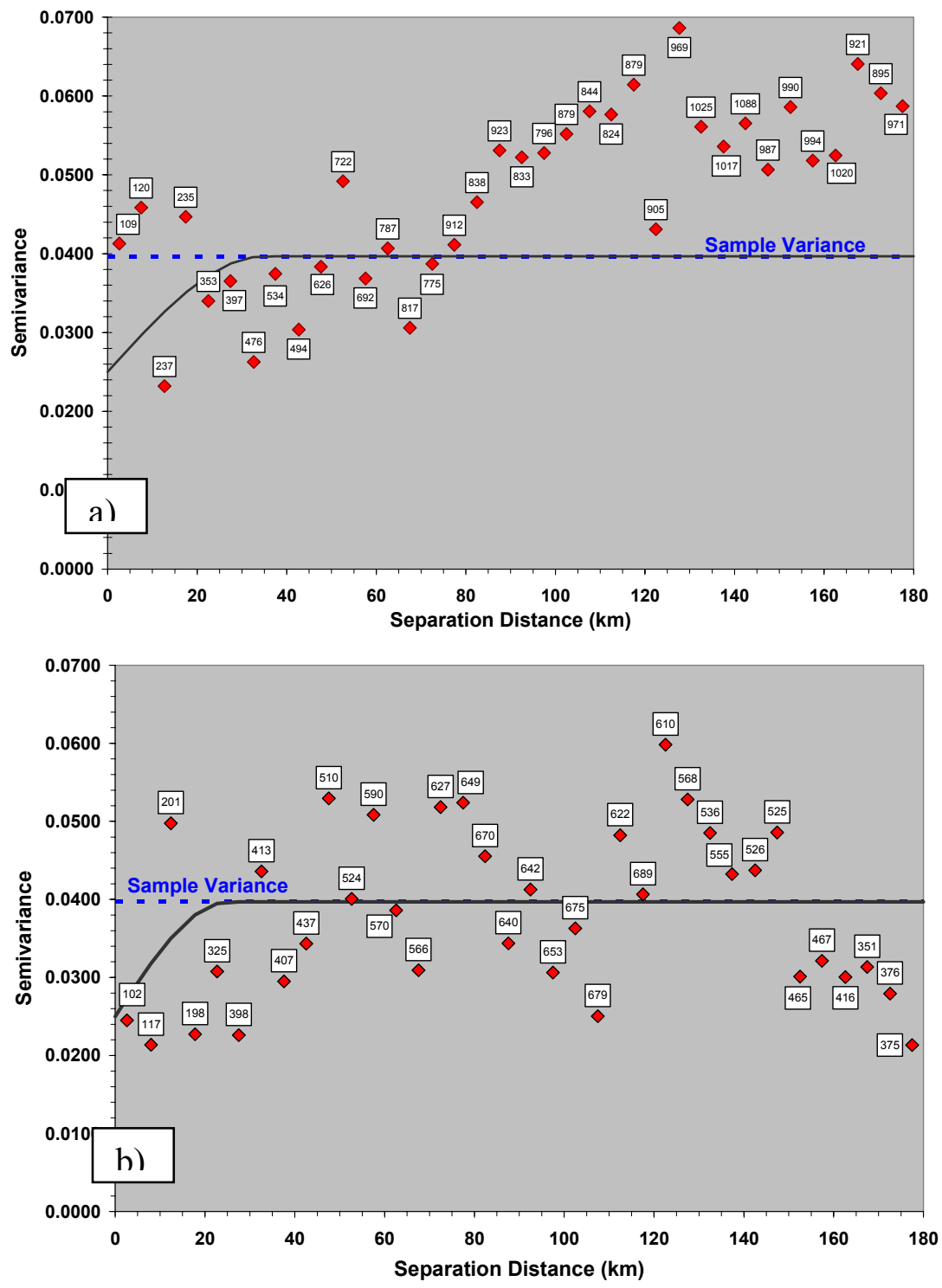

Figure 2

Anisotropic experimental variograms and best-fit models for a) maximum direction of continuity and b) minimum direction of continuity 


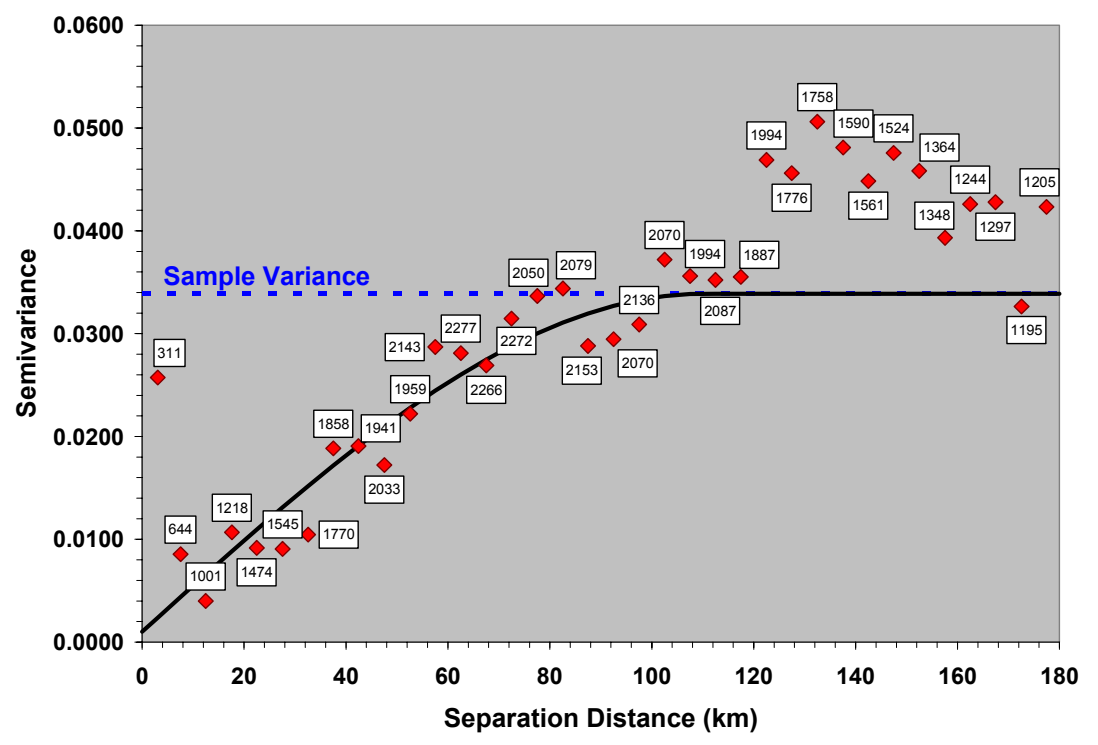

Figure 3

Isotropic experimental variogram and best-fit model for the Gulf of Guinea

The anisotropic variograms also show a degree of structure, but require a more rigorous analysis to determine the true patterns related to gas hydrate deposits, especially in the Gulf of Guinea study area. The maximum direction of spatial continuity identified in the Gulf of Mexico corresponds with the alignment of gas hydrate locations, suggesting an underlying structure control on gas hydrate formation. Gas hydrates have been reported to form localized deposits along faults and edges of salt diapers in the Gulf of Mexico.

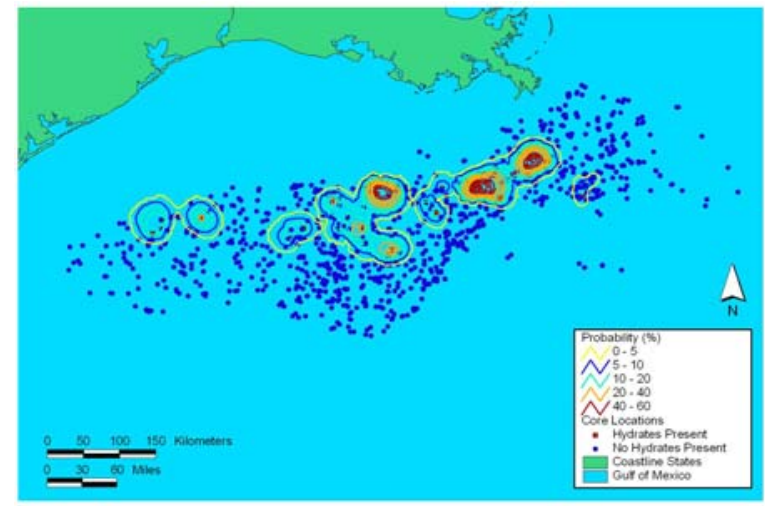

Figure 4

Isotropic conditional probability map of gas hydrate occurrences: Gulf of Mexico

\section{Conclusion}

Indicator variograms suggests a strong spatial continuity exists between gas hydrate locations as a function of both distance and direction from neighboring sampled locations. The nugget effect observed in the variograms is likely a result of bias sampling procedures. The nugget effect produces weights in the kriging process that are more equally distributed, inducing a source of error in the analysis. Additional analysis, and perhaps additional sampling, is required for investigations of anisotropy in the Gulf of Guinea case. Anisotropic variograms can be used to more accurately assign weights to sampled sites, increasing the weight for sites in 
the direction of maximum continuity. The stability of the spatial variance structure suggests a sufficient stationarity of hydrate forming processes that can be used for spatial prediction with stochastic models.

Basic knowledge of the nature of gas hydrate deposits contributes to an overall understanding of worldwide gas hydrate environments. Analysis of gas hydrate geology provides information for safe and efficient exploration and exploitation of economic resources and provides data for models of climatic change.

\section{LOGISTIC REGRESSION ANALYSIS OF GAS HYDRATE OCCURRENCES}

\section{Methods}

Logistic regression analysis was used to estimate the parameters of a regression model using a binary dependent variable. The dataset includes a subset of offshore gas hydrate samples compiled from published observations and measurements recorded from 1982-1994 (Booth and Rowe, 1996). The properties recorded for each observation include:

$>$ Geographic location

$>$ Water depth

$>$ Subbottom depth

$>$ Temperature at hydrate sample

The water depths, recorded directly from the referenced literature, are reported for each sample site. The subbottom depth refers to the vertical distance from the seafloor to the position of the gas hydrate sample or the top of the sampled gas hydrate zone. The temperature is reported at the depth of each gas hydrate sample, commonly based on downhole temperature data. Hydrates occurring at the seafloor report the bottom water temperature. The gas hydrate offshore database (Booth and Rowe, 1996) provided a total of 31 observations from 12 distinct regions.

Additional observations were compiled from log data obtained through the Ocean Drilling Program. These observations also include water depth, subbottom depth, and temperature measurements (downhole data) but denote locations where gas hydrates were not drilled. ODP records provided 100 observations selected at random. The analysis included a total of 131 observations.

Sampled locations were assigned values based on an indicator function (Isaaks and Srivastava, 1989):

$$
I(x)=I\left(V_{x} \mid H_{x}\right)=\left\{\begin{array}{ll}
1 & V_{x}=H_{x} \\
0 & \text { otherwise }
\end{array} .\right.
$$

An indicator value of one was assigned to locations where gas hydrates were present and a value of zero where gas hydrates were absent. The Excel program XLSTAT-Pro 6.1.9 (Addinsoft Software, 2003) was used to conduct the logistic regression analysis for multiple combinations of the three identified parameters.

Logistic regression is a non-linear transformation of the linear regression model. It is commonly used for (1) estimating the outcome of a categorical variable or (2) when the assumptions of classical linear regression do not apply to a dataset. The logistic regression model provides the conditional probability that an event (gas hydrate occurrence) will occur based on the following function:

$$
P\langle Y=1 \mid X\rangle=P=\frac{\exp (a+B X)}{1+\exp (a+B X)}
$$

where $a$ is the coefficient on the constant term, $B$ is the coefficient(s) on the independent variable(s), and $X$ is the independent variable(s) (Hosmer and Lemeshow, 1989). The logistic 
distribution is an S-shaped distribution function that constrains the estimated probabilities between 0 and 1 . The dependent variable of a logistic regression model is interpreted as the natural logarithm of the odds that the dependent binary variable is equal to 1 rather than 0 . The parameters of logistic regression are interpreted as the expected change in the "log of the odds" of the binary dependent variable. The antilogarithms of the coefficient values provide an expected change in the "odds" of the binary dependent variable associated with a one unit change in the independent variable (Hosmer and Lemeshow, 1989). The error associated with the logistic regression model follows a binomial distribution.

Model statistics used to determine the significance of each estimator and model include the chi-square statistic, log likelihood, Pearson's chi-square, log ratio chi-square, and a modified determination coefficient, $R^{2}$. The chi-square statistics are similar to the $F$ statistic used in ordinary regression analysis; the higher the statistic the more significant the estimator or model. Probabilities for the chi-square statistic provide the probability of being wrong when saying that the explanatory variables bring significant information to (1) explain the observed values or (2) compared to the independent model. The log likelihood value is the log of the likelihood function that is used to maximize the probability estimates. High log likelihood values indicate a more significant estimator or model. The McFadden Pseudo $R^{2}$ is the proportion of the variance in the dependent variable which is explained by the variance in the independent variables.

\section{Results}

Three models were tested in the logistic regression analysis to determine the most significant predictor variables for modeling the occurrence of gas hydrates. Table 3 describes each model and lists the resulting statistics used to choose the best-fit model.

Table 3

Logistic regression model statistics

\begin{tabular}{|c|c|c|c|c|c|c|c|c|}
\hline Model & Parameters & $\begin{array}{c}\text { P > } \\
\text { Chi- } \\
\text { square }\end{array}$ & $\begin{array}{c}\text { Log } \\
\text { Likelihood }\end{array}$ & $\begin{array}{l}\text { Pearson's } \\
\text { Chi-square }\end{array}$ & $\begin{array}{c}\text { P > } \\
\text { Chi- } \\
\text { square }\end{array}$ & $\begin{array}{c}\text { L.R. } \\
\text { Chi- } \\
\text { square }\end{array}$ & $\begin{array}{c}\text { P > } \\
\text { L.R. Chi- } \\
\text { square }\end{array}$ & $\begin{array}{c}\text { Pseudo } \\
\mathbf{R}^{2}\end{array}$ \\
\hline \multirow{3}{*}{$A$} & $\begin{array}{l}\text { Water Depth } \\
\text { (m) }\end{array}$ & 0.003 & \multirow{3}{*}{-60.718} & \multirow{3}{*}{278.427} & \multirow{3}{*}{$<0.0001$} & \multirow{3}{*}{21.925} & \multirow{3}{*}{$<0.0001$} & \multirow{3}{*}{0.152936} \\
\hline & $\begin{array}{l}\text { Subbottom } \\
\text { Depth }(\mathrm{m})\end{array}$ & 0.246 & & & & & & \\
\hline & Temp (C) & 0.091 & & & & & & \\
\hline \multirow[t]{2}{*}{$B$} & $\begin{array}{l}\text { Water Depth } \\
\text { (m) }\end{array}$ & 0.000 & \multirow{2}{*}{ 61.524355 } & \multirow[t]{2}{*}{270.709189} & \multirow[t]{2}{*}{$<0.0001$} & \multirow[t]{2}{*}{20.31174} & \multirow[t]{2}{*}{$<0.0001$} & \multirow[t]{2}{*}{0.141683} \\
\hline & Temp (C) & 0.029 & & & & & & \\
\hline \multirow{2}{*}{ C } & Temp (C) & 0.008 & \multirow{2}{*}{58.941668} & \multirow{2}{*}{286.917002} & \multirow{2}{*}{$<0.0001$} & \multirow{2}{*}{25.47712} & \multirow{2}{*}{$<0.0001$} & \multirow{2}{*}{0.177714} \\
\hline & $\begin{array}{l}\text { Log Pressure } \\
(\mathrm{MPa})\end{array}$ & $<0.0001$ & & & & & & \\
\hline
\end{tabular}

Model A describes a full model that includes all three parameters thought to influence gas hydrate deposition. The significance of each independent variable is evaluated by the $P>$ Chisquare value. The probability associated with subbottom depth is significantly higher compared to the water depth and temperature parameters. This suggests subbottom depth is not a statistically significant independent variables for predicting gas hydrate occurrences.

Model $B$ excludes the subbottom depth variable to examine the effect of the variable in the model. The test statistics and probabilities for Model B are similar to the results of Model A, 
showing only slight differences in the fit of the models. The subbottom depth variable used in Model A does not appear to increase the significance of the model and can be eliminated from the analysis.

Model $\mathrm{C}$ uses temperature and the log of pressure (derived from water depth). These parameters are commonly used to determine the phase boundary of gas hydrates. Model C tests whether these independent variables might also be useful in a regression analysis for determining gas hydrate presence. The significance of each independent variable suggests these parameters could accurately model hydrate occurrence, but the test statistics and probabilities show Model $\mathrm{C}$ to be less significant than Model A or Model B.

Figure 5 shows the standardized residuals for each model. The residuals are similar for each model, with an increase in error for observation numbers 27-31. Figure 6 considers the predictive ability of Model B. The conditional probabilities calculated from the model are compared to known observations with $100 \%$ corresponding to the presence of gas hydrate and $0 \%$ corresponding to the absence of gas hydrate. The overall accuracy of the model when applied to the dataset with which the model was developed was $89.3 \%$ (calculated as 117 correct predictions for the 131 observations within the dataset) (Table 4). The accuracy of the model was assessed by establishing an arbitrary $40 \%$ conditional probability threshold.

\section{Conclusions}

Model $\mathrm{B}$ is the recommended model to use for estimating the conditional probability of encountering gas hydrates given water depth and temperature. Model B produces the lowest Pvalues, a higher log likelihood value, the lowest Pearson's chi-square value, and comparable L.R. chi-square and Pseudo $R^{2}$ values. Model B produced the following probability equation for estimating the global occurrence of gas hydrate deposits:

$$
P=\frac{1}{1+\exp (-(1.029-7.425(\text { Water Depth })-0.123(\text { Temperature })))}
$$

with water depth measured in meters and temperature measured in ${ }^{\circ} \mathrm{C}$. An improved dataset including all recently discovered gas hydrates as well as more observations where gas hydrates have not been found would serve to further justify the use of a logistic regression model.

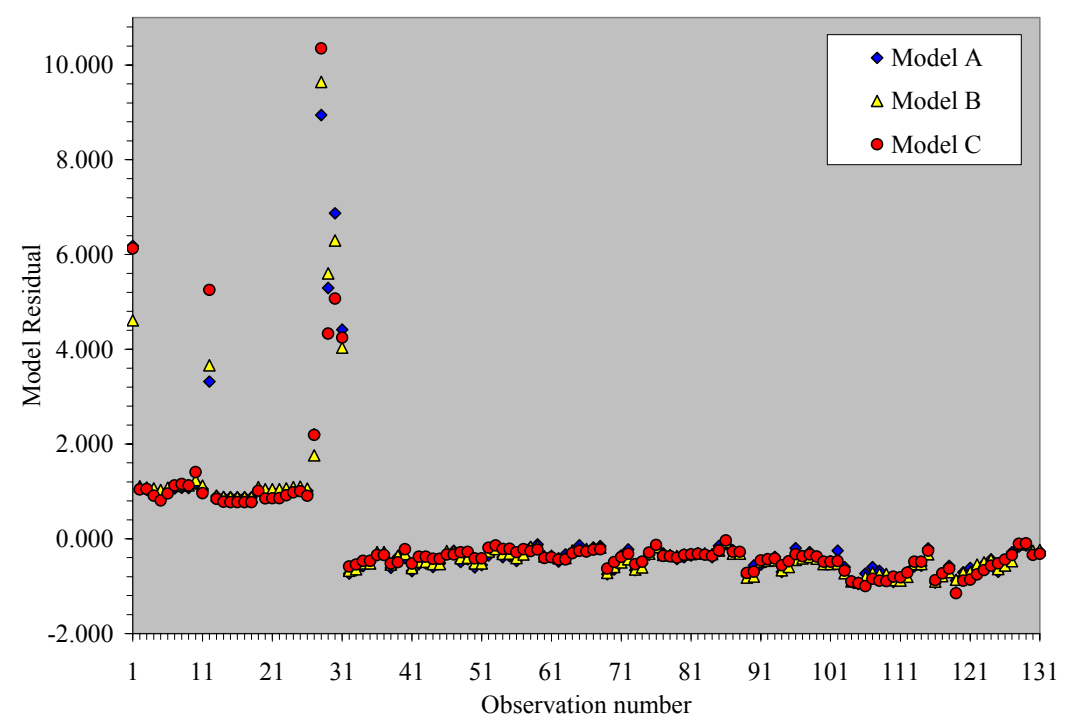

Figure 5: Residual plot for each logistic model considered 


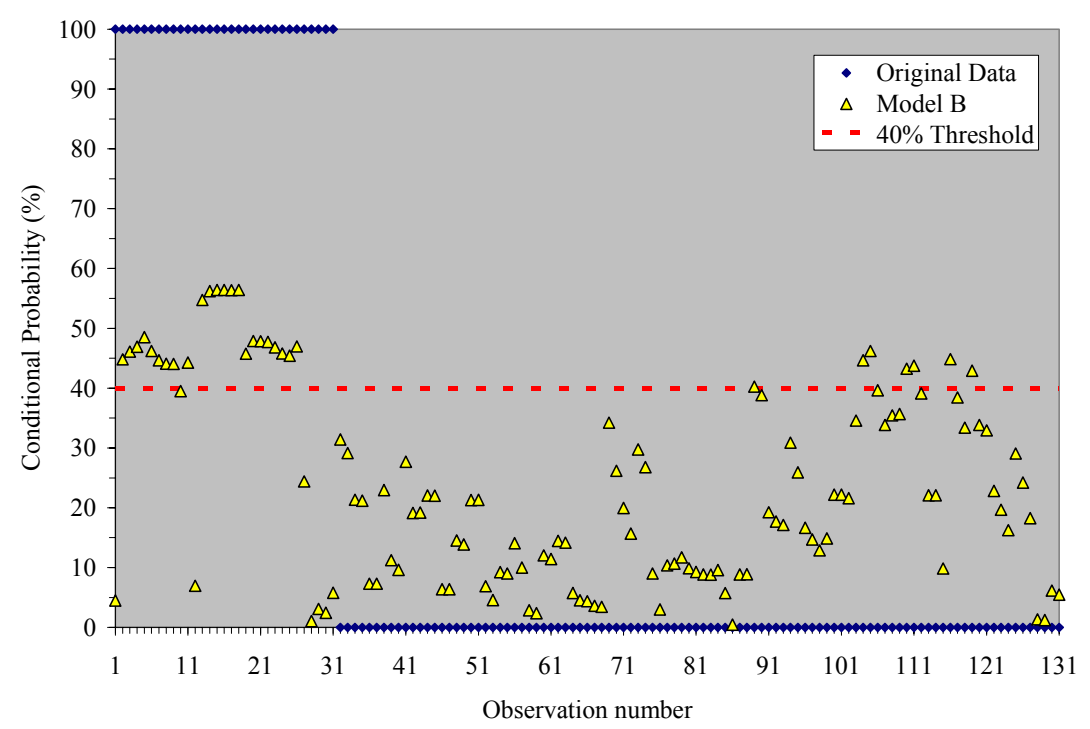

Figure 6: Predictive ability of the selected logistic regression model

Table 4: Classification table based on the logistic regression model

\begin{tabular}{|c|c|c|c|c|}
\hline & & \multicolumn{2}{|c|}{ Hydrate Occurrence } & $\begin{array}{l}\text { Accuracy of } \\
\text { Model }\end{array}$ \\
\hline \multirow{3}{*}{$\begin{array}{l}\overline{\frac{c}{0}} \\
\frac{0}{0} \\
\frac{0}{0} \\
\frac{0}{2} \\
\frac{0}{2} \\
\frac{d}{2}\end{array}$} & & YES & NO & \multirow{3}{*}{$89.3 \%$} \\
\hline & YES & 23 & 6 & \\
\hline & NO & 8 & 94 & \\
\hline
\end{tabular}

\section{REFERENCES}

Booth, J.S., and Rowe, M.M., 1996. Offshore gas hydrate sample database with an overview and preliminary analysis. USGS Open-File Report 96-272: Massachusetts, 17 p.

Booth, J. S., Winters, W.J., Dillon, W.P., Clennell, M.B., and Rowe, M.M., 1998, Major Occurrences and Reservoir Concepts of Marine Clathrate Hydrates: Implications of Field Evidence, in J. P. Henriet, and Mienhert, J., ed., Gas Hydrates: Relevance to World Margin Stability and Climate Change, London, Geological Society, p. 113-127.

Brooks, J. M., B.H. Cox, W.R. Bryant, M.C. Kennicutt III, R.G. Mann, T.J. McDonald, 1986, Association of Gas Hydrates and Oil Seepage in the Gulf of Mexico: Organic Geochemistry, v. 10, p. 221-234.

Brooks, J. M., Bryant, W.R., Bernard, B.B., and Cameron, N.R., 1999, The Nature of Gas Hydrates on the Nigerian Continental Slope: Annals of the New York Academy of Sciences, v. 912, p. 76-93. 
Brooks, J.M., Anderson, A.L., Sassen, R., MacDonald, I.R., Kennicutt, M.C. II, and Guinasso, N.L., Jr., 1994, Hydrate occurrences in shallow subsurface cores from continental slope sediments, in Sloan, E.D., Jr., Happel, John, Hnatow, and Miguel A., ed., International Conference on Natural Gas Hydrates: Annals of the New York Academy of Sciences, v. 715, p. 381-391

Cox, J. L., 1983, Natural Gas Hydrates: Properties, Occurrence, and Recovery: Woburn, Butterworth Publishers, $125 \mathrm{p}$.

Hosmer, David, and Lemeshow, Stanley, 1989. Applied Logistic Regression: New York, Wiley, 307p.

Isaaks, E. H., and Srivastava, M.R., 1989, An Introduction to Applied Geostatistics: New York City, Oxford University Press, $561 \mathrm{p}$.

Kaplan, I. R., 1974, Natural Gases in Marine Sediments, v. 3: New York, Plenum Press, 317 p.

Kvenvolden, K. A., Mark A. McMenamin, 1980, Hydrates of Natural Gas: A Review of Their Geologic Occurrence, Reston, U.S. Geological Survey.

Kvenvolden, K. A., Ginsburg, G.D., and Soloviev, V.A., 1993, Worldwide Distribution of Subaquatic Gas Hydrates: Geo-Marine Letters, v. 12, p. 32-40.

Malone, R., 1994, Overview: Gas Hydrate Geology and Geography: Annals of the New York Academy of Sciences, v. 715, p. 225-231.

Milkov, A. V., and Sassen, R., 2000, Thickness of the Gas Hydrate Stability Zone, Gulf of Mexico Continental Slope: Marine and Petroleum Geology, v. 17, p. 981-991.

Robertson, G. P., 2000, GS+: Geostatistics for the Environmental Sciences, Plainwell, Michigan, Gamma Design Software.

Sassen, R., I.R. MacDonald, 1994, Evidence of Structure H Hydrate, Gulf of Mexico Continental Slope: Organic Geochemistry, v. 22, p. 1029-1032.

Sassen, R., S.T. Sweet, A.V. Milkov, D.A. DeFreitas, G.G. Salata, E.C. McDade, 1999, Geology and Geochemistry of Gas Hydrates, Central Gulf of Mexico Continental Slope:

Transactions - Gulf Coast Association of Geological Societies, v. 49, p. 485-497.

Sassen, R., Sweet, S., Milkov, A. V., DeFreitas, D. A., Kennicutt II, M.C., and Roberts, H., 2001, Stability of Thermogenic Gas Hydrate in the Gulf of Mexico: Constraints on Models of Climate Change, in C. K. Paull, and Dillon, William P., ed., Natural Gas Hydrates: Occurrence, Distribution, and Detection: Geophysical Monograph Series, Washington, DC, American Geophysical Union, p. 131-143.

Tucholke, B. E., Bryan, George M., and Ewing, John, 1977, Gas Hydrate Horizons Detected in Seismic-Profiler Data from the Western North Atlantic: AAPG Bulletin, v. 61, p. 698700. 